\title{
imill metabolites
}

\section{Metabolism and Metabolomics of Liver in Health and Disease}

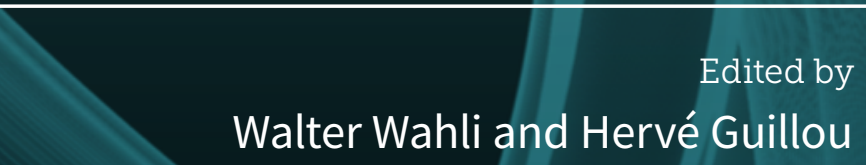

Printed Edition of the Special Issue Published in Metabolites 
Metabolism and Metabolomics of Liver in Health and Disease 



\section{Metabolism and Metabolomics of Liver in Health and Disease}

Editors

Walter Wahli

Hervé Guillou 


\section{Editors}

Walter Wahli

University of Lausanne

Switzerland
Hervé Guillou

INRA ToxAlim

France

\section{Editorial Office}

MDPI

St. Alban-Anlage 66

4052 Basel, Switzerland

This is a reprint of articles from the Special Issue published online in the open access journal Coatings (ISSN 2079-6412) (available at: https://www.mdpi.com/journal/metabolites/special_issues/liver).

For citation purposes, cite each article independently as indicated on the article page online and as indicated below:

LastName, A.A.; LastName, B.B.; LastName, C.C. Article Title. Journal Name Year, Article Number, Page Range.

ISBN 978-3-03943-635-4 (Hbk)

ISBN 978-3-03943-636-1 (PDF)

(C) 2021 by the authors. Articles in this book are Open Access and distributed under the Creative Commons Attribution (CC BY) license, which allows users to download, copy and build upon published articles, as long as the author and publisher are properly credited, which ensures maximum dissemination and a wider impact of our publications.

The book as a whole is distributed by MDPI under the terms and conditions of the Creative Commons license CC BY-NC-ND. 


\section{Contents}

About the Editors $\ldots \ldots \ldots \ldots \ldots \ldots \ldots \ldots \ldots \ldots \ldots \ldots \ldots$

Preface to "Metabolism and Metabolomics of Liver in Health and Disease" . . . . . . . . ix

Lorraine Smith, Joran Villaret-Cazadamont, Sandrine P. Claus, Cécile Canlet, Hervé Guillou, Nicolas J. Cabaton and Sandrine Ellero-Simatos

Important Considerations for Sample Collection in Metabolomics Studies with a Special Focus on Applications to Liver Functions

Reprinted from: Metabolites 2020, 10, 104, doi:10.3390/metabo10030104 . . . . . . . . . . 1

Aurélien Amiel, Marie Tremblay-Franco, Roselyne Gautier, Simon Ducheix,

Alexandra Montagner, Arnaud Polizzi, Laurent Debrauwer, Hervé Guillou,

Justine Bertrand-Michel and Cécile Canlet

Proton NMR Enables the Absolute Quantification of Aqueous Metabolites and Lipid Classes in

Unique Mouse Liver Samples

Reprinted from: Metabolites 2020, 10, 9, doi:10.3390/metabo10010009 _ . . . . . . . . . . 19

Matthias Cuykx, Charlie Beirnaert, Robim Marcelino Rodrigues, Kris Laukens,

Tamara Vanhaecke and Adrian Covaci

Exposure of HepaRG Cells to Sodium Saccharin Underpins the Importance of Including

Non-Hepatotoxic Compounds When Investigating Toxicological Modes of Action

Using Metabolomics

Reprinted from: Metabolites 2019, 9, 265, doi:10.3390/metabo9110265 . . . . . . . . . . 39

Vivaldy Prinville, Leanne Ohlund and Lekha Sleno

Targeted Analysis of 46 Bile Acids to Study the Effect of Acetaminophen in Rat by LC-MS/MS

Reprinted from: Metabolites 2020, 10, 26, doi:10.3390/metabo10010026 . . . . . . . . . . . . 49

Ana Margarida Araújo, Maria Enea, Félix Carvalho, Maria de Lourdes Bastos, Márcia Carvalho and Paula Guedes de Pinho

Hepatic Metabolic Derangements Triggered by Hyperthermia: An In Vitro Metabolomic Study

Reprinted from: Metabolites 2019, 9, 228, doi:10.3390/metabo9100228 . . . . . . . . . . 61

Fabienne Rajas, Amandine Gautier-Stein and Gilles Mithieux

Glucose-6 Phosphate, a Central Hub for Liver Carbohydrate Metabolism

Reprinted from: Metabolites 2019, 9, 282, doi:10.3390/metabo9120282 . . . . . . . . . . . . 75

Sandra Steensels, Jixuan Qiao and Baran A. Ersoy

Transcriptional Regulation in Non-Alcoholic Fatty Liver Disease

Reprinted from: Metabolites 2020, 10, 283, doi:10.3390/metabo10070283 . . . . . . . . . . 8

George N. Ioannou, G. A. Nagana Gowda, Danijel Djukovic and Daniel Raftery

Distinguishing NASH Histological Severity Using a Multiplatform Metabolomics Approach

Reprinted from: Metabolites 2020, 10, 168, doi:10.3390/metabo10040168 . . . . . . . . . . . 123

Maria Guarino and Jean-François Dufour

Nicotinamide and NAFLD: Is There Nothing New Under the Sun?

Reprinted from: Metabolites 2019, 9, 180, doi:10.3390/metabo9090180 . . . . . . . . . . . . 139 
Manuel García-Jaramillo, Kelli A. Lytle, Melinda H. Spooner and Donald B. Jump

A Lipidomic Analysis of Docosahexaenoic Acid $(22: 6, \omega 3)$ Mediated Attenuation of Western Diet Induced Nonalcoholic Steatohepatitis in Male $\mathrm{Ldlr} \%$ Mice

Reprinted from: Metabolites 2019, 9, 252, doi:10.3390/metabo9110252 . . . . . . . . . . 157

Xiangjin Meng, Xin Guo, Jing Zhang, Junji Moriya, Junji Kobayashi, Reimon Yamaguchi and Sohsuke Yamada

Acupuncture on ST36, CV4 and KI1 Suppresses the Progression of Methionine- and Choline-Deficient Diet-Induced Nonalcoholic Fatty Liver Disease in Mice

Reprinted from: Metabolites 2019, 9, 299, doi:10.3390/metabo9120299 . . . . . . . . . . . . . 18

Diren Beyoğlu and Jeffrey R. Idle

Metabolomic and Lipidomic Biomarkers for Premalignant Liver Disease Diagnosis and Therapy

Reprinted from: Metabolites 2020, 10, 50, doi:10.3390/metabo10020050 . . . . . . . . . . . . . 197 


\section{About the Editors}

Walter Wahli is a Professor emeritus at the Center for Integrative Genomics, University of Lausanne, Switzerland and Visiting Professor of Metabolic Disease at the Lee Kong Chian School of Medicine, Nanyang Technological University, Singapore. Until recently, he was also the President of the Council of the Nestle Foundation for the Study of Problems of Nutrition in the World. He is internationally recognized as a leader in the field of molecular endocrinology and metabolism and discovered the nuclear hormone receptors peroxisome proliferator-activated receptors $\beta$ and $\gamma$ $(\operatorname{PPAR} \beta$ and $\operatorname{PPAR} \gamma)$. He has received several awards for the elucidation of key functions of these receptors, which are activated by fatty acids and fatty acid derivatives. Synthetic PPAR agonists are drugs used mainly for lowering triglycerides and blood sugar. Prior to his present appointments, Walter Wahli was a visiting associate at the National Cancer Institute, National Institutes of Health, Bethesda, USA. He then became a Professor and Director of the Institute of Animal Biology at the University of Lausanne. He was the Vice-Rector for Research and Continuing Education of the university and founded the Center of Integrative Genomics, which he directed for several years. He was also a member of the Swiss National Science Foundation Research Council and presided over the Biology and Medicine Division.

Hervé Guillou was trained in molecular biology and lipid biochemistry during his Ph.D. at Agrocampus Rennes. He was then a post-doctoral fellow in the Len Stephens and Phill Hawkins lab at the Babraham Institute working in the field of signaling by phosphatidylinositols in neutrophils. He was then recruited as a junior investigator and became a group leader in INRA Toulouse where he started to investigate the transcriptional control of liver physiology by nuclear receptors. 



\section{Preface to "Metabolism and Metabolomics of Liver in Health and Disease"}

The liver is a key organ, which has a multitude of functions. In fact, it is thought to be in charge of more than 500 processes ranging from protein synthesis, carbohydrate and lipid metabolism, detoxification of various natural and synthetic compounds, to the production of molecules promoting whole-body homeostasis, to name but a few. Most of these functions are carried out by hepatocytes. In line with its multitasking physiology, the liver consumes plenty of oxygen, up to about $20 \%$ of resting total body consumption, half of which is provided by the hepatic portal vein, while the other half is met by the hepatic arteries. The liver plays an essential role in the breaking down of nutrients, such as carbohydrates, lipids, and proteins derived from feeding on plants and animals to convert them to substances that are essential to the body. Furthermore, it is key in catabolizing or modifying toxic substances such as nicotine, alcohol, toxins, and drugs and environmental xenobiotics in a process called detoxication. The liver also plays an important role in the immune system. Finally, the regeneration capacity of the liver is astonishing. More than half of the liver can be removed and it will rapidly go back to normal size.

Such a complex organ can be affected by many types of dysregulations causing diseases. Liver diseases represent a major global health problem in developed and developing countries. Most deaths are due to two conditions: hepatitis B infection and non-alcoholic fatty liver disease (NAFLD). Following an acute infection, hepatitis B becomes chronic in $5-20 \%$ of adults and causes severe health problems. NAFLD, which is quite common all over the globe is characterized by too much fat in the liver, which is often caused by lifestyle mainly overnutrition and insufficient physical activity. In adults, NAFLD is often associated with diabetes and obesity. Importantly, due to the progression of childhood obesity and children presenting increased vulnerability to genetic and environmental factors, NAFLD currently affects up to $20 \%$ of the pediatric population. The pathogenesis of NAFLD is very complex and plenty of hepatic mechanisms are implicated, such as alterations in glucose and lipid metabolism as well as insulin signaling. Furthermore, dysfunctional cross-talks between the liver and other organs, such as the adipose tissues and the gut and its microbiota also participate in NAFLD development. There is currently a strong interest in a better understanding of NAFLD, not least to assist in the development of treatments as there is no approved pharmacological therapy for NAFLD. In addition to hepatitis B infection and NAFLD, there is a huge range of rare liver diseases often difficult to accurately diagnose, which include glycogenosis, porphyria, congenital hepatic fibrosis, polycystic liver diseases, genetic cholestatic diseases, and several others. In brief, the complexity of liver diseases represents an immense challenge for research and clinical work.

The above description of liver diseases makes it easy to understand the need and importance of exploring the hepatic metabolome to investigate hepatic physiology both in health and the mentioned illnesses. Metabolomics is the most recently developed omics technology after genomics, transcriptomics, and proteomics. It has huge potential to identify specific and sensitive biomarker candidates in isolated liver cells, whole-liver tissues, and biofluids for future evaluation. It is a global approach that can identify and measure the levels of a very large number of metabolites, thereby, providing a precise metabolic readout of healthy physiological or disease states. The most used approaches in the characterization of metabolomes comprise targeted analysis and profiling of metabolites and metabolic fingerprinting. Mass spectrometry (MS)-based and nuclear magnetic resonance (NMR)-based approaches have become routine. To fully benefit from the contributions of 
metabolomics, it has to be associated with sophisticated bioinformatics analyses allowing metabolic snapshots in the course of physiological changes, disease progression, and treatment effects. Certainly, much work remains to be done to clarify the multifaceted functions of the liver in health and disease than has been described in this book and elsewhere.

This book would not have been possible without the efforts of so many authors who have generously shared their findings and knowledge in liver metabolomics for the benefit of all. We express our deepest gratitude for supporting our Special Issue with their excellent contributions. Our gratitude also goes to the reviewers for their constructive and valuable suggestions to improve the papers submitted for publication. We also deeply appreciate the collaboration from the Metabolites editorial team, with a very special mention going to Ms. Yi Zhang for assisting in liaising with authors and keeping us updated about the progression of this Special Issue on "Metabolism and Metabolomics of Liver in Health and Disease".

Walter Wahli , Hervé Guillou 


Review

\title{
Important Considerations for Sample Collection in Metabolomics Studies with a Special Focus on Applications to Liver Functions
}

\author{
Lorraine Smith ${ }^{1}$, Joran Villaret-Cazadamont ${ }^{1}$, Sandrine P. Claus ${ }^{2}$, Cécile Canlet ${ }^{1}$, \\ Hervé Guillou ${ }^{1}$, Nicolas J. Cabaton ${ }^{1}$ and Sandrine Ellero-Simatos ${ }^{1, *}$ \\ 1 Toxalim (Research Center in Food Toxicology), Université de Toulouse, INRAE, ENVT, INP-Purpan, UPS, \\ 31300 Toulouse, France; lorraine.smith@inrae.fr (L.S.); joran.villaret-cazadamont@inrae.fr (J.V.-C.); \\ cecile.canlet@inrae.fr (C.C.); herve.guillou@inrae.fr (H.G.); nicolas.cabaton@inrae.fr (N.J.C.) \\ 2 LNC Therapeutics, 17 place de la Bourse, 33076 Bordeaux, France; sandrine.claus@lnc.bio \\ * Correspondence: sandrine.ellero-simatos@inrae.fr
}

Received: 11 February 2020; Accepted: 7 March 2020; Published: 12 March 2020

\begin{abstract}
Metabolomics has found numerous applications in the study of liver metabolism in health and disease. Metabolomics studies can be conducted in a variety of biological matrices ranging from easily accessible biofluids such as urine, blood or feces, to organs, tissues or even cells. Sample collection and storage are critical steps for which standard operating procedures must be followed. Inappropriate sample collection or storage can indeed result in high variability, interferences with instrumentation or degradation of metabolites. In this review, we will first highlight important general factors that should be considered when planning sample collection in the study design of metabolomic studies, such as nutritional status and circadian rhythm. Then, we will discuss in more detail the specific procedures that have been described for optimal pre-analytical handling of the most commonly used matrices (urine, blood, feces, tissues and cells).
\end{abstract}

Keywords: metabolomics; standard operating procedures; urine; blood; feces; tissue; cells; liver function

\section{Introduction}

Metabolomics refers to the high-throughput quantification and characterization of small molecules (metabolites) in tissues or biofluids. Such biochemical profiles contain latent information relating to inherent parameters, such as the genotype, and environmental factors, including the diet, exposure to xenobiotics and gut microbiota. The liver is the heaviest organ in the human body, with a wide array of functions that can be divided into intermediary metabolism (including a central role in carbohydrate, lipid and nitrogen metabolism), immunological activity, secretion of bile, synthesis of various serum proteins, degradation of hormones, and detoxification of xenobiotics. Hepatic lipid catabolism plays a crucial role during fasting and/or prolonged exercise. Upon lowering of blood glucose, the liver increases glucose production by augmenting gluconeogenesis and glycogenolysis to maintain blood glucose levels; increases fatty acid oxidation and ketogenesis to provide extra-hepatic tissues with ketone bodies; and decreases lipogenesis to attenuate triglyceride storage. In the fed state, the opposite occurs and the liver increases glucose uptake to feed glycogenesis; limits lipid oxidation to favor lipogenesis and promotes saving of fatty acids in the form of triglycerides that are packaged in lipoproteins for remote storage in the white adipose tissue. Hence, the liver plays an essential role in the regulation of energy metabolism. Dysregulation of these metabolic pathways leads to metabolic diseases among which non-alcoholic fatty liver disease (NAFLD), which is diagnosed when more than $5 \%$ of hepatocytes are steatotic in patients who do not consume excessive alcohol. The disease severity 
ranges from simple steatosis to steatohepatitis, advanced fibrosis and cirrhosis. NAFLD epidemic represents a major public health burden [1] and remains an unmet medical need [2].

Metabolomics has found numerous applications in the study of liver functions in health and disease. Among others, these include: Non-invasive biomarker investigations to discriminate between the different stages of progression of NAFLD using non-invasive biofluids (urine and plasma) [3,4]; investigation of mechanisms underlying hepatic disease progression such as acute-on-chronic liver failure using serum metabolic profiling [5] or fibrosis [6]; characterization of the gut microbiota metabotypes in urine of NAFLD patients [4,7]; nutrimetabolomics studies to unravel hepatic pathways dysregulated directly in liver samples upon various nutritional challenges [8,9]; discovery of new metabolic functions for nuclear receptors, that are important regulators of liver physiology using direct hepatic metabolomics or other informative fluids such as urine and bile [10-13]; identification of patients at risk for idiosyncratic drug-induced liver injury (IDILI) before drug administration, a concept named "pharmaco-metabolomics", that was first demonstrated in urine of animal models [14] and is now extended to human biofluids (urine and serum) [15,16]; study of mechanisms of action for pharmaceutical drugs in urine and fecal samples [17] and environmental contaminants in HepG2 cells and animal biofluids and tissues $[18,19]$.

There are many sources of variation in metabolomic studies, some of which are directly related to the pre-analytical handling steps. Pre-analytical questions are indeed a crucial part of metabolomics study designs since inadequate sample collection, pre-treatment or storage can significantly affect sample quality and result interpretation. The reliability of the metabolomics approach requires inactivation of ongoing metabolism, metabolite stabilization and maintenance of sample integrity. In consideration of this, it is useful to have standard operating procedures (SOP) for pre-analytical handling of samples before starting a metabolomic study. In this review, we discuss the influence of sample collection pre-analytical handling procedures and storage conditions on the metabolomic profiles of the biological matrices that are most commonly used to investigate liver functions, namely urine, blood, feces, tissues and cells. Of note, metabolic profiling of several other matrices such as bile and ascitic fluids can provide interesting information about liver functions $[10,20,21]$ but will not be further discussed in this review given the paucity of data regarding sample collection and stability.

\section{Overview of the Pre-Analytical Handling Procedures of the Most Commonly Used Biological Matrices in Metabolomics}

\subsection{Time of Collection}

\subsubsection{Considering Nutritional Status}

The choice of time of collection is a crucial step for a successful metabolomics study and will depend on the research question under examination. Nutritional status of the experimental subjects greatly influences the circulating, urinary, fecal and tissue metabolomes and has to be carefully chosen. If one aims to identify a biomarker specifically associated with a food item, then acute postprandial urine will certainly be collected. Criteria for good biomarkers of habitual nutritional intake are metabolites that are metabolically inert and rapidly absorbed within $1.0-1.5 \mathrm{~h}$ of consumption in the upper gastrointestinal tract. Such markers are subsequently excreted 1.5-2.5 h later [22]. Plasma is more reflective of modulations in endogenous metabolism as a result of the food metabolome and it should be noted that perturbations of the plasma metabolic profile arise when homeostatic function is impaired. Therefore, fasting plasma samples are usually used to explore how systemic metabolism differs between populations with different dietary habits [23]. Of note, in rodents, 16-h fasting has been shown to affect $1 / 3$ to $1 / 2$ of monitored serum metabolites, with an increase in fatty and bile acids and a significant decrease in diet- and gut microbiota-derived metabolites [24]. Nutritional status also has a significant effect on the tissue metabolome. Especially in the liver, $77 \%$ of the hepatic metabolome has been shown to be sensitive to a nutritional high-fat-diet challenge at all times of day. Amino acids, xenobiotics and nucleotides were especially affected and decreased in HFD-fed mice at all time 
points [25]. Finally, the fecal (or cecal) metabolome is increasingly considered as a functional readout of the gut microbiome and can be used as an intermediate phenotype mediating host-microbiome interactions $[4,26]$. Although the microbial metabolome still represents an analytical challenge and many microbial metabolites still remains unknown, it is known that the fecal metabolome is highly sensitive to nutritional challenges $[27,28]$ and influences the host hepatic metabolism $[4,29,30]$.

\subsubsection{Considering Circadian Rhythm}

Circadian rhythms govern a large variety of behavioral, physiological and metabolic processes [31]. Recent advances reveal that a very large fraction of mammalian metabolism undergoes circadian oscillations. Many metabolic pathways are under circadian control and, in turn, may feedback to the clock system to assist in circadian timekeeping [32]. Transcriptomics studies have extensively illustrated a substantial fraction of the genome controlled by the molecular clock [33]. Metabolomics studies have also highlighted the circadian oscillations of metabolites in humans independently of sleep or feeding [34]. In mice, more than $40 \%$ of the serum metabolome and $45 \%$ of the liver metabolome have been shown to be sensitive to time, with both matrices providing different and complementary information. For example, more than $30 \%$ of the serum lipids were not found in the liver and more than half of them oscillated across the circadian cycle, while only $30 \%$ of the hepatic lipids oscillated [25]. Moreover, a high-fat challenge induced a loss of serum metabolite rythmicity, compared with the liver [25]. Therefore, when collecting samples for a metabolomics study, one should be aware that a tissue-specific and time-dependent disruption of metabolic homeostasis exists independently of feeding, but also in response to altered nutrition. Time of collection therefore needs to be carefully chosen, and if sample collection is spread between several days, time of collection should be homogenous between the collection days [35].

\subsection{Common Sources of Variation in Pre-Analytical Handling of Main Biological Matrices}

Specific SOPs have been described for collection, preparation and storage of metabolomics samples and will be described along the specificity of each biological matrix in the following sections. Several features of the pre-analytical steps are however shared between the different matrices. First, the numbers, weights or volumes of the samples are important points to anticipate before collection. Second, during collection, samples have to be kept at the lowest temperature possible, and immediate snap freezing is recommended in order to quench any rapid degradation activity such as oxidation of labile metabolites as well as enzymatic reactions.

Third, aliquoting the samples should also be considered whenever possible. This important step will avoid repeated freeze-thaw cycles that lead to progressive loss in sample quality. Finally, long term storage at $-80^{\circ} \mathrm{C}$ or less is recommended before analysis. These general recommendations, as well as matrix-specific pre-analytical factors that influence the results of metabolomics studies are summarized in Figure 1.

\subsection{Urine}

Urine is a biofluid commonly used for both human and animal metabolomics studies because sample collection is non-invasive. The simplicity of the collection allows multiple collections for kinetic studies and ensures reliability of the analysis. Urinary profiles contain signals derived from both endogenous and environmental sources, including diet and gut microbiota metabolic activity, and can therefore provide an overall measure of the metabolic phenotype. It is a collection of waste and biological by-products that reflects a large panel of metabolic processes that may have occurred over time and provides the researcher with a historical overview of the global metabolic events. In addition, it may contain cells (erythrocytes, leucocytes, urothelial cells, and epithelial cells), bacteria, fungi and non-cellular components including urates and mucus filaments [36]. Thus, it is a non-inert fluid and residual cellular or enzymatic activities could significantly change the metabolic composition of the 
samples. It is, therefore, necessary to remove cells and bacteria and/or to quench the ongoing enzymatic or metabolic activities in urine samples.

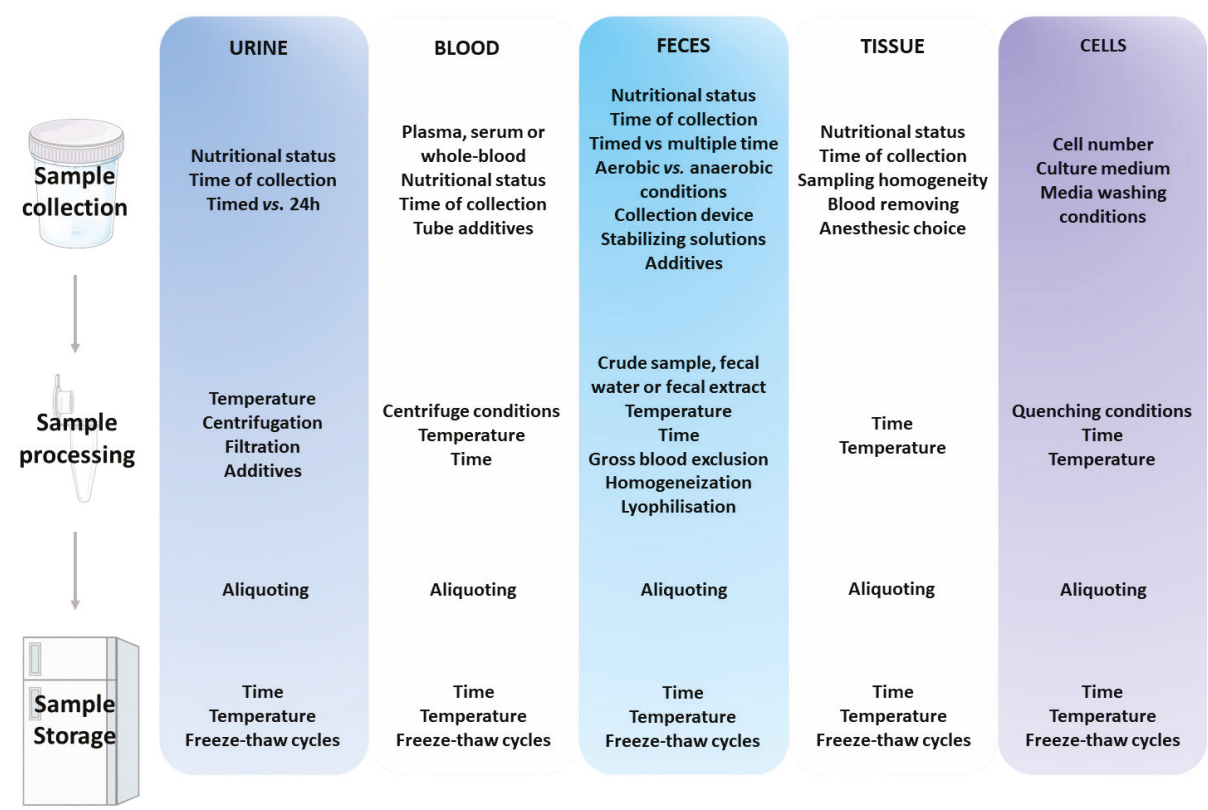

Figure 1. Summary of pre-analytical factors that can affect metabolite profiles in various matrices.

\subsubsection{Timed vs. 24 -Hour Collection}

The first main consideration in urine collection is to choose the appropriate sampling time: $24-\mathrm{h}$ collection or timed collection. It has been shown that there is a large variability depending of the collecting time (day vs. night, morning vs. afternoon) caused by the circadian rhythm regulating the energy metabolism and the gut microbiota metabolism and also due to a difference in physical activity and feeding state [37]. Therefore, 24-h sampling will be preferred if one aims at eliminating the day-time variability in metabolic profiles. Another advantage of $24-\mathrm{h}$ sampling might be that it minimizes variation in urine concentrations compared to timed samples. Indeed, unlike blood where metabolite concentration is tightly maintained, urine concentration can vary drastically from sample to sample, thereby influencing the urine metabolome. In a recent review, Stevens et al. propose that pre-analytical normalization of urine (for e.g., to osmolarity) may improve the reliability of metabolomics analyses [38]. However, 24-h sampling is not always feasible, especially in humans. In rodents, specific individual metabolic cages or use of hydrophobic sand are required, in which mice are isolated and therefore mildly stressed [39,40]. 24-h sampling might also not be appropriate. For example, a timed sampling is needed to study a time-related trend and a kinetic sampling can be done to monitor the evolution of a targeted compound or the overall effect on the metabolism after drug or nutrient intake. In timed sampling, the time of collection is a very important point to ensure the reproducibility and quality of the study.

\subsubsection{Sample Collection}

The most commonly used preservation methods are filtration, centrifugation or addition of bacteriostatics. Saude et al. showed that spinning urine samples at $112 \mathrm{~g}$ for $10 \mathrm{~min}$ was less effective in conserving the metabolome composition than filtration through a $0.22 \mu \mathrm{m}$ filter [41]. Bernini et al. have shown that a mild pre-centrifugation (between 1000 and $3000 \mathrm{~g}$ ) combined with filtration is the 
safest way to avoid contamination of the metabolic profiles attributed to bacterial removal without leading to an additional contamination due to cell damages or breaking (higher centrifugation speed induced partial breaking of cells and lower centrifugation was not effective to eliminate bacteria) [36]. Boric acid and sodium azide $\left(\mathrm{NaN}_{3}\right)$ are the two most commonly used antimicrobial preservatives. It has been shown that the addition of $200 \mathrm{mM}$ of boric acid or $10 \mathrm{mM}$ of $\mathrm{NaN}_{3}$ for 24-h samples or 2-20 mM of boric acid or $0.1-1 \mathrm{mM}$ of $\mathrm{NaN}_{3}$ for a timed sample are equally efficient to prevent bacterial overgrowth [42]. Nevertheless, boric acid is rarely used, as it induces formation of chemical complexes with endogenous metabolites [43]. Bernini et al. compared the use of $\mathrm{NaN}_{3}$ to a $0.2 \mu \mathrm{m}$ filtration, and showed the latter to be superior for sample stability over time due to bacterial removal [36].

To summarize, filtration showed superior ability to preserve the urinary metabolites during storage in comparison with unfiltered samples. Moreover, the metabolic profiles of centrifuged samples are more stable than non-centrifuged samples after one week storage at $-80{ }^{\circ} \mathrm{C}$, with this effect being less severe in samples that are rapidly frozen in liquid nitrogen to avoid cell breaking. A mild pre-centrifugation plus a filtration seems to be the best method to avoid sample degradation.

\subsubsection{Sample Storage}

For short-term storage, Gika et al. have shown that the storage of urine samples at $4{ }^{\circ} \mathrm{C}$ for up to $48 \mathrm{~h}$ maintained the metabolic integrity of the samples [44]. However, it is important to minimize sample storage at $4{ }^{\circ} \mathrm{C}$ as it has been shown that samples stored for more than 9 months will present an altered metabolome when compared to samples stored at $-20^{\circ} \mathrm{C}$ [45]. For long-term storage, metabolic profiles of urine samples stored at either -20 or $-80^{\circ} \mathrm{C}$ for 6 months did not show any significant differences [44]. This study, however, did not confirm whether or not the stored samples were identical to the original samples.

Freeze-thaw cycles have been shown to significantly modify the urine sample composition. Urine samples stored at $-80^{\circ} \mathrm{C}$ and thawed twice a week for 4 weeks ( 8 freeze-thaw cycles) indeed displayed a reduced metabolic stability in comparison to non-thawed ones stored at the same temperature. Metabolites deriving from bacterial metabolism (acetate, benzoate, succinate) increased [41]. Trivedi et al. showed that urinary metabolic profiles could be maintained only up to 3 freeze-thaw cycles using HILIC (Hydrophilic interaction liquid chromatography) mass-spectrometry [45].

\subsection{Blood}

Collecting blood is slightly more invasive than collecting urine, and the metabolic profiles of blood fractions provide a different, but complementary, metabolic information compared to the ones obtained with urine. Blood metabolic profiles are dynamic and vary continuously in response to changes in gene expression or changes induced by exogenous metabolites such as those provided by nutrients or drugs. Blood metabolic profiling is therefore widely used to study the dynamic variations of the endogenous metabolism in response to drug or food intake. Disruption in plasma metabolic profiles arises when homeostatic function is impaired. Serum and plasma are the most commonly used matrices, but other matrices do exist, such as platelet-free plasma (PFP), platelet-rich plasma (PRP) and whole blood, this latter receiving a growing interest.

Blood consists of two main components: plasma, which is a clear extracellular fluid containing clotting factors, proteins, glucose, minerals, and gases; and cellular elements, which are made up of blood cells (white blood cells, red blood cells) and platelets. Serum is the liquid fraction of whole blood, obtained by allowing the sample to clot naturally followed by a centrifugation step. The resulting supernatant is serum free of cells and of clotting factors such as the fibrinogen proteins. Plasma is prepared by collecting the whole blood into anticoagulant-treated tubes followed by a centrifugation step at $4{ }^{\circ} \mathrm{C}$ to separate blood cells. The supernatant designated as plasma is then immediately transferred into a clean tube. Plasma is a mixture of platelets, proteins, nutrients, hormones and gases. In some studies, further identification was given by naming it platelet-poor plasma (PPP) in opposition to platelet-free plasma or platelet-rich plasma by adding one or more additional centrifugation steps. 
Depending on the aim of the experiment, for example, if one wants to take into consideration the influence of growth factors or cytokines released by the platelets, the platelet content of the sample has to be carefully accounted for. Various manual, semi-automatic, and fully automated commercial systems have become available to prepare PFP, PPP and PRP [46].

\subsubsection{Sample Collection}

Several studies have addressed a direct comparison of plasma vs. serum and have been recently reviewed [38]. The conclusions of this review highlight that both matrices are appropriate for blood metabolomics with minor differences between them.

The metabolomics analysis of serum is known to present a higher sensitivity of metabolites compared to plasma due to the lack of big particles. However, its processing time has the disadvantage of introducing variations due to enzymatic conversion and degradation processes, and to influence the metabolite composition [47]. Moreover, the reproducibility of serum is not as good as that of the whole blood because hemolysis can occur during collection or processing, leading to the presence of free hemoglobulin in the samples that influences the metabolic profiles [48].

In comparison, there is a better reproducibility in plasma due to the absence of the blood-clotting step. Moreover, it has been suggested that the absence of platelets and the lower protein content could be beneficial to small molecule analysis, because of a reduced competition [49]. For plasma preparation, the choice of anti-coagulant addition is critical and needs to be carefully accounted for before sample collection. Several anticoagulant collection tubes are available. The three most common additives are: heparin, ethylene diamine tetra acetic acid (EDTA) and citrate. They have often been compared with opposing conclusions depending on the analytical platform used. Actually, additives found in collection tubes can affect the ionization process during the MS run, thereby suppressing metabolite ionization and/or introducing interfering peaks. Bari et al. have compared heparin, EDTA and citrate anticoagulants using an untargeted UPLC-MS analysis. They noticed subtle metabolite differences between the different plasma preparations mainly due to ion suppression or enhancement caused by citrate and EDTA. Heparin did not cause interferences and was therefore recommended by the authors [50]. On the contrary, Yin et al. analyzed heparin, citrate, and EDTA collection tubes using a non-targeted LC-MS approach and they noticed that heparin led to chemical noise in the mass spectra. Citrate and heparin showed few additional signals. They recommended avoiding heparin, preferring EDTA [48]. As for NMR analysis, heparin is usually recommended, as EDTA, citrate and other stabilizers give additional signals in the NMR spectra [51]. The choice of collection tube for plasma preparation is therefore critical, should be consistent throughout the experiment and should be adapted according to the analytical platform used for subsequent analysis.

\subsubsection{Sample Preparation}

After collection, samples should be quickly stored on ice. The time between collection and cell separation should be long enough to allow complete clot formation but short enough to avoid compositional changes. In general, it is recommended that the time before separation of blood cells should not exceed $30 \mathrm{~min}$ to minimize further metabolism or active and passive transport of analytes between the intra- and extracellular compartments. As for urine, whenever possible, samples should be stored as aliquots, allowing the use of fresh samples for each experiment and avoiding the introduction of bias due to repeated freeze-thaw cycles.

\subsubsection{Sample Storage}

It is well established that serum and plasma contain high levels of enzymes, that are efficiently active at $37^{\circ} \mathrm{C}$. A reduced temperature decreases enzymatic activity, but it should be noted that this activity is not completely inhibited until temperatures below $-56^{\circ} \mathrm{C}$ are reached [52]. Lipids and lipoproteins are especially sensitive due to lipase activity [35]. Small changes have been observed in 
the plasma metabolic profiles after one-month storage at $-20^{\circ} \mathrm{C}[35,53]$, while storage at $-80{ }^{\circ} \mathrm{C}$ for 4 years had minimal effects $[54,55]$.

Data regarding the number of freeze-thaw cycles acceptable are variable [42,44,46]. Unfractionated serum samples can be stored frozen for later quantitative lipid analysis as minor effects occur on quantitative lipid composition for most of the biologically relevant lipid species in humans, even with one to three freeze-thaw cycles. At the opposite freezing prior to lipoprotein fractionation significantly introduce a large variability in high-density lipoprotein and low-density lipoprotein cholesterol as well as in very low-density lipoprotein free fatty acids compared with fresh samples: density-based fractionation should preferably be undertaken in fresh serum [39].

\subsection{Feces}

Feces represents a growing interest in metabolomics studies, as fecal metabolic profiles reflect the metabolic interplay between the host and its gut microbiota [56]. The fecal metabolome has been shown to largely reflect gut microbiota composition in humans (explaining on average $67.7 \%$ of its variance), and is considered to be a functional readout of the microbiome [26]. Despite the rising popularity of fecal metabolomics, the methods for collecting, preparing and analyzing fecal samples are still far from being standardized. In a recent review, Karu et al. provided the state of knowledge with regards to the protocols and technologies in human fecal metabolite analysis [57]. They also present a comprehensive database that contains over 6000 identified human fecal metabolites, thereby highlighting the potential richness of the information contained in the metabolomics analysis of fecal samples. While the first metabolomics study of human feces used headspace GC-MS to study volatile organic compounds (VOCs) [58,59], it is now recognized that the majority of fecal metabolites are non-volatile [57].

The largest part of stool is made up of water $(60-80 \%$, depending on fiber intake), while the dry matter contains bacteria (both alive and dead, representing $25-54 \%$ of biomass) derived from the gastro-intestinal microbiota, colonic epithelial cells, macromolecules, undigested food residues, and thousands of metabolites including sugars, organic acids and amino acids, that constitute the fecal metabolome [60]. The latter includes both compounds derived from the metabolic activity of the gut microbiota and various host endogenous metabolites such as signaling peptides or bile acids [61].

\subsubsection{Sample Selection}

Timed vs. multiple-timed sampling: Much information contained within the fecal metabolome derives from dietary inputs and biochemical events that have occurred during their digestion. Thus, there is inherent variability in fecal samples depending upon feeding state and bowel activity. Both the gut microbiota composition and metabolic activity have been shown to be highly circadian $[62,63]$. Therefore, as for urine, it can be expected that timed collection vs. 24-h collection will provide different information. In animal studies, both timed [64] and 24-h [65] fecal sampling are commonly used for specific biochemical assays such as sterol and bile acid profiling; however, to our knowledge, no direct and systemic comparison of timed vs. 24-h fecal metabolome has been performed yet. In humans, it might not be feasible or relevant to collect 24-h samples. However, it was shown that the ${ }^{1} \mathrm{H}-\mathrm{NMR}$-based fecal metabolic profiles from single time samples greatly varied within one individual (day to day variation), and multiple day sampling and pooling has been proposed to minimize errors arising from day to day variation [66].

Presence of blood in stools: Gut bleeding is a clinically prevalent phenomenon associated with many gastro-intestinal diseases. The impact of blood in stool on the fecal metabolome has been shown to be minimal if the level of contamination is low (occult blood). However, gross (visible) blood in the fecal sample significantly contaminates the fecal metabolome [67]. Therefore, Karu et al. recommend visually inspecting samples and considering excluding the fecal samples or portions of fecal samples with gross blood [57]. 


\subsubsection{Sample Collection}

Feces collection presents the advantage of being non-invasive. In animals, fecal samples can be directly obtained from the intestine after euthanasia or collected from alive subject and pooled if necessary. Twenty-four hour feces can easily be obtained using metabolic cages; however, a mild but significant increase in fecal output was observed when housing rats in metabolic cages [39]. In humans, fecal samples can be directly collected in a falcon tube, in a plastic container, in a sterile bag [68,69], or in special anaerobic pouch systems [70]. Stabilizing solutions such as nucleotide stabilizers present in some stool collection kits should be avoided because they interfere with subsequent metabolomic analysis. Similarly, if stool samples are to be collected prior to colonoscopy, Bezabeh et al. recommend collection before patients start taking the solutions used for colonoscopy [71]. Indeed, most of these solutions contain polyethylene glycol, which produces strong interfering signals in the ${ }^{1} \mathrm{H}-\mathrm{NMR}$ spectrum.

Sample type and amount have to be decided beforehand and standardized. Samples for metabolomics study can be intact (crude) feces (usually for analysis of VOC), fecal water (the water fraction of an intact feces, obtained by ultracentrifugation of the stool), or a fecal aqueous extract (obtained after the addition of an aqueous buffer or of water to the stool, followed by homogenization and centrifugation). Fecal water generates a different metabolic coverage from feces and GC-MS analysis from crude feces samples yielded detection of more peaks than analysis of fecal water samples [67]. Stool samples are highly heterogeneous, and the topological position from the fecal sample has been shown to influence the fecal metabolome [72]. Therefore, it is sometimes recommended to collect as much sample as possible and to homogenize it before preparing aliquots, enabling a non-selective and more reproducible method [57,71]. The metabolic stability of aqueous extracts was shown to be higher than that of crude feces samples and it was recommended that fresh samples should be refrigerated and aqueous extraction conducted ideally within $1 \mathrm{~h}$ (and not longer than $24 \mathrm{~h}$ ) after collection before aliquoting and freezing [57,72].

Exposure to aerobic conditions and room temperature might quickly change the fecal metabolome due to microbial fermentation. Some researchers therefore place their fecal samples in an anaerobic chamber within $10 \mathrm{~min}$ of collection [73]. Couch et al. investigated the differences between home-based self-collection (ex vivo) samples and lab-based endoscopic collection (in vivo) samples in healthy subjects [74]. Using GC-MS, they found modest differences in the overall chemical distribution with a slight bias toward oxidized metabolites in the ex vivo samples. Further investigation revealed significant differences in the VOC metabolomes between the two groups. The effect of post-collection storage is much more drastic in fecal samples than in any other biological matrix, and most researchers store their fecal samples at $4{ }^{\circ} \mathrm{C}$ or lower immediately after collection. Fecal metabolites have indeed been shown to be highly unstable upon several storage conditions. For example, using GC-MS analysis, Phua et al. showed that, over 268 analytes, only $28 \%$ remained stable when crude feces were stored for one day at $4{ }^{\circ} \mathrm{C}$, and this declined to $10 \%$ at room temperature $\left(29^{\circ} \mathrm{C}\right)$ [67]. Immediate cooling of fecal samples is not always feasible, especially when human feces are collected at home or in clinics. Using LC-MS, Loftfield et al. compared several methods allowing preserving sample quality and demonstrated that crude fecal samples collected in $95 \%$ ethanol were stable for up to $96 \mathrm{~h}$ at room temperature [75]. Interestingly, these ethanol-preserved samples exhibited a metabolic profile more akin to fresh samples compared to immediately frozen-feces. This protocol represents an interesting alternative when immediate freezing of samples is not possible.

Water content in feces is variable (60-85\% in human) and this can sometimes create a bias to compare experimental groups. Immediately after collection, or before metabolites extraction, it is possible to lyophilize or freeze dry the samples to remove the water present in the feces. This improves sample weight precision, reduces bias due to the volume of solvent and/or derivation reagents added to samples during the metabolite extraction steps and allows quantitative metabolite data to be given in units per dry matter weight. This latter point is especially important for meta-analysis of metabolomics results and to establish reference levels for clinical use. While working with dried samples is less laborious, more reproducible and prevents bacterial growth, it also results in a loss of 
detected metabolites, especially VOCs. Indeed, the effect of lyophilization has been compared with the use of fresh sample and results in a decrease in short chain fatty acids [76,77]. Therefore, Karu et al. suggest that, unless volatile compounds are specifically targeted for quantification, fecal samples should be dried and weighed prior to storage or analysis. However, some researchers recommend not using it to minimize the number of preparation steps and guarantee quantifiable levels of short-chain fatty acids among others.

\subsubsection{Sample Storage}

While the analysis of fresh fecal samples is therefore recommended, the use of frozen samples could be more convenient. Two NMR studies investigated the effects of freezing on crude feces and/or aqueous fecal extracts and showed higher levels of several amino acids (namely branched-chain and aromatic amino acids) and glucose upon a single freeze-thaw cycle [72,76]. This observation has been confirmed using GC-MS analysis. Phua et al. indeed observed that only 33\% of the initial metabolites remained after one cycle, and $18 \%$ after two and three cycles [67]. In the same study, the metabolites also exhibited a poor stability at $-80{ }^{\circ} \mathrm{C}$, with only $24 \%$ of stable metabolites after 1 and 6 weeks of storage. The identity of the impacted metabolites was not given.

To summarize, although the immediate processing of fresh fecal samples is recommended, the use of frozen samples is often much more convenient. According to the last recommendations [57] and our present review, it seems that good practices would require fecal samples to be homogenized and aliquoted prior to freezing, while minimizing handling time at the lowest temperature possible. Very importantly, freeze-thaw cycle and storage duration should be minimized.

\subsection{Tissue}

Most metabolomics studies are based on non-invasive or minimally invasive sample types, such as blood, urine and feces. However, tissue analyses are also important, as the tissue represents the first place where the metabolic changes owing to a disease take place. Metabolomic studies have been conducted on almost every tissue (liver, intestine, muscle, adipose tissues from various locations, whole brain or selected brain areas, etc.). Most tissues are not homogenous. For example the liver has five different lobes, and within them the portal and periportal vascular regions are known to display different levels of some enzymatic systems such as those involved in glycolysis and gluconeogenesis [78-80]. Even when the tissue is composed of the same cell types, regional differences in composition may still exist. Such factors may result in increased biological variability, which should be taken into account during sampling [81].

\subsubsection{Sample Collection}

There are several critical points to be aware of during the handling step of tissues to avoid bias due to the collection procedure. The first important consideration is to ensure sample collection homogeneity throughout the experiment by always collecting samples from the same region to avoid bias due to biological variability. Whenever possible, it is also advisable that the same person collects the tissue samples throughout the experiment to minimize variability. Furthermore, to avoid contamination with blood metabolites, it is possible to wash the samples with cold deionized water or PBS after collection [82]. In some cases, saline in $\mathrm{D}_{2} \mathrm{O}$ or PBS is injected into the organ-associated artery before the collection to remove residual blood in organs [83]. One should also be aware that contaminant signals can result from the anesthetic used during the experiment, or even from surgical instrument cleaning solutions, especially ethanol that produces additional signals in the ${ }^{1} \mathrm{H}-\mathrm{NMR}$ spectra, thereby masking signals from endogenous metabolites.

\subsubsection{Sample Storage}

To obtain useful global metabolic profiles, sampling must be performed as rapidly as possible and samples should be either processed immediately or snap-frozen in liquid nitrogen to minimize further 
metabolism [84]. A good practice is to cut each sample into small pieces and to freeze the organs as rapidly as possible. For large prospective biobanking studies, this might not always be feasible and tissue samples might undergo several cycles of "storage-near-retrieval" due to storage constraints of adjacent samples for example. Testing different scenarios of storage-retrieval cycles in human liver tissues demonstrated that storage temperature affected metabolite concentrations only little, while there was a linear dependence on the number of temperature change cycles [85]. Metabolic changes induced by thawing were shown to be almost identical for all organs, with a marked increase in overall metabolite levels caused by increased protein and cell degradation [86].

\subsection{Cells}

Metabolomics analysis of cultured cells has emerged as an important technology for studying cellular biochemistry that provides an instantaneous snapshot of ongoing cellular metabolism. The major bottlenecks associated with metabolomics cell samples preparation workflow are efficient sampling, quenching and metabolites extraction in order to preserve the internal metabolite signatures [87].

\subsubsection{Sample Preparation}

Metabolomics analyses have been performed on a broad range of adherent cell numbers, ranging from $1 \times 10^{4}$ up to $4 \times 10^{7}$ cells [88]. Depending of the cells and the technology used to process the cell extractions, the seeding number must be optimized in order to get enough signal in NMR and/or MS, to be able to detect small metabolites that are present at low concentration but that still may be important for biological purposes, especially when global metabolomics is performed. Regarding the hepatic HepG2 cells for instance, tests have been performed to optimize the number of cells needed to obtain NMR metabolic fingerprints allowing to detect and identify the metabolite content in the cells. The number of $10^{6}$ cells was selected, as this seeding allowed the detection of the subtle modulations occurring after exposing the cells to xenobiotics such as estradiol or bisphenol A [18]. For cell extracts, it is usually recommended to work in very low volumes and concentrate the extract in a maximum of $50 \mathrm{uL}$. Microvolumes are easily handled using MS-based metabolomics or can be transferred to NMR microtubes or capillary tubes for analysis.

Culture media composition is also important to consider as some media contain interfering anions such as $\mathrm{Cl}^{-}, \mathrm{SO}_{4}{ }^{-}$, and $\mathrm{PO}_{4}{ }^{-}$, and depending on cultivated cells, amino acids, Good's buffers, organic acids, and complex biological mixtures such as fetal bovine serum. These components can cause substantial electrospray ionization suppression, additional signals in ${ }^{1} \mathrm{H}-\mathrm{NMR}$ spectra or contaminate the intracellular metabolite pool [88]. With regard to NMR-based metabolomics, culture media that contain HEPES should be avoided or carefully removed, since HEPES gives many broad additional signals. For these reasons, cells have to be separated from the medium before analysis. The washing step is therefore very important and should be performed with caution to effectively remove all the extracellular media components. Kapoore et al. (2017) performed five different washing protocols on the breast cancer cell line MDA-MB-231, using either PBS or distilled water. Based on a few metabolites detected by GC-MS, these authors suggested that in their conditions, a single washing step with PBS followed by quenching using $60 \%$ methanol supplemented with $70 \mathrm{mM}$ HEPES $\left(-50{ }^{\circ} \mathrm{C}\right)$ was the best condition for minimizing intracellular metabolites leakage. More recently, three-dimensional multicellular tumor spheroids have been used to perform metabolomics analysis. The washing steps consisted of a rapid washing of the spheroids directly on the cultivation plate only once using PBS [89].

Another crucial point for reducing the variability and improving the quality of sample preparation is to quench the cells before the extraction. This procedure aims at stopping cellular metabolism to prevent the turnover of metabolites, maintaining the metabolite concentrations at their physiological levels. Thus, the quenching method should immediately stop all cellular enzymatic activities or cellular changes in metabolite concentrations, without changing the cell environment, since metabolites are very sensitive to any variation of their environment. As for the other points mentioned above, 
several protocols have been tested and used by scientists and were improved over time. Previously, metabolic quenching and extraction were performed using drastic culture conditions changes (acidic condition, high temperature) to inactivate all enzymatic activities, but taking into account the loss of heat-sensitive and $\mathrm{pH}$-sensitive compounds [90]. Some papers reported quenching protocol using liquid nitrogen, for metabolomics studies, after detaching cells by trypsination [91]. In this case, cells are quenched and extracted at the same time [92]. This method substantially modifies the intra-cellular metabolic profiles because trypsin can induce cell stress, structural and protein disruption during cell detachment and consequently metabolite leakage [93]. Another method has been proposed to isolate the cells using filtration [88]. However, both filtration and centrifugation expose cells to a mechanical stress that can also modify the metabolic profiles. Recently, another method has been proposed to better preserve cell metabolism: after carefully removing the medium, cells are washed with PBS or deionized water at room temperature or at $37^{\circ} \mathrm{C}$. Finally, the cellular metabolism is quenched by adding liquid nitrogen or ice-cold methanol into the dishes. Cells can be stored at $-80^{\circ} \mathrm{C}$ or scraped with a cell lifter to be directly extracted. The solvents used for the quenching and metabolite extraction step is one critical step to anticipate, depending of which type of metabolites are targeted. It is admitted that up to now the choice of solvent was adapted to specific metabolite extractions. Usually, a mix of different proportions of solvents is used to quench, extract the cells and collect the metabolites. Mainly, methanol or acetonitrile are used as organic solvents, supplemented by water or acidified water [94]. Different ratios of organic solvents and water have been documented, such as methanol:water 50:50 (v/v) to perform global metabolomics [93] or acetonitrile:water 70:30 $(v / v)$ to perform targeted amine profiling [95]. Some protocols include only a cold mix freshly prepared of acetonile and deionized water to seek polar metabolites [96]. The choice of methanol, acetonitrile and water, even at different ratios, is efficient to recover a large panel of metabolite families such as amino acids, organic acids, nucleotides precursors, sugars ad sugar alcohols [97]. Potassium hydroxide or perchloric acid have also been used, but the results were less reproducible and less efficient for extracting nucleotides, sugars, sugar phosphates and organic acids. These authors concluded that acidic and alkaline extractions did not suit the requirements for a global metabolome analysis [97]. Research is ongoing to optimize conditions in order to get as many metabolites as possible from the same sample extracts using appropriate mix of solvents.

\subsubsection{Sample Storage}

After quenching and metabolite extraction, cell extracts are usually snap frozen in liquid nitrogen and quickly stored at $-80^{\circ} \mathrm{C}$ until further analysis to prevent metabolite degradation. Similarly to animal tissues, freeze-thaw cycles must be avoided and the thawing step should be performed on ice to increase gradually the temperature of the samples.

\section{Conclusions}

Metabolomics offers detailed insights into the metabolic phenotype of the liver and each of the associated organs and biofluids (including the gut microbiota). It is, therefore, nowadays one of the most promising tools in systems biology in hepatology, and is expected to help especially in non-invasive biomarker discovery and identification of biological pathways operating in the liver in physiology and pathology. Metabolomics studies have therefore been performed in a variety of biological matrices to study liver functions. Depending on the research question, several points have to be carefully considered before analytical handling of the samples, including: type and time of sampling, sampling conditions, quenching of ongoing metabolism, use of preservatives, aliquoting and storage conditions. SOPs vary according to the biological matrices used but aim to enhance metabolite recovery and stability to optimize metabolic pathways investigations in liver functions. This raises the question of international consensus protocols and international committees working on continuous improvement of standardized pre-analytical issues. 
Author Contributions: Writing—original draft preparation, L.S., J.V.-C., N.J.C. and S.E.-S.; writing-review and editing, S.P.C., C.C., H.G., N.J.C. and S.E.-S.; supervision, H.G., N.J.C. and S.E.-S.; project administration, H.G., N.J.C. and S.E.-S.; funding acquisition, H.G. and S.E.-S. All authors have read and agreed to the published version of the manuscript."

Funding: L.S. is supported by grants from the Agence Française pour la Biodiversité and the French Institute for Agricultural and Environmental Research (INRAE) AlimH department. This work was supported by grants from ANSES, Agence Nationale de la Recherche (ANR), Fond Européen de Développement Régional (FEDER), and Région Occitanie.

Conflicts of Interest: The authors declare no conflict of interest. There is no connection between LNC Therapeutics and the subject of this manuscript.

\section{References}

1. Estes, C.; Razavi, H.; Loomba, R.; Younossi, Z.; Sanyal, A.J. Modeling the epidemic of nonalcoholic fatty liver disease demonstrates an exponential increase in burden of disease. Hepatology 2018, 67, 123-133. [CrossRef] [PubMed]

2. Sanyal, A.J. Past, present and future perspectives in nonalcoholic fatty liver disease. Nat. Rev. Gastroenterol. Hepatol. 2019, 16, 377-386. [CrossRef]

3. Kalhan, S.C.; Guo, L.; Edmison, J.; Dasarathy, S.; McCullough, A.J.; Hanson, R.W.; Milburn, M. Plasma metabolomic profile in nonalcoholic fatty liver disease. Metab Clin. Exp. 2011, 60, 404-413. [CrossRef] [PubMed]

4. Hoyles, L.; Fernández-Real, J.-M.; Federici, M.; Serino, M.; Abbott, J.; Charpentier, J.; Heymes, C.; Luque, J.L.; Anthony, E.; Barton, R.H.; et al. Molecular phenomics and metagenomics of hepatic steatosis in non-diabetic obese women. Nat. Med. 2018, 24, 1070-1080. [CrossRef] [PubMed]

5. Moreau, R.; Clària, J.; Aguilar, F.; Fenaille, F.; Lozano, J.; Junot, C.; Colsch, B.; Caraceni, P.; Trebicka, J.; Pavesi, M.; et al. Blood metabolomics uncovers inflammation-associated mitochondrial dysfunction as a potential mechanism underlying ACLF. J. Hepatol. 2019. [CrossRef] [PubMed]

6. Chang, M.-L.; Yang, S.-S. Metabolic Signature of Hepatic Fibrosis: From Individual Pathways to Systems Biology. Cells 2019, 8, 1423. [CrossRef]

7. Dumas, M.-E.; Barton, R.H.; Toye, A.; Cloarec, O.; Blancher, C.; Rothwell, A.; Fearnside, J.; Tatoud, R.; Blanc, V.; Lindon, J.C.; et al. Metabolic profiling reveals a contribution of gut microbiota to fatty liver phenotype in insulin-resistant mice. PNAS 2006, 103, 12511-12516. [CrossRef]

8. Soltis, A.R.; Kennedy, N.J.; Xin, X.; Zhou, F.; Ficarro, S.B.; Yap, Y.S.; Matthews, B.J.; Lauffenburger, D.A.; White, F.M.; Marto, J.A.; et al. Hepatic Dysfunction Caused by Consumption of a High-Fat Diet. Cell Rep. 2017, 21, 3317-3328. [CrossRef]

9. Torre Della, S.; Mitro, N.; Meda, C.; Lolli, F.; Pedretti, S.; Barcella, M.; Ottobrini, L.; Metzger, D.; Caruso, D.; Maggi, A. Short-Term Fasting Reveals Amino Acid Metabolism as a Major Sex-Discriminating Factor in the Liver. Cell Metab. 2018, 28, 256-267. [CrossRef]

10. Li, F.; Patterson, A.D.; Krausz, K.W.; Tanaka, N.; Gonzalez, F.J. Metabolomics reveals an essential role for peroxisome proliferator-activated receptor $\alpha$ in bile acid homeostasis. J. Lipid Res. 2012, 53, 1625-1635. [CrossRef]

11. Cho, J.-Y.; Kang, D.W.; Ma, X.; Ahn, S.-H.; Krausz, K.W.; Luecke, H.; Idle, J.R.; Gonzalez, F.J. Metabolomics reveals a novel vitamin E metabolite and attenuated vitamin E metabolism upon PXR activation. J. Lipid Res. 2009, 50, 924-937. [CrossRef] [PubMed]

12. Montagner, A.; Korecka, A.; Polizzi, A.; Lippi, Y.; Blum, Y.; Canlet, C.; Tremblay-Franco, M.; Gautier-Stein, A.; Burcelin, R.; Yen, Y.-C.; et al. Hepatic circadian clock oscillators and nuclear receptors integrate microbiome-derived signals. Sci. Rep. 2016, 6, 20127. [CrossRef]

13. Lukowicz, C.; Ellero-Simatos, S.; Régnier, M.; Oliviero, F.; Lasserre, F.; Polizzi, A.; Montagner, A.; Smati, S.; Boudou, F.; Lenfant, F.; et al. Dimorphic metabolic and endocrine disorders in mice lacking the constitutive androstane receptor. Sci. Rep. 2019, 9, 20169. [CrossRef] [PubMed]

14. Clayton, T.A.; Lindon, J.C.; Cloarec, O.; Antti, H.; Charuel, C.; Hanton, G.; Provost, J.-P.; Le Net, J.-L.; Baker, D.; Walley, R.J.; et al. Pharmaco-metabonomic phenotyping and personalized drug treatment. Nature 2006, 440, 1073-1077. [CrossRef] [PubMed] 
15. Clayton, T.A.; Baker, D.; Lindon, J.C.; Everett, J.R.; Nicholson, J.K. Pharmacometabonomic identification of a significant host-microbiome metabolic interaction affecting human drug metabolism. Proc. Natl. Acad. Sci. USA 2009, 106, 14728-14733. [CrossRef] [PubMed]

16. Zhang, L.; Niu, M.; Wei, A.-W.; Tang, J.-F.; Tu, C.; Bai, Z.-F.; Zou, Z.-S.; Xiao, X.-H.; Liu, Y.-P.; Wang, J.-B. Risk profiling using metabolomic characteristics for susceptible individuals of drug-induced liver injury caused by Polygonum multiflorum. Arch. Toxicol. 2019, 295, 113. [CrossRef]

17. Yip, L.Y.; Aw, C.C.; Lee, S.H.; Hong, Y.S.; Ku, H.C.; Xu, W.H.; Chan, J.M.X.; Cheong, E.J.Y.; Chng, K.R.; $\mathrm{Ng}$, A.H.Q.; et al. The liver-gut microbiota axis modulates hepatotoxicity of tacrine in the rat. Hepatology 2018, 67, 282-295. [CrossRef]

18. Cabaton, N.J.; Poupin, N.; Canlet, C.; Tremblay-Franco, M.; Audebert, M.; Cravedi, J.-P.; Riu, A.; Jourdan, F.; Zalko, D. An Untargeted Metabolomics Approach to Investigate the Metabolic Modulations of HepG2 Cells Exposed to Low Doses of Bisphenol A and 17ß-Estradiol. Front. Endocrinol. 2018, 9, 571. [CrossRef]

19. Lukowicz, C.; Ellero-Simatos, S.; Régnier, M.; Polizzi, A.; Lasserre, F.; Montagner, A.; Lippi, Y.; Jamin, E.L.; Martin, J.-F.; Naylies, C.; et al. Metabolic Effects of a Chronic Dietary Exposure to a Low-Dose Pesticide Cocktail in Mice: Sexual Dimorphism and Role of the Constitutive Androstane Receptor. Environ. Health Perspect. 2018, 126, 067007. [CrossRef]

20. Weiss, J.M.; Davies, L.C.; Karwan, M.; Ileva, L.; Ozaki, M.K.; Cheng, R.Y.; Ridnour, L.A.; Annunziata, C.M.; Wink, D.A.; McVicar, D.W. Itaconic acid mediates crosstalk between macrophage metabolism and peritoneal tumors. J. Clin. Invest. 2018, 128, 3794-3805. [CrossRef]

21. Su, L.; Mao, J.; Hao, M.; Lu, T.; Mao, C.; Ji, D.; Tong, H.; Fei, C. Integrated Plasma and Bile Metabolomics Based on an UHPLC-Q/TOF-MS and Network Pharmacology Approach to Explore the Potential Mechanism of Schisandra chinensis-Protection From Acute Alcoholic Liver Injury. Front. Pharmacol. 2019, 10, 1543. [CrossRef] [PubMed]

22. Claus, S.P.; Swann, J.R. Nutrimetabonomics: Applications for nutritional sciences, with specific reference to gut microbial interactions. Annu. Rev. Food Sci. Technol. 2013, 4, 381-399. [CrossRef] [PubMed]

23. Ivey, K.L.; Rimm, E.B.; Kraft, P.; Clish, C.B.; Cassidy, A.; Hodgson, J.; Croft, K.; Wolpin, B.; Liang, L. Identifying the metabolomic fingerprint of high and low flavonoid consumers. J. Nutr. Sci. 2017, 6, e34. [CrossRef]

24. Robertson, D.G.; Ruepp, S.U.; Stryker, S.A.; Hnatyshyn, S.Y.; Shipkova, P.A.; Aranibar, N.; Mcnaney, C.A.; Fiehn, O.; Reily, M.D. Metabolomic and transcriptomic changes induced by overnight $(16 \mathrm{~h})$ fasting in male and female Sprague-Dawley rats. Chem. Res. Toxicol. 2011, 24, 481-487. [CrossRef] [PubMed]

25. Abbondante, S.; Eckel-Mahan, K.L.; Ceglia, N.J.; Baldi, P.; Sassone-Corsi, P. Comparative Circadian Metabolomics Reveal Differential Effects of Nutritional Challenge in the Serum and Liver. J. Biol. Chem. 2016, 291, 2812-2828. [CrossRef] [PubMed]

26. Zierer, J.; Jackson, M.A.; Kastenmüller, G.; Mangino, M.; Long, T.; Telenti, A.; Mohney, R.P.; Small, K.S.; Bell, J.T.; Steves, C.J.; et al. The fecal metabolome as a functional readout of the gut microbiome. Nat. Genet. 2018, 50, 790-795. [CrossRef]

27. Beaumont, M.; Portune, K.J.; Steuer, N.; Lan, A.; Cerrudo, V.; Audebert, M.; Dumont, F.; Mancano, G.; Khodorova, N.; Andriamihaja, M.; et al. Quantity and source of dietary protein influence metabolite production by gut microbiota and rectal mucosa gene expression: A randomized, parallel, double-blind trial in overweight humans. Am. J. Clin. Nutr. 2017, 106, 1005-1019. [CrossRef]

28. Zhang, X.; Grosfeld, A.; Williams, E.; Vasiliauskas, D.; Barretto, S.; Smith, L.; Mariadassou, M.; Philippe, C.; Devime, F.; Melchior, C.; et al. Fructose malabsorption induces cholecystokinin expression in the ileum and cecum by changing microbiota composition and metabolism. FASEB J. 2019, 33, 7126-7142. [CrossRef]

29. Choi, W.; Namkung, J.; Hwang, I.; Kim, H.; Lim, A.; Park, H.J.; Lee, H.W.; Han, K.-H.; Park, S.; Jeong, J.-S.; et al. Serotonin signals through a gut-liver axis to regulate hepatic steatosis. Nat. Commun. 2018, 9, 4824. [CrossRef]

30. Koh, A.; Molinaro, A.; Ståhlman, M.; Khan, M.T.; Schmidt, C.; Mannerås-Holm, L.; Wu, H.; Carreras, A.; Jeong, H.; Olofsson, L.E.; et al. Microbially Produced Imidazole Propionate Impairs Insulin Signaling through mTORC1. Cell 2018, 175, 947-961. [CrossRef]

31. Huang, W.; Ramsey, K.M.; Marcheva, B.; Bass, J. Circadian rhythms, sleep, and metabolism. J. Clin. Invest. 2011, 121, 2133-2141. [CrossRef] [PubMed]

32. Ramsey, K.M.; Yoshino, J.; Brace, C.S.; Abrassart, D.; Kobayashi, Y.; Marcheva, B.; Hong, H.-K.; Chong, J.L.; Buhr, E.D.; Lee, C.; et al. Circadian clock feedback cycle through NAMPT-mediated NAD+ biosynthesis. Science 2009, 324, 651-654. [CrossRef] [PubMed] 
33. Hughes, M.E.; DiTacchio, L.; Hayes, K.R.; Vollmers, C.; Pulivarthy, S.; Baggs, J.E.; Panda, S.; Hogenesch, J.B. Harmonics of circadian gene transcription in mammals. PLoS Genet. 2009, 5, e1000442. [CrossRef] [PubMed]

34. Dallmann, R.; Viola, A.U.; Tarokh, L.; Cajochen, C.; Brown, S.A. The human circadian metabolome. Proc. Natl. Acad. Sci. USA 2012, 109, 2625-2629. [CrossRef]

35. Deprez, S.; Sweatman, B.C.; Connor, S.C.; Haselden, J.N.; Waterfield, C.J. Optimisation of collection, storage and preparation of rat plasma for $1 \mathrm{H}$ NMR spectroscopic analysis in toxicology studies to determine inherent variation in biochemical profiles. J. Pharm. Biomed. Anal. 2002, 30, 1297-1310. [CrossRef]

36. Bernini, P.; Bertini, I.; Luchinat, C.; Nincheri, P.; Staderini, S.; Turano, P. Standard operating procedures for pre-analytical handling of blood and urine for metabolomic studies and biobanks. J. Biomol. NMR 2011, 49, 231-243. [CrossRef]

37. Slupsky, C.M.; Rankin, K.N.; Wagner, J.; Fu, H.; Chang, D.; Weljie, A.M.; Saude, E.J.; Lix, B.; Adamko, D.J.; Shah, S.; et al. Investigations of the effects of gender, diurnal variation, and age in human urinary metabolomic profiles. Anal. Chem. 2007, 79, 6995-7004. [CrossRef]

38. Stevens, V.L.; Hoover, E.; Wang, Y.; Zanetti, K.A. Pre-Analytical Factors that Affect Metabolite Stability in Human Urine, Plasma, and Serum: A Review. Metabolites 2019, 9, 156. [CrossRef]

39. Eriksson, E.; Royo, F.; Lyberg, K.; Carlsson, H.-E.; Hau, J. Effect of metabolic cage housing on immunoglobulin A and corticosterone excretion in faeces and urine of young male rats. Exp. Physiol. 2004, 89, 427-433. [CrossRef]

40. Hoffman, J.F.; Fan, A.X.; Neuendorf, E.H.; Vergara, V.B.; Kalinich, J.F. Hydrophobic Sand Versus Metabolic Cages: A Comparison of Urine Collection Methods for Rats (Rattus norvegicus). J. Am. Assoc. Lab. Anim. Sci. 2018, 57, 51-57.

41. Saude, E.J.; Sykes, B.D. Urine stability for metabolomic studies: Effects of preparation and storage. Metabolomics 2007, 3, 19-27. [CrossRef]

42. Thongboonkerd, V.; Saetun, P. Bacterial overgrowth affects urinary proteome analysis: Recommendation for centrifugation, temperature, duration, and the use of preservatives during sample collection. J. Proteome Res. 2007, 6, 4173-4181. [CrossRef] [PubMed]

43. Fernández-Peralbo, M.A.; Luque de Castro, M.D. Preparation of urine samples prior to targeted or untargeted metabolomics mass-spectrometry analysis. TrAC Trends Anal. Chem. 2012, 41, 75-85. [CrossRef]

44. Gika, H.G.; Theodoridis, G.A.; Wilson, I.D. Liquid chromatography and ultra-performance liquid chromatography-mass spectrometry fingerprinting of human urine: Sample stability under different handling and storage conditions for metabonomics studies. J. Chromatogr. A 2008, 1189, 314-322. [CrossRef] [PubMed]

45. Trivedi, D.K.; Jones, H.; Shah, A.J.; Iles, R.K. Development of Zwitterionic Hydrophilic Liquid Chromatography (ZIC®HILIC-MS) metabolomics method for Shotgun analysis of human urine. J. Chromatogr. Sep. Tech. 2012, 3. [CrossRef]

46. Rachita Dhurat, M.S. Principles and Methods of Preparation of Platelet-Rich Plasma: A Review and Author's Perspective. J. Cutan. Aesthetic Surg. 2014, 7, 189-197. [CrossRef]

47. Teahan, O.; Gamble, S.; Holmes, E.; Waxman, J.; Nicholson, J.K.; Charlotte Bevan, A.; Keun, H.C. Impact of Analytical Bias in Metabonomic Studies of Human Blood Serum and Plasma. Anal. Chem. 2006, 78, 4307-4318. [CrossRef]

48. Yin, P.; Lehmann, R.; Xu, G. Effects of pre-analytical processes on blood samples used in metabolomics studies. Anal. Bioanal. Chem. 2015, 407, 4879-4892. [CrossRef]

49. Denery, J.R.; Nunes, A.A.K.; Dickerson, T.J. Characterization of differences between blood sample matrices in untargeted metabolomics. Anal. Chem. 2011, 83, 1040-1047. [CrossRef]

50. Barri, T.; Dragsted, L.O. UPLC-ESI-QTOF/MS and multivariate data analysis for blood plasma and serum metabolomics: Effect of experimental artefacts and anticoagulant. Anal. Chim. Acta 2013, 768, 118-128. [CrossRef]

51. Beckonert, O.; Keun, H.C.; Ebbels, T.M.D.; Bundy, J.; Holmes, E.; Lindon, J.C.; Nicholson, J.K. Metabolic profiling, metabolomic and metabonomic procedures for NMR spectroscopy of urine, plasma, serum and tissue extracts. Nat. Protoc. 2007, 2, 2692-2703. [CrossRef] [PubMed]

52. Dunn, W.B.; Broadhurst, D.; Begley, P.; Zelena, E.; Francis-McIntyre, S.; Anderson, N.; Brown, M.; Knowles, J.D.; Halsall, A.; Haselden, J.N.; et al. Human Serum Metabolome (HUSERMET) Consortium Procedures for large-scale metabolic profiling of serum and plasma using gas chromatography and liquid chromatography coupled to mass spectrometry. Nat. Protoc. 2011, 6, 1060-1083. [CrossRef] [PubMed] 
53. Pinto, J.; Domingues, M.R.M.; Galhano, E.; Pita, C.; Almeida, M.D.C.; Carreira, I.M.; Gil, A.M. Human plasma stability during handling and storage: Impact on NMR metabolomics. Analyst 2014, 139, 1168-1177. [CrossRef] [PubMed]

54. Mitchell, B.L.; Yasui, Y.; Li, C.I.; Fitzpatrick, A.L.; Lampe, P.D. Impact of freeze-thaw cycles and storage time on plasma samples used in mass spectrometry based biomarker discovery projects. Cancer Inform 2005, 1, 98-104. [CrossRef] [PubMed]

55. Hernandes, V.V.; Barbas, C.; Dudzik, D. A review of blood sample handling and pre-processing for metabolomics studies. Electrophoresis 2017, 38, 2232-2241. [CrossRef]

56. Schroeder, B.O.; Bäckhed, F. Signals from the gut microbiota to distant organs in physiology and disease. Nat. Med. 2016, 22, 1079-1089. [CrossRef]

57. Karu, N.; Deng, L.; Slae, M.; Guo, A.C.; Sajed, T.; Huynh, H.; Wine, E.; Wishart, D.S. A review on human fecal metabolomics: Methods, applications and the human fecal metabolome database. Anal. Chim. Acta 2018, 1030, 1-24. [CrossRef]

58. Probert, C.S.J.; Jones, P.R.H.; Ratcliffe, N.M. A novel method for rapidly diagnosing the causes of diarrhoea. Gut 2004, 53, 58-61. [CrossRef]

59. Garner, C.E.; Smith, S.; de Lacy Costello, B.; White, P.; Spencer, R.; Probert, C.S.J.; Ratcliffe, N.M. Volatile organic compounds from feces and their potential for diagnosis of gastrointestinal disease. FASEB J. 2007, 21, 1675-1688. [CrossRef]

60. Rose, C.; Parker, A.; Jefferson, B.; Cartmell, E. The Characterization of Feces and Urine: A Review of the Literature to Inform Advanced Treatment Technology. Crit. Rev. Environ. Sci. Technol. 2015, 45, 1827-1879. [CrossRef]

61. Matysik, S.; Le Roy, C.I.; Liebisch, G.; Claus, S.P. Metabolomics of fecal samples: A practical consideration. Trends Food Sci. Technol. 2016, 57, 244-255. [CrossRef]

62. Liang, X.; Bushman, F.D.; FitzGerald, G.A. Rhythmicity of the intestinal microbiota is regulated by gender and the host circadian clock. Proc. Natl. Acad. Sci. USA 2015, 112, 10479-10484. [CrossRef] [PubMed]

63. Leone, V.; Gibbons, S.M.; Martinez, K.; Hutchison, A.L.; Huang, E.Y.; Cham, C.M.; Pierre, J.F.; Heneghan, A.F.; Nadimpalli, A.; Hubert, N.; et al. Effects of diurnal variation of gut microbes and high-fat feeding on host circadian clock function and metabolism. Cell Host Microbe 2015, 17, 681-689. [CrossRef] [PubMed]

64. Duparc, T.; Plovier, H.; Marrachelli, V.G.; Van Hul, M.; Essaghir, A.; Ståhlman, M.; Matamoros, S.; Geurts, L.; Pardo-Tendero, M.M.; Druart, C.; et al. Hepatocyte MyD88 affects bile acids, gut microbiota and metabolome contributing to regulate glucose and lipid metabolism. Gut 2016, 66, 620-632. [CrossRef]

65. Sberna, A.L.; Assem, M.; Gautier, T.; Grober, J.; Guiu, B.; Jeannin, A.; Pais de Barros, J.-P.; Athias, A.; Lagrost, L.; Masson, D. Constitutive androstane receptor activation stimulates faecal bile acid excretion and reverse cholesterol transport in mice. J. Hepatol. 2011, 55, 154-161. [CrossRef] [PubMed]

66. Lamichhane, S.; Sundekilde, U.K.; Blædel, T.; Dalsgaard, T.K.; Larsen, L.H.; Dragsted, L.O.; Astrup, A.; Bertram, H.C. Optimizing sampling strategies for NMR-based metabolomics of human feces: Pooled vs. unpooled analyses. Anal. Methods 2017, 9, 4476-4480. [CrossRef]

67. Phua, L.C.; Koh, P.K.; Cheah, P.Y.; Ho, H.K.; Chan, E.C.Y. Global gas chromatography/time-of-flight mass spectrometry (GC/TOFMS)-based metabonomic profiling of lyophilized human feces. J. Chromatogr. B 2013, 937, 103-113. [CrossRef]

68. Lamichhane, S.; Yde, C.C.; Schmedes, M.S.; Jensen, H.M.; Meier, S.; Bertram, H.C. Strategy for NuclearMagnetic-Resonance-Based Metabolomics of Human Feces. Anal. Chem. 2015, 87, 5930-5937. [CrossRef]

69. Le Gall, G.; Noor, S.O.; Ridgway, K.; Scovell, L.; Jamieson, C.; Johnson, I.T.; Colquhoun, I.J.; Kemsley, E.K.; Narbad, A. Metabolomics of fecal extracts detects altered metabolic activity of gut microbiota in ulcerative colitis and irritable bowel syndrome. J. Proteome Res. 2011, 10, 4208-4218. [CrossRef]

70. Armstrong, C.W.; McGregor, N.R.; Lewis, D.P.; Butt, H.L.; Gooley, P.R. The association of fecal microbiota and fecal, blood serum and urine metabolites in myalgic encephalomyelitis/chronic fatigue syndrome. Metabolomics 2016, 13, 8. [CrossRef]

71. Bezabeh, T.; Somorjai, R.L.; Smith, I.C.P. MR metabolomics of fecal extracts: Applications in the study of bowel diseases. Magn. Reson. Chem. 2009, 47, S54-S61. [CrossRef] [PubMed]

72. Gratton, J.; Phetcharaburanin, J.; Mullish, B.H.; Williams, H.R.T.; Thursz, M.; Nicholson, J.K.; Holmes, E.; Marchesi, J.R.; Li, J.V. Optimized Sample Handling Strategy for Metabolic Profiling of Human Feces. Anal. Chem. 2016, 88, 4661-4668. [CrossRef] [PubMed] 
73. Yen, S.; McDonald, J.A.K.; Schroeter, K.; Oliphant, K.; Sokolenko, S.; Blondeel, E.J.M.; Allen-Vercoe, E.; Aucoin, M.G. Metabolomic Analysis of Human Fecal Microbiota: A Comparison of Feces-Derived Communities and Defined Mixed Communities. J. Proteome Res. 2015, 14, 1472-1482. [CrossRef]

74. Couch, R.D.; Navarro, K.; Sikaroodi, M.; Gillevet, P.; Forsyth, C.B.; Mutlu, E.; Engen, P.A.; Keshavarzian, A. The Approach to Sample Acquisition and Its Impact on the Derived Human Fecal Microbiome and VOC Metabolome. PLoS ONE 2013, 8, e81163. [CrossRef]

75. Loftfield, E.; Vogtmann, E.; Sampson, J.N.; Moore, S.C.; Nelson, H.; Knight, R.; Chia, N.; Sinha, R. Comparison of Collection Methods for Fecal Samples for Discovery Metabolomics in Epidemiologic Studies. Cancer Epidemiol. Biomark. Prev. 2016, 25, 1483-1490. [CrossRef]

76. Saric, J.; Wang, Y.; Li, J.; Coen, M.; Utzinger, J.; Marchesi, J.R.; Keiser, J.; Veselkov, K.; Lindon, J.C.; Nicholson, J.K.; et al. Species variation in the fecal metabolome gives insight into differential gastrointestinal function. J. Proteome Res. 2008, 7, 352-360. [CrossRef] [PubMed]

77. Moosmang, S.; Pitscheider, M.; Sturm, S.; Seger, C.; Tilg, H.; Halabalaki, M.; Stuppner, H. Metabolomic analysis-Addressing NMR and LC-MS related problems in human feces sample preparation. Clin. Chim. Acta 2017, 489, 169-176. [CrossRef] [PubMed]

78. Jungermann, K.; Katz, N. Functional specialization of different hepatocyte populations. Physiol. Rev. 1989, 69, 708-764. [CrossRef]

79. Braeuning, A.; Ittrich, C.; Köhle, C.; Hailfinger, S.; Bonin, M.; Buchmann, A.; Schwarz, M. Differential gene expression in periportal and perivenous mouse hepatocytes. FEBS J. 2006, 273, 5051-5061. [CrossRef]

80. Isse, K.; Lesniak, A.; Grama, K.; Maier, J.; Specht, S.; Castillo-Rama, M.; Lunz, J.; Roysam, B.; Michalopoulos, G.; Demetris, A.J. Preexisting epithelial diversity in normal human livers: A tissue-tethered cytometric analysis in portal/periportal epithelial cells. Hepatology 2013, 57, 1632-1643. [CrossRef] [PubMed]

81. Want, E.J.; Masson, P.; Michopoulos, F.; Wilson, I.D.; Theodoridis, G.; Plumb, R.S.; Shockcor, J.; Loftus, N.; Holmes, E.; Nicholson, J.K. Global metabolic profiling of animal and human tissues via UPLC-MS. Nat. Protoc. 2013, 8, 17-32. [CrossRef]

82. Hu, Z.-P.; Browne, E.R.; Liu, T.; Angel, T.E.; Ho, P.C.; Chan, E.C.Y. Metabonomic profiling of TASTPM transgenic Alzheimer's disease mouse model. J. Proteome Res. 2012, 11, 5903-5913. [CrossRef]

83. Garrod, S.; Humpfer, E.; Spraul, M.; Connor, S.C.; Polley, S.; Connelly, J.; Lindon, J.C.; Nicholson, J.K.; Holmes, E. High-resolution magic angle spinning $1 \mathrm{H}$ NMR spectroscopic studies on intact rat renal cortex and medulla. Magn. Reson. Med. 1999, 41, 1108-1118. [CrossRef]

84. Zhou, L.; Zhang, W.; Xie, W.; Chen, H.; Yu, W.; Li, H.; Shen, G. Tributyl phosphate impairs the urea cycle and alters liver pathology and metabolism in mice after short-term exposure based on a metabonomics study. Sci. Total Environ. 2017, 603-604, 77-85. [CrossRef] [PubMed]

85. Abuja, P.M.; Ehrhart, F.; Schoen, U.; Schmidt, T.; Stracke, F.; Dallmann, G.; Friedrich, T.; Zimmermann, H.; Zatloukal, K. Alterations in Human Liver Metabolome during Prolonged Cryostorage. J. Proteome Res. 2015, 14, 2758-2768. [CrossRef] [PubMed]

86. Torell, F.; Bennett, K.; Cereghini, S.; Rännar, S.; Lundstedt-Enkel, K.; Moritz, T.; Haumaitre, C.; Trygg, J.; Lundstedt, T. Tissue sample stability: Thawing effect on multi-organ samples. Metabolomics 2015, 12, 19. [CrossRef]

87. Kapoore, R.V.; Coyle, R.; Staton, C.A.; Brown, N.J.; Vaidyanathan, S. Influence of washing and quenching in profiling the metabolome of adherent mammalian cells: A case study with the metastatic breast cancer cell line MDA-MB-231. Analyst 2017, 142, 2038-2049. [CrossRef]

88. Lorenz, M.A.; Burant, C.F.; Kennedy, R.T. Reducing time and increasing sensitivity in sample preparation for adherent mammalian cell metabolomics. Anal. Chem. 2011, 83, 3406-3414. [CrossRef]

89. Rusz, M.; Rampler, E.; Keppler, B.K.; Jakupec, M.A.; Koellensperger, G. Single Spheroid Metabolomics: Optimizing Sample Preparation of Three-Dimensional Multicellular Tumor Spheroids. Metabolites 2019, 9, 304. [CrossRef]

90. Shryock, J.C.; Rubio, R.; Berne, R.M. Extraction of adenine nucleotides from cultured endothelial cells. Anal. Biochem. 1986, 159, 73-81. [CrossRef]

91. Kořínek, M.; Šístek, V.; Mládková, J.; Mikeš, P.; Jiráček, J.; Selicharová, I. Quantification of homocysteine-related metabolites and the role of betaine-homocysteine S-methyltransferase in HepG2 cells. Biomed. Chromatogr. 2013, 27, 111-121. [CrossRef] [PubMed] 
92. Ibáñez, C.; Simó, C.; Valdés, A.; Campone, L.; Piccinelli, A.L.; García-Cañas, V.; Cifuentes, A. Metabolomics of adherent mammalian cells by capillary electrophoresis-mass spectrometry: HT-29 cells as case study. J. Pharm. Biomed. Anal. 2015, 110, 83-92. [CrossRef] [PubMed]

93. Dettmer, K.; Nürnberger, N.; Kaspar, H.; Gruber, M.A.; Almstetter, M.F.; Oefner, P.J. Metabolite extraction from adherently growing mammalian cells for metabolomics studies: Optimization of harvesting and extraction protocols. Anal. Bioanal. Chem. 2011, 399, 1127-1139. [CrossRef] [PubMed]

94. León, Z.; García-Cañaveras, J.C.; Donato, M.T.; Lahoz, A. Mammalian cell metabolomics: Experimental design and sample preparation. Electrophoresis 2013, 34, 2762-2775. [CrossRef]

95. Yuan, W.; Anderson, K.W.; Li, S.; Edwards, J.L. Subsecond absolute quantitation of amine metabolites using isobaric tags for discovery of pathway activation in mammalian cells. Anal. Chem. 2012, 84, 2892-2899. [CrossRef]

96. Poupin, N.; Corlu, A.; Cabaton, N.J.; Dubois-Pot-Schneider, H.; Canlet, C.; Person, E.; Bruel, S.; Frainay, C.; Vinson, F.; Maurier, F.; et al. Large-Scale Modeling Approach Reveals Functional Metabolic Shifts during Hepatic Differentiation. J. Proteome Res. 2019, 18, 204-216. [CrossRef]

97. Villas-Bôas, S.G.; Højer-Pedersen, J.; Akesson, M.; Smedsgaard, J.; Nielsen, J. Global metabolite analysis of yeast: Evaluation of sample preparation methods. Yeast 2005, 22, 1155-1169. [CrossRef]

(C) 2020 by the authors. Licensee MDPI, Basel, Switzerland. This article is an open access article distributed under the terms and conditions of the Creative Commons Attribution (CC BY) license (http://creativecommons.org/licenses/by/4.0/). 

Article

\title{
Proton NMR Enables the Absolute Quantification of Aqueous Metabolites and Lipid Classes in Unique Mouse Liver Samples
}

\author{
Aurélien Amiel ${ }^{1,2}$, Marie Tremblay-Franco ${ }^{1,2}$, Roselyne Gautier ${ }^{1,2}$, Simon Ducheix ${ }^{1}$, \\ Alexandra Montagner ${ }^{1}$, Arnaud Polizzi ${ }^{1}$, Laurent Debrauwer ${ }^{1,2}$, Hervé Guillou ${ }^{1}$, \\ Justine Bertrand-Michel ${ }^{3}$ and Cécile Canlet ${ }^{1,2, *}$ \\ 1 Toxalim-Research Centre in Food Toxicology, Toulouse University, INRAE UMR 1331, ENVT, INP-Purpan, \\ Paul Sabatier University, F-31027 Toulouse, France; aurelien.amiel@yahoo.fr (A.A.); \\ marie.tremblay-franco@inra.fr (M.T.-F.); roselyne.gautier@inra.fr (R.G.); \\ simon.ducheix@univ-nantes.fr (S.D.); alexandra.montagner@inserm.fr (A.M.); arnaud.polizzi@inra.fr (A.P.); \\ laurent.debrauwer@inra.fr (L.D.); herve.guillou@inra.fr (H.G.) \\ 2 Metatoul-AXIOM platform, National Infrastructure for Metabolomics and Fluxomics, MetaboHUB, Toxalim, \\ INRAE UMR 1331, F-31027 Toulouse, France \\ 3 Metatoul-Lipidomic Core Facility, MetaboHUB, I2MC U1048, INSERM, F-31432 Toulouse, France; \\ justine.bertrand-michel@inserm.fr \\ * Correspondence: cecile.canlet@inra.fr
}

Received: 20 November 2019; Accepted: 19 December 2019; Published: 21 December 2019

\begin{abstract}
Hepatic metabolites provide valuable information on the physiological state of an organism, and thus, they are monitored in many clinical situations. Typically, monitoring requires several analyses for each class of targeted metabolite, which is time consuming. The present study aimed to evaluate a proton nuclear magnetic resonance $\left({ }^{1} \mathrm{H}-\mathrm{NMR}\right)$ method for obtaining quantitative measurements of aqueous and lipidic metabolites. We optimized the extraction protocol, the standard samples, and the organic solvents for the absolute quantification of lipid species. To validate the method, we analyzed metabolic profiles in livers of mice fed three different diets. We compared our results with values obtained with conventional methods and found strong correlations. The ${ }^{1} \mathrm{H}-\mathrm{NMR}$ protocol enabled the absolute quantification of 29 aqueous metabolites and eight lipid classes. Results showed that mice fed a diet enriched in saturated fatty acids had higher levels of triglycerides, cholesterol ester, monounsaturated fatty acids, lactate, 3-hydroxy-butyrate, and alanine and lower levels of glucose, compared to mice fed a control diet. In conclusion, proton NMR provided a rapid overview of the main lipid classes (triglycerides, cholesterol, phospholipids, fatty acids) and the most abundant aqueous metabolites in liver.
\end{abstract}

Keywords: metabolomics quantitative profiling; lipidomics; ${ }^{1} \mathrm{H}-\mathrm{NMR}$ spectroscopy; liver; steatosis

\section{Introduction}

The liver is among the most metabolically diverse organs of the body, and it is involved in many metabolic processes. The liver plays a central physiological role in lipid metabolism; e.g., it hosts cholesterol synthesis, cholesterol degradation to bile acids, triglyceride production, and lipoprotein synthesis. The liver may be affected by many pathological aggressions. Associated with the obesity epidemic, Non Alcoholic Fatty Liver Diseases (NAFLD) is currently a major public health concern [1]. NAFLD ranges from benign fat accumulation to inflammatory steatohepatitis that may promote irreversible damage [1]. The current methods of diagnostic mostly rely on liver biopsies [2]. However, metabolomic approaches are extensively used for biomarker identification as well as for identification of metabolic pathways involved in the progression of lipid accumulation [3,4]. Therefore, there is 
a lot of interest in methods allowing the integration of both soluble metabolites and lipids from a single sample.

Metabolomics is currently established as a powerful investigation tool that provides rich information on metabolic disturbances in human disease. Mass spectrometry (MS) and nuclear magnetic resonance (NMR) are the two most widely used techniques in metabolomics. NMR spectroscopy has several advantages over MS, including high reproducibility, non-destructive analysis, a simple quantification approach, and minimal sample preparation [5]. ${ }^{1} \mathrm{H}-\mathrm{NMR}$-based metabolomics is currently widely used to gain insights into liver disease mechanisms [6,7] or to evaluate drug hepatotoxicity [8,9] and environmental contaminants [10]. In most studies that used proton NMR-based metabolomics for liver samples, analyses have been performed on either aqueous extracts or lipidic extracts. Those analyses employed spectral binning, followed by multivariate statistical analyses to highlight changes in metabolite composition due to disease [11-14], alcohol consumption [15-17], or contaminant exposure [18].

Lipids are a diverse, ubiquitous group of compounds, which have many key biological functions. Many diseases alter lipid metabolism; thus, a better understanding of these pathologies can be gained by analyzing lipid composition. Due to the structural diversity among lipid molecules, lipidomic profiling is complex. For the least abundant lipids (i.e., free fatty acids, cholesterol, oxylipids), we typically choose powerful, targeted, but time-consuming approaches, like liquid chromatography (LC) or gas chromatography (GC) coupled to mass spectrometry (MS) techniques (i.e., LC-MS or GC-MS), which can provide absolute quantitative results, under certain conditions [19]. However, to evaluate the most abundant lipids (i.e., phospholipids, sphingolipids, and triacylglycerides), we typically use LC-MS approaches that are not targeted, even though they do not provide quantitative results [20]. NMR is an alternative method that provides rapid, and in particular, quantitative analyses of hepatic lipids. Some studies have analyzed intact liver samples (biopsies) with high-resolution magic angle spinning (HR-MAS) NMR spectroscopy to study metabolic disruptions in human chronic hepatitis and cirrhosis [21] or to study non-alcoholic fatty liver disease (NAFLD) in murine models [22]. Those studies were carried out directly in the solid tissues, without any extraction. With HR-MAS, both lipid and aqueous metabolites can be simultaneous observed in the same spectrum, but the resolution is low, and absolute quantification is complex, due to overlapping signals. Other studies have performed metabolic analyses on tissue extracts to study human hepatocellular carcinoma (HCC) associated with NAFLD or cirrhosis [23] or to study the progression from hepatic steatosis to nonalcoholic steatohepatitis (NASH) in mouse models [24]. NMR analyses of tissue extracts require sample processing and separate analyses for lipidic and aqueous extracts. However, liquid state NMR provides spectra with a better resolution than those recorded with the HRMAS technique, and absolute quantification can be performed when a standard is used at a known concentration. Only a few studies have reported the absolute quantification of lipidic metabolites in liver samples, based on ${ }^{1} \mathrm{H}-\mathrm{NMR}$ spectroscopy [24]. Indeed, lipid species contain many long-chain fatty acids, and therefore, many overlapping proton signals (e.g., $\left(\mathrm{CH}_{2}\right)_{n}$ in fatty acids), which makes a detailed characterization of lipid species unfeasible. However, with ${ }^{1} \mathrm{H}$ NMR spectroscopy, it is possible to identify and quantify different classes of lipids, such as cholesterol, triglycerides, phospholipids, mono-unsaturated fatty acids (MUFAs), and poly-unsaturated fatty acids (PUFAs).

The present study aimed to evaluate ${ }^{1} \mathrm{H}-\mathrm{NMR}$ spectroscopy for the identification and absolute quantification of polar and non-polar metabolites in the same liver sample. First, we compared two extraction methods to optimize aqueous and lipidic metabolite extractions. Then, we optimized the absolute quantification of lipid species on mixtures of lipid standards, by comparing internal vs. external standards and various organic solvents. Finally, the optimized method was applied to investigate the effects of a diet deficient in essential fatty acids on liver mouse metabolism. Hepatic lipids were quantified with both ${ }^{1} \mathrm{H}-\mathrm{NMR}$ spectroscopy and conventional methods to compare the results and validate the methodology. 


\section{Results}

\subsection{Comparison of Extraction Methods}

Sample preparation represents a crucial step in metabolomic studies. In this study, we aimed to obtain the best preparation for both polar and lipid molecules. Several classical solvent systems have been developed for liver extractions [25]. Among the various possibilities, we chose Bligh and Dyer [26] and Folch [27] extractions, because these two methods resulted in a biphasic solvent system. Moreover, these were the main extraction methods used in our lab for targeted lipidomics, performed with conventional methods, and for NMR-based metabolomics [28]. With these extraction methods, the upper phase contained the polar (aqueous) fraction, and the bottom phase contained the lipids. When we tested the Bligh and Dyer extraction method with $50 \mathrm{mg}$ of liver, we noticed the presence of an emulsion, which made it difficult to separate the aqueous and organic phases. With the Folch extraction method, the organic phase was washed with a saturated $\mathrm{NaCl}$ solution, and the phases were well separated.

A visual assessment of the ${ }^{1} \mathrm{H}-\mathrm{NMR}$ spectra revealed that the two extraction procedures produced similar peak coverages and intensities for the extracted lipids (Figure 1). For the aqueous extracts, the intensities were higher in the aromatic region $(\delta 9.0-6.0 \mathrm{ppm})$ with the Folch extraction method than with the Bligh and Dyer method (Figure 2).

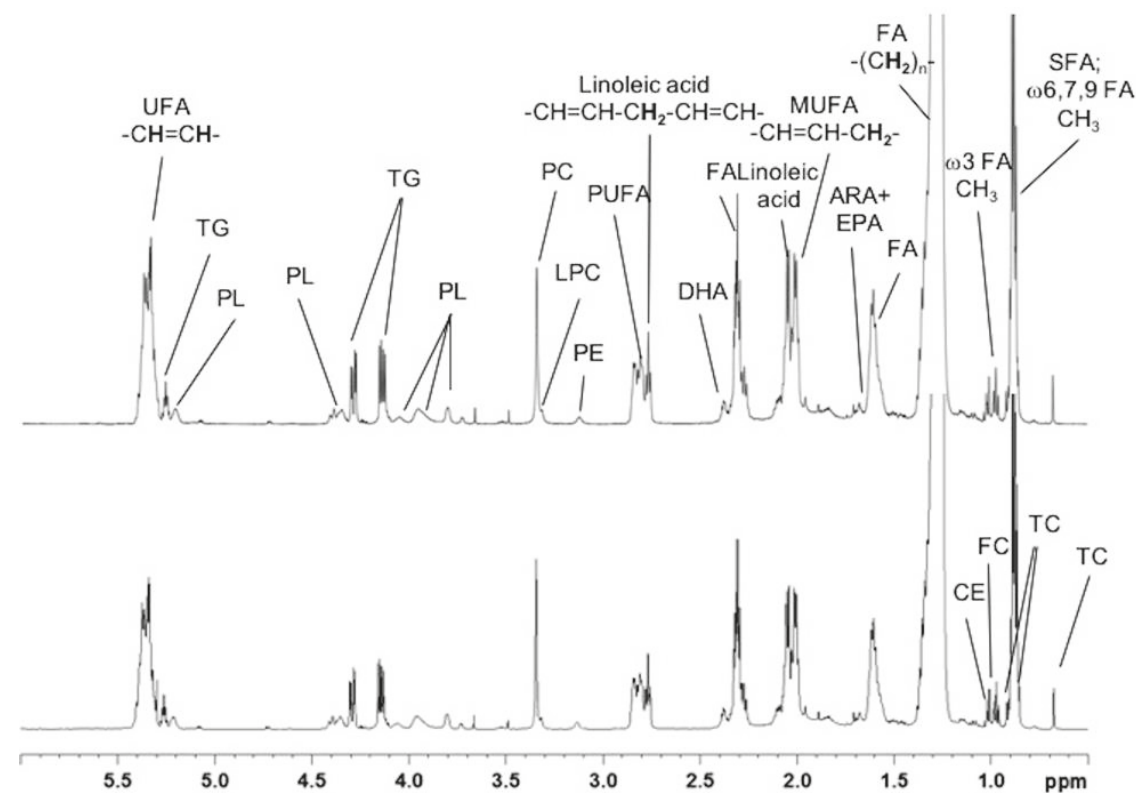

Figure 1. $600 \mathrm{MHz}{ }^{1} \mathrm{H}-\mathrm{NMR}$ spectra of lipophilic extracts from mouse liver samples. The Folch extraction method (top) and the Bligh and Dyer extraction method (bottom) show the same peaks. The peaks are labeled in only one panel for clarity, as follows: FC, free cholesterol; CE, cholesterol ester; TC, total cholesterol; FA, fatty acids; SFA, saturated fatty acids; ARA, arachidonic acid; EPA, eicosapentaenoic acid; MUFA, monounsaturated fatty acids; DHA, docosahexaenoic acid; PUFA, polyunsaturated fatty acid; PE, phosphatidylethanolamine; LPC, lysophosphatidylcholine; PC, phosphatidylcholine; PL, phospholipids; TG, triglycerides; UFA, unsaturated fatty acids. 


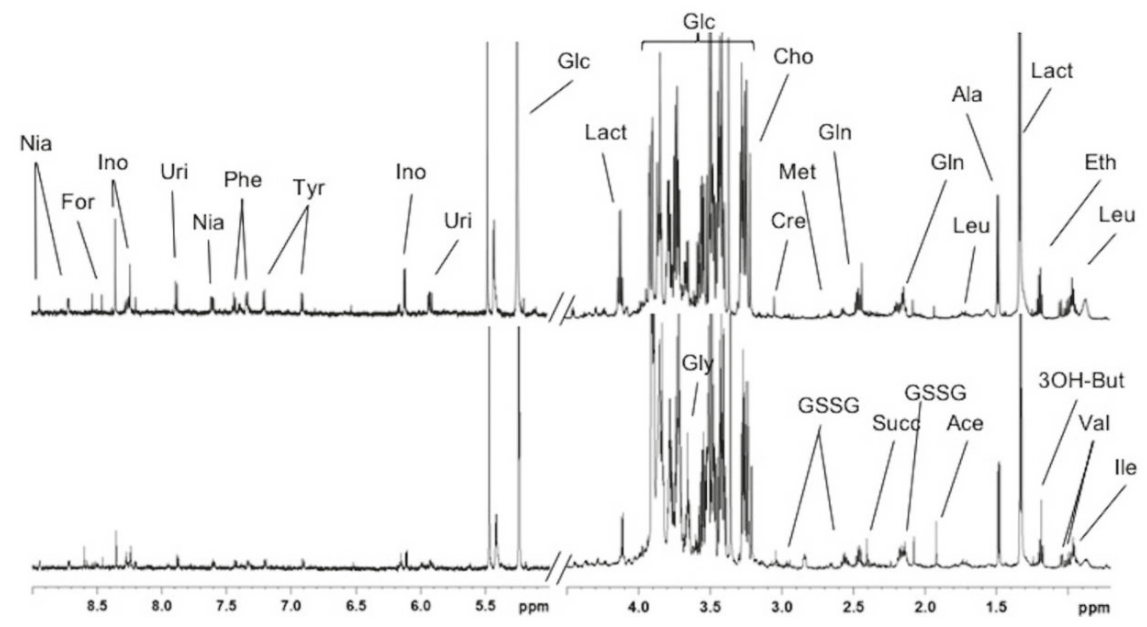

Figure 2. $600 \mathrm{MHz}{ }^{1} \mathrm{H}-\mathrm{NMR}$ spectra of aqueous extracts from mouse liver samples. The Folch extraction method (top) revealed more peaks than the Bligh and Dyer extraction method (bottom). The peaks are labeled in only one panel for clarity, as follows: Ile, isoleucine; Val, valine; $3 \mathrm{OH}-\mathrm{But}$, 3-hydroxybutyrate; Ace, acetate; GSSG, glutathione oxidized; Succ, succinate; Gly, glycine; Leu, leucine; Eth, ethanol; Lact, lactate; Ala, alanine; Gln, glutamine; Met, methionine; Cre, creatine; Cho, choline; Glc, glucose; Uri, uridine; Ino, inosine; Tyr, tyrosine; Phe, phenylalanine; Nia, niacinamide; For, formate.

For each method, the buckets representing metabolites with signal-to-noise ratios above 10 $(\mathrm{SNR}>10)$ were annotated by comparing the chemical shifts in the $1 \mathrm{D}^{1} \mathrm{H}-\mathrm{NMR}$ spectra with those of reference spectra recorded under the same conditions and reference spectra deposited in the Biological Magnetic Resonance Databank [29] and the Human Metabolome Database [30]. We could identify 29 metabolites present in the polar fraction and eight lipid classes present in the non-polar fraction. ${ }^{1} \mathrm{H}-\mathrm{NMR}$ resonance assignments of aqueous and lipidic metabolites are shown in Tables S1 and S2, respectively, with the chemical shifts, multiplicity, and coupling constants of the signals elucidated in the ${ }^{1} \mathrm{H}-\mathrm{NMR}$ spectra for both the water- and lipid-soluble extracts from mouse liver. For the lipidic extracts, among the selected 52 buckets, 50 were detected with an SNR > 10 with both extraction methods, which suggested that there was no significant difference between the two extraction methods, based on this criterion. For the aqueous extracts, among the selected 80 buckets, 40 and 58 were detected with an SNR > 10 with the Bligh and Dyer and the Folch extraction methods, respectively. This finding confirmed that the Folch method provided better extraction of the aqueous metabolites.

We performed a multivariate analysis combined with a principal component analysis (PCA) of the NMR buckets that described components of the extracts from both methods. We found that, for lipidic extracts, the two extraction methods were separated along the second principal component, which explained $11 \%$ of the variability. For aqueous extracts, the samples were separated along the first principal component, which explained $72.1 \%$ of the variability. These results suggested that the aqueous metabolites extracted were significantly different between these two extraction methods (Figure 3). The loading plot for aqueous liver extracts showed signals that contributed to the separation between extraction methods (Figure 4). Taurine, lactate, glucose, choline, alanine, glutathione and others amino acids are elevated in Folch extraction, and glycogen and adenosinemonophosphate (AMP) are elevated in Blye and Dyer extraction. 

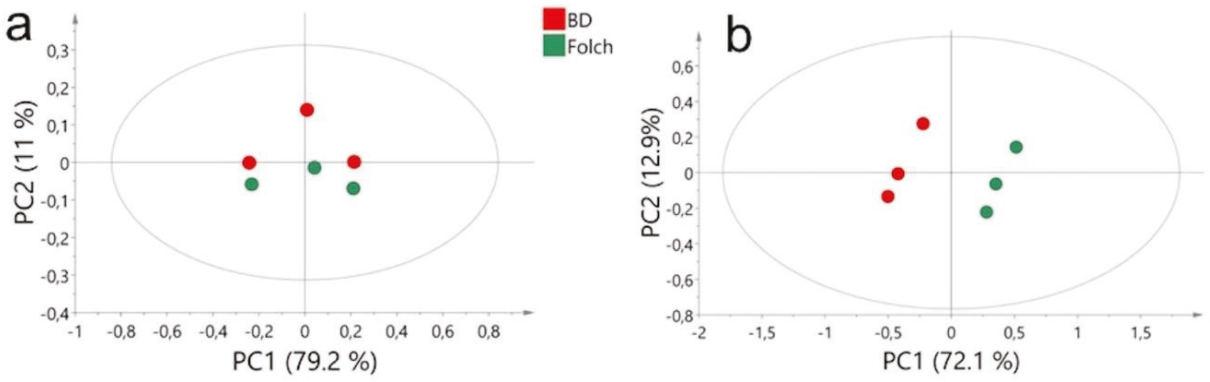

Figure 3. Principal component analysis score plot of the two extraction methods. (a) Liver lipidic extracts $(n=3)$; (b) liver aqueous extracts $(n=3)$. BD = Bligh and Dyer extraction method (red); Folch $=$ Folch extraction method (green). Ellipses indicate the $95 \%$ confidence region .

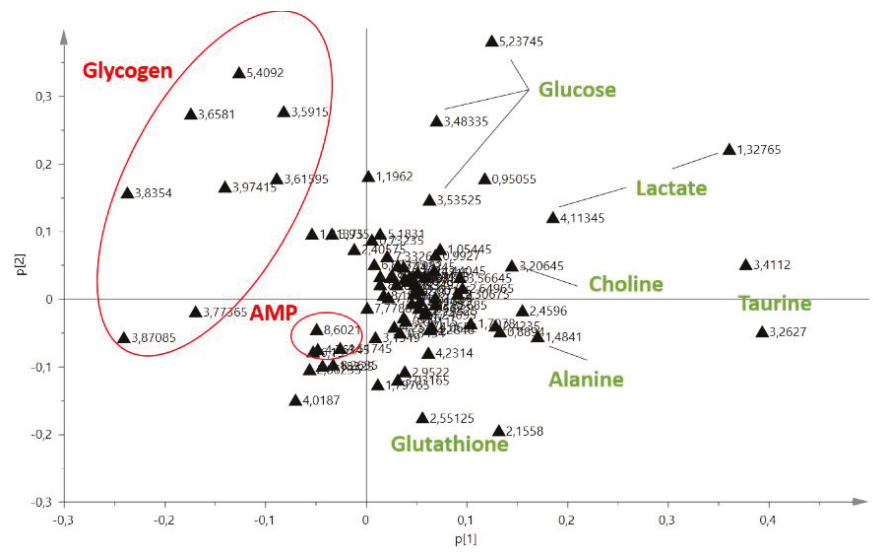

Figure 4. Principal component analysis loading plot of the two extraction methods for aqueous liver extracts. NMR buckets with the highest absolute loadings contributed the most to the separation between the two extraction methods and were annotated. Metabolites in red were elevated in Blye and Dyer extraction and metabolites in green were elevated in Folch extraction.

Generally, when the two classical Bligh and Dyer and Folch lipid extraction methods were used, most studies focused either on the analysis of lipid extracts using MS techniques [31] or on the analysis of aqueous extracts by NMR spectroscopy [32,33], but few studied reported the analysis of both extracts. Our results showed that, in this case, the Folch extraction method was more efficient in extracting aqueous metabolites than the Bligh and Dyer method, but the two extraction methods showed no differences in extracting lipidic metabolites. The variability was similar with both extraction methods. Therefore, we selected the Folch extraction method for all subsequent analyses in this study.

\subsection{Absolute Quantification of Lipidic and Aqueous Metabolites}

In NMR, absolute quantification requires the use of an internal or external standard at a known concentration. A number of reference compounds are available for quantitative NMR analysis. The most widely used reference compounds for chemical shift referencing and quantitative analysis are tetramethylsilane (TMS, organic solubility), 3-(trimethylsilyl)-1-propane sulfonic acid sodium salt (DSS, aqueous solubility), and 3-(trimethylsilyl)propionic acid sodium salt (TSP, aqueous solubility) [34]. In this study, for aqueous extracts, we used TSP as an internal standard, TSP was directly dissolved in the sample. Signals used for absolute quantification of aqueous metabolites were indicated in 
bold in Table S1. For lipid quantifications, TMS was directly dissolved in the sample as an internal standard; as an external standard, TSP was dissolved in deuterated water $\left(\mathrm{D}_{2} \mathrm{O}\right)$ in a coaxial capillary tube for mixtures of lipid standards. The final solvent in an NMR experiment is another key point: in the literature, deuterated chloroform $\left(\mathrm{CDCl}_{3}\right)$ and a mixture of $\mathrm{CDCl}_{3}$ and deuterated methanol $\left(\mathrm{CD}_{3} \mathrm{OD}\right)$ are mostly used for lipidomic analyses. In the present study, we tested both pure deuterated chloroform $\left(\mathrm{CDCl}_{3}\right)$ and a $4: 1(v / v)$ mixture of $\mathrm{CDCl}_{3}$ and $\mathrm{CD}_{3} \mathrm{OD}$ for the final solvent. The lipid extracts and standards were dissolved in these solvents.

The metabolites were quantified according to the following expression:

$$
C x=\frac{\frac{I x \times C S}{N x}}{\frac{I s}{N s}} \times \frac{V}{M}
$$

where $C x$ is the metabolite concentration, $I x$ is the integral of the metabolite proton peak, $N x$ is the number of protons in the metabolite proton peak, Cs is the standard concentration, Is is the integral of the standard proton peak, Ns is the number of protons in the standard proton peak, $V$ is the volume of the analyzed extract, and $M$ is the weight of liver tissue analyzed.

The quantification of lipid species was simple for isolated peaks without any signal overlapping, such as the total cholesterol (TC, singlet, $3 \mathrm{H}, 0.68 \mathrm{ppm}), \omega-3$ fatty acids ( $\omega-3 \mathrm{FAs}$, triplet, $3 \mathrm{H}$, $0.97 \mathrm{ppm}$ ), arachidonic and eicosapentaenoic acids (ARA+EPA, multiplet, 2H, $1.68 \mathrm{ppm}$ ), MUFAs (multiplet, 4H, $2.01 \mathrm{ppm}$ ), docosahexaenoic acid (DHA, multiplet, 4H, $2.38 \mathrm{ppm}$ ), linoleic acid (triplet, $2 \mathrm{H}, 2.27 \mathrm{ppm}$ ), phosphatidylethanolamine (PE, multiplet, $2 \mathrm{H}, 3.12 \mathrm{ppm}$ ), phosphatidylcholine and lysophosphatidylcholine (PC+LPC, singlet, 9H, 3.20 ppm), triglycerides (doublet of doublets, 2H, $4.29 \mathrm{ppm}$ ), and sphingomyelin (SM, multiplet, $1 \mathrm{H}, 5.70 \mathrm{ppm}$ ). Free cholesterol (FC) and cholesterol ester (CE) could be quantified based on the signals at 1.01 and $1.02 \mathrm{ppm}$, respectively, with the deconvolution algorithm available in Topspin software (Bruker, Rheinstetten, Germany). PUFAs could not be quantified directly, because the number of protons that corresponded to the signals at $2.82 \mathrm{ppm}$ could not be determined precisely. As previously mentioned by Vidal et al. [35], it was possible to determine the molar percentage of unsaturated fatty acids (UFA) with the following equation:

$$
\operatorname{UFA}(\%)=\frac{100 \times((2 \times A 7)+A 9))}{((2 \times A 9)+(4 \times A 8))}
$$

where $A 7$ is the signal integration between 1.92 and $2.15 \mathrm{ppm}$ corresponding to the functional group $-\mathrm{CH}_{2}-\mathrm{CH}=\mathrm{CH}$ - acyl group except for $-\mathrm{CH}_{2}$ - of DHA acyl group; $A 8$ is the signal integration between 2.25 and $2.36 \mathrm{ppm}$ corresponding to the functional group -OCO-CH$-\mathrm{H}_{2}$-acyl group except for DHA; and $A 9$ is the signal integration between 2.36 and $2.42 \mathrm{ppm}$ corresponding to the functional group $-\mathrm{OCO}-\mathrm{CH}_{2}-\mathrm{CH}_{2}$ - of DHA acyl group.

The total fatty acid concentration could be determined as the sum of the signals at $0.97 \mathrm{ppm}(\omega-3$ FAs) and $0.88 \mathrm{ppm}$. From this value, we could calculate the concentrations of UFAs, saturated fatty acids (SFAs), and PUFAs.

To validate our quantification method, we analyzed five mixtures of five lipid standards (triglycerides C17:0; FC; oleate cholesterol; DHA; and linoleic acid) and five mixtures of three phospholipid standards (PC, PE, and SM) at different concentrations (Table S3). These 10 mixtures represented most of the common signals in ${ }^{1} \mathrm{H}$ NMR spectra of lipophilic extracts from liver samples. Annotated NMR spectra of mixture of lipid standards (mix3) and mixture of phospholipid standards (mix6) are presented in Figures S1 and S2 respectively. The concentrations of lipid species were determined by calibrating to the internal and external standards in the two organic solvents. Tables 1 and 2 list the correlations between the concentrations obtained with NMR signal integration and their respective real concentrations, obtained by internal or external standard calibrations. The corresponding scatter plots and linear regressions are available in Figures S3 and S4 (TSP and $\mathrm{CDCl}_{3}$ ), Figures S5 and S6 (TMS and $\mathrm{CDCl}_{3}$ ), Figures S7 and S8 (TSP and $\mathrm{CDCl}_{3}-\mathrm{CD}_{3} \mathrm{OD}$ mixture), and 
Figures $\mathrm{S} 9$ and $\mathrm{S} 10$ (TMS and $\mathrm{CDCl}_{3}-\mathrm{CD}_{3} \mathrm{OD}$ mixture). All hypotheses underlying the linear regression (linearity, independence, homogeneity) were validated by the residue analysis. The results showed that the external standard and the solvent mixture $\mathrm{CDCl}_{3} / \mathrm{CD}_{3} \mathrm{OD}(4: 1)$ provided the best correlations $(r>0.9)$ and linearity in the regression analyses (i.e., slopes that approximated 1$).$

Table 1. Pearson's $r$ correlations and regression slopes indicate the similarity between NMR quantifications and real lipid concentrations for lipid mixtures dissolved in the solvent, $\mathrm{CDCl}_{3}$.

\begin{tabular}{ccccccc}
\hline \multirow{2}{*}{ Lipid Species } & \multicolumn{3}{c}{ External Standard (TSP) } & \multicolumn{3}{c}{ Internal Standard (TMS) } \\
\cline { 2 - 6 } & Pearson's r & $p$-value ${ }^{\mathbf{a}}$ & Slope & Pearson's r $^{\text {r }}$ & $p$-value ${ }^{\text {a }}$ & Slope \\
\hline Total FA & 0.98 & 0.002 & 1.3 & 0.82 & 0.089 & 0.8 \\
Saturated FA & 0.98 & 0.002 & 1.2 & 0.97 & 0.006 & 1.0 \\
$\omega$-3 FA & 0.98 & 0.002 & 1.0 & 0.94 & 0.019 & 1.8 \\
MUFA & 0.98 & 0.001 & 1.2 & 0.93 & 0.019 & 2.0 \\
PUFA & 0.92 & 0.03 & 0.8 & 0.95 & 0.015 & 1.7 \\
UFA & 0.94 & 0.017 & 0.8 & 0.95 & 0.014 & 1.7 \\
DHA & 0.99 & 0.0001 & 1.1 & 0.93 & 0.024 & 1.9 \\
Linoleic acid & 0.98 & 0.002 & 0.9 & 0.93 & 0.025 & 1.7 \\
TC & 0.99 & 0.0003 & 1.2 & 0.89 & 0.04 & 2.5 \\
FC & 0.95 & 0.011 & 1.0 & 0.89 & 0.04 & 1.7 \\
CE & 0.99 & 0.00007 & 0.8 & 0.98 & 0.004 & 1.4 \\
Triglycerides & 0.99 & 0.001 & 1.2 & 0.98 & 0.004 & 1.0 \\
PC & 0.95 & 0.012 & 1.2 & 0.96 & 0.009 & 1.2 \\
PE & 0.55 & 0.33 & 0.1 & 0.99 & 0.0001 & 0.2 \\
SM & 0.94 & 0.018 & 0.8 & 0.98 & 0.002 & 0.9 \\
Total PL & 0.91 & 0.03 & 0.9 & 0.95 & 0.015 & 1.0 \\
\hline
\end{tabular}

${ }^{\mathrm{a}} \mathrm{p}$ value of the Pearson test (H0: $\left.r=0\right)$; Abbreviations: TSP, 3-(trimethylsilyl)propionic acid sodium salt; TMS, tetramethylsilane; FA, fatty acids; MUFA, monounsaturated fatty acids; PUFA, monounsaturated fatty acids; UFA, unsaturated fatty acids; DHA, docosahexaenoic acid; TC, total cholesterol; FC, free cholesterol; CE, cholesterol ester; PC, phosphatidylcholine; PE, phosphatidylethanolamine; SM, sphingomyelin; PL, phospholipids.

Table 2. Pearson's r correlations and regression slopes indicate the similarity between NMR quantifications and real lipid concentrations for lipid mixtures dissolved in the mixture of solvents, $\mathrm{CDCl}_{3} / \mathrm{CD}_{3} \mathrm{OD}(4: 1)$.

\begin{tabular}{ccccccc}
\hline \multirow{2}{*}{ Lipid Species } & \multicolumn{3}{c}{ External Standard (TSP) } & \multicolumn{3}{c}{ Internal Standard (TMS) } \\
\cline { 2 - 6 } & Pearson's r & $p$-value a & Slope & Pearson's r & $p$-value a & Slope \\
\hline Total FA & 0.99 & $5 \times 10^{-5}$ & 1.0 & 0.98 & $2 \times 10^{-3}$ & 1.2 \\
Saturated FA & 0.99 & $2 \times 10^{-5}$ & 1.0 & 0.98 & $2 \times 10^{-3}$ & 1.2 \\
$\omega$-3 FA & 0.99 & $5 \times 10^{-5}$ & 1.0 & 0.98 & $2 \times 10^{-3}$ & 1.0 \\
MUFA & 0.99 & $3 \times 10^{-5}$ & 1.1 & 0.99 & $4 \times 10^{-4}$ & 1.0 \\
PUFA & 0.99 & $5 \times 10^{-4}$ & 1.0 & 0.97 & $6 \times 10^{-3}$ & 1.5 \\
UFA & 0.99 & $3 \times 10^{-4}$ & 1.0 & 0.97 & $5 \times 10^{-3}$ & 1.4 \\
DHA & 0.99 & $1 \times 10^{-5}$ & 1.1 & 0.99 & $4 \times 10^{-5}$ & 0.7 \\
Linoleic acid & 0.99 & $8 \times 10^{-6}$ & 0.9 & 0.99 & $2 \times 10^{-4}$ & 1.0 \\
TC & 0.98 & $2 \times 10^{-3}$ & 1.1 & 0.96 & $9 \times 10^{-3}$ & 1.6 \\
FC & 0.99 & $6 \times 10^{-4}$ & 1.0 & 0.93 & $2 \times 10^{-2}$ & 1.5 \\
CE & 0.99 & $8 \times 10^{-4}$ & 0.9 & 0.98 & $2 \times 10^{-3}$ & 0.9 \\
Triglycerides & 0.99 & $3 \times 10^{-6}$ & 1.0 & 0.99 & $7 \times 10^{-4}$ & 1.0 \\
PC & 0.99 & $5 \times 10^{-5}$ & 1.2 & 0.99 & $2 \times 10^{-4}$ & 1.1 \\
PE & 0.98 & $3 \times 10^{-3}$ & 0.6 & 0.91 & $3 \times 10^{-2}$ & 0.5 \\
SM & 0.99 & $8 \times 10^{-4}$ & 0.9 & 0.96 & $8 \times 10^{-3}$ & 0.8 \\
Total PL & 0.99 & $3 \times 10^{-5}$ & 1.0 & 0.99 & $2 \times 10^{-5}$ & 1.2 \\
\hline
\end{tabular}

${ }^{a}$ pvalue of the Pearson test (H0: $r=0$ ); Abbreviations: TSP, 3-(trimethylsilyl)propionic acid sodium salt; TMS, tetramethylsilane; FA, fatty acids; MUFA, monounsaturated fatty acids; PUFA, monounsaturated fatty acids; UFA, unsaturated fatty acids; DHA, docosahexaenoic acid; TC, total cholesterol; FC, free cholesterol; CE, cholesterol ester; PC, phosphatidylcholine; PE, phosphatidylethanolamine; SM, sphingomyelin; PL, phospholipids. 
These results also suggested that TMS was unsuitable for quantitative analyses and that the nature of the solvent was important. Phospholipids contain polar headgroups and nonpolar fatty acyl residues, which lead to line broadening in ${ }^{1} \mathrm{H}-\mathrm{NMR}$ spectroscopy; thus, the quantification was not accurate. Accordingly, we used an external standard and the solvent mixture, $\mathrm{CDCl}_{3} / \mathrm{CD}_{3} \mathrm{OD}(4: 1 \mathrm{v} / \mathrm{v})$, for the biological study.

\subsection{Analytical Validation with Liver Samples in a Dietary Intervention Study}

We evaluated the use of ${ }^{1} \mathrm{H}-\mathrm{NMR}$ spectroscopy for quantifying aqueous and lipidic metabolites in liver samples from mice fed the following diets: COCO (deficient in essential fatty acids, with 5\% saturated FA-rich oil), REF (balanced diet with 5\% REF oil), and FISH (n-3 PUFA-enriched diet, with 5\% PUFA-rich oil). Lipids were quantified with ${ }^{1} \mathrm{H}-\mathrm{NMR}$ spectroscopy and an external standard dissolved in the mixture of solvents, $\mathrm{CDCl}_{3} / \mathrm{CD}_{3} \mathrm{OD}(4: 1 \mathrm{v} / \mathrm{v})$.

\subsubsection{Comparison with GC-FID Data}

We compared ${ }^{1} \mathrm{H}-\mathrm{NMR}$ spectroscopy results to those obtained with GC with a flame ionization detector (GC-FID). We took the GC-FID data as the reference method for quantifying fatty acids and neutral lipids. Table 3 shows the correlation between NMR quantifications (expressed in nmol/mg of liver) and GC-FID quantifications. The correlations were good for all tested lipids ( $\mathrm{r}>0.8, p<0.01)$, except for ARA+EPA.

Table 3. Pearson's r correlations indicate the similarity between NMR and GC-FID quantifications of lipid concentrations in livers of mice in the dietary study.

\begin{tabular}{ccc}
\hline Lipid Species & Pearson's r & $p$-value \\
\hline a \\
\hline Total FA & 0.93 & $8.8 \times 10^{-8}$ \\
Saturated FA & 0.85 & $1.43 \times 10^{-5}$ \\
w-3 FA & 0.80 & $1.1 \times 10^{-4}$ \\
MUFA & 0.96 & $4.5 \times 10^{-10}$ \\
PUFA & 0.80 & $9.6 \times 10^{-5}$ \\
ARA+EPA & 0.69 & $2 \times 10^{-3}$ \\
DHA & 0.95 & $3.4 \times 10^{-9}$ \\
Linoleic acid & 0.96 & $6.7 \times 10^{-10}$ \\
MUFA/PUFA & 0.89 & $1.3 \times 10^{-6}$ \\
Total cholesterol & 0.99 & $1.2 \times 10^{-14}$ \\
Free cholesterol & 0.91 & $1.3 \times 10^{-6}$ \\
Cholesterol ester & 0.98 & $6.6 \times 10^{-12}$ \\
Triglycerides & 0.98 & $7.7 \times 10^{-12}$ \\
\hline
\end{tabular}

a $\mathrm{p}$ value of the Pearson test (H0: $r=0)$; Abbreviations: FA, fatty acids; MUFA, monounsaturated fatty acids; PUFA, polyunsaturated fatty acids; DHA, docosahexaenoic acid.

\subsubsection{Comparison with LC-MS Data}

The quantification of phospholipids with LC-MS provided relative values, due to the unavailability of standards for calibrating LC-MS quantifications. Thus, for phospholipids, we could only compare relative differences between diets. Table 4 shows the ratios of phospholipid concentrations between the test and reference diets. These ratios were calculated with results from the ${ }^{1} \mathrm{H}-\mathrm{NMR}$ and LC-MS analyses to compare the two methods. The relative error between LC-MS and NMR values were smaller than $6 \%$ for PE, PC+LPC and total PC except for SM (10\%). We can assume that the quantification ratios provided by the ${ }^{1} \mathrm{H}-\mathrm{NMR}$ analyses were similar to the quantification ratios provided by the LC-MS analyses. For SM ratio, the relative error was larger because the NMR signal was weak. 
Table 4. Comparison between ${ }^{1} \mathrm{H}-\mathrm{NMR}$ and LC-MS methods for determining ratios of phospholipid concentrations in livers from mice fed different diets in the dietary study.

\begin{tabular}{ccccccccc}
\hline \multirow{2}{*}{ Concentration Ratio } & \multicolumn{2}{c}{ PE } & \multicolumn{2}{c}{ PC + LPC } & \multicolumn{2}{c}{ SM } & \multicolumn{2}{c}{ Total PL, Except LPC } \\
\cline { 2 - 9 } & LC-MS & NMR & LC-MS & NMR & LC-MS & NMR & LC-MS & NMR \\
\hline COCO/REF & 0.94 & 0.95 & 1.07 & 1.01 & 0.90 & 0.81 & 1.05 & 1.03 \\
FISH/REF & 1.24 & 1.17 & 1.13 & 1.06 & 1.00 & 1.10 & 1.12 & 1.08 \\
\hline
\end{tabular}

Abbreviations: COCO, a diet with 5\% saturated FA-rich oil; REF: a diet with 5\% reference oil (control); FISH: a diet with 5\% n-3 long-chain PUFA-rich oil; PE, phosphatidylethanolamine; PC, phosphatidylcholine; LPC, lysophosphatidylcholine; PL, phospholipids;.

\subsubsection{Comparison with LipSpin Results}

LipSpin is a bioinformatic tool for the automatic quantification of lipid species, which uses ${ }^{1} \mathrm{H}$ NMR spectra of biological matrices [36]. Lipid quantifications rely on line-shape fitting analyses of spectral regions, from which individual signal areas are obtained. The user can select a signal pattern optimized for blood serum, which is provided by the algorithm; however, we designed our own signal pattern and imported our standard spectra, because our experimental conditions were different (i.e., solvents and acquisition parameters). This tool is user-friendly, fast, and requires only the import of NMR spectra. LipSpin provided the integration values for SFA, $\omega-3$ FA, MUFA, ARA+EPA, linoleic acid, FC, EC, triglyceride, PL, PE, SM, PC, and LPC signals. The concentration was calculated for each lipid species based on the total cholesterol concentration determined with GC-FID. Table 5 shows the correlations between LipSpin quantifications (expressed in nmol/mg liver) and our NMR quantifications. We found good correlations for $\omega-3$ FA, MUFA, linoleic acid, EC, and triglycerides $(\mathrm{r}>0.8, p<0.01)$, but low correlation values were obtained for other lipid species.

Table 5. Pearson's r correlations between LipSpin and NMR quantifications of lipid concentrations in livers from mice fed different diets in the dietary study.

\begin{tabular}{ccc}
\hline Lipid Species & Pearson's r & $p$-value ${ }^{\mathbf{a}}$ \\
\hline Saturated FA & 0.66 & $5.7 \times 10^{-3}$ \\
$\omega$-3 FA & 0.98 & $7.4 \times 10^{-12}$ \\
MUFA & 0.97 & $3.0 \times 10^{-10}$ \\
ARA+EPA & 0.001 & 0.99 \\
DHA & 0.31 & 0.24 \\
Linoleic acid & 0.95 & $2.8 \times 10^{-8}$ \\
Free cholesterol & 0.54 & 0.031 \\
Esterified cholesterol & 0.80 & $1.8 \times 10^{-4}$ \\
Triglycerides & 0.97 & $9.2 \times 10^{-10}$ \\
Total phospholipids & 0.27 & 0.32 \\
PE & 0.58 & 0.017 \\
SM & 0.14 & 0.59 \\
PC+LPC & 0.37 & 0.15 \\
\hline
\end{tabular}

${ }^{a} \mathrm{p}$ value of the Pearson test (H0: $\left.r=0\right)$; Abbreviations: FA, fatty acids; MUFA, monounsaturated fatty acids; ARA, arachidonic acid; EPA, eicosapentaenoic acid; DHA, docosahexaenoic acid; PE, phosphatidylethanolamine; SM, sphingomyelin; PC, phosphatidylcholine; LPC, lysophosphatidylcholine. 


\subsubsection{Biological Results}

Figure 5 shows the mean concentrations of lipid species in the livers of mice fed each diet obtained from ${ }^{1} \mathrm{H}-\mathrm{NMR}$ spectra. Lipid modifications were in good agreement with the results previously obtained with the standard GC-FID method [37]. The ${ }^{1} \mathrm{H}-\mathrm{NMR}$ and GC-FID methods also showed comparable significant increases in triglycerides and CE in mice fed the COCO diet compared to mice fed the REF diet. In addition, the ${ }^{1} \mathrm{H}-\mathrm{NMR}$ method highlighted significant increases in fatty acids, MUFAs, and SFAs, and significant decreases in PUFAs (DHA, EPA, linoleic acid, and $\omega-3$ FA) in mice fed the COCO diet compared to mice fed the REF diet. Moreover, we observed significant increases in DHA and $\omega-3$ FAs in mice fed the FISH diet compared to mice fed the REF diet. Although we did not compare the results on aqueous metabolites with results obtained with other methods, we found no significant changes in aqueous metabolites among the three diets, based on absolute concentrations.

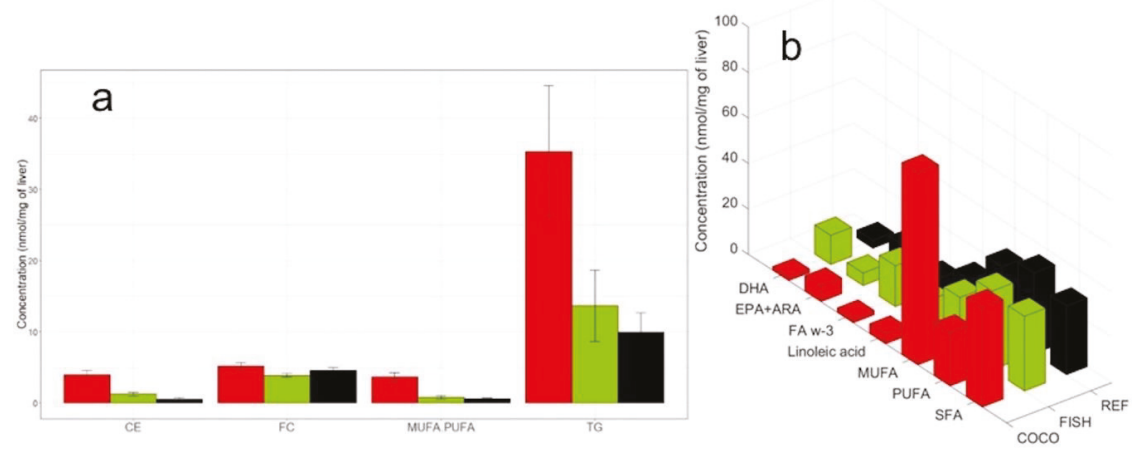

Figure 5. The quality of dietary fatty acids (FAs) affected hepatic lipid composition. Mouse liver lipid compositions were measured after 12-week diets of COCO (red) or FISH (green), compared to a reference (black) diet as revealed by ${ }^{1} \mathrm{H}-\mathrm{NMR}$ spectra. (a) Liver cholesterol (CE and FC) contents, triglyceride (TG) contents, and MUFA/PUFA ratios. (b) Liver fatty acid contents. Abbreviations: COCO, a diet with 5\% saturated FA-rich oil; REF, a diet with 5\% reference oil; FISH, a diet with 5\% n-3 long-chain PUFA-rich oil; CE, cholesterol ester; FC, free cholesterol; MUFA, monounsaturated FAs; PUFA, polyunsaturated FAs; TG, triglycerides; DHA, docosahexaenoic acid; EPA, eicosapentaenoic acid; ARA, arachidonic acid; FA w-3, omega-3 FAs; SFA, saturated FAs.

Absolute quantification of metabolites (i.e., both aqueous and lipidic metabolites) requires knowing the number of protons under each signal, which is given after all NMR signals are identified. Thus, it is important to work with well-resolved signals with no overlap to ensure the integration of pure signals. Because it was difficult to identify all the signals in a NMR spectrum of a biological sample, due to numerous potential line overlaps, we applied the binning method to NMR data, so as to use the entire spectrum to compare metabolic profiles between the three groups of animals.

To compare the metabolic profiles of livers from mice fed different diets, we performed a partial least squares-discriminant analysis (PLS-DA), based on the ${ }^{1} \mathrm{H}-\mathrm{NMR}$ spectra. Mice were fed COCO, REF, and FISH diets. For the lipidic extracts, mice fed the COCO diet were well separated on the first component from mice fed the REF and FISH diets (Figure 6a). This component could explain 47.7\% of the variability. In addition, mice fed the FISH diet were separated from mice fed the REF diet on the second component, which explained $38.9 \%$ of the variability. For aqueous extracts, prior to PLS-DA modeling, we applied an orthogonal signal correction to the data to filter out variations that were unrelated to the diet. Figure $6 \mathrm{~b}$ shows that mice fed the COCO or FISH diets were clearly separated from mice fed the REF diet on the first component, which explained $42.1 \%$ of the variability. We found eight lipid species and fifteen aqueous metabolites that were responsible for the separation between 
diets (Table 6). From binning data, we observed significant increases in triglyceride, and MUFA levels in mice fed the COCO diet compared to mice fed the REF diet. We also observed changes in aqueous metabolites between the COCO and REF diets. Compared to the REF diet, the COCO diet caused significant increases in 3-hydroxybutyrate, alanine, glycerophosphocholine, inosine, lactate, leucine, phenylalanine, succinate, threonine, tyrosine, and valine and a significant decrease in glucose (Table 6).
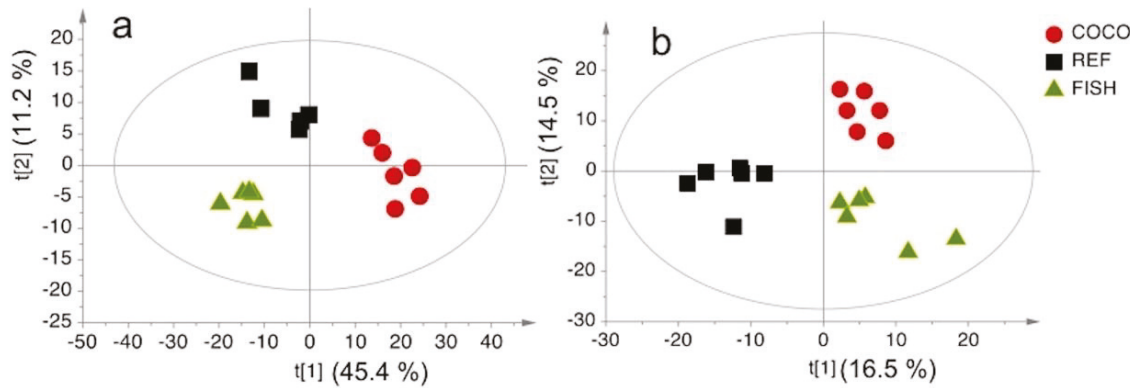

Figure 6. Partial Least Squares-Discriminant Analysis score plot for ${ }^{1} \mathrm{H}-\mathrm{NMR}$ data. (a) Liver lipidic extracts $\left(R^{2}=98.5 \%\right.$; $\left.Q^{2}=0.884\right)$; (b) liver aqueous extracts (orthogonal signal correction filtration, $53.4 \%$ of the remaining variability, $\mathrm{R}^{2}=98.7 \% ; \mathrm{Q}^{2}=0.883$ ). Ellipses indicate the $95 \%$ confidence regions. Abbreviations: COCO, a diet with 5\% saturated FA-rich oil; REF, a diet with 5\% reference oil; FISH, a diet with 5\% n-3 long-chain PUFA-rich oil.

Table 6. Fold-change of discriminants metabolites from binning data in mouse liver extracts induced by the COCO and FISH diets compared to the REF diet.

\begin{tabular}{ccc}
\hline Metabolites $^{\text {a }}$ & FC $^{\text {b }}$ COCO & FC $^{\mathbf{b}}$ FISH \\
\hline FA $\left(\mathrm{CH}_{2}\right)_{\mathrm{n}}$ & $0.71^{*}$ & 0.82 \\
EPA+ARA & $0.67^{*}$ & 0.80 \\
FA CH3 & $0.73^{*}$ & 0.80 \\
Linoleic acid & $0.28^{*}$ & 0,92 \\
MUFA & $1.99^{*}$ & 0.84 \\
PC+LPC+SM & $0.61^{*}$ & 0.94 \\
PL (Except LPC) & $0.65^{*}$ & 1.15 \\
TG & $1.63^{*}$ & 0.98 \\
3-Hydroxybutyrate & $1.21^{*}$ & 1.10 \\
Alanine & $1.30^{*}$ & 0.97 \\
Choline & 1.02 & $1.68^{*}$ \\
Glucose & $0.88^{*}$ & $0.88^{*}$ \\
Glutamine & 1 & $1.18^{*}$ \\
Glutathione & 1.07 & $1.30 *$ \\
GPC & $1.39^{*}$ & $1.61 *$ \\
Inosine & $1.24^{*}$ & 1 \\
Lactate & $1.27^{*}$ & $1.14 *$ \\
Leucine & $1.23^{*}$ & $1.11 *$ \\
Phenylalanine & $1.32^{*}$ & 1.15 \\
Succinate & $1.50^{*}$ & $1.38^{*}$ \\
Threonine & $1.28^{*}$ & 1.03 \\
Tyrosine & $1.47^{*}$ & $1.28^{*}$ \\
Valine & $\uparrow 1.21^{*}$ & 1.07
\end{tabular}

a Metabolites that were significantly different between groups; relative integrations of buckets were compared between groups with the Kruskal-Wallis test and a multiple test correction $(p<0.05)$. ${ }^{b} \mathrm{FC}$, Fold Change $=$ test diet/control diet. * indicates a significative difference between diets. Abbreviations: COCO, a diet with $5 \%$ saturated FA-rich oil; REF, a diet with 5\% reference oil; FISH, a diet with 5\% n-3 long-chain PUFA-rich oil; FA, fatty acids; EPA, eicosapentaenoic acid; ARA, arachidonic acid; MUFA, monounsaturated FAs; PC, phosphatidylcholine; LPC, lysophosphatidylcholine; SM, sphingomyelin; PL, phospholipids; TG, triglycerides; GPC, glycerophosphocholine. 


\section{Discussion}

\subsection{Lipid Quantification: Comparison to Other Methods}

In this study, we evaluated the usefulness of an NMR method for quantifying lipid species, based on peak integration and a linear combination of integrals with a standard. We started by optimizing the standard (either internal or external), the organic solvent in which the lipid species would be dissolved, and a mixture of standard lipids. We found that an external standard, TSP, dissolved in $\mathrm{D}_{2} \mathrm{O}$ in a coaxial tube provided better results than the internal standard, which was TMS dissolved in the sample. Because TMS is a volatile compound, it was difficult to determine its exact concentration after completing the sample preparation process. With an external standard, quantification was more accurate, because there was no interaction between the standard solution in the coaxial tube and the molecules in the sample. In previous studies, several organic solvents were used to analyze lipidic extracts with $\mathrm{NMR}$, including $\mathrm{CDCl}_{3}$ alone [38] or mixtures of solvents, such as $\mathrm{CDCl}_{3} / \mathrm{CD}_{3} \mathrm{OD}$ [17] or $\mathrm{CDCl}_{3} / \mathrm{CD}_{3} \mathrm{OD} / \mathrm{D}_{2} \mathrm{O}-\mathrm{EDTA}$ [39]. Because phospholipids contain polar headgroups and nonpolar fatty acyl residues, they form bilayers in an aqueous environment and 'inverse' micelles in an organic solvent, which are characterized by line broadening in ${ }^{1} \mathrm{H}-\mathrm{NMR}$ spectroscopy. When a solvent mixture contains both nonpolar and polar solvents, stable micelles are formed, which results in well resolved NMR signals and more accurate quantification [39].

The NMR method developed here for lipid quantification provided good correlations with the quantifications of different lipid classes determined with GC-FID and LC-MS. Both GC-FID and LC-MS are time-consuming, because they require an extraction and analysis method per class of lipids, as well as calibration curves for absolute quantification. In ${ }^{1} \mathrm{H}-\mathrm{NMR}$ spectroscopy, deconvolution or line fitting is useful for overlapping signals. We compared our ${ }^{1} \mathrm{H}-\mathrm{NMR}$ spectroscopy method with the LipSpin method for the quantification of lipid species [36]. The LipSpin tool is user-friendly, fast, requires only the import of NMR spectra, and it automatically provides the integration value for each lipid species. We obtained good correlations between our method and the LipSpin method for $\omega-3$ FA, MUFA, linoleic acid, EC, and triglycerides. For the other lipid species, the correlations between quantification values obtained with the two methods were not very good, likely due to the lower resolution of our spectra; indeed, some peaks, like PC, LPC, and SM, were not well resolved. In addition, we had not optimized all the parameters available in the LipSpin tool.

\subsection{Metabolic Differences between Livers of Mice Fed an Essential Fatty Acid-Deficient Diet or a Control Diet}

In this study, we investigated the metabolic disturbances that occurred during the development of steatosis induced by an essential PUFA deficiency. Deficiencies in essential fatty acids are well-known to promote de novo lipogenesis through transcriptional processes [40] involving the transcription factors, SREBP1c [41], ChREBP [42], and LXR [37]. These processes increase the expression and activity of hepatic enzymes involved in de novo lipogenesis (ACC, FAS), elongation (ELOVL6), and desaturation (SCD1). In turn, the elevated activities of these enzymes cause an increase in triglycerides enriched in MUFAs, such as oleic acid (C18:1n-9). Our metabolomics approach revealed that, with the COCO diet, liver triglycerides and MUFAs were up-regulated, as expected, but we also observed up-regulated levels of liver CEs, 3-hydroxybutyrate, alanine, glycerophosphocholine, inosine, lactate, leucine, phenylalanine, succinate, threonine, tyrosine, and valine, compared to mice fed the REF diet. In addition, we observed down-regulated levels of phospholipids and glucose in livers of mice fed the COCO diet compared to mice fed the REF diet. A similar increase in CE was previously reported in lipidic extracts of livers from mice with NASH [43]. That study also highlighted a reduced PUFA to MUFA ratio in NASH, but they observed no difference in triglyceride levels, compared to healthy mice. A reduced PUFA/MUFA ratio is considered a marker of lipid peroxidation in association with oxidative stress. In cancer tissues, such as HCC, a glycolytic shift was observed, with high levels of lactate and low levels of glucose $[23,44]$. Liver mitochondria produce 3-hydroxybutyrate during fatty acid oxidation. High levels of 3-hydroxybutyrate were also found in human HCC tissues. The aromatic 
amino acids, phenylalanine and tyrosine, are oxidized in the TCA cycle, after conversion into fumarate. Therefore, accumulations of aromatic amino acids and 3-hydroxybutyrate suggested that mitochondrial function and inflammatory status were impaired in the livers of mice fed the COCO diet.

The present study also revealed changes in amino acid metabolism associated with the COCO diet. Previous studies have shown that changes in branched chain amino acid levels in human liver occurred in hepatic pathologies, such as NASH or alcohol-related liver damage [45]. We observed elevated hepatic concentrations of leucine, and valine, consistent with previous reports that hepatic amino acid metabolism was abnormally regulated, and branched chain amino acid oxidation was reduced in these pathologies. Elevations in alanine were also described in hepatic pathologies [6].

\subsection{Advantages and Limitations of ${ }^{1} H-N M R$ Spectroscopy for Metabolic Profiling in Liver}

We evaluated a simple protocol for the simultaneous characterization of lipidic and aqueous metabolic profiles in mouse liver tissues. We used NMR spectroscopy to identify and quantify both polar and non-polar metabolites. ${ }^{1} \mathrm{H}-\mathrm{NMR}$ spectroscopy is often used to quantify aqueous metabolites in metabolomic studies, but it has rarely been used for quantifying lipid species. Currently, there is much interest in using this technique for obtaining absolute quantifications of complex lipids, such as phospholipids or sphingolipids, which cannot be obtained with MS. Furthermore, NMR is a non-destructive technique; therefore, the analyzed sample can subsequently be used in MS analyses, which can provide a molecular species characterization for each family. ${ }^{1} \mathrm{H}$ NMR has some drawbacks, such as low sensitivity, signal overlapping, and low resonance discrimination. Nevertheless, this technique can provide a rapid quantitative overview of the major lipid classes (fatty acids, triglycerides, phospholipids, and cholesterol) with a simple, single extraction, without extensive sample preparation, and due to its spectral linearity, without the need of multiple internal standards for quantitative estimations.

\section{Materials and Methods}

\subsection{Animals}

In vivo studies were conducted under E.U. guidelines for the use and care of laboratory animals, and they were approved by an independent Ethics Committee (TOXCOM/0043/NL AP). To address the relative contribution of the quantity and quality of dietary FAs to triglyceride accumulation, we fed 6-week old male C57BL6 mice (Charles River, Les Oncins, France) different diets for 12 weeks ( $n=6$ in each group). The diets were as follows: one contained 5\% saturated FA-rich oil (COCO), a second contained 5\% reference oil (REF), and a third contained 5\% n-3 long-chain PUFA-rich oil (FISH) [37]. At sacrifice, the liver was collected and immediately cut into samples that were snap-frozen in liquid nitrogen and stored at $-80^{\circ} \mathrm{C}$ until use for NMR and GC analysis.

\subsection{Extraction Procedure}

Liver samples (100-120 mg) were homogenized in $1.2 \mathrm{~mL}$ methanol in the Fastprep-24 homogenizer (MP Biomedicals, Irvine, CA, USA). For the comparison of extraction methods, a homogenate that corresponded to $50 \mathrm{mg}$ of tissue was extracted, with modifications, according to the method described by Bligh and Dyer [26], in dichloromethane/methanol/water (2.5:2.5:2.1, v/v/v), and a second homogenate from the same sample that corresponded to $50 \mathrm{mg}$ of tissue was extracted according to the method described by Folch [27] in dichloromethane/ methanol/ $\mathrm{NaCl} 0.9 \%$ in water $(2: 1: 0.2, v / v / v)$. After centrifugation $\left(1000 \times g, 15 \mathrm{~min}, 4^{\circ} \mathrm{C}\right)$, the solutions separated into an upper methanol/water phase (with polar metabolites) and a lower dichloromethane phase (with lipophilic compounds), with an intermediate phase of protein and cellular debris. The aqueous and organic phases were collected and evaporated to dryness. Chloroform was replaced by dichloromethane for security reason [46]. For the dietary intervention study, liver samples (100-120 mg) were homogenized in $1.2 \mathrm{~mL}$ methanol in a Fastprep-24 homogenizer (MP Biomedicals, Irvine, CA, USA). A homogenate that corresponded to 
$50 \mathrm{mg}$ of tissue was extracted as described above for NMR analysis, and two homogenates from the same sample that corresponded to $1 \mathrm{mg}$ of tissue were extracted for GC and LC-MS analyses.

\subsection{GC Analysis of Neutral Lipids and Fatty Acids}

To analyze neutral lipids, we introduced three internal standards ( $3 \mu \mathrm{g}$ of stigmasterol, $3 \mu \mathrm{g}$ of cholesteryl heptadecanoate, and $15 \mu \mathrm{g}$ of glyceryl trinonadecanoate) before extracting lipids from the homogenates. The dichloromethane phases were evaporated to dryness and dissolved in $20 \mu \mathrm{L}$ ethyl acetate. Then, $1 \mu \mathrm{L}$ of the lipid extract was analyzed with GC on a FOCUS-FID system (Thermo Electron, Waltham, MA, USA) equipped with a Zebron-1 fused silica capillary column (Phenomenex, Torrance, CA, USA; $5 \mathrm{~m} \times 0.32 \mathrm{~mm}$ i.d., $0.50 \mu \mathrm{m}$ film thickness) [47]. The oven temperature was programmed to increase from $200{ }^{\circ} \mathrm{C}$ to $350{ }^{\circ} \mathrm{C}$ at a rate of $5{ }^{\circ} \mathrm{C}$ per min, and the carrier gas was hydrogen $(0.5 \mathrm{bar})$. The injector and detector were maintained at $315{ }^{\circ} \mathrm{C}$ and $345^{\circ} \mathrm{C}$, respectively.

To analyze fatty acid methyl ester (FAME), we introduced the internal standard, glyceryl tri heptadecanoate $(2 \mu \mathrm{g})$, before extracting lipids from the homogenates. The lipid extracts were hydrolyzed in $\mathrm{KOH}\left(0.5 \mathrm{M}\right.$ in methanol) at $50{ }^{\circ} \mathrm{C}$ for $30 \mathrm{~min}$, and transmethylated in a $10 \%$ boron trifluoride methanol solution (1 mL, Sigma-Aldrich, St. Louis, MO, USA) and hexane $(1 \mathrm{~mL})$ at $80^{\circ} \mathrm{C}$ for one hour. After adding water $(1 \mathrm{~mL})$ to the crude solution, FAMEs were extracted with hexane $(3 \mathrm{~mL})$, evaporated to dryness, and dissolved in ethyl acetate $(20 \mu \mathrm{L})$. FAMEs $(1 \mu \mathrm{L})$ were analyzed with gas-liquid chromatography [48] on a Clarus 600-FID system (Perkin Elmer, Waltham, MA, USA) equipped with a Famewax fused silica capillary column (RESTEK, Lisses, France; $30 \mathrm{~m} \times 0.32 \mathrm{~mm}$ i.d. $0.25 \mu \mathrm{m}$ film thickness). The oven temperature was programmed to increase from $130^{\circ} \mathrm{C}$ to $220^{\circ} \mathrm{C}$ at a rate of $2{ }^{\circ} \mathrm{C}$ per min, and the carrier gas was hydrogen $(0.5$ bar). The injector and the detector were maintained at $225^{\circ} \mathrm{C}$ and $245^{\circ} \mathrm{C}$, respectively.

\subsection{HPLC-MS Analysis of Phospholipids}

Lipids were extracted from $1 \mathrm{mg}$ of liver with a method adapted from that described by Bligh and Dyer [26]. Extractions were performed in dichloromethane/methanol (2\% acetic acid)/water $(2.5: 2.5: 2 \mathrm{v} / \mathrm{v} / \mathrm{v})$, in the presence of six internal standards, including ceramides (Cer, d18:1/15:0, $16 \mathrm{ng})$; phosphatidylethanolamine (PE 12:0/12:0, $180 \mathrm{ng}$ ); phosphatidylcholine (PC, 13:0/13:0, 16 ng); SM (d18:1/12:0, 16 ng); phosphatidylinositol (PI, 16:0/17:0, 30 ng); and phosphatidylserine (PS, 12:0/12:0, $156.25 \mathrm{ng}$ ). The solution was centrifuged at $1500 \mathrm{rpm}$ for $3 \mathrm{~min}$. The organic phase was collected and dried under nitrogen, then dissolved in $50 \mu \mathrm{l}$ methanol. The extract $(5 \mu \mathrm{L})$ was analyzed with an Agilent 1290 UPLC system coupled to a G6460 triple quadrupole spectrometer (Agilent Technologies, Santa Clara, CA, USA) and equipped with MassHunter software, for data acquisition and analysis. A Kinetex HILIC column (Phenomenex, $50 \times 4.6 \mathrm{~mm}, 2.6 \mu \mathrm{m}$ ) was used for LC separations. The column temperature was controlled at $40^{\circ} \mathrm{C}$. The flow rate of the mobile phase was $0.3 \mathrm{~mL} / \mathrm{min}$. The mobile phase contained two parts: A was acetonitrile; and B was $10 \mathrm{mM}$ ammonium formate in water, $\mathrm{pH}$ 3.2. The gradient was prepared with the following specifications: from $10 \%$ to $30 \% \mathrm{~B}$ in $10 \mathrm{~min}$; $10-12 \mathrm{~min}$ in $100 \% \mathrm{~B}$; and then, at $13 \mathrm{~min}$, back to $10 \% \mathrm{~B}$ for 1-min re-equilibrium, prior to the next injection. An electrospray source was employed in positive ion mode for Cer, PE, PC, and SM analyses and in negative ion mode for PI and PS analyses. The collision gas was nitrogen. The needle voltage was set at $+4000 \mathrm{~V}$. Several scan modes were used. First, to obtain the natural masses of different species, we analyzed cellular lipid extracts with precursor ion scans of $184 \mathrm{~m} / \mathrm{z}, 241 \mathrm{~m} / \mathrm{z}$, and $264 \mathrm{~m} / \mathrm{z}$ for PC/SM, PI, and Cer, respectively; and neutral loss scans of 141 and 87 for PE and PS, respectively. The collision energy optimums for Cer, PE, PC, SM, PI, and PS were $25 \mathrm{eV}, 20 \mathrm{eV}, 30 \mathrm{eV}, 25 \mathrm{eV}, 45 \mathrm{eV}$, and $22 \mathrm{eV}$, respectively. Then, the corresponding SRM transitions were used to quantify different phospholipid species in each class. Two MRM acquisitions were necessary, due to important differences between phospholipid classes. Data were analyzed with QqQ Quantitative (vB.05.00) and Qualitative analysis software (vB.04.00). 


\section{5. ${ }^{1} \mathrm{H}-\mathrm{NMR}$ Measurements}

All NMR experiments were performed on a Bruker Avance spectrometer (Bruker Biospin, Rheinstetten, Germany), operating at a proton frequency of $600.13 \mathrm{MHz}$, with an inverse detection $5-\mathrm{mm}{ }^{1} \mathrm{H}-{ }^{13} \mathrm{C}-{ }^{15} \mathrm{~N}$ cryoprobe attached to a Cryoplatform (the preamplifier unit).

Dry lipid extracts were reconstituted in $500 \mu \mathrm{CDCl}_{3} / \mathrm{CD}_{3} \mathrm{OD}(4: 1, v / v)$ and transferred into 5-mm NMR tubes. ${ }^{1} \mathrm{H}-\mathrm{NMR}$ spectra were recorded in the presence of a reusable coaxial capillary tube that contained $120 \mu \mathrm{l}$ TSP $(1.17 \mathrm{mM})$ in $\mathrm{D}_{2} \mathrm{O}$, which also served as an internal standard for quantitative estimations. Dry aqueous extracts were reconstituted in $600 \mu \mathrm{l} \mathrm{D} \mathrm{O}_{2}$ containing TSP $(0.70 \mathrm{mM})$ and transferred into 5-mm NMR tubes.

A T1 (spin-lattice relaxation) measurement experiment was performed on the entire liver lipid extract (with TSP solution in the coaxial capillary as reference) in a sealed tube using an inversion-recovery sequence. The T1 values for TC (0.68 ppm), FC (1.01 ppm), CE (1.02 ppm), TG (5.27 ppm), TG (4.16 and $4.32 \mathrm{ppm}), \mathrm{PL}$ (5.23 ppm), UFA (5.35 ppm), linoleic acid (2.76 ppm), PUFA (2.82 ppm), FA (0.88 ppm), w3 UFA (0.98 ppm), MUFA (2.02 ppm), ARA+EPA (2.10 ppm), DHA (2.38 ppm) and TSP were 0.792, 0.792, 0.792, 1.44, 0.576, 1.44, 2.88, 1.58, 1.73, 3.17, 3.17, 1.44, 1.44, 1.15 and $3.45 \mathrm{~s}$, respectively.

${ }^{1} \mathrm{H}-\mathrm{NMR}$ spectra of liver lipid extracts were obtained with a one-pulse sequence, with a spectral width of 10 ppm, and the time domain data had 32,000 data points. The flip angle of the radio-frequency pulse was $30^{\circ}$, and the total relaxation delay was $15 \mathrm{~s}$ to ensure complete recovery of the magnetization between scans of the lipid components and for the external reference TSP. For each sample, 256 scans were accumulated, and data were Fourier-transformed, after multiplying by an exponential window function with a line-broadening function of $0.3 \mathrm{~Hz}$ to the free induction decays (FIDs).

${ }^{1} \mathrm{H}-\mathrm{NMR}$ spectra of aqueous liver extracts were acquired at $300 \mathrm{~K}$ with a conventional presaturation pulse sequence for water suppression, based on the first increment of the nuclear Overhauser effect spectroscopy (NOESY) pulse sequence. Solvent presaturation was applied during a recycling delay and mixing time $(100 \mathrm{~ms})$ to suppress residual water. A total of 256 transients were collected into 32,000 data points with a spectral width of $12 \mathrm{ppm}$ and a relaxation delay of $15 \mathrm{~s}$. Prior to the Fourier transform procedure, we applied an exponential line-broadening of $0.3 \mathrm{~Hz}$ to the FIDs.

\subsection{Data Processing and Multivariate Analysis}

NMR spectra were phase- and baseline-corrected, then calibrated (TSP, 0.0 ppm for aqueous extracts and TC, $0.68 \mathrm{ppm}$ for lipidic extracts) with Topspin software (version 2.1, Bruker). Next, NMR data were reduced with AMIX software (version 3.9, Bruker) to integrate 0.01 ppm-wide regions that corresponded to the $\delta 10.0-0.5 \mathrm{ppm}$ and the $\delta 6.4-0.6 \mathrm{ppm}$ regions for aqueous and lipidic extracts, respectively. The 5.1-4.5 ppm region, which included water resonance, was excluded in the NMR spectra of aqueous extracts. We included 757 and 552 NMR buckets in the data matrices for aqueous and lipidic extracts, respectively. To account for differences in sample volumes, each integration region was normalized to the total spectral area.

Multivariate pattern-recognition techniques were applied to study the effects of diet on the metabolome. First, we performed a PCA to reveal intrinsic clusters and detect eventual outliers. We then performed a PLS-DA to model the relationship between diet and NMR data. For aqueous extracts, prior to PLS-DA modeling, we applied orthogonal signal correction [49] to remove confounding variability; i.e., variability that was not linked to the diet (e.g., physiological, experimental, or instrumental variability). Data were Pareto-scaled (the square root of the standard deviation is used as the scaling factor). For all plots of scores, we performed Hotelling's T2 statistics to construct $95 \%$ confidence ellipses. The $\mathrm{R}^{2} \mathrm{Y}$ parameter represented the explained variance. Seven-fold cross validation was used to determine the number of latent variables that should be included in the PLS-DA model and to estimate the predictive ability (or predicted variance, $\mathrm{Q}^{2}$ parameter) of the fitted model. PLS-DA models with $\mathrm{Q}^{2}$ values higher than 0.4 were considered valid [50]. In addition, the robustness of PLS-DA models was assessed with a permutation test (number of permutations $=200$ ). In the 
permutation plot, a $\mathrm{Q}^{2}$ intercept $<0.05$ indicated a robust model [51]. Discriminant variables were determined with the Variable Importance in the Projection (VIP) value, a global measure of the influence of each variable in the PLS components. Variables with VIPs $>1$ were considered discriminants. Finally, we tested the significance of relative integration differences between groups, based on the non-parametric Kruskal-Wallis test. The false discovery rate (FDR) was applied to account for multiple testing. NMR variables that showed FDR-adjusted $p$-values $<0.05$ were considered significantly different. We used SIMCA-P software (V13, Umetrics, Umea, Sweden) to perform the multivariate analyses, and we used R (https://www.r-project.org/) for univariate testing.

\subsection{Statistical Analysis}

We performed analytical validations of ${ }^{1} \mathrm{H}-\mathrm{NMR}$ lipid quantifications by comparing the results with analogous measurements obtained with other methods. Comparisons were performed with linear regression and Pearson's (r) correlation.

Linear regression analyses were used to compare known concentrations of lipidic species and signal integrations obtained with ${ }^{1} \mathrm{H}-\mathrm{NMR}$. A known concentration was used as the independent variable, and the NMR-predicted concentration was used as the response variable. Hypotheses of linear regression were assessed based on the residuals: the Durbin-Watson, Shapiro-Wilks, and Breush-Pagan tests were applied, respectively, to test for the independence, normality, and homogeneity of residuals.

Pearson's correlation was used to compare LipSpin-computed and NMR-computed quantifications of lipids and to compare GC-FID-computed and NMR-computed quantifications. We set 0.05 as the threshold for significance. Univariate analyses were performed with $\mathrm{R}$ software (https: //www.r-project.org/).

\section{Conclusions}

The current study showed the potential and limitations of ${ }^{1} \mathrm{H}-\mathrm{NMR}$ spectroscopy for quantifying aqueous, and specially, lipid metabolites in the liver. We demonstrated that ${ }^{1} \mathrm{H}-\mathrm{NMR}$ spectra could provide a rapid overview of the major lipid classes and the most abundant aqueous metabolites. To achieve better extractions of aqueous and lipid metabolites and more accurate quantifications, we recommend the Folch extraction method, an external standard, and the $\mathrm{CDCl}_{3} / \mathrm{CD}_{3} \mathrm{OD}$ mixture of solvents. We found that LipSpin was a good alternative for lipid quantification, but the parameters must be optimized. Our metabolomics analysis allowed us to discriminate between livers of mice fed a diet deficient in essential fatty acids from livers of mice fed a balanced diet. The COCO dietary challenge was mainly associated with disturbances in lipid and energy metabolism, accompanied by altered amino acid metabolism.

Supplementary Materials: The following are available online at http://www.mdpi.com/2218-1989/10/1/9/s1, Table S1: Compounds identified in the polar extract of mouse liver tissue from NMR data: assigned chemical shifts and multiplicities, Table S2: Compounds identified in the non-polar extract of mouse liver tissue from NMR data: assigned chemical shifts and multiplicities, Table S3: Composition of lipid mixtures, Table S4: real lipid concentrations and concentrations computed from NMR data for lipid mixtures of Table S3, Table S5: lipid concentrations computed from GC and NMR data in mice livers, Table S6: NMR bucketing table for aqueous liver samples, Table S7: NMR bucketing table for lipidic liver samples, Figure S1: $600 \mathrm{MHz}{ }^{1} \mathrm{H}-\mathrm{NMR}$ spectrum of a mixture of five lipid standards (TG C17:0; FC; oleate cholesterol; DHA; linoleic acid), Figure S2: $600 \mathrm{MHz}$ ${ }^{1} \mathrm{H}-\mathrm{NMR}$ spectrum of a mixture of three lipid standards (PC, PE, SM), Figure S3: Scatter plot and linear regressions of NMR quantifications and lipid concentrations in lipid mixtures of Table S3 dissolved in $\mathrm{CDCl}_{3}$ and using an external standard (TSP) for neutral lipids and phospholipids, Figure S4: Scatter plot and linear regressions of NMR quantifications and lipid concentrations in lipid mixtures of Table S3 dissolved in CDCl3 and using an external standard (TSP) for fatty acids, Figure S5: Scatter plot and linear regressions of NMR quantifications and lipid concentrations in lipid mixtures of Table S3 dissolved in $\mathrm{CDCl}_{3}$ and using an internal standard (TMS) for neutral lipids and phospholipids, Figure S6: Scatter plot and linear regressions of NMR quantifications and lipid concentrations in lipid mixtures of Table S3 dissolved in $\mathrm{CDCl}_{3}$ and using an internal standard (TMS) for fatty acids, Figure S7: Scatter plot and linear regressions of NMR quantifications and lipid concentrations in lipid mixtures of Table $\mathrm{S} 3$ dissolved in $\mathrm{CDCl}_{3}-\mathrm{CD}_{3} \mathrm{OD}$ mixture and using an external standard (TSP) for neutral lipids and phospholipids, Figure S8: Scatter plot and linear regressions of NMR quantifications and lipid concentrations in lipid mixtures of Table $\mathrm{S} 3$ dissolved in $\mathrm{CDCl}_{3}-\mathrm{CD}_{3} \mathrm{OD}$ mixture and using an external standard (TSP) for fatty 
acids, Figure S9: Scatter plot and linear regressions of NMR quantifications and lipid concentrations in lipid mixtures of Table $\mathrm{S} 1$ dissolved in $\mathrm{CDCl}_{3}-\mathrm{CD}_{3} \mathrm{OD}$ mixture and using an internal standard (TMS) for neutral lipids and phospholipids, Figure S10: Scatter plot and linear regressions of NMR quantifications and lipid concentrations in lipid mixtures of Table $\mathrm{S} 1$ dissolved in $\mathrm{CDCl}_{3}-\mathrm{CD}_{3} \mathrm{OD}$ mixture and using an internal standard (TMS) for neutral lipids and phospholipids.

Author Contributions: Conceptualization, L.D., J.B.-M., and H.G.; methodology, C.C.; validation, C.C.; formal analysis, A.A. and M.T.-F.; investigation, A.A., R.G., and C.C.; resources, S.D., A.M., A.P., and H.G.; data curation, A.A. and C.C.; writing—original draft preparation, C.C.; writing—review and editing, H.G., J.B.-M., L.D., M.T.-F., A.M; supervision, L.D.; project administration, L.D. All authors have read and agreed to the published version of the manuscript. All authors have read and agreed to the published version of the manuscript.

Funding: This research was funded by Région Occitanie (H.G., A.P., A.M., S.D.), ANR Hepadialogue ANR-17-CE14-0015 (H.G., A.P., A.M., S.D.), and the French National Infrastructure for Metabolomics and Fluxomics MetaboHUB ANR-11-INBS-010 (C.C., A.A., L.D., M.T.-F., R.G., J.B.-M.).

Conflicts of Interest: The authors declare no conflict of interest

\section{References}

1. Friedman, S.L.; Neuschwander-Tetri, B.A.; Rinella, M.; Sanyal, A.J. Mechanisms of NAFLD development and therapeutic strategies. Nat. Med. 2018, 24, 908-922. [CrossRef] [PubMed]

2. Younossi, Z.; Tacke, F.; Arrese, M.; Chander Sharma, B.; Mostafa, I.; Bugianesi, E.; Wai-Sun Wong, V.; Yilmaz, Y.; George, J.; Fan, J.; et al. Global Perspectives on Nonalcoholic Fatty Liver Disease and Nonalcoholic Steatohepatitis. Hepatology 2019, 69, 2672-2682. [CrossRef] [PubMed]

3. Dumas, M.-E.; Kinross, J.; Nicholson, J.K. Metabolic phenotyping and systems biology approaches to understanding metabolic syndrome and fatty liver disease. Gastroenterology 2014, 146, 46-62. [CrossRef] [PubMed]

4. Mardinoglu, A.; Bjornson, E.; Zhang, C.; Klevstig, M.; Söderlund, S.; Ståhlman, M.; Adiels, M.; Hakkarainen, A.; Lundbom, N.; Kilicarslan, M.; et al. Personal model-assisted identification of NAD+ and glutathione metabolism as intervention target in NAFLD. Mol. Syst. Biol. 2017, 13, 916. [CrossRef] [PubMed]

5. Emwas, A.-H.; Roy, R.; McKay, R.T.; Tenori, L.; Saccenti, E.; Gowda, G.A.N.; Raftery, D.; Alahmari, F.; Jaremko, L.; Jaremko, M.; et al. NMR Spectroscopy for Metabolomics Research. Metabolites 2019, 9, 123. [CrossRef] [PubMed]

6. Le Moyec, L.; Triba, M.N.; Nahon, P.; Bouchemal, N.; Hantz, E.; Goossens, C.; Amathieu, R.; Savarin, P. Nuclear magnetic resonance metabolomics and human liver diseases: The principles and evidence associated with protein and carbohydrate metabolism. Biomed. Rep. 2017, 6, 387-395. [CrossRef] [PubMed]

7. Amathieu, R.; Triba, M.N.; Goossens, C.; Bouchemal, N.; Nahon, P.; Savarin, P.; Le Moyec, L. Nuclear magnetic resonance based metabolomics and liver diseases: Recent advances and future clinical applications. World J. Gastroenterol. 2016, 22, 417-426. [CrossRef]

8. Jiang, L.; Si, Z.H.; Li, M.H.; Zhao, H.; Fu, Y.H.; Xing, Y.X.; Hong, W.; Ruan, L.Y.; Li, P.M.; Wang, J.S. 1H NMR-based metabolomics study of liver damage induced by ginkgolic acid (15:1) in mice. J. Pharm. Biomed. Anal. 2017, 136, 44-54. [CrossRef]

9. Dagla, I.; Benaki, D.; Baira, E.; Lemonakis, N.; Poudyal, H.; Brown, L.; Tsarbopoulos, A.; Skaltsounis, A.L.; Mikros, E.; Gikas, E. Alteration in the liver metabolome of rats with metabolic syndrome after treatment with Hydroxytyrosol. A Mass Spectrometry and Nuclear Magnetic Resonance-based metabolomics study. Talanta 2018, 178, 246-257. [CrossRef]

10. Bonvallot, N.; Canlet, C.; Blas-Y-Estrada, F.; Gautier, R.; Tremblay-Franco, M.; Chevolleau, S.; Cordier, S.; Cravedi, J.P. Metabolome disruption of pregnant rats and their offspring resulting from repeated exposure to a pesticide mixture representative of environmental contamination in Brittany. PLoS ONE 2018, 13, e0198448. [CrossRef]

11. Chen, M.; Zheng, H.; Xu, M.; Zhao, L.; Zhang, Q.; Song, J.; Zhao, Z.; Lu, S.; Weng, Q.; Wu, X.; et al. Changes in hepatic metabolic profile during the evolution of STZ-induced diabetic rats via an $1 \mathrm{H}$ NMR-based metabonomic investigation. Biosci. Rep. 2019, 39. [CrossRef] [PubMed] 
12. Ghosh, S.; Sengupta, A.; Sharma, S.; Sonawat, H.M. Metabolic fingerprints of serum, brain, and liver are distinct for mice with cerebral and noncerebral malaria: $\mathrm{A}^{1} \mathrm{H}$ NMR spectroscopy-based metabonomic study. J. Proteome Res. 2012, 11, 4992-5004. [CrossRef] [PubMed]

13. Ruiz-Rodado, V.; Nicoli, E.R.; Probert, F.; Smith, D.A.; Morris, L.; Wassif, C.A.; Platt, F.M.; Grootveld, M. 1H NMR-Linked Metabolomics Analysis of Liver from a Mouse Model of NP-C1 Disease. J. Proteome Res. 2016, 15, 3511-3527. [CrossRef] [PubMed]

14. Zheng, H.; Cai, A.; Shu, Q.; Niu, Y.; Xu, P.; Li, C.; Lin, L.; Gao, H. Tissue-Specific Metabolomics Analysis Identifies the Liver as a Major Organ of Metabolic Disorders in Amyloid Precursor Protein/Presenilin 1 Mice of Alzheimer's Disease. J. Proteome Res. 2019, 18, 1218-1227. [CrossRef] [PubMed]

15. Fernando, H.; Bhopale, K.K.; Kondraganti, S.; Kaphalia, B.S.; Ansari, G.A.S. Lipidomic Changes in Rat Liver after Long-Term Exposure to Ethanol. Toxicol. Appl. Pharmacol. 2011, 255, 127-137. [CrossRef]

16. Fernando, H.; Bhopale, K.K.; Kondraganti, S.S.; Kaphalia, B.S.; Ansari, G.A.S. Alcohol-Induced Hepatic Steatosis: A Comparative Study to Identify Possible Indicator(s) of Alcoholic Fatty Liver Disease. J. Drug Alcohol Res. 2018, 7. [CrossRef]

17. Fernando, H.; Kondraganti, S.; Bhopale, K.K.; Volk, D.E.; Neerathilingam, M.; Kaphalia, B.S.; Luxon, B.A.; Boor, P.J.; Ansari, G.A.S. 1H and 31P NMR Lipidome of Ethanol-Induced Fatty Liver. Alcohol. Clin. Exp. Res. 2010, 34, 1937-1947. [CrossRef]

18. Cabaton, N.J.; Poupin, N.; Canlet, C.; Tremblay-Franco, M.; Audebert, M.; Cravedi, J.P.; Riu, A.; Jourdan, F.; Zalko, D. An Untargeted Metabolomics Approach to Investigate the Metabolic Modulations of HepG2 Cells Exposed to Low Doses of Bisphenol A and 17ß-Estradiol. Front. Endocrinol. 2018, 9, 571. [CrossRef]

19. Wei, F.; Lamichhane, S.; Orešič, M.; Hyötyläinen, T. Lipidomes in health and disease: Analytical strategies and considerations. TrAC Trends Anal. Chem. 2019, 120, 115664. [CrossRef]

20. Khoury, S.; Canlet, C.; Lacroix, M.Z.; Berdeaux, O.; Jouhet, J.; Bertrand-Michel, J. Quantification of Lipids: Model, Reality, and Compromise. Biomolecules 2018, 8, 174. [CrossRef]

21. Martínez-Granados, B.; Morales, J.M.; Rodrigo, J.M.; Del Olmo, J.; Serra, M.A.; Ferrández, A.; Celda, B.; Monleón, D. Metabolic profile of chronic liver disease by NMR spectroscopy of human biopsies. Int. J. Mol. Med. 2011, 27, 111-117. [CrossRef] [PubMed]

22. Cobbold, J.F.L.; Anstee, Q.M.; Goldin, R.D.; Williams, H.R.T.; Matthews, H.C.; North, B.V.; Absalom, N.; Thomas, H.C.; Thursz, M.R.; Cox, R.D.; et al. Phenotyping murine models of non-alcoholic fatty liver disease through metabolic profiling of intact liver tissue. Clin. Sci. Lond. Engl. 2009, 116, 403-413. [CrossRef] [PubMed]

23. Teilhet, C.; Morvan, D.; Joubert-Zakeyh, J.; Biesse, A.S.; Pereira, B.; Massoulier, S.; Dechelotte, P.; Pezet, D.; Buc, E.; Lamblin, G.; et al. Specificities of Human Hepatocellular Carcinoma Developed on Non-Alcoholic Fatty Liver Disease in Absence of Cirrhosis Revealed by Tissue Extracts ${ }^{1} \mathrm{H}-\mathrm{NMR}$ Spectroscopy. Metabolites 2017, 7, 49. [CrossRef] [PubMed]

24. Vinaixa, M.; Rodríguez, M.A.; Rull, A.; Beltrán, R.; Bladé, C.; Brezmes, J.; Cañellas, N.; Joven, J.; Correig, X. Metabolomic assessment of the effect of dietary cholesterol in the progressive development of fatty liver disease. J. Proteome Res. 2010, 9, 2527-2538. [CrossRef] [PubMed]

25. Lin, C.Y.; Wu, H.; Tjeerdema, R.S.; Viant, M.R. Evaluation of metabolite extraction strategies from tissue samples using NMR metabolomics. Metabolomics 2007, 3, 55-67. [CrossRef]

26. Bligh, E.G.; Dyer, W.J. A rapid method of total lipid extraction and purification. Can. J. Biochem. Physiol. 1959, 37, 911-917. [CrossRef]

27. Folch, J.; Lees, M.; Sloane Stanley, G.H. A simple method for the isolation and purification of total lipides from animal tissues. J. Biol. Chem. 1957, 226, 497-509.

28. Beckonert, O.; Keun, H.C.; Ebbels, T.M.D.; Bundy, J.; Holmes, E.; Lindon, J.C.; Nicholson, J.K. Metabolic profiling, metabolomic and metabonomic procedures for NMR spectroscopy of urine, plasma, serum and tissue extracts. Nat. Protoc. 2007, 2, 2692-2703. [CrossRef]

29. Ulrich, E.L.; Akutsu, H.; Doreleijers, J.F.; Harano, Y.; Ioannidis, Y.E.; Lin, J.; Livny, M.; Mading, S.; Maziuk, D.; Miller, Z.; et al. BioMagResBank. Nucleic Acids Res. 2008, 36, D402-D408. [CrossRef] 
30. Wishart, D.S.; Tzur, D.; Knox, C.; Eisner, R.; Guo, A.C.; Young, N.; Cheng, D.; Jewell, K.; Arndt, D.; Sawhney, S.; et al. HMDB: The Human Metabolome Database. Nucleic Acids Res. 2007, 35, D521-D526. [CrossRef]

31. Reis, A.; Rudnitskaya, A.; Blackburn, G.J.; Mohd Fauzi, N.; Pitt, A.R.; Spickett, C.M. A comparison of five lipid extraction solvent systems for lipidomic studies of human LDL. J. Lipid Res. 2013, 54, 1812-1824. [CrossRef] [PubMed]

32. Wu, H.; Southam, A.D.; Hines, A.; Viant, M.R. High-throughput tissue extraction protocol for NMR-and MS-based metabolomics. Anal. Biochem. 2008, 372, 204-212. [CrossRef] [PubMed]

33. Viant, M.R.; Ebbels, T.M.D.; Beger, R.D.; Ekman, D.R.; Epps, D.J.T.; Kamp, H.; Leonards, P.E.G.; Loizou, G.D.; MacRae, J.I.; van Ravenzwaay, B.; et al. Use cases, best practice and reporting standards for metabolomics in regulatory toxicology. Nat. Commun. 2019, 10, 1-10. [CrossRef] [PubMed]

34. Bharti, S.K.; Roy, R. Quantitative 1H NMR spectroscopy. TrAC Trends Anal. Chem. 2012, 35, 5-26. [CrossRef]

35. Vidal, N.P.; Manzanos, M.J.; Goicoechea, E.; Guillén, M.D. Quality of farmed and wild sea bass lipids studied by (1) H NMR: Usefulness of this technique for differentiation on a qualitative and a quantitative basis. Food Chem. 2012, 135, 1583-1591. [CrossRef] [PubMed]

36. Barrilero, R.; Gil, M.; Amigó, N.; Dias, C.B.; Wood, L.G.; Garg, M.L.; Ribalta, J.; Heras, M.; Vinaixa, M.; Correig, X. LipSpin: A New Bioinformatics Tool for Quantitative 1H NMR Lipid Profiling. Anal. Chem. 2018, 90, 2031-2040. [CrossRef]

37. Ducheix, S.; Montagner, A.; Polizzi, A.; Lasserre, F.; Marmugi, A.; Bertrand-Michel, J.; Podechard, N.; Al Saati, T.; Chétiveaux, M.; Baron, S.; et al. Essential fatty acids deficiency promotes lipogenic gene expression and hepatic steatosis through the liver X receptor. J. Hepatol. 2013, 58, 984-992. [CrossRef]

38. Jiang, C.; Yang, K.; Yang, L.; Miao, Z.; Wang, Y.; Zhu, H. A 1H NMR-Based Metabonomic Investigation of Time-Related Metabolic Trajectories of the Plasma, Urine and Liver Extracts of Hyperlipidemic Hamsters. PLoS ONE 2013, 8, e66786. [CrossRef]

39. Li, J.; Vosegaard, T.; Guo, Z. Applications of nuclear magnetic resonance in lipid analyses: An emerging powerful tool for lipidomics studies. Prog. Lipid Res. 2017, 68, 37-56. [CrossRef]

40. Botolin, D.; Wang, Y.; Christian, B.; Jump, D.B. Docosahexaneoic acid (22:6, n-3) regulates rat hepatocyte SREBP-1 nuclear abundance by Erk-and 26S proteasome-dependent pathways. J. Lipid Res. 2006, 47, 181-192. [CrossRef]

41. Jump, D.B. Fatty acid regulation of hepatic lipid metabolism. Curr. Opin. Clin. Nutr. Metab. Care 2011, 14, 115-120. [CrossRef] [PubMed]

42. Dentin, R.; Benhamed, F.; Pégorier, J.P.; Foufelle, F.; Viollet, B.; Vaulont, S.; Girard, J.; Postic, C. Polyunsaturated fatty acids suppress glycolytic and lipogenic genes through the inhibition of ChREBP nuclear protein translocation. J. Clin. Investig. 2005, 115, 2843-2854. [CrossRef] [PubMed]

43. Klein, M.S.; Dorn, C.; Saugspier, M.; Hellerbrand, C.; Oefner, P.J.; Gronwald, W. Discrimination of steatosis and NASH in mice using nuclear magnetic resonance spectroscopy. Metabolomics 2011, 7, 237-246. [CrossRef]

44. Yang, Y.; Li, C.; Nie, X.; Feng, X.; Chen, W.; Yue, Y.; Tang, H.; Deng, F. Metabonomic studies of human hepatocellular carcinoma using high-resolution magic-angle spinning $1 \mathrm{H}$ NMR spectroscopy in conjunction with multivariate data analysis. J. Proteome Res. 2007, 6, 2605-2614. [CrossRef]

45. Schofield, Z.; Reed, M.A.; Newsome, P.N.; Adams, D.H.; Günther, U.L.; Lalor, P.F. Changes in human hepatic metabolism in steatosis and cirrhosis. World J. Gastroenterol. 2017, 23, 2685-2695. [CrossRef]

46. Chen, I.S.; Shen, C.S.J.; Sheppard, A.J. Comparison of methylene chloride and chloroform for the extraction of fats from food products. J. Am. Oil Chem. Soc. 1981, 58, 599-601. [CrossRef]

47. Barrans, A.; Collet, X.; Barbaras, R.; Jaspard, B.; Manent, J.; Vieu, C.; Chap, H.; Perret, B. Hepatic lipase induces the formation of pre-beta 1 high density lipoprotein (HDL) from triacylglycerol-rich HDL2. A study comparing liver perfusion to in vitro incubation with lipases. J. Biol. Chem. 1994, 269, 11572-11577.

48. Lillington, J.M.; Trafford, D.J.; Makin, H.L. A rapid and simple method for the esterification of fatty acids and steroid carboxylic acids prior to gas-liquid chromatography. Clin. Chim. Acta Int. J. Clin. Chem. 1981, 111, 91-98. [CrossRef]

49. Wold, S.; Antti, H.; Lindgren, F.; Öhman, J. Orthogonal signal correction of near-infrared spectra. Chemom. Intell. Lab. Syst. 1998, 44, 175-185. [CrossRef] 
50. McCombie, G.; Browning, L.M.; Titman, C.M.; Song, M.; Shockcor, J.; Jebb, S.A.; Griffin, J.L. omega-3 oil intake during weight loss in obese women results in remodelling of plasma triglyceride and fatty acids. Metab. Off. J. Metab. Soc. 2009, 5, 363-374. [CrossRef]

51. Lapins, M.; Eklund, M.; Spjuth, O.; Prusis, P.; Wikberg, J.E. Proteochemometric modeling of HIV protease susceptibility. BMC Bioinform. 2008, 9, 181. [CrossRef] [PubMed]

(C) 2019 by the authors. Licensee MDPI, Basel, Switzerland. This article is an open access article distributed under the terms and conditions of the Creative Commons Attribution (CC BY) license (http://creativecommons.org/licenses/by/4.0/). 
Article

\title{
Exposure of HepaRG Cells to Sodium Saccharin Underpins the Importance of Including Non-Hepatotoxic Compounds When Investigating Toxicological Modes of Action Using Metabolomics
}

\author{
Matthias Cuykx ${ }^{1,2, *}$, Charlie Beirnaert ${ }^{3,4}$, Robim Marcelino Rodrigues ${ }^{2}$, Kris Laukens ${ }^{3,4}$, \\ Tamara Vanhaecke ${ }^{2,+}$ and Adrian Covaci ${ }^{1, *,+}$ \\ 1 Toxicological Centre, University of Antwerp, Universiteitsplein 1, 2610 Wilrijk, Belgium \\ 2 Research Group In Vitro Toxicology and Dermato-Cosmetology (IVTD), Vrije Universiteit Brussel, \\ Laarbeeklaan 103, 1090 Jette, Belgium; Robim.marcelino.rodrigues@vub.be (R.M.R.); \\ Tamara.vanhaecke@vub.be (T.V.) \\ 3 Department of Mathematics \& Computer Science, University of Antwerp, Middelheimlaan 1, \\ 2020 Antwerp, Belgium; Charlie.beirnaert@uza.be (C.B.); Kris.laukens@uantwerpen.be (K.L.) \\ 4 Biomedical Informatics Network Antwerpen (Biomina), University of Antwerp, Middelheimlaan 1, \\ 2020 Antwerp, Belgium \\ * Correspondence: matthiascuykx@gmail.com (M.C.); Adrian.Covaci@uantwerpen.be (A.C.) \\ + These authors contributed equally to this work.
}

Received: 10 October 2019; Accepted: 30 October 2019; Published: 4 November 2019

\begin{abstract}
Metabolites represent the most downstream information of the cellular organisation. Hence, metabolomics experiments are extremely valuable to unravel the endogenous pathways involved in a toxicological mode of action. However, every external stimulus can introduce alterations in the cell homeostasis, thereby obscuring the involved endogenous pathways, biasing the interpretation of the results. Here we report on sodium saccharin, which is considered to be not hepatotoxic and therefore can serve as a reference compound to detect metabolic alterations that are not related to liver toxicity. Exposure of HepaRG cells to high levels of sodium saccharin $(>10 \mathrm{mM})$ induced cell death, probably due to an increase in the osmotic pressure. Yet, a low number $(n=15)$ of significantly altered metabolites were also observed in the lipidome, including a slight decrease in phospholipids and an increase in triacylglycerols, upon daily exposure to $5 \mathrm{mM}$ sodium saccharin for $72 \mathrm{~h}$. The observation that a non-hepatotoxic compound can affect the metabolome underpins the importance of correct experimental design and data interpretation when investigating toxicological modes of action via metabolomics.
\end{abstract}

Keywords: in vitro; HepaRG; sodium saccharin; reference toxicants

\section{Introduction}

Since the introduction of metabolomics as a new "-omics" domain in 1999, the field of research has proved to be a valuable source of information for the actual phenotype or state of organisms [1]. Metabolomics is defined as the study of the biochemical profile of small molecules in an organism [1]. Because the metabolome is the most downstream level in the biomolecular organisation of a system, metabolomics fingerprints are very dynamic and alterations may be induced even by small external triggers [2,3]. Many of these stimuli, such as gender, age (young vs. old), dietary status (e.g., fasting vs. fed) and activity (rested vs. active) potentially form biases that obscure the metabolic signature related to exposure. These biases are important, but can be anticipated through strict subject selection criteria and proper randomisation [4]. 
An effect often not considered is the exposure itself. Although this placebo effect is well-known in medicine, precautions are often not taken during metabolomics studies. The "exposure bias" is relevant when combining metabolomics with in vitro experiments, since it is one of the main potential sources of bias. Although the biological variation and bias is reduced in cell culture experiments, confounding factors still pose a risk during metabolomics investigations. Indeed, the mere presence of a xenobiotic may theoretically cause a metabolic shift, even though this compound is not considered harmful. This confounding factor of "exposure" can generate results falsely interpreted to be related to toxicity [5].

Sodium saccharin is an artificial sweetener that has been used for over a century. Except for a case study describing an idiosyncratic reaction after exposure to different pharmaceutical products containing the sweetener, no evidence of human hepatotoxicity has been reported so far $[6,7]$. The safe characteristics of sodium saccharin make it a good candidate to investigate potential metabolic alterations triggered in vitro upon exposure to a non-hepatotoxic molecule.

\section{Materials and Methods}

\subsection{Materials and Methods}

Materials, exposure and acquisition methods have been performed as described previously [8]. A brief description highlighting the principles is mentioned in-text and full details concerning the experimental protocols are provided in the Supplementary Materials SM-1 to SM-5.

\subsection{Determination of Testing Concentrations}

Seven days after initial cell seeding, the wells were divided into two negative control groups and eight groups that were exposed to sodium saccharin at different concentrations ranging from 0.40 to $40 \mathrm{mM}$ for a period of $72 \mathrm{~h}$ in a repeated dose exposure, in which the medium was refreshed every $24 \mathrm{~h}$. Viability was assessed using the neutral red uptake (NRU) assay [9,10]. Full details are available in the Supplementary Materials SM-2.

\subsection{Metabolomics Experiments}

\subsubsection{Seeding of the HepaRG ${ }^{\circledR}$ Cells and Exposure to Sodium Saccharin}

Cryopreserved differentiated HepaRG ${ }^{\circledR}$ cells were thawed and seeded in collagen-coated two-well Lab-Tek chamber slides at a density of $1.03 \times 10^{6}$ cells/well. Two additional blank chamber slides were treated identically to serve as blanks during further analysis. After seven days of cultivation, the cell cultures were visually checked for hepatocyte/biliary cell ratio and block randomised in three groups: a negative control group in comparison to a dose of sodium saccharin at a concentration of $5.5 \mathrm{mM}$ (high dose) and a 1/10 dilution of the high dose, i.e., $0.5 \mathrm{mM}$ (low dose). Higher concentrations of sodium saccharin were not applied because of hyperosmotic toxic effects (additional osmotic pressure $>20 \mathrm{mOsm} / \mathrm{L}$ ). Each group contained six replicates, which is often considered an adequate sample size for in vitro metabolomics experiments [5,11]. The cell cultures were exposed for $72 \mathrm{~h}$ with a medium refreshment every $24 \mathrm{~h}$. The exposure experiment has been performed twice to reduce false positive results [8].

\subsubsection{Sample Preparation}

The cell cultures were harvested according to previously described protocols, full details are available in SM-3 [12,13]. Briefly, cells were prepared for extraction with a wash in phosphate buffered saline $\left(37^{\circ} \mathrm{C}\right)$ followed by freezing on liquid nitrogen. Cells were scraped from the surface three times with $200 \mu \mathrm{L}$ of a cooled $\left(-80^{\circ} \mathrm{C}\right) 80 \%(\mathrm{v} / \mathrm{v})$ methanol $(\mathrm{MeOH}) / \mathrm{milliQ}$ water solution. Liquid/liquid extraction was performed using ultrapure water, methanol, and chloroform. Quality control $(\mathrm{QC})$ pools 
were generated through the collection of aliquots of all samples for the polar and non-polar phases [14]. Both fractions were evaporated to dryness and reconstituted in LC-MS-compatible solvents.

\subsubsection{LC-MS Analysis}

LC-MS analysis was performed using separation mechanisms described in SM-4 $[8,15]$. The non-polar fraction was analysed using reversed phase chromatography on a Kinetex XB-C18 $(150 \times 2.1 \mathrm{~mm} ; 1.7 \mu \mathrm{m}$ particle size, Phenomenex, Utrecht, the Netherlands). Mobile phase compositions were mixtures of methanol, isopropanol (IPA) and water with ammonium acetate $(\mathrm{pH} 6.7)$ and of acetonitrile $(\mathrm{ACN})$, IPA and water with an acetate buffer $(\mathrm{pH} 4.2)$ for negative and positive ionisation modes, respectively.

The polar fractions were analysed using HILIC systems using an iHILIC column $(100 \times 2.1 \mathrm{~mm}$; $1.8 \mu \mathrm{m}$ particle size, HILICON, Umea, Sweden) with $\mathrm{ACN}, \mathrm{MeOH}$ and water with an ammonium formate buffer ( $\mathrm{pH}$ 3.15) for the positive ionisation mode, and a polymeric iHILIC Fusion Column $(100 \times 2.1 \mathrm{~mm}, 5 \mu \mathrm{m}$ particle size, HILICON) in combination with $\mathrm{ACN}, \mathrm{MeOH}$ and water with an ammonium carbonate $\left(\left(\mathrm{NH}_{4}\right)_{2} \mathrm{CO}_{3}\right)$ buffer $(\mathrm{pH}$ 9.0) for the negative ionisation mode. LC-separation was performed on an Agilent Infinity 1290 UPLC (Agilent Technologies, Santa Clara, CA, USA), connected to an Agilent 6530 QTOF with Agilent Jet Stream nebuliser (Agilent Technologies). The LC-MS system was equilibrated using $15 \mathrm{QC}$-injections at the start of the data acquisition. The injection order of the samples was block-randomised to prevent bias related to instrumental drift. One QC injection was performed after every four sample injections to monitor instrumental drifts.

\subsubsection{Data Analysis}

\section{Data Quality Control}

Internal standards were used to evaluate the precision of the retention time and $\mathrm{m} / \mathrm{z}$-accuracy within and between experimental batches. The raw data were searched for the internal standards using the Find by Formula algorithm (Agilent Technologies) with the following parameters: formula matching $\pm 10 \mathrm{ppm}$, expected variation $2 \mathrm{mDa} \pm 8 \mathrm{ppm}$. Samples were only considered when internal standards were detected and the number of molecular features was comparable to those of the other samples. The absence of internal standards and/or molecular features in an acquired LC-MS run may indicate analytical issues during the run and, therefore, the removal of the failed runs was considered to improve the quality of the final dataset. The results of internal standard quality control were used to set the parameters for further data processing.

Data Pretreatment

Acquired data were imported on the MassHunter Qualitative software (Agilent Technologies, $\mathrm{v} 2.06 .00$ ) and converted to centroid $\mathrm{m} / \mathrm{z}$ data. The generic datafiles were processed using the XCMS package in the $\mathrm{R}$ workspace [16,17]. Features representing the ions of the extracted metabolites were searched using the centWave algorithm. Features were aligned with the Obiwarp algorithm and grouped by density [18]. Missing peaks were re-extracted using the fillPeaks algorithm [17].

The dataset was cleaned by removing isotopes and features present in blank samples. Other applied filters were based on a high number of missing values and within-group variability. All preprocessing functions were executed using the MetaboMeeseeks package [19]. A principal component analysis (PCA) was performed and outliers were removed for further analysis $(n=4)$ (Figure S6). After outlier removal, the filter process was re-iterated and samples were normalised using BatchCorr normalisation [20]. Missing values imputation was considered but not applied, since it had no positive impact on the within-group variance. The final dataset was once more evaluated using a PCA to assess important trends and their potential impact on the subsequent multivariate analysis. All parameters of feature extraction and data clean-up are mentioned in SM-5. 


\section{Statistical Analysis}

Univariate statistical analysis was performed through the non-parametric Mann-Whitney U test with a Benjamini-Hochberg correction for multiple testing using the multtest package in R [21]. In addition, a partial least squares discriminant analysis (PLS-DA) and a random forest classification were performed as multivariate analyses [19,22]. Performances were checked using leave-one-out cross-validation. Metabolic alterations were defined based on significance in the univariate tests ( $q$-value $<0.05$ ) and on importance in the multivariate models (based on the covariance of the latent values of the first component of the PLS-DA and the bimodal distribution of the variable importance measure (VIM) of the random forest classifier model). The raw signals of the selected signals were manually checked to confirm the result.

\section{Metabolite Annotation}

The details of metabolite annotation are mentioned in SM-6. Briefly, annotation was performed in Mass Hunter using the molecular feature extractor algorithm: the signals corresponding to the altered metabolite were selected, the complete result set was extracted and the Molecular Formula Generator (MFG) generated a list of possible chemical formulas. The identification was based on the $\mathrm{m} / \mathrm{z}$-value, the isotope pattern, the measured retention time and the fragmentation spectra acquired during the equilibration runs. Results were reported according to the standards of the CAWG and MSI $[23,24]$. Level 2 and level 3 identifications were considered of sufficient quality to infer a biological interpretation to the outcome of the experiments. All metabolites, including molecular features with lower levels of confidence in annotation (levels 4-5), are reported in Table S4.

\section{Results}

\subsection{Experimental Observations}

The dose-response curves of the viability assay in Figure 1 showed a clear decrease in viability from $10 \mathrm{mM}$ sodium saccharin onwards. Indeed, concentrations of $10 \mathrm{mM}$ increased the osmotic pressure over $20 \mathrm{mOsm} / \mathrm{L}$ to supra-physiological ranges [25]. Hyperosmolarity is a form of toxicity not related to physiological hepatotoxicity, and such high exposures are therefore not considered as a good reference for investigating the chemical hazard of the product. A high-dose exposure of $5 \mathrm{mM}$ induced an osmotic pressure of $\pm 10 \mathrm{mOsm} / \mathrm{L}$, yet no cytotoxic effects were observed.

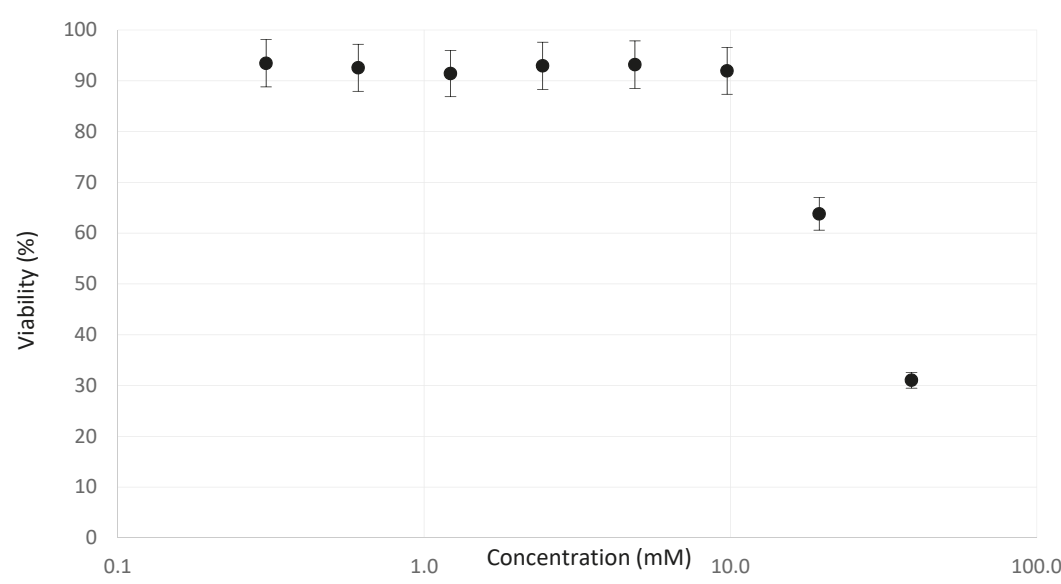

Figure 1. Averaged viability curve for the neutral red uptake (NRU)-assay upon daily exposure to sodium saccharin for a period of $72 \mathrm{~h}$. Cytotoxicity is observed from $10 \mathrm{mM}$ onwards, which is equal to an osmotic pressure of $\pm 20 \mathrm{mOsm} / \mathrm{L}$. 


\subsection{Data Quality}

The injections that were not considered during statistical analysis are reported in Table S2. For the non-polar fraction, injections QC-1 and QC-2 were excluded from the analysis because of failed injections, reflected by the absence of internal standards in the chromatogram and the absence of the typical chromatogram.

As shown in Table S3, standard deviations were higher during the first experimental batch due to autosampler thermostatic issues. The high mRSDs for QCs in comparison to all other experiments using lipidomics approaches can be explained by the shift in retention times between the two experimental batches. To correctly match corresponding peaks, the parameters for alignment and grouping were less strict, which introduced extra noise in the data.

\subsection{Selection of Potential Endogenous Markers of Exposure}

As shown in the PCA plots in Figure 2 and Figures S7-S10, no clear distinction between all exposure groups was observed. The overlap between the different exposure groups indicated that the source of variation in the dataset was probably not related to the exposure. This was also reflected by the poor performance of both multivariate and univariate tests: all AUCs for the random forest classifiers reported in Table 1 were below 0.7, except for the high-dose exposure conditions. According to the $\mathrm{R}^{2}$ and $\mathrm{Q}^{2}$ values in Table 2, the PLS-models overfit, with $\mathrm{Q}^{2}$ values $<0.2$. The $\mathrm{R}^{2}$ of the non-polar fraction in positive mode was good $(>0.8)$, but the cross-validation showed this was an overfit value, and the $\mathrm{Q}^{2}$ was reduced to 0.40 .

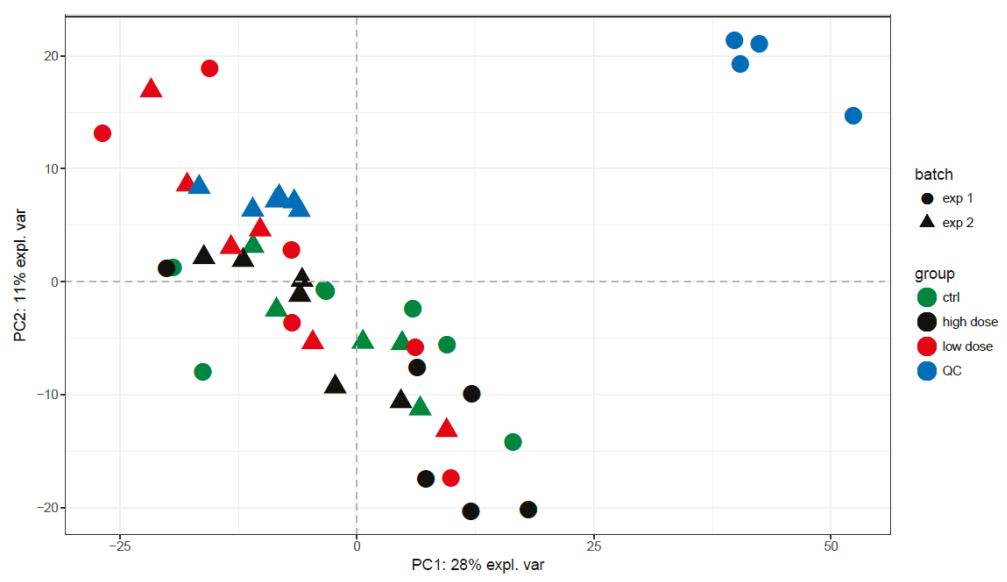

Figure 2. PCA plots of the non-polar fraction in positive mode during the 72-h exposure showing PC1 vs. PC2. There is strong overlapping of the different exposure groups in all principal components, indicating the variance is not related to exposure. Only a slight trend is visible between the negative control group and the group exposed to the higher dose.

Univariate tests did not reveal major differences between the exposure groups and the negative control group. Only 15 features were observed to be significantly different between the negative control group and the high-dose exposure group, and only for the non-polar fraction in positive mode.

The identified features given in Table S4 represent a decreased presence of six phosphatidylethanolamines, two phosphatidylinositols and one sphingomyelin. Further differences included the increase of two low-saturated triacylglycerols. Their respective boxplots are represented in Figure S10. 
Table 1. AUCs of the random forest classifiers comparing the negative control group against the different exposures.

\begin{tabular}{ccccc}
\hline Exposure & Non-Polar Positive & Non-Polar Negative & Polar Positive & Polar Negative \\
\hline Low Dose & 0.51 & 0.55 & 0.59 & 0.17 \\
High Dose & 0.95 & 0.40 & 0.69 & 0.21 \\
\hline
\end{tabular}

Table 2. $R^{2}$ and $Q^{2}$ values for the PLS-DA discrimination between the exposure groups and the negative control group.

\begin{tabular}{ccccccccc}
\hline \multirow{2}{*}{ Exposure } & \multicolumn{2}{c}{ Non-Polar Positive } & \multicolumn{2}{c}{ Non-Polar Negative } & \multicolumn{2}{c}{ Polar Positive } & \multicolumn{2}{c}{ Polar Negative } \\
\cline { 2 - 9 } & $\mathbf{R}^{\mathbf{2}}$ & $\mathbf{Q}^{\mathbf{2}}$ & $\mathbf{R}^{\mathbf{2}}$ & $\mathbf{Q}^{\mathbf{2}}$ & $\mathbf{R}^{\mathbf{2}}$ & $\mathbf{Q}^{\mathbf{2}}$ & $\mathbf{R}^{\mathbf{2}}$ & $\mathbf{Q}^{\mathbf{2}}$ \\
\hline Low Dose & 0.01 & 0.01 & 0.1 & 0.06 & 0.22 & 0.14 & 0.21 & 0.05 \\
High Dose & 0.84 & 0.4 & 0.42 & 0.10 & 0.08 & 0.03 & 0.33 & 0.04 \\
\hline
\end{tabular}

\section{Discussion}

The metabolome is a dynamic level of the cellular organisation. External stimuli theoretically invoke a response of the cell, resulting in a change of the metabolome. Sodium saccharin is considered to be a non-hepatotoxic chemical, which makes it an ideal compound to select markers of exposure not necessarily related to hepatotoxicity $[8,9]$.

The acquisition of the metabolome of cells exposed to a hepatotoxicant in comparison to vehicle only would reveal alterations that would all be addressed to a toxic mode of action. The main consequence of this assumption is the questionable predictive value of the observed metabolic alterations, especially in small-scale experiments (one dose, one time point exposure of a single chemical exposure). This consideration stresses the importance of an exposure to a non-toxic negative control during the experiment.

Few features of the lipidome were changed significantly $(n=15)$ and included the presence of triacylglycerols and a lower presence of phospholipids. The absence of significant changes in the polar fraction suggested that the cell culture did not implement major adaptations in metabolism as a response to the external stimuli.

Effects related to hyperosmolarity have also been described in human cell cultures, which showed the increased presence of monosaccharides and amino acids to retain the osmotic balance [26]. However, it is possible that these effects were not observed in this current experiment due to the deliberate choice to avoid these hyperosmolar (non-physiological) concentrations. The choice of a supra-physiological dose would imply a bias to select metabolic alterations related to modes of action not relevant in physiological conditions. This bias is especially relevant in in vitro techniques, as exposure concentrations can be increased to unrealistic, non-physiological levels. A potential prevention is the use of toxicokinetic data to confirm the plausibility of the exposure conditions.

García-Cañaveras et al. [27] and Ramirez et al. [28] classified different toxicants according to the mode of action, observing specific fingerprints for different end-points of toxicity. Ramirez et al. described the downregulation of carnitine, creatine, phosphocreatine, and pantothenic acid during exposure to peroxisome proliferating agents, the decrease of oleic acid, galactose and acetyl aspartate in combination with an increase of tryptophan and alanine during exposure to enzyme-inducing xenobiotics [28]. Garcia et al. compared the fingerprint of xenobiotics inducing oxidative stress, steatosis and phospholipidosis, in which they observed alterations in glutamate levels, oxido-reductive status, lysophospholipid/phospholipid ratio and lipid accumulation [27]. The inclusion of non-hepatotoxic compounds in their experimental design, such as citrate and ketotifen, states the importance of reference compounds for non-hepatotoxicity as metabolic changes were observed, albeit with a different fingerprint.

A qualitative comparison of the metabolic alterations for steatosis and cholestasis obtained in previous experiments are presented in Table $3[8,29]$. The clear alterations of the metabolome during 
hepatotoxic modes of action can be discriminated from the exposure to a non-hepatotoxic toxicant. Next to significant differences in the polar metabolome, the lipidome of a cell culture exposed to hepatotoxic compounds showed clear and strong alterations, with multiple lipid species of several classes involved in the downstream effect, whereas the effects during exposure to sodium saccharin were not substantial.

Table 3. Heat map for the endogenous markers of toxicity for hepatotoxicants inducing steatosis (sodium valproate) [8] and cholestasis (bosentan) [29] showing their up- (red) or down-regulation (green) in comparison to a negative control group (sodium saccharin). Common alterations indicate the importance of the use of non-hepatotoxic reference compounds as a negative control to prevent an exposure bias, especially in the lipidomics group.

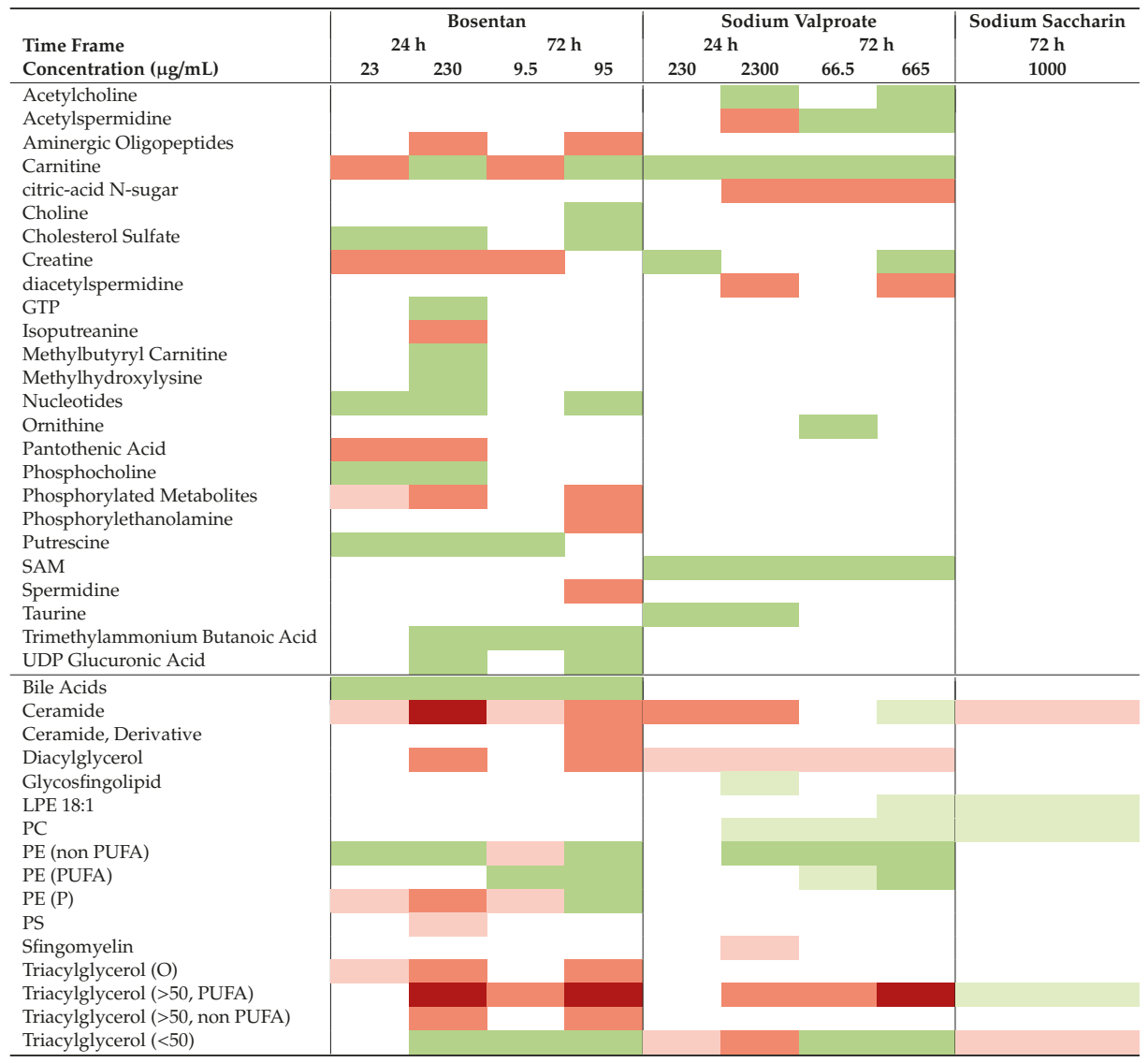

(A)

Abbreviations: GTP, guanosyl triphosphate; LPE, lysophosphatidylethanolamine; PC, phosphatidylcholine; PE, phosphatidylethanolamine; PI, phosphaditylinositol; PS, phosphatidylserine; SAM, S-adenosyl methionine; TG, triacylglycerol.

\begin{tabular}{ccccccc}
\hline Colour & & & & & & \\
\hline Number of Lipid Species & $>3$ & $1-3$ & 0 & $1-3$ & $4-10$ & $>10$ \\
\hline Signal Abundance & Lower & Lower & N/A & Higher & Higher & Higher \\
\hline
\end{tabular}

(B)

A mechanistic interpretation based on the observed metabolic alterations provides an additional value to the localisation of the specific molecular initiating event and the potential adverse outcome, as 
this observation is an additional argument for a toxic cascade. Although metabolic alterations were observed upon exposure to sodium saccharin, no specific affected pathway could be identified. Based on all observations, the use of a non-hepatotoxic compound instead of a vehicle-only negative control group may help reducing false positive results without jeopardising the sensitivity of toxic insults.

\section{Conclusions}

The exposure of HepaRG cells to high levels of sodium saccharin induced cell death, possibly due to osmotic pressure. The exposure concentration should not solely be determined from viability curves, but should also be checked for physiological relevance to prevent unrealistic exposure scenarios. Although sodium saccharin is not considered to be a hepatotoxicant, minor changes were observed in the lipidome, including a slight decrease in phospholipids and an increase in saturated triacylglycerols. The metabolome was altered upon exposure to non-hepatotoxic compounds, indicating the importance of reference compounds when investigating toxicological insults. The metabolic changes were less pronounced than those of reference hepatotoxicants and can therefore be used as a background response to prevent false positive results related to the exposure bias.

Supplementary Materials: The following are available online at http://www.mdpi.com/2218-1989/9/11/265/s1, Figure S1: Viability curves for the NRU-assay $72 \mathrm{~h}$ of exposure., Figure S2: Extracted ion chromatogram for all samples in experiment 1 for $\mathrm{m} / \mathrm{z} 162.1139$ at a retention time of 8.7. Figure S3: Boxplots for $\mathrm{m} / \mathrm{z} 162$ at $\mathrm{rt} 8.7 \mathrm{~min}$. Figure S4: Annotation of the molecular formula using the Agilent Molecular Formula Generator reveals a match for the proton, sodium and potassium adduct of a molecule with formula $\mathrm{C}_{7} \mathrm{H}_{15} \mathrm{NO}_{3}$, Figure S5: MS/MS spectrum of the molecular feature (up), which matches with the MS/MS spectrum of L-carnitine in the METLIN database (down), Figure S6: PCA reflecting the outlier position of QC-1 and IC10-6 for the dataset of the non-polar fraction in negative ionisation mode, Figure S7: PCA plots of the non-polar fraction in negative mode during the $72 \mathrm{~h}$ exposure showing PC1 vs. PC2 (upper) and PC3 vs. PC4 (lower), Figure S8: PCA plots of the non-polar fraction in positive mode during the $72 \mathrm{~h}$ exposure showing PC1 vs. PC2 (upper) and PC3 vs. PC4 (lower), Figure S9: PCA plots of the polar fraction in negative mode during the $72 \mathrm{~h}$ exposure PC1 vs. PC2 (upper) and PC3 vs. PC4 (lower), Figure S10: PCA plots of the polar fraction in positive mode during the $72 \mathrm{~h}$ exposure showing PC1 vs. PC2 (upper) and PC3 vs. PC4 (lower), Table S1: Concentration range (in $\mu \mathrm{g} / \mathrm{mL}$ ) for the NRU-assay of sodium saccharin on HepaRG cells for a period of 72 h, Table S2: Samples which did not meet QC criteria., Table S3: Median relative standard deviations (mRSDs) for all subgroups in the experiments, Table S4: AUCs of the random forest classifiers comparing the negative control group against the different exposures, Table S5: $R^{2}$ and $Q^{2}$ values for the PLS-DA discrimination between the exposure groups and the negative control group, Table S6: Metabolites identified as potential metabolites of interest for all exposure models.

Author Contributions: Conceptualization, R.M.R. and T.V.; Data curation, C.B. and K.L.; Investigation, M.C., T.V. and A.C.; Methodology, M.C., C.B. and R.M.R.; Software, C.B.; Supervision, R.M.R., K.L., T.V. and A.C.; Writing—original draft, M.C.; Writing—review \& editing, K.L., T.V. and A.C.

Funding: Matthias Cuykx has been funded by the Fonds voor Wetenschappelijk Onderzoek (FWO, Belgium; fellowships $12 \mathrm{H} 2216 \mathrm{~N}$ and $11 \mathrm{Z} 3318 \mathrm{~N})$. Further funding of this research has been provided by the Vrije Universiteit Brussel (VUB, Belgium) and the University of Antwerp (UA, Belgium).

Conflicts of Interest: The authors declare no conflict of interest.

\section{References}

1. Nicholson, J.K.; Lindon, J.C.; Holmes, E. "Metabonomics": Understanding the metabolic responses of living systems to pathophysiological stimuli via multivariate statistical analysis of biological NMR spectroscopic data. Xenobiotica 1999, 29, 1181-1189. [CrossRef]

2. Balcke, G.U.; Kolle, S.N.; Kamp, H.; Bethan, B.; Looser, R.; Wagner, S.; Landsiedel, R.; van Ravenzwaay, B. Linking energy metabolism to dysfunctions in mitochondrial respiration-A metabolomics in vitro approach. Toxicol. Lett. 2011, 203, 200-209. [CrossRef]

3. Ramirez, T.; Daneshian, M.; Kamp, H.; Bois, F.Y.; Clench, M.R.; Coen, M.; Donley, B.; Fischer, S.M.; Ekman, D.R.; Fabian, E.; et al. Metabolomics in toxicology and preclinical research. ALTEX 2013, 30, 209-225. [CrossRef]

4. Broadhurst, D.I.; Kell, D.B. Statistical strategies for avoiding false discoveries in metabolomics and related experiments. Metabolomics 2006, 2, 171-196. [CrossRef]

5. Cuykx, M.; Rodrigues, R.M.; Laukens, K.; Vanhaecke, T.; Covaci, A. In vitro assessment of hepatotoxicity by metabolomics: A review. Arch. Toxicol. 2018, 92, 3007-3029. [CrossRef] [PubMed] 
6. Negro, F.; Mondardine, A.; Palmas, F. Hepatotoxicity of saccharin. N. Engl. J. Med. 1994, 330, $134-135$. [CrossRef] [PubMed]

7. US National Library of Medicine Toxnet: Hazardous Substance Database-Saccharin. Available online: http://toxnet.nlm.nih.gov (accessed on 1 October 2018).

8. Cuykx, M.; Claes, L.; Rodrigues, R.M.; Vanhaecke, T.; Covaci, A. Metabolomics profiling of steatosis progression in HepaRG ${ }^{\circledR}$ cells using sodium valproate. Toxicol. Lett. 2018, 286, 22-30. [CrossRef] [PubMed]

9. Zhang, S.-Z.; Lipsky, M.M.; Trump, B.F.; Hsu, I.-C. Neutral red (NR) assay for cell viability and xenobiotic-induced cytotoxicity in primary cultures of human and rat hepatocytes. Cell Biol. Toxicol. 1990, 6, 219-234. [CrossRef]

10. Ates, G.; Vanhaecke, T.; Rogiers, V.; Rodrigues, R.M. Assaying cellular viability using the Neutral Red Uptake assay. Cell Viability Assays. In Methods in Molecular Biology; Humana Press: New York, NY, USA, 2017; pp. 19-26.

11. 1Hayton, S.; Maker, G.L.; Mullaney, I.; Trengove, R.D. Experimental design and reporting standards for metabolomics studies of mammalian cell lines. Cell. Mol. Life Sci. 2017, 74, 4421-4441. [CrossRef]

12. $\mathrm{Wu}, \mathrm{H}$.; Southam, A.D.; Hines, A.; Viant, M.R. High-throughput tissue extraction protocol for NMR- and MS-based metabolomics. Anal. Biochem. 2008, 372, 204-212. [CrossRef]

13. Cuykx, M.; Mortelé, O.; Rodrigues, R.M.; Vanhaecke, T.; Covaci, A. Optimisation of in vitro sample preparation for LC-MS metabolomics applications on HepaRG cell cultures. Anal. Methods 2017, 9, 3704-3712. [CrossRef]

14. Dunn, W.B.; Wilson, I.D.; Nicholls, A.W.; Broadhurst, D. The importance of experimental design and QC samples in large-scale and MS-driven untargeted metabolomic studies of humans. Bioanalysis 2012, 4, 2249-2264. [CrossRef] [PubMed]

15. Cuykx, M.; Negreira, N.; Beirnaert, C.; Van den Eede, N.; Rodrigues, R.; Vanhaecke, T.; Laukens, K.; Covaci, A. Tailored LC-MS analysis improves the coverage of the intracellular metabolome of HepaRG cells. J. Chromatogr. 2017, 1487, 168-178. [CrossRef] [PubMed]

16. R Core Team. R: A Language and Environment for Statistical Computing; R Foundation for Statistical Computing: Vienna, Austria, 2014.

17. Smith, C.A.; Want, E.J.; Maille, G.O.; Abagyan, R.; Siuzdak, G. XCMS: Processing mass spectrometry data for metabolite profiling using nonlinear peak alignment, matching, and identification. ACS Publ. 2006, 78, 779-787. [CrossRef]

18. Prince, J.T.; Marcotte, E.M. Chromatographic alignment of ESI-LC-MS proteomics data sets by ordered bijective interpolated warping. Anal. Chem. 2006, 78, 6140-6152. [CrossRef]

19. Beirnaert, C.; Cuykx, M.; Bijtebier, S. MetaboMeeseeks: Helper functions for metabolomics analysis. R Package. 2019. version 0.1.10044. Available online: https://github.com/Beirnaert/MetaboMeeseeks (accessed on 25 September 2019).

20. Wehrens, R.; Hageman, J.A.; van Eeuwijk, F.; Kooke, R.; Flood, P.J.; Wijnker, E.; Keurentjes, J.J.; Lommen, A.; van Eekelen, H.D.; Hall, R.D.; et al. Improved batch correction in untargeted MS-based metabolomics. Metabolomics 2016, 12, 88. [CrossRef]

21. Pollard, K.S.; Dudoit, S.; Van der Laan, M.J. Multiple Testing Procedures: The multtest Package and Applications to Genomics. In Bioinformatics and Computational Biology Solutions Using $R$ and Bioconductor; Springer: New York, NY, USA, 2005; pp. 249-271.

22. Rohart, F.; Gautier, B.; Singh, A.; le Cao, K.-A. Le mixOmics: An R package for 'omics feature selection and multiple data integration. PLoS Comput. Biol. 2017, 13, e1005752. [CrossRef]

23. Schymanski, E.L.; Jeon, J.; Gulde, R.; Fenner, K.; Ruff, M.; Singer, H.P.; Hollender, J. Identifying small molecules via high resolution mass spectrometry: Communicating confidence. Environ. Sci. Technol. 2014, 48, 2097-2098. [CrossRef]

24. Sumner, L.W.; Amberg, A.; Barrett, D.; Beale, M.H.; Beger, R.; Daykin, C.A.; Fan, T.W.M.; Fiehn, O.; Goodacre, R.; Griffin, J.L.; et al. Proposed minimum reporting standards for chemical analysis: Chemical Analysis Working Group (CAWG) Metabolomics Standards Initiative (MSI). Metabolomics 2007, 3, 211-221. [CrossRef]

25. Burtis, C.; Ashwood, E.; Bruns, D. Tietz Textbook of Clinical Chemistry and Molecular Diagnostics; Elsevier: St. Louis, MO, USA, 2006; ISBN 13 978-0-7216-0189-2. 
26. Sévin, D.C.; Stählin, J.N.; Pollak, G.R.; Kuehne, A.; Sauer, U. Global metabolic responses to salt stress in fifteen species. PLoS ONE 2016, 11, e0148888. [CrossRef]

27. García-Cañaveras, J.C.; Castell, J.V.; Donato, M.T.; Lahoz, A. A metabolomics cell-based approach for anticipating and investigating drug-induced liver injury. Sci. Rep. 2016, 6, 27239. [CrossRef] [PubMed]

28. Ramirez, T.; Strigun, A.; Verlohner, A.; Huener, H.A.; Peter, E.; Herold, M.; Bordag, N.; Mellert, W.; Walk, T.; Spitzer, M.; et al. Prediction of liver toxicity and mode of action using metabolomics in vitro in HepG2 cells. Arch. Toxicol. 2017, 92, 839-906. [CrossRef] [PubMed]

29. Cuykx, M.; Beirnaert, C.; Rodrigues, R.M.; Laukens, K.; Vanhaecke, T.; Covaci, A. Untargeted liquid chromatography-mass spectrometry metabolomics to assess drug-induced cholestatic features in HepaRG ${ }^{\circledR}$ cells. Toxicol. Appl. Pharmacol. 2019, 379, 114666. [CrossRef] [PubMed]

(C) 2019 by the authors. Licensee MDPI, Basel, Switzerland. This article is an open access article distributed under the terms and conditions of the Creative Commons Attribution (CC BY) license (http://creativecommons.org/licenses/by/4.0/). 
Article

\title{
Targeted Analysis of 46 Bile Acids to Study the Effect of Acetaminophen in Rat by LC-MS/MS
}

\author{
Vivaldy Prinville, Leanne Ohlund and Lekha Sleno * \\ Department of Chemistry, Université du Québec à Montréal (UQAM), P.O. Box 8888 Downtown Station, \\ Montreal, QC H3C 3P8, Canada; vivaldyp@hotmail.com (V.P.); ohlund@gmail.com (L.O.) \\ * Correspondence: sleno.lekha@uqam.ca
}

Received: 30 September 2019; Accepted: 6 January 2020; Published: 7 January 2020

\begin{abstract}
Bile acids represent a large class of steroid acids synthesized in the liver and further metabolized by many bacterial and mammalian enzymes. Variations in bile acid levels can be used as a measure of liver function. There still exists, however, a need to study the variation of individual circulating bile acids in the context of hepatotoxity or liver disease. Acetaminophen (APAP), a drug commonly taken to relieve pain and decrease fever, is known to cause acute liver failure at high doses. We have developed a targeted liquid chromatography-tandem mass spectrometry method to monitor the effects of different doses of APAP on the bile acid plasma profile in a rat model. The analysis method was optimized to ensure chromatographic resolution of isomeric species using a mixture of 46 standard bile acids, and 14 isotopically-labeled internal standard (IS) compounds detected in multiple reaction monitoring (MRM) mode on a triple quadrupole mass spectrometer. Four doses of acetaminophen were studied, the highest of which shows signs of hepatotoxicity in rats. This targeted method revealed that high dose APAP has an important effect on bile acid profiles. Changes were seen in several unconjugated bile acids as well as glycine conjugates; however, no obvious changes were apparent for taurine-conjugated species.
\end{abstract}

Keywords: bile acids; metabolomics; rat plasma; tandem mass spectrometry; liquid chromatography; acetaminophen; hepatotoxicity

\section{Introduction}

Bile acids play many roles crucial for metabolism and liver health. They are formed from cholesterol through a series of enzymatic reactions and they represent the primary pathway for cholesterol catabolism [1]. In addition, bile acids emulsify fat from our diet and help absorb lipids and cholesterol [2]. Primary bile acids, such as cholic acid (CA) and chenodeoxycholic acid (CDCA) in humans and $\alpha$-muricholic acid $(\alpha-\mathrm{MCA})$ and $\beta$-muricholic acid ( $\beta$-MCA) in rodents, are synthesized in the liver. Before being secreted by the liver, bile acids can be conjugated to taurine or glycine amino acids. In the intestines, bile acids are unconjugated and converted into secondary bile acids, such as deoxycholic acid (DCA) and lithocholic acid. Most bile acids are reabsorbed in the liver, conjugated again, and excreted in the bile to complete the enterohepatic circulation [2,3].

An increased plasma concentration of bile acids is a sign of liver disease [4]. High concentrations are toxic, though the potential for toxicity depends on the bile acid profile. For example, it has been reported that chenodeoxycholic acid and lithocholic acid, as well as their conjugates, can damage hepatic cells and induce mitochondrial malfunction, oxidative stress, and apoptosis [5-8]. Bile acids can also damage cells within the colon $[9,10]$. The different physiological functions of bile acids and their implication in pathological processes highlight the importance of understanding circulating bile acid profiles in drug-induced hepatotoxicity.

Acetaminophen (APAP) is a drug commonly used to relieve pain and decrease fever. When taken in therapeutics doses, APAP is considered a very safe drug. With excessive doses, APAP can become 
highly toxic [11]. In North America, it is the main cause of acute liver failure, and often requires liver transplantation if too severe or not treated rapidly enough [12]. In extreme cases, APAP toxicity can cause death within $48 \mathrm{~h}$. Previous studies have shown APAP interferes with bile acid synthesis [13-15].

Different LC-MS based methods to measure bile acids exist [16,17], but a gap still remains with regards to the wide range of bile acid derivatives that exist and their changing profiles with APAP dose. By studying the effect on individual bile acids, specific reactions related to bile acid metabolism can be assessed as being relevant to follow altered hepatic metabolism. The goal of this study was to develop an optimized and semi-quantitative method to evaluate the effects of APAP on numerous bile acids, including free and conjugated forms. Liquid chromatography coupled to tandem mass spectrometry is a powerful technique that offers many advantages for selective detection of individual bile acids, which are uniquely challenging due to the presence of many isomers. Bile acids can be difficult to analyze due to the similarities between the structures. In this study, we developed a rapid method to monitor 46 bile acids by LC-MS/MS on a triple quadrupole platform in multiple reaction monitoring (MRM) mode.

\section{Results and Discussion}

A targeted liquid chromatography-multiple reaction monitoring (LC-MRM) method was developed to monitor 46 bile acids in rat plasma following a simple sample preparation to evaluate the effect of increasing APAP dose. Bile acids were extracted by protein precipitation using methanol, following the addition of an isotopically labeled internal standard mix. A reverse-phase solid-core C18 column was employed to separate the 46 bile acids with excellent resolution and peak shape using acidified water and acetonitrile as mobile phase, within a 45 min gradient. As shown in Figure 1, all 46 bile acids in the standard mix were well resolved, including many bile acid isomers (e.g., UDCA, CDCA, and DCA). For example, LC-MRM chromatograms for $\alpha$-TMCA, $\beta$-TMCA, and TCA in rat plasma show good resolution obtained and highlight the usefulness of this method to monitor these isomers. The list of bile acids assessed in this study was based on the availability of a standard mix as well as multiple isotopically-labeled bile acids for relative quantitation, through a generous gift from MRM Proteomics Inc. The separation of these internal standard (IS) compounds is shown in Figure 2.

LC-MRM analyses in negative ion mode yielded better results than in positive ion mode in terms of sensitivity (data not shown), though both were optimized. In positive mode, precursor ions were often associated to in-source water losses and had limited sensitivity as compared to negative mode. In negative mode, unconjugated bile acids were monitored with two transitions, the highest signal coming from monitoring the pseudo-MRM transition of precursor ion to precursor ion, since their fragmentation resulted in a complex mix of fragments, thus limiting sensitivity for more specific fragment ions [18]. For conjugated bile acids, fragment ions resulting from the taurine and glycine moieties were employed as product ions. For each bile acid, however, secondary transitions were monitored for confirmatory purposes. In rat plasma samples, 39 of the 46 bile acids were measurable, with peaks having signal-to-noise of at least 10 and retention time matching that of the standard mix. No peak was observed for GDHCA, TDHCA, IDCA, DHCA, TLCA, AILCA and ILCA in rat plasma samples. DHCA is a synthetic product of the oxidation of CA and is mainly converted into 3 - $\alpha$-hydroxylated-oxo bile acids [19]. It is therefore normal that the conjugated bile acids of DHCA (GDHCA and TDHCA) are not present in rat plasma either. Iso-bile acids (IDCA, AILCA and ILCA) are excreted in the feces of animals [20]. Of the 39 bile acids remaining, several had very small peaks that did not yield any statistically-significant changes between APAP doses, including GHCA, GLCA, GUDCA, NCA, NUDCA, DHLCA, LCA, di-oxo-LCA and 6,7 diketo-LCA. 

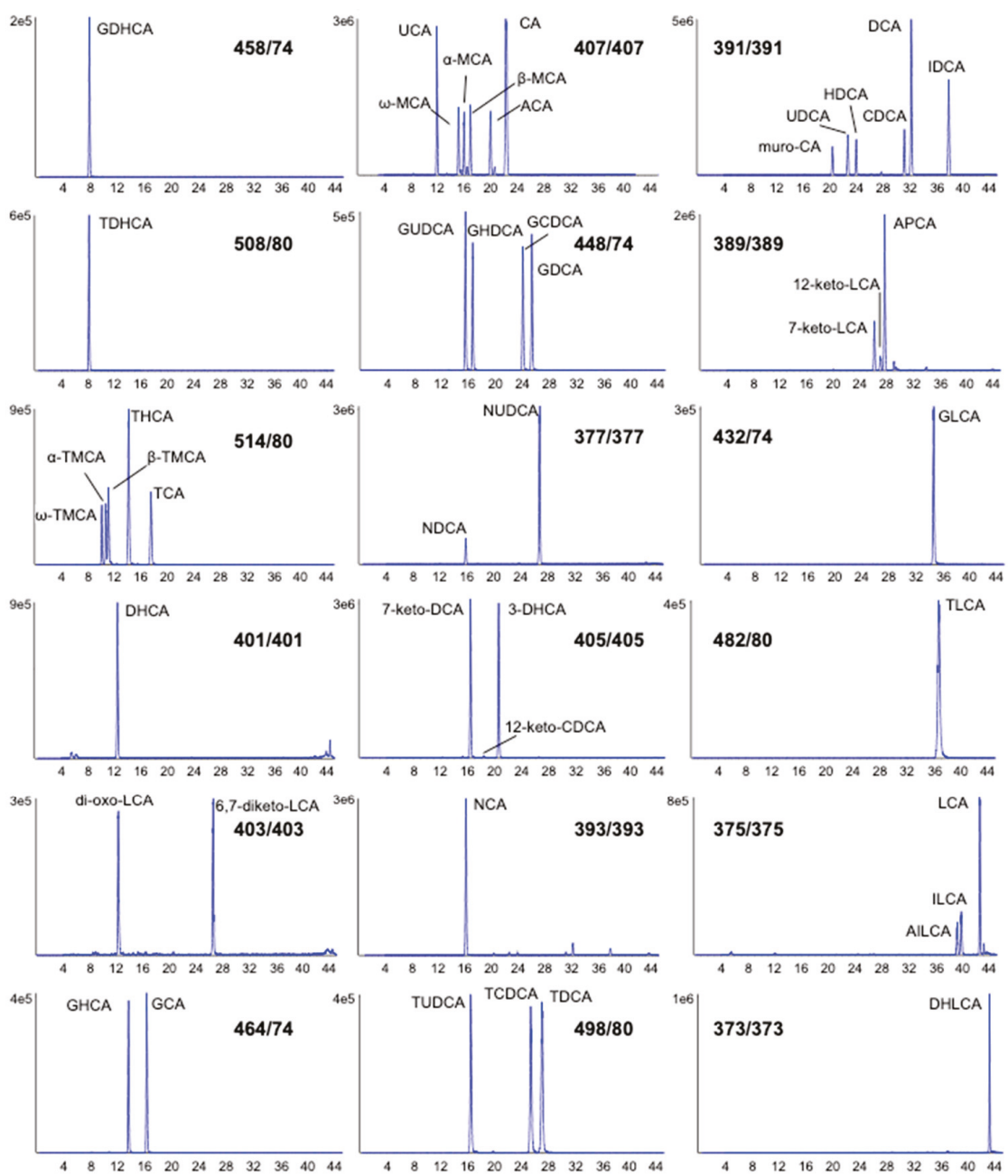

Figure 1. Representative LC-MRM chromatograms in negative mode of a standard mixture containing 46 bile acids (using the most sensitive transition for each bile acid, as shown). Acronyms for each bile acid species are listed in the Abbreviations (and Methods) section.

The highest APAP dose administered in this study significantly influenced the peaks corresponding to several bile acids (Figure 3). Table S1 shows the $p$-values and fold changes seen for each of these changing bile acids at each of the dosing levels compared to the lowest dose. This table also shows the integration data considering both MRM transitions monitored for each of these bile acids, and confirms that for all except two which were too small to properly integrate, these secondary transitions correlated well with the first (more sensitive) transition. Each MRM peak was also investigated for saturation effects. Although no linear ranges were determined directly, based on the peak heights of these bile acids, it was confirmed that we would be able to detect changes in terms of fold change (up or down). It is, however, very important to state here that fold changes of peak area ratios do not directly translate into concentration fold changes. These results are reported to determine which bile acids of the 46 from the standard mix were well observed in rat plasma samples and which were altered significantly with increased APAP dose. Thirteen bile acids of the 30 having significant signal-to-noise in our samples 
were shown to have statistically-relevant changes between the lowest and highest dose given in this study, with a $p$-value of lower than 0.05 , six of which had $p$-values lower than 0.01 . Increasing the APAP dose affected the concentration of some bile acids more than others. The bile acids with the most significant changes (with $p<0.01$ ) were GCA, GDCA, 7-keto-DCA, APCA, CA and DCA. The graphs in Figure 3 show the peak area ratios at all four doses of APAP. The taurine conjugates monitored did not show any statistically relevant changes with APAP dose. An important effect was seen, however, for several conjugated glycine conjugates. All four glycine conjugates having adequate peak size (GCA, GCDCA, GDCA and GHDCA) were found to significantly increase between 75 and $600 \mathrm{mg} / \mathrm{kg}$ APAP. The three with less obvious quantitative changes were notably much smaller peaks in the rat plasma extracts. For example, the peak area ratio for GDCA was 10.1 times higher (with a $p$-value of 0.0024 ) with $600 \mathrm{mg} / \mathrm{kg}$ compared with $75 \mathrm{mg} / \mathrm{kg}$ APAP, while the corresponding taurine conjugate, TDCA, did not show any effect at the highest dose. Since the conjugation of bile acids is an important pathway for their secretion by the liver, our results indicate that APAP could influence the transfer pathway of bile acids from the liver to the bloodstream.

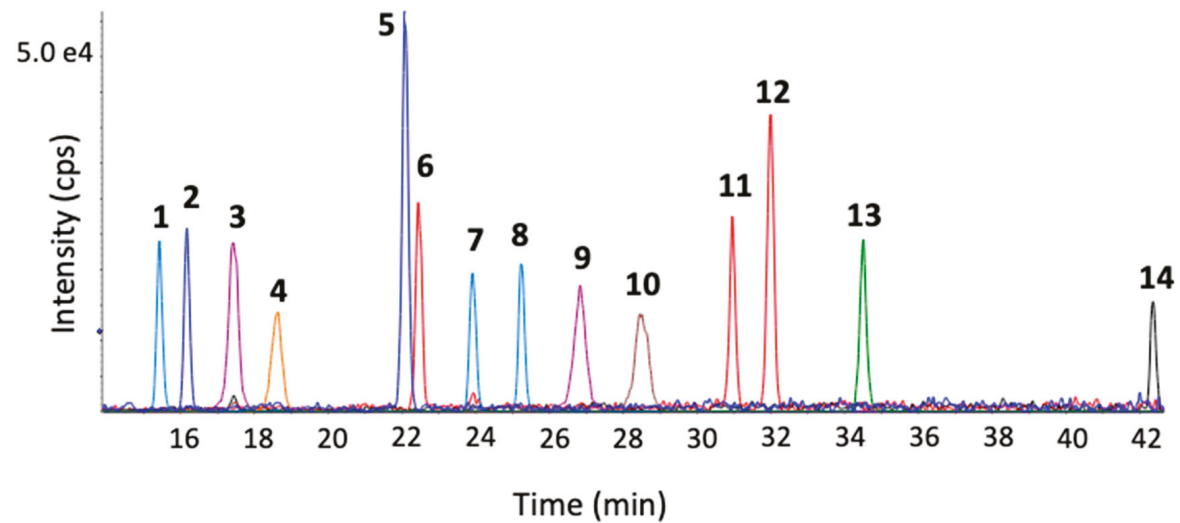

Figure 2. Representative LC-MRM chromatograms from a $20 \mu \mathrm{L}$ injection of IS mix containing 0.013-0.1 $\mu \mathrm{M}$ final concentration of each IS compound. (1) d4-GUDCA (2) d4-GCA (3) d4-TUDCA (4) d4-TCA (5) d4-CA (6) d4-UDCA (7) d4-GCDCA (8) d4-GDCA (9) d4-TCDCA (10) d6-TDCA (11) d4-CDCA (12) d4-DCA (13) d4-GLCA (14) d4-LCA.

We found that for the two primary bile acids, CDCA and CA, only CA was found to have a statistically significant increase with APAP dose levels (fold change of 1.8 and $p$-value of 0.005 at highest dose). Peak area ratios for $\alpha$-MCA, and $\omega$-MCA had increased by 4.6-, and 7.4-fold ( $p$-value of 0.0268 , and 0.0322 ), respectively. Given that CDCA is transformed by $6 \beta$-hydroxylase in rat liver into $\alpha$-MCA, $\beta-\mathrm{MCA}$, and $\omega$-MCA, it is likely that CDCA is mostly converted into different MCA isomers [21]. The peak area ratio of DCA increased 5.6-fold, (with a $p$-value of 0.0003). Interestingly, DCA has been reported to induce both early apoptosis and necrosis, thus affecting cell development [22]. The fold changes between different individual bile acids cannot be directly compared, of course, since the relative response and sensitivity of each compound by LC-MS/MS is unique. We are not assuming that a larger fold change from this data set gives a stronger change in actual concentration. This would need a follow-up study for absolute quantitation of individual bile acids, with calibration curves for each. This is quite difficult, however, considering we are not able to construct traditional calibration curves for endogenous metabolites in complex biological matrices, such as plasma, as is done for therapeutic drug monitoring. 

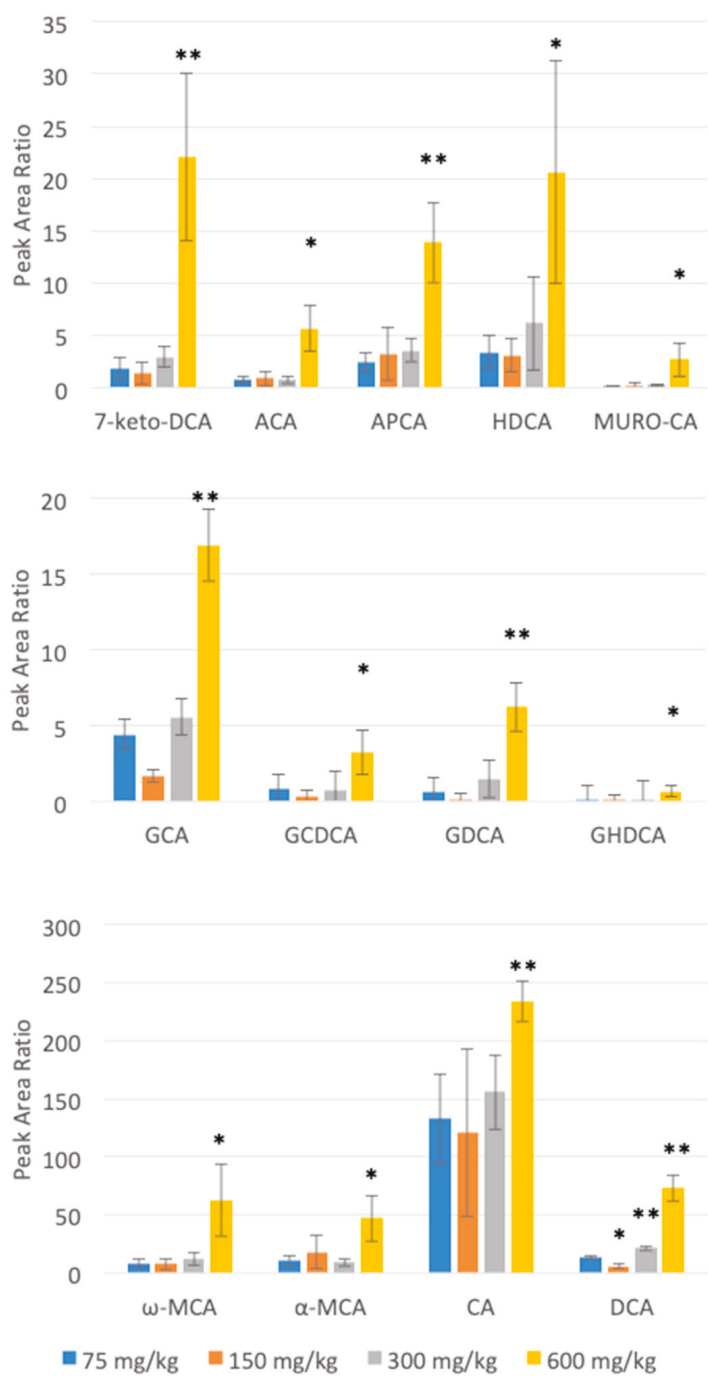

Figure 3. Peak area ratios for bile acids having significant changes between $75 \mathrm{mg} / \mathrm{kg}$ and $600 \mathrm{mg} / \mathrm{kg}$ APAP dosing, as measured in rat plasma after $24 \mathrm{~h}$. ${ }^{*} p$-value $<0.05,{ }^{* *} p$-value $<0.01$.

The LC-MRM data was imported into metabolomics software (MarkerView ${ }^{\mathrm{TM}}$ ) to perform statistical analyses (Student's t-test, as shown previously) and also to visualize data presented within a principal component analysis (PCA). Figure 4 shows the PCA plot of the first two principal components (PC1 vs. PC2), with Pareto scaling to alleviate bias to highest peaks. This plot shows clearly that the highest dose of $600 \mathrm{mg} / \mathrm{kg}$ clusters separately to the three lower doses $(75,150$, and $300 \mathrm{mg} / \mathrm{kg}$ ), as was evident from the t-testing results of the individual bile acids. The PCA plot, which used all features from the LC-MRM data, following supervised peak integration, serves to show that the high dose had a marked effect compared to the three lower doses, instead of seeing a gradual shift between the four doses. 
Scores for PC1 (43.8\%) versus PC2 (15.2\%), Log | Pareto

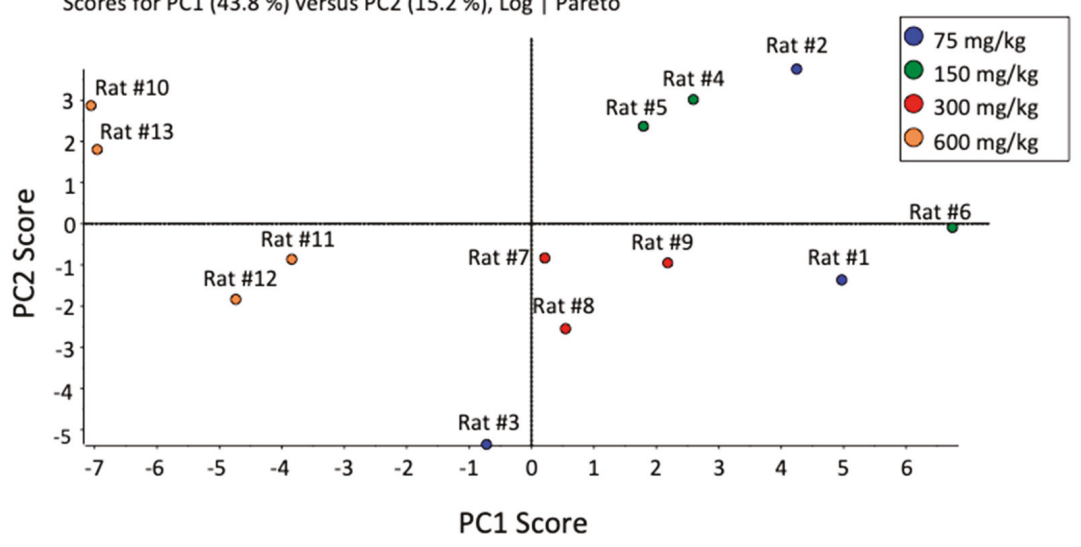

Figure 4. Unsupervised principal component analysis (PCA) of 39 bile acids detected in rat plasma samples (comparing four APAP dosing groups).

A higher throughput method could be devised to assess the specific bile acids perturbed by APAP in a follow-up study, for a more rapid assessment of changes in a clinical setting, for instance. It is important to note, however, that there exists many isomers of bile acids in biological samples and that even if we are interested in targeting a finite list of specific ones for a follow-up assay, we would still need to ensure proper separation of all these isomers. The study presented here focused specifically on evaluation the 46 bile acids available from a known standard mix. This method is not presented for the purpose of being a clinical assay, since it would likely not be high throughput enough considering the chromatographic separation needed to access all these different isomers. It also does not serve to accurately quantify each bile acid (in terms of concentration), rather it looks at relative amounts of bile acids (e.g., their profiles) in a biological matrix (rat plasma) to look for specific effects of APAP dose on individual bile acids. Therefore, this work should not be considered as a new validated method, as per US FDA guidelines. It would be interesting in a future study to validate a method for the bile acids specifically perturbed by high dose APAP. This is quite challenging in the case of endogenous metabolites since it would necessitate stable isotope standards for each metabolite to be quantified, as well as a suitable "blank" biological matrix to be used for preparing calibration curves for each analyte. Additionally, a non-targeted metabolomics approach using high-resolution tandem mass spectrometry would be able to access many more bile acid isomers, as well as sulfate and glucuronide metabolites, without the need for optimizing MS/MS parameters for MRM detection.

\section{Materials and Methods}

\subsection{Materials}

HPLC-grade acetonitrile (ACN) and methanol (MeOH), as well as LC-MS-grade formic acid were purchased from Sigma-Aldrich (Oakville, ON, Canada). Purified water was prepared in-house. MetaboloMetrics ${ }^{\mathrm{TM}}$ bile acids analysis kits were obtained from MRM Proteomics Inc. (Montreal, QC, Canada). Kits contained a mix of 46 bile acids and an IS mix of 14 deuterated isotope-labeled internal standards. Sprague-Dawley rats were dosed (IP) with 75, 150, 300, and $600 \mathrm{mg} / \mathrm{kg}$ APAP, in triplicate, and plasma was collected after $24 \mathrm{~h}$ at INRS Centre de Biologie Experimentale (Laval, QC, Canada), within standard ethical practices. The protocol was approved by the Ethics Committee of the INRS Centre de Biologie Experimentale under the ethical practices of the Canadian Council on Animal Care (project UQLK.14.02). These samples were collected in February 2014 and stored at $-80{ }^{\circ} \mathrm{C}$ until proceeding with sample preparation. 
- The standard mix containing 46 bile acids (each at $2.5 \mathrm{nmol}$ ), except for deoxycholic acid (5 nmol) and taurohyocholic acid $(6.5 \mathrm{nmol})$ was provided as a dried sample (Tube A). The bile acids in the standard mix were as follows: glycodehydrocholic acid (GDHCA), taurodehydrocholic acid (TDHCA), tauro- $\omega$-muricholic acid ( $\omega$-TMCA), tauro- $\alpha$-muricholic acid ( $\alpha$-TMCA), tauro- $\beta$-muricholic acid ( $\beta$-TMCA), taurohyocholic acid (THCA), taurocholic acid (TCA), dehydrocholic acid (DHCA), dioxolithocholic acid (di-oxo-LCA), 6,7-diketolithocholic acid (6,7-diketo-LCA), glycohyocholic acid (GHCA), glycocholic acid (GCA), ursocholic acid (UCA), $\omega$-muricholic acid ( $\omega$-MCA), $\alpha$-muricholic acid ( $\alpha$-MCA), $\beta$-muricholic acid ( $\beta$-MCA), allocholic acid (ACA), cholic acid (CA), glycoursodeoxycholic acid (GUDCA), glycohyodeoxycholic acid (GHDCA), glycochenodeoxycholic acid (GCDCA), glycodeoxycholic acid (GDCA), nordeoxycholic acid (NDCA), norursodeoxycholic acid (NUDCA), 7-ketodeoxycholic acid (7-keto-DCA), 12-ketodeoxycholic acid (12-keto-DCA), 3-dehydrocholic acid (3-DHCA), norcholic acid (NCA), tauroursodeoxycholic acid (TUDCA), taurochenodeoxycholic acid (TCDCA), taurodeoxycholic acid (TDCA), murocholic acid (muro-CA), ursodeoxycholic acid (UDCA), hyodeoxycholic acid (HDCA), chenodeoxycholic acid (CDCA), deoxycholic acid (DCA), isodeoxycholic acid (IDCA), 7-ketolithocholic acid (7-keto-LCA), 12-ketolithocholic acid (12-keto-LCA), apocholic acid (APCA), glycolithocholic acid (GLCA), taurolithocholic acid (TLCA), alloisolithocholic acid (AILCA), isolithocholic acid (ILCA), lithocholic acid (LCA) and dehydrolithocholic acid (DHLCA).

- Isotopically labeled bile acids were provided as an IS mix for normalization purposes. The labeled bile acids were present at between $0.1-0.75 \mathrm{nmol}$, as a dried sample (Tube B). The labeled bile acids in the IS mix were as follows: glycoursodeoxycholic acid- $\mathrm{d}_{4}\left(\mathrm{~d}_{4}\right.$-GUDCA), glycocholic acid- $\mathrm{d}_{4}\left(\mathrm{~d}_{4}-\mathrm{GCA}\right)$, tauroursodeoxycholic acid- $\mathrm{d}_{4}\left(\mathrm{~d}_{4}-\mathrm{TUDCA}\right)$, taurocholic acid- $\mathrm{d}_{4}\left(\mathrm{~d}_{4}-\mathrm{TCA}\right)$, cholic acid- $\mathrm{d}_{4}\left(\mathrm{~d}_{4}-\mathrm{CA}\right)$, ursodeoxycholic acid- $\mathrm{d}_{4}\left(\mathrm{~d}_{4}\right.$-UDCA), glycochenodeoxycholic acid- $\mathrm{d}_{4}$ $\left(\mathrm{d}_{4}\right.$-GCDCA), glycodeoxycholic acid- $\mathrm{d}_{4}\left(\mathrm{~d}_{4}-5\right.$ GDCA $)$, taurochenodeoxycholic acid- $\mathrm{d}_{4}\left(\mathrm{~d}_{4}-\mathrm{TCDCA}\right)$, taurodeoxycholic acid- $\mathrm{d}_{6}\left(\mathrm{~d}_{6}-\mathrm{TDCA}\right)$, chenodeoxycholic acid- $\mathrm{d}_{4}\left(\mathrm{~d}_{4}-\mathrm{CDCA}\right)$, deoxycholic acid- $\mathrm{d}_{4}$ $\left(\mathrm{d}_{4}\right.$-DCA), glycolithocholic acid- $\mathrm{d}_{4}\left(\mathrm{~d}_{4}\right.$-GLCA) and lithocholic acid- $\mathrm{d}_{4}\left(\mathrm{~d}_{4}-\mathrm{LCA}\right)$.

\subsection{Sample Preparation}

For the standard mix preparation, $250 \mu \mathrm{L}$ ACN was added to Tube A, followed by a 1:10 dilution with $40 \%$ ACN. This standard mix was injected $(2 \mu \mathrm{L})$ with the LC-MRM method described (shown in Figure 1) for retention time matching of bile acids in samples. For the internal standard (IS) mix, $7.5 \mathrm{~mL}$ of $40 \%$ ACN was added to Tube B. Plasma samples $(50 \mu \mathrm{L})$ were mixed with $50 \mu \mathrm{L}$ of the reconstituted (Tube B) internal standard solution. The extraction of bile acids from rat plasma was then performed by adding $300 \mu \mathrm{L} \mathrm{MeOH}$ to precipitate proteins. Samples were vortexed and sonicated for $15 \mathrm{~min}$, then centrifuged at $14,000 \mathrm{rpm}$ for $8 \mathrm{~min}$. Supernatants $(300 \mu \mathrm{L})$ were transferred to new microtubes, dried under nitrogen and reconstituted with $150 \mu \mathrm{L} \mathrm{50 \%} \mathrm{MeOH}$ prior to analysis. Extracts were stored at $-20^{\circ} \mathrm{C}$ until LC-MS analysis.

\section{3. $L C-M S / M S$}

Extracted plasma and standards were separated on an Aeris ${ }^{\mathrm{TM}}$ PEPTIDE XB-C18 column $(1.7 \mu \mathrm{m}$, $100 \mathrm{~mm} \times 2.1 \mathrm{~mm})\left(\right.$ Phenomenex ${ }^{\circledR}$, Torrance, CA, USA) using a Nexera ultra high performance liquid chromatography (UHPLC) system (Shimadzu, Columbia, MD, USA) at $50{ }^{\circ} \mathrm{C}$ with gradient elution using water and $\mathrm{ACN}$, each containing $0.1 \%$ formic acid as mobile phase $\mathrm{A}$ and $\mathrm{B}$, respectively, at a flow rate of $0.400 \mathrm{~mL} / \mathrm{min}$ and injection volume of $15 \mu \mathrm{L}$. The gradient started at $10 \% \mathrm{~B}$ and was held for $1 \mathrm{~min}$ increased linearly to $25 \%$ for $2 \mathrm{~min}$, to $35 \%$ over $17 \mathrm{~min}$, to $50 \%$ over $20 \mathrm{~min}$, to $60 \%$ over $2 \mathrm{~min}$ and $90 \%$ for $1 \mathrm{~min}$, followed by a $10 \mathrm{~min}$ column re-equilibration time at starting conditions. MS data was collected using a QTRAP 5500 system (Sciex, Concord, ON, Canada). Electrospray ionization (ESI) in negative ion mode and multiple reaction monitoring (MRM) was used. The MRM parameters (first and second transitions with collision energies (CE)) for all 46 bile acids are listed in Table 1). Each transition was monitored throughout the chromatogram with a dwell time of $7 \mathrm{~ms}$. 
Because of the fragmentation behavior of the deprotonated unconjugated bile acids, the first transitions chosen for the unconjugated forms were simply precursor to precursor ions, as has been done in many previous reports on bile acid analysis [17,23-25]. This was necessary for ensuring the best sensitivity of detection for these unconjugated forms. For glycine conjugated bile acids, there was a specific and sensitive common fragment ion at $m / z 74$, corresponding to the deprotonated glycine moiety being lost. Similarly, taurine conjugates yielded a common fragment at $m / z$ 80, corresponding to the $\mathrm{HSO}_{3}{ }^{-}$ ion from the taurine group. The secondary transitions were used for confirmation. For those bile acids found to be altered significantly upon APAP dose, the secondary transition was also integrated and compared.

Table 1. Optimized MRM transitions and collision energies for 46 standard bile acids along with their respective retention times.

\begin{tabular}{|c|c|c|c|c|}
\hline Bile Acid & RT (min) & 1st Transition (CE) & 2nd Transition (CE) & IS \\
\hline GDHCA & 7.9 & $458.2 / 74.0(-66)$ & $458.2 / 348.1(-41)$ & CDCA- $d_{4}$ \\
\hline TDHCA & 8.2 & $508.2 / 80.0(-123)$ & $508.2 / 124.1(-67)$ & CDCA-d 4 \\
\hline T- $\omega-\mathrm{MCA}$ & 10.1 & $514.2 / 80.0(-135)$ & $514.2 / 107.0(-82)$ & TCA-d 4 \\
\hline T- $\alpha-\mathrm{MCA}$ & 10.7 & $514.2 / 80.0(-135)$ & $514.2 / 107.0(-82)$ & TCA- $\mathrm{d}_{4}$ \\
\hline T- $\beta-\mathrm{MCA}$ & 11.1 & $514.2 / 80.0(-135)$ & $514.2 / 124.0(-65)$ & TCA-d 4 \\
\hline UCA & 11.9 & $407.2 / 407.2(-15)$ & $407.2 / 343.1(-46)$ & CA- $\mathrm{d}_{4}$ \\
\hline di-oxo-LCA & 12.3 & $403.2 / 403.2(-18)$ & $403.2 / 385.2(-40)$ & CDCA- $\mathrm{d}_{4}$ \\
\hline DHCA & 12.4 & $401.2 / 401.2(-18)$ & $401.2 / 331.1(-36)$ & CDCA- $\mathrm{d}_{4}$ \\
\hline GHCA & 13.6 & $464.2 / 74.0(-82)$ & $464.2 / 354.1(-56)$ & GCA-d 4 \\
\hline THCA & 14.1 & $514.2 / 80.0(-135)$ & $514.2 / 107.0(-82)$ & TCA-d 4 \\
\hline$\omega-\mathrm{MCA}$ & 15.1 & $407.2 / 407.2(-15)$ & $407.2 / 371.1(-43)$ & CA-d ${ }_{4}$ \\
\hline GUDCA & 15.5 & $448.2 / 74.0(-83)$ & $448.2 / 386.1(-59)$ & GUDCA-d $\mathrm{d}_{4}$ \\
\hline NDCA & 15.8 & $377.2 / 377.2(-15)$ & $377.2 / 331.1(-46)$ & CDCA-d 4 \\
\hline$\alpha-\mathrm{MCA}$ & 16.0 & $407.2 / 407.2(-15)$ & $407.2 / 371.1(-43)$ & CA-d 4 \\
\hline $\mathrm{NCA}$ & 16.1 & $393.2 / 393.2(-15)$ & $393.2 / 375.1(-45)$ & CDCA- $\mathrm{d}_{4}$ \\
\hline GCA & 16.2 & $464.2 / 74.0(-82)$ & $464.2 / 402.1(-46)$ & GCA-d 4 \\
\hline 7-keto-DCA & 16.4 & $405.2 / 405.2(-18)$ & $405.2 / 289.1(-51)$ & CDCA-d 4 \\
\hline TUDCA & 16.4 & $498.2 / 80.0(-130)$ & $498.2 / 107(-82)$ & TUDCA- $_{4}$ \\
\hline GHDCA & 16.6 & $448.2 / 74.0(-83)$ & $448.2 / 386.1(-59)$ & GUDCA-d 4 \\
\hline$\beta$-MCA & 16.9 & $407.2 / 407.2(-15)$ & $407.2 / 371.1(-43)$ & CA-d ${ }_{4}$ \\
\hline TCA & 17.5 & $514.2 / 80.0(-135)$ & $514.2 / 124.0(-65)$ & TCA-d 4 \\
\hline 12-keto-CDCA & 18.4 & $405.2 / 405.2(-18)$ & $405.2 / 387.1(-45)$ & CDCA- $\mathrm{d}_{4}$ \\
\hline ACA & 19.9 & $407.2 / 407.2(-15)$ & $407.2 / 371.1(-43)$ & CA-d 4 \\
\hline muro-CA & 20.3 & $391.2 / 391.2(-15)$ & $391.2 / 343.1(-53)$ & UDCA-d 4 \\
\hline 3-DHCA & 20.5 & $405.2 / 405.2(-18)$ & $405.2 / 289.1(-51)$ & CDCA- $\mathrm{d}_{4}$ \\
\hline $\mathrm{CA}$ & 22.2 & $407.2 / 407.2(-15)$ & $407.2 / 343.1(-46)$ & CA-d ${ }_{4}$ \\
\hline UDCA & 22.6 & $391.2 / 391.2(-15)$ & $391.2 / 373.2(-48)$ & UDCA-d 4 \\
\hline HDCA & 23.9 & $391.2 / 391.2(-15)$ & $391.2 / 373.2(-48)$ & CDCA-d ${ }_{4}$ \\
\hline GCDCA & 24.0 & $448.2 / 74.0(-83)$ & $448.2 / 404.2(-46)$ & GCDCA-d 4 \\
\hline TCDCA & 25.3 & $498.2 / 80.0(-130)$ & $498.2 / 124(-64)$ & TCDCA-d 4 \\
\hline
\end{tabular}


Table 1. Cont.

\begin{tabular}{ccccc}
\hline Bile Acid & RT (min) & 1st Transition (CE) & 2nd Transition (CE) & IS \\
\hline GDCA & 25.3 & $448.2 / 74.0(-83)$ & $448.2 / 404.2(-46)$ & GDCA-d $_{4}$ \\
\hline 7-keto-LCA & 26.1 & $389.2 / 389.2(-18)$ & $389.2 / 354.1(-43)$ & LCA-d $_{4}$ \\
\hline 6,7-diketo-LCA & 26.5 & $403.2 / 403.2(-18)$ & $403.2 / 347.1(-39)$ & LCA-d $_{4}$ \\
\hline NUDCA & 26.7 & $377.2 / 377.2(-15)$ & $377.2 / 359.1(-45)$ & CDCA-d $_{4}$ \\
\hline TDCA & 27.0 & $498.2 / 80.0(-130)$ & $498.2 / 124(-64)$ & TDCA-d $_{6}$ \\
\hline 12-keto-LCA & 27.1 & $389.2 / 389.2(-18)$ & $389.2 / 354.1(-43)$ & LCA-d $_{4}$ \\
\hline APCA & 27.6 & $389.2 / 389.2(-18)$ & $389.2 / 371.1(-43)$ & CDCA-d $_{4}$ \\
\hline CDCA & 31.1 & $391.2 / 391.2(-15)$ & $391.2 / 373.2(-48)$ & CDCA-d \\
\hline DCA & 32.1 & $391.2 / 391.2(-15)$ & $391.2 / 343.1(-53)$ & DCA-d 4 \\
\hline GLCA & 34.6 & $432.2 / 74.0(-66)$ & $432.2 / 388.1(-45)$ & GLCA-d $_{4}$ \\
\hline TLCA & 36.6 & $482.2 / 80.0(-135)$ & $482.2 / 107(-80)$ & LCA-d $_{4}$ \\
\hline IDCA & 37.8 & $391.2 / 391.2(-15)$ & $391.2 / 345.1(-45)$ & DCA-d $_{4}$ \\
\hline AILCA & 39.1 & $375.2 / 375.2(-15)$ & $375.2 / 45(-50)$ & LCA-d $_{4}$ \\
\hline ILCA & 39.7 & $375.2 / 375.2(-15)$ & $375.2 / 45(-50)$ & LCA-d $_{4}$ \\
\hline LCA & 42.5 & $375.2 / 375.2(-15)$ & $375.2 / 45(-50)$ & LCA-d $_{4}$ \\
\hline DHLCA & 43.1 & $373.2 / 373.2(-18)$ & $373.2 / 45(-50)$ & LCA-d $_{4}$ \\
\hline
\end{tabular}

\subsection{Statistical Analysis}

A mixture of 14 deuterated bile acids was added to the plasma samples prior to metabolite extraction, for normalization of data as peak area ratios (analyte/IS) (see Table 2). For those bile acids without corresponding deuterated analogs, the closest eluting deuterated analog was used as IS, as noted in Table 1. Standards were used to confirm the identity of each bile acid, based on retention and MRM signal. Peak integration was performed using Multiquant ${ }^{\mathrm{TM}} 2.1$ (Sciex). Statistical analyses were done using MarkerView ${ }^{\mathrm{TM}}$ 1.2.1 (Sciex). This software was used to perform Student's t-tests, yielding $p$-values and fold changes between different dosing groups, for each bile acid detected in rat plasma samples. Within Markerview software, principal component analysis was performed on the integrated LC-MRM data, without weighting and using Pareto scaling (unsupervised).

Table 2. Optimized MRM transitions and collision energies for 14 internal standards compounds, along with their respective retention times.

\begin{tabular}{ccccc}
\hline & RT (min) & Q1 $(\mathrm{m} / \mathbf{z})$ & Q3 $(\mathrm{m} / \mathbf{z})$ & CE (V) \\
\hline GUDCA-d $_{4}$ & 15.5 & 452.3 & 74.0 & -41 \\
GCA-d $_{4}$ & 16.2 & 468.3 & 74.0 & -45 \\
TUDCA-d $_{4}$ & 17.4 & 502.3 & 80.0 & -73 \\
TCA-d $_{4}$ & 18.7 & 518.3 & 80.0 & -80 \\
CA-d & 22.1 & 411.3 & 411.3 & -15 \\
UDCA-d & 22.5 & 395.3 & 395.3 & -15 \\
GCDCA-d $_{4}$ & 23.8 & 452.3 & 74.0 & -37 \\
GDCA-d & 25.3 & 452.3 & 74.0 & -41 \\
TCDCA-d & 26.8 & 502.3 & 80.0 & -80 \\
TDCA-d & 28.7 & 504.3 & 80.0 & -80 \\
CDCA-d & 31 & 395.3 & 395.3 & -15 \\
DCA-d & 32 & 395.3 & 395.3 & -15 \\
GLCA-d & 34.5 & 436.3 & 74.0 & -41 \\
LCA-d & 42.4 & 379.3 & 379.3 & -15 \\
\hline
\end{tabular}




\section{Conclusions}

In this study, we have developed a targeted metabolomics method to gain a better understanding of the effects of APAP on circulating bile acid profiles. A simple protein precipitation procedure in rat plasma was employed rapidly prepare samples for analysis. A standard mix of 46 bile acids was successfully resolved by LC-MRM, 39 of which were detected in rat plasma samples. These analyses highlighted significant changes in bile acid profiles with increasing APAP dose in rats. In general, these results indicate that APAP can have an important effect on the metabolism of bile acids. Depending on the dose level, exposure to high or repeated APAP doses has the potential to induce serious health problems, related to bile acid metabolism and excretion. The specificity of these biomarkers to APAP-related toxicity would still need to be investigated. Certain of these bile acids can also serve as biomarkers to establish the level of hepatotoxicity; however, more work would be needed to validate specific bile acid biomarkers for clinical use.

Supplementary Materials: The following are available online at http://www.mdpi.com/2218-1989/10/1/26/s1, Table S1: Comparison of p-values and fold-changes between the four doses of APAP administered in this study (using two MRM transitions for each peak) of known bile acids showing significant differences between the lowest and highest dose APAP.

Author Contributions: Conceptualization, L.S.; methodology, V.P., L.O., L.S.; formal analysis, V.P.; data curation, V.P.; writing—original draft preparation, V.P., L.S.; writing-review and editing, L.S.; supervision, L.S.; funding acquisition, L.S. All authors have read and agreed to the published version of the manuscript.

Funding: This research was funded by the Natural Sciences and Engineering Research Council of Canada (NSERC), discovery grant program (grant number 355933-2016).

Acknowledgments: We would like to thank MRM Proteomics Inc. for the generous gift of the bile acid kit, containing the standard and IS mix.

Conflicts of Interest: The authors declare no conflict of interest. The funders had no role in the design of the study; in the collection, analyses, or interpretation of data; in the writing of the manuscript, or in the decision to publish the results.

$\begin{array}{ll}\text { Abbreviations } & \\ \text { GDHCA } & \begin{array}{l}\text { glycodehydrocholic acid } \\ \text { taurodehydrocholic acid }\end{array} \\ \text { TDHCA } & \text { tauro- } \omega \text {-muricholic acid } \\ \alpha \text {-TMCA } & \text { tauro- } \alpha \text {-muricholic acid } \\ \beta \text {-TMCA } & \text { tauro- } \beta \text {-muricholic acid } \\ \text { THCA } & \text { taurohyocholic acid } \\ \text { TCA } & \text { taurocholic acid } \\ \text { DHCA } & \text { dehydrocholic acid } \\ \text { di-oxo-LCA } & \text { dioxolithocholic acid } \\ 6,7-\text { diketo-LCA } & 6,7 \text {-diketolithocholic acid } \\ \text { GHCA } & \text { glycohyocholic acid } \\ \text { GCA } & \text { glycocholic acid } \\ \text { UCA } & \text { ursocholic acid } \\ \omega-M C A & \omega \text {-muricholic acid } \\ \alpha \text {-MCA } & \alpha \text {-muricholic acid } \\ \beta \text {-MCA } & \beta \text {-muricholic acid } \\ \text { ACA } & \text { allocholic acid } \\ \text { CA } & \text { cholic acid } \\ \text { GUDCA } & \text { glycoursodeoxycholic acid } \\ \text { GHDCA } & \text { glycohyodeoxycholic acid } \\ \text { GCDCA } & \text { glycochenodeoxycholic acid } \\ \text { GDCA } & \text { glycodeoxycholic acid } \\ \text { NDCA } & \text { nordeoxycholic acid } \\ \text { NUDCA } & \text { norursodeoxycholic acid } \\ & \end{array}$




$\begin{array}{ll}\text { 7-keto-DCA } & \text { 7-ketodeoxycholic acid } \\ \text { 12-keto-DCA } & \text { 12-ketodeoxycholic acid } \\ \text { 3-DHCA } & \text { 3-dehydrocholic acid } \\ \text { NCA } & \text { norcholic acid } \\ \text { TUDCA } & \text { tauroursodeoxycholic acid } \\ \text { TCDCA } & \text { taurochenodeoxycholic acid } \\ \text { TDCA } & \text { taurodeoxycholic acid } \\ \text { muro-CA } & \text { murocholic acid } \\ \text { UDCA } & \text { ursodeoxycholic acid } \\ \text { HDCA } & \text { hyodeoxycholic acid } \\ \text { CDCA } & \text { chenodeoxycholic acid } \\ \text { DCA } & \text { deoxycholic acid } \\ \text { IDCA } & \text { isodeoxycholic acid } \\ \text { 7-keto-LCA } & \text { 7-ketolithocholic acid } \\ \text { 12-keto-LCA } & \text { 12-ketolithocholic acid } \\ \text { APCA } & \text { apocholic acid } \\ \text { GLCA } & \text { glycolithocholic acid } \\ \text { TLCA } & \text { taurolithocholic acid } \\ \text { AILCA } & \text { alloisolithocholic acid } \\ \text { ILCA } & \text { isolithocholic acid } \\ \text { LCA } & \text { lithocholic acid } \\ \text { DHLCA } & \text { dehydrolithocholic acid } \\ & \end{array}$

\section{References}

1. Russell, D.W. Fifty years of advances in bile acid synthesis and metabolism. J. Lipid Res. 2009, 50, S120-S125. [CrossRef] [PubMed]

2. Wilson, J.D. The role of bile acids in the overall regulation of steroid metabolism. Arch. Intern. Med. 1972, 130, 493-505. [CrossRef]

3. Boyer, J.L. Bile formation and secretion. Compr. Physiol. 2013, 3, 1035-1078. [CrossRef]

4. Hofmann, A.F. Bile Acids: The Good, the Bad, and the Ugly. News Physiol. Sci. 1999, 14, 24-29. [CrossRef] [PubMed]

5. Copple, B.L.; Jaeschke, H.; Klaassen, C.D. Oxidative stress and the pathogenesis of cholestasis. Semin. Liver Dis. 2010, 30, 195-204. [CrossRef] [PubMed]

6. Fang, Y.; Han, S.I.; Mitchell, C.; Gupta, S.; Studer, E.; Grant, S.; Hylemon, P.B.; Dent, P. Bile acids induce mitochondrial ROS, which promote activation of receptor tyrosine kinases and signaling pathways in rat hepatocytes. Hepatology 2004, 40, 961-971. [CrossRef] [PubMed]

7. Gupta, S.; Natarajan, R.; Payne, S.G.; Studer, E.J.; Spiegel, S.; Dent, P.; Hylemon, P.B. Deoxycholic acid activates the c-Jun N-terminal kinase pathway via FAS receptor activation in primary hepatocytes: Role of acidic sphingomyelinase-mediated ceramide generation in FAS receptor activation. J. Biol. Chem. 2004, 279, 5821-5828. [CrossRef] [PubMed]

8. Jaeschke, H.; Gores, G.J.; Cederbaum, A.I.; Hinson, J.A.; Pessayre, D.; Lemasters, J.J. Mechanisms of hepatotoxicity. Toxicol. Sci. 2002, 65, 166-176. [CrossRef]

9. Reddy, B.S.; Weisburger, J.H.; Wynder, E.L. Effects of high risk and low risk diets for colon carcinogenesis on fecal microflora and steroids in man. J. Nutr. 1975, 105, 878-884. [CrossRef]

10. Nagengast, F.M.; Grubben, M.J.A.L.; van Munster, I.P. Role of bile acids in colorectal carcinogenesis. Eur. J. Cancer 1995, 31, 1067-1070. [CrossRef]

11. Larson, A.M.; Polson, J.; Fontana, R.J.; Davern, T.J.; Lalani, E.; Hynan, L.S.; Reisch, J.S.; SchiØdt, F.V.; Ostapowicz, G.; Shakil, A.O.; et al. Acetaminophen-induced acute liver failure: Results of a United States multicenter, prospective study. Hepatology 2005, 42, 1364-1372. [CrossRef] [PubMed]

12. Lee, W.M. Acute liver failure in the United States. Semin. Liver Dis. 2003, 23, 217-226. [CrossRef]

13. Woolbright, B.L.; McGill, M.R.; Staggs, V.S.; Winefield, R.D.; Gholami, P.; Olyaee, M.; Sharpe, M.R.; Curry, S.C.; Lee, W.M.; Jaeschke, H.; et al. Glycodeoxycholic acid levels as prognostic biomarker in acetaminophen-induced acute liver failure patients. Toxicol. Sci. 2014, 142, 436-444. [CrossRef] 
14. James, L.; Yan, K.; Pence, L.; Simpson, P.; Bhattacharyya, S.; Gill, P.; Letzig, L.; Kearns, G.; Beger, R. Comparison of Bile Acids and Acetaminophen Protein Adducts in Children and Adolescents with Acetaminophen Toxicity. PLoS ONE 2015, 10, e0131010. [CrossRef]

15. Bhushan, B.; Borude, P.; Edwards, G.; Walesky, C.; Cleveland, J.; Li, F.; Ma, X.; Apte, U. Role of bile acids in liver injury and regeneration following acetaminophen overdose. Am. J. Pathol. 2013, 183, 1518-1526. [CrossRef]

16. Scherer, M.; Gnewuch, C.; Schmitz, G.; Liebisch, G. Rapid quantification of bile acids and their conjugates in serum by liquid chromatography-tandem mass spectrometry. J. Chromatogr. B 2009, 877, 3920-3925. [CrossRef]

17. Suzuki, Y.; Kaneko, R.; Nomura, M.; Naito, H.; Kitamori, K.; Nakajima, T.; Ogawa, T.; Hattori, H.; Seno, H.; Ishii, A. Simple and rapid quantitation of 21 bile acids in rat serum and liver by UPLC-MS-MS: Effect of high fat diet on glycine conjugates of rat bile acids. Nagoya J. Med. Sci. 2013, 75, 57-72. [CrossRef]

18. Han, J.; Liu, Y.; Wang, R.; Yang, J.; Ling, V.; Borchers, C.H. Metabolic profiling of bile acids in human and mouse blood by LC-MS/MS in combination with phospholipid-depletion solid-phase extraction. Anal. Chem. 2015, 87, 1127-1136. [CrossRef]

19. Yousef, I.M.; Mignault, D.; Weber, A.M.; Tuchweber, B. Influence of dehydrocholic acid on the secretion of bile acids and biliary lipids in rats. Digestion 1990, 45, 40-51. [CrossRef] [PubMed]

20. Shefe, S.; Salen, G.; Hauser, S.; Dayal, B.; Batta, A.K. Metabolism of Iso-Bile Acids in the Rat. J. Biol. Chem. 1982, 257, 1401-1406.

21. Voigt, W.; Thomas, P.J.; Hsia, S.L. Enzymatic studies of bile acid metabolism. I. 6Beta-hydroxylation of chenodeoxycholic and taurochenodeoxycholic acids by microsomal preparations of rat liver. J. Biol. Chem. 1968, 243, 3493-3499. [PubMed]

22. Shiraki, K.; Ito, T.; Sugimoto, K.; Fuke, H.; Inoue, T.; Miyashita, K.; Yamanaka, T.; Suzuki, M.; Nabeshima, K.; Nakano, T.; et al. Different effects of bile acids, ursodeoxycholic acid and deoxycholic acid, on cell growth and cell death in human colonic adenocarcinoma cells. Int. J. Mol. Med. 2005, 16, 729-733. [PubMed]

23. Cai, X.; Liu, Y.; Zhou, X.; Navaneethan, U.; Shen, B.; Guo, B. An LC-ESI-MS method for the quantitative analysis of bile acids composition in fecal materials. Biomed. Chromatogr. 2012, 26, 101-108. [CrossRef] [PubMed]

24. Tagliacozzi, D.; Mozzi, A.F.; Casetta, B.; Bertucci, P.; Bernardini, S.; Di Ilio, C.; Urbani, A.; Federici, G. Quantitative analysis of bile acids in human plasma by liquid chromatography-electrospray tandem mass spectrometry: A simple and rapid one-step method. Clin. Chem. Lab. Med. 2003, 41, 1633-1641. [CrossRef]

25. Garcia-Canaveras, J.C.; Donato, M.T.; Castell, J.V.; Lahoz, A. Targeted profiling of circulating and hepatic bile acids in human, mouse, and rat using a UPLC-MRM-MS-validated method. J. Lipid Res. 2012, 53, 2231-2241. [CrossRef]

(C) 2020 by the authors. Licensee MDPI, Basel, Switzerland. This article is an open access article distributed under the terms and conditions of the Creative Commons Attribution (CC BY) license (http://creativecommons.org/licenses/by/4.0/). 


\title{
Hepatic Metabolic Derangements Triggered by Hyperthermia: An In Vitro Metabolomic Study
}

\author{
Ana Margarida Araújo ${ }^{1, *}$, Maria Enea ${ }^{1}$, Félix Carvalho ${ }^{1}$, Maria de Lourdes Bastos ${ }^{1}$, \\ Márcia Carvalho ${ }^{1,2, *}$ and Paula Guedes de Pinho ${ }^{1, *}$ \\ 1 UCIBIO, REQUIMTE, Laboratory of Toxicology, Faculty of Pharmacy, University of Porto, \\ Rua Jorge Viterbo Ferreira, 228, 4050-313 Porto, Portugal; eneavmaria@gmail.com (M.E.); \\ felixdc@ff.up.pt (F.C.); mlbastos@ff.up.pt (M.d.L.B.) \\ 2 UFP Energy, Environment and Health Research Unit (FP-ENAS), University Fernando Pessoa, \\ Praça Nove de Abril, 349, 4249-004 Porto, Portugal \\ * Correspondence: ana.margarida.c.araujo@gmail.com (A.M.A.); mcarv@ufp.edu.pt (M.C.); \\ pguedes@ff.up.pt (P.G.d.P.); Tel.: +351-220-428-500 (A.M.A. \& P.G.d.P.); +351-225-071-300 (M.C.)
}

Received: 20 August 2019; Accepted: 11 October 2019; Published: 15 October 2019

\begin{abstract}
Background and aims: Liver toxicity is a well-documented and potentially fatal adverse complication of hyperthermia. However, the impact of hyperthermia on the hepatic metabolome has hitherto not been investigated. Methods: In this study, gas chromatography-mass spectrometry (GC-MS)-based metabolomics was applied to assess the in vitro metabolic response of primary mouse hepatocytes (PMH, $n=10$ ) to a heat stress stimulus, i.e., after $24 \mathrm{~h}$ exposure to $40.5^{\circ} \mathrm{C}$. Metabolomic profiling of both intracellular metabolites and volatile metabolites in the extracellular medium of PMH was performed. Results: Multivariate analysis showed alterations in levels of 22 intraand 59 extracellular metabolites, unveiling the capability of the metabolic pattern to discriminate cells exposed to heat stress from cells incubated at normothermic conditions $\left(37^{\circ} \mathrm{C}\right)$. Hyperthermia caused a considerable loss of cell viability that was accompanied by significant alterations in the tricarboxylic acid cycle, amino acids metabolism, urea cycle, glutamate metabolism, pentose phosphate pathway, and in the volatile signature associated with the lipid peroxidation process. Conclusion: These results provide novel insights into the mechanisms underlying hyperthermia-induced hepatocellular damage.
\end{abstract}

Keywords: heat stress; primary mouse hepatocytes; metabolic profile; GC-MS; multivariate statistical analysis

\section{Introduction}

Thermoregulation is a complex process, crucial for body homeostasis and survival, that is meticulously orchestrated by the thermoregulatory center in the hypothalamus [1]. A failure in hypothalamic regulation leads to an imbalance between heat accumulation (either due to extreme environmental temperatures and/or body heat generation) and heat dissipation, and may cause a huge increase in body temperature above that considered physiologically normal-this condition is commonly referred as hyperthermia [2]. A wide variety of xenobiotics can affect the thermal homeostasis, triggering or exacerbating the hyperthermia-induced damage, both by the increased metabolic heat production (e.g., sympathomimetic agents) or by an impairment of heat-dissipating effector mechanisms (e.g., anticholinergic agents) [3]. This disruption will consequently affect many other homeostatic systems and may result in several life-threatening complications such as disseminated intravascular coagulation, hyperkalemia, metabolic acidosis, multi-organ failure, and rhabdomyolysis [4-6].

Hepatocellular injury is a well-documented adverse complication of heat stroke, and oxidative stress has been identified as the main mechanism underlying hyperthermia-induced liver toxicity [7-9]. Earlier in vitro studies have provided convincing evidence that hyperthermia per se stimulates an 
aggressive pro-oxidant state in freshly isolated rat and mouse hepatocytes and in rat liver, making liver cells more vulnerable to prooxidant species that subsequently lead to lipid peroxidation and cellular damage [9-12]. Furthermore, it is also known that variations in the cellular temperature affect the effectiveness of various enzymes and alter membrane stability and diffusion capacity, which disrupts a great number of critical cellular functions, such as energy use and membrane ion fluxes [13]. However, specific hepatic metabolic pathways altered by hyperthermia remain largely unknown.

The aim of this study was to improve our understanding of how hyperthermia affects the cellular metabolome of primary mouse hepatocytes (PMH). For this, a gas chromatography-mass spectrometry (GC-MS) untargeted metabolomic approach was used to analyze the metabolic profile of primary mouse hepatocytes at hyperthermic $\left(40.5^{\circ} \mathrm{C}\right)$ conditions and compare it with normothermic $\left(37^{\circ} \mathrm{C}\right)$ conditions. The analysis of the metabolites released from (extracellular metabolome) or existing within the cells (intracellular metabolome) was performed in order to obtain a more detailed metabolic characterization profile. As far as we know, this is the first metabolomic study to investigate the metabolic derangements triggered by hyperthermia in hepatic cells.

\section{Results}

\subsection{Hyperthermic Conditions Affect the Viability of PMH}

Changes in viability of PMH triggered by hyperthermic conditions were evaluated using the MTT reduction and lactate dehydrogenase (LDH) leakage assays. The data presented in Figure 1A show that a temperature rise from 37 to $40.5^{\circ} \mathrm{C}$, after $24 \mathrm{~h}$, caused a significant reduction in cell viability as compared with control (about $40 \%$ decrease in viability according to the MTT reduction assay, $p<0.0001$ ) and also significantly affected the cellular membrane integrity, according to the LDH release assay (about $35 \%$ of the cells were affected, $p<0.0001$ ) (Figure 1B). In order to ensure that $\mathrm{pH}$ of the culture medium was not affected by temperature, and therefore contributed to the observed cell death, this factor was measured, and no significant differences were found between groups.
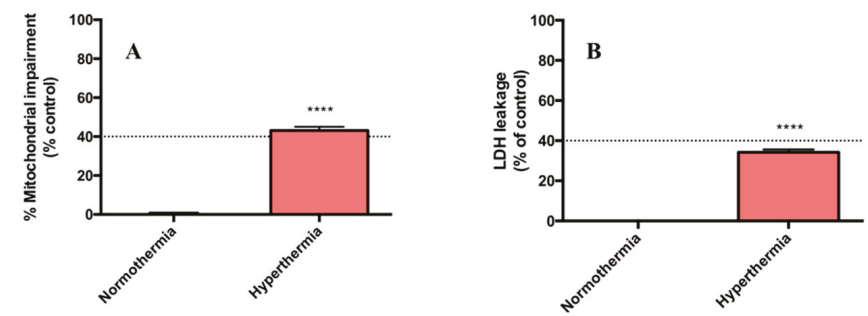

Figure 1. Cell viability measured by (A) MTT reduction and (B) lactate dehydrogenase (LDH) leakage, $24 \mathrm{~h}$ after exposure of primary mouse hepatocytes to normothermic $\left(37^{\circ} \mathrm{C}\right)$ and hyperthermic $\left(40.5^{\circ} \mathrm{C}\right)$ conditions. Results were obtained from 10 independent experiments, performed in triplicate. $* * * * \quad p<1.00 \times 10^{-4}$ (hyperthermic vs. normothermic conditions).

\subsection{Hyperthermia Significantly Alters the Metabolic Profile of PMH}

Data obtained in this study showed that all quality control $(\mathrm{QC})$ samples and the internal standards used in the analysis of the intracellular and extracellular (volatile organic compounds, VOCs and volatile carbonyl compounds, VCCs) metabolome had good reproducibility over the acquisition time (Figure S1), the chromatographic datasets being considered robust and qualified for statistical analyses. Unsupervised multivariate analysis revealed that the exposure of PMH to heat stress resulted in significant alterations in the intra- and extra-cellular metabolome, since the separation between cells under normothermic and hyperthermic conditions was already apparent in all principal component analysis (PCA) score plots (Figure S2), indicating a unique metabolite profile of each group. This separation was maximized in the orthogonal projections to latent structures discriminant 
analysis (OPLS-DA) models (Figure $2 \mathrm{~A}-\mathrm{C}$ ) which presented good quality parameters $\left(\mathrm{R}^{2} \mathrm{X}=0.72\right.$, $\mathrm{R}^{2} \mathrm{Y}=0.77, \mathrm{Q}^{2}=0.54$, and $p$-value $=1.5 \times 10^{-2}$ for intracellular data; $\mathrm{R}^{2} \mathrm{X}=0.54, \mathrm{R}^{2} \mathrm{Y}=0.88, \mathrm{Q}^{2}=0.74$, and $p$-value $=2.3 \times 10^{-4}$ for VOCs, and $\mathrm{R}^{2} \mathrm{X}=0.54, \mathrm{R}^{2} \mathrm{Y}=0.92, \mathrm{Q}^{2}=0.84$, and $p$-value $=6.5 \times 10^{-6}$ for VCCs). Furthermore, the robustness of all OPLS-DA models was confirmed through a permutation test (Figure $2 \mathrm{D}-\mathrm{F}$ ), since all $\mathrm{R}^{2}$ and $\mathrm{Q}^{2}$ values of the permuted classes are lower than the original classes.
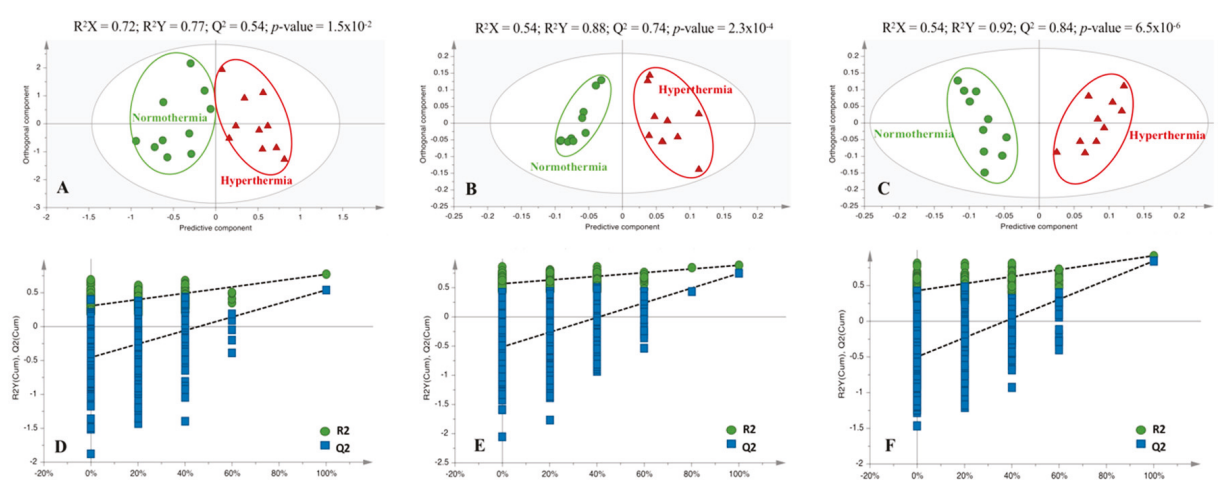

Figure 2. Orthogonal projections to latent structures discriminant analysis (OPLS-DA) score scatter plots obtained for the chromatograms corresponding to cells exposed to normothermic $(n=10$, -) and hyperthermic $(n=10, \mathbf{\Delta})$ conditions, after analysis of the (A) intracellular metabolome, (B) volatile organic compound (VOC) and (C) volatile carbonyl compound (VCC) in extracellular metabolome. (D-F) Statistical validation of the respective OPLS-DA models obtained by permutation tests (500 permutations).

Through the analysis of the corresponding loading S-plot, the variables with VIP $>1$ combined with $\mid p($ corr $) \mid>0.5$ were selected for integration in order to assess the magnitude and significance of metabolic variations caused by hyperthermia (Figure 3). A total of 28 intracellular metabolites were identified as potentially discriminant $(|\mathrm{p}(\mathrm{corr})|>0.5$ and VIP $>1)$, including several amino acids and derivatives, organic acids, carbohydrates, and fatty acids derivatives. In brief, only 22 intracellular metabolites appeared significantly $(p<0.05)$ affected by the temperature increase, of which 15 were significantly decreased (namely 1,5-anhydrohexitol, fumarate, malate, 2-ketoglutarate, aspartate, glutamate, ornithine, mannitol, myo-inositol, ribose, and five unidentified metabolites) and seven significantly increased (including valine, phenylalanine, isoleucine, docosahexaenoic acid, 2-monostearin, glycerol monostearate, and one unidentified metabolite) (Figure 3A). In parallel, the univariate analysis revealed that of a total of 31 potentially discriminating VOCs ( $|\mathrm{p}(\mathrm{corr})|>0.5$ and VIP $>1)$, 30 varied significantly $(p<0.05)$ between the two groups (hyperthermia vs. normothermia). Significantly altered VOCs include one alkane, one ester, two aldehydes, five alkanals, seven ketones, seven alcohols, and seven unidentified metabolites. In general, VOCs appeared to be significantly up-regulated, with exception of 1,1-dimethylpropyl acetate, cyclohexanol, and an unknown compound which appeared to be significantly down-regulated after temperature rise (Figure 3B). Regarding VCCs analysis, the metabolites found significantly altered in the hyperthermic conditions compared to the normothermic condition are summarized in Figure 3C. 

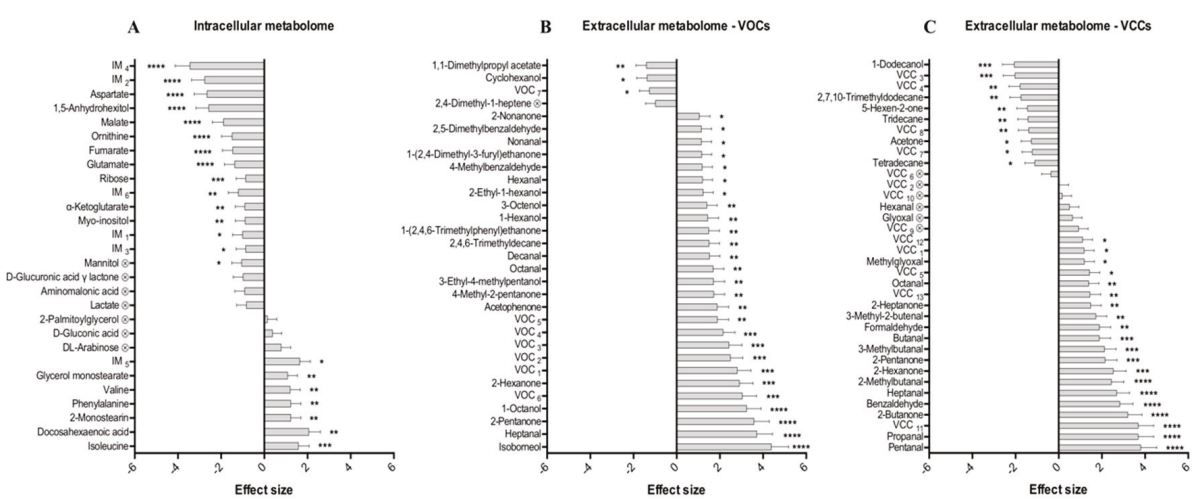

Figure 3. Effect size of the metabolites altered by heat stress, evaluated by comparison of cells exposed to hyperthermic vs. normothermic conditions in the (A) intracellular metabolome and (B and C) extracellular metabolome (VOCs and VCCs, respectively). Unidentified compounds are reported as ' $\mathrm{IM}_{\mathrm{i}}{ }^{\prime},{ }^{\prime} \mathrm{VOC}_{\mathrm{i}}{ }^{\prime}$ and ' $\mathrm{VCC}_{\mathrm{i}}{ }^{\prime}(\mathrm{i}=1,2,3 \ldots)$ according to the ascending order of their retention time (RT) values. Metabolites marked with $\otimes$ are not statistically significant after false discovery rate (FDR) correction (FDR corrected $p$-value: $3.93 \times 10^{-2}$ for intracellular metabolome, $4.84 \times 10^{-2}$ for VOCs, and $4.12 \times 10^{-2}$ for VCCs). ${ }^{*} p<5.00 \times 10^{-2},{ }^{* *} p<1.00 \times 10^{-2},{ }^{* * *} p<1.00 \times 10^{-3},{ }^{* * * *} p<1.00 \times 10^{-4}$ (hyperthermic vs. normothermic conditions).

The corresponding loading S-plot indicates 36 VCCs as potentially discriminant ( $\mid \mathrm{p}$ (corr) $\mid>0.5$ and VIP $>1)$, of which 30 were found significantly altered $(p<0.05)$. Significant metabolites detected after VCCs analysis include an alcohol, an alkenal, a dicarbonyl, an aromatic aldehyde, three alkanes, six ketones, eight alkanals, and nine unidentified metabolites. Univariate analysis indicated that tetradecane, tridecane, 2,7,10-trimethyldodecane, acetone, 1-dodecanol, and four unidentified compounds appeared to be significantly down-regulated in the medium after temperature rise, while all other VCCs appeared to be significantly up-regulated in hyperthermic conditions. It is noteworthy that in the analysis of VOCs and VCCs five common compounds were found (hexanal, heptanal, octanal, 2-pentanone, and 2-hexanone) with the same alteration trends (up-regulation under hyperthermia).

\subsection{Discriminant Metabolites Identified}

A total of 28 intracellular metabolites and 67 extracellular metabolites (31 VOCs and 36 VCCs) were indicated as potentially altered under hyperthermic conditions. A complete list with the information used for the identification of these discriminant metabolites (such as retention time (RT), characteristic ions $(m / z)$, retention indexes (RI), reverse match score, Human Metabolome Database (HMDB) and Kyoto Encyclopedia of Genes and Genomes (KEGG) identification codes) as well as their identification level is summarized in Tables S1 and S2 [14]. The identification of 44 metabolites was unequivocally confirmed with analytical standards (level 1), 25 metabolites were putatively identified based on commercial spectral libraries (level 2), a compound class was attributed to four metabolites (level 3), and 22 metabolites were not yet identified (level 4) (Table S1 and S2).

\subsection{Hepatic Biochemical Pathways Affected by Hyperthermia}

To aid in the identification of the major disturbed metabolic pathways, metabolites significantly altered by temperature $(p<0.05)$ were analyzed using the MetaboAnalyst 4.0 software. Our analysis revealed that phenylalanine and tyrosine metabolism, aspartate metabolism, urea cycle, tricarboxylic acid (TCA) cycle, the transference of acetyl groups into the mitochondria and malate-aspartate shuttle were the hepatic pathways more affected by hyperthermia (Figure 4). 


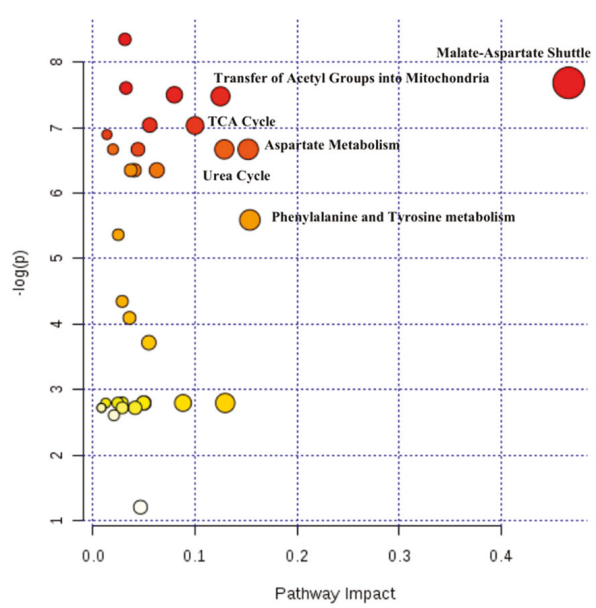

Figure 4. Overview of the dysregulated metabolic pathways based on metabolites alteration caused by hyperthermia. The node color is based on the $p$ value, where a dark circle color indicates a more significant pathway. The node radius corresponds to the pathway impact value. Pathways were annotated when $p<0.05$ and pathway impact $>0.1$.

Due to the limitations associated with the software database and to overcome the lack of knowledge about the role of some compounds in the metabolic pathways (especially volatile compounds), Spearman's correlation indexes were calculated using all discriminant metabolites $(p<0.01)$ (Figure 5). Taking into account a $\mid$ correlation index $\mid \geq 0.90$ and $p<0.0001$, data revealed the existence of several strong positive correlations, namely between 2-pentanone and $\mathrm{VCC}_{11}(\mathrm{r}=0.90), 2$-pentanone and 2-butanone $(\mathrm{r}=0.90)$, 2-hexanone and $\mathrm{VOC}_{1}(\mathrm{r}=0.90)$, fumarate and 2-ketoglutarate $(\mathrm{r}=0.91)$, glycerol monostearate and 2-monostearin $(\mathrm{r}=0.91)$, 2-butanone and $\mathrm{VCC}_{11}(\mathrm{r}=0.91)$, 2-pentanone and isoborneol $(\mathrm{r}=0.91), \mathrm{VOC}_{2}$ and $\mathrm{VOC}_{3}(\mathrm{r}=0.91)$, malate and ornithine $(\mathrm{r}=0.92)$, 2-pentanone and 2-hexanone $(r=0.92)$, malate and 1,5-anhydrohexitol $(r=0.93)$, 2-hexanone and $\mathrm{VCC}_{11}(\mathrm{r}=0.93)$, 2-methylbutanal and 3-methylbutanal $(r=0.93)$, heptanal and benzaldehyde $(r=0.93)$, 2-ketoglutarate and ornithine $(r=0.94)$, valine and phenylalanine $(r=0.94)$, 2-hexanone and isoborneol $(r=0.94)$, fumarate and malate $(r=0.96)$, phenylalanine and isoleucine $(r=0.96)$, fumarate and ornithine $(r=0.97)$, and finally between valine and isoleucine $(r=0.97)$. These correlation magnitudes suggest that these metabolites may share the same metabolic pathway or some common regulatory mechanism. Moreover, the Spearman's rank correlation coefficient suggests that $\mathrm{VCC}_{11}$ may also be a ketone derivative due to their positive correlations with 2-butanone, 2-pentanone and 2-hexanone as well as common characteristic MS fragments (Table S2). 


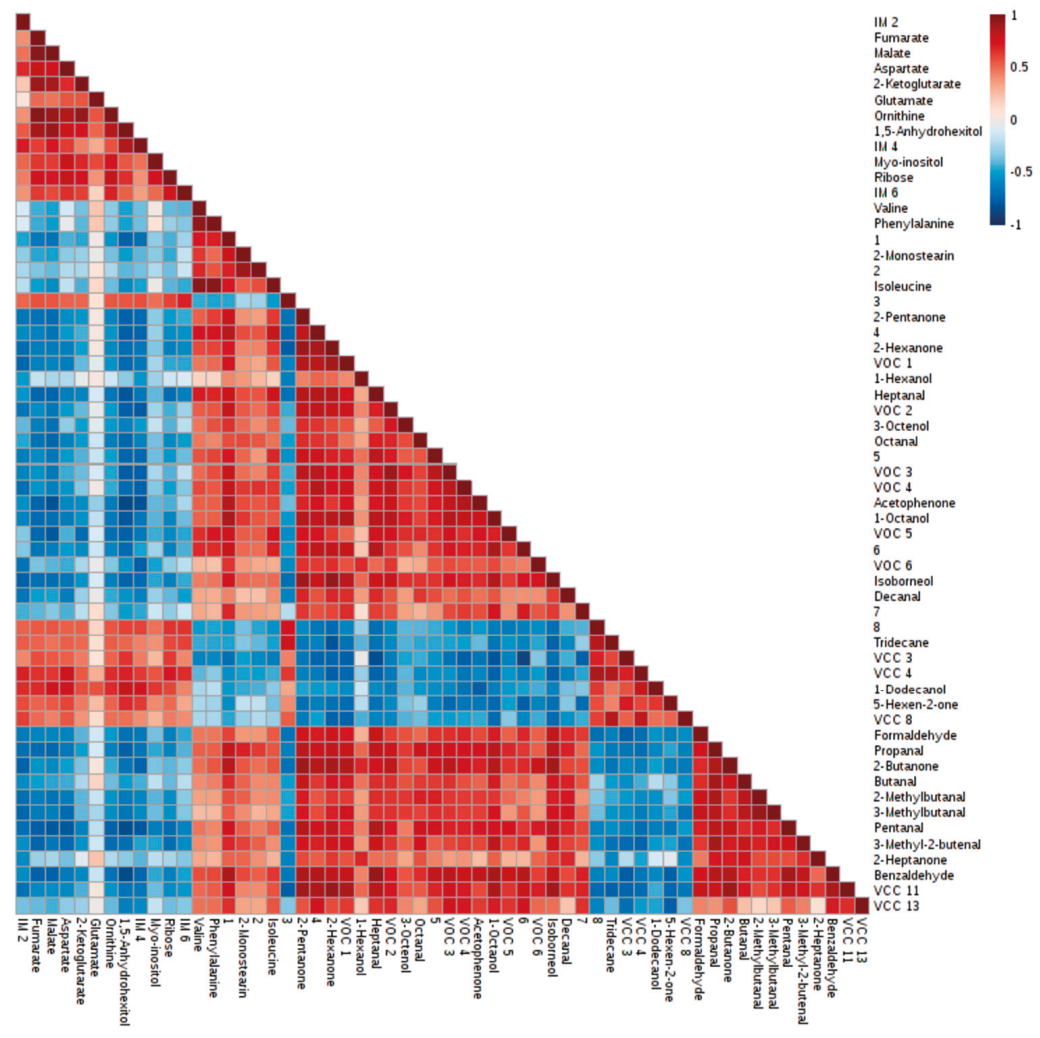

Figure 5. Heatmap representing the Spearman's correlations between the metabolites significantly altered $(p<0.01)$ after a thermal insult. 1. Docosahexaenoic acid; 2. Glycerol monostearate; 3. 1,1-Dimethylpropyl acetate; 4. 4-Methyl-2-pentanone; 5. 3-Ethyl-4-methylpentanol; 6. 2,4,6-Trimethyldecane; 7. 1-(2,4,6-Trimethylphenyl)ethanone; 8. 2,7,10-Trimethyldodecane.

\section{Discussion}

The number of metabolomic studies based on mass spectrometry methodologies has increased exponentially due to its high sensitivity, selectivity, and rapid data acquisition [15]. In this work, different GC-MS approaches were implemented to study the intracellular and extracellular metabolome in order to better characterize the changes caused by hyperthermia in mice isolated hepatocytes. Hyperthermia induced a significant loss of cell viability under our experimental conditions (approximately 35\%, according to the LDH assay). This may represent a limitation of the present study as the metabolome reflects both viable and dead cells, but may also be more representative of the real consequences derived from a hyperthermic state, as the resulting liver diseases are also associated with the death of hepatic cells. The combination of intracellular and extracellular datasets clearly demonstrated that an increase in temperature from physiological $\left(37^{\circ} \mathrm{C}\right)$ to hyperthermic $\left(40.5^{\circ} \mathrm{C}\right)$ conditions, after $24 \mathrm{~h}$, induces profound changes in the hepatic metabolome, reflected in the level of 22 intracellular metabolites and 59 extracellular volatile metabolites ( 30 VOCs and 29 VCCs). This high number of metabolites suggests that several metabolic pathways may be altered and contribute to the liver damage. Therefore, the main hepatic changes described in this study as being induced by hyperthermia will be discussed below. 
First of all, our metabolomic study revealed that one of the major alterations caused by hyperthermia was the significant decrease of some TCA cycle intermediates, namely 2-ketoglutarate $(p<0.001)$, fumarate and malate $(p<0.0001)$, suggesting a mitochondrial dysfunction and consequently an energetic failure. Heat stress has been shown to cause mitochondrial protein denaturation, specifically the pyruvate decarboxylase complex subunits and the TCA cycle enzymes, which could explain these decreased levels [16]. Additionally, cells exposed to a thermal insult showed alterations in the levels of some glucogenic and/or ketogenic amino acids, with glutamate and aspartate having a significant depletion $(p<0.0001)$ and phenylalanine $(p=0.0034)$, valine $(p=0.0040)$, and isoleucine $(p=0.0005)$ a significant increase. Protein denaturation may also justify the accumulation of phenylalanine, valine, and isoleucine, since they are not being used to restore the basal levels of TCA cycle intermediates, possibly due to an ineffective activity of transaminases. This enzymatic alteration may contribute to the impairment of the energetic pathway, and although a positive correlation between phenylalanine, valine, and isoleucine support the existence of a common regulatory pathway, further studies are needed to confirm this theory. Moreover, despite the impossibility of contributing to the restoration of the TCA cycle intermediates via transamination, glutamate and aspartate seem to be used in other metabolic pathways, since their intracellular levels are reduced.

As suggested by the pathway analysis, the urea cycle appears to be also affected, since a significant decrease $(p<0.0001)$ in ornithine levels was observed after a rise in the incubation temperature from 37 to $40.5^{\circ} \mathrm{C}$. However, it is possible that the ornithine levels may have been grossly estimated, since arginine can be converted into ornithine during the derivatization reaction and, therefore, some caution in the interpretation of this result is necessary [17].

Another remarkable change observed in this study was the significant decrease of glutamate levels $(p<0.0001)$ in hyperthermic conditions. This metabolite is crucial for the glutathione (GSH) synthesis, since its first biosynthesis phase requires an ATP enzymatic step that leads to the formation of $\gamma$-glutamylcysteine from glutamate and cysteine [18]. Thus, low levels of glutamate coupled with the breakdown in ATP production indicate that the ability of hepatocytes to synthesize GSH may be compromised [18]. Glutathione plays several vital functions, including detoxification of xenobiotics and/or their metabolites and cell signaling, and is also a major cellular antioxidant crucial in protecting cells against oxidative stress [18]. Since hyperthermia is a pro-oxidant aggressive condition, decreased levels of hepatic GSH may compromise cellular antioxidant defenses and render cells more susceptible to the deleterious effects of reactive oxygen and nitrogen species (ROS/RNS) formed within the cell [9]. Consistent with our results, a significant reduction of GSH was observed in freshly isolated rat hepatocytes and in the HepG2 cell line after exposure to heat stress $[10,19]$. Furthermore, it has been demonstrated that GSH depletion triggered by hyperthermic conditions is in fact coupled with an increased ROS/RNS production [19]. Owing to their high reactivity, ROS/RNS can interact with the lipids present in the cell and cause their oxidative damage with consequent lipid peroxidation and impairment of cell membrane functions [20]. Lipid peroxidation gives rise to diverse secondary end-products capable of reacting with several intracellular targets and exert adverse biological effects [20]. The study of the volatile fraction of the extracellular metabolic profile developed in this work detected several of these secondary end-products, where hydrocarbons, alcohols, ketones, and aldehydes appeared as the main classes significantly altered under hyperthermic conditions. Hydrocarbons are a class of compounds typically generated by polyunsaturated fatty acid (PUFA) peroxidation [21,22]. However, in our study their levels were significantly decreased after a temperature increase. Such decrease may be explained by a possible up-regulation of cytochrome P450 enzymes responsible for hydroxylating alkanes and lead to the production of the corresponding alcohols, which, with the exception of cyclohexanol and 1-dodecanol, appear increased under hyperthermic conditions [21,22]. On the other hand, alcohols can be converted into ketones or aldehydes, which could justify the reduced levels of cyclohexanol and 1-dodecanol and the significant increase of some of the ketone and aldehyde metabolites found in the extracellular environment of cells exposed to hyperthermia [21]. The increase in aldehydes may also have a direct origin in the reduction of 
hydroperoxides by cytochrome P450 [21,22]. Most of the volatile compounds detected in our study are positively correlated, which may suggest a common metabolic origin. These results suggest that lipid peroxidation is responsible for most of the metabolic changes that hyperthermia causes in the volatile fraction of surrounding medium of PMH. In fact, this harmful process had already been associated with the hepatotoxicity caused by heat stress [9].

Finally, another volatile compound found significantly increased after exposure to hyperthermia was formaldehyde $(p=0.0230$ ). Formaldehyde may be endogenously formed through the L-methionine, histamine, methanol, and methylamine metabolic pathway, and its catabolism involves its conversion to $\mathrm{CO}_{2}$ through reactions involving glutathione $[23,24]$. However, since increasing temperature seems to compromise the glutathione metabolism, this could explain the endogenous accumulation of formaldehyde, that can rapidly react with nucleophilic groups present in nucleic acids and proteins, leading to mutagenesis and cell death [23]. Low glutathione levels may also represent a limiting factor for methylglyoxal detoxification, which was another volatile compound found with significantly increased levels in the extracellular environment of cells exposed to hyperthermia $(p=0.0212)$ [25]. Although the main production pathway of methylglyoxal is associated with glycolysis, in pathological conditions the oxidation of ketone bodies is also an important source of this compound [26]. In fact, this study showed that this methylglyoxal formation route seems to be activated, since hyperthermia led to a significant decrease $(p=0.0129)$ in acetone levels compared to the basal levels found in normothermia. Methylglyoxal is one of the most potent and reactive glycating agents present in cells, so its accumulation causes several deleterious effects, including genotoxic effects [25]. Evidence suggests that high levels of methylglyoxal causes interstrand cross-links in duplex DNA, strand breaks, and increased mutation frequency [26]. Thus, taking into account that hyperthermia considerably affects DNA repair mechanisms, PMH will be more sensitive to methylglyoxal's genotoxic effects [16].

In addition, our metabolomic results also suggest a dysfunction of the pentose phosphate pathway since a significant decrease of the ribose reserves was observed $(p=0.0003)$, an intermediate metabolite essential for the nucleotide biosynthesis and that can consequently affect DNA repair mechanisms [27].

In several in vivo studies where the global heat stress was evaluated in different matrices (serum, plasma, urine, milk, liver), alterations in the amino acid metabolism, TCA cycle, and nucleotide metabolism were also found [28-30]. Our findings are in agreement with the changes found in in vivo studies, thereby suggesting translatability of our results.

\section{Materials and Methods}

\subsection{Chemicals}

All reagents were of analytical grade or of the highest grade available. Antibiotic mixture of penicillin/streptomycin $(10,000 \mathrm{U} / \mathrm{mL} / 10,000 \mathrm{mg} / \mathrm{mL})$, fungizone $(250 \mathrm{mg} / \mathrm{mL})$, and heat-inactivated fetal bovine serum (FBS) were obtained from GIBCO Invitrogen (Barcelona, Spain). Collagen $\mathrm{G}$ was obtained from Merck (Darmstadt, Germany). 4-Fluorobenzaldehyde ( $\geq 98 \%$ ), collagenase from Clostridium histolyticum Type IA, desmosterol ( $\geq 84 \%$ ), dexamethasone, ethylene glycol-bis-(2-aminoethylether)- $N$, $N, N^{\prime}, N^{\prime}$-tetraacetic acid (EGTA), gentamicin, insulin solution from bovine pancreas $(10 \mathrm{mg} / \mathrm{mL})$, methoxyamine hydrochloride ( $\geq 98 \%), N, O$-bis(trimethylsilyl)trifluoroacetamide with $1 \%$ trimethylchlorosilane (BSTFA $+1 \%$ TMCS), O-(2,3,4,5,6-pentafluorobenzyl)hydroxylamine hydrochloride (PFBHA, $\geq 99 \%$ ), sodium chloride $(\mathrm{NaCl}, \geq 99.5 \%$ ), thiazolyl blue tetrazolium bromide (MTT, $\geq 98 \%$ ), thymol ( $\geq 98.5 \%)$, Triton X-100, trypan blue solution, Williams' E medium, and all standards used throughout the work were purchased from Sigma-Aldrich (St. Louis, Missouri, USA). Methanol ( $\geq 99.9 \%$ ) and pyridine ( $\geq 99 \%$ ) were purchased from VWR (Leuven, Belgium). 


\subsection{Isolation and Primary Culture of Mouse Hepatocytes}

Ten male CD-1 mice (7-9 weeks old) were used in these experiments. Animal housing and experimental procedures were performed in accordance with Portuguese legislation (Decree-Law No. 113/2013, of August 7th), and approved by the Ethical Committee of the Faculty of Pharmacy of University of Porto (protocol number P158/2016) and by the Portuguese National Authority for Animal Health (reference number 0421/000/000/2017). Isolation of hepatocytes was performed using a modified collagenase perfusion method, as described by Godoy et al. [31]. Surgical procedures were performed under isoflurane anesthesia and carried out between 10.00 and 11.00 a.m. The initial viability of the isolated mouse hepatocytes was estimated by the trypan blue exclusion test and was always greater than $80 \%$. Subsequently, a suspension containing $0.5 \times 10^{6}$ viable cells $/ \mathrm{mL}$ was prepared in complete culture medium (William's E medium supplemented with 10\% FBS, $100 \mathrm{U} / \mathrm{mL}$ penicillin, $100 \mathrm{mg} / \mathrm{mL}$ streptomycin, $100 \mu \mathrm{g} / \mathrm{mL}$ gentamicin, $5 \mu \mathrm{g} / \mathrm{mL}$ insulin, $50 \mathrm{nM}$ dexamethasone, and $2.5 \mu \mathrm{g} / \mathrm{mL}$ fungizone) and seeded into a collagen-coated 35-mm Petri dishes (for metabolomic studies) and 96-well culture plates (for cell viability assays). The cells were then incubated overnight at $37^{\circ} \mathrm{C}$ with $5 \% \mathrm{CO}_{2}$ to allow cell adhesion. After seeding, the maintenance media was replaced by serum-free medium and the cells were incubated for $24 \mathrm{~h}$ under normothermic $\left(37^{\circ} \mathrm{C}\right)$ or hyperthermic $\left(40.5^{\circ} \mathrm{C}\right)$ conditions. For each 96 -well plate, a positive control (1\% Triton X-100) was also considered.

\subsection{Cell Viability Assays}

The effect of temperature on metabolic activity of PMH was determined using the MTT reduction assay, as described in a previous work [32]. In order to evaluate the effect of temperature in the cell membrane disruption, the release of lactate dehydrogenase (LDH) to the extracellular medium was assessed using a protocol previously described by Valente et al. [33]. For both assays data were normalized to a no-effect $\left(\mathrm{PMH}\right.$ at $\left.37^{\circ} \mathrm{C}\right)$ and a maximum-effect $(\mathrm{PMH}$ lysed with $1 \%$ Triton X-100) controls.

\subsection{Collection, Preparation, and Analysis of Samples for Metabolomic Analysis}

The collection of samples was performed according to a protocol used in a previous study [32]. Briefly, for the analysis of the extracellular volatile fraction, the culture medium from each well was collected on ice and subsequently centrifuged $\left(2000 \times \mathrm{g}, 5 \mathrm{~min}, 4{ }^{\circ} \mathrm{C}\right)$ to eliminate possible cellular fragments. Adherent cells were washed twice with $0.9 \% \mathrm{NaCl}$, and then an ice-cold methanol:water solution $(80: 20, v / v)$ was added to extract the intracellular metabolites. In sequence, cells were scraped, harvested, sonicated on ice for a few seconds, and centrifuged for $10 \mathrm{~min}$ at $3000 \times g$ at $4{ }^{\circ} \mathrm{C}$. The supernatant was collected in a glass vial for further intracellular metabolome analysis. For each GC-MS procedure, quality control (QC) samples were prepared by pooling the same amount of each sample used in the study. All samples were kept at $-80{ }^{\circ} \mathrm{C}$ until analysis.

The analysis of volatile fraction of the extracellular metabolome was performed by two methodologies based on headspace solid-phase microextraction (HS-SPME) coupled to GC-MS previously optimized by our group [34]. The analysis VOCs was carried out directly in the headspace of the cell culture medium, while VCCs were determined after a previous derivatization step. Sample preparation and GC-MS analysis of samples is described in detail in previous studies of our group [32,34].

\subsection{GC-MS Data Pre-Processing}

The GC-MS data were converted to the CDF file format using the software MASSTransit 3.0.1.16 (Palisade Corp, Newfield, NY) and pre-processed using the software MZmine 2.23 [35]. The parameters used in the pre-processing steps were set as follows: RT range 4.3-24.5 $\mathrm{min}, \mathrm{m} / \mathrm{z}$ range 50-400, MS data noise level $3 \times 10^{4}, \mathrm{~m} / \mathrm{z}$ tolerance 0.5 , baseline level $8 \times 10^{4}$ and peak duration range $0.02-0.35 \mathrm{~min}$ for the intracellular analysis; RT range $2.1-25.0 \mathrm{~min}, \mathrm{~m} / \mathrm{z}$ range $40-300$, MS data noise level $1 \times 10^{5}, \mathrm{~m} / \mathrm{z}$ 
tolerance 0.5 , baseline level $4 \times 10^{4}$ and peak duration range $0.02-0.3$ for the VOCs analysis; and RT range 10.5-35.5 $\mathrm{min}, \mathrm{m} / \mathrm{z}$ range 50-500, MS data noise level $1 \times 10^{5}, \mathrm{~m} / \mathrm{z}$ tolerance 0.5 , baseline level $2 \times 10^{4}$ and peak duration range $0.02-0.5 \mathrm{~min}$ for the VCCs analysis. After pre-processing steps, data were normalized by total chromatogram area to eliminate systematic and biological bias [36]. All known artefacts including peaks from the chromatographic column, SPME fibers (e.g., phthalates and siloxanes) and plasticizers, as well as chromatographic peaks with a signal to noise less than three and with relative standard deviation (RSD) higher than $30 \%$ across all QCs, were not considered in the statistical analysis.

\subsection{Multivariate and Univariate Statistical Analysis}

The final matrices were imported into the SIMCA-P 13.0.3 software (Umetrics Umea, Sweden) for multivariate analysis. Principal component analysis (PCA) and orthogonal projections to latent structures discriminant analysis (OPLS-DA) were applied to Pareto scaled data, with a default 7-fold internal cross validation, from which $R^{2}$ and $Q^{2}$ values reflect, respectively, the explained variance and the predictive capability of the models [37]. Simultaneously, all OPLS-DA models were validated through permutation test (500 permutations) and CV-ANOVA $p$-value (cross-validated analysis of variance) were also performed to determine the level of significance of group separation, a $p$-value $<0.05$ being indicative of a significant model [37]. The variables $(\mathrm{m} / \mathrm{z}$-RT pairs) relevant for groups separation were assessed through the inspection of loading $S$-plots. Only the variables corresponding to the metabolite fingerprint (based on relative abundance and selectivity) and that simultaneously presented variables importance to the projection (VIP) $>1$ and $p($ corr $)>|0.5|$ were used in subsequent univariate analysis [37]. In addition, metabolites that resulted in multiple chromatographic peaks as a consequence of derivatization reactions were summed, as suggested by Mastrangelo et al. [38]. The statistical significance between the mean of two groups under study (PMH under normothermic vs. hyperthermic conditions) was assessed for the relevant metabolites ( $|\mathrm{p}(\mathrm{corr})|>0.5$ and VIP $>1$ ) in GraphPad Prism version 6 (GraphPad Software, San Diego, CA, USA). The $p$-value was determined through an unpaired student $t$-test for normal distribution data or an unpaired Mann-Whitney test for a non-normal distribution. False discovery rate (FDR) corrected $p$-values were considered in the assessment of statistical significance [39]. Additionally, the effect size (ES), corrected for a small number of samples, were also determined for each relevant metabolite, according to equations provided in the literature [40].

\subsection{Identification of Discriminant Metabolites}

The identification of discriminant metabolites was done according to the Metabolomics Standards Initiative (MSI) guidelines, being based on the comparison of the retention index (RI) determined for each metabolite with the RI described in the literature and by comparing the retention time (RTs) and mass spectrum of the discriminant metabolite with spectra accessible in the National Institute of Standards and Technology (NIST14) mass spectral library [14]. Only for reverse match factors greater than 700, the tentative metabolite identification was considered. Whenever possible, the identification was unequivocally confirmed with authentic reference standards injected under the same chromatographic conditions. Metabolites that do not meet these identification criteria are reported throughout the paper according to their crescent $\mathrm{RT}$ values as ' $\mathrm{IM}_{\mathrm{i}}$ ' (for the intracellular metabolites), 'VOC ${ }_{\mathrm{i}}^{\prime}$ or ${ }^{\prime} \mathrm{VCC}_{\mathrm{i}}$ ' $(\mathrm{i}=1,2,3 \ldots)$.

\subsection{Biochemical Interpretation}

Metabolic pathway analysis was used to identify biochemical pathways associated with alterations caused by the temperature increase. Metabolites significantly altered $(p<0.05)$ with Human Metabolome Database (HMDB) codes were imported into a Metaboanalyst 4.0 software (http://www.metaboanalyst. ca) and were searched against Mus musculus database [41]. Biochemical pathways were selected according to the $p$-value $(p<0.05)$ and pathway impact value $(>0.1)$. The Human Metabolome Database 
(HMDB, www.hmdb.ca) and Kyoto Encyclopedia of Genes and Genomes (KEGG, www.kegg.jp) were also checked to support the biochemical interpretation. Furthermore, to search for possible correlations between metabolites significantly altered $(p<0.01)$, Spearman's rank correlation coefficient was also calculated and represented in a heatmap.

\section{Conclusions}

Heat stress is a life-threatening condition capable of disturbing cellular homeostasis. In this work, we presented a metabolomic study of the liver following hyperthermia in an in vitro model. Our data revealed that GC-MS metabolomic profiling can be successfully used to visualize the hyperthermia-induced disorders, since in the present study prominent derangements were observed in the intra and extracellular hepatic metabolome. Multivariate and univariate statistical analysis revealed a high number of compromised metabolites that are essentially associated with the energetic pathway, synthesis of antioxidant defenses, and with the lipid peroxidation process. Taking into account the results obtained, it is our belief that this metabolomic study may represent an interesting platform to evaluate and understand the deleterious effects of heat stroke in humans.

Supplementary Materials: The following are available online at http://www.mdpi.com/2218-1989/9/10/228/s1, Figure S1: Principal component analysis (PCA) score scatter plots obtained for the GC-MS chromatograms of the three distinct procedures ((A) intracellular metabolite profiling, (B) extracellular metabolite profiling-VOCs and (C) extracellular metabolite profiling-VCCs) to evaluate data quality. Each sample is represented in the score's scatter plot as an individual variable, namely quality control $(\mathrm{QC})$ samples $(\bullet)$ and the intracellular/extracellular content of all primary mouse hepatocytes samples (๑). (D) Boxplot of the three internal standards used in the different metabolomics studies. Data are expressed as the mean and standard deviation (SD) of the normalized peak area by total area of the chromatogram. All internal standards presented a variation coefficient inferior to 20\%.; Figure S2: PCA score scatter plots obtained for the chromatograms corresponding to cells exposed to normothermic $(n=10, \bullet)$ and hyperthermic $(n=10, \mathbf{\Delta})$ conditions, after analysis of the (A) intracellular metabolome, as well as (B) VOCs and (C) VCCs present in the extracellular metabolome.; Table S1. Identification of discriminant intracellular metabolites selected from OPLS-DA loading $S$-plots (VIP $>1$ and $\mid p($ corr $) \mid>0.5$ ). The identification of the metabolites was done according to the Metabolomics Standards Initiative (MSI) levels. They were characterized by retention time $(\mathrm{RT})$, characteristic ions $(\mathrm{m} / \mathrm{z})$, retention index (from the literature $\left(R I_{\text {lit }}\right)$ and compared with the calculated $\left(R I_{\text {calc }}\right)$ for the same chromatographic column), reverse match factor from National Institute of Standards and Technology (NIST) and Human Metabolome Database (HMDB) and Kyoto Encyclopedia of Genes and Genomes (KEGG) code (when available). Table S2. Identification of discriminant volatile extracellular metabolites (VOCs and VCCs) selected from OPLS-DA loading S-plots (VIP > 1 and $\mid \mathrm{p}($ corr)| $>0.5)$. The identification of the metabolites was done according to the MSI levels. They were characterized by retention time (RT), characteristic ions $(\mathrm{m} / \mathrm{z})$, retention index (from the literature $\left(R I_{\text {lit }}\right)$ and compared with the calculated $\left(R I_{\text {calc }}\right)$ for the same chromatographic column), reverse match factor from NIST and HMDB and KEGG code (when available).

Author Contributions: A.M.A. was responsible for the execution of the experimental work, data analysis and writing the manuscript. M.E. helped with the isolation of primary mouse hepatocytes. F.C., M.d.L.B., M.C. and P.G.d.P. contributed to the design and management of the study. All authors critically commented on and approved the final submitted version of the paper.

Funding: This research was funded by European Union (FEDER funds POCI/01/0145/FEDER/007728) and National Funds (FCT/MEC, Fundação para a Ciência e a Tecnologia and Ministério da Educação e Ciência) under the Partnership Agreement PT2020 UID/MULTI/04378/2019. The study is a result of the project NORTE-01-0145-FEDER-000024, supported by Norte Portugal Regional Operational Program (NORTE 2020), under the PORTUGAL 2020 Partnership Agreement (DESignBIOtecHealth-New Technologies for three Health Challenges of Modern Societies: Diabetes, Drug Abuse and Kidney Diseases), through the European Regional Development Fund (ERDF). A. M. Araújo and M. Enea thank FCT for their PhD fellowships (SFRH/BD/107708/2015 and PD/BD/109634/2015, respectively) and M. Carvalho also acknowledges FCT through the UID/MULTI/04546/2019 project.

Conflicts of Interest: The authors declare no conflict of interest.

\section{References}

1. Dao, C.K.; Nowinski, S.M.; Mills, E.M. The heat is on: Molecular mechanisms of drug-induced hyperthermia. Temperature 2014, 1, 183-191. [CrossRef] [PubMed]

2. Roth, J.; Rummel, C.; Barth, S.W.; Gerstberger, R.; Hubschle, T. Molecular aspects of fever and hyperthermia. Neurol. Clin. 2006, 24, 421-439. [CrossRef] [PubMed] 
3. Hayes, B.D.; Martinez, J.P.; Barrueto, F., Jr. Drug-induced hyperthermic syndromes: Part I. Hyperthermia in overdose. Emerg. Med. Clin. N. Am. 2013, 31, 1019-1033. [CrossRef] [PubMed]

4. Jamshidi, N.; Dawson, A. The hot patient: Acute drug-induced hyperthermia. Aust. Prescr. 2019, 42, 24-28. [CrossRef] [PubMed]

5. Coenen, S.; Tran, K.; de Haan, J.; de Man, R. Liver transplantation for non-exertional heat stroke-related acute liver failure. BMJ Case Rep. 2017, 2017. [CrossRef] [PubMed]

6. White, M.G.; Luca, L.E.; Nonner, D.; Saleh, O.; Hu, B.; Barrett, E.F.; Barrett, J.N. Cellular mechanisms of neuronal damage from hyperthermia. Prog. Brain Res. 2007, 162, 347-371. [CrossRef]

7. Davis, B.C.; Tillman, H.; Chung, R.T.; Stravitz, R.T.; Reddy, R.; Fontana, R.J.; McGuire, B.; Davern, T.; Lee, W.M.; Acute Liver Failure Study Group. Heat stroke leading to acute liver injury \& failure: A case series from the Acute Liver Failure Study Group. Liver Int. 2017, 37, 509-513. [CrossRef]

8. Garcin, J.M.; Bronstein, J.A.; Cremades, S.; Courbin, P.; Cointet, F. Acute liver failure is frequent during heat stroke. World J. Gastroenterol. 2008, 14, 158-159. [CrossRef]

9. Skibba, J.L.; Powers, R.H.; Stadnicka, A.; Cullinane, D.W.; Almagro, U.A.; Kalbfleisch, J.H. Oxidative stress as a precursor to the irreversible hepatocellular injury caused by hyperthermia. Int. J. Hyperth. 1991, 7, 749-761. [CrossRef]

10. Carvalho, F.; Remiao, F.; Soares, M.E.; Catarino, R.; Queiroz, G.; Bastos, M.L. d-Amphetamine-induced hepatotoxicity: Possible contribution of catecholamines and hyperthermia to the effect studied in isolated rat hepatocytes. Arch. Toxicol. 1997, 71, 429-436. [CrossRef]

11. Carvalho, M.; Carvalho, F.; Bastos, M.L. Is hyperthermia the triggering factor for hepatotoxicity induced by 3,4-methylenedioxymethamphetamine (ecstasy)? An in vitro study using freshly isolated mouse hepatocytes. Arch. Toxicol. 2001, 74, 789-793. [CrossRef] [PubMed]

12. Ando, M.; Katagiri, K.; Yamamoto, S.; Asanuma, S.; Usuda, M.; Kawahara, I.; Wakamatsu, K. Effect of hyperthermia on glutathione peroxidase and lipid peroxidative damage in liver. J. Therm. Biol. 1994, 19, 177-185. [CrossRef]

13. Morrison, S.F.; Nakamura, K. Central neural pathways for thermoregulation. Front. Biosci. 2011, 16, 74-104. [CrossRef] [PubMed]

14. Sumner, L.W.; Amberg, A.; Barrett, D.; Beale, M.H.; Beger, R.; Daykin, C.A.; Fan, T.W.; Fiehn, O.; Goodacre, R.; Griffin, J.L.; et al. Proposed minimum reporting standards for chemical analysis Chemical Analysis Working Group (CAWG) Metabolomics Standards Initiative (MSI). Metabolomics 2007, 3, 211-221. [CrossRef] [PubMed]

15. Gowda, G.A.; Djukovic, D. Overview of mass spectrometry-based metabolomics: Opportunities and challenges. Methods Mol. Biol. 2014, 1198, 3-12. [CrossRef]

16. Slimen, I.B.; Najar, T.; Ghram, A.; Dabbebi, H.; Ben Mrad, M.; Abdrabbah, M. Reactive oxygen species, heat stress and oxidative-induced mitochondrial damage. A review. Int. J. Hyperth. 2014, 30, 513-523. [CrossRef]

17. Halket, J.M.; Waterman, D.; Przyborowska, A.M.; Patel, R.K.P.; Fraser, P.D.; Bramley, P.M. Chemical derivatization and mass spectral libraries in metabolic profiling by GC/MS and LC/MS/MS. J. Exp. Bot. 2005, 56, 210-243. [CrossRef]

18. Lu, S.C. Glutathione synthesis. Biochim. Biophys. Acta 2013, 1830, 3143-3153. [CrossRef]

19. Da Silva, D.D.; Silva, E.; Carmo, H. Combination effects of amphetamines under hyperthermia-The role played by oxidative stress. J. Appl. Toxicol. 2014, 34, 637-650. [CrossRef]

20. Ayala, A.; Munoz, M.F.; Arguelles, S. Lipid peroxidation: Production, metabolism, and signaling mechanisms of malondialdehyde and 4-hydroxy-2-nonenal. Oxid. Med. Cell. Longev. 2014, 2014, 360438. [CrossRef]

21. Hakim, M.; Broza, Y.Y.; Barash, O.; Peled, N.; Phillips, M.; Amann, A.; Haick, H. Volatile organic compounds of lung cancer and possible biochemical pathways. Chem. Rev. 2012, 112, 5949-5966. [CrossRef] [PubMed]

22. Broza, Y.Y.; Mochalski, P.; Ruzsanyi, V.; Amann, A.; Haick, H. Hybrid volatolomics and disease detection. Angew. Chem. Int. Ed. Engl. 2015, 54, 11036-11048. [CrossRef] [PubMed]

23. Reingruber, H.; Pontel, L.B. Formaldehyde metabolism and its impact on human health. Curr. Opin. Toxicol. 2018, 9, 28-34. [CrossRef]

24. Gulec, M.; Gurel, A.; Armutcu, F. Vitamin E protects against oxidative damage caused by formaldehyde in the liver and plasma of rats. Mol. Cell. Biochem. 2006, 290, 61-67. [CrossRef] [PubMed]

25. Allaman, I.; Belanger, M.; Magistretti, P.J. Methylglyoxal, the dark side of glycolysis. Front. Neurosci. 2015, 9, 23. [CrossRef] [PubMed] 
26. Sousa Silva, M.; Gomes, R.A.; Ferreira, A.E.; Ponces Freire, A.; Cordeiro, C. The glyoxalase pathway: The first hundred years... and beyond. Biochem. J. 2013, 453, 1-15. [CrossRef]

27. Lane, A.N.; Fan, T.W. Regulation of mammalian nucleotide metabolism and biosynthesis. Nucleic Acids Res. 2015, 43, 2466-2485. [CrossRef]

28. Liao, Y.; Hu, R.; Wang, Z.; Peng, Q.; Dong, X.; Zhang, X.; Zou, H.; Pu, Q.; Xue, B.; Wang, L. Metabolomics Profiling of Serum and Urine in Three Beef Cattle Breeds Revealed Different Levels of Tolerance to Heat Stress. J. Agric. Food Chem. 2018, 66, 6926-6935. [CrossRef]

29. Fan, C.; Su, D.; Tian, H.; Li, X.; Li, Y.; Ran, L.; Hu, R.; Cheng, J. Liver metabolic perturbations of heat-stressed lactating dairy cows. Asian-Australas. J. Anim. Sci. 2018, 31, 1244-1251. [CrossRef]

30. Cui, Y.; Wang, C.; Hao, Y.; Gu, X.; Wang, H. Chronic Heat Stress Induces Acute Phase Responses and Serum Metabolome Changes in Finishing Pigs. Animals 2019, 9, 395. [CrossRef]

31. Godoy, P.; Hewitt, N.J.; Albrecht, U.; Andersen, M.E.; Ansari, N.; Bhattacharya, S.; Bode, J.G.; Bolleyn, J.; Borner, C.; Bottger, J.; et al. Recent advances in 2D and 3D in vitro systems using primary hepatocytes, alternative hepatocyte sources and non-parenchymal liver cells and their use in investigating mechanisms of hepatotoxicity, cell signaling and ADME. Arch. Toxicol. 2013, 87, 1315-1530. [CrossRef] [PubMed]

32. Araujo, A.M.; Bastos, M.L.; Fernandes, E.; Carvalho, F.; Carvalho, M.; Guedes de Pinho, P. GC-MS metabolomics reveals disturbed metabolic pathways in primary mouse hepatocytes exposed to subtoxic levels of 3,4-methylenedioxymethamphetamine (MDMA). Arch. Toxicol. 2018, 92, 3307-3323. [CrossRef]

33. Valente, M.J.; Araujo, A.M.; Silva, R.; Bastos Mde, L.; Carvalho, F.; Guedes de Pinho, P.; Carvalho, M. 3,4-Methylenedioxypyrovalerone (MDPV): In vitro mechanisms of hepatotoxicity under normothermic and hyperthermic conditions. Arch. Toxicol. 2016, 90, 1959-1973. [CrossRef] [PubMed]

34. Araujo, A.M.; Moreira, N.; Lima, A.R.; Bastos, M.L.; Carvalho, F.; Carvalho, M.; de Pinho, P.G. Analysis of extracellular metabolome by HS-SPME/GC-MS: Optimization and application in a pilot study to evaluate galactosamine-induced hepatotoxicity. Toxicol. Lett. 2018, 295, 22-31. [CrossRef] [PubMed]

35. Pluskal, T.; Castillo, S.; Villar-Briones, A.; Oresic, M. MZmine 2: Modular framework for processing, visualizing, and analyzing mass spectrometry-based molecular profile data. BMC Bioinform. 2010, 11, 395. [CrossRef] [PubMed]

36. Hutschenreuther, A.; Kiontke, A.; Birkenmeier, G.; Birkemeyer, C. Comparison of extraction conditions and normalization approaches for cellular metabolomics of adherent growing cells with GC-MS. Anal. Methods 2012, 4. [CrossRef]

37. Wheelock, A.M.; Wheelock, C.E. Trials and tribulations of 'omics data analysis: Assessing quality of SIMCA-based multivariate models using examples from pulmonary medicine. Mol. Biosyst. 2013, 9, 2589-2596. [CrossRef]

38. Mastrangelo, A.; Ferrarini, A.; Rey-Stolle, F.; Garcia, A.; Barbas, C. From sample treatment to biomarker discovery: A tutorial for untargeted metabolomics based on GC-(EI)-Q-MS. Anal. Chim. Acta 2015, 900, 21-35. [CrossRef]

39. Benjamini, Y.; Hochberg, Y. Controlling the false discovery rate: A practical and powerful approach to multiple testing. J. R. Statist. Soc. B 1995, 57, 289-300. [CrossRef]

40. Berben, L.; Sereika, S.M.; Engberg, S. Effect size estimation: Methods and examples. Int. J. Nurs. Stud. 2012, 49, 1039-1047. [CrossRef]

41. Chong, J.; Soufan, O.; Li, C.; Caraus, I.; Li, S.; Bourque, G.; Wishart, D.S.; Xia, J. MetaboAnalyst 4.0: Towards more transparent and integrative metabolomics analysis. Nucleic Acids Res. 2018, 46, W486-W494. [CrossRef] [PubMed] 



\title{
Glucose-6 Phosphate, a Central Hub for Liver Carbohydrate Metabolism
}

\author{
Fabienne Rajas 1,2,3,*, Amandine Gautier-Stein 1,2,3 and Gilles Mithieux 1,2,3 \\ 1 Institut National de la Santé et de la Recherche Médicale, U1213, F-69008 Lyon, France; \\ amandine.gautier-stein@univ-lyon1.fr (A.G.-S.); gilles.mithieux@univ-lyon1.fr (G.M.) \\ 2 Université de Lyon, F-69008 Lyon, France \\ 3 Université Lyon 1, F-69622 Villeurbanne, France \\ * Correspondence: fabienne.rajas@univ-lyon1.fr
}

Received: 17 October 2019; Accepted: 18 November 2019; Published: 20 November 2019

\begin{abstract}
Cells efficiently adjust their metabolism according to the abundance of nutrients and energy. The ability to switch cellular metabolism between anabolic and catabolic processes is critical for cell growth. Glucose- 6 phosphate is the first intermediate of glucose metabolism and plays a central role in the energy metabolism of the liver. It acts as a hub to metabolically connect glycolysis, the pentose phosphate pathway, glycogen synthesis, de novo lipogenesis, and the hexosamine pathway. In this review, we describe the metabolic fate of glucose- 6 phosphate in a healthy liver and the metabolic reprogramming occurring in two pathologies characterized by a deregulation of glucose homeostasis, namely type 2 diabetes, which is characterized by fasting hyperglycemia; and glycogen storage disease type I, where patients develop severe hypoglycemia during short fasting periods. In these two conditions, dysfunction of glucose metabolism results in non-alcoholic fatty liver disease, which may possibly lead to the development of hepatic tumors. Moreover, we also emphasize the role of the transcription factor carbohydrate response element-binding protein (ChREBP), known to link glucose and lipid metabolisms. In this regard, comparing these two metabolic diseases is a fruitful approach to better understand the key role of glucose- 6 phosphate in liver metabolism in health and disease.
\end{abstract}

Keywords: de novo lipogenesis; carbohydrate response element-binding protein; ChREBP; diabetes; glucose production; glycogen; glycolysis; glycogen storage disease type I; hexosamine; nonalcoholic fatty liver disease; NAFLD; pentose phosphate pathway; steatosis

\section{Introduction}

The liver plays a crucial role in the maintenance of glucose homeostasis by extracting glucose from the blood and then storing it after a meal, and also by producing glucose in post-absorptive state. When its concentration increases in the bloodstream, glucose enters the hepatocytes mainly through the glucose transporter 2 (GLUT2). Within the cells, free glucose is immediately phosphorylated on the sixth carbon by glucokinase (also named hexokinase IV), producing glucose-6 phosphate (G6P) and consuming one molecule of ATP. Contrary to the other hexokinases, glucokinase has relatively low affinity for glucose and is not inhibited by G6P [1]. Glucokinase expression is transcriptionally regulated by hormones [induced by insulin through the transcription factor SREBP1c (Sterol Response Element-Binding Protein 1c) and inhibited by glucagon] and metabolites of glucose and glucokinase activity is dependent on its binding to a specific inhibitor named glucokinase regulatory protein (GKRP) (see [2] for a review of glucokinase regulation). Other binding proteins such as 6-phosphofructo-2-kinase/fructose 2,6 biphosphatase (PFK2/FBP2) are also able to activate glucokinase by direct interaction with this enzyme [3].

The phosphorylation of glucose by glucokinase adds a charged phosphate group to this molecule. Consequently, G6P cannot cross the cell membrane, preventing the diffusion of free glucose out of the 
cells. Thanks to this phosphorylation step, glucokinase enables hepatocytes to trap glucose. During fasting periods, G6P is also produced after isomerization of glucose-1 phosphate during the breakdown of glycogen and by gluconeogenesis in the hepatocyte. It should be noted that a limited amount of free glucose can be directly released from glycogen through the action of the debranching enzyme $\alpha-1,6$-glucosidase (AGL) and/or the lysosomal acid $\alpha-1,4$ glucosidase (also known as acid maltase) [4].

Within the cells, G6P has many possible fates and therefore it represents a central hub for carbohydrate metabolism (Figure 1). After isomerization, it initiates major metabolic pathways, i.e., glycolysis, pentose phosphate pathway (PPP), glycogen synthesis, hexosamine pathway, and glucose production according to the nutritional or hormonal states. This review is focused on the key metabolic roles of G6P in cell signaling in the healthy or pathological liver. Here, we will highlight the metabolic reprogramming taking place in two metabolic diseases characterized by a dysfunction of glucose metabolism, namely type 2 diabetes and glycogen storage disease type I (GSDI). Interestingly, type 2 diabetes is an epidemic disease characterized by hyperglycemia, while GSDI is a rare genetic disease due to a loss of endogenous glucose production leading to severe hypoglycemia during short fasting. In type 2 diabetes, hyperglycemia is responsible for an increase in metabolic pathways downstream of G6P, while in GSDI the blockage of glucose production leads to the accumulation of G6P in the hepatocytes, which also increases all the metabolic pathways downstream of G6P (Figure 2). These two diseases are characterized by an accumulation of ectopic lipids in the liver, which leads to the development of hepatic steatosis and promotes hepatic tumorigenesis over time [5]. In this review, we will also consider the well-established role of the Carbohydrate-Responsive Element-Binding Protein (ChREBP) as the carbohydrate sensor that coordinates glucose and lipid metabolism in the liver according to nutritional states. 


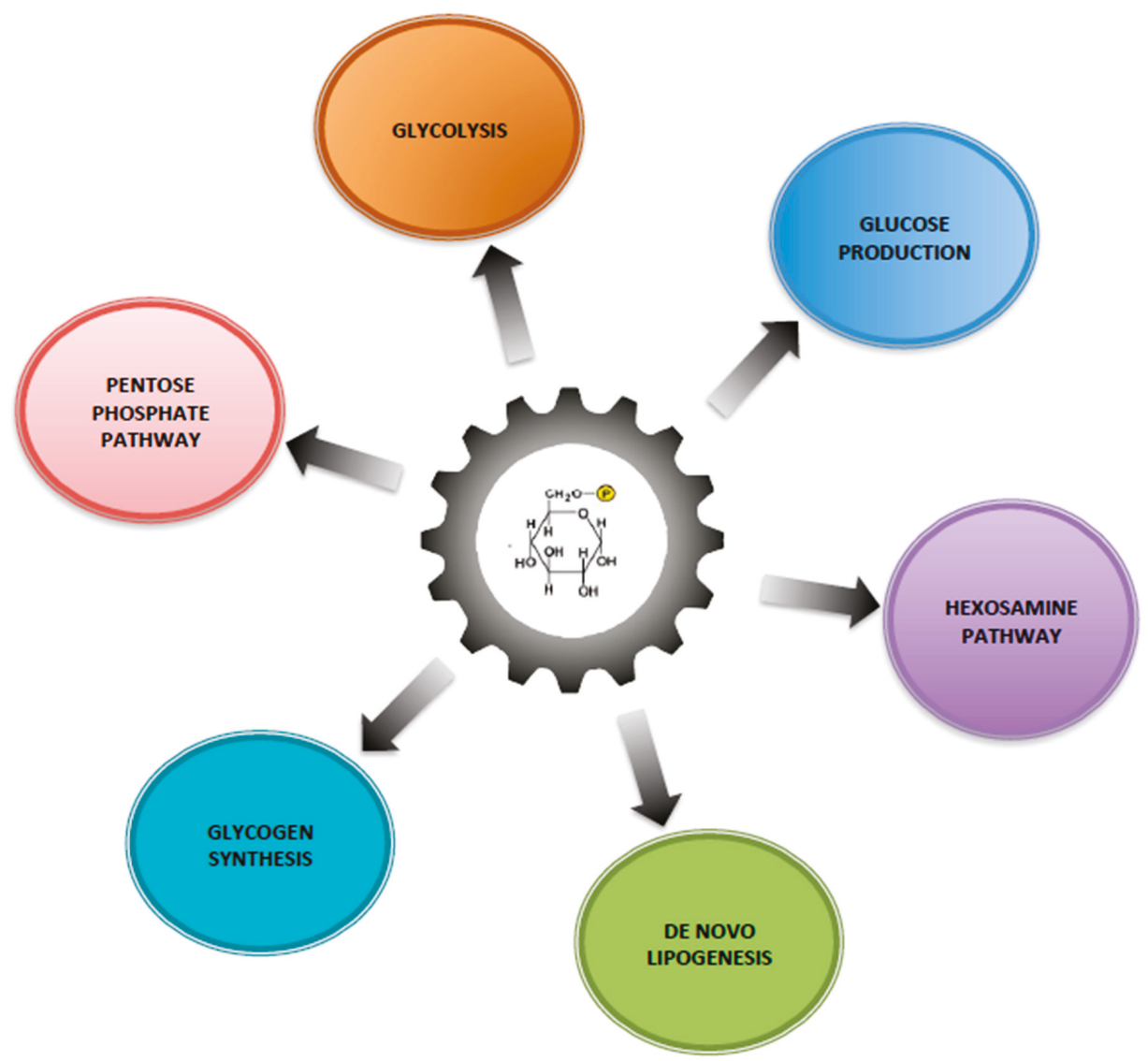

Figure 1. Glucose-6 phosphate, a central hub for liver carbohydrate metabolism. The increase of flux through G6P is responsible for increasing glycogen synthesis, glycolysis, pentose phosphate pathway (PPP), hexosamine pathway and de novo lipogenesis. 


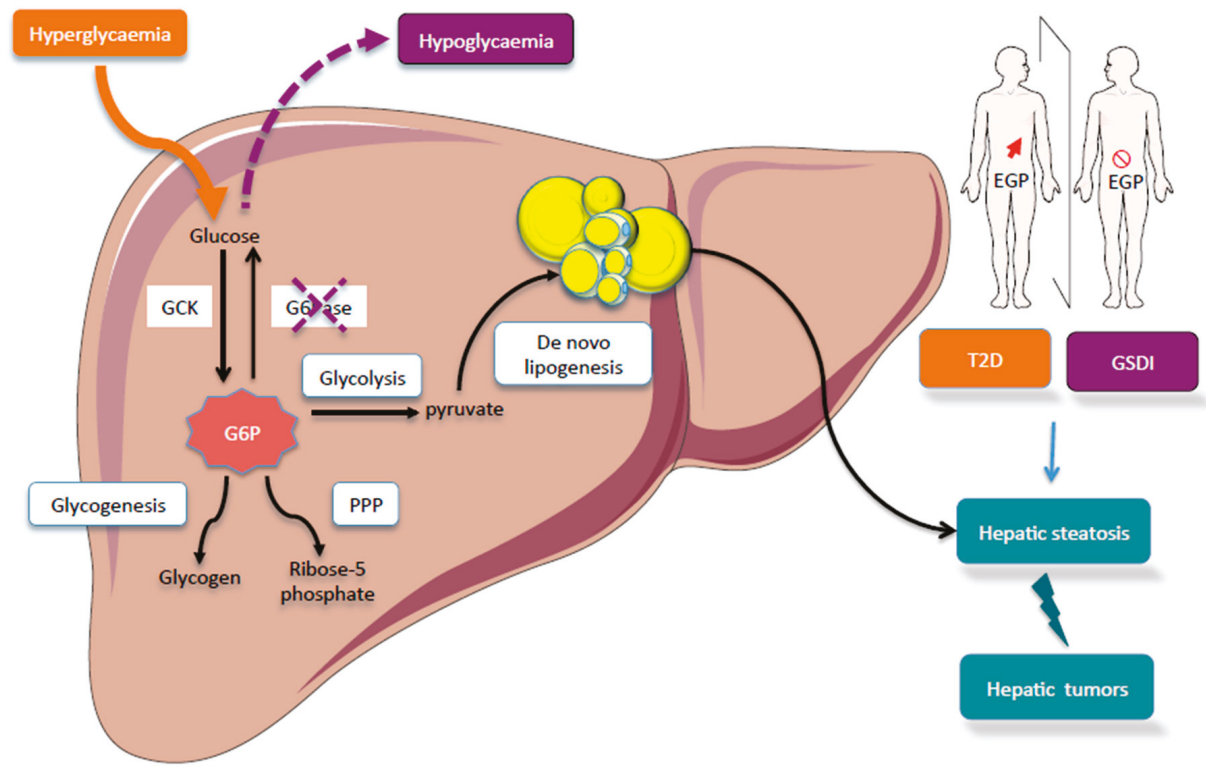

Figure 2. Comparison of hepatic glucose metabolism in glycogen storage disease type I (GSDI) or type 2 diabetes. Type 2 diabetes is characterized by an increase in endogenous glucose production (EGP) while GSDI is due to an absence of EGP. In GSDI, the absence of G6Pase activity is responsible for G6P accumulation in the hepatocyte. In diabetes, hyperglycemia is responsible for the increase flux through G6P. In both cases, this leads to a metabolic reprogramming characterized by the activation of glycolysis, PPP, and de novo lipogenesis. This metabolic reprogramming promotes hepatic steatosis in type 2 diabetes and GSDI, in which the risk of liver tumorigenesis is increased. Figures were drawn using Sevier Medical Art images.

\section{Metabolic Fate of Glucose-6 Phosphate in the Healthy Liver}

In order to control cell metabolism and proliferation, G6P enters different metabolic pathways to provide energy and/or precursors for biomolecule synthesis needed to sustain these processes. First, glucose concentrations fluctuate between the fed state and fasting periods. The liver plays a crucial role in maintaining blood glucose levels by its capacity to produce glucose during fasting periods. Moreover, in the case of overnutrition, excessive G6P is converted into fatty acids via de novo lipogenesis in the liver. Secondly, during fasting periods, glucose should be preserved to supply precursors for maintaining biomass, especially for cell renewal. Ketone bodies then become a major energy source for most tissues. Thus, the liver plays a central role by coordinating the storage and synthesis of glucose and the redistribution of nutrients, through the G6P metabolism.

\subsection{Glucose and Lipid Storage}

After a meal, a large portion of the excess carbohydrates (approximately $30-40 \%$ of the glucose ingested) is stored as glycogen in the liver, inside the hepatocytes, and in muscles (glycogenesis). In healthy individuals, hepatic glycogen represents around $5 \%$ of the liver weight. Glycogen is a polymer of glucose residues linked by $\alpha-(1,4)$ and $\alpha-(1,6)$-glycosidic bonds. To synthesize glycogen, G6P is isomerized into glucose- 1 phosphate and then converted into UDP-glucose. For de novo glycogen synthesis, UDP-glucose molecules are attached to a protein known as glycogenin. Once a linear chain of 10-20 glucose moieties is formed, glycogen synthase extends the glycogen chain, forming $\alpha-1-4$ glycosidic links, and a branching enzyme introduces a branch point. The branching enzyme transfers a glycosyl chain of 6 to 8 units to the glycogen thread forming an $\alpha-1-6$ linkage [6]. G6P is a precursor 
for glycogen synthesis but it also plays a huge role in regulating the activities of glycogen synthase and glycogen phosphorylase. Indeed, G6P is an allosteric inhibitor of glycogen phosphorylase and an allosteric activator of glycogen synthase, thus favoring hepatic glycogen increase [7]. In addition, glycogen synthesis/degradation is tightly regulated by hormones and nutritional states, which has been extensively described (see [7] for a review). It is of note that the presence of high insulin level after a meal favors glycogen synthesis.

Importantly, the capacity to store glycogen in the liver is limited. In case of excessive feeding of carbohydrates or in pathological states such as GSDI, glycogen turnover allows to continually breakdown glycogen to limit glycogen accumulation $[4,8]$. Moreover, the excess of dietary glucose that cannot be stored as glycogen is converted into fat by de novo lipogenesis (see below) [9]. A deregulation of glycogen storage or metabolic dysfunctions leading to abnormal glycogen storage in the liver results in hepatic glycogen storage diseases, which are metabolic inherited diseases characterized by hypoglycemia. GSDI belongs to this group of hepatic diseases, representing about $30 \%$ of GSD cases [10].

In the liver, triglycerides can be packed into very low density lipoproteins (VLDL) and secreted into the circulation, stored as lipid droplets, or be metabolized by the $\beta$-oxidation pathway. Excessive G6P is converted into fatty acids via de novo lipogenesis using the acetyl-CoA generated from glycolysis-driven pyruvate and NADPH derived from PPP. After glucose load, lipogenesis is markedly increased at the expense of glycogen synthesis; conversely, low carbohydrate diets reduce de novo lipogenesis [9]. Interestingly, insulin secreted in response to elevated blood glucose levels and glucose can induce hepatic lipogenesis through the synergistically activation of SREBP-1c and ChREBP, respectively [11]. Thus, increased consumption of simple sugars leads to the ectopic accumulation of lipids in the liver and increases the risk of metabolic diseases such as obesity, type 2 diabetes and nonalcoholic fatty liver disease (NAFLD).

\subsection{Maintenance of Glycaemia and Endogenous Glucose Production}

The liver plays a key role in the maintenance of blood glucose, particularly during the beginning of fasting periods. Just after the intestinal glucose absorption from food is completed, hepatic G6P is mainly derived from glycogen breakdown, while gluconeogenesis becomes the major source of G6P after more prolonged fasting. Indeed, hepatic glycogen stores are depleted after a $12 \mathrm{~h}$-fasting in mice and an overnight fasting period in Humans [12,13]. Glycogenolysis requires the intervention of two different enzymes: glycogen phosphorylase that degrades the glycogen chain down to a chain length of 4 units into glucose- 1 phosphate, and glycogen debranching enzyme (GDE) that first transfers 3 glucose units to the terminal end of another chain and then cleaves off the final glucose unit, releasing it as free glucose. Glucose-1-phosphate must further be converted by phosphoglucomutase into G6P to enter the metabolism mainstream. During a longer fast or starvation, the liver synthetizes glucose de novo mainly from lactate, alanine, and glycerol while glutamine is a predominant gluconeogenic substrate in the kidney and intestine $[14,15]$. Interestingly, the contribution of hepatic glucose production decreases during fasting [16]. This decrease is compensated by glucose production from the kidneys and intestine, which are especially capable of producing glucose thanks to gluconeogenesis and to participate in the maintenance of blood glucose when fasting is prolonged [17-20]. The significance of the renal and intestinal gluconeogenesis has been firmly demonstrated in mice that are incapable to produce glucose by the liver (Liver-specific G6pc knockout mice- L.G6pc ${ }^{-/}$) [21]. Indeed, despite a drop in blood sugar levels in the post-prandial period, L.G6 $\mathrm{pc}^{-/-}$mice regulate their blood sugar similarly to control mice after several hours of fasting thanks to an induction of gluconeogenic genes in the kidney and the intestine $[13,22]$.

To be released as glucose into the bloodstream, G6P has to be dephosphorylated into glucose by glucose-6 phosphatase (G6Pase), which is expressed only in the liver, kidneys, and intestine. G6P is first translocated into the endoplasmic reticulum by the G6Pase transporter subunit (G6PT) and subsequently hydrolyzed into free glucose and inorganic phosphate by the G6Pase catalytic subunit 
(G6PC). Glucose is finally released from the cytosol into the bloodstream through GLUT2. Thus, the liver, kidneys, and intestine play a central role in maintaining blood glucose levels at around $1 \mathrm{~g} / \mathrm{L}$ $(5 \mathrm{mM})$ since most mammals, including Humans, are incapable of tolerating hypoglycemia for more than a few minutes. Failure to activate these physiological pathways results in severe hypoglycemia that can be fatal, especially in GSDI or in diabetic patients treated with inappropriate doses of insulin.

\subsection{Glucose-6 Phosphate: A Source of Energy and Carbon Skeletons}

In feeding periods, glucose can be oxidized to $\mathrm{CO}_{2}$ through a series of metabolic pathways, namely glycolysis in the cytosol, followed by the tricarboxylic acid cycle and the respiratory chain in the mitochondria. The first step of glycolysis is the isomerization of G6P into fructose-6 phosphate to produce triose-phosphate, then resulting in the generation of 2 pyruvate molecules and a small amount of ATP (net gain of 2 ATP molecules). The oxidation of pyruvate then generates the bulk of ATP under aerobic conditions in quiescent differentiated cells (Figure 3).

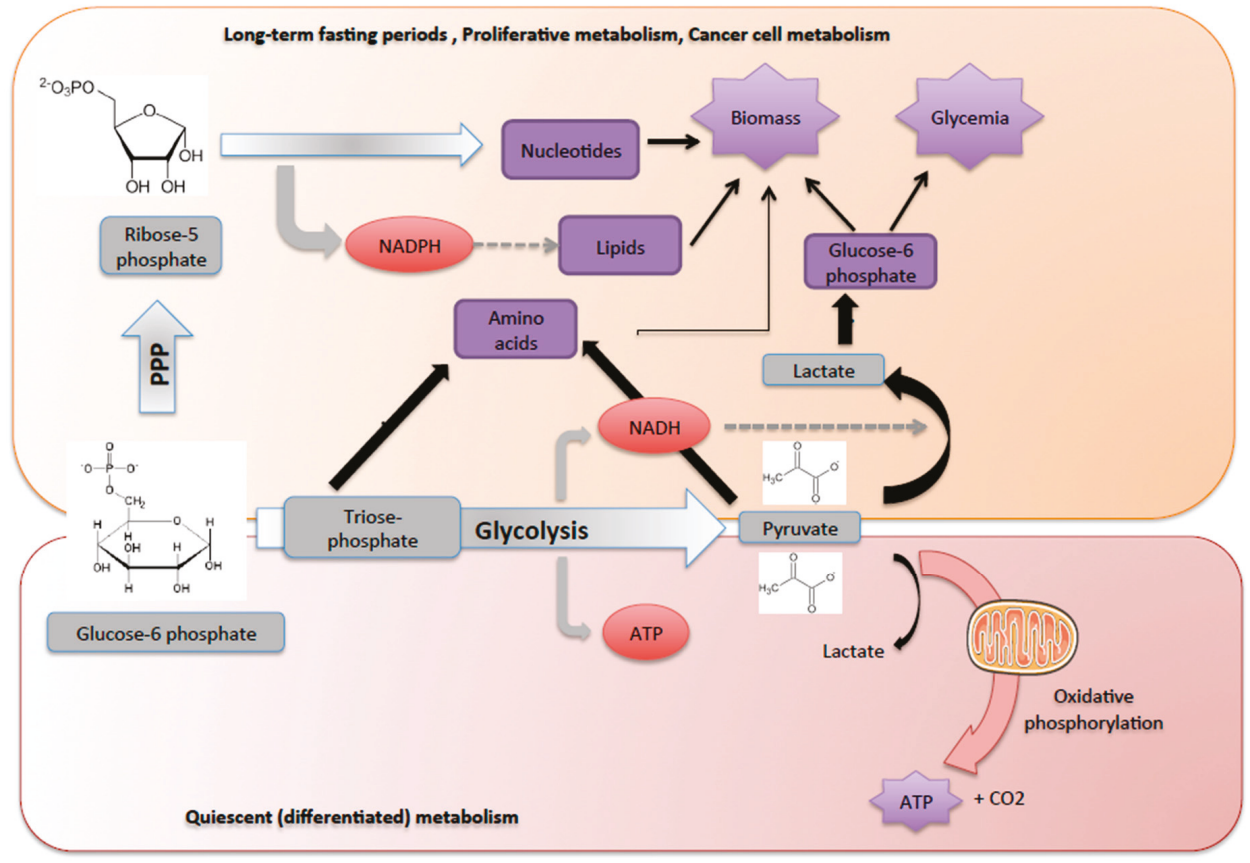

Figure 3. Glucose-6 phosphate: a source of energy and carbon skeletons. The G6P is metabolized either through the glycolytic pathway or PPP, which are tightly connected, depending on metabolic demands. Non-dividing normal differentiated cells mainly depend on mitochondrial oxidative phosphorylation of pyruvate, which is produced from glycolysis, to generate ATP. During cell proliferation or starvation periods, G6P is preferentially metabolized via PPP to maintain carbon homeostasis and produce biomass. In this case, glycolysis produces pyruvate and lactate as final metabolites and becomes inefficient in producing ATP. Indeed, G6P is preferentially metabolized via PPP to provide precursors for nucleotide and amino acid biosynthesis and to provide reducing molecules in the form of NADPH used in reductive biosynthesis reactions within cells (e.g., fatty acid synthesis). Lactate is also used by the hepatocyte to produce glucose and maintain glycaemia.

While glucose is generally considered to be the main source of cell energy, it is above all a major provider of carbon skeletons for cell growth and survival [16]. Indeed, glucose oxidation to $\mathrm{CO}_{2}$ to produce energy should be avoided to permit to supply essential functions in some 
situations, in particular during long-term fasting or during cell proliferation. Glycolysis supplies 3 carbon-compounds, such as triose-phosphate, pyruvate and lactate that can be used to maintain cellular homeostasis and produce biomass (Figure 3). Hence, global glucose turnover decreases and glucose is used to supply PPP that provides the carbon skeletons needed for the synthesis of nucleotides, chromosomal duplication and cell proliferation (Figure 3). PPP is an important metabolic pathway known to provide reducing equivalents (NADPH) for anabolism and it plays a pivotal role in counteracting oxidative stress. Indeed, during oxidative stress, NADPH is needed for the generation of reduced glutathione. In the first step of PPP, G6P is oxidized into gluconolactone and carbon dioxide by glucose-6 phosphate dehydrogenase and 6-phosphogluconic dehydrogenase (oxidative branch). Ribulose- 5 phosphate yielded is then isomerized to ribose- 5 phosphate, which is the critical precursor for de novo ribonucleotide synthesis or epimerized into xylulose- 5 phosphate. Additionally, a series of reversible reactions that recruit additional glycolytic intermediates, such as fructose- 6 phosphate and glyceraldehyde-3-phosphate, can be converted into pentose phosphates and vice versa (non-oxidative branch). Transketolase (TKT) and transaldolase (TALDO) are the two major reversible enzymes that mediate the non-oxidative PPP and determine the diversion of metabolite flux in the PPP (Figure 4). Thus, in proliferative cells, TKT and TALDO divert fructose-6 phosphate and glyceraldehyde-3-phosphate from glycolysis to generate additional ribonucleotides. Interestingly, cancer cells can accelerate non-oxidative PPP by elevating the expression of these enzymes [23], while deficiency in TALDO can prevent HCC [24].

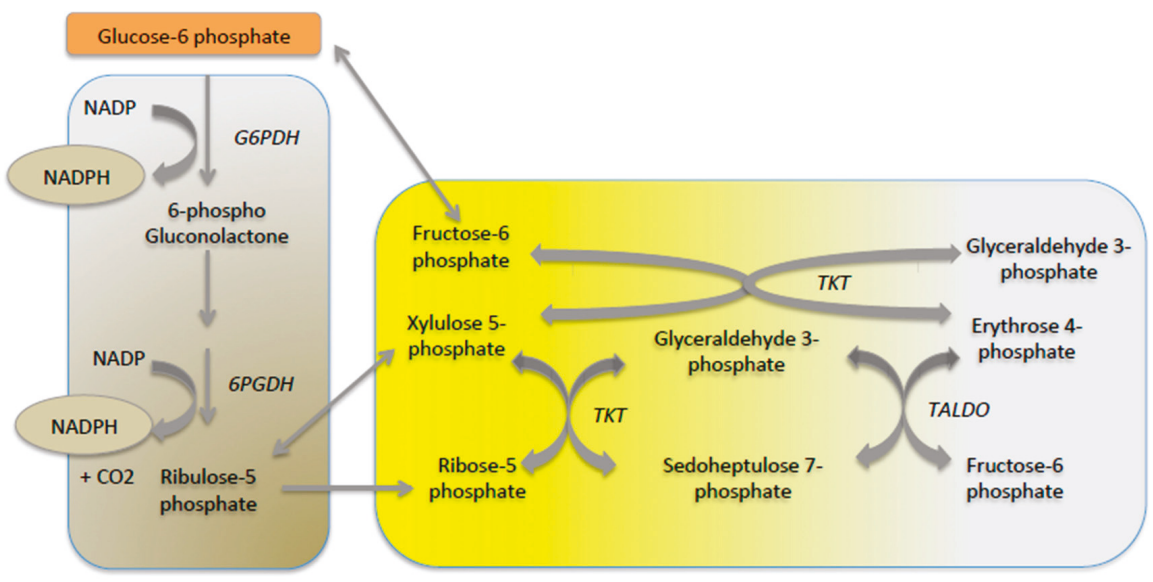

Figure 4. Scheme of the pentose phosphate pathway. The oxidative branch of PPP is highlighted in the brown part and the non-oxidative branch is represented in the yellow part of the figure. G6PDH: glucose-6 phosphate dehydrogenase; 6PGDH: 6-phosphogluconic dehydrogenase; TKT: Transketolase; TALDO: transaldolase.

Thus, the ability to switch the glucose metabolism from a catabolic to an anabolic process is critical for cells to thrive, especially during long fasting periods. This capacity is also an advantage for cancer cells that can grow and multiply by using glucose as a carbon source to build proteins and nucleotides rather than as an energy source, thanks to the Warburg effect [25].

\subsection{Hexosamine Pathway}

When G6P is increased, the hexosamine pathway produces carbohydrate units for glycosylation of proteins and contributes to the synthesis of complex molecules such as glycolipids, proteoglycans and glycosylphosphatidylinositol anchors. First, G6P is converted into fructose-6 phosphate, which may either enter the hexosamine pathway in combination with glutamine to produce 
UDP-Nacetylglucosamine or it can follow the glycolytic pathway. The hexosamine pathway usually accounts for only $2-5 \%$ of total glucose metabolism. Interestingly, O-GlcNAcylation of different key transcription factors involved in energy metabolism, including ChREBP and the nuclear receptor Farnesoid X receptor (FXR), requires the hexosamine pathway [26].

\section{ChREBP: A Glucose Sensor}

In the hepatocyte, the transcriptional effects of glucose on gene expression are mediated by the transcription factor ChREBP, which in interaction with Max-like protein (Mlx) binds conserved consensus sequences (Carbohydrate Response Element, ChoRE). Indeed, in response to increased glucose concentration, ChREBP is translocated to the nucleus and it activates several genes involved in glucose and lipid metabolism [such as liver-Pyruvate kinase (L-PK), Fatty Acid Synthase (FAS) acetyl-CoA carboxylase (ACC), and stearoyl-CoA desaturase (SCD1)], but also genes involved in insulin signaling [27-29]. Recently, ChREBP was pointed out as a potential regulator of VLDL secretion in the liver [30]. Thus, it is assumed that ChREBP has important roles in the development of liver diseases including NAFLD [31]. In consequence, inactivation of ChREBP or liver-specific inhibition of ChREBP led to a decrease in glycolytic and lipogenic gene expression and a decrease in hepatic steatosis in mice $[29,32,33]$. On the contrary, the overexpression of ChREBP led to the development of hepatic steatosis without concomitant insulin resistance [27]. More recently, a key regulatory role for ChREBP in hepatic tumorigenesis was also suggested, since ChREBP expression was found to be increased in non-tumorous surrounding tissue in liver samples and further increased in HCC in Humans [34]. In addition, a recent study reported the importance of ChREBP in HCC [35]. The genetic deletion of ChREBP in mice impaired hepatocarcinogenesis driven by protein kinase B/Akt overexpression [36]. Furthermore, in vitro studies of ChREBP silencing in hepatoma cells resulted in a metabolic switch from aerobic glycolysis to mitochondrial oxidative phosphorylation, concomitantly with a reduction of cell proliferation [37]. In GSDI, the overexpression of ChREBP has been linked to glucose and lipid metabolism reprogramming $[38,39]$. In this context, enhanced ChREBP could partly account for increased proliferation of hepatocytes by favoring cancer cell-like metabolism. Further investigation is required to unravel the exact role of ChREBP in hepatocarcinogenesis in the context of NAFLD.

Two isoforms of ChREBP have been recently described originating from an alternative first exon promoter - ChREBP $\alpha$ and $\beta$ [40]. The presence of a ChoRE sequence in the exon promoter $1 \beta$ suggests that $C h R E B P \alpha$ directly regulates the expression of $C h R E B P \beta$, considered as a constitutively active isoform (due to the loss of a regulatory inhibitory domain). Consequently, the response to glucose under hyperglycemic conditions could be exacerbated. The regulation of ChREBP activity by glucose is complex and the relative role of xylulose- 5 phosphate, G6P or other glucose metabolites on the triggering of ChREBP activation is still discussed [41]. It was shown that xylulose-5 phosphate activates PP2A promoting dephosphorylation of ChREBP and its nuclear translocation and activation [42]. However, G6P seems to have a central role for the increase in ChREBP activity, especially by favoring ChREBP translocation to the nucleus and transactivation [28]. The key role of G6P was supported by the identification of a putative G6P recognition motif in the transactivation domain, called glucose-activation conserved element (GRACE), suggesting the possibility of an allosteric regulation of ChREBP by G6P [43]. High glucose also stimulates ChREBP activity and affinity to ChoRE sequences through acetylation and/or O-GlcNacetylation [44,45]. Finally, during fasting periods, phosphorylation of ChREBP by AMPK, in response to glucagon or to an increase in cellular AMP, is responsible for its cytoplasmic retention and/or for its decreased binding to target promoters [46,47].

In conclusion, ChREBP is a carbohydrate-signaling transcription factor, which masters, in the liver, the storage of lipids in feeding response. Recent studies have also supported the importance of ChREBP in the regulation of fructose metabolism $[48,49]$. 


\section{Imbalance of Glucose-6 Phosphate Metabolism Leads to Metabolic Diseases and Promotes Hepatocarcinogenesis}

In this review, we have chosen to illustrate two different pathological states characterized by impaired glucose metabolism. Firstly, type 2 diabetes is a chronic disease with the status of a global pandemic that is closely linked to overnutrition and obesity [50,51]. The main hallmark of diabetes is hyperglycemia due to insulin resistance and an overproduction of glucose by the body [52]. Hyperglycemia results in non-enzymatic glycosylation (glycation) and thus loss of function of proteins, glucose-induced oxidative damage and other adverse effects such as macrovascular and microvascular complications [53]. The second metabolic disease characterized by carbohydrate metabolism disruption is GSDI. This is a rare genetic disease (1 birth over 100,000) due to mutations in G6PC (that cause GSDIa) or G6PT (that cause GSDIb) leading to a loss of G6Pase activity and endogenous glucose production. In consequence, patients develop severe hypoglycemia during short fasting periods [54]. Thus, although type 2 diabetes and GSDI appear to be opposite diseases in terms of glucose production and insulin sensitivity, the liver is chronically exposed to either hyperglycemia or G6P accumulation, respectively, leading the same metabolic consequences, in particular hepatic steatosis (Figure 2). Comparing type 2 diabetes and GSDI will allow us to highlight metabolic perturbations that promote tumour development in relation to the ectopic accumulation of lipids in the liver.

In both type 2 diabetes and GSDI, liver metabolism is characterized by an increased metabolic flux downstream of G6P (Figure 2). Even if glucose uptake is impaired in obese and/or diabetic mice or patients, high blood glucose levels are responsible for the activation of all G6P-dependent pathways previously described [7]. Subsequently, one major metabolic consequence is an increase in triglyceride synthesis by the liver leading to hepatic steatosis [55,56]. Indeed, up to $70 \%$ of diabetic subjects may present NAFLD [56,57] and all GSDI develop a NAFLD-like pathology [54]. In insulin-resistant states, hyperglycemia and hyperinsulinemia are in part responsible for enhancing de novo lipogenesis through the activation of both ChREBP and SREBP1c. Interestingly, both transcription factors are also induced in the context of a high carbohydrate feeding independently of insulin signaling [58]. As previously mentioned, it has been shown that the global or liver-specific inhibition of ChREBP protected mice against carbohydrate-induced hepatic steatosis [29,33]. However, the effects of ChREBP inhibition on hepatic insulin sensitivity are still controversial. In GSDI, the absence of G6Pase activity leads to the accumulation of G6P in the liver and consequently the accumulation of glycogen and lipids, responsible for hepatomegaly and hepatic steatosis. Contrarily to diabetes, lipid synthesis is activated by ChREBP but independently of liver X receptor (LXR) and SREBP-1c [59]. The lack of SREBP-1c activation is probably due to a low intensity of insulin signaling in GSDI [37]. De novo lipogenesis is not the only process contributing to fatty liver. Indeed, the accumulation of lipid is also caused by an unbalanced diet, elevated non-esterified fatty acid due to a decreased inhibition of adipose tissue lipolysis, and reduced hepatic VLDL export [60,61]. All these disturbances contribute to hypertriglyceridemia and hypercholesteridemia observed in diabetes and GSDI. In conclusion, the liver metabolism of diabetes and GSDI is very similar, albeit exacerbated in GSDI, with G6P being at the metabolic crossroad as a main responsible for metabolic reprogramming [39,62].

Interestingly, both diabetes and GSDI patients are prone to the development of hepatic tumors. In diabetes, NAFLD can progress to liver fibrosis associated with inflammation i.e., non-alcoholic steatohepatitis NASH, cirrhosis and finally to the development of HCC. However, an important fraction of obese/diabetic patients develop HCC in the absence of liver cirrhosis $[63,64]$. Interestingly, GSDI subjects develop simple hepatic steatosis, which was long considered as a benign reversible condition. Nonetheless, lipid accumulation in the liver is a fertile ground for the development of hepatic tumors and most of patients with GSDI develop hepatocellular adenomas (HCA) that can later progress into HCC [54]. Despite the important accumulation of glycogen and lipids, GSDI patients present only low-grade hepatic inflammation and no hepatic injuries (namely normal hepatic transaminase levels and absence of liver failure). It is noteworthy that in obese/diabetic patients a part of HCA arises at the state of NAFLD characterized by a low-grade inflammation and may progress to HCC [65]. 
The comparison of HCC occurrence in NAFLD and GSDI livers argues for a dominant role of metabolic reprogramming in the molecular induction of tumor development.

Interestingly, tumor cells are metabolically reprogrammed to fuel cell proliferation, mostly by increasing glucose uptake and flux through aerobic glycolysis (Warburg effect) and anabolic pathways (PPP and de novo lipogenesis). The Warburg effect is characterized by high rates of glycolysis and lactic acid fermentation that occur in the cytosol regardless of the oxygen level. This provides essential bioenergetic substrates for cell growth and replication, i.e., components needed for cellular membrane biogenesis and amino acids and nucleotide synthesis for cell division. Recently, we showed that GSDI hepatocytes exhibit the main characteristics of cancer cell metabolism, with a Warburg-like metabolic reprogramming that predisposes GSDIa livers to tumor development [15]. Indeed, we observed a hyperactivation of the glycolysis pathway notably characterized by an overexpression of the M2 isoform of pyruvate kinase in the tumors and an increase in lactate production. Moreover, OXPHOS analyses revealed a decrease in mitochondrial respiration with a reduction of pyruvate oxidation [39].

A rational therapeutic approach for the treatment of NAFLD is to increase hepatic energy expenditure and thereby increase hepatic fat oxidation. Recently, we showed that the use of PPAR- $\alpha$ agonists, in particular fenofibrate, prevented NAFLD and hepatic injuries in GSDI, as previously described in diabetes [66-68]. Interestingly, the activation of $\beta$-oxidation by fenofibrate promoted the utilization of G6P through lipid metabolism, avoiding the accumulation of glycogen [66]. In diabetes, thyroid hormone receptor- $\beta$ agonist combined with glucagon treatment, or glucacon like peptide 1 agonist-gastric inhibitory peptide-glucagon tri-conjugate [69], or liver targeted mitochondrial protonophores [70,71] were shown to reverse NAFLD in preclinical studies. Interestingly, glucose-lowering medications such as metformin also reduce the risk of HCC in diabetes, suggesting that better control of hepatic glucose metabolism should permit prevention of carcinogenesis.

To conclude, the activation of G6P-mediated metabolism is a hallmark of both GSDI and diabetes that causes hepatic steatosis and may promote cell proliferation and liver cancer. Thus, an optimal metabolic control, thanks to a strict diet with a reduced consumption of simple carbohydrates, should prevent tumor occurrence in GSDI [54]. In diabetes, better control of hyperglycemia should also permit better control of glucose/G6P metabolism and its possible consequences in hepatocytes. Thus, comparing these two metabolic diseases is a useful approach to better understand the key role of G6P in the liver both in health and pathological conditions.

Author Contributions: Wrote, reviewed and edited the manuscript, F.R.; Reviewed the manuscript, A.G.-S. and G.M.

Funding: This research received no external funding.

Acknowledgments: The authors thank all past and current colleagues in the laboratory who contributed to the work reviewed. We would particularly like to thank Monika Gjorgjieva for her kind contribution to the manuscript.

Conflicts of Interest: The authors declare that they have no conflict of interest.

\section{References}

1. Van Schaftingen, E.; Detheux, M.; Veiga da Cunha, M. Short-term control of glucokinase activity: Role of a regulatory protein. FASEB J. 1994, 8, 414-419. [CrossRef] [PubMed]

2. Agius, L. Hormonal and Metabolite Regulation of Hepatic Glucokinase. Annu. Rev. Nutr. 2016, 36, 389-415. [CrossRef] [PubMed]

3. Baltrusch, S.; Lenzen, S.; Okar, D.A.; Lange, A.J.; Tiedge, M. Characterization of glucokinase-binding protein epitopes by a phage-displayed peptide library identification of 6-phosphofructo-2-kinase/fructose2,6-bisphosphatase as a novel interaction partner. J. Biol. Chem. 2001, 276, 43915-43923. [CrossRef] [PubMed]

4. Hijmans, B.S.; Boss, A.; van Dijk, T.H.; Soty, M.; Wolters, H.; Mutel, E.; Groen, A.K.; Derks, T.G.J.; Mithieux, G.; Heerschap, A.; et al. Hepatocytes contribute to residual glucose production in a mouse model for glycogen storage disease type Ia. Hepatology 2017, 66, 2042-2054. [CrossRef]

5. Rajas, F.; Labrune, P.; Mithieux, G. Glycogen storage disease type 1 and diabetes: Learning by comparing and contrasting the two disorders. Diabetes Metab. 2013, 39, 377-387. [CrossRef] 
6. Adeva-Andany, M.M.; González-Lucán, M.; Donapetry-García, C.; Fernández-Fernández, C.; Ameneiros-Rodríguez, E. Glycogen metabolism in humans. BBA Clin. 2016, 5, 85-100. [CrossRef]

7. Petersen, M.C.; Vatner, D.F.; Shulman, G.I. Regulation of hepatic glucose metabolism in health and disease. Nat. Rev. Endocrinol. 2017, 13, 572-587. [CrossRef]

8. Magnusson, I.; Rothman, D.L.; Jucker, B.; Cline, G.W.; Shulman, R.G.; Shulman, G.I. Liver glycogen turnover in fed and fasted humans. Am. J. Physiol. 1994, 266, E796-E803. [CrossRef]

9. McDevitt, R.M.; Bott, S.J.; Harding, M.; Coward, W.A.; Bluck, L.J.; Prentice, A.M. De novo lipogenesis during controlled overfeeding with sucrose or glucose in lean and obese women. Am. J. Clin. Nutr. 2001, 74, 737-746. [CrossRef]

10. Ozen, H. Glycogen storage diseases: New perspectives. World J. Gastroenterol. 2007, 13, 2541-2553. [CrossRef]

11. Linden, A.G.; Li, S.; Choi, H.Y.; Fang, F.; Fukasawa, M.; Uyeda, K.; Hammer, R.E.; Horton, J.D.; Engelking, L.J.; Liang, G. Interplay between ChREBP and SREBP-1c coordinates postprandial glycolysis and lipogenesis in livers of mice. J. Lipid Res. 2018, 59, 475-487. [CrossRef]

12. Xu, K.; Morgan, K.T.; Todd Gehris, A.; Elston, T.C.; Gomez, S.M. A Whole-Body Model for Glycogen Regulation Reveals a Critical Role for Substrate Cycling in Maintaining Blood Glucose Homeostasis. PLoS Comput. Biol. 2011, 7, e1002272. [CrossRef] [PubMed]

13. Mutel, E.; Gautier-Stein, A.; Abdul-Wahed, A.; Amigó-Correig, M.; Zitoun, C.; Stefanutti, A.; Houberdon, I.; Tourette, J.A.; Mithieux, G.; Rajas, F. Control of Blood Glucose in the Absence of Hepatic Glucose Production During Prolonged Fasting in Mice. Diabetes 2011, 60, 3121-3131. [CrossRef] [PubMed]

14. Mithieux, G. New data and concepts on glutamine and glucose metabolism in the gut. Curr. Opin. Clin. Nutr. Metab. Care 2001, 4, 267-271. [CrossRef] [PubMed]

15. Mithieux, G.; Rajas, F.; Gautier-Stein, A. A novel role for glucose 6-phosphatase in the small intestine in the control of glucose homeostasis. J. Biol. Chem. 2004, 279, 44231-44234. [CrossRef] [PubMed]

16. Soty, M.; Gautier-Stein, A.; Rajas, F.; Mithieux, G. Gut-Brain Glucose Signaling in Energy Homeostasis. Cell Metab. 2017, 25, 1231-1242. [CrossRef]

17. Pillot, B.; Soty, M.; Gautier-Stein, A.; Zitoun, C.; Mithieux, G. Protein feeding promotes redistribution of endogenous glucose production to the kidney and potentiates its suppression by insulin. Endocrinology 2009, 150, 616-624. [CrossRef]

18. Gerich, J.E.; Meyer, C.; Woerle, H.J.; Stumvoll, M. Renal gluconeogenesis: Its importance in human glucose homeostasis. Diabetes Care 2001, 24, 382-391. [CrossRef]

19. Croset, M.; Rajas, F.; Zitoun, C.; Hurot, J.M.; Montano, S.; Mithieux, G. Rat small intestine is an insulin-sensitive gluconeogenic organ. Diabetes 2001, 50,740-746. [CrossRef]

20. Mithieux, G.; Gautier-Stein, A.; Rajas, F.; Zitoun, C. Contribution of intestine and kidney to glucose fluxes in different nutritional states in rat. Comp. Biochem. Physiol. B Biochem. Mol. Biol. 2006, 143, 195-200. [CrossRef]

21. Mutel, E.; Abdul-Wahed, A.; Ramamonjisoa, N.; Stefanutti, A.; Houberdon, I.; Cavassila, S.; Pilleul, F.; Beuf, O.; Gautier-Stein, A.; Penhoat, A.; et al. Targeted deletion of liver glucose- 6 phosphatase mimics glycogen storage disease type 1a including development of multiple adenomas. J. Hepatol. 2011, 54, 529-537. [CrossRef] [PubMed]

22. Penhoat, A.; Fayard, L.; Stefanutti, A.; Mithieux, G.; Rajas, F. Intestinal gluconeogenesis is crucial to maintain a physiological fasting glycemia in the absence of hepatic glucose production in mice. Metab. Clin. Exp. 2014, 63, 104-111. [CrossRef] [PubMed]

23. Heinrich, P.C.; Morris, H.P.; Weber, G. Behavior of transaldolase (EC 2.2.1.2) and transketolase (EC 2.2.1.1) Activities in normal, neoplastic, differentiating, and regenerating liver. Cancer Res. 1976, 36, 3189-3197. [PubMed]

24. Hanczko, R.; Fernandez, D.R.; Doherty, E.; Qian, Y.; Vas, G.; Niland, B.; Telarico, T.; Garba, A.; Banerjee, S.; Middleton, F.A.; et al. Prevention of hepatocarcinogenesis and increased susceptibility to acetaminophen-induced liver failure in transaldolase-deficient mice by $\mathrm{N}$-acetylcysteine. J. Clin. Investig. 2009, 119, 1546-1557. [CrossRef]

25. Koppenol, W.H.; Bounds, P.L.; Dang, C.V. Otto Warburg's contributions to current concepts of cancer metabolism. Nat. Rev. Cancer 2011, 11, 325-337. [CrossRef]

26. Benhamed, F.; Filhoulaud, G.; Caron, S.; Lefebvre, P.; Staels, B.; Postic, C. O-GlcNAcylation Links ChREBP and FXR to Glucose-Sensing. Front. Endocrinol. 2015, 5, 230. [CrossRef] 
27. Benhamed, F.; Denechaud, P.D.; Lemoine, M.; Robichon, C.; Moldes, M.; Bertrand-Michel, J.; Ratziu, V.; Serfaty, L.; Housset, C.; Capeau, J.; et al. The lipogenic transcription factor ChREBP dissociates hepatic steatosis from insulin resistance in mice and humans. J. Clin. Investig. 2012, 122, 2176-2194. [CrossRef]

28. Dentin, R.; Tomas-Cobos, L.; Foufelle, F.; Leopold, J.; Girard, J.; Postic, C.; Ferré, P. Glucose 6-phosphate, rather than xylulose 5-phosphate, is required for the activation of ChREBP in response to glucose in the liver. J. Hepatol. 2012, 56, 199-209. [CrossRef]

29. Iizuka, K.; Miller, B.; Uyeda, K. Deficiency of carbohydrate-activated transcription factor ChREBP prevents obesity and improves plasma glucose control in leptin-deficient (ob/ob) mice. Am. J. Physiol. Endocrinol. Metab. 2006, 291, E358-E364. [CrossRef]

30. Niwa, H.; Iizuka, K.; Kato, T.; Wu, W.; Tsuchida, H.; Takao, K.; Horikawa, Y.; Takeda, J. ChREBP Rather Than SHP Regulates Hepatic VLDL Secretion. Nutrients 2018, 10, 321. [CrossRef]

31. Poungvarin, N.; Chang, B.; Imamura, M.; Chen, J.; Moolsuwan, K.; Sae-Lee, C.; Li, W.; Chan, L. Genome-Wide Analysis of ChREBP Binding Sites on Male Mouse Liver and White Adipose Chromatin. Endocrinology 2015, 156, 1982-1994. [CrossRef] [PubMed]

32. Dentin, R.; Benhamed, F.; Hainault, I.; Fauveau, V.; Foufelle, F.; Dyck, J.R.B.; Girard, J.; Postic, C. Liver-specific inhibition of ChREBP improves hepatic steatosis and insulin resistance in ob/ob mice. Diabetes 2006, 55, 2159-2170. [CrossRef] [PubMed]

33. Jois, T.; Chen, W.; Howard, V.; Harvey, R.; Youngs, K.; Thalmann, C.; Saha, P.; Chan, L.; Cowley, M.A.; Sleeman, M.W. Deletion of hepatic carbohydrate response element binding protein (ChREBP) impairs glucose homeostasis and hepatic insulin sensitivity in mice. Mol. Metab. 2017, 6, 1381-1394. [CrossRef] [PubMed]

34. Calvisi, D.F.; Wang, C.; Ho, C.; Ladu, S.; Lee, S.A.; Mattu, S.; Destefanis, G.; Delogu, S.; Zimmermann, A.; Ericsson, J.; et al. Increased lipogenesis, induced by AKT-mTORC1-RPS6 signaling, promotes development of human hepatocellular carcinoma. Gastroenterology 2011, 140, 1071-1083. [CrossRef] [PubMed]

35. Ribback, S.; Sonke, J.; Lohr, A.; Frohme, J.; Peters, K.; Holm, J.; Peters, M.; Cigliano, A.; Calvisi, D.F.; Dombrowski, F. Hepatocellular glycogenotic foci after combined intraportal pancreatic islet transplantation and knockout of the carbohydrate responsive element binding protein in diabetic mice. Oncotarget 2017, 8 , 104315-104329. [CrossRef] [PubMed]

36. Ribback, S.; Che, L.; Pilo, M.G.; Cigliano, A.; Latte, G.; Pes, G.M.; Porcu, A.; Pascale, R.M.; Li, L.; Qiao, Y.; et al. Oncogene-dependent addiction to carbohydrate-responsive element binding protein in hepatocellular carcinoma. Cell Cycle 2018, 17, 1496-1512. [CrossRef]

37. Tong, X.; Zhao, F.; Mancuso, A.; Gruber, J.J.; Thompson, C.B. The glucose-responsive transcription factor ChREBP contributes to glucose-dependent anabolic synthesis and cell proliferation. Proc. Natl. Acad. Sci. USA 2009, 106, 21660-21665. [CrossRef]

38. Abdul-Wahed, A.; Gautier-Stein, A.; Casteras, S.; Soty, M.; Roussel, D.; Romestaing, C.; Guillou, H.; Tourette, J.A.; Pleche, N.; Zitoun, C.; et al. A link between hepatic glucose production and peripheral energy metabolism via hepatokines. Mol. Metab. 2014, 3, 531-543. [CrossRef]

39. Gjorgjieva, M.; Calderaro, J.; Monteillet, L.; Silva, M.; Raffin, M.; Brevet, M.; Romestaing, C.; Roussel, D.; Zucman-Rossi, J.; Mithieux, G.; et al. Dietary exacerbation of metabolic stress leads to accelerated hepatic carcinogenesis in glycogen storage disease type Ia. J. Hepatol. 2018, 69, 1074-1087. [CrossRef]

40. Eissing, L.; Scherer, T.; Tödter, K.; Knippschild, U.; Greve, J.W.; Buurman, W.A.; Pinnschmidt, H.O.; Rensen, S.S.; Wolf, A.M.; Bartelt, A.; et al. De novo lipogenesis in human fat and liver is linked to ChREBP- $\beta$ and metabolic health. Nat. Commun. 2013, 4, 1528. [CrossRef]

41. Filhoulaud, G.; Guilmeau, S.; Dentin, R.; Girard, J.; Postic, C. Novel insights into ChREBP regulation and function. Trends Endocrinol. Metab. 2013, 24, 257-268. [CrossRef] [PubMed]

42. Kabashima, T.; Kawaguchi, T.; Wadzinski, B.E.; Uyeda, K. Xylulose 5-phosphate mediates glucose-induced lipogenesis by xylulose 5-phosphate-activated protein phosphatase in rat liver. Proc. Natl. Acad. Sci. USA 2003, 100, 5107-5112. [CrossRef] [PubMed]

43. McFerrin, L.G.; Atchley, W.R. A novel N-terminal domain may dictate the glucose response of Mondo proteins. PLoS ONE 2012, 7, e34803. [CrossRef] [PubMed]

44. Bricambert, J.; Miranda, J.; Benhamed, F.; Girard, J.; Postic, C.; Dentin, R. Salt-inducible kinase 2 links transcriptional coactivator p300 phosphorylation to the prevention of ChREBP-dependent hepatic steatosis in mice. J. Clin. Investig. 2010, 120, 4316-4331. [CrossRef] 
45. Guinez, C.; Filhoulaud, G.; Rayah-Benhamed, F.; Marmier, S.; Dubuquoy, C.; Dentin, R.; Moldes, M.; Burnol, A.F.; Yang, X.; Lefebvre, T.; et al. O-GlcNAcylation Increases ChREBP Protein Content and Transcriptional Activity in the Liver. Diabetes 2011, 60, 1399-1413. [CrossRef]

46. Davies, M.N.; O'Callaghan, B.L.; Towle, H.C. Glucose activates ChREBP by increasing its rate of nuclear entry and relieving repression of its transcriptional activity. J. Biol. Chem. 2008, 283, 24029-24038. [CrossRef]

47. Sato, S.; Jung, H.; Nakagawa, T.; Pawlosky, R.; Takeshima, T.; Lee, W.R.; Sakiyama, H.; Laxman, S.; Wynn, R.M.; Tu, B.P.; et al. Metabolite Regulation of Nuclear Localization of Carbohydrate-response Element-binding Protein (ChREBP): ROLE OF AMP AS AN ALLOSTERIC INHIBITOR. J. Biol. Chem. 2016, 291, 10515-10527. [CrossRef]

48. Ortega-Prieto, P.; Postic, C. Carbohydrate Sensing Through the Transcription Factor ChREBP. Front. Genet. 2019, 10, 472. [CrossRef]

49. Kim, M.S.; Krawczyk, S.A.; Doridot, L.; Fowler, A.J.; Wang, J.X.; Trauger, S.A.; Noh, H.L.; Kang, H.J.; Meissen, J.K.; Blatnik, M.; et al. ChREBP regulates fructose-induced glucose production independently of insulin signaling. J. Clin. Investig. 2016, 126, 4372-4386. [CrossRef]

50. Unnikrishnan, R.; Pradeepa, R.; Joshi, S.R.; Mohan, V. Type 2 Diabetes: Demystifying the Global Epidemic. Diabetes 2017, 66, 1432-1442. [CrossRef]

51. Chen, L.; Magliano, D.J.; Zimmet, P.Z. The worldwide epidemiology of type 2 diabetes mellitus-present and future perspectives. Nat. Rev. Endocrinol. 2012, 8, 228-236. [CrossRef] [PubMed]

52. Lin, H.V.; Accili, D. Hormonal regulation of hepatic glucose production in health and disease. Cell Metab. 2011, 14, 9-19. [CrossRef] [PubMed]

53. Brownlee, M. Biochemistry and molecular cell biology of diabetic complications. Nature 2001, 414, 813-820. [CrossRef] [PubMed]

54. Kishnani, P.S.; Austin, S.L.; Abdenur, J.E.; Arn, P.; Bali, D.S.; Boney, A.; Chung, W.K.; Dagli, A.I.; Dale, D.; Koeberl, D.; et al. Diagnosis and management of glycogen storage disease type I: A practice guideline of the American College of Medical Genetics and Genomics. Genet. Med. 2014, 16, e1. [CrossRef] [PubMed]

55. Smith, B.W.; Adams, L.A. Non-alcoholic fatty liver disease. Crit. Rev. Clin. Lab. Sci. 2011, 48, 97-113. [CrossRef]

56. Hazlehurst, J.M.; Woods, C.; Marjot, T.; Cobbold, J.F.; Tomlinson, J.W. Non-alcoholic fatty liver disease and diabetes. Metab. Clin. Exp. 2016, 65, 1096-1108. [CrossRef]

57. Williamson, R.M.; Price, J.F.; Glancy, S.; Perry, E.; Nee, L.D.; Hayes, P.C.; Frier, B.M.; Van Look, L.A.F.; Johnston, G.I.; Reynolds, R.M.; et al. Prevalence of and risk factors for hepatic steatosis and nonalcoholic Fatty liver disease in people with type 2 diabetes: The Edinburgh Type 2 Diabetes Study. Diabetes Care 2011, 34, 1139-1144. [CrossRef]

58. Matsuzaka, T.; Shimano, H. Insulin-dependent and -independent regulation of sterol regulatory element-binding protein-1c. J. Diabetes Investig. 2013, 4, 411-412. [CrossRef]

59. Grefhorst, A.; Schreurs, M.; Oosterveer, M.H.; Cortés, V.A.; Havinga, R.; Herling, A.W.; Reijngoud, D.J.; Groen, A.K.; Kuipers, F. Carbohydrate-response-element-binding protein (ChREBP) and not the liver $\mathrm{X}$ receptor $\alpha(\mathrm{LXR} \alpha)$ mediates elevated hepatic lipogenic gene expression in a mouse model of glycogen storage disease type 1. Biochem. J. 2010, 432, 249-254. [CrossRef]

60. Postic, C.; Girard, J. Contribution of de novo fatty acid synthesis to hepatic steatosis and insulin resistance: Lessons from genetically engineered mice. J. Clin. Investig. 2008, 118, 829-838. [CrossRef]

61. Bandsma, R.H.J.; Prinsen, B.H.; de Sain-van der Velden, M.; Rake, J.P.; Boer, T.; Smit, G.P.A.; Reijngoud, D.J.; Kuipers, F. Increased de novo Lipogenesis and Delayed Conversion of Large VLDL into Intermediate Density Lipoprotein Particles Contribute to Hyperlipidemia in Glycogen Storage Disease Type 1a. Pediatr. Res. 2008, 63, 702-707. [CrossRef] [PubMed]

62. Gjorgieva, M.; Oosterveer, M.H.; Mithieux, G.; Rajas, F. Mechanisms by Which Metabolic Reprogramming in GSD1 Liver Generates a Favorable Tumorigenic Environment. J. Inborn Errors Metab. Screen. 2016, 4. [CrossRef]

63. Calzadilla Bertot, L.; Adams, L.A. The Natural Course of Non-Alcoholic Fatty Liver Disease. Int. J. Mol. Sci. 2016, 17, 774. [CrossRef] [PubMed]

64. Sanyal, A.; Poklepovic, A.; Moyneur, E.; Barghout, V. Population-based risk factors and resource utilization for HCC: US perspective. Curr. Med. Res. Opin. 2010, 26, 2183-2191. [CrossRef] [PubMed] 
65. Bengtsson, B.; Stål, P.; Wahlin, S.; Björkström, N.K.; Hagström, H. Characteristics and outcome of hepatocellular carcinoma in patients with NAFLD without cirrhosis. Liver Int. 2019, 39, 1098-1108. [CrossRef] [PubMed]

66. Monteillet, L.; Gjorgjieva, M.; Silva, M.; Verzieux, V.; Imikirene, L.; Duchampt, A.; Guillou, H.; Mithieux, G.; Rajas, F. Intracellular lipids are an independent cause of liver injury and chronic kidney disease in non alcoholic fatty liver disease-like context. Mol. Metab. 2018, 16, 100-115. [CrossRef]

67. Musso, G.; Cassader, M.; Gambino, R. Non-alcoholic steatohepatitis: Emerging molecular targets and therapeutic strategies. Nat. Rev. Drug Discov. 2016, 15, 249-274. [CrossRef]

68. Waskowicz, L.R.; Zhou, J.; Landau, D.J.; Brooks, E.D.; Lim, A.; Yavarow, Z.A.; Kudo, T.; Zhang, H.; Wu, Y.; Grant, S.; et al. Bezafibrate induces autophagy and improves hepatic lipid metabolism in glycogen storage disease type Ia. Hum. Mol. Genet. 2018, 28, 143-154. [CrossRef]

69. Finan, B.; Clemmensen, C.; Zhu, Z.; Stemmer, K.; Gauthier, K.; Müller, L.; De Angelis, M.; Moreth, K.; Neff, F.; Perez-Tilve, D.; et al. Chemical Hybridization of Glucagon and Thyroid Hormone Optimizes Therapeutic Impact for Metabolic Disease. Cell 2016, 167, 843-857. [CrossRef]

70. Perry, R.J.; Zhang, D.; Zhang, X.M.; Boyer, J.L.; Shulman, G.I. Controlled-release mitochondrial protonophore reverses diabetes and steatohepatitis in rats. Science 2015, 347, 1253-1256. [CrossRef]

71. Perry, R.J.; Kim, T.; Zhang, X.M.; Lee, H.Y.; Pesta, D.; Popov, V.B.; Zhang, D.; Rahimi, Y.; Jurczak, M.J.; Cline, G.W.; et al. Reversal of hypertriglyceridemia, fatty liver disease, and insulin resistance by a liver-targeted mitochondrial uncoupler. Cell Metab. 2013, 18, 740-748. [CrossRef] [PubMed]

(C) 2019 by the authors. Licensee MDPI, Basel, Switzerland. This article is an open access article distributed under the terms and conditions of the Creative Commons Attribution (CC BY) license (http://creativecommons.org/licenses/by/4.0/). 


\title{
Transcriptional Regulation in Non-Alcoholic Fatty Liver Disease
}

\author{
Sandra Steensels ${ }^{\dagger}$, Jixuan Qiao ${ }^{\dagger}$ and Baran A. Ersoy * \\ Joan \& Sanford I. Weill Department of Medicine, Weill Cornell Medical College, New York, NY 10021, USA; \\ sas2906@med.cornell.edu (S.S.); jiq2001@med.cornell.edu (J.Q.) \\ * Correspondence: bersoy@med.cornell.edu \\ + These authors have contributed equally to this work.
}

Received: 30 May 2020; Accepted: 2 July 2020; Published: 9 July 2020

\begin{abstract}
Obesity is the primary risk factor for the pathogenesis of non-alcoholic fatty liver disease (NAFLD), the worldwide prevalence of which continues to increase dramatically. The liver plays a pivotal role in the maintenance of whole-body lipid and glucose homeostasis. This is mainly mediated by the transcriptional activation of hepatic pathways that promote glucose and lipid production or utilization in response to the nutritional state of the body. However, in the setting of chronic excessive nutrition, the dysregulation of hepatic transcriptional machinery promotes lipid accumulation, inflammation, metabolic stress, and fibrosis, which culminate in NAFLD. In this review, we provide our current understanding of the transcription factors that have been linked to the pathogenesis and progression of NAFLD. Using publicly available transcriptomic data, we outline the altered activity of transcription factors among humans with NAFLD. By expanding this analysis to common experimental mouse models of NAFLD, we outline the relevance of mouse models to the human pathophysiology at the transcriptional level.
\end{abstract}

Keywords: non-alcoholic fatty liver disease; non-alcoholic steatohepatitis; transcription factors; inflammation; metabolic stress; fibrosis; lipid homeostasis; glucose homeostasis

\section{Introduction}

Obesity often results in the dysregulation of lipid and glucose metabolism and is therefore the primary risk factor for the pathogenesis of metabolic disorders, including cardiovascular disease, type 2 diabetes mellitus (T2DM), and non-alcoholic fatty liver disease (NAFLD) [1]. The global prevalence of NAFLD, which was $15 \%$ in 2005 , has quickly escalated to $24 \%$ by 2016 in a parallel trend to obesity [2]. NAFLD encompasses a spectrum of pathologies ranging from hepatocellular lipid accumulation (steatosis) to non-alcoholic steatohepatitis (NASH) characterized by steatosis and inflammation. In addition, chronic inflammation activates hepatic stellate cells (HSC), which promote fibrosis by secreting type I and III collagen and fibronectin into the extracellular matrix (ECM) [3]. When fibrotic NASH remains untreated, it can lead to cirrhosis and hepatocellular carcinoma (HCC) [4]. Despite alarming increases in prevalence, the treatment strategy of NAFLD remains limited to weight loss regiments and requires a more complete understanding of diet-induced pathogenesis of NAFLD in obese patients [5]. 
The pathogenesis of NAFLD is complex, and evolving theories have culminated in a two-hit versus multiple-hit hypotheses [6]. In the 'two-hit hypothesis', the first hit originates from the accumulation of more than $5 \%$ hepatic steatosis, during which insulin resistance emerges as a pathogenic contributor. This makes the liver more susceptible to a second hit, including oxidative stress, the production of pro-inflammatory cytokines, and apoptosis, which progress the disease to the necro-inflammatory stage defined as NASH [7]. In contrast, the 'multiple-hit' hypothesis encompasses the interplay of multiple factors whereby genetics, environment, unhealthy dietary habits, insulin resistance, adipocyte differentiation, and the intestinal microbiota together contribute to disease development and progression [8]. Regardless of the source of the hit (s), hepatic responses to extrahepatic stimuli are controlled by well-described transcriptionally regulated pathways that help transcribe the relevant biological machinery to maintain energy homeostasis. However, obesity-induced maladaptive activation or the inhibition of these transcriptional regulators often exacerbates lipid accumulation, insulin resistance, inflammation, and fibrosis [9].

The efforts toward identifying the promoters of obesity-induced NAFLD have relied heavily on rodent models due to limited access to and the variability within human samples arising from differences in disease stage, age, sex, medication, body weight, and other lifestyle choices such as alcohol consumption. However, rodent models do not capture all the features of the human pathophysiology. The rodent NAFLD models described in this review are categorized by their mode of induction using diet, chemicals, or genetic alteration (Box 1). For the diet-induced models, we highlight high-fat diet (HFD), Western diet (WD), methionine- and choline-deficient diet (MCD), choline-deficient L-amino acid-defined (CDAA) diet, and fructose-palmitate-cholesterol and trans-fat (FPC or NASH) diet. The chemically induced models include the combination of HFD with streptozotocin (STZ) supplementation or the use of carbon tetrachloride $\left(\mathrm{CCl}_{4}\right)$. For genetic models, we highlight the APOE2 knock-in (APOE2-KI) mouse [10], hepatocyte-specific phosphatase and tensin homolog (PTEN) knockout model [11], and Mice expressing urokinase-type plasminogen activator (uPA) under the control of the major urinary protein (MUP) promoter (MUP-uPA mice) [12].

In this review, we discuss our current understanding of the transcription factors that have been linked to the pathogenesis and progression of NAFLD. Transcription factors that are associated with obesity-induced liver injury and the pathogenesis and progression of NAFLD often serve essential biological functions in the maintenance of energy homeostasis and stress response. Furthermore, recent studies have indicated that the gut microbiota may contribute to NAFLD by altering the production of endogenous substrates that control the activity of hepatic transcription factors. Therefore, we have categorized these transcriptional regulators under lipid and glucose metabolism, inflammation, metabolic stress, fibrosis, and microbiome dysbiosis. Key transcriptional regulators that play significant roles in multiple metabolic responses have been addressed in all relevant categories. 
Box 1. Mouse models of non-alcoholic fatty liver disease (NAFLD).

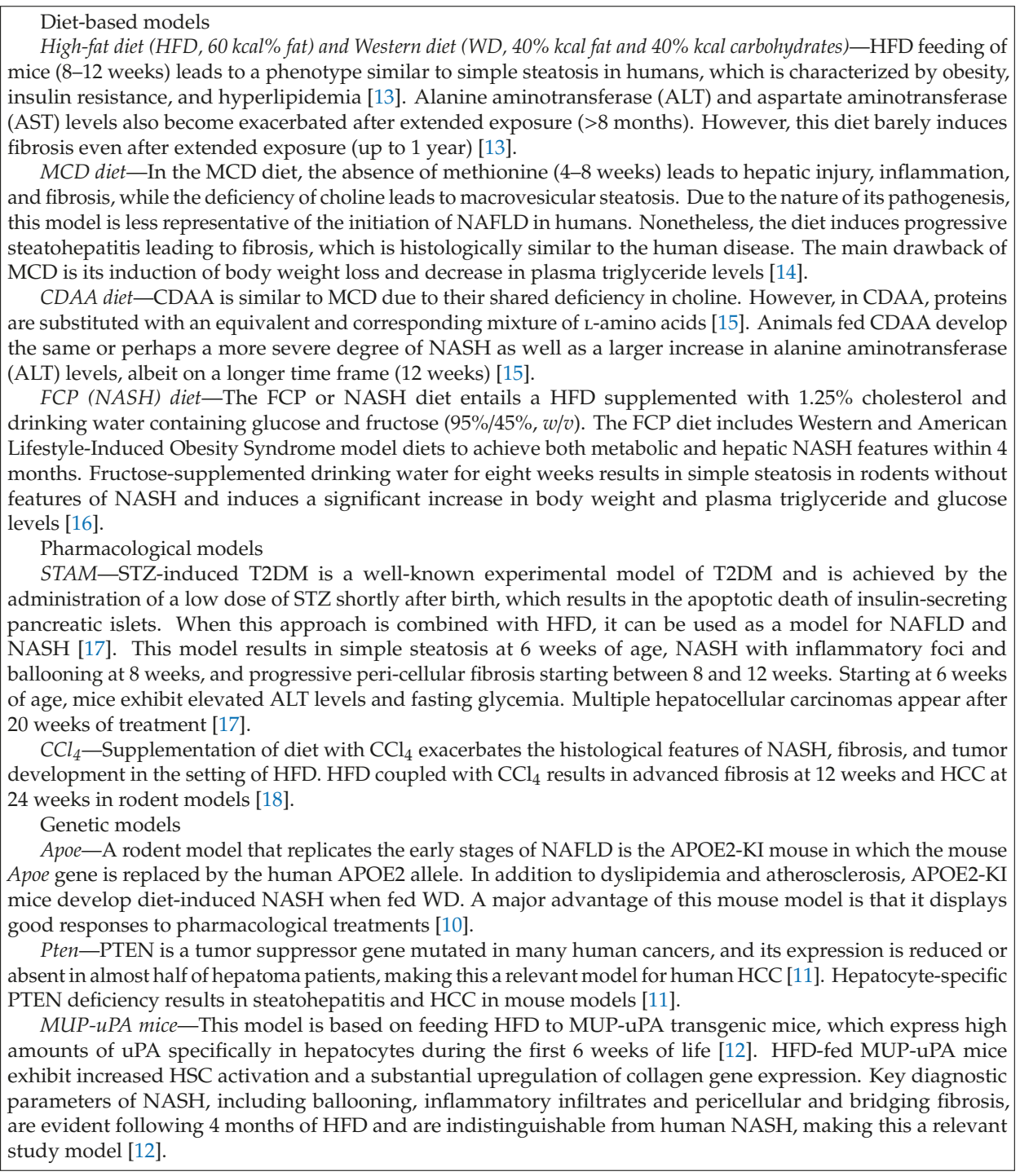

\section{Lipid Metabolism}

Hepatic steatosis is a consequence of increased hepatic lipid uptake, increased de novo lipogenesis, and reduced lipid clearance. Excessive nutrition, accompanied by hyperinsulinemia and hyperglycemia, drives steatosis by promoting de novo lipogenesis in the liver, which contributes substantially to the accumulation of triglycerides and other lipid species [19]. Hepatic lipid homeostasis is mainly regulated by peroxisome proliferator-activated receptor alpha (PPAR $\alpha), \operatorname{PPAR} \gamma, \operatorname{PPAR} \delta$ and sterol regulatory element binding protein $1 \mathrm{c}$ (SREBP1c), which coordinate transcriptional responses to altered metabolic conditions such as feeding and fasting to promote fat storage or catabolism, respectively. Other transcription factors of lipid metabolism that are altered in the setting of NAFLD include the constitutive androstane receptor (CAR), liver X receptor (LXR), Cyclic AMP-responsive element-binding 
protein $\mathrm{H}(\mathrm{CREBH})$, Farnesoid $X$ receptor (FXR), signal transducer and activator of transcription 5 (STAT5), and CCAAT/enhancer binding protein alpha (C/EBP $\alpha)($ Table 1$)$.

Table 1. Changes in the activity of transcription factors that regulate glucose and lipid metabolism, inflammation and fibrosis in the setting of NAFLD in humans and mice.

\begin{tabular}{|c|c|c|c|c|}
\hline Factor & Model & Pathway & Regulation & Reference \\
\hline $\operatorname{PPAR} \alpha$ & Humans, mice & $\begin{array}{l}\text { Lipid metabolism, inflammation, } \\
\text { fibrosis }\end{array}$ & Upregulation & {$[20]$} \\
\hline $\operatorname{PPAR} \gamma$ & Humans, mice & $\begin{array}{l}\text { Lipid metabolism, inflammation, } \\
\text { fibrosis }\end{array}$ & Upregulation & [20] \\
\hline SREBP Family & Humans, mice & Lipid metabolism & $\begin{array}{c}\text { Genetic variations increase risk } \\
\text { of NAFLD }\end{array}$ & [21] \\
\hline ChREBP & Humans, mice & Lipid metabolism & Upregulation & {$[22]$} \\
\hline CAR & Humans, mice & Lipid metabolism, inflammation & Upregulation & [23] \\
\hline LXR & Humans & Lipid metabolism, inflammation & Upregulation & {$[24]$} \\
\hline FXR & Humans & Lipid metabolism & Downregulation & [25] \\
\hline STAT5 & Humans & Lipid metabolism & Upregulated & [26] \\
\hline $\mathrm{C} / \mathrm{EBP} \alpha$ & Mice & Lipid metabolism & Upregulation & {$[27]$} \\
\hline PGC1 $\alpha$ & Mice & Glucose homeostasis & Downregulation & [28] \\
\hline FoxO & Humans & Glucose homeostasis & Upregulation & [29] \\
\hline $\mathrm{HNF} 4 \alpha$ & Humans & $\begin{array}{l}\text { Central regulator, Glucose } \\
\text { homeostasis }\end{array}$ & Downregulation & {$[25]$} \\
\hline NF-kB & Humans, mice & Inflammation & Upregulation & {$[30]$} \\
\hline IRFs & Mice & Inflammation & Upregulation & {$[31]$} \\
\hline STAT1/3 & Mice & Inflammation & Upregulation & [32] \\
\hline AP-1 and c-Jun & Humans, mice & Inflammation, fibrosis & Upregulation & {$[30,33]$} \\
\hline SHP & Humans, mice & Inflammation & Downregulation & [34] \\
\hline Nrf2 & Mice & Inflammation & Upregulation & {$[35]$} \\
\hline Runx2 & Mice & Inflammation & Upregulation & [36] \\
\hline $\mathrm{C} / \mathrm{EBP} \beta$ & & Inflammation & & \\
\hline IRE1 $\alpha$ & Human & Metabolic stress & Upregulation & [37] \\
\hline Xbp1 & Mice & Metabolic stress & Upregulation & {$[38]$} \\
\hline $\mathrm{eIF} 2 \alpha$ & Mice & Metabolic stress & Upregulation & [39] \\
\hline ATF4 & Humans & Metabolic stress & Upregulation & [40] \\
\hline ATF6 & Humans & Metabolic stress & Upregulation & [41] \\
\hline Smad & Humans, mice & Fibrosis & Upregulation & {$[42]$} \\
\hline TGF $\beta$ & Humans, mice & Fibrosis & Upregulation & [42] \\
\hline AEBP1 & Humans, mice & Fibrosis & Upregulation & {$[43]$} \\
\hline AATF/che-1 & Humans, mice & Fibrosis & Upregulation & {$[44]$} \\
\hline YAP & Humans, mice & Fibrosis & Upregulation & [45] \\
\hline
\end{tabular}

Abbreviations: peroxisome proliferator-activated receptor (PPAR), sterol regulatory element binding protein (SREBP), carbohydrate-responsive element-binding protein (ChREBP), constitutive androstane receptor (CAR), liver X receptor (LXR), farnesoid X receptor (FXR), signal transducer and activator of transcription (STAT), CCAAT/enhancer binding protein $(\mathrm{C} / \mathrm{EBP})$, PPAR $\gamma$ coactivator 1 alpha $(\mathrm{PGC1} \alpha)$, forkhead protein $\mathrm{O}(\mathrm{FoxO})$, hepatocyte nuclear factor (HNF), nuclear factor of the $\mathrm{k}$ light chain enhancer of $\mathrm{B}$ cells (NF- $\mathrm{kB}$ ), interferon regulatory factors (IRFs), activator protein 1 (AP-1), small heterodimer partner (SHP), nuclear factor erythroid 2-related factor 2 (Nrf2), runt-related transcription factor 2 (Runx2), inositol-requiring enzyme $1 \alpha$ (IRE1 $\alpha$ ), X box-binding protein 1 (Xbp1), eukaryotic translation initiation factor $2 \alpha(\mathrm{eIF} 2 \alpha)$, activating transcription factor (ATF), transcription factors against decapentaplegic homolog (Smad), transforming growth factor $\beta$ (TGF $\beta$ ), adipocyte enhancer binding protein 1 (AEBP1), apoptosis antagonizing transcription factor (AATF/che-1), yes-associated protein (YAP).

\subsection{PPAR $\alpha$}

PPAR $\alpha$ belongs to the PPAR nuclear receptor family. PPAR $\alpha$ is mostly expressed in hepatocytes where it becomes activated upon binding by fatty acids (FAs) and promotes FA uptake and utilization through $\beta$-oxidation and ketogenesis [46]. Hepatic PPAR $\alpha$ expression is increased in male mice and both male and female humans with NAFLD [20,46]. Suggestive of a protective function, mice lacking 
PPAR $\alpha$ expression exhibit more severe steatosis [47]. Therefore, NAFLD-induced increases in PPAR $\alpha$ abundance can be further enhanced by its pharmacological activation: the PPAR $\alpha$ agonist WY-14643 protects mice against steatosis and steatohepatitis by preventing intrahepatic lipid and lipoperoxide accumulation [47]. Since WY-14643 causes toxicity in humans, other fibrates have been extensively used in the treatment of hypertriglyceridemia. However, these studies failed to establish benefits against NASH, which is most likely due to the widespread extrahepatic expression of PPAR $\alpha$ [48].

\subsection{PPAR $\gamma$}

Another member of the PPAR family, PPAR $\gamma$ is also activated by FA ligands and promotes lipogenesis and lipid accumulation. In humans and mice, two isoforms of PPAR $\gamma$ exist: PPAR $\gamma 1$ is found in nearly all tissues except muscle, while PPAR $\gamma 2$ is mostly expressed in adipose tissue and the intestine. PPAR $\gamma 2$ expression is upregulated in the liver and adipose tissue of obese humans and high-fat diet (HFD)-fed mice, whereas the PPAR $\gamma 1$ expression remains unchanged under these conditions [49]. In hepatocytes, PPAR $\gamma 1$ increases the transcription of genes that are required for FA uptake and de novo lipogenesis [50]. Meanwhile, lipidomic analyses suggest that PPAR 2 plays an important anti-lipotoxic role when induced ectopically in liver and muscle by facilitating the deposition of lipid droplets and preventing the accumulation of reactive lipid species, such as ceramides and pro-inflammatory lysophosphatidylcholine [51]. HFD-fed mice with a hepatocyte specific loss of PPAR $\gamma$ expression exhibit a reduction of hepatic lipid vacuoles as well as the downregulation of genes involved in de novo lipogenesis [52]. Furthermore, the liver-specific ablation of PPAR $\gamma$ in $o b / o b$ mice reduces hepatic triglycerides despite increasing serum FAs [53]. Livers of NAFLD patients have increased hepatic PPAR $\gamma$ expression $[20,46]$. Whereas increased PPAR $\gamma$ activity within hepatocytes would be expected to contribute to steatosis [54,55], the treatment of patients with the PPAR $\gamma$ agonists rosiglitazone or pioglitazone result in reduced hepatic steatosis [56-58]. This alleviation could be explained by the extrahepatic effects of PPAR $\gamma$ activation in the adipose tissue where it promotes the storage of excess energy in the form of lipid droplets, thereby limiting exposure of the liver to excess lipids.

\subsection{PPARS}

Similar to other PPARs, PPAR $\delta$ binds to the PPAR response element (PPRE) to initiate or repress the expression of target genes [59]. PPAR $\delta$ is ubiquitously expressed and is activated by polyunsaturated fatty acids and their metabolites. In mouse livers, PPAR $\delta$ prevents lipid accumulation by increasing $\beta$-oxidation and autophagy. In addition, the activation of PPAR $\delta$ in the adipose tissue of mice upregulates the expression of genes involved in $\beta$-oxidation and energy dissipation $[60,61]$. Recent clinical studies using PPAR $\delta$ agonists atorvastatin and cardarine reduced hepatic fat content in overweight patients with mixed dyslipidemia [61,62].

\subsection{SREBP}

The SREBP family transcription factors consist of three isoforms: SREBP1a, SREBP1c, and SREBP2. Each isoform exhibits a different tissue expression pattern and metabolic control [63]. SREBP1a is the predominant isoform in the intestine, spleen, and cultured cells, while SREBP1c and SREBP2 exhibit higher abundance in the liver [63]. SREBP1a is a potent activator of genes that mediate the synthesis of cholesterol, fatty acids, and triglycerides. The roles of SREBP1c and SREBP2 are more restricted than those of SREBP1a. SREBP1c promotes the transcription of genes involved in lipogenesis, such as acetyl-coenzyme A (CoA) carboxylase (ACC), FA synthase (FASN), and steroyl-CoA desaturase in response to insulin and high-energy state [64]. By contrast, hepatic markers for energy deprivation, such as glucagon signaling (protein kinase A [PKA], AMP activated protein kinase [AMPK]) and the deacetylase sirtuin1 (SIRT1) inhibit SREBP1c, suggesting that SREBP1c does not promote hepatic lipid synthesis in the setting of starvation [65]. Among the genes involved in lipogenesis, SREBP1c also promotes the transcription of patatin-like phospholipase3 (PNPLA3), which in turn stimulates lipid 
accumulation [66]. Independent studies in humans have confirmed that PNPLA3 variants are strongly associated with the severity of NAFLD and NASH [67-69]. SREBP1c is upregulated in the livers of humans and mice with NAFLD [70]. Interestingly, there is also a positive correlation between single nucleotide polymorphisms (i.e., rs2297508) as well as rare variants of SREBP1 with the risk of developing NAFLD [21]. Unlike SREBP1c, SREBP2 preferentially activates cholesterol synthesis [71]. In mice, SREBP2 contributes to the onset of NASH by triggering cholesterol accumulation [72]. Increased hepatic SREBP2 is also associated with increased free cholesterol in NASH patients [73].

\subsection{CAR}

CAR is a member of the nuclear receptor superfamily [74]. It mainly functions as a sensor of endobiotic and xenobiotic substances, as CAR-activated genes regulate drug metabolism and enhance bilirubin clearance [74]. Unlike most nuclear receptors, this transcriptional regulator is constitutively active in the absence of a ligand. CAR activity is anti-obesogenic and improves insulin sensitivity [75]. The metabolic benefits of CAR activation stem from the combined effects of reduced lipogenesis, very low-density lipoprotein (VLDL) secretion, and gluconeogenesis, as well as increased peripheral fat mobilization for thermogenesis in brown adipose tissue [75]. The anti-steatotic effect of CAR was first demonstrated using a mouse model with the genetic ablation of cytoplasmic CAR retention protein (CCRP), which isolates CAR to the cytosol and inactivates it. Subsequent CAR activation represses lipogenic gene expression and increases $\beta$-oxidation [76]. Similar to mouse models of NAFLD, CAR is also upregulated in the livers of patients with NAFLD [23].

\subsection{LXR}

LXR is a member of the nuclear receptor family of transcription factors that is closely related to PPARs [77]. LXR forms heterodimers with the obligate partner retinoid $X$ receptor (RXR), which is activated by retinoic acid and cholesterol derivatives. LXR is an important regulator of cholesterol, FA, and glucose homeostasis [77]. LXR activation increases hepatic triglyceride accumulation and cholesterol metabolism in both humans and mice and initiates bile acid degradation in mice [78]. Humans express two LXR family members, namely LXR $\alpha$ (NR1H3) and LXR $\beta$ (NR1H2). LXR $\alpha$ expression increases by 2- and 3-fold in the livers of NAFLD and NASH patients, respectively, compared to healthy controls [24]. Furthermore, $\mathrm{LXR} \alpha$ expression positively correlates with the amount of hepatic fat and hepatic expression of the cholesterol transporter ATP-binding cassette sub-family G member 5 (ABCG5/8), the FA transporter cluster of differentiation 36 (CD36), and SREBP1c [24].

\subsection{CREBH}

CREBH is primarily expressed in the endoplasmic reticulum (ER) of cells in the liver and small intestine [79,80]. CREBH expression increases in response to fasting through glucagon signaling [81]. CREBH expression is also controlled by the binding of glucocorticoid or PPRE to its promotor region [82]. Therefore, CREBH expression can be induced by a variety of PPAR $\alpha$ agonists such as palmitate and oleate [82]. ER-anchored CREBH becomes activated in response to hepatic lipid accumulation and VLDL assembly. The activation of CREBH requires ER-to-golgi trafficking followed by proteolytic cleavage and nuclear translocation $[81,83,84]$. CREBH activates a group of genes that are involved in TG and lipoprotein production $[85,86]$. CREBH also binds to and functions as a co-activator for both PPAR $\alpha$ and LXR $\alpha$ to promote FA uptake and utilization [86]. CREBH-deficient mice are susceptible to hepatic steatosis following fasting [81] or diets with high-fat content [79,86]. Interestingly, the livers of CREBH-deficient mice exhibit the reduced expression of genes that promote de novo lipogenesis and FA elongation [86]. Observed steatosis most likely arises from the reduced hepatic expression of genes involved in FA oxidation and increased lipolysis in the adipose tissue, resulting in an increased flow of FA from adipose tissue to the liver [79]. Furthermore, fibroblast growth factor (FGF) 21 is a critical CREBH target that reduces hepatic lipid storage. CREBH overexpression in the livers of mice suppresses hepatic lipid accumulation by increasing FGF21 secretion [87]. 


\subsection{FXR}

FXR is a major member of the ligand-activated nuclear receptor superfamily [78]. The family consists of four isoforms namely, FXR $\alpha 1$, FXR $\alpha 2$, FXR $\beta 1$, and FXR $\beta 2$ [88]. Similar to LXR, bile acids are natural ligands for FXR, which plays an important role in regulating bile acid homeostasis, glucose and lipid metabolism, intestinal bacterial growth, and hepatic regeneration [89]. While LXR facilitates the storage of carbohydrate- and fat-derived energy, FXR decreases TG levels and improves glucose metabolism [90]. One of the primary functions of FXR activation is the suppression of CYP7A1, the rate-limiting enzyme in bile acid synthesis from cholesterol [91]. FXR expression is decreased in NASH patients [25], which can aggravate the development of steatosis and NASH: (1) FXR activation represses hepatic lipogenesis via the FXR-SHP-SREBP1c pathway (see below for more on small heterodimer partner [SHP]), (2) FXR activation promotes $\beta$-oxidation by stimulating the expression of PPAR $\alpha$ and CPT1, and (3) FXR activation reduces hepatic FA uptake by reducing the expression of CD36 [89].

\subsection{STAT5}

STAT5 belongs to a family of intracellular transcription factors that are activated by membrane receptor-associated Janus kinases (JAK). The growth hormone (GH)-mediated activation of STAT5 [92] plays an important role in hepatic fat metabolism through the downregulation of CD36 [93]. The liver-specific loss of STAT5 in mice induces hepatic steatosis following a HFD [92]. These mice also exhibit hyperglycemia, hyperinsulinemia, hyperleptinemia, and elevated free FA and cholesterol concentrations following HFD. At the transcriptional stage, the loss of STAT5 results in the transcription of genes involved in lipid uptake (CD36), VLDL uptake (very low-density lipoprotein receptor), and lipogenesis (stearoyl-CoA desaturase and PPAR $\gamma$ ) [93]. However, it is unclear whether STAT5 directly regulates the expression of these factors. In addition, its relevance in human steatosis associated with GH-deficiency is yet to be established.

\subsection{0. $C / E B P \alpha$}

$\mathrm{C} / \mathrm{EBP} \alpha$ belongs to a transcription factor family of six members which are involved in a variety of cellular responses [94]. C/EBP $\alpha$ plays a role in lipogenic gene expression by inducing the expression of $\operatorname{PPAR} \gamma$ [95]. The liver-specific ablation of C/EBP $\alpha$ reduces lipogenic gene expression and triglycerides in the livers of leptin-deficient $o b / o b$ mice, which otherwise display severe steatosis [95]. These findings were confirmed by a similar observation of reduced hepatic gene expression following siRNA-mediated inhibition of $\mathrm{C} / \mathrm{EBP} \alpha$ expression in the livers of leptin receptor-deficient $(d b / d b)$ mice [27].

\section{Glucose Metabolism}

The liver does not only play a central role in systemic lipid homeostasis but also regulates the glucose balance in circulation. This is mediated by the activation of carbohydrate-responsive element-binding protein (ChREBP) in response to increases in plasma glucose and the nuclear localization of PPAR $\gamma$ coactivator 1 alpha (PGC1 $\alpha$ ), cAMP response element binding protein (Creb), CREBH, forkhead protein O1 (FOXO1), and hepatocyte nuclear factor $4 \alpha$ (HNF4 $\alpha$ ) in response to fasting to promote hepatic glucose production [96] (Table 1). Furthermore, PPAR $\delta$ also plays a role in glucose homeostasis. The exacerbation of hepatic glucose production coupled with hyperglycemia and insulin resistance play an important pathogenic role in NAFLD.

\subsection{ChREBP}

ChREBP consists of ChREBP $\alpha$, the full-length isoform, or ChREBP- $\beta$, the truncated isoform [97]. $\mathrm{ChREBP} \alpha$ is directly activated by glucose, independently from insulin signaling [98]. Little is known about ChREBP $\beta$, which was reported to be expressed in a glucose- and ChREBP-dependent manner whereby glucose-activated ChREBP $\alpha$ initiates ChREBP $\beta$ transcription from an alternate promoter [99]. 
In the liver, ChREBP promotes glycolysis and lipogenesis. ChREBP expression is increased in the livers of NASH patients with advanced steatosis [100]. By contrast, decreased ChREBP expression is associated with severe insulin resistance [22]. This pattern indicates that ChREBP is essential for the storage of excess glucose as triglycerides. In fact, mice that overexpress ChREBP exhibit improved insulin sensitivity and glucose tolerance despite having more pronounced hepatic steatosis. Together, these studies have demonstrated that increased ChREBP activity improves insulin sensitivity by promoting simple steatosis without lipotoxicity [22].

\subsection{PGC1a}

The PGC1 family of transcriptional co-activators play a central role in the regulation of metabolism. The PGC1 family consists of three members, namely PGC1 $\alpha$, PGC1 $\beta$, and the PGC-related co-activator (PRC), which interact with transcription factors and nuclear receptors to exert their biological functions. PGC1 $\alpha$ expression is induced by metabolic cues such as exercise, cold, and fasting [101]. The activation of PGC1 $\alpha$ in the liver drives the expression of genes that are essential to gluconeogenesis, FA oxidation, lipid transport, and mitochondrial biogenesis. The activity of PGC1 $\alpha$ becomes impaired in the setting of liver injury and steatosis in mice, and the loss of PGC1 $\alpha$ has been linked to the increased susceptibility to NAFLD in HFD-fed mice [102]. PGC1 $\alpha$ haploinsufficiency in mouse liver inhibits $\beta$-oxidation and increases triglyceride synthesis, leading to hepatic steatosis and insulin resistance. Similarly, PGC1 $\alpha$ overexpression in rat hepatocytes results in reduced concentrations of hepatic triglycerides in vitro and in vivo, due to increased $\beta$-oxidation [28].

\subsection{CREB}

CREB becomes activated in response to glucagon-mediated increases in cellular cAMP. The knockdown of CREB dramatically reduces fasting plasma glucose concentrations in several rodent models for obesity and type 2 diabetes, including Zucker diabetic fatty (ZDF) rats, STZ-treated/HFD-fed rats, and $o b / o b$ mice. CREB does not only promote the expression of gluconeogenic genes but also increases plasma TG and cholesterol concentrations as well as hepatic steatosis by activating de novo lipogenesis in the liver [103].

\subsection{CREBH}

CREBH was reported to bind and upregulate genes that contain cAMP-responsive elements, including phosphoenolpyruvate carboxykinase 1 (Pck1) and glucose-6-phosphatase (G6Pase) [80,81], which are essential promoters of gluconeogenesis. CREBH also upregulates the rate-limiting enzyme for hepatic glycogenolysis, namely glycogen phosphorylase (Pygl) [81]. Consequently, CREBH overexpression in the livers of mice increases plasma glucose levels, while its knockdown reduces circulating glucose [81].

\subsection{FOXO}

The forkhead protein family comprises of more than 100 members in humans and are enumerated FOXA to FOXR based on their sequence similarity [104]. The members of the FOXO subfamily, which consists of FOXO1, FOXO3, FOXO4, and FOXO6, are regulated by insulin signaling whereby Akt-mediated phosphorylation sequesters FOXOs within the cytosol, inhibiting their transcriptional activity in the nucleus [105]. FOXO family members mediate the expression of genes that play a role in cell death, DNA repair, glucose, and energy metabolism [106]. Hepatic FOXO1 regulates the expression of both gluconeogenic and lipogenic genes. Under fasting conditions, FOXO1 drives the expression of gluconeogenic enzymes. In addition, FOXO1 induces the transcription of genes involved in the hepatic assembly of VLDL, reducing hepatic steatosis [106]. The genetic ablation of FOXO increases susceptibility to NAFLD and NASH in mice [105]. Specifically, the deletion of FOXO1/3 or FOXO1/3/4 genes in mouse livers leads to mild or moderate hepatic steatosis, even when mice are maintained on a regular chow diet [105]. Exposing the mice to HFD supplemented with cholesterol further exacerbates 
steatosis in FOXO1/3/4-deficient mice [105]. Conversely, the overexpression of a constitutively active FOXO1 reduces hepatic triglycerides [105]. On the other hand, livers of NASH patients exhibit a greater expression of FOXO1 compared to patients with simple steatosis as well as metabolically healthy patients with and without obesity [29]. More investigation into FOXO1 activity during different stages of human liver disease is needed to establish mechanisms and physiological relevance.

\section{6. $H N F 4 \alpha$}

HNF $4 \alpha$ is a member of the nuclear hormone receptor superfamily and has been shown to play an essential role in maintaining bile acid, lipid, and glucose homeostasis. HNF4 $\alpha$ translates extracellular endocrine signals and intracellular stress and nutritional state onto transcriptional responses in the liver. HNF $4 \alpha$ is regulated by growth hormone, glucocorticoids, thyroid hormone, insulin, transforming growth factor beta (TGF $\beta$ ), estrogen, and cytokines [107]. HNF4 $\alpha$ target genes have been identified in the liver, pancreas, and colon. In the liver, the targets include genes involved in glucose (PEPCK, glucose-6-phosphatase (G6Pase)), bile (CYP7A1), xenobiotics and drug metabolism (CYP3A4, CYP2D6, and CYP2E1) [108]. HNF4 $\alpha$ also regulates circulating levels of cholesterol and triglycerides by inducing the transcription of genes that encode for apolipoproteins. HFD-induced oxidative stress promotes hepatic steatosis by blocking the activity of HNF4 $\alpha$ in mice [109]. The expression of HNF4 $\alpha$ is decreased in NASH patients [25]. Furthermore, a systematic integrative analysis of gene transcription has identified $\mathrm{HNF} 4 \alpha$ as 'the central gene' in the NASH pathogenesis [25].

\subsection{PPARS}

In addition to its activation of fatty acid oxidation, PPAR $\delta$ improves glucose homeostasis and protects from insulin resistance by promoting insulin secretion in the pancreatic islet $\beta$-cells $[110,111]$ and by increasing energy utilization [112]. Mice lacking PPAR $\delta$ expression have reduced energy expenditure and are glucose-intolerant. In contrast, receptor activation by GW50516, a PPAR $\delta$-specific agonist, suppresses hepatic glucose output, improves insulin sensitivity and increases glucose disposal in mice [112]. This increase in energy disposal has been linked to increased $\beta$-oxidation in the skeletal muscle of mice following GW50516 treatment [113].

\section{Inflammation}

The hepatic inflammatory response is an important driving force for NASH progression as it promotes sustained hepatic fibrogenesis. Transcription factors activated in response to inflammatory stimuli mainly belong to the family of nuclear factor of the $k$ light chain enhancer of B cells (NF- $k B s$ ), interferon regulatory factors (IRFs), STAT, and activator protein 1 (AP-1) [114]. Other factors that have also been implicated in the transcriptional regulation of the inflammatory response include apoptosis antagonizing transcription factor (AATF, synonym: che-1), SHP, Runt-related transcription factor 2 (Runx2), and C/EBP $\beta$. In addition to inflammation-specific regulators, transcriptional regulators of lipid homeostasis PPAR $\alpha, \operatorname{PPAR} \gamma, \mathrm{CAR}$, and LXR also affect the hepatic inflammatory state (Table 1).

\section{1. $N F-\kappa B$}

$\mathrm{NF}-\mathrm{KB}$ is a protein complex that controls cytokine production and cell survival, and as such, it plays a key role in the immune response to infection. NF- $\mathrm{kB}$ is also critical for the development of inflammation in various metabolic disorders such as T2DM [115] and is highly activated in both mice and patients with NASH $[30,116]$. The pharmacological inhibition of NF- $\mathrm{KB}$ signaling protects MCD-fed mice from the pathogenesis of NASH with significant reductions in hepatocellular injury and hepatic inflammation. Furthermore, the stage of inflammation and fibrosis in livers of NASH patients correlates with the expression of the $\mathrm{p} 65$ subunit of NF-kB [117]. 


\subsection{IRFS}

IRFs are a family of transcription factors that regulate the transcription of interferons and consist of nine members. Most IRFs are involved in innate immunity and defense against pathogens. IRF family members impose variable impacts on inflammation in the pathogenesis of NAFLD. Studies using mice with the deletion of IRF7 expression indicated that IRF7 promotes weight gain, hepatic fat deposition, and insulin resistance in the setting of HFD [118]. In contrast, a similar study using IRF9-deficient mice demonstrated that IRF9 promotes insulin sensitivity and attenuates inflammation and hepatic steatosis [119]. Interestingly, IRF9 was shown to interact with PPAR $\alpha$ and activate its target genes [119].

\subsection{STAT}

STAT family members with inflammatory biological functions (STAT1 and STAT3) have been associated with NAFLD and NASH. The oxidative hepatic environment in obesity inhibits the STAT1 and STAT3 phosphatase, T cell protein tyro-sine phosphatase (TCPTP), which results in increased STAT1 and STAT3 signaling. This in turn increases the risk of developing NASH and HCC in the setting of excessive nutrition [32]. Furthermore, the inactivation of TCPTP, coupled with increased STAT1 and STAT3 signaling, are easily detectable events in the livers of humans with NASH [32].

\subsection{AP1}

AP1 activation requires the synthesis of c-Jun and c-Fos proteins and c-Jun phosphorylation by c-Jun N-terminal kinase (JNK) for the full transactivation of target genes. Obese patients with NASH exhibit an enhanced hepatic expression of AP1 targets [30]. JNK activation and the extent of c-Jun nuclear localization correlates very well with the pathogenesis and progression of NASH in humans and mouse models [33]. Activated c-Jun promotes nuclear accumulation of JNK, which provides a positive feedback loop to further enhance AP1 transcriptional activity and exacerbate NASH progression [120,121].

\subsection{AATF}

AATF mediates cell proliferation and survival [122-124]. Its expression in the liver increases with simple steatosis [44]. Indicative of a role in inflammation, AATF expression increases in response to tumor necrosis factor $\alpha(\mathrm{TNF} \alpha)$-mediated activation of SREBP1 in cultured cells. In turn, AATF induces the expression of the inflammatory cytokine monocyte chemotactic protein 1 (MCP1) by activating STAT3. Hepatic AATF expression does not increase any further with disease progression to NASH [44], suggesting that it plays a role in exacerbating simple steatosis toward the pathogenesis of inflammatory stages of steatohepatitis. However, its contribution to the progression of NASH to advanced stages remains unclear.

\subsection{SHP}

SHP is technically not a transcription factor, since it lacks a DNA binding domain but is still classified as such due to its sequence homology to other nuclear receptor families. The principal role of SHP is the repression of other nuclear receptors by binding and forming a dysfunctional heterodimer. SHP is a critical repressor of various genes involved in glucose and lipid metabolism and bile acid synthesis [125]. Several factors indicate a role in inflammation: first, SHP inhibits inflammatory responses that are triggered by the Toll-like receptor (TLR) [126] as well as the NLR family pyrin domain containing 3 (NLRP3) inflammasome, which consists of a multimeric protein complex that triggers inflammatory cell death and the release of pro-inflammatory cytokines interleukin (IL)-1 $\beta$ and IL-18 [127]. In addition, SHP suppresses inflammation by inhibiting transcription of the chemokine CCL2 whose biological function is to recruit macrophages and promote inflammation [34]. The SHP-mediated mitigation of inflammatory responses could play a protective role in NASH. SHP expression is drastically decreased in the livers of a mouse model of NASH and in the livers of 
NASH patients compared healthy or steatotic livers [34]. The rescue of SHP expression in the livers of mice prevents the progression of NAFLD to NASH [34]. Mechanistically, the reduction of SHP expression in NASH was linked to inhibitory binding of c-Jun to the SHP promoter, suggesting that the JNK/SHP/NF-KB/CCL2 axis is a promising target for NASH prevention and treatment.

\subsection{Runx2}

Runx2 plays an important role in atherosclerosis. It has been indicated that atherosclerosis shares a similar histopathology with NASH with respect to macrophage infiltration. Indeed, experiments in mouse primary HSCs have elucidated a mechanism whereby Runx 2 within HSCs promotes macrophage infiltration by increasing the transcription of MCP1 [36].

\section{8. $C / E B P \beta$}

$\mathrm{C} / \mathrm{EBP} \beta$ was originally identified as nuclear factor interleukin-6 (NFIL6) because of its inducibility by IL- 6 and its important role in the activation of acute inflammatory response genes in human hepatoma cells [128]. The livers of mice lacking C/EBP $\beta$ express reduced markers of inflammation and endoplasmic reticulum (ER) stress and exhibit decreased steatosis following an MCD diet. By contrast, C/EBP $\beta$ overexpression increases the hepatic prevalence of PPAR $\gamma$, ER stress, NF- $\mathrm{KB}$ activation, and steatosis [27].

\subsection{PPAR $\alpha$}

In addition to its role in the regulation of metabolism, PPAR $\alpha$ also exhibits anti-inflammatory effects through its regulation of NF-KB [129]. The treatment of non-steatotic mice with the PPAR $\alpha$ agonist WY-14643 decreases the hepatic inflammatory gene expression profile, suggesting a direct anti-inflammatory effect of PPAR $\alpha$ independent of changes in liver triglycerides [130].

\subsection{PPAR $\gamma$}

Hepatic PPAR $\gamma$ does not only regulate hepatocyte metabolism but also plays an important regulatory role in liver-resident macrophages (Kupffer cells), where it acts as an inhibitor of macrophage activation and cytokine production. This regulation is mediated through the PPAR $\gamma 1$-mediated inhibition of AP-1, STAT, and NF- $\mathrm{KB}$, which are the major regulators of macrophage activation and TNF $\alpha$ synthesis [131]. Mice with Kupffer cell-specific loss of PPAR $\gamma$ expression exhibit increased hepatic expression of inflammatory cytokines TNF $\alpha$ and IL1 $\beta$ and fibrosis in response to $\mathrm{CCl}_{4}$-induced liver injury [132]. Conversely, PPAR $\gamma$ induction by rosiglitazone decreases the number of hepatic Kupffer cells, attenuating the inflammatory response as well as steatosis in a diet-induced mouse model of NAFLD [133].

\subsection{CAR}

CAR activation can potentially be used to delay or reduce the progression of NAFLD due to its dual anti-steatotic and anti-inflammatory effects. In the MCD mouse model of NASH, the administration of the CAR agonist 2,2'-[1,4-phenylenebis(oxy)]bis[3,5-dichloro]-pyridine (TCPOBOP) reduces inflammation and hepatocellular apoptosis by reducing the accumulation of Kupffer cells and enhancing the hepatic clearance of pro-inflammatory leukotriene B4 [134]. On the other hand, CAR knockout mice exhibit improved lipid peroxidation and hepatic fibrosis after exposure to the MCD diet [135]. Therefore, the precise role of CAR in the pathophysiology of NASH requires additional studies.

\subsection{2. $L X R$}

Although LXR promotes inflammation, its impact on obesity and steatosis is inconsistent. Mouse models with the deletion of LXR expression have indicated that LXR decreases inflammation by inhibiting the transcription of TNF $\alpha$, IL-6, and IL-1 $\beta$ but increases steatosis [136]. On the other 
hand, the treatment of mice with the LXR antagonist SR9238 generates both anti-steatotic [137] and anti-fibrotic effects [138] with a dramatic reduction in steatosis, inflammation, and collagen disposition in the livers of mice. Overall, all studies indicate that LXR could be a valuable target for the treatment and prevention of NASH.

\section{Metabolic Stress}

The pathogenesis of NAFLD does not only depend on energy metabolism and inflammation but has also been mechanistically linked to increased cellular stress. Upon excessive nutrition, the ER cannot meet high metabolic demands and initiates the unfolded protein response (UPR) by activating three transmembrane factors located on the ER membrane: protein kinase R-like ER kinase (PERK), inositol-requiring enzyme $1 \alpha$ (IRE1 $\alpha$ ), and activating transcription factor 6 (ATF6). PERK activates eukaryotic translation initiation factor $2 \alpha$ (eIF2 $\alpha$ ), which in turn activates ATF4. Meanwhile, IRE1 $\alpha$ splices the mRNA of X box-binding protein 1 (Xbp1) to its active isoform Xbp1s. ATF4, ATF6, and Xbp1 together initiate transcriptional events to resolve ER stress. However, excessive reactive oxygen species (ROS) and ER stress due to excessive lipid accumulation in the liver can lead to inflammation and hepatocyte death [139]. For instance, increased CYP2E1 expression promotes ROS production and the progression of NAFLD. In contrast, stress-induced activation of nuclear erythroid 2-related factor 2 (NRF2) protects against oxidative stress and the pathogenesis of NAFLD [140]. Furthermore, unresolved ER stress results in the activation of apoptotic transcription factor CCAAT/enhancer binding protein $(\mathrm{CHOP})$. The prolonged activation of IRE1 $\alpha$ also leads to the activation of the inflammatory transcription factors c-Jun and NF-kB. Thus, the transcription factors involved in stress-induced responses may contribute to the development of NASH (Table 1).

\section{1. $\mathrm{Xbp1}$}

High caloric stress leads to the splicing and nuclear localization of Xbp1, which in turn transcribes factors that improve protein folding as well as lipogenesis [38]. However, obesity-induced chronic stress limits the nuclear localization of Xbp1 and aggravates ER stress and insulin resistance [141]. The rescue of Xbp1 activity in HFD-fed or $o b / o b$ mice improves glucose homeostasis and reduces hepatic steatosis, which is associated with reductions in the expression of lipogenic genes [142]. Whether the improvement of hepatic steatosis in these mouse models is a direct outcome of transcriptional regulation by $\mathrm{Xbp} 1$ or a secondary consequence of resolved metabolic stress and improved insulin sensitivity remains unclear. Nonetheless, the lipogenic role of $\mathrm{Xbp} 1$ is demonstrated using a mouse model with a liver-specific ablation of Xbp1 following WD, whereby the loss of Xbp1 is associated with reduced steatosis but enhanced liver injury and fibrosis with the upregulation of type-I collagen $\alpha 1$ (Col $\alpha 1)$, TGF $\beta 1$, CHOP, and p-JNK $[38,143]$.

\subsection{ATF4}

In NASH patients, the mRNA expression of ATF4 and $\mathrm{CHOP}$ and protein expression of $\mathrm{CHOP}$ are significantly elevated compared to liver samples from patients with simple steatosis [40]. ATF4 depletion protects mice from high fructose-induced hepatic steatosis by reducing lipogenesis through the reduced hepatic expression of PPAR $\gamma$, SREBP1c, ACC, and FASN [144].

\subsection{ATF6}

Hepatic ATF6 knockdown or overexpression of its dominant-negative form by adenovirus in WD-fed mice exacerbates insulin resistance and hepatic steatosis with reduced transcriptional activity of the PPAR $\alpha /$ RXR complex. Conversely, overexpression of the cleaved active from of ATF6 protects mice from hepatic steatosis and promotes hepatic FA oxidation. Experiments in hepatocytes have shown that ATF6 promotes hepatic FA oxidation by enhancing PPAR $\alpha$ transcriptional activity through direct interaction and activates its downstream targets such as carnitine palmitoyltransferase 1 alpha 
(CPT1 $\alpha$ ) and medium-chain acyl-CoA dehydrogenase (MCAD) [145]. Activated ATF6 also interacts with SREBP2 and inhibits SREBP2 target genes in hepatocytes [146].

\subsection{NRF2}

NRF2 is the primary driver of gene expression via the antioxidative response elements (ARE). In response to oxidative damage such as lipid peroxidation and DNA damage, NRF2 increases the transcription of antioxidative factors, including $[140,147]$ NRF2, which suppresses inflammation by preventing the increased transcription of pro-inflammatory cytokines [140]. Specifically, NRF2 interferes with the lipopolysaccharide-induced transcriptional upregulation of IL- 6 and IL- $1 \beta$. Accumulating evidence supports a protective role of NRF2 in NASH [148]. In rats and mice with diet-induced NASH, NRF2 activation improves glucose homeostasis and inhibits hepatic steatosis, inflammation, and fibrosis by decreasing lipid synthesis and upregulating $\beta$-oxidation and lipoprotein assembly [35,149]. In contrast, the loss of NRF2 exacerbates hepatic steatosis and accelerates the development of NASH in mice fed an HFD or MCD [150,151]. Mechanistically, the oxidative stress due to the deletion of NRF2 in these mice activates NF- $\mathrm{kB}$ and leads to the upregulation of the inflammatory cytokines IL- 6 and TNF $\alpha$.

\subsection{CYP2E1}

Although it is not a transcription factor, it is important to include CYP2E1, which becomes activated following insulin resistance and lipotoxicity [152] and promotes ROS production in the setting of NAFLD [153]. CYP2E1 plays key metabolic roles in gluconeogenesis and fatty acid metabolism. It controls the formation of lactate or glucose from the ketone body acetone [154]. Furthermore, CYP2E1 carries out the omega hydroxylation of fatty acids, increasing lipotoxicity and inflammation [155], which represent major pathophysiological mechanisms in NAFLD progression [156]. The role of CYP2E1 in liver injury was first identified following the alcohol-induced induction of CYP2E1 protein. However, clear differences exist between alcoholic liver disease (ALD)- and NAFLD-induced activation of CYP2E1: while alcohol consumption only stabilizes the CYP2E1 protein without changes in mRNA expression, excessive nutrition increases both protein stability and mRNA abundance [157]. Although the transcriptional regulation of CYP2E1 has been linked to the activities of HNF1 $\alpha$ [158], HNF4 $\alpha$ [108], SP1 [159], and C/EBP [154], the mechanisms by which obesity and NAFLD exacerbate CYP2E1 activity requires additional studies [153].

\section{Fibrosis}

Fibrosis is the strongest predictor of adverse clinical outcomes for NASH. Fibrogenesis during liver injury is initiated by the activation of HSCs in the liver $[160,161]$. Established inducers of fibrogenesis and HSC activation include adipocyte enhancer binding protein 1 (AEBP1), AATF, yes-associated protein (YAP), and transforming growth factor beta-(TGF $\beta$ )-mediated activation of transcription factors against decapentaplegic homolog (SMAD). In addition to these fibrosis-specific regulators, the main transcriptional regulators of lipid homeostasis (including PPAR $\alpha$ and PPAR $\gamma$ ) and inflammation (RUNX2 and c-Jun) have also been reported to dictate the fibrotic stage in NASH (Table 1).

\section{1. $T G F \beta / S M A D$ axis}

TGF $\beta$ is secreted from activated HSC and is a potent inducer of fibrogenesis. Its pro-fibrogenic effect is mainly mediated by the TGF $\beta$ receptor (TGF $\beta$ R)-dependent activation of the SMAD family in HSC: the phosphorylated SMAD2/3 complex binds to SMAD4 and translocates to the nucleus to promote the transcription of fibrogenic genes including $\operatorname{Co} 1 \alpha 1$, $\operatorname{Co} 3 \alpha 1$, smooth muscle alpha 2 actin $(\alpha \mathrm{SMA})$, and TGF $\beta$ as well as the production of tissue inhibitor of metalloproteinases (TIMPs) [162], which promote fibrosis by inhibiting matrix degradation [163]. In contrast, Smad7 inhibits the regulation of the TGF $\beta$ signaling by recruiting ubiquitin E3 ligases that promote the degradation of TGF $\beta R 1$ and by recruiting the protein phosphatase PP1C, which inactivates TGF $\beta R 1$ [164]. The livers of NASH patients as well as a mouse model of NASH exhibit increased nuclear localization of the 
SMAD2/3/4 complex and the reduced expression of SMAD7, which all together contribute to increased TGF $\beta$, Co1 $\alpha 1$, and $\alpha$ SMA [42]. The regulation of SMAD2/3 has also highlighted the role of additional transcription factors in mediating TGF $\beta$-mediated fibrogenesis: the interactions of the transcriptional coactivators CREB binding protein (CBP) and p300 with SMAD2/3 promotes histone acetylation and increased transcriptional activity [165]. Supporting the pathophysiological relevance of this axis, the AMPK-mediated degradation of p300 results in the inhibition of TGF $\beta / S M A D 3-$ mediated fibrogenesis in HSC [166]. Finally, the transcription factor v-ets avian erythroblastosis virus E26 oncogene homolog 1 (ETS1), which is elevated in a NASH mouse model, enhances TGF $3 /$ SMAD signaling by directly binding to SMAD3 and preventing its ubiquitination and degradation [167].

\subsection{AEBP1}

AEBP1 plays a role in adipogenesis [168,169], myofibroblast differentiation [170], and macrophage cholesterol homeostasis [171]. AEBP1 was identified as a key transcription factor during the transition from simple steatosis to NASH using a co-regulatory network approach, which assessed AEBP1 expression in NASH fibrosis versus other NAFLD histological classes using pairwise comparisons [172]. In support of this database analysis, AEBP1 expression increases in the setting of NASH compared to simple steatosis in the livers of $A p o E^{+/-}$mice. A recent clinical study demonstrated that AEBP1 is specifically expressed in HSC and at a greater extent in the livers of patients with NASH [43]. The ablation of AEBP1 only in the HSC of mice protects against high fat and high cholesterol diet-induced fibrosis. Mechanistically, AEBP1 activates Wnt signaling by specifically binding frizzled-8 and low-density lipoprotein-related receptor 6, which blocks the PPAR $\gamma$-dependent inhibition of activated HSC. Another study confirmed that hepatic AEBP1 is directly associated with the degree of steatosis, lobular inflammation, and fibrosis in NASH patients [168]. This study also found that AEBP1 upregulates the expression of genes identified as part of an algorithm-predicted AEBP1-associated NASH co-regulatory network [168]. These target genes include the regulators of fibrosis (AKR1B10, CCDC80, DPT, EFEMP1, ITGBL1, LAMC3, MOXD1, SPP1, and STMN2), ECM production and maintenance (COL4A2 and MARCO), and myofibroblast transition (ACTA2, COL1A1, COL1A2, SERPINE1 and PLAU). Taken together, these findings strongly implicate AEBP1 in the diagnosis and treatment of NASH.

\subsection{YAP}

The Hippo pathway and its effector YAP are particularly important for controlling liver size by regulating proliferation and growth [173]. The expression of YAP is barely detectable in healthy livers of humans and mice but becomes activated in the setting of NASH [45]. YAP is expressed in hepatocytes and activates the expression of proteins that promote fibrosis (ColL1 $\alpha 1$, TIMP1, TGF $\beta 2$ ) and inflammation (TNF $\alpha, \mathrm{IL}-1 \beta)$, which stimulate the expansion of myofibroblasts and the recruitment of immune cells, exacerbating liver fibrosis [174]. YAP is also activated in Kupffer cells by the lipopolysaccharides (LPS)/TLR4 signaling pathway, where it promotes the development of NASH by enhancing the production of pro-inflammatory cytokines [175]. Further gain and loss of function experiments have shown that the activation of the YAP/transcriptional co-activator with PDZ-binding motif (TAZ) axis leads to the expression of a key matricellular chemokine (CYR61), which stimulates and recruits extrahepatic macrophages to promote liver fibrosis.

\subsection{PPAR $\alpha$}

In addition to beneficial effects on steatosis and inflammation, PPAR $\alpha$ agonist treatment also reverses fibrosis by targeting PPAR $\alpha$ in HSC, which decreases the expression of fibrogenic factors including Col1 $\alpha 1$ and TIMPs and reduces the number of activated HSC. The protective effect of PPAR $\alpha$ was further demonstrated by treating fibrotic $\mathrm{APOE}_{2} \mathrm{KI} 1811 \mathrm{~A}$ mice with the PPAR $\alpha$ agonist fenofibrate, which protected mice from NASH by reducing both steatosis and hepatic macrophage 
accumulation [10]. By contrast, mice with a genetic ablation of PPAR $\alpha$ display increased susceptibility to NASH $[130,176]$.

\subsection{PPAR $\gamma$}

In humans, growth factors activate HSC that display decreased PPAR $\gamma$ expression during the progression of NAFLD to NASH [160]. On the other hand, livers with simple steatosis exhibit increased PPAR $\gamma$ expression. The treatment of rats with NASH with the PPAR $\gamma$ agonist pioglitazone prevents hepatic fibrosis and reduces the expression of TIMPs [177]. Indicating that the inhibition of PPAR $\gamma$ in HSC is responsible for the increased transcription of TIMPs, the overexpression of PPAR $\gamma$ reduces the expression of TIMP1, TIMP2, and alpha smooth muscle actin ( $\alpha$ SMA) and reverses hepatic fibrosis. By contrast, the HSC-specific ablation of PPAR $\gamma$ aggravates $\mathrm{CCl}_{4}$-induced liver fibrosis and increases $\alpha$ SMA expression [132]. Collectively, these findings clearly link decreased PPAR $\gamma$ activity in HSC to hepatic fibrosis. Accordingly, pioglitazone ameliorates only moderate pericentrilobular fibrosis in rats with no effect on severe bridging fibrosis, which is most likely due to the reduced PPAR $\gamma$ availability for pioglitazone to target under the advanced stages of the disease [178]. On the other hand, the effect of TZDs on fibrosis in humans have been less clear. Unlike in rats, a meta-analysis of TZD effects from eight randomized trials $(n=516)$ on NASH-associated liver fibrosis found pioglitazone to significantly improve fibrosis, particularly in the advanced fibrosis stage with bridging fibrosis and cirrhosis compared to NASH with mild perisinusoidal/periportal fibrosis [179]. This effect could have been independent of $\operatorname{PPAR} \gamma$, as TZDs can bind alternative targets such as the mitochondrial pyruvate carrier [179]. In fact, the inhibition of the mitochondrial pyruvate carrier by a next-generation TZD (MSDC0602) was found to reverse hepatic fibrosis in mice, supporting the mitochondria pyruvate carrier as a relevant treatment target $[180,181]$. Nonetheless, the relevance of targeting PPAR $\gamma$ for the treatment of advanced fibrosis in humans remains unclear.

\subsection{RUNX2}

Studies have shown that Runx2 acts as a fibrogenic or tumorigenic transcription factor in hepatic fibrosis or hepatocellular carcinoma [182,183]. Runx2 is expressed in the non-parenchymal cells of the liver but not in the hepatocytes. In a mouse model of NAFLD/NASH, Runx2 becomes upregulated in the HSCs during the development of NAFLD [163].

\section{7. c-Jun}

The impact of c-Jun on fibrogenesis depends on the liver cell type. The deletion of c-Jun only in hepatocytes reduces steatosis but increases fibrosis, whereas its deletion in both hepatocytes and non-parenchymal cells protects against MCD-induced fibrosis in mice [142]. This was linked to reductions in the pro-inflammatory cytokine osteopontin (Opn, also known as SPP1), which is an established marker of a regenerative response called the ductular reaction (DR), which is an essential driver of fibrogenesis. Additional investigations using $\mathrm{Opn}^{-/-}$mice established that c-Jun expression in NPLC promotes NASH-related DR and subsequent fibrosis by upregulating Opn expression [39,184].

\section{Microbiome Dysbiosis}

The contribution of obesity-induced changes in the gut microbiome to the pathogenesis and progression of NAFLD [185] was initially established using germ-free mice and fecal transplant from lean [186] and diet-induced obese mice [187]. Furthermore, the inoculation of germ-free mice with the gut microbiota of obese humans [188] and NASH patients [189] leads to the onset of hepatic steatosis and NASH, respectively. These findings formed the base or microbiota-based therapies for NAFLD such as pre- and probiotics and fecal microbiota transplantation [190-192]. 
The gut microbiota can influence the progression of NAFLD through several pathways, which has been reviewed extensively elsewhere [193]. Briefly, these pathways include changes in gut permeability, low-grade inflammation and immune balance, the modulation of dietary choline and bile acid metabolism, and the production of endogenous substrates [186]. In this review, we highlight that the microbiota, through the production of endogenous substrates, may alter the transcriptional profile of the liver. Major metabolites that are linked to alterations in the gut microbiota include bile acids [194-196], short-chain fatty acids (SCFA) [197] and lipopolysaccharides (LPS) [189]. These metabolites can play an important role in NAFLD progression by mediating the gut-liver axis [198]. Products derived from bile acid metabolism act on FXR to decrease hepatic triglyceride levels and improve glucose metabolism [90]. Specifically, the HFD-induced remodeling of the gut microbiota increases the production of bile salt hydrolase (BSH), which is a bacterial enzyme that hydrolyzes and inactivates tauro- $\beta$-muricholic acid (T- $\beta$-MCA) [199]. T- $\beta$-MCA inhibits intestinal FXR signaling, which suppresses ceramide synthesis [200]. Therefore, microbiome dysbiosis results in increased FXR signaling and ceramide production, which in turn promotes SREBP1c activity and steatosis in the liver [201].

SCFAs have been shown to increase the AMPK activity in liver and muscle tissue [202]. The activation of AMPK triggers PGC- $1 \alpha$ expression, which controls the transcriptional activity of PPAR $\alpha$, PPAR $\gamma$, PPAR $\delta$, LXR, and FXR, which are important transcriptional regulators of cholesterol, lipid, and glucose metabolism [203]. LPS has been shown to activate NF-KB in cultured hepatocytes [204], which plays a major role in the development of inflammation during NAFLD progression [91] and is highly activated in both mice and patients with NASH. Furthermore, LPS can induce MAP kinase kinase-3 (MKK3) activation, which in turn stimulates C/EBP $\beta$ and C/EBP $\delta$ binding elements to promote the transcription of CYP2E1 and induce oxidative stress [154].

\section{Prediction of Transcriptional Regulators by Database Analyses}

\subsection{Prognostic Biomarkers for Human NAFLD and NASH}

Many transcriptomic studies have been conducted to elucidate novel biomarkers for the different stages of NAFLD, including steatosis, ballooning, and fibrosis. To elucidate the transcriptional changes that are associated with human NAFLD, we procured publicly available human NAFLD/NASH transcriptome data from the Gene Expression Omnibus (GEO) and subjected them to Ingenuity Pathway Analysis for the prediction of changes in upstream factors (Table 2). Predictions were based on two GEO datasets with strong power analysis (Table 2) as well as a previously published Ingenuity Pathway Analysis (IPA)-based prediction analysis $[7,205,206]$. The activation of PPAR $\gamma$ was the only consistent prediction for simple steatosis, whereas the onset of fibrosis was associated with changes in a larger number of transcription factors, which were consistent in at least half of the datasets. These included the activation of inflammation (NF-kB, RELA, JUN, IRF1, IRF3, STAT1, SP1), glucose production (FOXO1), and lipogenesis (SREBP1), as well as the inhibition of PPAR $\alpha, \operatorname{PPAR} \gamma$, and RXR $\alpha$. The activation of C/EBP $\beta, C T N N B 1$, and SMAD3 and the inhibition of HNF4 $\alpha$ and SMAD7 were also associated with NASH and NASH-induced HCC, suggesting that these factors might contribute to the pathogenesis of advanced stage fibrosis. 
Metabolites 2020, 10, 283

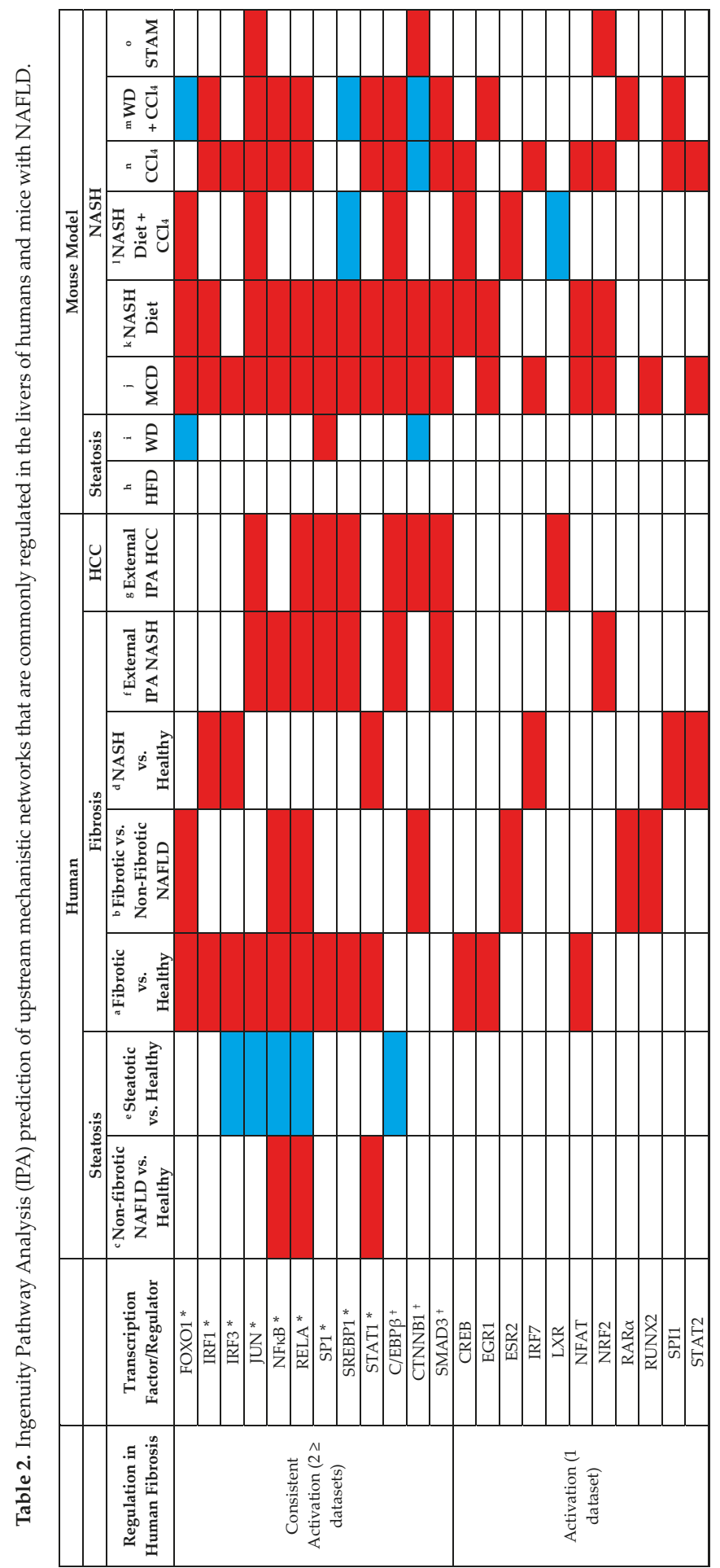




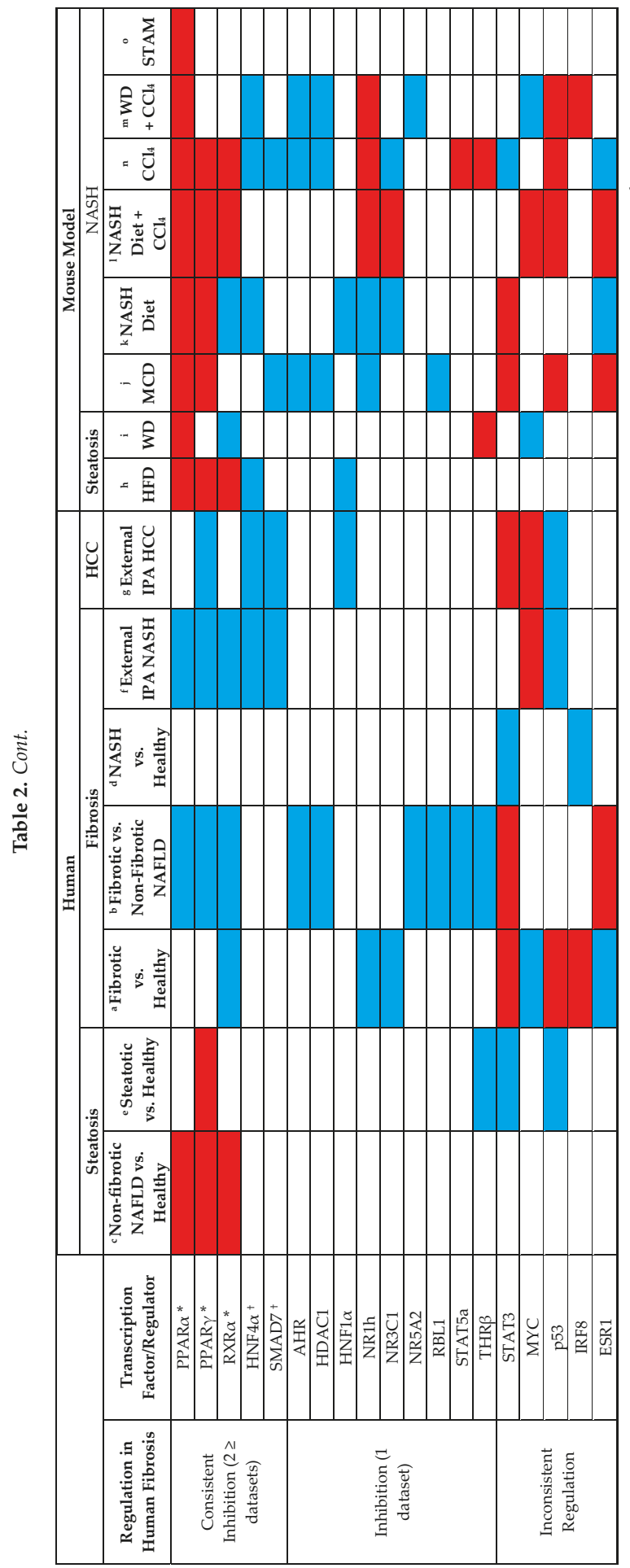

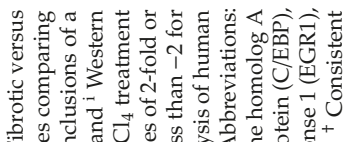
死 को

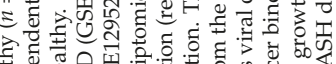
至 कृ

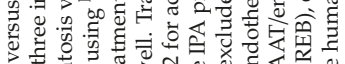
O. 付

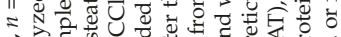

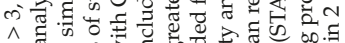

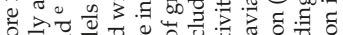

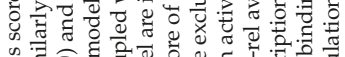

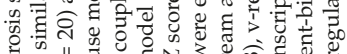

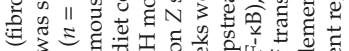

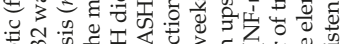

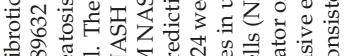

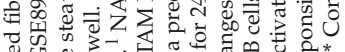

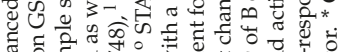

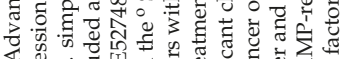

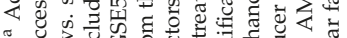

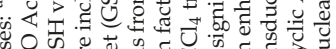

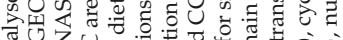

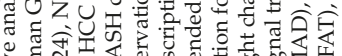

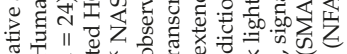

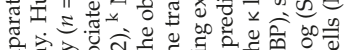
결

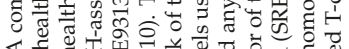

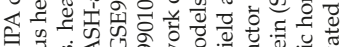
考 跑

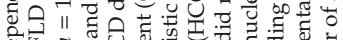

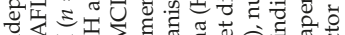

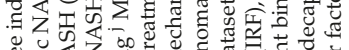

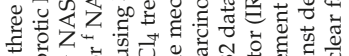

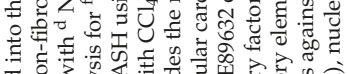

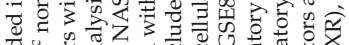
势

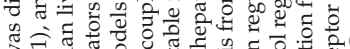

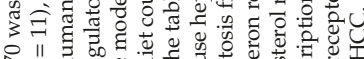
尺 II

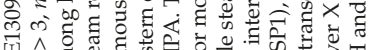

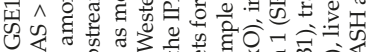

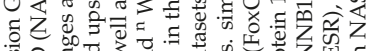

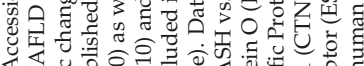

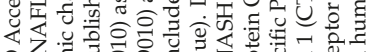

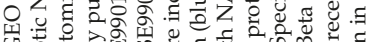

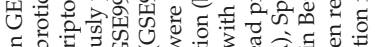

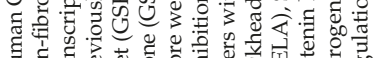

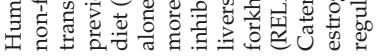




\subsection{Altered Transcription Factors in Mouse Models of NAFLD and NASH}

The same strategy was applied to predict the changes in the activity of transcription factors in mouse models of NAFLD/NASH. We performed IPA upstream activity prediction analysis for publicly available liver transcriptome data from mouse models of steatosis that were established by feeding high caloric diets $[18,207]$ as well as mouse models of NASH that were established by feeding an MCD diet [207], NASH diet, or a combination of high caloric diets coupled with $\mathrm{CCl}_{4}$ treatment $[18,208]$. Additionally, we included the observations of a previously published STZ-induced NASH and HCC model (STAM) [7]. Transgenic mouse models that involve genetic manipulations were excluded, since our comparative analysis was not aimed at delineating the transcriptional consequences of rare gene variants in humans and mice. As anticipated, the activation of most consistent pathways in humans (Table 2) were also confirmed in most mouse models. However, in contrast to the downregulation of $\operatorname{PPAR} \alpha, \operatorname{PPAR} \gamma$, and $\mathrm{RXR} \alpha$ in human livers with NASH, these pathways were upregulated in most mouse models of NASH (Table 2). The activity regulation of PPAR $\alpha$, PPAR $\gamma$, and RXR $\alpha$ in mouse models of NASH was instead more representative of their activity in human livers with simple steatosis (Table 2).

IPA analysis of the mouse models also identified pathways that were not predicted to be affected among human NASH datasets. Among these, the activation of SMAD2, SMAD4, YAP1, NOTCH1, EP300, p63, and the inhibition of nuclear receptor corepressor (NCOR) has been previously linked to human NASH by independent studies (Table 3) $[42,166,173,209-212]$. Therefore, most mouse models mimic the transcriptional signature of human NASH for these transcription factors. However, the consistent inhibition of SREBP2 and the activation of fos proto-oncogene (FOS) and PGC1 $\alpha$ in mouse NASH models mimicked human steatosis but were absent in the setting of human fibrosis (Table 3) $[102,213,214]$. Furthermore, increased FOS mRNA in fibrotic versus non-fibrotic NAFLD patients was in line with increased EGR1 activity, which promotes FOS expression, as previously published (Table 2). However, IPA did not predict FOS activation, as this would be anticipated to inhibit NF- $\mathrm{KB}$ and SP1 and activate CEBBP, which was in contrast with the regulation in fibrotic livers (Table 2). Other frequently altered transcriptional mechanisms in mouse NASH models, which were not previously associated with human NASH, included PPAR $\delta, H I F 1 \alpha$, MED1, NCOA1, NCOA2, SMARCA4, FOXO3, HDAC2, STAT5b, and STAT6 (Table 4). Individual transcriptomic datasets from mouse livers also predicted the regulation of unique pathways for each dataset, which were not predicted to be regulated in other mouse models (Table 4). Since IPA prediction of upstream factors in human NASH also failed to identify changes in the activity of these sets of transcription factors in Tables 3 and 4, studying their relevance in the pathogenesis of NASH in humans would be beneficial prior to investigating their roles in pre-clinical rodent models of NASH. It is worth mentioning that the activation of ChREBP and C/EBP $\alpha$ were only confirmed in the mouse models using MCD and WD coupled with $\mathrm{CCl}_{4}$, respectively, but they were not detected in human fibrosis datasets. Another category of altered transcription factors belonged to those that were altered in a single human dataset but not in any of the mouse models (Table 5). Although the activity regulation of these factors could be relevant in the pathogenesis of NASH, corroborating evidence is lacking.

To determine how the transcriptional activity of popular NASH mouse models faired against human NASH, we implemented a scoring strategy ranging from +2 to -2 for each transcription factor: +2 for the confirmation of a transcriptional activity in a mouse model, which was observed in more than one human NASH dataset (i.e., NF- $\mathrm{kB}$ ); -2 for the reversal of a transcriptional activity, which was observed in more than one human NASH dataset (i.e., PPAR $\gamma$ ); +1 for the confirmation of a transcriptional activity, which was observed only in one human NASH dataset (i.e., SMAD7); -1 for the reversal of a transcriptional activity, which was observed only in one human NASH dataset (i.e., THR $\beta$ for $\mathrm{CCl}_{4}$ model); -1 when multiple confirmations for a transcriptional activity among human datasets remained unchanged in a mouse model (i.e., NF-kB for NASH diet $+\mathrm{CCl}_{4}$ model); 0 for a lack of transcriptional activity in a mouse model, which was also observed in some of the human NASH datasets (i.e., $\mathrm{THRb}$ for all models except $\mathrm{CCl}_{4}$ ); and 0 when a transcriptional activity was predicted 
to be inconsistently regulated among different human NASH datasets regardless of the state of the activity of that transcription factor within the mouse models (i.e., STAT3). From a possible maximum score of +47 for all the common transcription factors (Table 2), the NASH diet, MCD diet, NASH diet + $\mathrm{CCl}_{4}, \mathrm{CCl}_{4}$ alone, and $\mathrm{WD}+\mathrm{CCl}_{4}$ netted total scores of 23, 27,-10, 12 and 7 , respectively, suggesting that the NASH diet and MCD diet exhibit transcriptional activity profiles that are more representative of human $\mathrm{NASH}$, whereas the models that involved $\mathrm{CCl}_{4}$ treatment did not. We excluded the STAM mouse model due to the low number of predicted matches.

Table 3. IPA prediction of upstream regulators that were detected in the livers of mouse models of NASH but not in human NASH cohorts.

\begin{tabular}{|c|c|c|c|c|c|c|}
\hline & ${ }^{\mathrm{j}} \mathrm{MCD}$ & k NASH Diet & ${ }^{1} \mathrm{NASH}$ Diet $+\mathrm{CCl}_{4}$ & ${ }^{\mathrm{n}} \mathrm{CCl}_{4}$ & ${ }^{\mathrm{m}} \mathrm{WD}+\mathrm{CCl}_{4}$ & Association with Human Fibrosis \\
\hline SMAD4 & & & & & & NASH \\
\hline SMAD2 & & & & & & NASH \\
\hline YAP1 & & & & & & NASH \\
\hline NOTCH1 & & & & & & NASH \\
\hline EP300 & & & & & & NASH \\
\hline NCOR & & & & & & NASH \\
\hline p63 & & & & & & NASH, steatosis \\
\hline SREBP2 & & & & & & Steatosis \\
\hline CAR & & & & & & Steatosis \\
\hline FOS & & & & & & Steatosis, insulin resistance \\
\hline PGC1 $\alpha$ & & & & & & Steatosis, insulin resistance \\
\hline PPAR $\delta$ & & & & & & $\mathrm{N} / \mathrm{A}$ \\
\hline HIF1 $\alpha$ & & & & & & N/A \\
\hline MED1 & & & & & & N/A \\
\hline NCOA1 & & & & & & N/A \\
\hline SMARCA4 & & & & & & N/A \\
\hline NCOA2 & & & & & & N/A \\
\hline FOXO3 & & & & & & N/A \\
\hline HDAC2 & & & & & & N/A \\
\hline STAT5b & & & & & & N/A \\
\hline STAT6 & & & & & & N/A \\
\hline
\end{tabular}

Mouse models of NASH using ${ }^{j}$ MCD diet (GSE93132), ${ }^{k}$ NASH diet (GSE52748), ${ }^{1}$ NASH diet coupled with $\mathrm{CCl}_{4}$ treatment (GSE129525), ${ }^{\mathrm{m}} \mathrm{CCl}_{4}$ treatment alone (GSE99010) and ${ }^{\mathrm{n}}$ Western diet coupled with $\mathrm{CCl}_{4}$ treatment (GSE99010). Abbreviations: transcription factors against decapentaplegic homolog (SMAD), yes-associated protein (YAP), notch receptor 1 (NOTCH1), Histone Acetyltransferase P300 (EP300), nuclear receptor corepressor (NCOR), sterol regulatory element binding protein (SREBP), constitutive androstane receptor (CAR), fos proto-oncogene (FOS), PPAR $\gamma$ coactivator 1 alpha (PGC1 $\alpha$ ), peroxisome proliferator-activated receptor (PPAR), hypoxia inducible factor $1 \alpha(\mathrm{HIF} 1 \alpha)$, mediator complex subunit 1 (MED1), nuclear receptor coactivator (NCOA), SWI/SNF related, matrix associated, actin dependent regulator of chromatin subfamily A member 4 (SMARCA4), forkhead protein O (FoxO), histone deacetylase (HDAC), signal transducer and activator of transcription (STAT). 
Table 4. IPA prediction of upstream mechanistic networks that are unique to each mouse model.

\begin{tabular}{|c|c|c|c|c|c|}
\hline & ${ }^{\mathrm{j}} \mathrm{MCD}$ & k NASH Diet & ${ }^{1} \mathrm{NASH}$ Diet $+\mathrm{CCl}_{4}$ & ${ }^{\mathrm{n}} \mathrm{CCl}_{4}$ & ${ }^{\mathrm{m}} \mathrm{WD}+\mathrm{CCl}_{4}$ \\
\hline \multicolumn{6}{|l|}{$\mathrm{AR}$} \\
\hline \multicolumn{6}{|l|}{ ARNTL } \\
\hline \multicolumn{6}{|l|}{ CDKN2A } \\
\hline \multicolumn{6}{|l|}{$\mathrm{C} / \mathrm{EBP} \alpha$} \\
\hline \multicolumn{6}{|l|}{ ChREBP } \\
\hline \multicolumn{6}{|l|}{ CIITA } \\
\hline \multicolumn{6}{|l|}{ E2F } \\
\hline \multicolumn{6}{|l|}{ FOXO4 } \\
\hline \multicolumn{6}{|l|}{ HNF1 $\alpha$} \\
\hline \multicolumn{6}{|l|}{ HSF1 } \\
\hline \multicolumn{6}{|l|}{ IRF9 } \\
\hline \multicolumn{6}{|l|}{ KLF4 } \\
\hline \multicolumn{6}{|l|}{ MAX } \\
\hline \multicolumn{6}{|l|}{ MYB } \\
\hline \multicolumn{6}{|l|}{ PGR } \\
\hline \multicolumn{6}{|l|}{ RARB } \\
\hline \multicolumn{6}{|l|}{ RB1 } \\
\hline \multicolumn{6}{|l|}{ RORA } \\
\hline \multicolumn{6}{|l|}{ SNAI } \\
\hline \multicolumn{6}{|l|}{ SP3 } \\
\hline \multicolumn{6}{|l|}{ STAT4 } \\
\hline \multicolumn{6}{|l|}{ TCF7L2 } \\
\hline \multicolumn{6}{|l|}{$\mathrm{THR} \alpha$} \\
\hline \multicolumn{6}{|l|}{ VDR } \\
\hline \multicolumn{6}{|l|}{ WT1 } \\
\hline \multicolumn{6}{|l|}{ Ybx1 } \\
\hline ZNFn1a1 & & & & & \\
\hline
\end{tabular}

Mouse models of NASH using ${ }^{j} \mathrm{MCD} \operatorname{diet}(\mathrm{GSE} 93132),{ }^{\mathrm{k}} \mathrm{NASH} \operatorname{diet}\left(\mathrm{GSE52748)},{ }^{1} \mathrm{NASH}\right.$ diet coupled with $\mathrm{CCl}_{4}$ treatment (GSE129525), ${ }^{\mathrm{m}} \mathrm{CCl}_{4}$ treatment alone (GSE99010) and ${ }^{\mathrm{n}}$ Western diet coupled with $\mathrm{CCl}_{4}$ treatment (GSE99010). Abbreviations: androgen receptor (AR), aryl hydrocarbon receptor nuclear translocator like (ARNTL), cyclin dependent kinase inhibitor 2A (CDKN2A), CCAAT/enhancer binding protein (C/EBP), class ii major histocompatibility complex transactivator (CITA), forkhead protein $\mathrm{O}$ (FoxO), hepatocyte nuclear factor (HNF), heat shock transcription factor 1 (HSF1), interferon regulatory factor (IRF), kruppel like factor 4 (KLF4), MYC associated factor X (MAX), myb proto-oncogene (MYB), progesterone receptor (PGR), retinoic acid receptor beta (RARB), retinoblastoma transcriptional corepressor 1 (RB1), retinoic acid receptor-related orphan receptor alpha (RORA), snail family transcriptional repressor (SNAI), specificity protein 3 (SP3), signal transducer and activator of transcription (STAT), transcription factor 7 like 2 (TCF7L2), thyroid hormone receptor $\alpha$ (THR $\alpha$ ), vitamin D receptor (VDR), Wilms' tumor protein (WT1), Y box-binding protein 1 (Ybx1), IKAROS family zinc finger 1 (ZNFn1a1).

Table 5. IPA prediction of upstream mechanistic networks that are unique to each human NASH cohort.

\begin{tabular}{|c|l|l|l|l|}
\hline & ${ }^{a}$ Fibrotic vs. Healthy & $\begin{array}{c}\text { b Fibrotic vs. } \\
\text { Non-Fibrotic NAFLD }\end{array}$ & ${ }^{d}$ NASH vs. Healthy & f External IPA NASH \\
\hline CCND1 & & & & \\
\hline CCNE1 & & & & \\
\hline HMGB1 & & & & \\
\hline IRF2 & & & & \\
\hline IRF5 & & & & \\
\hline KLF2 & & & & \\
\hline NRIP1 & & & & \\
\hline SOX2 & & & & \\
\hline
\end{tabular}

Human GEO Accession GSE130970 was divided into three independent IPA comparative analyses: ${ }^{a}$ Advanced fibrotic (fibrosis score $>3, n=16$ ) versus healthy $(n=8)$, ${ }^{b}$ Fibrotic versus non-fibrotic NAFLD (NAS $>3, n=11$ ), and ${ }^{\mathrm{d}}$ non-fibrotic NAFLD versus healthy. ${ }^{\mathrm{f}}$ Previously reported IPA of NASH (Kakehashi et al.). Abbreviations: cyclin D1 (CCND1), cyclin E1 (CCNE1), high mobility group box 1 (HMGB1), interferon regulatory factor (IRF), kruppel like factor 2 (KLF2), nuclear receptor interacting protein 1 (NRIP1), sex determining region Y box transcription factor 2 (SOX2). 


\section{Conclusions}

Maladaptive responses to obesity results in the activation of inflammatory and fibrogenic pathways in the liver. Here, we reviewed the transcription factors, the activity of which have been commonly associated with obesity-induced NAFLD and NASH. The development of NAFLD and NASH strongly correlates with the dysregulation of transcriptional regulators that play a role in lipid metabolism, inflammation, metabolic stress, and fibrosis. Interestingly, the review of gluconeogenic transcription factors indicated a protective function against steatosis and NASH, since their loss often resulted in disease. The field of main regulators will continue to increase with heightened focus on delineating new pathways in the pathogenesis of NAFLD, as each of the areas discussed in this review are still being actively researched and adding to our understanding of the transcriptional regulation of NAFLD.

Our review also indicates that none of the diet-based rodent models replicate all the features of the human pathophysiology. Our observations suggested that the FCP diet and MCD diet exhibit transcriptional activity profiles that are more representative of human NASH, whereas the models that involved chemical induction, such as $\mathrm{CCl}_{4}$ treatment, did not. The generation of novel experimental models that more accurately reproduce human pathophysiology, including mice with humanized livers [215], will be central to the discovery of tractable targets for the management of NAFLD.

Author Contributions: Conceptualized the outline of the review: S.S.; performed literature search: S.S., J.Q. and B.A.E.; wrote and edited the manuscript: S.S., J.Q. and B.A.E.; conceptualized the outline: B.A.E., directed and validated the review: B.A.E.; analyzed publicly available datasets: B.A.E. All authors have read and agreed to the published version of the manuscript.

Funding: This review article did not receive any external funding.

Conflicts of Interest: The authors declare no conflict of interest.

\section{References}

1. Machado, M.V.; Cortez-Pinto, H. Non-alcoholic fatty liver disease: What the clinician needs to know. World J. Gastroenterol. 2014, 20, 12956-12980. [CrossRef] [PubMed]

2. Younossi, Z.; Anstee, Q.M.; Marietti, M.; Hardy, T.; Henry, L.; Eslam, M.; George, J.; Bugianesi, E. Global burden of NAFLD and NASH: Trends, predictions, risk factors and prevention. Nat. Rev. Gastroenterol. Hepatol. 2017, 15, 11-20. [CrossRef]

3. Iwaisako, K.; Brenner, D.A.; Kisseleva, T. What's new in liver fibrosis? The origin of myofibroblasts in liver fibrosis. J. Gastroenterol. Hepatol. 2012, 27, 65-68. [CrossRef] [PubMed]

4. Benedict, M.; Zhang, X. Non-alcoholic fatty liver disease: An expanded review. World J. Hepatol. 2017, 9, 715-732. [CrossRef] [PubMed]

5. Oseini, A.M.; Sanyal, A.J. Therapies in non-alcoholic steatohepatitis (NASH). Liver Int. 2017, 37, 97-103. [CrossRef] [PubMed]

6. Paschos, P.; Paletas, K. Non alcoholic fatty liver disease and metabolic syndrome. Hippokratia 2009, 13, 9-19.

7. Kakehashi, A.; Stefanov, V.E.; Ishii, N.; Okuno, T.; Fujii, H.; Kawai, K.; Kawada, N.; Wanibuchi, H. Proteome Characteristics of Non-Alcoholic Steatohepatitis Liver Tissue and Associated Hepatocellular Carcinomas. Int. J. Mol. Sci. 2017, 18, 434. [CrossRef]

8. Buzzetti, E.; Pinzani, M.; Tsochatzis, E.A. The multiple-hit pathogenesis of non-alcoholic fatty liver disease (NAFLD). Metabolism 2016, 65, 1038-1048. [CrossRef]

9. Zaret, K.S.; Carroll, J.S. Pioneer transcription factors: Establishing competence for gene expression. Genes Dev. 2011, 25, 2227-2241. [CrossRef]

10. Shiri-Sverdlov, R.; Wouters, K.A.M.; Van Gorp, P.; Gijbels, M.J.; Noël, B.; Buffat, L.; Staels, B.; Maeda, N.; Van Bilsen, M.; Hofker, M.H. Early diet-induced non-alcoholic steatohepatitis in APOE2 knock-in mice and its prevention by fibrates. J. Hepatol. 2006, 44, 732-741. [CrossRef]

11. Horie, Y.; Suzuki, A.; Kataoka, E.; Sasaki, T.; Hamada, K.; Sasaki, J.; Mizuno, K.; Hasegawa, G.; Kishimoto, H.; Iizuka, M.; et al. Hepatocyte-specific Pten deficiency results in steatohepatitis and hepatocellular carcinomas. J. Clin. Investig. 2004, 113, 1774-1783. [CrossRef] [PubMed] 
12. Nakagawa, H.; Umemura, A.; Taniguchi, K.; Font-Burgada, J.; Dhar, D.; Ogata, H.; Zhong, Z.; Valasek, M.A.; Seki, E.; Hidalgo, J.; et al. ER stress cooperates with hypernutrition to trigger TNF-dependent spontaneous HCC development. Cancer Cell 2014, 26, 331-343. [CrossRef] [PubMed]

13. Ito, M.; Suzuki, J.; Tsujioka, S.; Sasaki, M.; Gomori, A.; Shirakura, T.; Hirose, H.; Ito, M.; Ishihara, A.; Iwaasa, H.; et al. Longitudinal analysis of murine steatohepatitis model induced by chronic exposure to high-fat diet. Hepatol. Res. 2007, 37, 50-57. [CrossRef] [PubMed]

14. Stephenson, K.; Kennedy, L.; Hargrove, L.; Demieville, J.; Thomson, J.; Alpini, G.; Francis, H.L. Updates on Dietary Models of Nonalcoholic Fatty Liver Disease: Current Studies and Insights. Gene Expr. 2018, 18, 5-17. [CrossRef]

15. Nakae, D.; Mizumoto, Y.; Andoh, N.; Tamura, K.; Horiguchi, K.; Endoh, T.; Kobayashi, E.; Tsujiuchi, T.; Denda, A.; Lombardi, B.; et al. Comparative Changes in the Liver of Female Fischer-344 Rats after Short-Term Feeding of a Semipurified or a Semisynthetic L-Amino Acid-Defined Choline-Deficient Diet. Toxicol. Pathol. 1995, 23, 583-590. [CrossRef]

16. Mamikutty, N.; Thent, Z.; Suhaimi, F. Fructose-Drinking Water Induced Nonalcoholic Fatty Liver Disease and Ultrastructural Alteration of Hepatocyte Mitochondria in Male Wistar Rat. BioMed Res. Int. 2015, 2015, 1-7. [CrossRef]

17. Fujii, M.; Shibazaki, Y.; Wakamatsu, K.; Honda, Y.; Kawauchi, Y.; Suzuki, K.; Arumugam, S.; Watanabe, K.; Ichida, T.; Asakura, H.; et al. A murine model for non-alcoholic steatohepatitis showing evidence of association between diabetes and hepatocellular carcinoma. Med. Mol. Morphol. 2013, 46, 141-152. [CrossRef]

18. Tsuchida, T.; Lee, Y.A.; Fujiwara, N.; Ybanez, M.; Allen, B.; Martins, S.; Fiel, M.I.; Goossens, N.; Chou, H.-I.; Hoshida, Y.; et al. A simple diet- and chemical-induced murine NASH model with rapid progression of steatohepatitis, fibrosis and liver cancer. J. Hepatol. 2018, 69, 385-395. [CrossRef]

19. Sinton, M.; Hay, D.C.; Drake, A.J. Metabolic control of gene transcription in non-alcoholic fatty liver disease: The role of the epigenome. Clin. Epigenet. 2019, 11, 104. [CrossRef]

20. Montagner, A.; Polizzi, A.; Fouché, E.; Ducheix, S.; Lippi, Y.; Lasserre, F.; Barquissau, V.; Régnier, M.; Lukowicz, C.; Benhamed, F.; et al. Liver PPAR $\alpha$ is crucial for whole-body fatty acid homeostasis and is protective against NAFLD. Gut 2016, 65, 1202-1214. [CrossRef]

21. Ponugoti, B.; Kemper, J.K.; Fang, S. Functional Interaction of Hepatic Nuclear Factor-4 and Peroxisome Proliferator-Activated Receptor- Gamma Coactivator 1 alpha in CYP7A1 Regulation Is Inhibited by a Key Lipogenic Activator, Sterol Regulatory Element-Binding Protein-1c. Mol. Endocrinol. 2007, 21, 2698-2712. [CrossRef] [PubMed]

22. Benhamed, F.; Denechaud, P.-D.; Lemoine, M.; Robichon, C.; Moldes, M.; Bertrand-Michel, J.; Ratziu, V.; Serfaty, L.; Housset, C.; Capeau, J.; et al. The lipogenic transcription factor ChREBP dissociates hepatic steatosis from insulin resistance in mice and humans. J. Clin. Investig. 2012, 122, 2176-2194. [CrossRef] [PubMed]

23. Tanaka, N.; Aoyama, T.; Kimura, S.; Gonzalez, F.J. Targeting nuclear receptors for the treatment of fatty liver disease. Pharmacol. Ther. 2017, 179, 142-157. [CrossRef] [PubMed]

24. Ahn, S.B.; Jang, K.; Jun, D.W.; Lee, B.H.; Shin, K.J. Expression of Liver X Receptor Correlates with Intrahepatic Inflammation and Fibrosis in Patients with Nonalcoholic Fatty Liver Disease. Dig. Dis. Sci. 2014, 59, 2975-2982. [CrossRef] [PubMed]

25. Baciu, C.; Pasini, E.; Angeli, M.; Schwenger, K.; Afrin, J.; Humar, A.; Fischer, S.; Patel, K.; Allard, J.; Bhat, M. Systematic integrative analysis of gene expression identifies HNF4A as the central gene in pathogenesis of non-alcoholic steatohepatitis. PLOS ONE 2017, 12, e0189223. [CrossRef]

26. Kaltenecker, D.; Themanns, M.; Mueller, K.M.; Spirk, K.; Suske, T.; Merkel, O.; Kenner, L.; Luís, A.; Kozlov, A.; Haybaeck, J.; et al. Hepatic growth hormone-JAK2-STAT5 signalling: Metabolic function, non-alcoholic fatty liver disease and hepatocellular carcinoma progression. Cytokine 2019, 124, 154569. [CrossRef]

27. Rahman, S.M.; Janssen, R.C.; Jiang, H.; Qadri, I.; MacLean, K.N.; Friedman, J.E.; Schroeder-Gloeckler, J.M. CCAAT/enhancing binding protein $\beta$ deletion in mice attenuates inflammation, endoplasmic reticulum stress, and lipid accumulation in diet-induced nonalcoholic steatohepatitis. Hepatology 2007, 45, 1108-1117. [CrossRef]

28. Piccinin, E.; Villani, G.; Moschetta, A. Metabolic aspects in NAFLD, NASH and hepatocellular carcinoma: The role of PGC1 coactivators. Nat. Rev. Gastroenterol. Hepatol. 2018, 16, 160-174. [CrossRef] 
29. Valenti, L.; Rametta, R.; Dongiovanni, P.; Maggioni, M.; Fracanzani, A.L.; Zappa, M.; Lattuada, E.; Roviaro, G.; Fargion, S. Increased Expression and Activity of the Transcription Factor FOXO1 in Nonalcoholic Steatohepatitis. Diabetes 2008, 57, 1355-1362. [CrossRef]

30. Videla, L.; Tapia, G.; Rodrigo, R.; Pettinelli, P.; Haim, D.; Santibáñez, C.; Araya, A.V.; Smok, G.; Csendes, A.; Gutiérrez, L.; et al. Liver NF-кB and AP-1 DNA Binding in Obese Patients. Obesity 2009, 17, 973-979. [CrossRef]

31. Severa, M.; Islam, S.A.; Waggoner, S.N.; Jiang, Z.; Kim, N.D.; Ryan, G.; Kurt-Jones, E.; Charo, I.; Caffrey, D.R.; Boyartchuk, V.L.; et al. The transcriptional repressor BLIMP1 curbs host defenses by suppressing expression of the chemokine CCL8. J. Immunol. 2014, 192, 2291-2304. [CrossRef] [PubMed]

32. Grohmann, M.; Wiede, F.; Dodd, G.T.; Gurzov, E.N.; Ooi, G.J.; Butt, T.; Rasmiena, A.A.; Kaur, S.; Gulati, T.; Goh, P.K.; et al. Obesity Drives STAT-1-Dependent NASH and STAT-3-Dependent HCC. Cell 2018, 175, 1289.e20-1306.e20. [CrossRef] [PubMed]

33. Dorn, C.; Engelmann, J.C.; Saugspier, M.; Koch, A.; Hartmann, A.; Müller-Nurasyid, M.; Spang, R.; Bosserhoff, A.K.; Hellerbrand, C. Increased expression of c-Jun in nonalcoholic fatty liver disease. Lab. Investig. 2014, 94, 394-408. [CrossRef] [PubMed]

34. Zou, A.; Magee, N.; Deng, F.; Lehn, S.; Zhong, C.; Zhang, Y. Hepatocyte nuclear receptor SHP suppresses inflammation and fibrosis in a mouse model of nonalcoholic steatohepatitis. J. Biol. Chem. 2018, 293, 8656-8671. [CrossRef]

35. Shimozono, R.; Asaoka, Y.; Yoshizawa, Y.; Aoki, T.; Noda, H.; Yamada, M.; Kaino, M.; Mochizuki, H. Nrf2 Activators Attenuate the Progression of Nonalcoholic Steatohepatitis-Related Fibrosis in a Dietary Rat Model. Mol. Pharmacol. 2013, 84, 62-70. [CrossRef]

36. Zhong, L.; Huang, L.; Xue, Q.; Liu, C.; Xu, K.; Shen, W.; Deng, L. Cell-specific elevation of Runx2 promotes hepatic infiltration of macrophages by upregulating MCP-1 in high-fat diet-induced mice NAFLD. J. Cell. Biochem. 2019, 120, 11761-11774. [CrossRef]

37. Lebeaupin, C.; Vallee, D.; Rousseau, D.; Patouraux, S.; Bonnafous, S.; Adam, G.; Luciano, F.; Luci, C.; Anty, R.; Iannelli, A.; et al. Bax inhibitor-1 protects from nonalcoholic steatohepatitis by limiting inositol-requiring enzyme 1 alpha signaling in mice. Hepatology 2018, 68, 515-532. [CrossRef]

38. Lee, A.-H.; Scapa, E.F.; Cohen, D.E.; Glimcher, L.H. Regulation of Hepatic Lipogenesis by the Transcription Factor XBP1. Science 2008, 320, 1492-1496. [CrossRef]

39. Cazanave, S.; Podtelezhnikov, A.; Jensen, K.; Seneshaw, M.; Kumar, D.P.; Min, H.-K.; Santhekadur, P.K.; Banini, B.; Mauro, A.G.; Oseini, A.M.; et al. The Transcriptomic Signature of Disease Development and Progression of Nonalcoholic Fatty Liver Disease. Sci. Rep. 2017, 7, 17193. [CrossRef]

40. Gonzalez-Rodriguez, A.; Mayoral, R.; Agra, N.; Valdecantos, M.P.; Pardo, V.; E Miquilena-Colina, M.; Vargas-Castrillón, J.; Iacono, O.L.; Corazzari, M.; Fimia, G.M.; et al. Impaired autophagic flux is associated with increased endoplasmic reticulum stress during the development of NAFLD. Cell Death Dis. 2014, 5, e1179. [CrossRef]

41. Lee, S.; Kim, S.; Hwang, S.; Cherrington, N.J.; Ryu, D.-Y. Dysregulated expression of proteins associated with ER stress, autophagy and apoptosis in tissues from nonalcoholic fatty liver disease. Oncotarget 2017, 8, 63370-63381. [CrossRef] [PubMed]

42. Dattaroy, D.; Pourhoseini, S.; Das, S.; Alhasson, F.; Seth, R.; Nagarkatti, M.; Michelotti, G.A.; Diehl, A.M.; Chatterjee, S. Micro-RNA 21 inhibition of SMAD7 enhances fibrogenesis via leptin-mediated NADPH oxidase in experimental and human nonalcoholic steatohepatitis. Am. J. Physiol. Liver Physiol. 2014, 308, G298-G312. [CrossRef] [PubMed]

43. Teratani, T.; Tomita, K.; Suzuki, T.; Furuhashi, H.; Irie, R.; Nishikawa, M.; Yamamoto, J.; Hibi, T.; Miura, S.; Minamino, T; et al. Aortic carboxypeptidase-like protein, a WNT ligand, exacerbates nonalcoholic steatohepatitis. J. Clin. Investig. 2018, 128, 1581-1596. [CrossRef] [PubMed]

44. Kumar, D.P.; Santhekadur, P.K.; Seneshaw, M.; Mirshahi, F.; Tuculescu, C.U.; Sanyal, A.J. A Regulatory Role of Apoptosis Antagonizing Transcription Factor in the Pathogenesis of Nonalcoholic Fatty Liver Disease and Hepatocellular Carcinoma. Hepatology 2019, 69, 1520-1534. [CrossRef]

45. Patel, S.H.; Camargo, F.D.; Yimlamai, D. Hippo Signaling in the Liver Regulates Organ Size, Cell Fate, and Carcinogenesis. Gastroenterology 2016, 152, 533-545. [CrossRef] [PubMed]

46. Souza-Mello, V. Peroxisome proliferator-activated receptors as targets to treat non-alcoholic fatty liver disease. World J. Hepatol. 2015, 7, 1012-1019. [CrossRef] 
47. Ip, E.; Farrell, G.; Robertson, G.; Hall, P.; Kirsch, R.; Leclercq, I. Central role of PPAR $\alpha$-dependent hepatic lipid turnover in dietary steatohepatitis in mice. Hepatology 2003, 38, 123-132. [CrossRef]

48. Musso, G.; Gambino, R.; Cassader, M.; Pagano, G. A meta-analysis of randomized trials for the treatment of nonalcoholic fatty liver disease. Hepatology 2010, 52, 79-104. [CrossRef]

49. Lee, Y.K.; Park, J.E.; Lee, M.; Hardwick, J.P. Hepatic lipid homeostasis by peroxisome proliferator-activated receptor gamma 2认. Liver Res. 2018, 2, 209-215. [CrossRef]

50. Greenstein, A.W.; Majumdar, N.; Yang, P.; Subbaiah, P.V.; Kineman, R.D.; Cordoba-Chacon, J. Hepatocyte-specific, PPAR $\gamma$-regulated mechanisms to promote steatosis in adult mice. J. Endocrinol. 2016, 232, 107-121. [CrossRef]

51. Medina-Gómez, G.; Gray, S.L.; Yetukuri, L.; Shimomura, K.; Virtue, S.; Campbell, M.; Curtis, R.K.; Jimenez-Liñan, M.; Blount, M.; Yeo, G.S.H.; et al. PPAR gamma 2 Prevents Lipotoxicity by Controlling Adipose Tissue Expandability and Peripheral Lipid Metabolism. PLoS Genet. 2007, 3, e64. [CrossRef] [PubMed]

52. Morán-Salvador, E.; López-Parra, M.; García-Alonso, V.; Titos, E.; Martínez-Clemente, M.; González-Périz, A.; López-Vicario, C.; Barak, Y.; Arroyo, V.; Claria, J. Role for PPAR $\gamma$ in obesity-induced hepatic steatosis as determined by hepatocyte- and macrophage-specific conditional knockouts. FASEB J. 2011, 25, 2538-2550. [CrossRef] [PubMed]

53. Matsusue, K.; Haluzík, M.; Lambert, G.; Yim, S.-H.; Gavrilova, O.; Ward, J.M.; Brewer, B.; Reitman, M.L.; Gonzalez, F.J. Liver-specific disruption of PPAR $\gamma$ in leptin-deficient mice improves fatty liver but aggravates diabetic phenotypes. J. Clin. Investig. 2003, 111, 737-747. [CrossRef]

54. Pettinelli, P.; Videla, L. Up-Regulation of PPAR- $\gamma$ mRNA Expression in the Liver of Obese Patients: An Additional Reinforcing Lipogenic Mechanism to SREBP-1c Induction. J. Clin. Endocrinol. Metab. 2011, 96, 1424-1430. [CrossRef] [PubMed]

55. Pan, X.; Wang, P.; Luo, J.; Wang, Z.; Song, Y.; Ye, J.; Hou, X. Adipogenic changes of hepatocytes in a high-fat diet-induced fatty liver mice model and non-alcoholic fatty liver disease patients. Endocrine 2014, 48, 834-847. [CrossRef] [PubMed]

56. Ratziu, V.; Charlotte, F.; Bernhardt, C.; Giral, P.; Halbron, M.; Lenaour, G.; Hartmann-Heurtier, A.; Bruckert, E.; Poynard, T.; LIDO Study Group. Long-term efficacy of rosiglitazone in nonalcoholic steatohepatitis: Results of the fatty liver improvement by rosiglitazone therapy (FLIRT 2) extension trial. Hepatology 2009, 51, 445-453. [CrossRef]

57. Ratziu, V.; Giral, P.; Jacqueminet, S.; Charlotte, F.; Hartemann-Heurtier, A.; Serfaty, L.; Podevin, P.; Lacorte, J.; Bernhardt, C.; Bruckert, E.; et al. Rosiglitazone for Nonalcoholic Steatohepatitis: One-Year Results of the Randomized Placebo-Controlled Fatty Liver Improvement With Rosiglitazone Therapy (FLIRT) Trial. Gastroenterology 2008, 135, 100-110. [CrossRef]

58. Cusi, K.; Orsak, B.; Bril, F.; Lomonaco, R.; Hecht, J.; Ortiz-Lopez, C.; Tio, F.; Hardies, J.; Darland, C.; Musi, N.; et al. Long-Term Pioglitazone Treatment for Patients With Nonalcoholic Steatohepatitis and Prediabetes or Type 2 Diabetes Mellitus. Ann. Intern. Med. 2016, 165, 305. [CrossRef]

59. Feige, J.N.; Gelman, L.; Tudor, C.; Engelborghs, Y.; Wahli, W.; Desvergne, B. Fluorescence Imaging Reveals the Nuclear Behavior of Peroxisome Proliferator-activated Receptor/Retinoid X Receptor Heterodimers in the Absence and Presence of Ligand. J. Biol. Chem. 2005, 280, 17880-17890. [CrossRef]

60. Kliewer, S.A.; Forman, B.M.; Blumberg, B.; Ong, E.S.; Borgmeyer, U.; Mangelsdorf, D.J.; Umesono, K.; Evans, R.M. Differential expression and activation of a family of murine peroxisome proliferator-activated receptors. Proc. Natl. Acad. Sci. USA 1994, 91, 7355-7359. [CrossRef]

61. Bays, H.E.; Schwartz, S.; Littlejohn, T.; Kerzner, B.; Krauss, R.M.; Karpf, D.B.; Choi, Y.-J.; Wang, X.; Naim, S.; Roberts, B.K. MBX-8025, A Novel Peroxisome Proliferator Receptor-? Agonist: Lipid and Other Metabolic Effects in Dyslipidemic Overweight Patients Treated with and without Atorvastatin. J. Clin. Endocrinol. Metab. 2011, 96, 2889-2897. [CrossRef] [PubMed]

62. Riserus, U.; Sprecher, D.; Johnson, T.; Olson, E.; Hirschberg, S.; Liu, A.; Fang, Z.; Hegde, P.; Richards, D.; Sarov-Blat, L.; et al. Activation of Peroxisome Proliferator-Activated Receptor (PPAR) Promotes Reversal of Multiple Metabolic Abnormalities, Reduces Oxidative Stress, and Increases Fatty Acid Oxidation in Moderately Obese Men. Diabetes 2007, 57, 332-339. [CrossRef] [PubMed]

63. Shimano, H. SREBPs: Physiology and pathophysiology of the SREBP family. FEBS J. 2008, 276, 616-621. [CrossRef] [PubMed] 
64. Fatehi-Hassanabad, Z.; Chan, C.B. Transcriptional regulation of lipid metabolism by fatty acids: A key determinant of pancreatic $\beta$-cell function. Nutr. Metab. 2005, 2, 1. [CrossRef] [PubMed]

65. Walker, A.K.; Yang, F.; Jiang, K.; Ji, J.-Y.; Watts, J.L.; Purushotham, A.; Boss, O.; Hirsch, M.L.; Ribich, S.; Smith, J.J.; et al. Conserved role of SIRT1 orthologs in fasting-dependent inhibition of the lipid/cholesterol regulator SREBP. Genes Dev. 2010, 24, 1403-1417. [CrossRef]

66. Bruschi, F.V.; Tardelli, M.; Claudel, T.; Trauner, M. PNPLA3 expression and its impact on the liver: Current perspectives. Hepatic Med. 2017, 9, 55-66. [CrossRef] [PubMed]

67. Cohen, J.C.; Horton, J.D.; Hobbs, H.H. Human Fatty Liver Disease: Old Questions and New Insights. Science 2011, 332, 1519-1523. [CrossRef]

68. Dongiovanni, P.; Donati, B.; Fares, R.; Lombardi, R.; Mancina, R.M.; Romeo, S.; Valenti, L. PNPLA3 I148M polymorphism and progressive liver disease. World J. Gastroenterol. 2013, 19, 6969-6978. [CrossRef]

69. Sookoian, S.; Pirola, C.J. Meta-analysis of the influence of I148M variant of patatin-like phospholipase domain containing 3 gene (PNPLA3) on the susceptibility and histological severity of nonalcoholic fatty liver disease. Hepatology 2011, 53, 1883-1894. [CrossRef]

70. Kohjima, M.; Higuchi, N.; Kato, M.; Kotoh, K.; Yoshimoto, T.; Fujino, T.; Yada, M.; Yada, R.; Harada, N.; Enjoji, M.; et al. SREBP-1c, regulated by the insulin and AMPK signaling pathways, plays a role in nonalcoholic fatty liver disease. Int. J. Mol. Med. 2008, 21, 507-511. [CrossRef]

71. Horton, J.D.; Goldstein, J.L.; Brown, M.S. SREBPs: Activators of the complete program of cholesterol and fatty acid synthesis in the liver. J. Clin. Investig. 2002, 109, 1125-1131. [CrossRef]

72. Kim, J.Y.; Garcia-Carbonell, R.; Yamachika, S.; Zhao, P.; Dhar, D.; Loomba, R.; Kaufman, R.J.; Saltiel, A.R.; Karin, M. ER Stress Drives Lipogenesis and Steatohepatitis via Caspase-2 Activation of S1P. Cell 2018, 175, 133-145.e15. [CrossRef] [PubMed]

73. Caballero, F.; Fernandez, A.; De Lacy, A.M.; Fernándezcheca, J.C.; Caballería, J.; García-Ruiz, C. Enhanced free cholesterol, SREBP-2 and StAR expression in human NASH. J. Hepatol. 2009, 50, 789-796. [CrossRef] [PubMed]

74. Ueda, A.; Hamadeh, H.K.; Webb, H.K.; Yamamoto, Y.; Sueyoshi, T.; Afshari, C.A.; Lehmann, J.M.; Negishi, M. Diverse roles of the nuclear orphan receptor CAR in regulating hepatic genes in response to phenobarbital. Mol. Pharmacol. 2002, 61, 1-6. [CrossRef] [PubMed]

75. Gao, J.; He, J.; Zhai, Y.; Wada, T.; Xie, W. The Constitutive Androstane Receptor Is an Anti-obesity Nuclear Receptor That Improves Insulin Sensitivity*. J. Biol. Chem. 2009, 284, 25984-25992. [CrossRef]

76. Dong, B.; Saha, P.K.; Huang, W.; Chen, W.; Abu-Elheiga, L.; Wakil, S.J.; Stevens, R.D.; Ilkayeva, O.; Newgard, C.B.; Chan, L.; et al. Activation of nuclear receptor CAR ameliorates diabetes and fatty liver disease. Proc. Natl. Acad. Sci. USA 2009, 106, 18831-18836. [CrossRef] [PubMed]

77. Patel, M.B.; Oza, N.A.; Anand, I.S.; Deshpande, S.S.; Patel, C.N. Liver X Receptor: A Novel Therapeutic Target. Indian J. Pharm. Sci. 2008, 70, 135-144. [CrossRef]

78. Han, C.Y. Update on FXR Biology: Promising Therapeutic Target? Int. J. Mol. Sci. 2018, 19, 2069. [CrossRef]

79. Park, J.-G.; Xu, X.; Cho, S.; Hur, K.Y.; Lee, M.-S.; Kersten, S.; Lee, A.-H. CREBH-FGF21 axis improves hepatic steatosis by suppressing adipose tissue lipolysis. Sci. Rep. 2016, 6, 27938. [CrossRef]

80. Nakagawa, Y.; Shimano, H. CREBH Regulates Systemic Glucose and Lipid Metabolism. Int. J. Mol. Sci. 2018, 19, 1396. [CrossRef]

81. Lee, M.-W.; Chanda, D.; Yang, J.; Oh, H.; Kim, S.S.; Yoon, Y.-S.; Hong, S.; Park, K.; Lee, I.-K.; Choi, C.S.; et al. Regulation of Hepatic Gluconeogenesis by an ER-Bound Transcription Factor, CREBH. Cell Metab. 2010, 11, 331-339. [CrossRef]

82. Danno, H.; Ishii, K.-A.; Nakagawa, Y.; Mikami, M.; Yamamoto, T.; Yabe, S.; Furusawa, M.; Kumadaki, S.; Watanabe, K.; Shimizu, H.; et al. The liver-enriched transcription factor CREBH is nutritionally regulated and activated by fatty acids and PPAR $\alpha$. Biochem. Biophys. Res. Commun. 2010, 391, 1222-1227. [CrossRef] [PubMed]

83. Xu, X.; Park, J.-G.; So, J.-S.; Hur, K.Y.; Lee, A.-H. Transcriptional regulation of apolipoprotein A-IV by the transcription factor CREBH. J. Lipid Res. 2014, 55, 850-859. [CrossRef] [PubMed]

84. Cheng, N.; Xu, X.; Simon, T.; Boudyguina, E.; Deng, Z.; Verhague, M.; Lee, A.-H.; Shelness, G.S.; Weinberg, R.B.; Parks, J.S. Very Low Density Lipoprotein Assembly Is Required for cAMP-responsive Element-binding Protein H Processing and Hepatic Apolipoprotein A-IV Expression. J. Biol. Chem. 2016, 291, 23793-23803. [CrossRef] [PubMed] 
85. Lee, J.H.; Giannikopoulos, P.; Duncan, S.A.; Wang, J.; Johansen, C.T.; Brown, J.D.; Plutzky, J.; Hegele, R.A.; Glimcher, L.H.; Lee, A.-H. The transcription factor cyclic AMP-responsive element-binding protein $\mathrm{H}$ regulates triglyceride metabolism. Nat. Med. 2011, 17, 812-815. [CrossRef] [PubMed]

86. Zhang, C.; Wang, G.; Zheng, Z.; Maddipati, K.R.; Zhang, X.; Dyson, G.; Williams, P.; Duncan, S.A.; Kaufman, R.J.; Zhang, K. Endoplasmic reticulum-tethered transcription factor cAMP responsive element-binding protein, hepatocyte specific, regulates hepatic lipogenesis, fatty acid oxidation, and lipolysis upon metabolic stress in mice. Hepatology 2012, 55, 1070-1082. [CrossRef] [PubMed]

87. Satoh, A.; Han, S.-I.; Araki, M.; Nakagawa, Y.; Ohno, H.; Mizunoe, Y.; Kumagai, K.; Murayama, Y.; Osaki, Y.; Iwasaki, H.; et al. CREBH Improves Diet-Induced Obesity, Insulin Resistance, and Metabolic Disturbances by FGF21-Dependent and FGF21-Independent Mechanisms. iScience 2020, 23, 100930. [CrossRef] [PubMed]

88. Zhang, Y.; Kast-Woelbern, H.R.; Edwards, P.A. Natural Structural Variants of the Nuclear Receptor Farnesoid $X$ Receptor Affect Transcriptional Activation. J. Biol. Chem. 2002, 278, 104-110. [CrossRef]

89. $\mathrm{Xi}, \mathrm{Y}$;; $\mathrm{Li}, \mathrm{H}$. Role of farnesoid $\mathrm{X}$ receptor in hepatic steatosis in nonalcoholic fatty liver disease. Biomed. Pharmacother. 2020, 121, 109609. [CrossRef]

90. Kalaany, N.Y.; Mangelsdorf, D.J. LXRS AND FXR: The Yin and Yang of Cholesterol and Fat Metabolism. Annu. Rev. Physiol. 2006, 68, 159-191. [CrossRef]

91. Jiao, Y.; Lu, Y.; Li, X.-Y. Farnesoid X receptor: A master regulator of hepatic triglyceride and glucose homeostasis. Acta Pharmacol. Sin. 2014, 36, 44-50. [CrossRef] [PubMed]

92. Barclay, J.; Nelson, C.N.; Ishikawa, M.; Murray, L.A.; Kerr, L.M.; McPhee, T.R.; Powell, E.E.; Waters, M.J. GH-Dependent STAT5 Signaling Plays an Important Role in Hepatic Lipid Metabolism. Endocrinology 2011, 152, 181-192. [CrossRef] [PubMed]

93. Baik, M.; Nam, Y.S.; Piao, M.; Kang, H.J.; Park, S.J.; Lee, J.-H. Liver-specific deletion of the signal transducer and activator of transcription 5 gene aggravates fatty liver in response to a high-fat diet in mice. J. Nutr. Biochem. 2016, 29, 56-63. [CrossRef] [PubMed]

94. Ko, C.-Y.; Chang, W.-C.; Wang, J.-M. Biological roles of CCAAT/Enhancer-binding protein delta during inflammation. J. Biomed. Sci. 2015, 22, 6. [CrossRef] [PubMed]

95. Matsusue, K.; Gavrilova, O.; Lambert, G.; Brewer, H.B.; Ward, J.M.; Inoue, Y.; Leroith, D.; Gonzalez, F.J. Hepatic CCAAT/Enhancer Binding Protein $\alpha$ Mediates Induction of Lipogenesis and Regulation of Glucose Homeostasis in Leptin-Deficient Mice. Mol. Endocrinol. 2004, 18, 2751-2764. [CrossRef] [PubMed]

96. Yoon, J.C.; Puigserver, P.; Chen, G.; Donovan, J.; Wu, Z.; Rhee, J.; Adelmant, G.; Stafford, J.M.; Kahn, C.R.; Granner, D.K.; et al. Control of hepatic gluconeogenesis through the transcriptional coactivator PGC-1. Nature 2001, 413, 131-138. [CrossRef] [PubMed]

97. Yu, Y.; Maguire, T.G.; Alwine, J.C. ChREBP, a glucose-responsive transcriptional factor, enhances glucose metabolism to support biosynthesis in human cytomegalovirus-infected cells. Proc. Natl. Acad. Sci. USA 2014, 111, 1951-1956. [CrossRef]

98. Kim, M.-S.; Krawczyk, S.A.; Doridot, L.; Fowler, A.J.; Wang, J.X.; Trauger, S.A.; Noh, H.-L.; Kang, H.J.; Meissen, J.K.; Blatnik, M.; et al. ChREBP regulates fructose-induced glucose production independently of insulin signaling. J. Clin. Investig. 2016, 126, 4372-4386. [CrossRef]

99. Herman, M.A.; Peroni, O.D.; Villoria, J.; Schön, M.R.; Abumrad, N.A.; Blüher, M.; Klein, S.; Kahn, B.B. A novel ChREBP isoform in adipose tissue regulates systemic glucose metabolism. Nature 2012, 484, 333-338. [CrossRef]

100. Xu, X.; So, J.-S.; Park, J.-G.; Lee, A.-H. Transcriptional control of hepatic lipid metabolism by SREBP and ChREBP. Semin. Liver Dis. 2013, 33, 301-311. [CrossRef]

101. Austin, S.; St-Pierre, J. PGC1 and mitochondrial metabolism-Emerging concepts and relevance in ageing and neurodegenerative disorders. J. Cell Sci. 2012, 125, 4963-4971. [CrossRef] [PubMed]

102. Aharoni-Simon, M.; Hann-Obercyger, M.; Pen, S.; Madar, Z.; Tirosh, O. Fatty liver is associated with impaired activity of PPAR $\gamma$-coactivator $1 \alpha(\mathrm{PGC} 1 \alpha)$ and mitochondrial biogenesis in mice. Lab. Investig. 2011, 91, 1018-1028. [CrossRef] [PubMed]

103. Erion, D.M.; Ignatova, I.D.; Yonemitsu, S.; Nagai, Y.; Chatterjee, P.; Weismann, D.; Hsiao, J.J.; Zhang, D.; Iwasaki, T.; Stark, R.; et al. Prevention of Hepatic Steatosis and Hepatic Insulin Resistance by Knockdown of cAMP Response Element-Binding Protein. Cell Metab. 2009, 10, 499-506. [CrossRef] [PubMed]

104. Carter, M.E.; Brunet, A. FOXO transcription factors. Curr. Biol. 2007, 17, R113-R114. [CrossRef] 


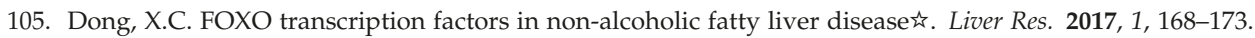
[CrossRef]

106. Sparks, J.; Dong, H.H. FoxO1 and hepatic lipid metabolism. Curr. Opin. Lipidol. 2009, 20, 217-226. [CrossRef]

107. Lu, H. Crosstalk of HNF4 $\alpha$ with extracellular and intracellular signaling pathways in the regulation of hepatic metabolism of drugs and lipids. Acta Pharm. Sin. B 2016, 6, 393-408. [CrossRef]

108. Liu, H.; Lou, G.; Li, C.; Wang, X.; Cederbaum, A.I.; Gan, L.; Xie, B. HBx Inhibits CYP2E1 Gene Expression via Downregulating HNF4 $\alpha$ in Human Hepatoma Cells. PLoS ONE 2014, 9, e107913. [CrossRef]

109. Yu, D.; Chen, G.; Pan, M.; Zhang, J.; He, W.; Liu, Y.; Nian, X.; Sheng, L.; Xu, B. High fat diet-induced oxidative stress blocks hepatocyte nuclear factor $4 \alpha$ and leads to hepatic steatosis in mice. J. Cell. Physiol. 2018, 233, 4770-4782. [CrossRef]

110. Ravnskjaer, K.; Frigerio, F.; Boergesen, M.; Nielsen, T.; Maechler, P.; Mandrup, S. PPAR $\delta$ is a fatty acid sensor that enhances mitochondrial oxidation in insulin-secreting cells and protects against fatty acid-induced dysfunction. J. Lipid Res. 2009, 51, 1370-1379. [CrossRef]

111. Iglesias, J.; Barg, S.; Vallois, D.; Lahiri, S.; Roger, C.; Yessoufou, A.; Pradevand, S.; McDonald, A.; Bonal, C.; Reimann, F.; et al. PPAR $\beta / \delta$ affects pancreatic $\beta$ cell mass and insulin secretion in mice. J. Clin. Investig. 2012, 122, 4105-4117. [CrossRef] [PubMed]

112. Lee, C.-H.; Olson, P.; Hevener, A.; Mehl, I.; Chong, L.-W.; Olefsky, J.M.; Gonzalez, F.J.; Ham, J.; Kang, H.; Peters, J.M.; et al. PPAR $\delta$ regulates glucose metabolism and insulin sensitivity. Proc. Natl. Acad. Sci. USA 2006, 103, 3444-3449. [CrossRef] [PubMed]

113. Tanaka, T.; Yamamoto, J.; Iwasaki, S.; Asaba, H.; Hamura, H.; Ikeda, Y.; Watanabe, M.; Magoori, K.; Ioka, R.X.; Tachibana, K.; et al. Activation of peroxisome proliferator-activated receptor $\delta$ induces fatty acid $\beta$-oxidation in skeletal muscle and attenuates metabolic syndrome. Proc. Natl. Acad. Sci. USA 2003, 100, 15924-15929. [CrossRef] [PubMed]

114. Smale, S.T.; Natoli, G. Transcriptional Control of Inflammatory Responses. Cold Spring Harb. Perspect. Biol. 2014, 6, a016261. [CrossRef]

115. Karin, M.; Yamamoto, Y.; Wang, Q.M. The IKK NF-kB system: A treasure trove for drug development. Nat. Rev. Drug Discov. 2004, 3, 17-26. [CrossRef]

116. Pena, A.D.; Leclercq, I.; Field, J.; George, J.; Jones, B.; Farrell, G. NF-kB Activation, Rather Than TNF, Mediates Hepatic Inflammation in a Murine Dietary Model of Steatohepatitis. Gastroenterology 2005, 129, 1663-1674. [CrossRef]

117. Ribeiro, P.S.; Cortez-Pinto, H.; Solá, S.; Castro, R.E.; Ramalho, R.; Baptista, A.; Moura, M.C.; Camilo, M.E.; Rodrigues, C.M.P.; Baptista, A. Hepatocyte Apoptosis, Expression of Death Receptors, and Activation of NF-кB in the Liver of Nonalcoholic and Alcoholic Steatohepatitis Patients. Am. J. Gastroenterol. 2004, 99, 1708-1717. [CrossRef]

118. Wang, X.-A.; Zhang, R.; Zhang, S.; Deng, S.; Jiang, D.; Zhong, J.; Yang, L.; Wang, T.; Hong, S.; Guo, S.; et al. Interferon regulatory factor 7 deficiency prevents diet-induced obesity and insulin resistance. Am. J. Physiol. Metab. 2013, 305, E485-E495. [CrossRef]

119. Iyer, S.; Upadhyay, P.K.; Majumdar, S.S.; Nagarajan, P. Animal Models Correlating Immune Cells for the Development of NAFLD/NASH. J. Clin. Exp. Hepatol. 2015, 5, 239-245. [CrossRef]

120. Ferreira, D.M.S.; Castro, R.E.; Machado, M.V.; Evangelista, T.; Silvestre, A.; Costa, A.; Coutinho, J.; Carepa, F.; Cortez-Pinto, H.; Rodrigues, C.M.P. Apoptosis and insulin resistance in liver and peripheral tissues of morbidly obese patients is associated with different stages of non-alcoholic fatty liver disease. Diabetology 2011, 54, 1788-1798. [CrossRef]

121. Schreck, I.; Al-Rawi, M.; Mingot, J.-M.; Scholl, C.; Diefenbacher, M.E.; O’Donnell, P.; Bohmann, D.; Weiss, C. c-Jun localizes to the nucleus independent of its phosphorylation by and interaction with JNK and vice versa promotes nuclear accumulation of JNK. Biochem. Biophys. Res. Commun. 2011, 407, 735-740. [CrossRef] [PubMed]

122. Bruno, T.; Iezzi, S.; De Nicola, F.; Di Padova, M.; DeSantis, A.; Scarsella, M.; Di Certo, M.G.; Leonetti, C.; Floridi, A.; Passananti, C.; et al. Che-1 activates XIAP expression in response to DNA damage. Cell Death Differ. 2007, 15, 515-520. [CrossRef]

123. DeSantis, A.; Bruno, T.; Catena, V.; De Nicola, F.; Goeman, F.; Iezzi, S.; Sorino, C.; Ponzoni, M.; Bossi, G.; Federico, V.; et al. Che-1-induced inhibition of mTOR pathway enables stress-induced autophagy. EMBO J. 2015, 34, 1214-1230. [CrossRef] [PubMed] 
124. DeSantis, A.; Bruno, T.; Catena, V.; De Nicola, F.; Goeman, F.; Iezzi, S.; Sorino, C.; Gentileschi, M.P.; Germoni, S.; Monteleone, V.; et al. Che-1 modulates the decision between cell cycle arrest and apoptosis by its binding to p53. Cell Death Dis. 2015, 6, e1764. [CrossRef] [PubMed]

125. Zhang, K.; Wang, S.; Malhotra, J.; Hassler, J.R.; Back, S.H.; Wang, G.; Chang, L.; Xu, W.; Miao, H.; Leonardi, R.; et al. The unfolded protein response transducer IRE1 $\alpha$ prevents ER stress-induced hepatic steatosis. EMBO J. 2011, 30, 1357-1375. [CrossRef] [PubMed]

126. Yuk, J.-M.; Shin, N.-M.; Lee, H.-M.; Kim, J.-J.; Kim, S.-W.; Jin, H.S.; Yang, C.-S.; Park, K.A.; Chanda, D.; Kim, N.-K.; et al. The orphan nuclear receptor SHP acts as a negative regulator in inflammatory signaling triggered by Toll-like receptors. Nat. Immunol. 2011, 12, 742-751. [CrossRef]

127. Yang, C.-S.; Kim, J.-J.; Kim, T.S.; Jo, E.-K.; Kim, S.Y.; Lee, H.-M.; Shin, N.-M.; Nguyen, L.T.; Lee, M.-S.; Jin, H.S.; et al. Small heterodimer partner interacts with NLRP3 and negatively regulates activation of the NLRP3 inflammasome. Nat. Commun. 2015, 6, 6115. [CrossRef] [PubMed]

128. Poli, V. The Role of C/EBP Isoforms in the Control of Inflammatory and Native Immunity Functions. J. Biol. Chem. 1998, 273, 29279-29282. [CrossRef]

129. Berghe, W.V.; Vermeulen, L.; Delerive, P.; De Bosscher, K.; Staels, B.; Haegeman, G. A Paradigm for Gene Regulation: Inflammation, NF-kB and PPAR. Adv. Exp. Med. Biol. 2003, 544, 181-196. [CrossRef]

130. Stienstra, R.; Mandard, S.; Patsouris, D.; Maass, C.; Kersten, S.; Muller, M. Peroxisome Proliferator-Activated Receptor $\alpha$ Protects against Obesity-Induced Hepatic Inflammation. Endocrinology 2007, 148, 2753-2763. [CrossRef]

131. Ricote, M.; Li, A.C.; Willson, T.M.; Kelly, C.J.; Glass, C.K. The peroxisome proliferator-activated receptor- $\gamma$ is a negative regulator of macrophage activation. Nature 1998, 391, 79-82. [CrossRef] [PubMed]

132. Morán-Salvador, E.; Titos, E.; Rius, B.; González-Périz, A.; García-Alonso, V.; López-Vicario, C.; Miquel, R.; Barak, Y.; Arroyo, V.; Claria, J. Cell-specific PPAR $\gamma$ deficiency establishes anti-inflammatory and anti-fibrogenic properties for this nuclear receptor in non-parenchymal liver cells. J. Hepatol. 2013, 59, 1045-1053. [CrossRef]

133. Luo, W.; Xu, Q.; Wang, Q.; Wu, H.; Hua, J. Effect of modulation of PPAR- $\gamma$ activity on Kupffer cells M1/M2 polarization in the development of non-alcoholic fatty liver disease. Sci. Rep. 2017, 7, 44612. [CrossRef] [PubMed]

134. Baskin-Bey, E.S.; Anan, A.; Isomoto, H.; Bronk, S.F.; Gores, G.J. Constitutive androstane receptor agonist, TСРОВОР, attenuates steatohepatitis in the methionine choline-deficient diet-fed mouse. World J. Gastroenterol. 2007, 13, 5635-5641. [CrossRef] [PubMed]

135. Yamazaki, Y.; Kakizaki, S.; Horiguchi, N.; Sohara, N.; Sato, K.; Takagi, H.; Mori, M.; Negishi, M. The role of the nuclear receptor constitutive androstane receptor in the pathogenesis of non-alcoholic steatohepatitis. Gut 2006, 56, 565-574. [CrossRef] [PubMed]

136. Venteclef, N.; Jakobsson, T.; Ehrlund, A.; Damdimopoulos, A.; Mikkonen, L.; Ellis, E.; Nilsson, L.-M.; Parini, P.; Jänne, O.A.; Gustafsson, J.-Å.; et al. GPS2-dependent corepressor/SUMO pathways govern anti-inflammatory actions of LRH-1 and LXR $\beta$ in the hepatic acute phase response. Genes Dev. 2010, 24, 381-395. [CrossRef]

137. Griffett, K.; Solt, L.A.; Elgendy, B.; Kamenecka, T.M.; Burris, T.P. A Liver-Selective LXR Inverse Agonist That Suppresses Hepatic Steatosis. ACS Chem. Biol. 2012, 8, 559-567. [CrossRef]

138. Griffett, K.; Welch, R.D.; Flaveny, C.A.; Kolar, G.R.; Neuschwander-Tetri, B.A.; Burris, T.P. The LXR inverse agonist SR9238 suppresses fibrosis in a model of non-alcoholic steatohepatitis. Mol. Metab. 2015, 4, 353-357. [CrossRef]

139. Kim, K.H.; Lee, M.-S. Pathogenesis of Nonalcoholic Steatohepatitis and Hormone-Based Therapeutic Approaches. Front. Endocrinol. 2018, 9, 485. [CrossRef]

140. Xu, D.; Xu, M.; Jeong, S.; Qian, Y.; Wu, H.; Xia, Q.; Kong, X. The Role of Nrf2 in Liver Disease: Novel Molecular Mechanisms and Therapeutic Approaches. Front. Pharmacol. 2019, 9, 9. [CrossRef]

141. Lee, J.; Sun, C.; Zhou, Y.; Lee, J.; Gökalp, D.; Herrema, H.; Park, S.W.; Davis, R.J.; Ozcan, U. p38 MAPK-mediated regulation of Xbp1s is crucial for glucose homeostasis. Nat. Med. 2011, 17, 1251-1260. [CrossRef] [PubMed]

142. Herrema, H.; Zhou, Y.; Zhang, D.; Lee, J.; Hernandez, M.A.S.; Shulman, G.I.; Ozcan, U. XBP1s Is an Anti-lipogenic Protein. J. Biol. Chem. 2016, 291, 17394-17404. [CrossRef] [PubMed] 
143. Liu, X.; Henkel, A.S.; Lecuyer, B.E.; Schipma, M.J.; Anderson, K.A.; Green, R.M. Hepatocyte X-box binding protein 1 deficiency increases liver injury in mice fed a high-fat/sugar diet. Am. J. Physiol. Liver Physiol. 2015, 309, G965-G974. [CrossRef] [PubMed]

144. Xiao, G.; Zhang, T.; Yu, S.; Lee, S.; Calabuig-Navarro, V.; Yamauchi, J.; Ringquist, S.; Dong, H.H. ATF4 Protein Deficiency Protects against High Fructose-induced Hypertriglyceridemia in Mice*. J. Biol. Chem. 2013, 288, 25350-25361. [CrossRef]

145. Chen, X.; Zhang, F.; Gong, Q.; Cui, A.; Zhuo, S.; Hu, Z.; Han, Y.; Gao, J.; Sun, Y.; Liu, Z.; et al. Hepatic ATF6 Increases Fatty Acid Oxidation to Attenuate Hepatic Steatosis in Mice through Peroxisome Proliferator-Activated Receptor Alpha. Diabetes 2016, 65, 1904-1915. [CrossRef]

146. Zeng, L.; Lu, M.; Mori, K.; Luo, S.; Lee, A.S.; Zhu, Y.; Shyy, J.Y.-J. ATF6 modulates SREBP2-mediated lipogenesis. EMBO J. 2004, 23, 950-958. [CrossRef]

147. Tang, W.; Jiang, Y.-F.; Ponnusamy, M.; Diallo, M. Role of Nrf2 in chronic liver disease. World J. Gastroenterol. 2014, 20, 13079-13087. [CrossRef]

148. Gupte, A.A.; Lyon, C.J.; Hsueh, W.A. Nuclear Factor (Erythroid-Derived 2)-Like-2 Factor (Nrf2), a Key Regulator of the Antioxidant Response to Protect Against Atherosclerosis and Nonalcoholic Steatohepatitis. Curr. Diabetes Rep. 2013, 13, 362-371. [CrossRef]

149. Sharma, R.S.; Harrison, D.J.; Kisielewski, D.; Cassidy, D.M.; McNeilly, A.D.; Gallagher, J.R.; Walsh, S.V.; Honda, T.; McCrimmon, R.J.; Dinkova-Kostova, A.T.; et al. Experimental Nonalcoholic Steatohepatitis and Liver Fibrosis Are Ameliorated by Pharmacologic Activation of Nrf2 (NF-E2 p45-Related Factor 2). Cell. Mol. Gastroenterol. Hepatol. 2017, 5, 367-398. [CrossRef]

150. Chowdhry, S.; Nazmy, M.H.; Meakin, P.J.; Dinkova-Kostova, A.T.; Walsh, S.V.; Tsujita, T.; Dillon, J.; Ashford, M.L.; Hayes, J. Loss of Nrf2 markedly exacerbates nonalcoholic steatohepatitis. Free Radic. Biol. Med. 2010, 48, 357-371. [CrossRef]

151. Liu, Z.; Dou, W.; Ni, Z.; Wen, Q.; Zhang, R.; Qin, M.; Wang, X.; Tang, H.; Cao, Y.; Wang, J.; et al. Deletion of Nrf2 leads to hepatic insulin resistance via the activation of NF-кB in mice fed a high-fat diet. Mol. Med. Rep. 2016, 14, 1323-1331. [CrossRef] [PubMed]

152. Woodcroft, K.J.; Hafner, M.S.; Novak, R.F. Insulin signaling in the transcriptional and posttranscriptional regulation of CYP2E1 expression. Hepatology 2002, 35, 263-273. [CrossRef] [PubMed]

153. Leung, T.-M.; Nieto, N. CYP2E1 and oxidant stress in alcoholic and non-alcoholic fatty liver disease. J. Hepatol. 2013, 58, 395-398. [CrossRef] [PubMed]

154. Kelicen, P.; Tindberg, N. Lipopolysaccharide Induces CYP2E1 in Astrocytes through MAP Kinase Kinase-3 and C/EBPbeta and -Delta. J. Biol. Chem. 2003, 279, 15734-15742. [CrossRef]

155. Daly, A.K. Relevance of CYP2E1 to Non-alcoholic Fatty Liver Disease. Membr. Biog. 2013, 67, $165-175$. [CrossRef]

156. Svegliati-Baroni, G.; Pierantonelli, I.; Torquato, P.; Marinelli, R.; Ferreri, C.; Chatgilialoglu, C.; Bartolini, D.; Galli, F. Lipidomic biomarkers and mechanisms of lipotoxicity in non-alcoholic fatty liver disease. Free Radic. Biol. Med. 2019, 144, 293-309. [CrossRef]

157. Aubert, J.; Begriche, K.; Knockaert, L.; Robin, M.; Fromenty, B. Increased expression of cytochrome P450 2E1 in nonalcoholic fatty liver disease: Mechanisms and pathophysiological role. Clin. Res. Hepatol. Gastroenterol. 2011, 35, 630-637. [CrossRef]

158. Liu, S.-Y.; Gonzalez, F.J. Role of the Liver-Enriched Transcription Factor HNF-1 $\alpha$ in Expression of theCYP2E1Gene. DNA Cell Biol. 1995, 14, 285-293. [CrossRef]

159. Jin, M.; Ande, A.; Kumar, A.; Kumar, S. Regulation of cytochrome P450 2e1 expression by ethanol: Role of oxidative stress-mediated pkc/jnk/sp1 pathway. Cell Death Dis. 2013, 4, e554. [CrossRef]

160. Tsuchida, T.; Friedman, S.L. Mechanisms of hepatic stellate cell activation. Nat. Rev. Gastroenterol. Hepatol. 2017, 14, 397-411. [CrossRef]

161. Koyama, Y.; Xu, J.; Liu, X.; Brenner, D.A. New Developments on the Treatment of Liver Fibrosis. Dig. Dis. 2016, 34, 589-596. [CrossRef]

162. Meng, X.-M.; Nikolic-Paterson, D.J.; Lan, H.-Y. TGF- $\beta$ : The master regulator of fibrosis. Nat. Rev. Nephrol. 2016, 12, 325-338. [CrossRef] [PubMed]

163. Wobser, H.; Dorn, C.; Weiss, T.S.; Amann, T.; Bollheimer, C.; Büttner, R.; Schölmerich, J.; Hellerbrand, C. Lipid accumulation in hepatocytes induces fibrogenic activation of hepatic stellate cells. Cell Res. 2009, 19, 996-1005. [CrossRef] [PubMed] 
164. Xu, P.; Liu, J.; Derynck, R. Post-translational regulation of TGF- $\beta$ receptor and Smad signaling. FEBS Lett. 2012, 586, 1871-1884. [CrossRef]

165. Inoue, Y.; Itoh, Y.; Abe, K.; Okamoto, T.; Daitoku, H.; Fukamizu, A.; Onozaki, K.; Hayashi, H. Smad3 is acetylated by p300/CBP to regulate its transactivation activity. Oncogene 2006, 26, 500-508. [CrossRef] [PubMed]

166. Lim, J.-Y.; Oh, M.-A.; Kim, W.H.; Sohn, H.-Y.; Park, S.I. AMP-activated protein kinase inhibits TGF-?-induced fibrogenic responses of hepatic stellate cells by targeting transcriptional coactivator p300. J. Cell. Physiol. 2011, 227, 1081-1089. [CrossRef] [PubMed]

167. Liu, E.; Liu, Z.; Zhou, Y.; Chen, M.; Wang, L.; Li, J. MicroRNA-142-3p inhibits trophoblast cell migration and invasion by disrupting the TGF- $\beta 1 /$ Smad3 signaling pathway. Mol. Med. Rep. 2019, 19, 3775-3782. [CrossRef]

168. Gerhard, G.S.; Hanson, A.; Wilhelmsen, D.; Piras, I.S.; Still, C.D.; Chu, X.; Petrick, A.T.; Distefano, J.K. AEBP1 expression increases with severity of fibrosis in NASH and is regulated by glucose, palmitate, and miR-372-3p. PLoS ONE 2019, 14, e0219764. [CrossRef]

169. Kim, S.-W.; Muise, A.M.; Lyons, P.J.; Ro, H.-S. Regulation of Adipogenesis by a Transcriptional Repressor That Modulates MAPK Activation. J. Biol. Chem. 2001, 276, 10199-10206. [CrossRef]

170. Jager, M.; Lee, M.-J.; Li, C.; Farmer, S.R.; Fried, S.K.; Layne, M.D. Aortic carboxypeptidase-like protein enhances adipose tissue stromal progenitor differentiation into myofibroblasts and is upregulated in fibrotic white adipose tissue. PLoS ONE 2018, 13, e0197777. [CrossRef]

171. Majdalawieh, A.; Zhang, L.; Fuki, I.V.; Rader, D.J.; Ro, H.-S. Adipocyte enhancer-binding protein 1 is a potential novel atherogenic factor involved in macrophage cholesterol homeostasis and inflammation. Proc. Natl. Acad. Sci. USA 2006, 103, 2346-2351. [CrossRef] [PubMed]

172. Lou, Y.; Chen, Y.-D.; Sun, F.-R.; Shi, J.-P.; Song, Y.; Yang, J. Potential Regulators Driving the Transition in Nonalcoholic Fatty Liver Disease: A Stage-Based View. Cell. Physiol. Biochem. 2017, 41, 239-251. [CrossRef] [PubMed]

173. Camargo, F.D.; Gokhale, S.; Johnnidis, J.B.; Fu, N.; Bell, G.W.; Jaenisch, R.; Brummelkamp, T.R. YAP1 Increases Organ Size and Expands Undifferentiated Progenitor Cells. Curr. Biol. 2007, 17, 2054-2060. [CrossRef] [PubMed]

174. Mooring, M.; Fowl, B.H.; Lum, S.Z.C.; Liu, Y.; Yao, K.; Softic, S.; Kirchner, R.; Bernstein, A.; Singhi, A.D.; Jay, D.G.; et al. Hepatocyte Stress Increases Expression of YAP and TAZ in Hepatocytes to Promote Parenchymal Inflammation and Fibrosis. Hepatology 2019. [CrossRef]

175. Song, K.; Kwon, H.; Han, C.; Chen, W.; Zhang, J.; Ma, W.; Dash, S.; Gandhi, C.R.; Wu, T. YAP in Kupffer cells enhances the production of pro-inflammatory cytokines and promotes the development of non-alcoholic steatohepatitis. Hepatology 2019. [CrossRef] [PubMed]

176. Abdelmegeed, M.A.; Yoo, S.-H.; Henderson, L.E.; Gonzalez, F.J.; Woodcroft, K.J.; Song, B.-J. PPARalpha expression protects male mice from high fat-induced nonalcoholic fatty liver. J. Nutr. 2011, 141, 603-610. [CrossRef]

177. Kawaguchi, K.; Sakaida, I.; Tsuchiya, M.; Omori, K.; Takami, T.; Okita, K. Pioglitazone prevents hepatic steatosis, fibrosis, and enzyme-altered lesions in rat liver cirrhosis induced by a choline-deficient l-amino acid-defined diet. Biochem. Biophys. Res. Commun. 2004, 315, 187-195. [CrossRef]

178. Leclercq, I.A.; Sempoux, C.; Stärkel, P.; Horsmans, Y. Limited therapeutic efficacy of pioglitazone on progression of hepatic fibrosis in rats. Gut 2006, 55, 1020-1029. [CrossRef]

179. Colca, J.R.; McDonald, W.G.; Cavey, G.S.; Cole, S.L.; Holewa, D.D.; Brightwell-Conrad, A.S.; Wolfe, C.L.; Wheeler, J.S.; Coulter, K.R.; Kilkuskie, P.M.; et al. Identification of a Mitochondrial Target of Thiazolidinedione Insulin Sensitizers (mTOT)—Relationship to Newly Identified Mitochondrial Pyruvate Carrier Proteins. PLoS ONE 2013, 8, e61551. [CrossRef]

180. McCommis, K.S.; Hodges, W.T.; Brunt, E.M.; Nalbantoglu, I.; McDonald, W.G.; Holley, C.; Fujiwara, H.; Schaffer, J.E.; Colca, J.; Finck, B.N. Targeting the mitochondrial pyruvate carrier attenuates fibrosis in a mouse model of nonalcoholic steatohepatitis. Hepatology 2017, 65, 1543-1556. [CrossRef]

181. Skat-Rørdam, J.; Ipsen, D.H.; Lykkesfeldt, J.; Tveden-Nyborg, P. A role of peroxisome proliferator-activated receptor $\gamma$ in non-alcoholic fatty liver disease. Basic Clin. Pharmacol. Toxicol. 2019, 124, 528-537. [CrossRef] [PubMed] 
182. Hattori, S.; Dhar, D.K.; Hara, N.; Tonomoto, Y.; Onoda, T.; Ono, T.; Yamanoi, A.; Tachibana, M.; Tsuchiya, M.; Nagasue, N. FR-167653, a selective p38 MAPK inhibitor, exerts salutary effect on liver cirrhosis through downregulation of Runx2. Lab. Investig. 2007, 87, 591-601. [CrossRef]

183. Cao, Z.; Sun, B.; Zhao, X.; Zhang, Y.; Gu, Q.; Liang, X.; Dong, X.; Zhao, N. The Expression and Functional Significance of Runx2 in Hepatocellular Carcinoma: Its Role in Vasculogenic Mimicry and Epithelial-Mesenchymal Transition. Int. J. Mol. Sci. 2017, 18, 500. [CrossRef] [PubMed]

184. Sahai, A.; Malladi, P.; Melin-Aldana, H.; Green, R.M.; Whitington, P.F. Upregulation of osteopontin expression is involved in the development of nonalcoholic steatohepatitis in a dietary murine model. Am. J. Physiol. Liver Physiol. 2004, 287, G264-G273. [CrossRef]

185. Kolodziejczyk, A.A.; Zheng, D.; Shibolet, O.; Elinav, E. The role of the microbiome in NAFLD and NASH. EMBO Mol. Med. 2018, 11, e9302. [CrossRef] [PubMed]

186. Bäckhed, F.; Ding, H.; Wang, T.; Hooper, L.V.; Koh, G.Y.; Nagy, A.; Semenkovich, C.F.; Gordon, J.I. The gut microbiota as an environmental factor that regulates fat storage. Proc. Natl. Acad. Sci. USA 2004, 101, 15718-15723. [CrossRef] [PubMed]

187. Zeng, H.; Liu, J.; Jackson, M.I.; Zhao, F.-Q.; Yan, L.; Combs, G.F. Fatty liver accompanies an increase in lactobacillus species in the hind gut of C57BL/6 mice fed a high-fat diet. J. Nutr. 2013, 143, 627-631. [CrossRef] [PubMed]

188. Wang, R.; Li, H.; Yang, X.; Xue, X.; Deng, L.; Shen, J.; Zhang, M.; Zhao, L.; Zhang, C. Genetically Obese Human Gut Microbiota Induces Liver Steatosis in Germ-Free Mice Fed on Normal Diet. Front. Microbiol. 2018, 9. [CrossRef]

189. Chiu, C.-C.; Ching, Y.-H.; Li, Y.-P.; Liu, J.-Y.; Huang, Y.-T.; Huang, Y.-W.; Yang, S.-S.; Huang, W.-C.; Chuang, H.-L. Nonalcoholic Fatty Liver Disease Is Exacerbated in High-Fat Diet-Fed Gnotobiotic Mice by Colonization with the Gut Microbiota from Patients with Nonalcoholic Steatohepatitis. Nutrients 2017, 9, 1220. [CrossRef]

190. Lambert, J.E.; Parnell, J.A.; Eksteen, B.; Raman, M.; Bomhof, M.R.; Rioux, K.P.; Madsen, K.L.; Reimer, R.A. Gut microbiota manipulation with prebiotics in patients with non-alcoholic fatty liver disease: A randomized controlled trial protocol. BMC Gastroenterol. 2015, 15, 169. [CrossRef]

191. Perumpail, B.J.; Li, A.A.; John, N.; Sallam, S.; Shah, N.D.; Kwong, W.; Cholankeril, G.; Kim, D.; Ahmed, A. The Therapeutic Implications of the Gut Microbiome and Probiotics in Patients with NAFLD. Diseases 2019, 7, 27. [CrossRef]

192. Cheng, Y.-W.; Phelps, E.; Ganapini, V.; Khan, N.; Ouyang, F.; Xu, H.; Khanna, S.; Tariq, R.; Friedman-Moraco, R.J.; Woodworth, M.H.; et al. Fecal microbiota transplantation for the treatment of recurrent and severe Clostridium difficileinfection in solid organ transplant recipients: A multicenter experience. Arab. Archaeol. Epigr. 2018, 19, 501-511. [CrossRef]

193. Aron-Wisnewsky, J.; Gaborit, B.; Dutour, A.; Clément, K. Gut microbiota and non-alcoholic fatty liver disease: New insights. Clin. Microbiol. Infect. 2013, 19, 338-348. [CrossRef] [PubMed]

194. Arab, J.P.; Karpen, S.J.; Dawson, P.A.; Arrese, M.; Trauner, M. Bile acids and nonalcoholic fatty liver disease: Molecular insights and therapeutic perspectives. Hepatology 2016, 65, 350-362. [CrossRef] [PubMed]

195. Cruz-Ramón, V.; Chinchilla-López, P.; Ramírez-Pérez, O.; Méndez-Sánchez, N. Bile Acids in Nonalcoholic Fatty Liver Disease: New Concepts and Therapeutic Advances. Ann. Hepatol. 2017, 16, S58-S67. [CrossRef]

196. Chiang, J.Y. Bile acid metabolism and signaling in liver disease and therapy. Liver Res. 2017, 1, 3-9. [CrossRef]

197. Leung, C.; Rivera, L.; Furness, J.B.; Angus, P.W. The role of the gut microbiota in NAFLD. Nat. Rev. Gastroenterol. Hepatol. 2016, 13, 412-425. [CrossRef]

198. Wang, L.; Wan, Y.-J.Y. The role of gut microbiota in liver disease development and treatment. Liver Res. 2019, 3, 3-18. [CrossRef]

199. Jones, B.V.; Begley, M.; Hill, C.; Gahan, C.G.; Marchesi, J.R. Functional and comparative metagenomic analysis of bile salt hydrolase activity in the human gut microbiome. Proc. Natl. Acad. Sci. USA 2008, 105, 13580-13585. [CrossRef]

200. Xie, C.; Jiang, C.; Shi, J.; Gao, X.; Sun, D.; Sun, L.; Wang, T.; Takahashi, S.; Anitha, M.; Krausz, K.W.; et al. An Intestinal Farnesoid X Receptor-Ceramide Signaling Axis Modulates Hepatic Gluconeogenesis in Mice. Diabetes 2016, 66, 613-626. [CrossRef] 
201. Jiang, C.; Xie, C.; Li, F.; Zhang, L.; Nichols, R.G.; Krausz, K.W.; Cai, J.; Qi, Y.; Fang, Z.-Z.; Takahashi, S.; et al. Intestinal farnesoid $X$ receptor signaling promotes nonalcoholic fatty liver disease. J. Clin. Investig. 2014, 125, 386-402. [CrossRef] [PubMed]

202. Gao, Z.; Yin, J.; Zhang, J.; Ward, R.E.; Martin, R.J.; Lefevre, M.; Cefalu, W.T.; Ye, J. Butyrate Improves Insulin Sensitivity and Increases Energy Expenditure in Mice. Diabetes 2009, 58, 1509-1517. [CrossRef] [PubMed]

203. Besten, G.D.; Van Eunen, K.; Groen, A.K.; Venema, K.; Reijngoud, D.-J.; Bakker, B.M. The role of short-chain fatty acids in the interplay between diet, gut microbiota, and host energy metabolism. J. Lipid Res. 2013, 54, 2325-2340. [CrossRef] [PubMed]

204. Liu, S.; Gallo, D.J.; Green, A.M.; Williams, D.L.; Gong, X.; Shapiro, R.A.; Gambotto, A.A.; Humphris-Narayanan, E.; Vodovotz, Y.; Billiar, T.R. Role of Toll-Like Receptors in Changes in Gene Expression and NF-кB Activation in Mouse Hepatocytes Stimulated with Lipopolysaccharide. Infect. Immun. 2002, 70, 3433-3442. [CrossRef] [PubMed]

205. Hoang, S.A.; Oseini, A.; Feaver, R.E.; Cole, B.K.; Asgharpour, A.; Vincent, R.; Siddiqui, M.S.; Lawson, M.J.; Day, N.; Taylor, J.; et al. Gene Expression Predicts Histological Severity and Reveals Distinct Molecular Profiles of Nonalcoholic Fatty Liver Disease. Sci. Rep. 2019, 9, 12541. [CrossRef] [PubMed]

206. Arendt, B.M.; Comelli, E.M.; Ma, D.W.; Lou, W.; Teterina, A.; Kim, T.; Fung, S.K.; Wong, D.K.; McGilvray, I.D.; Fischer, S.E.; et al. Altered hepatic gene expression in nonalcoholic fatty liver disease is associated with lower hepatic n-3 and n-6 polyunsaturated fatty acids. Hepatology 2015, 61, 1565-1578. [CrossRef]

207. Cichocki, J.A.; Furuya, S.; Konganti, K.; Luo, Y.-S.; McDonald, T.J.; Iwata, Y.; Chiu, W.A.; Threadgill, D.W.; Pogribny, I.P.; Rusyn, I. Impact of Nonalcoholic Fatty Liver Disease on Toxicokinetics of Tetrachloroethylene in Mice. J. Pharmacol. Exp. Ther. 2017, 361, 17-28. [CrossRef]

208. Maeso-Diaz, R.; Boyer-Diaz, Z.; Lozano, J.J.; Ortega-Ribera, M.; Peralta, C.; Bosch, J.; Gracia-Sancho, J. New rat model of advanced nash mimicking pathophysiological features and transcriptomic signature of the human disease. Cells 2019, 8, 1062. [CrossRef]

209. Qin, G.; Wang, G.Z.; Guo, D.D.; Bai, R.-X.; Wang, M.; Du, S.Y. Deletion of Smad4 reduces hepatic inflammation and fibrogenesis during nonalcoholic steatohepatitis progression. J. Dig. Dis. 2018, 19, 301-313. [CrossRef]

210. Valenti, L.; Mendoza, R.M.; Rametta, R.; Maggioni, M.; Kitajewski, C.; Shawber, C.J.; Pajvani, U.B. Hepatic notch signaling correlates with insulin resistance and nonalcoholic fatty liver disease. Diabetes 2013, 62, 4052-4062. [CrossRef]

211. Liang, N.; Damdimopoulos, A.; Goni, S.; Huang, Z.; Vedin, L.L.; Jakobsson, T.; Giudici, M.; Ahmed, O.; Pedrelli, M.; Barilla, S.; et al. Hepatocyte-specific loss of GPS2 in mice reduces non-alcoholic steatohepatitis via activation of PPAR $\alpha$. Nat. Commun. 2019, 10, 1684. [CrossRef] [PubMed]

212. Porteiro, B.; Fondevila, M.F.; Delgado, T.C.; Iglesias, C.; Imbernon, M.; Iruzubieta, P.; Crespo, J.; Zabala-Letona, A.; Ferno, J.; Gonzalez-Teran, B.; et al. Hepatic p63 regulates steatosis via IKK $\beta /$ ER stress. Nat. Commun. 2017, 8, 15111. [CrossRef] [PubMed]

213. Li, Z.; Yu, P.; Wu, J.; Tao, F.; Zhou, J. Transcriptional regulation of early growth response gene-1 (EGR1) is associated with progression of nonalcoholic fatty liver disease (NAFLD) in patients with insulin resistance. Med. Sci. Monit. 2019, 25, 2293-3004. [CrossRef] [PubMed]

214. Cheng, C.; Deng, X.; Xu, K. Increased expression of sterol regulatory element binding protein-2 alleviates autophagic dysfunction in NAFLD. Int. J. Mol. Med. 2018, 41, 1877-1886. [CrossRef]

215. Kabbani, M.; Michailidis, E.; Steensels, S.; Zou, C.H.; Zeck, B.; Inna, R.L.; Fulmer, C.G.; Quirk, C.; Ashbrook, A.W.; Belkaya, S.; et al. PNPLA3-148M Overexpression in primary human hepatocytes exacerbates steatosis in tissue culture and chimeric mouse models of NAFLD. Hepatology 2019, 70, 1325A-1326A.

(C) 2020 by the authors. Licensee MDPI, Basel, Switzerland. This article is an open access article distributed under the terms and conditions of the Creative Commons Attribution (CC BY) license (http://creativecommons.org/licenses/by/4.0/). 



\title{
Distinguishing NASH Histological Severity Using a Multiplatform Metabolomics Approach
}

\author{
George N. Ioannou ${ }^{1,2,3, *}$, G. A. Nagana Gowda ${ }^{4}$, Danijel Djukovic ${ }^{4}$ and Daniel Raftery ${ }^{4,5,6, *}$ \\ 1 Division of Gastroenterology, Veterans Affairs Puget Sound Healthcare System and University of \\ Washington, Seattle, WA 98108, USA \\ 2 Department of Medicine, Veterans Affairs Puget Sound Healthcare System and University of Washington, \\ Seattle, WA 98108, USA \\ 3 Research and Development, Veterans Affairs Puget Sound Healthcare System, Seattle, WA 98108, USA \\ 4 Northwest Metabolomics Research Center, Anesthesiology and Pain Medicine, University of Washington, \\ Seattle, WA 98109, USA; ngowda@uw.edu (G.A.N.G.); djukovic@uw.edu (D.D.) \\ 5 Department of Chemistry, University of Washington, Seattle, WA 98195, USA \\ 6 Fred Hutchinson Cancer Research Center, Seattle, WA 98109, USA \\ * Correspondence: GeorgeI@medicine.washington.edu (G.N.I.); draftery@uw.edu (D.R.); \\ Tel.: +1-206-277-3136 (G.N.I.); +1-206-543-9709 (D.R.)
}

Received: 10 April 2020; Accepted: 21 April 2020; Published: 24 April 2020

\begin{abstract}
Nonalcoholic fatty liver disease (NAFLD) is categorized based on histological severity into nonalcoholic fatty liver (NAFL) or nonalcoholic steatohepatitis (NASH). We used a multiplatform metabolomics approach to identify metabolite markers and metabolic pathways that distinguish NAFL from early NASH and advanced NASH. We analyzed fasting serum samples from 57 prospectively-recruited patients with histologically-proven NAFLD, including 12 with NAFL, 31 with early NASH and 14 with advanced NASH. Metabolite profiling was performed using a combination of liquid chromatography-mass spectrometry (LC-MS) and nuclear magnetic resonance (NMR) spectroscopy analyzed with multivariate statistical and pathway analysis tools. We targeted 237 metabolites of which 158 were quantified. Multivariate analysis uncovered metabolite profile clusters for patients with NAFL, early NASH, and advanced NASH. Also, multiple individual metabolites were associated with histological severity, most notably spermidine which was more than 2-fold lower in advanced fibrosis vs. early fibrosis, in advanced NASH vs. NAFL and in advanced NASH vs. early NASH, suggesting that spermidine exercises a protective effect against development of fibrosing NASH. Furthermore, the results also showed metabolic pathway perturbations between early-NASH and advanced-NASH. In conclusion, using a combination of two reliable analytical platforms (LC-MS and NMR spectroscopy) we identified individual metabolites, metabolite clusters and metabolic pathways that were significantly different between NAFL, early-NASH, and advanced-NASH. These differences provide mechanistic insights as well as potentially important metabolic biomarker candidates that may noninvasively distinguish patients with NAFL, early-NASH, and advanced-NASH. The associations of spermidine levels with less advanced histology merit further assessment of the potential protective effects of spermidine in NAFLD.
\end{abstract}

Keywords: nonalcoholic fatty liver; nonalcoholic steatohepatitis; liquid chromatography-mass spectrometry; nuclear magnetic resonance spectroscopy; metabolic pathway

\section{Introduction}

Nonalcoholic fatty liver disease (NAFLD) is strongly associated with the metabolic syndrome and each of its components [1,2]. In fact, NAFLD is regarded as the hepatic manifestation of the 
metabolic syndrome [1]. By definition, all patients with NAFLD have excessive lipid deposition within lipid droplets in hepatocytes, a condition known as hepatic steatosis. However, patients with NAFLD can exhibit a very broad spectrum of hepatic inflammation and fibrosis ranging from almost none (a condition known as simple steatosis or "nonalcoholic fatty liver" (NAFL)) to variable degrees of inflammation and fibrosis (known as "nonalcoholic steatohepatitis" or "NASH") to established cirrhosis [3,4]. Patients with NAFL have a very low probability of progression to cirrhosis or hepatocellular carcinoma (HCC). On the other hand, patients with NASH have significantly higher probability of progression [4,5]. Furthermore, among the histological features that distinguish NASH, hepatic fibrosis is emerging as the feature most strongly associated with adverse liver-related outcomes and even all-cause mortality, rather than inflammation or the presence of balloon hepatocytes [6,7]. Therefore, there is a pressing clinical need to understand the factors that lead to the development of fibrosing NASH, rather than NAFL and to identify non-invasive tests that can distinguish the presence of NASH versus simple steatosis. Given the strong link between metabolic factors and NAFLD, we hypothesized that serum metabolite profiling using metabolomics could identify serum metabolites that distinguish NAFL from NASH.

The growing field of metabolomics describes the study of concentrations and fluxes of low molecular weight metabolites (molecular weight $<1000 \mathrm{Da}$ ) present in biofluids or tissue that provide detailed information for understanding biological phenotypes, deciphering mechanisms, and identifying biomarkers or drug targets for a variety of conditions [8-10]. Utilization of two powerful analytical platforms, liquid chromatography-mass spectrometry (LC-MS) [11] and nuclear magnetic resonance (NMR) [12] spectroscopy, leads to the quantitative analysis of hundreds of small molecules on a relatively routine basis. In the area of liver diseases, to date, several cross-sectional studies have demonstrated the potential for serum or plasma-derived metabolite biomarkers to distinguish different liver diseases and hepatocellular carcinoma (HCC) [13-19]. Metabolomics studies in this area have focused on both early biomarker detection as well as identification of altered metabolic pathways [20]. It has been shown that metabolite-based biomarkers can distinguish HCC from cirrhosis better than the conventional marker, alpha-fetoprotein (AFP) $[14,21]$. However, metabolomic investigations focused on distinguishing high risk NASH from NAFL are scarce.

In the current study, metabolite profiling of serum samples employing both MS and NMR spectroscopy methods, in combination with multivariate statistical methods, was performed to identify serum metabolites or metabolite profiles that distinguish simple steatosis from NASH, or even early NASH from advanced NASH. Our results show promise for developing metabolite profiles to stage patients as well as providing insights on the pathogenesis and progression of NAFLD.

\section{Results}

\subsection{Patient Characteristics}

Among 57 patients with biopsy-proven NAFLD included in this study, the mean age was 51 years and the majority were white and male-consistent with recruitment from a VA hospital (Table 1). As expected in patients with NAFLD, diabetes was very common, mean BMI was in the range of obesity and mean serum AST and ALT were mildly elevated (Table 1).

By study design, patients with early-NASH and advanced-NASH had progressively increasing NAS, ballooning degeneration, inflammation, steatosis, and fibrosis scores compared to patients with simple steatosis. Patients with early-NASH had a mean NAS score of $3.3 \pm 0.7$ and fibrosis stage of $0.8 \pm 0.6$. Patients with advanced-NASH had a mean NAS score of $5.7 \pm 0.6$ and fibrosis stage of $2.0 \pm 0.9$. 
Table 1. Demographic and clinical characteristics of study participants divided according to histological severity of nonalcoholic fatty liver disease (NAFLD) (simple steatosis, early-nonalcoholic steatohepatitis (NASH), advanced-NASH).

\begin{tabular}{|c|c|c|c|}
\hline Parameter & $\begin{array}{c}\text { Simple Steatosis } \\
n=12\end{array}$ & $\begin{array}{c}\text { Early NASH } \\
n=31\end{array}$ & $\begin{array}{c}\text { Advanced NASH } \\
n=14\end{array}$ \\
\hline Age (yrs), mean (SD) & $50.2(12)$ & $50.4(9.5)$ & $52.3(9.1)$ \\
\hline \multicolumn{4}{|l|}{ Race: } \\
\hline White, non-Hispanic (\%) & $100 \%$ & $73 \%$ & $87 \%$ \\
\hline Black, non-Hispanic (\%) & $0 \%$ & $7 \%$ & $0 \%$ \\
\hline Other $(\%)$ & $0 \%$ & $10 \%$ & $3 \%$ \\
\hline Not-declared (\%) & $0 \%$ & $10 \%$ & $10 \%$ \\
\hline Male (\%) & $92 \%$ & $93 \%$ & $80 \%$ \\
\hline Diabetes (\%) & $75 \%$ & $71 \%$ & $40 \%$ \\
\hline BMI $\left(\mathrm{Kg} / \mathrm{m}^{2}\right)$, mean $(\mathrm{SD})$ & $35(7)$ & $33(5)$ & $34(6)$ \\
\hline \multicolumn{4}{|l|}{ Serum Laboratory Tests, mean (SD): } \\
\hline AST (U/L) & $39(32)$ & $39(20)$ & $66(39)$ \\
\hline ALT (U/L) & $50(30)$ & $62(32)$ & $97(42)$ \\
\hline Albumin $(\mathrm{g} / \mathrm{dL})$ & $4.4(0.4)$ & $4.6(0.2)$ & $4.6(0.2)$ \\
\hline Bilirubin $(\mathrm{g} / \mathrm{dL})$ & $0.7(0.4)$ & $0.5(0.1)$ & $0.5(0.3)$ \\
\hline \multicolumn{4}{|l|}{ Liver Histology: } \\
\hline Steatosis Grade $0 / 1 / 2 / 3$ & $0 / 7 / 4 / 1$ & $0 / 19 / 11 / 1$ & $0 / 1 / 9 / 4$ \\
\hline Inflammation Grade 0/1/2/3 & $3 / 9 / 0 / 0$ & $0 / 30 / 1 / 0$ & $0 / 4 / 10 / 0$ \\
\hline Ballooning Degeneration 0/1/2 & $12 / 0 / 0$ & $0 / 27 / 4$ & $0 / 1 / 13$ \\
\hline Fibrosis Stage 0/1/2/3/4 & $12 / 0 / 0 / 0 / 0$ & $10 / 21 / 0 / 0 / 0$ & $0 / 4 / 6 / 3 / 1$ \\
\hline NAS Score (0-8), mean (SD) & $2.4(0.8)$ & $3.3(0.7)$ & $5.7(0.6)$ \\
\hline
\end{tabular}

\subsection{Serum Metabolite Profiles Cluster According to NAFL, Early-NASH and Advanced-NASH}

Serum metabolite profiling using LC-MS and NMR techniques targeted 237 metabolites. Of these, 158 metabolites were quantified, 106 metabolites by MS (Table S1) and 52 metabolites by NMR (Table S2). Partial least squares-discriminant analysis (PLS-DA) results are shown as score plots in Figure 1. Each circle or point in the score plot represents one patient. The points (also known as scores) for patients that exhibit similar metabolite profiles in serum appear closer to each other and vice versa. Ideally, the scores for patients with NAFL, early NASH and advanced NASH will form separate and distinct clusters owing to the potential differences in metabolic profiles between the groups. In our study, multivariate analysis showed clustering of the serum metabolite profiles of patients depending on the histological severity of NAFLD (NAFL vs. NASH and early-NASH, vs. advanced-NASH) as shown in Figure 1a-c. In particular, the metabolite profiles of NAFL and NASH showed a distinct clustering with minimal overlap of the scores for patients (Figure 1a); the dispersion of the scores was greater for NASH compared to NAFL. However, results for the analysis of the data after dividing NASH patients into early NASH and advanced NASH showed significant improvement in the separation; both early NASH vs. NAFL as well as advanced NASH vs. NAFL exhibited distinct clusters (Figure $1 b, c)$. 

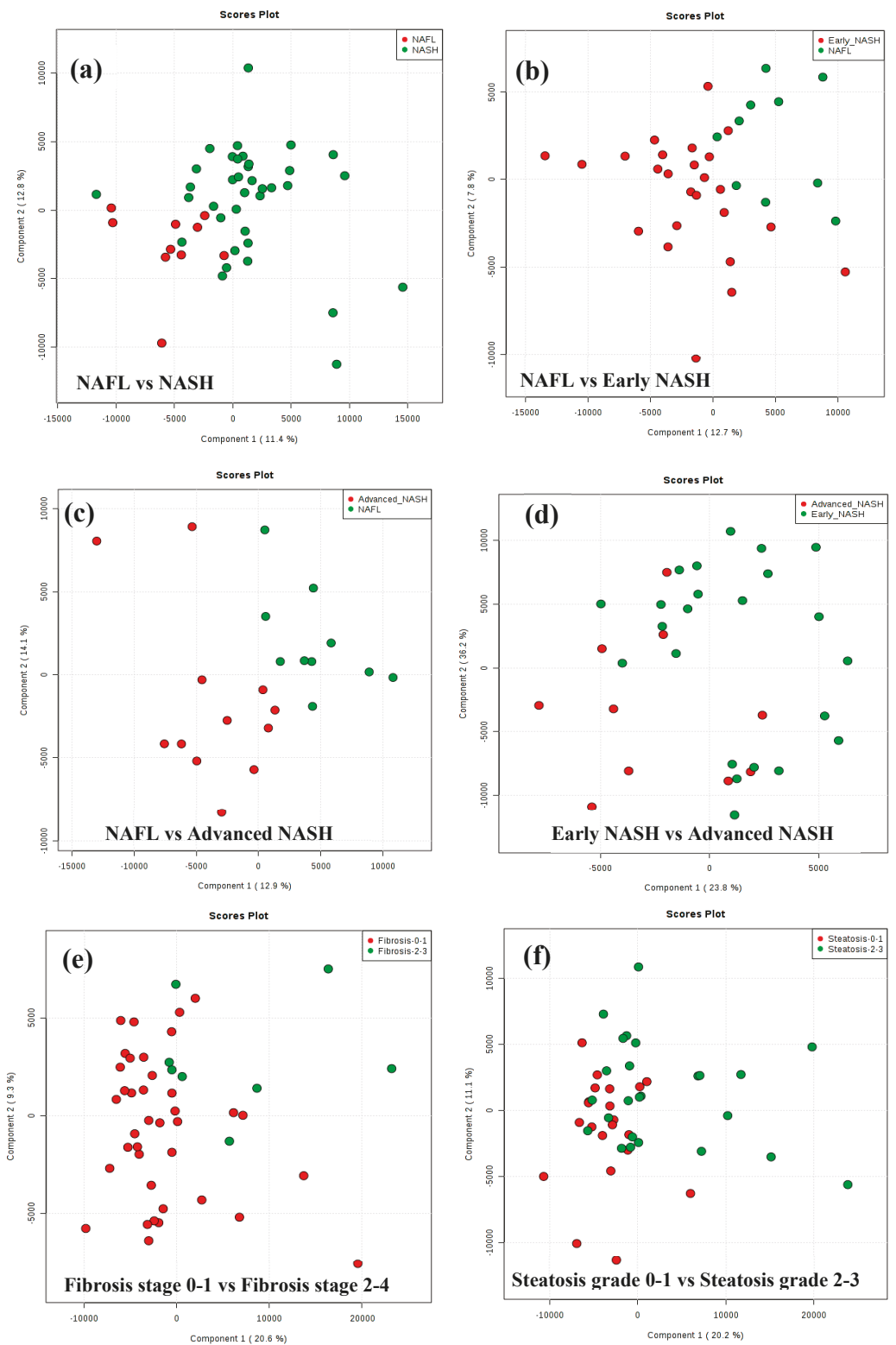

Figure 1. The results of PLS-DA analysis of metabolite levels derived from MS are shown as score plots. Each red or green point in the score plot represents one patient. In these plots, points (known as scores) for patients that exhibit similar metabolite profiles will appear closer to each other and vice versa. The score plots show good clustering and separation of the patients with (a) nonalcoholic fatty liver (NAFL) vs. NASH; (b) NAFL vs. early NASH; (c) NAFL vs. advanced NASH; (d) Early NASH vs. advanced NASH; (e) Fibrosis stage $0-1$ vs. Fibrosis stage $2-4$; and (f) Steatosis grade $0-1$ vs. Steatosis grade $2-3$. 
Multivariate statistical results also reflected the metabolic differences between early NASH and advanced NASH; a comparison between the two groups showed separate clusters, although there was some degree of overlap (Figure 1d). In addition, analysis performed after subdividing the patients according to fibrosis stage (0-1 vs. $2-4)$ or steatosis grade (0-1 vs. $2-3$ ) also showed clusters, although the two clusters were again partially overlapped (Figure 1e,f).

\subsection{Individual Metabolites were Associated with Histological Severity in NAFLD}

Univariate analysis identified 11 metabolites that were significantly different between NAFL and NASH with fold changes ranging from 0.57 to 3.13 (Table 2). Nine metabolites were significantly different between NAFL and early-NASH (with fold changes from 0.83 to 1.19) and ten were significantly different between NAFL and advanced-NASH (with FC from 0.33 to 2.38). Six metabolites were significantly different between early-NASH and advanced-NASH with FC from 0.49 to 1.33 ; further, the metabolites that distinguished early-NASH from advanced-NASH were largely different from the ones that distinguished NAFL from early-NASH (Table 2). In addition, we identified eight metabolites that were significantly different between fibrosis stages $0-1$ and fibrosis stages $2-4$ and four significantly different between steatosis grades $0-1$ and steatosis grades $2-3$. (Table 3 ). The most notable example was spermidine, which was more than 2-fold decreased in advanced fibrosis vs. early fibrosis, in advanced NASH vs. NAFL, and in advanced NASH vs. early NASH, suggesting that spermidine exercises a protective effect against development of fibrosing NASH.

Table 2. Metabolites that differed significantly between patients with NAFL $(n=12)$, early NASH $(n=31)$ and advanced NASH $(n=14)$.

\begin{tabular}{|c|c|c|c|c|c|c|c|}
\hline \multicolumn{4}{|c|}{ NASH vs. NAFL } & \multicolumn{4}{|c|}{ Early NASH vs. NAFL } \\
\hline Metabolite & $p$ Value & $\begin{array}{c}\text { Fold * } \\
\text { Change }\end{array}$ & Method & Metabolite & $p$ Value & $\begin{array}{c}\text { Fold * } \\
\text { Change }\end{array}$ & Method \\
\hline Acetylglycine & 0.03 & 0.57 & MS & Hydroxyphenylpyruvate & 0.002 & 0.83 & MS \\
\hline Cysteine & 0.04 & 0.88 & MS & Inositol & 0.03 & 0.86 & MS \\
\hline Alanine & 0.02 & 0.96 & NMR & Cysteine & 0.04 & 0.87 & MS \\
\hline Glucose & 0.04 & 1.16 & MS & Acetylcarnitine & 0.04 & 0.90 & MS \\
\hline Erythrose & 0.02 & 1.18 & MS & Phenylalanine & 0.03 & 1.12 & NMR \\
\hline Tyrosine & 0.01 & 1.18 & NMR & Tyrosine & 0.02 & 1.18 & NMR \\
\hline Isovaleric acid & 0.02 & 1.25 & MS & Erythrose & 0.04 & 1.18 & MS \\
\hline Leucic acid & 0.04 & 1.28 & MS & Alanine & 0.03 & 1.18 & NMR \\
\hline Xanthine & 0.02 & 1.49 & MS & Tryptophan & 0.04 & 1.19 & NMR \\
\hline Oxypurinol & 0.01 & 1.54 & MS & & & & \\
\hline Glycochenodeoxycholate & 0.04 & 3.13 & MS & & & & \\
\hline \multicolumn{4}{|c|}{ Advanced NASH vs. Early NASH } & \multicolumn{4}{|c|}{ Advanced NASH vs. NAFL } \\
\hline Metabolite & $p$ Value & $\begin{array}{c}\text { Fold * } \\
\text { Change }\end{array}$ & Method & Metabolite & $p$ Value & $\begin{array}{c}\text { Fold * } \\
\text { Change }\end{array}$ & Method \\
\hline Spermidine & 0.005 & 0.49 & MS & Spermidine & 0.005 & 0.33 & MS \\
\hline Oxaloacetate & 0.01 & 0.85 & MS & Acetylglycine & 0.01 & 0.48 & MS \\
\hline Orotate & 0.0009 & 0.85 & MS & Glucose & 0.04 & 1.20 & MS \\
\hline Linoleic acid & 0.01 & 1.32 & MS & Isovaleric acid & 0.04 & 1.30 & MS \\
\hline Linolenic acid & 0.01 & 1.33 & MS & Leucic acid & 0.02 & 1.30 & MS \\
\hline \multirow[t]{5}{*}{ 2-hydroxyglutarate } & 0.01 & 1.33 & MS & 2-hydroxyisovaleric acid & 0.03 & 1.49 & MS \\
\hline & & & & Xanthine & 0.04 & 2.08 & MS \\
\hline & & & & Oxypurinol & 0.04 & 2.17 & MS \\
\hline & & & & Glycocholate & 0.02 & 2.22 & MS \\
\hline & & & & Glycochenodeoxycholate & 0.01 & 2.38 & MS \\
\hline
\end{tabular}

* Fold changes shown are the ratios of NASH/ NAFL; Early NASH/ NAFL; Advanced NASH/ NAFL; Advanced NASH/ Early NASH. They are ordered from the lowest ratio (i.e., most "protective" against advanced disease) to the highest ratio (i.e., most highly associated with advanced disease). 
Table 3. Metabolites that differed significantly between patients with different levels of hepatic fibrosis (F0-1 $(n=43)$ vs. F2-4 $(n=14)$ or steatosis (grade $0-1(n=27)$ vs. grade $2-3(n=30))$.

\begin{tabular}{cccccccc}
\hline \multicolumn{3}{c}{ Fibrosis Stage 2-4 vs. Fibrosis Stage 0-1 } & \multicolumn{3}{c}{ Steatosis Grade 2-3 vs. Steatosis Grade 0-1 } \\
\hline Metabolite & $p$ Value & $\begin{array}{c}\text { Fold } \\
\text { Change } \\
\end{array}$ & Method & Metabolite & $p$ Value & $\begin{array}{c}\text { Fold } \\
\text { Change }\end{array}$ & Method \\
Spermidine & 0.0008 & 0.47 & MS & Erythrose & 0.01 & 1.19 & MS \\
N-acetylglycine & 0.001 & 0.63 & MS & Mannose & 0.002 & 1.33 & NMR \\
Oxaloacetate & 0.004 & 0.83 & MS & Isovaleric acid & 0.01 & 1.33 & MS \\
Orotate & 0.01 & 0.85 & MS & Glucose & $0.02 / 0.005$ & $1.37 / 1.22$ & NMR/MS \\
Adipic acid & 0.03 & 1.20 & MS & & & & \\
Sucrose & 0.04 & 1.20 & MS & & & & \\
Aconitate & 0.03 & 1.23 & MS & & & & \\
Azelaic acid & 0.04 & 1.25 & MS & & & & \\
\hline
\end{tabular}

* The fold changes are the ratios of Fibrosis stage 2-4/Fibrosis stage 0-1; Steatosis grade 2-3/Steatosis grade 0-1. They are ordered from the lowest ratio (i.e., most "protective" against advanced disease) to the highest ratio (i.e., most highly associated with advanced disease).

\subsection{Metabolic Pathways and Metabolite Enrichment Distinguished NAFL, Early-NASH, Advanced-NASH}

Metabolic pathway enrichment analysis was performed using the Metaboanalyst software platform [22-24]. The results are shown in Figure S1, where each pathway is shown as a circle. The color of the circle indicates the significance of the pathway with red being the most significant $(p<0.05)$ and the size of the circle indicates the impact of the pathway. Pathway analysis made based on 106 MS derived metabolite levels showed a total of 56 matched pathways and the analysis based on 52 NMR derived metabolites showed 48 matched metabolic pathways. A number of these pathways were significantly altered $(p<0.05)$ between different groups of patients (Table S3). All comparisons except NAFL vs. advanced NASH showed significant differences in at least one pathway. In particular, the differences between early-NASH and advanced-NASH, and between steatosis grade 0,1 and steatosis grade 2,3 were more significant than between the other groups; the former exhibited the most number of altered pathways and the latter exhibited the most number of pathways that were highly significant $(p \leq 0.003)$ (Table S3, Figure S1).

Separately, metabolite set enrichment analysis was performed using quantitative metabolite data. Both advanced NASH vs. NAFL and early NASH vs. advanced NASH identified 31 sets of metabolites (Figure 2; Figure S2). For advanced NASH vs. NAFL, two sets that correspond to beta-alanine metabolism and arginine and proline metabolism exhibited high significance; the $p$ value adjusted using FDR for both sets was 0.02 (Figure 2a). On the other hand, for early NASH vs. advanced NASH (Figure 2b), one set that corresponds to butanoate metabolism exhibited high significance; as shown in the figure, its $p$ value adjusted using FDR was 0.05 . 
(a) Advanced NASH vs. NAFL

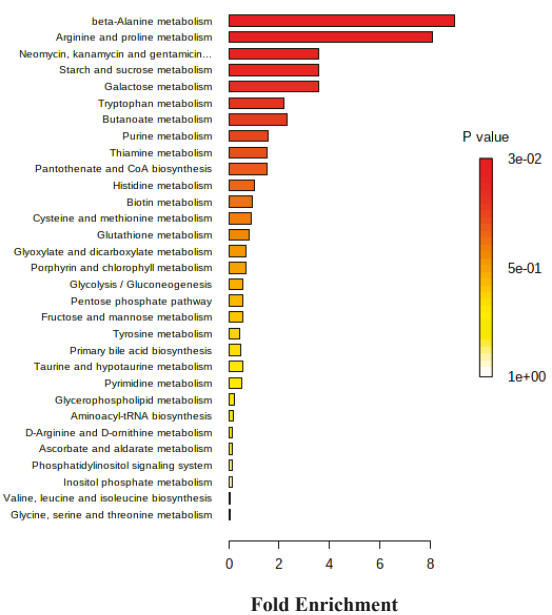

(b) Early NASH vs. Advanced NASH

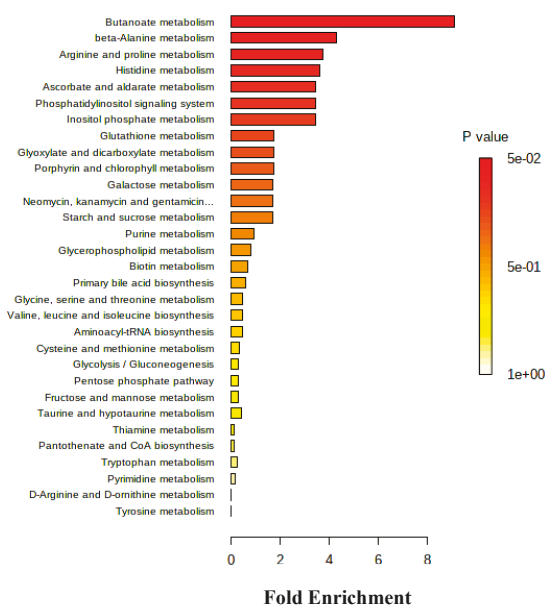

Figure 2. Biological patterns identified from metabolite set enrichment analysis. Metabolite data were derived from MS. The enrichment analysis combines functionally related metabolites to discern consistent changes among the related metabolites. The color and the bar length indicate $p$ value and the fold enrichment, respectively. (a) In the advanced NASH vs. NAFL comparison, the analysis identified 31 sets, of which two sets that correspond to beta-alanine metabolism and arginine and proline metabolism exhibited high significance; the $p$ value adjusted using false discovery rate for both sets was 0.02. (b) In the early NASH vs. advanced NASH comparison, 31 sets were also identified, of which one set that corresponds to butanoate metabolism exhibited high significance; its $p$ value adjusted using false discovery rate was 0.05 .

\subsection{Association of the GSG-Index with Histological Severity}

Neither the GSG-index nor its amino acids, individually, discriminated between NAFLD and NASH (data not shown). They also did not discriminate either between mild (stage 0,1 ) and advanced fibrosis (stage 2-4) or between mild (grade 0,1 ) and advanced steatosis (grade 2, 3). However, the alanine, aspartate, and glutamate metabolism pathway, which involves one of the amino acids of the GSG index was altered between early NASH and advanced NASH and between hepatic fibrosis stage $0-1$ and Fibrosis stage 2-3 (Table S3). In addition, glycine, serine, and threonine metabolism, which involves glycine as one of the amino acids of the GSG index was altered between hepatic fibrosis stage $0-1$ and Fibrosis stage $2-4$.

\subsection{Evaluation of the Confounding Effects of Gender and Diabetes on the Biomarkers of NASH/NAFL}

Female patients were minimally differentiated in the multivariate statistical analysis results as shown in the Supplementary Figure S3a. Univariate analysis results showed that 17 metabolites were statistically different $(p<0.05)$ between male vs. female patients (Table S4). However, none of these metabolites was common to the biomarkers of NASH/NAFL disease patients (Table 2). Similarly, evaluation of metabolite signatures that differ between diabetes and non-diabetes patients showed minor differences as seen from the partial overlap of the two clusters in the Supplementary Figure S3b. Ten metabolites were statistically different between diabetes and non-diabetes patients (Table S5), of which 4 metabolites (isovaleric acid, oxypurinol, xanthine and sucrose) were common to those observed for NASH/NAFL disease (Table 2). 


\section{Discussion}

There is great interest in identifying patients with NAFLD who are at risk of having, or progressing to, histologically advanced disease. Our goal was to identify serum metabolite profiles or metabolite pathways that distinguished NAFL, early-NASH and advanced-NASH using targeted LC-MS and quantitative NMR-based metabolomics approaches, combined with multivariate statistical methods. Serum samples obtained from patients after overnight fasting were used to avoid potential interferences from diet. For a global visualization of the data, multivariate statistical analysis was utilized and showed distinct clustering of patients, which provides evidence for the metabolic differences between NAFLD patients grouped by histological severity (Figure 1). In particular, the separation of the clusters between NAFL and NASH reveals a large difference in metabolism between these groups and the ability of the serum markers to identify the high risk NASH patients. We also found a significant difference between early NASH and advanced NASH, which indicates the potential to identify the patients at highest risk.

The results that many individual metabolite levels are significantly different $(p<0.05)$ between patients with NASH vs. NAFL not only substantiate the results of multivariate statistical analysis but also provide more specific information on altered cellular metabolism (Table 2). Among the many metabolites that were altered significantly, notable metabolic perturbations are the more than 2-fold reduction of spermidine and acetylglycine, and the more than 2-fold increase of oxypurinol, xanthine and bile acids (glycocholate and glycochenodeoxycholate) in advanced NASH compared to NAFL or early NASH (Table 2). Spermidine, which is found in mushrooms, aged cheese, soybeans, legumes and whole grains, has recently been shown to prevent liver fibrosis and hepatocellular carcinoma and to ameliorate fatty liver disease in mouse models [25]. However, we are not aware of other human studies suggesting a protective effect of spermidine against NASH. We found that levels of spermidine were more than two times lower consistently when comparing advanced fibrosis vs. early fibrosis, or advanced NASH vs. NAFL, or advanced NASH vs. early NASH. This suggests that spermidine might exercise a protective effect against development of fibrosing NASH and should be confirmed in future human studies.

A number of studies have shown the association of acetylglycine with liver disease; for example, its increased level was observed in liver tissue, serum as well as urine due to drug induced injury to the liver [26] or liver disease induced by alcohol $[27,28]$. Oxypurinol is an inhibitor of xanthine oxidase and is monitored to assess liver function [29]. The large changes observed for these metabolite levels in our study indicates their potential utility as distinguishing markers in clinical management of the NASH patients, as such large perturbations are more likely to hold upon independent validation.

Metabolic pathway analyses enabled global visualization of alterations in metabolism between the NAFLD patient groups. In particular, the results indicate that while the measured serum metabolites mapped to a large number of metabolic pathways, only six were significantly different between NASH and NAFL (Table S3). The most significant among the pathways is fatty acid biosynthesis. Our results indicate that the fatty acid synthesis pathway is associated with NAFLD, which is in accordance with previous findings that showed hepatic accumulation of triglycerides [30]. Taurine and hypotaurine metabolism is another pathway that distinguishes NASH from NAFL. It is well known that bile acid synthesis is one of the major functions of the liver and taurine conjugated bile acids are a major component in human bile [31]. Altered metabolism of taurine may indicate its association with altered bile acids synthesis in NASH. In accordance with the altered pathway, our univariate analysis results show that two primary bile acids, glycocholate and glycochenoxycholate differ significantly between advanced NASH and simple steatosis; both bile acids were higher in NASH by more than 2-fold (Table 2).

Metabolite set enrichment analysis enabled identification of biologically meaningful patterns for NAFLD patients. This analysis combines functionally related metabolites to discern consistent changes among the related metabolites. Although, patterns with high significance $(p<0.05)$ were identified for many groups of NAFLD patients, only two groups, advanced NASH vs. NAFL and early NASH vs. 
advanced NASH, showed patterns that exhibited high significance $(p<0.05)$ even after adjusting the $p$ value using the false discovery rate (FDR) (Figure 2; Figure S2). Beta-alanine metabolism and arginine and proline metabolism were significantly altered for advanced NASH vs. NAFL (Figure 2a), whereas butanoate metabolism was significantly altered for early NASH vs. advanced NASH (Figure 2b). In terms of specific metabolites, spermidine contributed the most for advanced NASH vs. NAFL, which is in agreement with its down regulation in advanced NASH vs. NAFL (Table 2). On the other hand, 2-hydroxyglutrate contributed the most for early NASH vs. advanced NASH, which is also in accordance with its upregulation in advanced NASH vs. early NASH (Table 2).

We also found that metabolism is altered significantly depending on the degree of hepatic fibrosis or steatosis. These differences were significant and observed in the: (A) multivariate statistical analysis results, where clusters were clearly visible (Figure 1e,f); (B) univariate analysis results, where many metabolites are significantly different (Table 3); and (C) metabolic pathway analysis, where several pathways were significantly impacted (Figure S1d,e, Table S3). Overall, the results indicate that metabolic profiling is sensitive to differences in fibrosis and steatosis, the two most important histological features of NAFLD.

Numerous studies indicate serum amino acids levels, particularly, glutamate, glycine and serine, are altered in liver diseases [32-37]. More recently, the ratio of glutamate to the sum of serine and glycine, termed the GSG-index, was tested for its association with blood levels of BCCAs, ALT, AST, and GGT [38]. Amino acids of the GSG-index are critical for the synthesis of glutathione (GSH), which is the major antioxidant that controls oxidative stress in the liver [39]. The GSG-index was moderately correlated with ALT $(r=0.34 \pm 0.13)$ and AST $(r=0.45 \pm 0.12)$. However, in our study the GSG-index did not discriminate between NAFLD vs. NASH or between mild (stage $0-1$ ) and more advanced (stage 2-4) liver fibrosis. In the prior study the GSG-index was shown to be significantly associated with stage $3-4$ vs. stage $0-2$ fibrosis (rather than stage $2-4$ vs. $0-1$ as we tested). Future, larger studies will need to further evaluate whether the GSG-index is a marker of histological severity in NAFLD.

Considering the lack of metabolomics studies that focus on identifying biomarkers that distinguish NASH from NAFLD, our results that demonstrate numerous altered metabolites and metabolic pathways between the two groups are potentially of high significance. In prior studies, urine and serum were used to distinguish different stages of NAFLD based on metabolomics [40]. Some of the findings, such as the higher levels of xanthine and tryptophan in NASH as well as the association of energy metabolism and amino acid metabolism with the pathological processes in NASH are in accordance with our findings (Table 2, Table S3). Another recent study using mice as well as patient samples identified several metabolites that distinguished simple steatosis from NASH [41]. Metabolic signatures including fatty acids, amino acids and bile acids that differentiate NAFL and NASH were detected, and these findings are somewhat in accordance with our results. A study of blood plasma, rather than serum, showed metabolic differences between controls vs. NAFLD as well as control vs. NASH; however, it could not identify a distinguishing metabolite profile between steatosis and NASH [32]. In that study, global LC/MS and GC-MS methods were used, while in our study the more quantitative targeted LC-MS and NMR platforms were used to analyze the determined pool of pre-identified metabolites, unambiguously. It is important to note that in the metabolomics field different analytical platforms have been used to investigate the same disease; depending on the sensitivity, selectivity, and reliability of metabolite identification, different analytical platforms enable access to different facets of the metabolome. In this study the combined use of the robust LC-MS and NMR methods for quantitative analysis of metabolites represents a broad based approach. In our study, apart from differentiating between NAFL and NASH, we have identified metabolites and metabolic pathways that differentiate various subtypes of NAFLD including early NASH and advanced NASH, as well as fibrosis stage and steatosis grade, and find many differentiating metabolites and pathways among the different histologies. Separately, analysis of the metabolite profiles showed that the confounding effects of demographics such as gender and diabetes are minimal in the biomarker identification for NAFL disease. To our knowledge, this is the first study to identify distinguishing metabolites and 
metabolic pathways for different liver histology associated with NAFL, combining targeted LC-MS and NMR based metabolomics methods.

Many other "non-metabolite", serum-based biomarkers (e.g., type 4 collagen, M2BPGi) or biomarker panels (e.g., Fibrosis-4 score, Enhanced Liver Fibrosis score, Fibrotest/Fibrosure, Fibrospect) have been described that may distinguish advanced NASH, early NASH and NAFL [42]. Future studies should compare the performance characteristics of these tests with the metabolites that we described in distinguishing disease severity in NAFLD.

There are several limitations to this study, most notably the moderate sample size. Our results of novel metabolite (e.g., spermidine) and metabolite pathways associated with NASH severity will need to be confirmed in future studies. The small number of subjects does not allow us to make conclusions as to the separate effects of diabetes and insulin resistance, as insulin resistance was not measured in this study. Although two widely used metabolomics platforms, MS and NMR, were used to profile serum metabolites, the number of metabolites profiled is still small compared to the breadth of the serum metabolite profile. Moreover, no independent validation of the results was made.

In conclusion, a comprehensive metabolite profiling combining data from two analytical platforms, MS and NMR, provides new insights into the underlying metabolites and metabolic pathways that distinguish NAFLD patients based on histological severity. In particular, numerous features distinguished high risk NASH from low risk NAFL that potentially offers new avenues to identify high-risk NASH patients in the clinical setting. Of significance is that apart from identifying many altered metabolic pathways, our study enabled the identification of metabolites such as spermidine, acytylglycine, oxypurinol, and bile acids that varied by as much as 2-fold or higher between NAFL and NASH. These findings are particularly remarkable considering the tight regulation of many blood metabolites.

\section{Materials and Methods}

\subsection{Study Population}

All subjects gave their informed consent for inclusion before they participated in the study. The study was conducted in accordance with the Declaration of Helsinki, and the protocol was approved by the Institutional Review Board (IRB) at the Veterans Affairs Puget Sound Healthcare System (IRB protocol \# 01010). Patients were identified from a prospective biorepository of chronic liver disease patients established at the Veterans Affairs Puget Sound Healthcare System, which recruited patients undergoing clinically indicated liver biopsy. We identified patients with NAFLD based on histological hepatic steatosis from liver biopsies in the absence of hepatitis $C$ virus (negative serum HCV antibody and HCV RNA), hepatitis B virus (negative serum HBV surface antigen), excessive alcohol consumption (dedicated alcohol questionnaire administered on the day of liver biopsy), iron overload (hepatic stain and serum iron markers), or markers of autoimmune liver diseases. The liver biopsies of these patients with NAFLD were prospectively reviewed by a single hepatopathologist who scored the grade of steatosis (1-3), inflammation (0-3) and ballooning degeneration (0-2) and the stage of fibrosis (0-4) according to the system proposed by Kleiner et al. [43]. Based on these scores we divided the patients with NAFLD a priori according to their histological severity into the following groups, without any knowledge of their metabolomic profiles:

a. $\quad \operatorname{NAFL~}(n=12)$ : Steatosis grade $1-3$ with no or minimal inflammation (grade $0-1)$, no ballooning degeneration (grade 0 ) and no fibrosis (stage $\mathrm{F} 0$ ).

b. Early-NASH $(n=31)$ : No or mild fibrosis (stage F0-F1) and NAFLD activity score (NAS) 3-4, including steatosis grade $\geq 1$, inflammation $\geq 1$, and ballooning degeneration score $\geq 1$. The NAS score is the sum of steatosis (0-3) plus inflammation (0-3) plus ballooning degeneration (0-2) grades and takes values ranging from 1-8 [43].

c. Advanced-NASH $(n=14)$ : Fibrosis score F1-F4 and NAS score 5-8, including ballooning degeneration score $=2$. 
The European Association for the Study of the Liver (EASL) defines "early" NASH as "no or mild fibrosis F0-F1" [44], which is consistent with the definition we chose above. However, there is no universally agreed definition of "advanced" NASH and we wanted to categorize NASH based on both fibrosis and NAS scores, hence we used the categories above.

In addition, we performed analyses based on subdividing patients by fibrosis stage ( $0-1$ vs. $2-4)$ and by steatosis grade ( $0-1$ vs. $2-3)$, given the fundamental importance of these histological features.

\subsection{Serum Metabolite Profiling: LC-MS}

A fasting serum specimen was prospectively collected from all patients between 7-9 am after an overnight fast just prior to liver biopsy and stored at $-80^{\circ} \mathrm{C}$ until the analysis.

Frozen serum samples were thawed at $4{ }^{\circ} \mathrm{C}$, after which protein precipitation and metabolite extraction was performed by adding $150 \mu \mathrm{L}$ of methanol; the mixture was then vortexed for $2 \mathrm{~min}$, stored at $-20^{\circ} \mathrm{C}$ for $20 \mathrm{~min}$ and centrifuged at $20,800 \times \mathrm{g}$ for $10 \mathrm{~min}$. The supernatant was collected into a new Eppendorf vial and dried using a Vacufuge Plus evaporator (Eppendorf, Hauppauge, NY, USA). The dried samples were stored at $-20{ }^{\circ} \mathrm{C}$ and were reconstituted in $500 \mu \mathrm{L}$ of $5 \mathrm{mM}$ ammonium acetate in $40 \%$ water $/ 60 \%$ acetonitrile $+0.2 \%$ acetic acid containing $5.13 \mu \mathrm{M}$ tyrosine $-{ }^{13} \mathrm{C}_{2}$ and $22.5 \mu \mathrm{M}$ sodium lactate- ${ }^{13} \mathrm{C}_{3}$. The two isotope-labeled internal standards were added to each sample to monitor the MS system performance. The samples were filtered through $0.45 \mu \mathrm{m}$ PVDF filters (Phenomenex, Torrance, CA, USA) prior to LC-MS analysis. A pooled human serum extracted using the same procedure as described above was used as the quality-control $(\mathrm{QC})$ sample and was analyzed once every 10 patient samples.

Targeted LC-MS analysis was performed using an AB Sciex QTrap 5500 mass spectrometer (AB Sciex, Toronto, ON, Canada) equipped with an electrospray ionization (ESI) source. The MS instrument was connected to an LC system composed of two Agilent 1260 binary pumps, an Agilent 1260 autosampler, and Agilent 1290 column compartment containing a column-switching valve (Agilent Technologies, Santa Clara, CA, USA). Two hydrophilic interaction chromatography (HILIC) columns (SeQuant ZIC-cHILIC columns; $150 \times 2.1$ mm, $3.0 \mu \mathrm{m}$ particle size, Merck KGaA, Darmstadt, Germany) connected in parallel were used. One column was used for positive ionization and the other, for negative ionization. Each sample was injected twice, once for positive ionization mode $(2 \mu \mathrm{L})$ and once for negative ionization mode $(10 \mu \mathrm{L})$. Targeted data acquisition was performed in multiple-reaction-monitoring (MRM) mode. The mobile phase, the gradient conditions and the MS parameters used have been described previously in detail [45]. The extracted MRM peaks were integrated using MultiQuant 2.1 software (AB Sciex).

\subsection{NMR Spectroscopy}

NMR analyses were performed on a Bruker Avance III $800 \mathrm{MHz}$ spectrometer equipped with a cryogenically cooled probe and Z-gradients suitable for inverse detection. The CPMG (Carr-Purcell-Meiboom-Gill) pulse sequence with water suppression using presaturation, was used with $9615 \mathrm{~Hz}$ spectral width, $32 \mathrm{k}$ time domain points, $6 \mathrm{~s}$ relaxation delay and 256 transients for each sample. Fourier transformation was performed using a spectral size of $32 \mathrm{k}$ data points after multiplying the raw data by an exponential window function with line broadening of $0.5 \mathrm{~Hz}$. Chemical shifts were referenced to the internal TSP signal for ${ }^{1} \mathrm{H} 1 \mathrm{D}$ spectra. Bruker Topspin software (version 3.0 or 3.1) was used for NMR data acquisition, processing and analyses. Peak assignments relied on established literature values [46]. Bruker AMIX software was used to quantitate metabolites.

\subsection{Statistical, Enrichment, and Pathway Analyses}

Relative peak integrals or (where available) absolute concentrations of metabolites obtained from MS and NMR were used for data analysis. The MS and NMR data were subjected to univariate (Student's $t$-test) analysis, multivariate analysis, principle component analysis (PCA), partial least squares discriminant analysis (PLS-DA), statistical correlations, and metabolic pathway analysis. Prior 
to statistical analysis the MS and NMR data were normalized using Pareto scaling, where each variable (concentration for each metabolite) was mean-centered and divided by the square root of standard deviation of each variable. Metabolites with $p<0.05$ were considered statistically significant. Pathway enrichment analysis was performed to identify pathways significantly associated with the different groups of NAFLD patients. For multivariate analysis, statistical correlations, enrichment analysis and pathway analysis, the Metaboanalyst software package version 4.0 [22-24] was used. We have also evaluated whether the confounding factors such as gender and diabetes affected the biomarkers of NAFLD.

\subsection{Evaluation of the GSG-Index}

The GSG-index, a newly developed parameter associated with NAFLD, refers to the ratio of glutamate to sum of serine and glycine [glutamate/(serine + glycine)] [38]. Amino acids of the GSG-index, glutamate, serine, and glycine, are critical for the synthesis of glutathione (GSH), which is the major antioxidant that controls oxidative stress in the liver. Several amino acids including those associated with the GSG-index, branched chain amino acids (BCCAs) and aromatic amino acids (AAAs) were shown to be altered in NAFLD [38]. Hence, we evaluated the associations of the GSG-index with histological severity of NAFLD.

Supplementary Materials: The following are available online at http://www.mdpi.com/2218-1989/10/4/168/s1, Figure S1: Matched pathways obtained from pathway enrichment analysis and the impact of the matched pathways obtained from pathway topology analysis, Figure S2: Network view of metabolite set enrichment analysis with labeling for some of the prominent sets, Figure S3: Results of PLS-DA of metabolites derived from MS and NMR for (a) male vs female; and (b) diabetes vs non-diabetes patients, Table S1: List of metabolites quantified by mass spectrometry (MS), Table S2: List of metabolites quantified by NMR spectroscopy, Table S3: Metabolic pathways that are significantly different between patients with NAFL, early-NASH or advanced-NASH, and between different fibrosis stages or steatosis grades, Table S4: Metabolites that differed significantly between male and female patients, Table S5: Metabolites that differed significantly between diabetes and non-diabetes patients.

Author Contributions: G.N.I. and D.R. are the guarantors of this paper; G.N.I. contributed to the study concept and design, interpretation of data, access to human biorepository specimens, critical revision of manuscript; G.A.N.G. contributed to the study concept and design, interpretation of data, execution of experimental analytical protocols, critical revision of manuscript and drafting of manuscript; D.D. contributed to the design and execution of mass spectrometry analysis; and D.R. contributed to the study concept and design, interpretation of data, execution of experimental analytical protocols, critical revision of manuscript, and drafting of the manuscript. All authors have read and agreed to the published version of the manuscript.

Funding: This work was supported by Veterans Affairs, Biomedical and Laboratory Research and Development, I01 BX002910, Diabetes Research Center (DRC), University of Washington, P30 DK017047, Nutrition and Obesity Center (NORC), University of Washington, P30 DK035816, Cancer Consortium Support Grant, University of Washington, P30 CA015704, and University of Washington Startup Funding.

Acknowledgments: The authors thank Natalie Hong for help with sample preparation for NMR analysis.

Conflicts of Interest: The authors declare no conflict of interest. Daniel Raftery reports holding equity and an executive position at Matrix Bio, Inc; however, the company was not involved in this research.

\section{References}

1. Marchesini, G.; Brizi, M.; Bianchi, G.; Tomassetti, S.; Bugianesi, E.; Lenzi, M.; McCullough, A.J.; Natale, S.; Forlani, G.; Melchionda, N. Nonalcoholic fatty liver disease: A feature of the metabolic syndrome. Diabetes 2001, 50, 1844-1850. [CrossRef] [PubMed]

2. Pagano, G.; Pacini, G.; Musso, G.; Gambino, R.; Mecca, F.; Depetris, N.; Cassader, M.; David, E.; Cavallo-Perin, P.; Rizzetto, M. Nonalcoholic steatohepatitis, insulin resistance, and metabolic syndrome: Further evidence for an etiologic association. Hepatology 2002, 35, 367-372. [CrossRef]

3. Chalasani, N.; Younossi, Z.; Lavine, J.E.; Charlton, M.; Cusi, K.; Rinella, M. The diagnosis and management of nonalcoholic fatty liver disease: Practice guidance from the American Association for the Study of Liver Diseases. Hepatology 2018, 67, 328-357. [CrossRef] [PubMed]

4. Matteoni, C.A.; Younossi, Z.M.; Gramlich, T.; Boparai, N.; Liu, Y.C.; McCullough, A.J. Nonalcoholic fatty liver disease: A spectrum of clinical and pathological severity. Gastroenterology 1999, 116, 1413-1419. [CrossRef] 
5. Bugianesi, E.; Leone, N.; Vanni, E.; Marchesini, G.; Brunello, F.; Carucci, P.; Musso, A.; De Paolis, P.; Capussotti, L.; Salizzoni, M.; et al. Expanding the natural history of nonalcoholic steatohepatitis: From cryptogenic cirrhosis to hepatocellular carcinoma. Gastroenterology 2002, 123, 134-140. [CrossRef] [PubMed]

6. Angulo, P.; Kleiner, D.E.; Dam-Larsen, S.; Adams, L.A.; Bjornsson, E.S.; Charatcharoenwitthaya, P.; Mills, P.R.; Keach, J.C.; Lafferty, H.D.; Stahler, A.; et al. Liver Fibrosis, but No Other Histologic Features, Is Associated With Long-term Outcomes of Patients With Nonalcoholic Fatty Liver Disease. Gastroenterology 2015, 149, 389-397. [CrossRef]

7. Ekstedt, M.; Hagström, H.; Nasr, P.; Fredrikson, M.; Stål, P.; Kechagias, S.; Hultcrantz, R. Fibrosis stage is the strongest predictor for disease-specific mortality in NAFLD after up to 33 years of follow-up. Hepatology 2015, 61, 1547-1554. [CrossRef]

8. Lindon, J.C.; Nicholson, J.K. The emergent role of metabolic phenotyping in dynamic patient stratification. Expert Opin. Drug Metab. Toxicol. 2014, 10, 915-919. [CrossRef]

9. Nagana Gowda, G.A.; Raftery, D. Biomarker Discovery and Translation in Metabolomics. Curr. Metab. 2013, 1, 227-240. [CrossRef]

10. Dang, N.H.; Singla, A.K.; Mackay, E.M.; Jirik, F.R.; Weljie, A.M. Targeted cancer therapeutics: Biosynthetic and energetic pathways characterized by metabolomics and the interplay with key cancer regulatory factors. Curr. Pharm. Des. 2014, 20, 2637-2647. [CrossRef]

11. Raftery, D. Mass Spectrometry in Metabolomics-Methods and Protocols. Methods in Molecular Biology; Walker, J., Ed.; Springer: New York, NY, USA, 2014.

12. Nagana Gowda, G.A.; Raftery, D. Recent Advances in NMR-Based Metabolomics. Anal. Chem. 2017, 89, 490-510. [CrossRef] [PubMed]

13. Wei, S.; Suryani, Y.; Nagana Gowda, G.A.; Skill, N.; Maluccio, M.; Raftery, D. Differentiating hepatocellular carcinoma from hepatitis C using metabolite profiling. Metabolites 2012, 2, 701-716. [CrossRef] [PubMed]

14. Baniasadi, H.; Nagana Gowda, G.A.; Gu, H.; Zeng, A.; Zhuang, S.; Skill, N.; Maluccio, M.; Raftery, D. Targeted metabolic profiling of hepatocellular carcinoma and hepatitis C using LC-MS/MS. Electrophoresis 2013, 34, 2910-2917. [CrossRef] [PubMed]

15. Bowers, J.; Hughes, E.; Skill, N.; Maluccio, M.; Raftery, D. Detection of hepatocellular carcinoma in hepatitis C patients: Biomarker discovery by LC-MS. J. Chromatogr. B Analyt. Technol. Biomed. Life Sci. 2014, 966, 154-162. [CrossRef] [PubMed]

16. Liu, Y.; Hong, Z.; Tan, G.; Dong, X.; Yang, G.; Zhao, L.; Chen, X.; Zhu, Z.; Lou, Z.; Qian, B.; et al. NMR and LC/MS-based global metabolomics to identify serum biomarkers differentiating hepatocellular carcinoma from liver cirrhosis. Int. J. Cancer 2014, 135, 658-668. [CrossRef]

17. Huang, Q.; Tan, Y.; Yin, P.; Ye, G.; Gao, P.; Lu, X.; Wang, H.; Xu, G. Metabolic characterization of hepatocellular carcinoma using nontargeted tissue metabolomics. Cancer Res. 2013, 73, 4992-5002. [CrossRef]

18. Tan, Y.; Yin, P.; Tang, L.; Xing, W.; Huang, Q.; Cao, D.; Zhao, X.; Wang, W.; Lu, X.; Xu, Z.; et al. Metabolomics study of stepwise hepatocarcinogenesis from the model rats to patients: Potential biomarkers effective for small hepatocellular carcinoma diagnosis. Mol. Cell Proteom. 2012, 11, M111 010694. [CrossRef]

19. Patterson, A.D.; Maurhofer, O.; Beyoglu, D.; Lanz, C.; Krausz, K.W.; Pabst, T.; Gonzalez, F.J.; Dufour, J.F.; Idle, J.R. Aberrant lipid metabolism in hepatocellular carcinoma revealed by plasma metabolomics and lipid profiling. Cancer Res. 2011, 71, 6590-6600. [CrossRef]

20. Safaei, A.; Arefi Oskouie, A.; Mohebbi, S.R.; Rezaei-Tavirani, M.; Mahboubi, M.; Peyvandi, M.; Okhovatian, F.; Zamanian-Azodi, M. Metabolomic analysis of human cirrhosis, hepatocellular carcinoma, non-alcoholic fatty liver disease and non-alcoholic steatohepatitis diseases. Gastroenterol. Hepatol. Bed. Bench. 2016, 9, 158-173.

21. Di Poto, C.; Ferrarini, A.; Zhao, Y.; Varghese, R.S.; Tu, C.; Zuo, Y.; Wang, M.; Nezami Ranjbar, M.R.; Luo, Y.; Zhang, C.; et al. Metabolomic Characterization of Hepatocellular Carcinoma in Patients with Liver Cirrhosis for Biomarker Discovery. Cancer Epidemiol. Biomark. Prev. 2017, 26, 675-683. [CrossRef]

22. Xia, J.; Wishart, D.S. Using MetaboAnalyst 3.0 for Comprehensive Metabolomics Data Analysis. Curr. Protoc. Bioinform. 2016, 55, 14.10.1-14.10.91. [CrossRef] [PubMed]

23. Xia, J.; Sinelnikov, I.V.; Han, B.; Wishart, D.S. MetaboAnalyst 3.0—making metabolomics more meaningful. Nucleic Acids Res. 2015, 43, W251-W257. [CrossRef]

24. Chong, J.; Soufan, O.; Li, C.; Caraus, I.; Li, S.; Bourque, G.; Wishart, D.S.; Xia, J. MetaboAnalyst 4.0: Towards more transparent and integrative metabolomics analysis. Nucleic Acids Res. 2018, 46, W486-W494. [CrossRef] [PubMed] 
25. Yue, F.; Li, W.; Zou, J.; Jiang, X.; Xu, G.; Huang, H.; Liu, L. Spermidine Prolongs Lifespan and Prevents Liver Fibrosis and Hepatocellular Carcinoma by Activating MAP1S-Mediated Autophagy. Cancer Res. 2017, 77, 2938-2951. [CrossRef] [PubMed]

26. Kim, J.W.; Ryu, S.H.; Kim, S.; Lee, H.W.; Lim, M.S.; Seong, S.J.; Kim, S.; Yoon, Y.R.; Kim, K.B. Pattern recognition analysis for hepatotoxicity induced by acetaminophen using plasma and urinary $1 \mathrm{H}$ NMR-based metabolomics in humans. Anal. Chem. 2013, 85, 11326-11334. [CrossRef] [PubMed]

27. Manna, S.K.; Patterson, A.D.; Yang, Q.; Krausz, K.W.; Idle, J.R.; Fornace, A.J.; Gonzalez, F.J. UPLC-MS-based urine metabolomics reveals indole-3-lactic acid and phenyllactic acid as conserved biomarkers for alcohol-induced liver disease in the Ppara-null mouse model. J. Proteome Res. 2011, 10, 4120-4133. [CrossRef]

28. Bradford, B.U.; O’Connell, T.M.; Han, J.; Kosyk, O.; Shymonyak, S.; Ross, P.K.; Winnike, J.; Kono, H.; Rusyn, I. Metabolomic profiling of a modified alcohol liquid diet model for liver injury in the mouse uncovers new markers of disease. Toxicol. Appl. Pharmacol. 2008, 232, 236-243. [CrossRef]

29. van Waeg, G.; Lööf, L.; Groth, T.; Niklasson, F. Allopurinol kinetics in humans as a means to assess liver function: Evaluation of an allopurinol loading test. Scand. J. Clin. Lab Invest. 1988, 48, 45-57. [CrossRef]

30. Barr, J.; Caballeria, J.; Martinez-Arranz, I.; Domínguez-DíezM, A.; Alonso, C.; Muntané, J.; Pérez-Cormenzana, M.; García-Monzón, C.; Mayo, R.; Martín-Duce, A.; et al. Obesitydependent metabolic signatures associated with nonalcoholic fatty liver disease progression. J. Proteome Res. 2012, 11, 2521-2532. [CrossRef]

31. Nagana Gowda, G.A.; Ijare, O.B.; Somashekar, B.S.; Sharma, A.; Kapoor, V.K.; Khetrapal, C.L. Single-step analysis of individual conjugated bile acids in human bile using $1 \mathrm{H}$ NMR spectroscopy. Lipids 2006, 41, 591-603. [CrossRef]

32. Kalhan, S.C.; Guo, L.; Edmison, J.; Dasarathy, S.; McCullough, A.J.; Hanson, R.W.; Milburn, M. Plasma metabolomic profile in nonalcoholic fatty liver disease. Metabolism 2011, 60, 404-413. [CrossRef] [PubMed]

33. Bianchi, G.; Marchesini, G.; Brunetti, N.; Manicardi, E.; Montuschi, F.; Chianese, R.; Zoli, M. Impaired insulin-mediated amino acid plasma disappearance in non-alcoholic fatty liver disease: A feature of insulin resistance. Dig. Liver Dis. 2003, 35, 722-727. [CrossRef]

34. Mardinoglu, A.; Agren, R.; Kampf, C.; Asplund, A.; Uhlen, M.; Nielsen, J. Genome-scale metabolic modelling of hepatocytes reveals serine deficiency in patients with non-alcoholic fatty liver disease. Nat. Commun. 2014, 5, 3083. [CrossRef] [PubMed]

35. Lake, A.D.; Novak, P.; Shipkova, P.; Aranibar, N.; Robertson, D.G.; Reily, M.D.; Lehman-McKeeman, L.D.; Vaillancourt, R.R.; Cherrington, N.J. Branched chain amino acid metabolism profiles in progressive human nonalcoholic fatty liver disease. Amino Acids 2015, 47, 603-615. [CrossRef]

36. Hyotylainen, T.; Jerby, L.; Petaja, E.M.; Mattila, I.; Jantti, S.; Auvinen, P.; Gastaldelli, A.; Yki-Järvinen, H.; Ruppin, E.; Orešič, M. Genome-scale study reveals reduced metabolic adaptability in patients with non-alcoholic fatty liver disease. Nat. Commun. 2016, 7, 8994. [CrossRef]

37. Mardinoglu, A.; Bjornson, E.; Zhang, C.; Klevstig, M.; Soderlund, S.; Stahlman, M.; Adiels, M.; Hakkarainen, A.; Lundbom, N.; Kilicarslan, M.; et al. Personal model-assisted identification of NAD ${ }^{+}$and glutathione metabolism as intervention target in NAFLD. Mol. Syst. Biol. 2017, 13, 916. [CrossRef]

38. Gaggini, M.; Carli, F.; Rosso, C.; Buzzigoli, E.; Marietti, M.; Della Latta, V.; Ciociaro, D.; Abate, M.L.; Gambino, R.; Cassader, M.; et al. Altered amino acid concentrations in NAFLD: Impact of obesity and insulin resistance. Hepatology 2018, 67, 145-158. [CrossRef]

39. Koliaki, C.; Szendroedi, J.; Kaul, K.; Jelenik, T.; Nowotny, P.; Jankowiak, F.; Herder, C.; Carstensen, M.; Krausch, M.; Knoefel, W.T.; et al. Adaptation of hepatic mitochondrial function in humans with non-alcoholic fatty liver is lost in steatohepatitis. Cell Metab. 2015, 21, 739-746. [CrossRef]

40. Dong, S.; Zhan, Z.Y.; Cao, H.Y.; Wu, C.; Bian, Y.Q.; Li, J.Y.; Cheng, G.H.; Liu, P.; Sun, M.Y. Urinary metabolomics analysis identifies key biomarkers of different stages of nonalcoholic fatty liver disease. World J. Gastroenterol. 2017, 23, 2771-2784. [CrossRef]

41. Alonso, C.; Fernández-Ramos, D.; Varela-Rey, M.; Martínez-Arranz, I.; Navasa, N.; Van Liempd, S.M.; Lavín Trueba, J.L.; Mayo, R.; Ilisso, C.P.; de Juan, V.G.; et al. Metabolomic Identification of Subtypes of Nonalcoholic Steatohepatitis. Gastroenterology 2017, 152, 1449-1461. [CrossRef]

42. Castera, L.; Friedrich-Rust, M.; Loomba, R. Noninvasive Assessment of Liver Disease in Patients with Nonalcoholic Fatty Liver Disease. Gastroenterology 2019, 156, 1264-1281 e4. [CrossRef] [PubMed] 
43. Kleiner, D.E.; Brunt, E.M.; Van Natta, M.; Behling, C.; Contos, M.J.; Cummings, O.W.; Ferrell, L.D.; Liu, Y.C.; Torbenson, M.S.; Unalp-Arida, A.; et al. Design and validation of a histological scoring system for nonalcoholic fatty liver disease. Hepatology 2005, 41, 1313-1321. [CrossRef] [PubMed]

44. European Association for the Study of the Liver (EASL). European Association for the Study of Diabetes (EASD); European Association for the Study of Obesity (EASO). EASL-EASD-EASO Clinical Practice Guidelines for the management of non-alcoholic fatty liver disease. J. Hepatol. 2016, 64, 1388-1402. [CrossRef] [PubMed]

45. Zhu, J.; Djukovic, D.; Deng, L.; Gu, H.; Himmati, F.; Chiorean, E.G.; Raftery, D. Colorectal cancer detection using targeted serum metabolic profiling. J. Proteome Res. 2014, 13, 4120-4130. [CrossRef]

46. Nagana Gowda, G.A.; Gowda, Y.N.; Raftery, D. Expanding the limits of human blood metabolite quantitation using NMR spectroscopy. Anal. Chem. 2015, 87, 706-715. [CrossRef]

(C) 2020 by the authors. Licensee MDPI, Basel, Switzerland. This article is an open access article distributed under the terms and conditions of the Creative Commons Attribution (CC BY) license (http://creativecommons.org/licenses/by/4.0/). 



\title{
Nicotinamide and NAFLD: Is There Nothing New Under the Sun?
}

\author{
Maria Guarino ${ }^{1,2}$ and Jean-François Dufour 1,3,* \\ 1 Hepatology, Department for BioMedical Research, University of Bern, 3008 Bern, Switzerland \\ 2 Gastroenterology, Department of Clinical Medicine and Surgery, University of Naples Federico II, \\ 80131 Naples, Italy \\ 3 University Clinic of Visceral Surgery and Medicine, Inselspital Bern, 3008 Bern, Switzerland \\ * Correspondence: jean-francois.dufour@dbmr.unibe.ch
}

Received: 8 August 2019; Accepted: 6 September 2019; Published: 10 September 2019

\begin{abstract}
Nicotinamide adenine dinucleotide (NAD) has a critical role in cellular metabolism and energy homeostasis. Its importance has been established early with the discovery of NAD's therapeutic role for pellagra. This review addresses some of the recent findings on NAD physiopathology and their effects on nonalcoholic fatty liver disease (NAFLD) pathogenesis, which need to be considered in the search for a better therapeutic approach. Reduced NAD concentrations contribute to the dysmetabolic imbalance and consequently to the pathogenesis of NAFLD. In this perspective, the dietary supplementation or the pharmacological modulation of NAD levels appear to be an attractive strategy. These reviewed studies open the doors to growing interest in NAD metabolism for NAFLD diagnosis, prevention, and treatment. Future rigorous clinical studies in humans will be necessary to validate these preliminary but promising results.
\end{abstract}

Keywords: nicotinamide; NAFLD; steatosis

\section{Introduction}

The global diabesity (diabetes and obesity) [1] epidemic has dramatically increased the prevalence of nonalcoholic fatty liver disease (NAFLD), such that it is the most frequent cause of chronic liver disease. NAFLD is considered to be the liver manifestation of the metabolic syndrome, because of its frequent association with dyslipidemia, cardiovascular disease, obstructive sleep apnea, vitamin D deficiency, and other components of the metabolic syndrome, and insulin resistance is central to its pathogenesis [2,3].

Liver steatosis is the hallmark histologic feature of NAFLD, and it is the result of triglyceride accumulation in the hepatocytes cytoplasm. Liver lipid accumulation arises from an imbalance between lipid accumulation and removal, which is linked to increased liver lipogenesis, increased lipid uptake, and/or reduced triglyceride export or $\beta$-oxidation [4,5]. Liver secretion of triglycerides as very low-density lipoprotein (VLDL) particles for delivery to peripheral tissues is a crucial pathway for the mobilization of hepatic fat. Defects in VLDL processing are directly linked to hepatic steatosis. Jiang et al. showed that non-alcoholic steatohepatitis (NASH) was related to an increment in VLDL particle size, while hepatic fibrosis was related to a reduction in the concentration of small VLDL particles [6]. Moreover, there is a relationship between choline deficiency and accumulation of liver lipid, which is why choline-deficient diets are often used to induce NAFLD in animal models. Within hepatocytes, choline may be oxidized for phosphatidylcholine synthesis. Liver phosphatidylcholine is used to build the monolayers of VLDL, and its deficiency increases de novo hepatic lipogenesis [7].

The present model for NAFLD pathophysiology, called "the multiple-hit hypothesis", defines NAFLD as the manifestation of environmental and genetic factors, including the dysfunction of different organs and organelles, together with the intricate interaction between hepatocytes and other 
cells (such as stellate cells and Kupffer) in the liver [8]. Additionally, the liver is a hub for several metabolic pathways defining NAFLD as a multistep, progressive systemic disease.

\section{NAD: Behind Its Metabolism}

Nicotinamide adenine dinucleotide (NAD) is a hydride acceptor producing the reduced NADH, as well as the derivate phosphorylated dinucleotide pair NADP/NADPH, which is required for many cellular biosynthetic pathways and for protecting cells from reactive oxygen species (ROS). The keystone function of NAD is to facilitate hydrogen transfer in metabolic pathways as enzyme cofactors dealing with hydrogen transfer in reductive or oxidative metabolic reaction. So, it plays a central role in basic energy metabolism such as assisting with mitochondrial electron transport, glycolysis, the oxidation of fatty acids and amino acids in mitochondria, and the citric acid cycle. NAD is also a substrate for signaling enzymes such as poly (ADP ribose) polymerase (PARP), sirtuins (SIRTs), and ADP ribosyl transferases, called "NAD consumers" [9] (Figure 1). For example, it is involved in repairing and maintaining genomic integrity, thanks to PARP, which transfers ADP-ribose from NAD to itself, histones, and other proteins at sites of DNA damage.

\section{De novo synthesis}

\section{Tryptophan}

Quinolonicacid

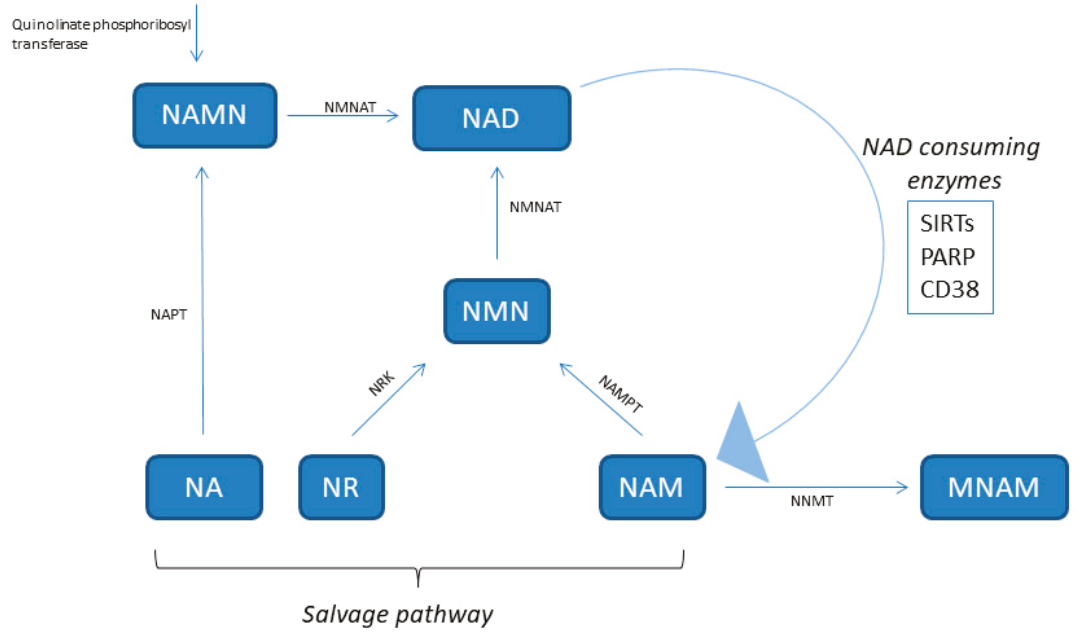

Figure 1. NAD synthesis pathways. NA, nicotinic acid; NAD, nicotinamide adenine dinucleotide; NAM, nicotinamide; NAMN, nicotinic acid mononucleotide; NAPT, nicotinic acid phosphoribosyltransferase; NMN, nicotinamide mononucleotide; NMNAT, nicotinamide nucleotide adenylyltransferase; NR, nicotinamide riboside; NRK, NR kinase; NNMT, nicotinamide-N-methyltransferase; PARP, poly (ADP ribose) polymerase; NNMT, nicotinamide N-methyltransferase; NAMPT, nicotinamide phosphoribosyltransferase; SIRT, sirtuin.

The cellular NAD pool is created by a balance between the activity of NAD-consuming and synthesizing enzymes [10-12]. NAD concentrations display the cell energy state and are modulated by physiological processes. In fact, during fasting, caloric restriction, and exercise, NAD levels increase. Conversely, caloric excess and aging diminish NAD levels [13]. 
NAD is synthesized from four distinct biosynthetic precursors in two different pathways (Figure 1). De novo synthesis (the deamidated pathway) uses as precursor the dietary amino acid tryptophan, which is metabolized to create biosynthetic intermediates. In particular, the creation of unstable $\alpha$-amino- $\beta$-carboxymuconate- $\varepsilon$-semialdehyde (ACMS) forms a branching point of the deaminated pathway. The ACMS is subjected to both non-enzymatic cyclization or complete enzymatic oxidation to quinolinic acid, and this is the first limiting step [14]. The second limiting mechanism involves the catalytic conversion of quinolinic acid to nicotinic acid mononucleotide (NAMN) by quinolinate phosphoribosyl transferase. Next, NAMN is transformed into NAD by the nicotinamide mononucleotide adenylyltransferase (NMNAT) enzyme. This pathway is recognized as the minor contributor to the total NAD pool [14].

Dietary vitamin B3 compounds, including nicotinic acid (NA), also known as niacin, NAM, and nicotinamide riboside (NR), supply as NAD biosynthetic precursors and are rescued from the diet (the amidated pathway) for generating cellular NAD. This salvage pathway is the most relevant for NAD homeostasis [15]. NA is converted to NAMN by nicotinic acid phosphoribosyltransferase (NAPT), which is afterward converted to NAD by NMNAT. The NAM and NR are transformed into NMN by nicotinamide phosphoribosyltransferase (NAMPT) and NR kinase (NRK) enzymes, respectively. Finally, NMN is enzymatically transformed into NAD by NMNAT [15].

NAMPT, also known with the name visfatin, is a highly conserved protein with cytokine functions, which is expressed in almost all tissues and cells (Figure 1) [16]. In particular, it is an essential regulator of the intracellular NAD pool by catalyzing the formation of nicotinamide mononucleotide (NMN) from nicotinamide and $5^{\prime}$-phosphoribosyl-1-pyrophosphate, which is the limiting step in the NAD salvage pathway [17]. NAMPT has both intracellular and extracellular forms in mammals. The extracellular NAMPT (eNAMPT) is secreted from adipocytes [18], hepatocytes [19], and leucocytes [20] and circulates in the blood where, additionally to its enzymatic function, it has also cytokine-like actions $[16,21,22]$. In virtue of its NAD biosynthetic activity, intracellular NAMPT (iNAMPT) controls the activity of NAD-dependent and consuming enzymes, such as SIRTs [23], the NADase CD38 (a cyclic ADP-ribose synthesis) [24], and PARPs [25], by which it controls mitochondrial biogenesis, cellular metabolism [26], and adaptive responses to oxidative, inflammatory, genotoxic, and proteotoxic stress [27]. Genotoxic stress and nutrient deprivation activate NAMPT, which protects cells from these stresses through the maintenance of the mitochondrial NAD level [23].

The NAD levels are also regulated by the cytosolic enzyme nicotinamide-N-methyltransferase (NNMT), which methylates nicotinamide to produce N1-methyl nicotinamide (MNAM) toward the universal methyl donor S-adenosylmethionine as a methyl donor (Figure 1). NNMT is mainly expressed in the liver, but also in other organs such as muscle, adipose tissue, and heart. An increase of NNMT expression has been observed in obesity and diabetes [28-30].

SIRTs are NAD-dependent deacylases [31]. SIRTs have key roles in response to environmental and nutritional perturbations, such as DNA damage, oxidative stress, and fasting. For this reason, SIRTs have to be considered as nutritional sensors that operate in regulating glucose and lipid homeostasis, inflammatory responses, and cell death [23,32-34]. Additionally, SIRTs influence cells' metabolism through the regulation of the circadian clock machinery with the deacetylation of central clock components in the liver $[35,36]$. Accordingly, NAD synthesis is controlled by the circadian machinery to furnish a crucial link from the clock oscillator to metabolic pathways [37]. NAD is synthesized with circadian oscillations, leading to a circadian schedule of SIRT activation and mitochondrial metabolism, such as the oxidation of fatty acids [38]. SIRTs' activity is dependent on its cofactor NAD and it is sensitive to the cellular NAD levels [39], designating NAD as a rate-limiting substrate for their reactions $[32,40,41]$. As NAM is the product of SIRT-catalyzed deacetylation reactions, high levels of NAM have been used as a SIRTs inhibitor [42]. This drives speculation that enzymes involved in NAD synthesis could control SIRTs' activity. For example, an increment in NAD was proposed by Lin et al. to mediate the health span and extension of life by dietary restriction [43], and recently, 
studies demonstrated that the activity of SIRTs declines with aging by a systemic reduction in NAD levels $[44,45]$.

\section{NAD Involvement in NAFLD Pathogenesis}

In the last years, an emerging role of NAD metabolism in protection against NAFLD stimulated a growing interest. Von Shönfels et al. performed a small-molecule metabolite screen of human hepatic tissue to find metabolic markers related to NASH histology. According to its concentration in liver tissue, they suggested a protective effect of NA, which was subsequently verified in a nutritional animal model of NAFLD showing a marked effect on steatosis and transaminases levels with NA supplementation [46]. NAD deficiency decreases the oxidation of fatty acids, promoting steatosis [47]. Usually, the triglycerides are broken down into glycerol and fatty acids, so they can enter into the mitochondria and proceed on with fatty acid oxidation. Fatty acids shift in this pathway as Coenzyme A (CoA) derivatives utilizing NAD. The acetyl groups created by the $\beta$-oxidation of the fatty acid take part in the activity of the Krebs cycle, causing the formation of NADH. The reduced coenzyme (NADH) is oxidized by leaving the protons and electrons to oxygen in the mitochondria to synthesize ATP in the electron transport system [48]. So, NAD deficiency causes a reduction of $\beta$-oxidation, and consequently the accumulation of triglycerides in the hepatocytes (steatosis).

The control of rate-limiting enzymes of NAD biosynthesis avoids the negative effects of high-fat diet (HFD) and keeps up insulin sensitivity and glucose homeostasis. Penke et al. [49] reported increased hepatic NAD levels in mice under HFD thanks to increased NAMPT expression. So, it seems that NAD deficiency is a crucial risk factor for NAFLD resulting from having compromised the NAMPT-controlled NAD salvage pathway in liver [50]. Plasma levels of eNAMPT may be closely linked to NAFLD, obesity, diabetes, and atherosclerosis [51-54]. Moreover, decreased NAMPT expression in NAMPT +/- mice, which reduced circulating NMN levels and decreased NAD levels in brown adipose tissue, impaired glucose-stimulated insulin secretion [22]. This event can be rescued by NMN supplementation, suggesting that the maintenance of NAD concentrations is critical for pancreatic function [22].

The mechanisms of NAMPT protecting the liver from HFD are depicted in Figure 2. NAMPT induces the production of NAD by activating the NAD salvage pathway, and consecutively, the augmented NAD (as a substrate) activates the SIRT 1 and 3 signaling pathways, alleviating HFD-induced hepatic steatosis. De novo lipogenesis (DNL) is known to be high in individuals with NAFLD, and provides about $26 \%$ of hepatic lipids $[55,56]$. The NAMPT is critical for the formation of acetyl-CoA and for the increase of fatty acid oxidation by providing NAD for SIRT3 with the activation of acetyl-CoA synthetase (ACS) [57]. At the same time, the activation of SIRT1 by NAMPT promotes the deacetylation of sterol regulatory element-binding protein 1 (SREBP1), which inhibits SREBP1 activity, resulting in the lower expression of lipogenesis genes, including fatty acid synthase (FAS) and acetyl-CoA carboxylase (ACC). Additionally, SIRT 1 directly activates AMP-activated protein kinase (AMPK), which further inhibits SREBP1 activity. All together, these results show that NAMPT modulates processes involved in NAFLD pathogenesis (such as de novo lipogenesis and fatty acid oxidation). Accordingly, Zhou et al. showed that dominant negative-NAMPT transgenic mice, under normal chow, display systemic NAD decrease and had a moderate NASH phenotype, with enhanced oxidative stress, lipid accumulation, impaired insulin sensitivity, and triggered inflammation in liver. These features deteriorate further under HFD [50]. 


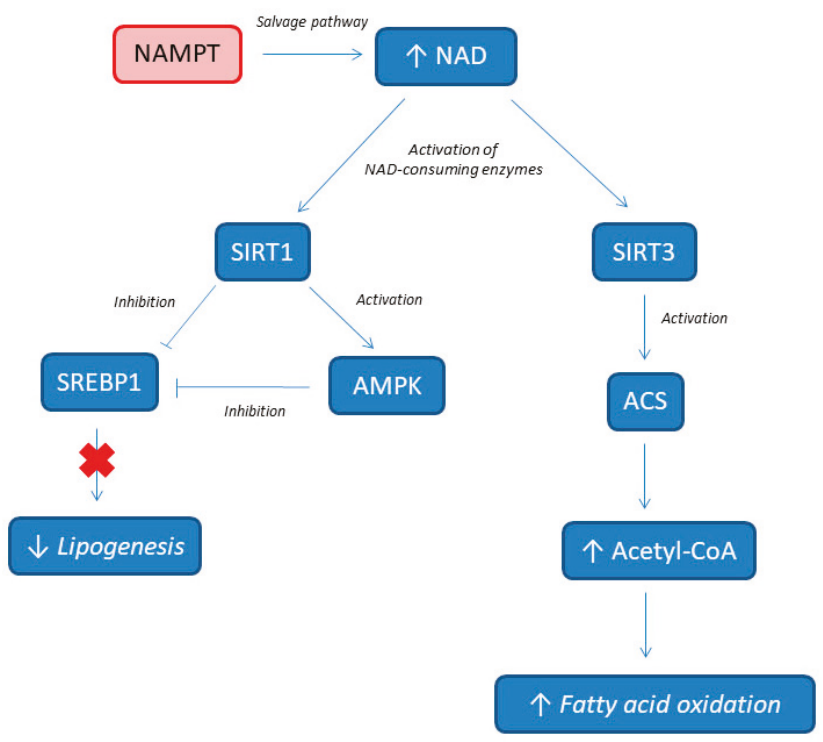

Figure 2. NAMPT involvement in lipids metabolism. NAMPT, nicotinamide phosphoribosyltransferase; NAD, nicotinamide adenine dinucleotide; ACS, acetyl-CoA synthetase; AMPK, AMP-activated protein kinase; SIRT, sirtuin; SREBP1, sterol regulatory element-binding protein 1.

NNMT has also been associated to the development of diabetes, obesity, and metabolic syndrome [28-30]. An increase of NNMT expression has been observed in obesity and diabetes [28-30], probably because NNMT controls lipid, cholesterol, and glucose metabolism by stabilizing SIRTs [58]. In humans, adipose tissue NNMT expression and its product MNAM correlate positively with insulin resistance. Kannt et al. [29] showed an increased expression of NNMT in the adipose tissue of diabetic patients according to the insulin resistance severity, suggesting that NNMT could be a "bad actor" limiting fuel oxidation and promoting fat storage. NNMT protein levels are upregulated in the liver and adipose tissue of mouse models of insulin resistance and obesity, and NNMT knockdown has a protective effect against the metabolic consequences of HFD [28], suggesting that NNMT may have a critical role in NAFLD pathogenesis. The dietary regulation of liver NNMT expression, the site of its major expression, shows some interesting patterns. The ketogenic diet suppresses liver NNMT expression, contributing to the increased liver and serum cholesterol levels in this model [59]. Conversely, caloric restriction increased NNMT liver expression, promoting SIRT1 protein stability, which mediates several metabolic effects of caloric restriction [60]. Liver NNMT expression inversely correlates with serum triglycerides (TGs), cholesterol, and free fatty acid levels, suggesting that increased liver NNMT expression is associated with a better metabolic profile, contrary to its expression in adipose tissue $[28,29]$. Furthermore, a genome-wide association study showed significant associations between the risk of developing NASH and a specific single-nucleotide polymorphisms (SNPs) in the NNMT gene (rs694539) [61]: in this case, subjects with the AA genotype showed a statistically significant increased NASH risk, while the GG genotype seemed to be protective. Similarly, Hasan et al. showed that the AA genotype correlates with the degree of steatosis as detected by the controlled attenuation parameter, even if it does not correlate with the degree of fibrosis detected by FibroScan [62].

\section{NAD as Biomarker for NAFLD Diagnosis}

The identification of non-invasive biomarkers has become a major focus of interest in NAFLD. Since the diagnosis of NASH is still a histological one, the dramatic increase in the prevalence of 
NAFLD and its severity spectrum mean that liver biopsy is not feasible for all patients. Current plasma biomarkers include predictive models for diagnosing or grading steatosis (such as the fatty liver index) or staging fibrosis (such as the NAFLD fibrosis score), and other ones specific to NAFLD (such as the BARD and NAFLD fibrosis scores), even if some have been initially developed in a hepatitis $C$ setting (AST/ALT ratio, APRI, FIB-4) [63].

Several studies evaluated the relationship between NAD metabolism and NAFLD [29,64-68] (Table 1). Human studies investigated how plasma and liver NAMPT protein levels are affected in subjects with steatosis and NAFLD [64-68]. Gaddipati et al. [64] showed that a significant reduction in the NAMPT levels of the visceral adipose tissue is associated to degree of steatosis in NAFLD patients. Similarly, Amirkalali et al. [68] showed that higher serum NAMPT is associated with lower liver DNL in female subjects (probably associated with a higher adipose tissue DNL according to the higher fat mass), while the only significant association in male subjects was between serum NAMPT and liver fat content, probably for the inflammatory role of NAMPT. Thus, the plasma NAMPT levels could have a different meaning for each sex because of the opposing effects of liver and adipose tissue DNL on NAFLD pathogenesis. Conversely, Kannt et al. [29] showed that NNMT mRNA in adipose tissue and 1-methylnicotinamide serum concentrations are higher in patients with insulin resistance and correlate with insulin resistance severity. An additional interesting result is that improvements of insulin sensitivity obtained with exercise and bariatric surgery are associated with a reduction of NNMT expression in adipose tissue and of 1-methylnicotinamide serum levels [29].

\section{NAD Supplementation for NAFLD Prevention}

The evidence for using dietary supplementation to prevent chronic disease is a longstanding issue of debate. Several evidences are emerging to support the hypothesis that supplementation with NAD precursors could protect against metabolic imbalance and liver steatosis (Table 2) [12,49,69-71]. A supplementation study with NMN showed its property to restore NAD levels either in nuclear and mitochondrial cells compartments and to prevent diet-induced and age-induced diabetes in C57BL/6 mice [12]. Tao et al. showed that NAMPT gives resistance to hepatic steatosis through NAD synthesis [69], and NR supplementation gives protection against steatosis in mice under high-fat/high-sucrose diet [70,71]. NAM supplementation protects hepatocytes from palmitate-induced cell death, and autophagy induction contributes to the anti-lipotoxic property of NAM through SIRT1 activation in hepatocytes. Additionally, NAM prevents hepatic alterations in glucose-6-phosphate dehydrogenase and the redox state, and attenuates increased serum FFA, oxidative stress, inflammation, and hepatic damage in high fructose or high glucose consumption-induced liver steatosis in rats [72]. Lastly, Komatsu et al. showed that NNMT and NAM supplementation causes liver steatosis and fibrosis, although increased lipid metabolism and decreased adiposity. NNMT overexpression induces genes for liver steatosis and fibrosis by decreasing tissue NAD content and methylation pool, suggesting that NNMT connects NAD and methionine metabolism and causes NAFLD progression [73]. Thus, NAD supplementation may represent a preventive treatment for metabolic dysfunctions such as diabetes, and NAFLD spectrum disease, from steatosis to NASH.

\section{NAD Supplementation for NAFLD Treatment}

The relevance of dietary NAD precursors in health is well known, thanks to the historical use of NA and NAM in the treatment of dietary tryptophan deficits (pellagra) and hyperlipidemia, although high-dose NA use is limited by painful flushing, while high-dose NAM is hepatotoxic [74,75]. In fact, the use of NA is associated with a flush of face and chest and a sensation of warmth or burning. NA causes flushing principally by releasing prostaglandins D2 and E2 from skin cells, which afterwards dilates skin arterioles [76,77]. The precursors NA, NMN, and NR, but also PARP or CD38 inhibitors, rise NAD levels in different mice cells and tissues [12,13,70]. Boosting NAD concentrations can be therapeutic in metabolic diseases such as diabetes [12,53] and NAFLD [70], and potentially protects against obesity [51] and age-related disorders (Table 3). 
Due to its ability to increase NAD synthesis without inducing side effects [44,70], NR has been used in mice to increase NAD metabolism and improve health in models of metabolic stress, showing that NR abolishes DNA damage in HFD-fed mice [70,78]. Canto et al. [70] treated mice with NR (400 mg/kg animal weight per day), demonstrating an increase of NAD levels in muscle and liver. Mice under HFD were protected from body weight increase and showed an improvement of mitochondrial function and fatty acids oxidation as a fuel source. In accordance with increases in tissue NAD levels, SIRT1 and SIRT3 were upregulated [70]. NR also ameliorated insulin sensitivity in weight-matched mice [70]. Similarly, Zhou et al. [50] demonstrated that the oral administration of NR corrects NAFLD phenotypes induced by NAD deficiency alone or combined with HFD. Trammell et al. [79] performed a clinical study enrolling 12 healthy subjects receiving three single doses of NR, demonstrating that NR supplementation safely induces NAD metabolism at all doses. They also demonstrated that NR is more orally bioavailable than NAM, which is more orally bioavailable than NA. The capability of NR to increase ADPR is threefold higher than NAM. This validates NR as the preferred NAD precursor vitamin for boosting NAD and NAD-consuming activities in liver. No dose-dependent side effects of NR have been reported, contrary to high-dose NAM, which may lead to liver damage [15]. Shi et al. [80] carried out a dose-response dietary intervention mice study using a wide range of NR (from 5 to $900 \mathrm{mg}$ NR per $\mathrm{kg}$ of an obesogenic diet), concluding that $30 \mathrm{mg} / \mathrm{kg}$ diet constitutes the best concentration to reinforce metabolic health. These studies showed the powerful biological effects of NR in mitigating the negative consequences of HFDs [70,71,81,82], suggesting that NAD substrates supplementation may be a promising therapeutic strategy for preventing and treating NAFLD/NASH.

Another possibility to modulate NAD levels consists of using NMN. Supplementation with NMN, an enzymatic product of NAMPT, improves diabetes [12,13] and other damages such as vascular dysfunction, oxidative stress [83], and cognitive impairment [84]. Yoshino et al. demonstrated that increasing NAD biosynthesis by the intraperitoneal injection of NMN improves glucose homeostasis in obese mice, and that NAMPT activity is altered by HFD and can cause diabetes [12]. Similarly, supplementation with MNAM significantly reduces hepatic cholesterol and triglycerides concentrations, by suppressing fatty acid and cholesterol synthesis and the expression of lipogenic and cholesterol synthesis genes [58]. MNAM supplementation produces a selective reduction in larger lipoprotein particles but not high-density lipoprotein, suggesting that MNAM or its derivatives could be used to reduce low-density lipoprotein levels [58].

Another attractive angle to modulate NAD levels consists in targeting the activity of NAD-consuming enzymes, such as CD38 [10] and PARPs [11]. Several studies showed that CD38 knockout $(\mathrm{KO})$ mice have higher NAD levels than Wild-type (WT) animals, and are protected against obesity and metabolic syndrome $[10,85]$. The treatment of obese mice with CD38 inhibitors augments intracellular NAD concentrations and improves glucose and lipid homeostasis [86]. Increased PARP activity causes an elevated consumption of cellular NAD, which is associated to increased ATP consumption, compromising energy balance and facilitating cell death [87]. Upon persistent PARP activation, decreased mitochondrial ATP production inhibits NAD re-synthesis, creating a feed-forward loop in ATP-consuming processes, and resulting in metabolic catastrophe and cell death. PARP inhibition causes an increase in NAD levels. Rucaparib (a PARP inhibitor) significantly increases hepatic NAD levels, as previously described with NAM treatment [88], while in PARP1 KO liver, NAD levels were similar to those in treated PARP1 WT liver. So, CD38 and PARP inhibition combined with NAD precursors may be an intriguing therapeutic perspective for NAFLD [13].

Finally, Katsiuba et al. presented an additional mechanism for increasing NAD levels toward the inhibition of the ACMS decarboxylase with a selective inhibitor recently developed, TES-991. ACMS decarboxylase inhibition in a mouse model of diet-induced NAFLD increased levels of NAD and the activation of SIRT1 with improvement of the NAFLD phenotype, without systemic side effects [14]. 


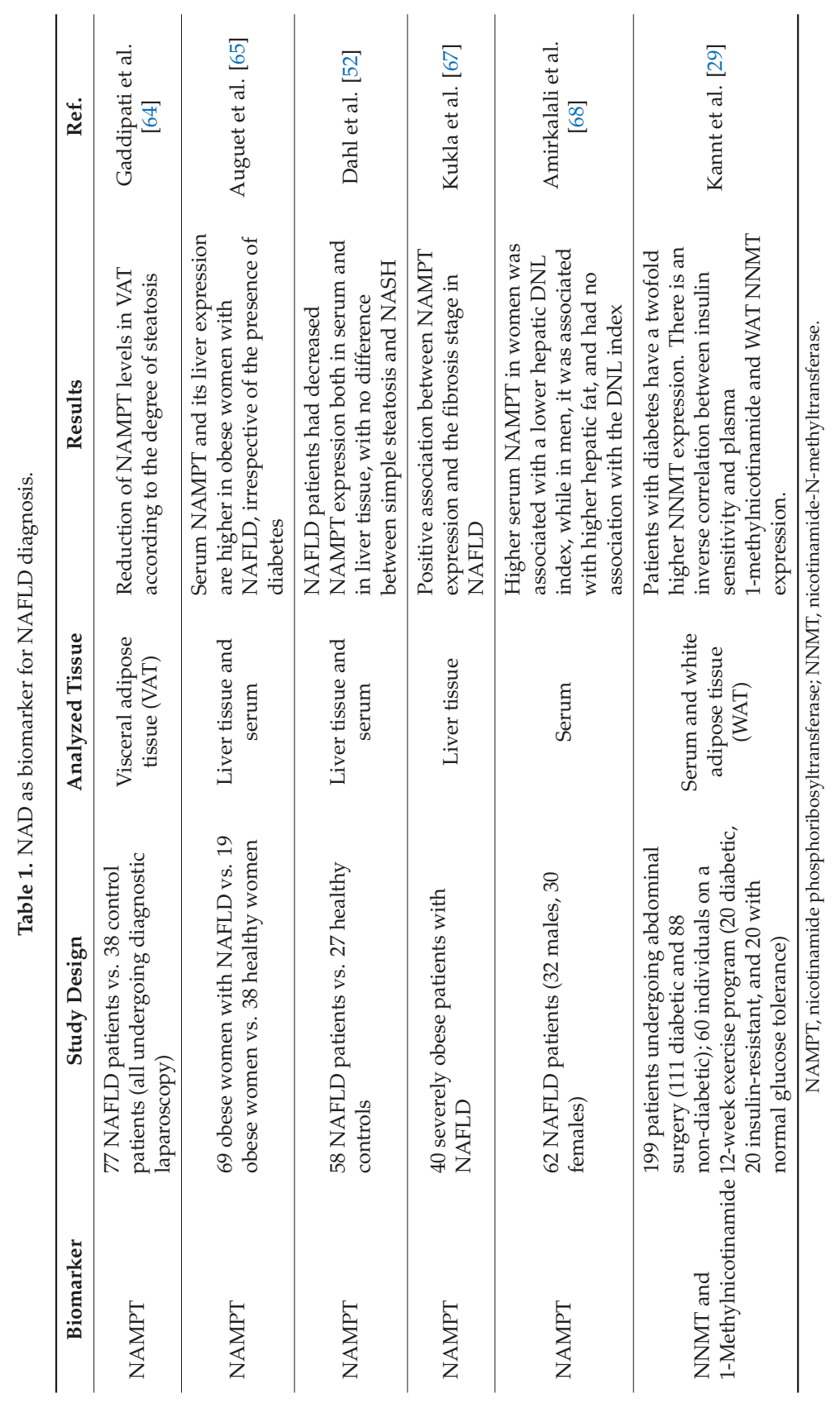




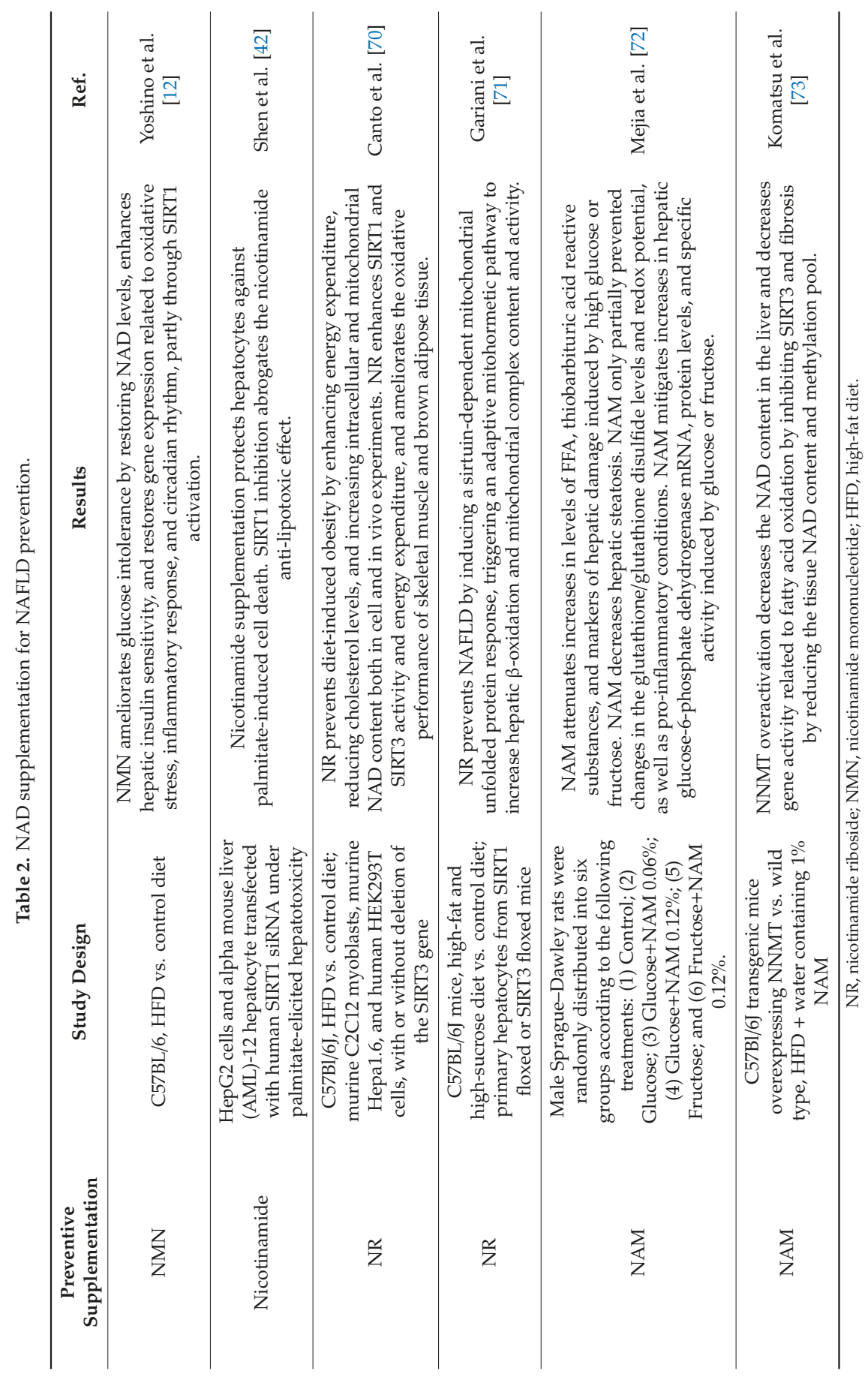




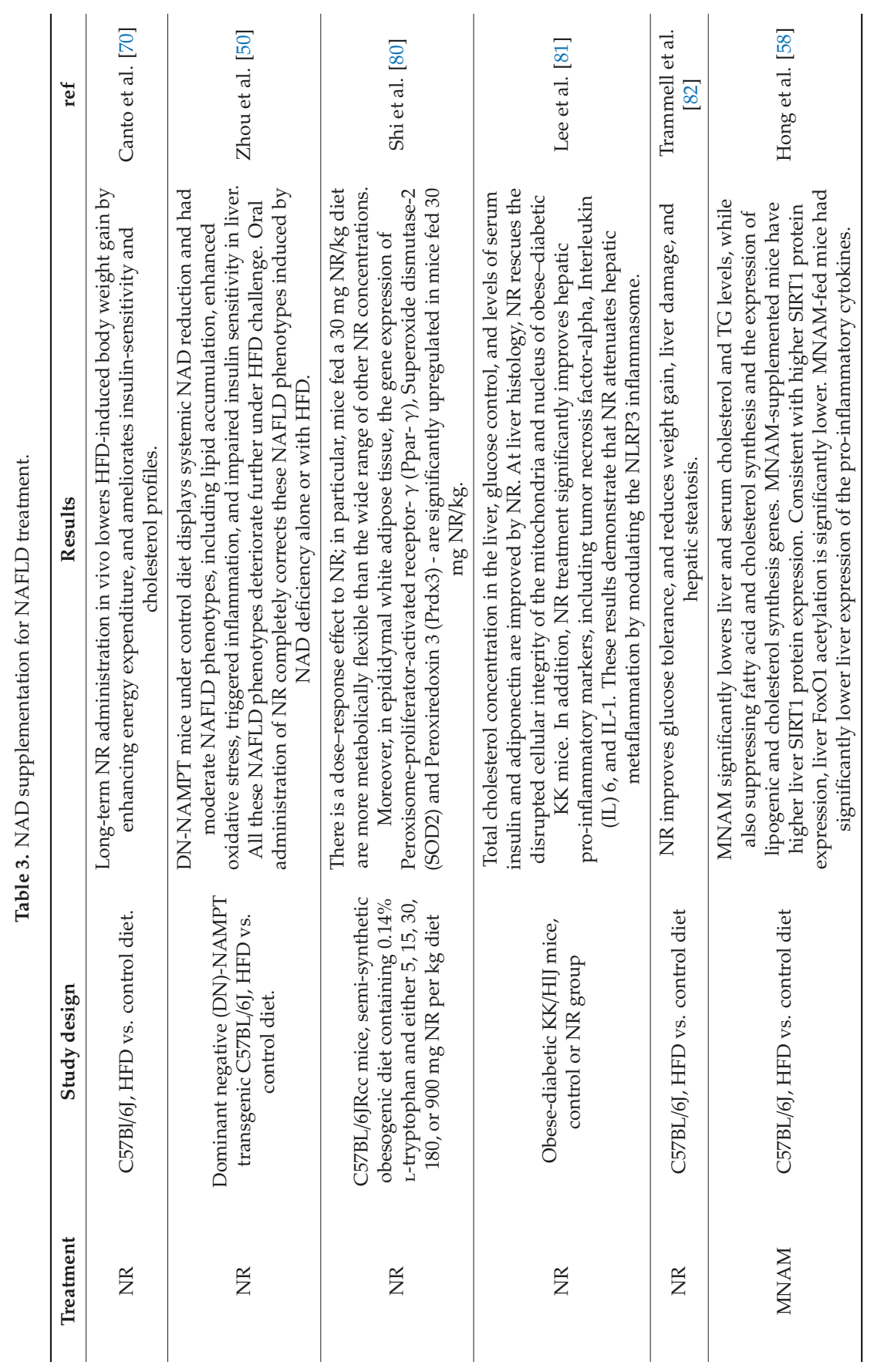




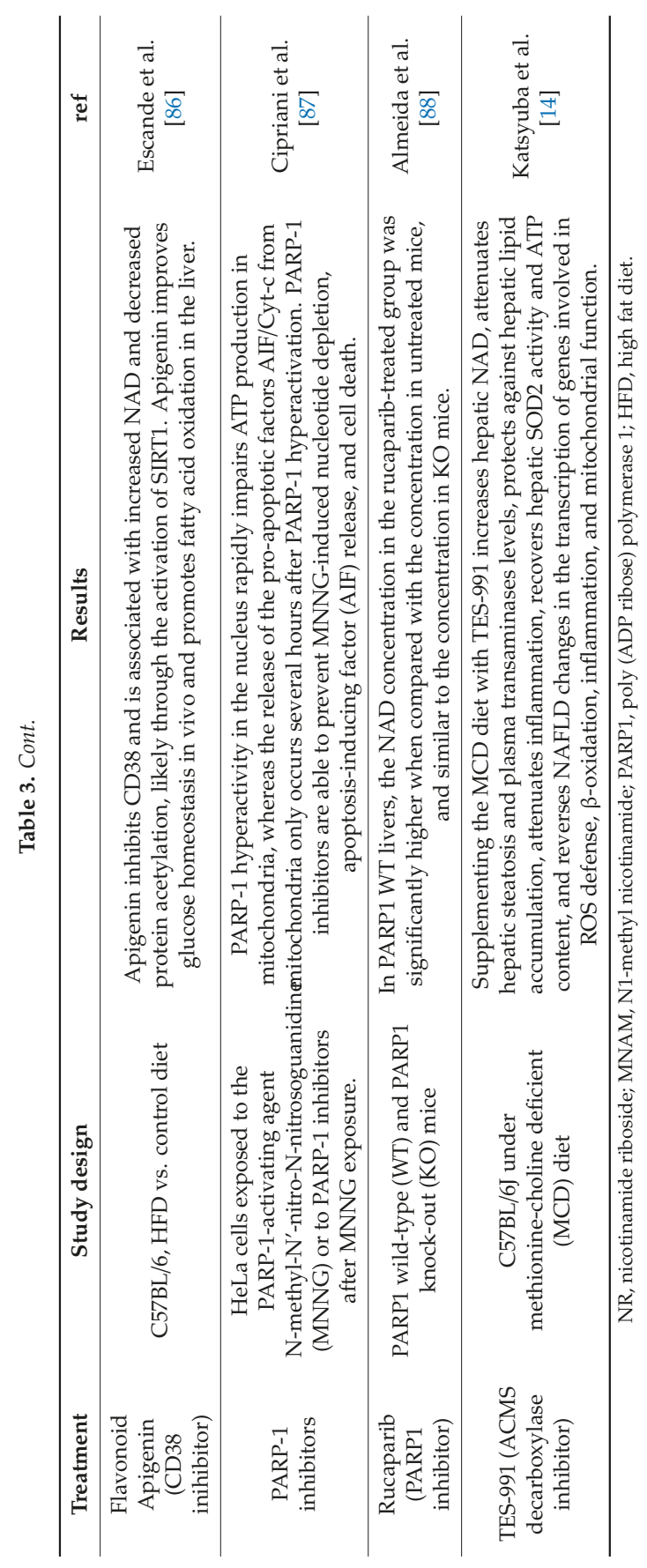




\title{
7. Conclusions
}

Until now, there is still no approved drug for the treatment of NAFLD, and although lifestyle modification appears beneficial in patients with NAFLD, no single approach is likely to be suitable for all patients. NAD reduction might be caused by the imbalance in NAD biosynthesis and depletion, both of which occur in NAFLD. NAD reduction may induce NAFLD through decreased SIRT activities in the nucleus and mitochondria. The supplementation of key NAD intermediates, such as NMN and NR, can ameliorate NAFLD.

Author Contributions: Conceptualization, J.-F.D. and M.G.; Literature Analysis, M.G.; Writing-Original Draft Preparation, M.G. and J.-F.D.; Writing-Review and Editing, M.G. and J.-F.D.

Funding: J.F.D is supported by the Swiss National Foundation (310030-185219).

Conflicts of Interest: JF Dufour: Advisory committee for Abbvie, Bayer, BMS, Fallk, Genfit, Genkyotex, Gilead, Heparegenerix, Intercept, Lilly, Merck, Novartis. M Guarino has no conflicts of interest to declare.

\begin{abstract}
Abbreviations
ACC, acetyl-CoA carboxylase; ACMS, amino- $\beta$-carboxymuconate- $\varepsilon$-semialdehyde; ACS, acetyl-CoA synthetase; ADPR, ADP ribose; AMPK, AMP-activated protein kinase; DNL, de novo lipogenesis; eNAMPT, extracellular NAMPT; FAS, fatty acid synthase; FFA, free fatty acid; HCC, hepatocellular carcinoma; HFD, high fat diet; iNAMPT, intracellular NAMPT; KO, knock-out; MNAM, N1-methyl nicotinamide; NA, nicotinic acid; NAD, nicotinamide adenine dinucleotide; NADP, phosphorylated nicotinamide adenine dinucleotide; NAFLD, nonalcoholic fatty liver disease; NAM, nicotinamide; NAMN, NA mononucleotide; NAMPT, nicotinamide phosphoribosyltransferase; NAPRT1, nicotinate phosphoribosyltransferase domain containing 1; NASH, non-alcoholic steatohepatitis; NMN, nicotinamide mononucleotide; NMNAT, nicotinamide nucleotide adenylyltransferase 3; NNMT, nicotinamide-N-methyltransferase; NR, nicotinamide riboside; NRK, NR kinase; PARP, poly (ADP ribose) polymerase; ROS, reactive oxygen species; SIRT, sirtuin; SREBP1, sterol regulatory element-binding protein 1; TNF- $\alpha$, tumor necrosis factor- $\alpha$; WT, wild-type.
\end{abstract}

\section{References}

1. Kalra, S. Diabesity. J. Pak. Med. Assoc. 2013, 63, 532-534.

2. Musso, G.; Gambino, R.; De Michieli, F.; Cassader, M.; Rizzetto, M.; Durazzo, M.; Fagà, E.; Silli, B.; Pagano, G. Dietary habits and their relations to insulin resistance and postprandial lipemia in nonalcoholic steatohepatitis. Hepatology 2003, 37, 909-916. [CrossRef]

3. Sookoian, S.; Burgueno, A.L.; Castano, G.; Pirola, C.J. Should nonalcoholic fatty liver disease be included in the definition of metabolic syndrome? A cross-sectional comparison with adult treatment panel III criteria in nonobese nondiabetic subjects: Response to Musso. Diabetes Care 2008, 31, e42. [CrossRef]

4. Kawano, Y.; Cohen, D.E. Mechanisms of hepatic triglyceride accumulation in non-alcoholic fatty liver disease. J. Gastroenterol. 2013, 48, 434-441. [CrossRef]

5. Koo, S.H. Nonalcoholic fatty liver disease: Molecular mechanisms for the hepatic steatosis. Clin. Mol. Hepatol. 2013, 19, 210-215. [CrossRef]

6. Jiang, Z.G.; Tapper, E.B.; Connelly, M.A.; Pimentel, C.F.; Feldbrügge, L.; Kim, M.; Krawczyk, S.; Afdhal, N.; Robson, S.C.; Herman, M.A.; et al. Steatohepatitis and liver fibrosis are predicted by the characteristics of very low density lipoprotein in nonalcoholic fatty liver disease. Liver Int. 2016, 36, 1213-1220. [CrossRef]

7. Sherriff, J.L.; O'Sullivan, T.A.; Properzi, C.; Oddo, J.L.; Adams, L.A. Choline, its potential role in nonalcoholic fatty liver disease, and the case for human and bacterial genes. Adv. Nutr. 2016, 7, 5-13. [CrossRef]

8. Buzzetti, E.; Pinzani, M.; Tsochatzis, E.A. The multiple-hit pathogenesis of non-alcoholic fatty liver disease (NAFLD). Metabolism 2016, 65, 1038-1048. [CrossRef]

9. Canto, C.; Auwerx, J. NAD ${ }^{+}$as a signaling molecule modulating metabolism. Cold Spring Harb. Symp. Quant. Biol. 2011, 76, 291-298. [CrossRef]

10. Barbosa, M.T.; Soares, S.M.; Novak, C.M.; Sinclair, D.; Levine, J.A.; Aksoy, P.; Chini, E.N. The enzyme CD38 (a NAD glycohydrolase, EC 3.2.2.5) is necessary for the development of diet-induced obesity. FASEB J. 2007, 21, 3629-3639. [CrossRef]

11. Bai, P.; Cantó, C.; Oudart, H.; Brunyánszki, A.; Cen, Y.; Thomas, C.; Yamamoto, H.; Huber, A.; Kiss, B.; Houtkooper, R.H.; et al. PARP-1 inhibition increases mitochondrial metabolism through SIRT1 activation. Cell Metab. 2011, 13, 461-468. [CrossRef] 
12. Yoshino, J.; Mills, K.F.; Yoon, M.J.; Imai, S.I. Nicotinamide mononucleotide, ia key NAD(+) intermediate, treats the pathophysiology of diet- and age-induced diabetes in mice. Cell Metab. 2011, 14, 528-536. [CrossRef]

13. Mouchiroud, L.; Houtkooper, R.H.; Auwerx, J. NAD ${ }^{+}$metabolism: A therapeutic target for age-related metabolic disease. Crit. Rev. Biochem. Mol. Biol. 2013, 48, 397-408. [CrossRef]

14. Katsyuba, E.; Mottis, A.; Zietak, M.; De Franco, F.; van der Velpen, V.; Gariani, K.; Ryu, D.; Cialabrini, L.; Matilainen, O.; Liscio, P.; et al. De novo $\mathrm{NAD}^{+}$synthesis enhances mitochondrial function and improves health. Nature 2018, 563, 354-359. [CrossRef]

15. Bogan, K.L.; Brenner, C. Nicotinic acid, nicotinamide, and nicotinamide riboside: A molecular evaluation of $\mathrm{NAD}^{+}$precursor vitamins in human nutrition. Annu. Rev. Nutr. 2008, 28, 115-130. [CrossRef]

16. Samal, B.; Sun, Y.; Stearns, G.; Xie, C.; Suggs, S.; McNiece, I. Cloning and characterization of the cDNA encoding a novel human pre-B-cell colony enhancing factor. Mol. Cell Biol. 1994, 14, 1431-1437. [CrossRef]

17. Rongvaux, A.; Shea, R.J.; Mulks, M.H.; Gigot, D.; Urbain, J.; Leo, O.; Andris, F. Pre-B cell colony-enhancing factor, whose expression is up-regulated in activated lymphocytes, is a nicotinamide phosphoribosyltransferase, a cytosolic enzyme involved in NAD biosynthesis. Eur. J. Immunol. 2002, 32, 3225-3234. [CrossRef]

18. Tanaka, M.; Nozaki, M.; Fukuhara, A.; Segawa, K.; Aoki, N.; Matsuda, M.; Komuro, R.; Shimomura, I. Visfatin is released from 3T3-L1 adipocytes via a non-classical pathway. Biochem. Biophys. Res. Commun. 2007, 359, 194-201. [CrossRef]

19. Garten, A.; Petzold, S.; Barnikol-Oettler, A.; Körner, A.; Thasler, W.E.; Kratzsch, J.; Kiess, W.; Gebhardt, R. Nicotinamide phosphoribosyltransferase (NAMPT/PBEF/visfatin) is constitutively released from human hepatocytes. Biochem. Biophys. Res. Commun. 2010, 391, 376-381. [CrossRef]

20. Friebe, D.; Neef, M.; Kratzsch, J.; Erbs, S.; Dittrich, K.; Garten, A.; Petzold-Quinque, S.; Blüher, S.; Reinehr, T.; Stumvoll, M.; et al. Leucocytes are a major source of circulating nicotinamide phosphoribosyl transferase (NAMPT)/pre-B cell colony (PBEF)/visfatin linking obesity and inflammation in humans. Diabetologia 2011, 54, 1200-1211. [CrossRef]

21. Yang, H.; Lavu, S.; Sinclair, D.A. Nampt/PBEF/Visfatin: A regulator of mammalian health and longevity? Exp. Gerontol. 2006, 41, 718-726. [CrossRef] [PubMed]

22. Revollo, J.R.; Körner, A.; Mills, K.F.; Satoh, A.; Wang, T.; Garten, A.; Dasgupta, B.; Sasaki, Y.; Wolberger, C.; Townsend, R.R.; et al. Nampt/PBEF/Visfatin regulates insulin secretion in beta cells as a systemic NAD biosynthetic enzyme. Cell Metab. 2007, 6, 363-375. [CrossRef] [PubMed]

23. Yang, H.; Yang, T.; Baur, J.A.; Perez, E.; Matsui, T.; Carmona, J.J.; Lamming, D.W.; Souza-Pinto, N.C.; Bohr, V.A.; Rosenzweig, A.; et al. Nutrient-sensitive mitochondrial NAD ${ }^{+}$levels dictate cell survival. Cell 2007, 130, 1095-1107. [CrossRef] [PubMed]

24. Bowlby, S.C.; Thomas, M.J.; D’Agostino, R.B., Jr.; Kridel, S.J. Nicotinamide phosphoribosyl transferase (Nampt) is required for de novo lipogenesis in tumor cells. PLoS ONE 2012, 7, e40195. [CrossRef] [PubMed]

25. Pillai, J.B.; Isbatan, A.; Imai, S.; Gupta, M.P. Poly(ADP-ribose) polymerase 1 dependent cardiac myocyte cell death during heart failure is mediated by $\mathrm{NAD}^{+}$depletion and reduced Sir2 $\alpha$ deacetylase activity. J. Biol. Chem. 2005, 280, 43121-43130. [CrossRef]

26. Rodgers, J.T.; Lerin, C.; Gerhart-Hines, Z.; Puigserver, P. Metabolic adaptations through the PGC $1 \propto$ and SIRT1 pathways. FEBS Lett. 2008, 582, 46-53. [CrossRef]

27. Luo, X.; Kraus, W.L. On PAR with PARP: Cellular stress signaling through poly (ADP-ribose) and PARP 1. Genes Dev. 2012, 26, 417-432. [CrossRef]

28. Kraus, D.; Yang, Q.; Kong, D.; Banks, A.S.; Zhang, L.; Rodgers, J.T.; Pirinen, E.; Pulinilkunnil, T.C.; Gong, F.; Wang, Y.C.; et al. Nicotinamide Nmethyltransferase knockdown protects against diet-induced obesity. Nature 2014, 508, 258-262. [CrossRef]

29. Kannt, A.; Pfenninger, A.; Teichert, L.; Tönjes, A.; Dietrich, A.; Schön, M.R.; Klöting, N.; Blüher, M. Association of nicotinamide-N-methyltransferase mRNA expression in human adipose tissue and the plasma concentration of its product, 1-methylnicotinamide, with insulin resistance. Diabetologia 2015, 58, 799-808. [CrossRef]

30. Giuliante, R.; Sartini, D.; Bacchetti, T.; Rocchetti, R.; Klöting, I.; Polidori, C.; Ferretti, G.; Emanuelli, M. Potential involvement of nicotinamide n-methyltransferase in the pathogenesis of metabolic syndrome. Metab. Syndr. Relat. Disord. 2015, 13, 165-170. [CrossRef] 
31. Imai, S.; Armstrong, C.M.; Kaeberlein, M.; Guarente, L. Transcriptional silencing and longevity protein Sir2 is an NAD-dependent histone, deacetylase. Nature 2000, 403, 795-800. [CrossRef] [PubMed]

32. Pfluger, P.T.; Herranz, D.; Velasco-Miguel, S.; Serrano, M.; Tschöp, M.H. Sirt1 protects against high-fat diet-induced metabolic damage. Proc. Natl. Acad. Sci. USA 2008, 105, 9793-9798. [CrossRef] [PubMed]

33. Houtkooper, R.H.; Pirinen, E.; Auwerx, J. Sirtuins as regulators of metabolism and healthspan. Nat. Rev. Mol. Cell Biol. 2012, 13, 225-238. [CrossRef] [PubMed]

34. Caron, A.Z.; He, X.; Mottawea, W.; Seifert, E.L.; Jardine, K.; Dewar-Darch, D.; Cron, G.O.; Harper, M.E.; Stintzi, A.; McBurney, M.W. The SIRT1 deacetylase protects mice against the symptoms of metabolic syndrome. FASEB J. 2014, 28, 1306-1316. [CrossRef] [PubMed]

35. Asher, G.; Gatfield, D.; Stratmann, M.; Reinke, H.; Dibner, C.; Kreppel, F.; Mostoslavsky, R.; Alt, F.W.; Schibler, U. SIRT1 regulates circadian clock gene expression through PER2 deacetylation. Cell 2008, 134, 317-328. [CrossRef] [PubMed]

36. Nakahata, Y.; Kaluzova, M.; Grimaldi, B.; Sahar, S.; Hirayama, J.; Chen, D.; Guarente, L.P.; Sassone-Corsi, P. The $\mathrm{NAD}^{+}$-dependent deacetylase SIRT1 modulates CLOCK-mediated chromatin remodeling and circadian control. Cell 2008, 134, 329-340. [CrossRef] [PubMed]

37. Imai, S. "Clocks" in the NAD World: NAD as a metabolic oscillator for the regulation of metabolism and aging. Biochim. Biophys. Acta 2010, 1804, 1584-1590. [CrossRef] [PubMed]

38. Peek, C.B.; Affinati, A.H.; Ramsey, K.M.; Kuo, H.Y.; Yu, W.; Sena, L.A.; Ilkayeva, O.; Marcheva, B.; Kobayashi, Y.; Omura, C.; et al. Circadian clock NAD ${ }^{+}$cycle drives mitochondrial oxidative metabolism in mice. Science 2013, 342, 1243417. [CrossRef]

39. Fulco, M.; Schiltz, R.L.; Iezzi, S.; King, M.T.; Zhao, P.; Kashiwaya, Y.; Hoffman, E.; Veech, R.L.; Sartorelli, V. Sir2 regulates skeletal muscle differentiation as a potential sensor of the redox state. Mol. Cell 2003, 12, 51-62. [CrossRef]

40. Kendrick, A.A.; Choudhury, M.; Rahman, S.M.; McCurdy, C.E.; Friederich, M.; Van Hove, J.L.; Watson, P.A.; Birdsey, N.; Bao, J.; Gius, D.; et al. Fatty liver is associated with reduced SIRT3 activity and mitochondrial protein hyperacetylation. Biochem. J. 2011, 433, 505-514. [CrossRef]

41. Ishikawa, S.; Li, G.; Takemitsu, H.; Fujiwara, M.; Mori, N.; Yamamoto, I.; Arai, T. Change in mRNA expression of sirtuin 1 and sirtuin 3 in cats fed on high fat diet. BMC Vet. Res. 2013, 9, 187. [CrossRef] [PubMed]

42. Shen, C.; Dou, X.; Ma, Y.; Ma, W.; Li, S.; Song, Z. Nicotinamide protects hepatocytes against palmitate-induced lipotoxicity via SIRT1-dependent autophagy induction. Nutr. Res. 2017, 40, 40-47. [CrossRef] [PubMed]

43. Lin, S.-J.; Ford, E.; Haigis, M.; Liszt, G.; Guarente, L. Calorie restriction extends yeast life span by lowering the level of NADH. Genes Dev. 2004, 18, 12-16. [CrossRef] [PubMed]

44. Zhang, H.; Ryu, D.; Wu, Y.; Gariani, K.; Wang, X.; Luan, P.; D'Amico, D.; Ropelle, E.R.; Lutolf, M.P.; Aebersold, R.; et al. NAD ${ }^{+}$repletion improves mitochondrial and stem cell function and enhances life span in mice. Science 2016, 352, 1436-1443. [CrossRef] [PubMed]

45. Satoh, A.; Stein, L.; Imai, S. The role of mammalian sirtuins in the regulation of metabolism, aging, and longevity. Handb. Exp. Pharmacol. 2011, 206, 125-162. [CrossRef]

46. Von Schönfels, W.; Patsenker, E.; Fahrner, R.; Itzel, T.; Hinrichsen, H.; Brosch, M.; Erhart, W.; Gruodyte, A.; Vollnberg, B.; Richter, K.; et al. Metabolomic tissue signature in human non-alcoholic fatty liver disease identifies protective candidate metabolites. Liver Int. 2015, 35, 207-214. [CrossRef] [PubMed]

47. Mukherjee, S.; Chellappa, K.; Moffitt, A.; Ndungu, J.; Dellinger, R.W.; Davis, J.G.; Agarwal, B.; Baur, J.A. Nicotinamide adenine dinucleotide biosynthesis promotes liver regeneration. Hepatology 2017, 65, 616-630. [CrossRef]

48. Shi, L.; Tu, B.P. Acetyl-CoA and the regulation of metabolism: Mechanisms and consequences. Curr. Opin. Cell Biol. 2015, 33, 125-131. [CrossRef]

49. Penke, M.; Larsen, P.S.; Schuster, S.; Dall, M.; Jensen, B.A.; Gorski, T.; Meusel, A.; Richter, S.; Vienberg, S.G.; Treebak, J.T.; et al. Hepatic NAD salvage pathway is enhanced in mice on a high-fat diet. Mol. Cell Endocrinol. 2015, 412, 65-72. [CrossRef]

50. Zhou, C.C.; Yang, X.; Hua, X.; Liu, J.; Fan, M.B.; Li, G.Q.; Song, J.; Xu, T.Y.; Li, Z.Y.; Guan, Y.F.; et al. Hepatic $\mathrm{NAD}(+)$ deficiency as a therapeutic target for non-alcoholic fatty liver disease in ageing. Br. J. Pharmacol. 2016, 173, 2352-2368. [CrossRef] 
51. Chang, Y.H.; Chang, D.M.; Lin, K.C.; Shin, S.J.; Lee, Y.J. Visfatin in overweight/obesity, type 2 diabetes mellitus, insulin resistance, metabolic syndrome and cardiovascular diseases: A meta-analysis and systemic review. Diabetes Metab. Res. Rev. 2011, 27, 515-527. [CrossRef] [PubMed]

52. Dahl, T.B.; Yndestad, A.; Skjelland, M.; Oie, E.; Dahl, A.; Michelsen, A.; Damas, J.K.; Tunheim, S.H.; Ueland, T.; Smith, C.; et al. Increased expression of visfatin in macrophages of human unstable carotid and coronary atherosclerosis: Possible role in inflammation and plaque, destabilization. Circulation 2007, 115, 972-980. [CrossRef] [PubMed]

53. El-Mesallamy, H.O.; Kassem, D.H.; El-Demerdash, E.; Amin, A.I. Vaspin and visfatin/Nampt are interesting interrelated adipokines playing a role in the pathogenesis of type 2 diabetes mellitus. Metabolism 2011, 60, 63-70. [CrossRef] [PubMed]

54. Aller, R.; de Luis, D.A.; Izaola, O.; Sagrado, M.G.; Conde, R.; Velasco, M.C.; Alvarez, T.; Pacheco, D.; Gonzalez, J.M. Influence of visfatin on histopathological changes of non-alcoholic fatty liver disease. Dig. Dis. Sci. 2009, 54, 1772-1777. [CrossRef] [PubMed]

55. Lee, J.J.; Lambert, J.E.; Hovhannisyan, Y.; Ramos-Roman, M.A.; Trombold, J.R.; Wagner, D.A.; Parks, E.J. Palmitoleic acid is elevated in fatty liver disease and reflects hepatic lipogenesis. Am. J. Clin. Nutr. 2015, 101, 34-43. [CrossRef] [PubMed]

56. Berlanga, A.; Guiu-Jurado, E.; Porras, J.A.; Auguet, T. Molecular pathways in non-alcoholic fatty liver disease. Clin. Exp. Gastroenterol. 2014, 7, 221-239. [CrossRef]

57. Hallows, W.C.; Lee, S.; Denu, J.M. Sirtuins deacetylate and activate mammalian acetyl-CoA synthetases. Proc. Natl. Acad. Sci. USA 2006, 103, 10230-10235. [CrossRef]

58. Hong, S.; Moreno-Navarrete, J.M.; Wei, X.; Kikukawa, Y.; Tzameli, I.; Prasad, D.; Lee, Y.; Asara, J.M.; Fernandez-Real, J.M.; Maratos-Flier, E.; et al. Nicotinamide N-methyltransferase regulates hepatic nutrient metabolism through Sirt1 protein stabilization. Nat. Med. 2015, 21, 887-894. [CrossRef]

59. Kennedy, A.R.; Pissios, P.; Otu, H.; Roberson, R.; Xue, B.; Asakura, K.; Furukawa, N.; Marino, F.E.; Liu, F.F.; Kahn, B.B.; et al. A high-fat, ketogenic diet induces a unique metabolic state in mice. Am. J. Physiol. 2007, 292, E1724-E1739. [CrossRef]

60. Bordone, L.; Cohen, D.; Robinson, A.; Motta, M.C.; van Veen, E.; Czopik, A.; Steele, A.D.; Crowe, H.; Marmor, S.; Luo, J.; et al. SIRT1 transgenic mice show phenotypes resembling calorie restriction. Aging Cell 2007, 6, 759-767. [CrossRef]

61. Sazci, A.; Ozel, M.D.; Ergul, E.; Aygun, C. Association of nicotinamide-N-methyltransferase gene rs694539 variant with patients with nonalcoholic steatohepatitis. Genet. Test. Mol. Biomark. 2013, 17, 849-853. [CrossRef] [PubMed]

62. Hasan, E.M.; Abd Al Aziz, R.A.; Sabry, D.; Darweesh, S.K.; Badary, H.A.; Elsharkawy, A.; Abouelkhair, M.M.; Yosry, A. Genetic Variants in nicotinamide-N-methyltransferase (NNMT) gene are related to the stage of non-alcoholic fatty liver disease diagnosed by controlled attenuation parameter (CAP)-fibroscan. J. Gastrointest. Liver Dis. 2018, 27, 265-272. [CrossRef]

63. Castera, L.; Friedrich-Rust, M.; Loomba, R. Noninvasive Assessment of Liver Disease in Patients with NAFLD. Gastroenterology 2019, 156, 1264-1281.e4. [CrossRef] [PubMed]

64. Gaddipati, R.; Sasikala, M.; Padaki, N.; Mukherjee, R.M.; Sekaran, A.; Jayaraj-Mansard, M.; Rabella, P.; Rao-Guduru, V.; Reddy-Duvvuru, N. Visceral adipose tissue visfatin in nonalcoholic fatty liver disease. Ann. Hepatol. 2010, 9, 266-270. [CrossRef]

65. Auguet, T.; Terra, X.; Porras, J.A.; Orellana-Gavaldà, J.M.; Martinez, S.; Aguilar, C.; Lucas, A.; Pellitero, S.; Hernández, M.; Del Castillo, D.; et al. Plasma visfatin levels and gene expression in morbidly obese women with associated fatty liver disease. Clin. Biochem. 2013, 46, 202-208. [CrossRef] [PubMed]

66. Dahl, T.B.; Haukeland, J.W.; Yndestad, A.; Ranheim, T.; Gladhaug, I.P.; Damås, J.K.; Haaland, T.; Løberg, E.M.; Arntsen, B.; Birkeland, K.; et al. Intracellular nicotinamide phosphoribosyl transferase protects against hepatocyte apoptosis and is down-regulated in nonalcoholic fatty liver disease. J. Clin. Endocrinol. Metab. 2010, 95, 3039-3047. [CrossRef] [PubMed]

67. Kukla, M.; Ciupińska-Kajor, M.; Kajor, M.; Wyleżoł, M.; Zwirska-Korczala, K.; Hartleb, M.; Berdowska, A.; Mazur, W. Liver visfatin expression in morbidly obese patients with nonalcoholic fatty liver disease undergoing bariatric surgery. Pol. J. Pathol. 2010, 61, 147-153. [PubMed] 
68. Amirkalali, B.; Sohrabi, M.R.; Esrafily, A.; Jalali, M.; Gholami, A.; Hosseinzadeh, P.; Keyvani, H.; Shidfar, F.; Zamani, F. Association between Nicotinamide Phosphoribosyltransferase and de novo Lipogenesis in Nonalcoholic Fatty Liver Disease. Med. Princ. Pract. 2017, 26, 251-257. [CrossRef]

69. Tao, R.; Wei, D.; Gao, H.; Liu, Y.; DePinho, R.A.; Dong, X.C. Hepatic FoxOs regulate lipid metabolism via modulation of expression of the nicotinamide phosphoribosyltransferase gene. J. Biol. Chem. 2011, 286, 14681-14690. [CrossRef]

70. Canto, C.; Houtkooper, R.H.; Pirinen, E.; Youn, D.Y.; Oosterveer, M.H.; Cen, Y.; Fernandez-Marcos, P.J.; Yamamoto, H.; Andreux, P.A.; Cettour-Rose, P.; et al. The NAD(+) precursor nicotinamide riboside enhances oxidative metabolism and protects against high-fat diet-induced obesity. Cell Metab. 2012, 15, 838-847. [CrossRef]

71. Gariani, K.; Menzies, K.J.; Ryu, D.; Wegner, C.J.; Wang, X.; Ropelle, E.R.; Moullan, N.; Zhang, H.; Perino, A.; Lemos, V.; et al. Eliciting the mitochondrial unfolded protein response by nicotinamide adenine dinucleotide repletion reverses fatty liver disease in mice. Hepatology 2016, 63, 1190-1204. [CrossRef] [PubMed]

72. Mejía, S.Á.; Gutman, L.A.B.; Camarillo, C.O.; Navarro, R.M.; Becerra, M.C.S.; Santana, L.D.; Cruz, M.; Pérez, E.H.; Flores, M.D. Nicotinamide prevents sweet beverage-induced hepatic steatosis in rats by regulating the G6PD, NADPH/NADP ${ }^{+}$and GSH/GSSG ratios and reducing oxidative and inflammatory, stress. Eur. J. Pharmacol. 2018, 818, 499-507. [CrossRef] [PubMed]

73. Komatsu, M.; Kanda, T.; Urai, H.; Kurokochi, A.; Kitahama, R.; Shigaki, S.; Ono, T.; Yukioka, H.; Hasegawa, K.; Tokuyama, H.; et al. NNMT activation can contribute to the development of fatty liver disease by modulating the NAD ${ }^{+}$metabolism. Sci. Rep. 2018, 8, 8637. [CrossRef] [PubMed]

74. Houtkooper, R.H.; Canto, C.; Wanders, R.J.; Auwerx, J. The secret life of NAD ${ }^{+}$: An old metabolite controlling new metabolic signaling pathways. Endocr. Rev. 2010, 31, 194-223. [CrossRef] [PubMed]

75. Knip, M.; Douek, I.F.; Moore, W.P.; Gillmor, H.A.; McLean, A.E.; Bingley, P.J.; Gale, E.A.; European Nicotinamide Diabetes Intervention Trial Group. Safety of high-dose nicotinamide: A review. Diabetologia 2000, 43, 1337-1345. [CrossRef] [PubMed]

76. Lukasova, M.; Hanson, J.; Tunaru, S.; Offermanns, S. Nicotinic acid (niacin): New lipid independent mechanisms of action and therapeutic potentials. Trends Pharmacol. Sci. 2011, 32, 700-707. [CrossRef] [PubMed]

77. Yadav, R.; France, M.; Younis, N.; Hama, S.; Ammori, B.J.; Kwok, S.; Soran, H. Extended release niacin with laropiprant: A review on efficacy, clinical effectiveness and safety. Expert Opin. Pharmacother. 2012, 13, 1345-1362. [CrossRef] [PubMed]

78. Gomes, A.L.; Teijeiro, A.; Burén, S.; Tummala, K.S.; Yilmaz, M.; Waisman, A.; Theurillat, J.P.; Perna, C.; Djouder, N. Metabolic Inflammation-Associated IL-17A Causes Non-alcoholic Steatohepatitis and Hepatocellular Carcinoma. Cancer Cell 2016, 30, 161-175. [CrossRef]

79. Trammell, S.A.; Schmidt, M.S.; Weidemann, B.J.; Redpath, P.; Jaksch, F.; Dellinger, R.W.; Li, Z.; Abel, E.D.; Migaud, M.E.; Brenner, C. Nicotinamide riboside is uniquely and orally bioavailable in mice and humans. Nat. Commun. 2016, 7, 12948. [CrossRef] [PubMed]

80. Shi, W.; Hegeman, M.A.; van Dartel, D.A.; Tang, J.; Suarez, M.; Swarts, H.; van der Hee, B.; Arola, L.; Keijer, J. Effects of a wide range of dietary nicotinamide riboside (NR) concentrations on metabolic flexibility and white adipose tissue (WAT) of mice fed a mildly obesogenic diet. Mol. Nutr. Food Res. 2017, 61. [CrossRef]

81. Lee, H.J.; Hong, Y.S.; Jun, W.; Yang, S.J. Nicotinamide riboside ameliorates hepatic metaflammation by modulating NLRP3 inflammasome in a rodent model of type 2 diabetes. J. Med. Food 2015, 18, 1207-1213. [CrossRef] [PubMed]

82. Trammell, S.A.; Weidemann, B.J.; Chadda, A.; Yorek, M.S.; Holmes, A.; Coppey, L.J.; Obrosov, A.; Kardon, R.H.; Yorek, M.A.; Brenner, C. Nicotinamide Riboside Opposes Type 2 Diabetes and Neuropathy in Mice. Sci. Rep. 2016, 6, 26933. [CrossRef] [PubMed]

83. De Picciotto, N.E.; Gano, L.B.; Johnson, L.C.; Martens, C.R.; Sindler, A.L.; Mills, K.F.; Imai, S.; Seals, D.R. Nicotinamide mononucleotide supplementation reverses vascular dysfunction and oxidative stress with aging in mice. Aging Cell 2016, 15, 522-530. [CrossRef] [PubMed]

84. Wang, X.; Hu, X.; Yang, Y.; Takata, T.; Sakurai, T. Nicotinamide mononucleotide protects against beta-amyloid oligomer-induced cognitive impairment and neuronal death. Brain Res. 2016, 1643, 1-9. [CrossRef] [PubMed]

85. Aksoy, P.; White, T.A.; Thompson, M.; Chini, E.N. Regulation of intracellular levels of NAD: A novel role for CD38. Biochem. Biophys. Res. Commun. 2006, 345, 1386-1392. [CrossRef] 
86. Escande, C.; Nin, V.; Price, N.L.; Capellini, V.; Gomes, A.P.; Barbosa, M.T.; O’Neil, L.; White, T.A.; Sinclair, D.A.; Chini, E.N. Flavonoid apigenin is an inhibitor of the NAD ${ }^{+}$ase CD38: Implications for cellular $\mathrm{NAD}^{+}$metabolism, protein acetylation, and treatment of metabolic syndrome. Diabetes 2013, 62, 1084-1109. [CrossRef]

87. Cipriani, G.; Rapizzi, E.; Vannacci, A.; Rizzuto, R.; Moroni, F.; Chiarugi, A. Nuclear poly(ADP-ribose) polymerase-1 rapidly triggers mitochondrial dysfunction. J. Biol. Chem. 2005, 280, 17227-17234. [CrossRef]

88. Almeida, G.S.; Bawn, C.M.; Galler, M.; Wilson, I.; Thomas, H.D.; Kyle, S.; Curtin, N.J.; Newell, D.R.; Maxwell, R.J. PARP inhibitor rucaparib induces changes in NAD levels in cells and liver tissues as assessed by MRS. NMR Biomed. 2017, 30. [CrossRef]

(C) 2019 by the authors. Licensee MDPI, Basel, Switzerland. This article is an open access article distributed under the terms and conditions of the Creative Commons Attribution (CC BY) license (http://creativecommons.org/licenses/by/4.0/). 

Article

\title{
A Lipidomic Analysis of Docosahexaenoic Acid (22:6, w3) Mediated Attenuation of Western Diet Induced Nonalcoholic Steatohepatitis in Male $\mathrm{Ldlr}^{-/-}$Mice
}

\author{
Manuel García-Jaramillo ${ }^{1,2,3}$, Kelli A. Lytle ${ }^{1,3,+}$, Melinda H. Spooner ${ }^{1,3}$ and Donald B. Jump ${ }^{1,3, *}$ \\ 1 Nutrition Program, School of Biological and Population Health Sciences, Oregon State University, Corvallis, \\ OR 97331, USA; Manuel.g.jaramillo@oregonstate.edu (M.G.-J.); Kelli.Lytle@mayo.edu (K.A.L.); \\ Spoonerm@oregonstate.edu (M.H.S.) \\ 2 Department of Chemistry, Oregon State University, Corvallis, OR 97331, USA \\ 3 The Linus Pauling Institute, Oregon State University, Corvallis, OR 97331, USA \\ * Correspondence: Donald.Jump@oregonstate.edu \\ † Current affiliation: Endocrine Research Unit, Mayo Clinic, Rochester, MN 55902, USA.
}

Received: 21 September 2019; Accepted: 22 October 2019; Published: 28 October 2019

\begin{abstract}
Nonalcoholic fatty liver disease (NAFLD) is a major public health problem worldwide. NAFLD ranges in severity from benign steatosis to nonalcoholic steatohepatitis (NASH), cirrhosis, and primary hepatocellular cancer (HCC). Obesity and type 2 diabetes mellitus (T2DM) are strongly associated with NAFLD, and the western diet (WD) is a major contributor to the onset and progression of these chronic diseases. Our aim was to use a lipidomic approach to identify potential lipid mediators of diet-induced NASH. We previously used a preclinical mouse (low density lipoprotein receptor null mouse, $\mathrm{Ldll}^{-}-$) model to assess transcriptomic mechanisms linked to WD-induced NASH and docosahexaenoic acid (DHA, 22:6, $\omega 3$ )-mediated remission of NASH. This report used livers from the previous study to carry out ultra-high-performance liquid chromatography coupled with tandem mass spectrometry (LC-MS/MS) and high-performance liquid chromatography coupled with dynamic multi-reaction monitoring (HPLC-dMRM) to assess the impact of the WD and DHA on hepatic membrane lipid and oxylipin composition, respectively. Feeding mice the WD increased hepatic saturated and monounsaturated fatty acids and arachidonic acid (ARA, 20:4, $\omega 6$ ) in membrane lipids and suppressed $\omega 3$ polyunsaturated fatty acids (PUFA) in membrane lipids and $\omega 3$ PUFA-derived anti-inflammatory oxylipins. Supplementing the WD with DHA lowered hepatic ARA in membrane lipids and ARA-derived oxylipins and significantly increased hepatic DHA and its metabolites in membrane lipids, as well as $\mathrm{C}_{20-22}$ w3 PUFA-derived oxylipins. NASH markers of inflammation and fibrosis were inversely associated with hepatic $\mathrm{C}_{20-22} \omega 3$ PUFA-derived Cyp2C- and Cyp2J-generated anti-inflammatory oxylipins (false discovery rate adjusted $p$-value; $q \leq 0.026$ ). Our findings suggest that dietary DHA promoted partial remission of WD-induced NASH, at least in part, by lowering hepatic pro-inflammatory oxylipins derived from ARA and increasing hepatic anti-inflammatory oxylipins derived from $\mathrm{C}_{20-22} \omega 3$ PUFA.
\end{abstract}

Keywords: nonalcoholic fatty liver disease; nonalcoholic steatohepatitis; arachidonic acid; docosahexaenoic acid; inflammation; fibrosis; lipidomics; mass spectrometry

\section{Introduction}

Nonalcoholic fatty liver disease (NAFLD) is the most common chronic fatty liver disease worldwide [1-3] and is defined as excessive neutral lipid deposition in the liver in individuals who consume little or no alcohol [4,5]. Obesity and type 2 diabetes mellitus (T2DM) are strongly associated with NAFLD [3,6-8]. In fact, $60 \%$ of patients with a BMI $>30$ display evidence of liver 
steatosis [9]. Based on estimates from the Centers for Disease Control, 93 million adults [10] and $\sim 14$ million children [11] in the US are obese. As such, both obese children and adults are at risk of developing NAFLD [12]. Lifestyle, diet, genetics, and endocrine status contribute to the onset of NAFLD and its progression to nonalcoholic steatohepatitis (NASH), cirrhosis, and primary hepatocellular cancer (HCC) [7,13]. Moreover, NAFLD is a risk factor for cardiovascular disease [14-16]. The top four risk factors for NAFLD are obesity, T2DM, dyslipidemia, and metabolic syndrome $[17,18]$.

The progression of benign steatosis to NASH is a multicellular and multi-hit process [19-23] that is associated with excessive lipid accumulation in hepatocytes leading to insulin resistance and hepatic injury involving endoplasmic reticulum stress, oxidative stress, and inflammation [24]. Hepatic injury leads to cell death and fibrosis [25-27]. The best strategies to prevent NAFLD and stop its progression from benign steatosis to NASH remain ill-defined [28-31]. While current strategies focus on lifestyle management (exercise and diet) [28,32-43], patient noncompliance remains a major concern when using lifestyle interventions to improve health outcomes [44-46]. Although targeted pharmacological agents are in development to treat NAFLD [46-48], adverse drug effects arising from off-target mechanisms often occur. To date, the Food and Drug Administration has not approved any specific therapies for NASH [49]. The absence of specific treatment strategies makes NAFLD a major public health concern [30].

While the clinical features of NAFLD are well described, the impact of diet, such as the western diet, on hepatic physiology and lipid metabolism remains poorly defined. Accordingly, we developed a preclinical NASH model using the low density lipoprotein (LDL)-receptor null ( Ldl $^{--}$) mouse and the western diet (WD). This model recapitulates human NASH in male and female mice [50-52]. Mice fed the WD become obese and the liver presents all the hallmarks of NASH, i.e., hepatosteatosis, leukocyte accumulation in the liver, centrilobular fibrosis, and increased expression of HCC markers.

A key outcome of our research established that the WD lowers hepatic content of $\mathrm{C}_{18-22}$ polyunsaturated fatty acids (PUFA, both $\omega 3$ and $\omega 6$ ). The WD is moderately high in saturated (SFA) and monounsaturated (MUFA) fatty acids, simple sugar and cholesterol, but low in essential fatty acids, e.g., linoleic acid (LA, 18:2,w6) and $\alpha$-linolenic acid (ALA, 18:3,w3) [50,53,54]. Interestingly, clinical studies have shown that NASH patients have low hepatic $\mathrm{C}_{18-22}$ PUFA when compared to patients with benign steatosis [55-57]. Moreover, PUFA and $\omega 3$ PUFA, specifically, affect whole body lipid metabolism by decreasing blood triglycerides, suppressing fatty acid synthesis, and promoting fatty acid oxidation. In contrast, dietary $\omega 6$ PUFAs are precursors to bioactive pro-inflammatory oxylipins [58,59]. As such, changes in the relative abundance of hepatic SFA, MUFA, PUFA, and the type of PUFA, i.e., $\omega 3$ versus $\omega 6$ PUFA, has the potential to affect whole body and liver health.

To reinforce the role of dietary PUFA in NAFLD development, we established that supplementing the WD with docosahexaenoic acid (DHA, 22:6, $\omega 3$ ) at $2 \%$ total calories restored hepatic $\mathrm{C}_{20-22} \omega 3$ PUFA, lowered arachidonic acid (ARA, 20:4,w6), a precursor to harmful pro-inflammatory ARA-derived oxylipins, and lowered histologic and transcriptomic markers of inflammation, oxidative stress, and fibrosis [52]. More recently, we used a lipidomic approach to assess the impact of the WD on hepatic membrane lipids and oxylipins in female $\mathrm{Ldlr}^{-1}$ mice [60]. These studies established that feeding mice the WD significantly changed the acyl chain composition of multiple hepatic membrane lipid classes and $\omega 3$ and $\omega 6$ PUFA-derived oxylipins. Specifically, the WD increased the hepatic membrane content of SFA and MUFA, as well as ARA and ARA-derived oxylipins. The hepatic abundance of $\omega 3$ PUFA-derived oxylipins, however, was low in mice fed the WD. This oxylipin profile was associated with increased hepatic markers of inflammation, oxidative stress, fibrosis, apoptosis, autophagy, notch and hedgehog signaling, and hepatic cancer [60].

Fatty acids and their derivatives are well-established regulators of cell function. The principal targets for this action include regulation of membrane lipid composition, oxylipin type and abundance, and regulation of cell signaling originating from the plasma membrane, as well as targeting nuclear receptors $[59,61]$. The rationale for using DHA to combat NASH is based on the well-established role of $\mathrm{C}_{20-22} \omega 3$ PUFA in the control of blood triglycerides and hepatic fatty acid synthesis and oxidation [62]. 
Moreover, $\mathrm{C}_{20-22} \omega 3$ PUFA interfere with ARA-derived oxylipin production and function [58]. Finally, clinical studies support the use of $\omega 3$ PUFA dietary supplementation to treat NAFLD [12,47]. Recent meta-analyses of clinical trials using $\mathrm{C}_{20-22} \omega 3$ PUFA dietary supplementation indicate significant improvement in several metabolic outcomes, including lowering plasma triglycerides and hepatic fat content [12,47]. The most consistent improvement in liver health is seen with dietary DHA [12] or the combination of DHA and eicosapentaenoic acid (EPA, 20:5, w3), e.g., Lovaza ${ }^{\mathrm{TM}}$, GlaxoSmithKline [63]. EPA treatment alone, however, has proven ineffective in improving liver health in NAFLD patients $[50,64]$.

In this report, we used liver samples from our previous study which documented the capacity of DHA to block NASH progression (Figure 1) [52]. This study included detailed gas chromatographic (GC) analysis of diet effects on hepatic lipids as well as extensive transcriptomic analysis of hepatic markers of inflammation and fibrosis. Herein, we expanded our lipidomic analysis by using ultra-high-performance liquid chromatography coupled with tandem mass spectrometry (UPLC-MS/MS) and high-performance liquid chromatography coupled with dynamic multi-reaction monitoring (HPLC-dMRM), as described [60]. Our aim was to document how the WD and DHA altered hepatic membrane lipid and non-esterified oxylipin composition in a preclinical NASH model. We then used a statistical approach to determine how these diet-induced changes in hepatic lipids correlated with changes in hepatic markers of inflammation and fibrosis.

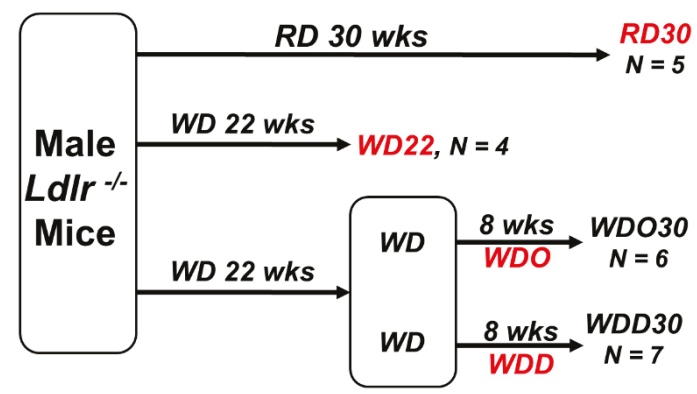

Figure 1. Study design for docosahexaenoic acid (DHA)-mediated nonalcoholic steatohepatitis (NASH) remission in male $\mathrm{Ldlr}^{-/}$mice. Liver samples used in this lipidomic analysis were obtained from our previously published study assessing the capacity of DHA to promote NASH remission [52]. Briefly, mice at $10 \mathrm{wks}$ of age were fed a chow diet (Purina Pico Lab diet 5053) and served as a reference diet (RD) group. The RD group was maintained on the RD for the duration of the study, i.e., 30 weeks (wks; RD30, number of animals $(N)=5$ ). Mice were also fed the western diet (WD) (Research Diets, D12079B) for 22 wks. At 22 wks, a group of WD-fed mice were euthanized for recovery of blood and liver. This group (WD22, $N=5$ ) served as a baseline for disease progression. The remaining WD-fed mice were switched to a WD supplemented with either olive oil (WDO30, $N=6$ ) or DHASCO (WDD30, $N=7$ ) and euthanized 8 weeks ( 8 wks) later. See Materials and Methods for more details.

\section{Results}

\subsection{Impact of the Western Diet (WD) on Membrane Lipids}

The study designed included 4 groups of male $\mathrm{Ldlr}^{--}$mice as described in Figure 1. A control group consisted of mice maintained on a reference diet (RD) for 30 weeks. The remaining mice were fed the WD for 22 weeks. At 22 weeks, WD-fed mice were split into three groups. One group was euthanized and served as the baseline group (WD22) for NASH progression analysis. The remaining mice were fed the WD supplemented with either olive oil (WDO) or DHASCO (WDD) for 8 weeks (see Materials and Methods). The WDO and WDD diets were matched for calories as fat. The dose of DHA used in these studies was equivalent to a human taking $4 \mathrm{~g}$ of Lovaza ${ }^{\mathrm{TM}}$ (GlaxoSmithKline)/day to treat hypertriglyceridemia [65]. After 8 weeks on the WDO and WDD diets, mice were euthanized for blood and liver collection. The group identifications for these mice are WDO30 and WDD30, respectively. 
Total hepatic lipids were extracted and fractionated using the UPLC-MS/MS approach described in Materials and Methods.

Our UPLC-MS/MS analysis identified 13 classes of membrane lipids (Figure 2). To assess the impact of diet on these lipids, we quantified the cumulative saturation index (CSI). The CSI reflects the amount of lipid within a lipid class and the level of saturation of the fatty acyl chains within each lipid class. Figure 2A represents the CSI across all major membrane lipids in mice maintained on the RD for 30 weeks (RD30). The highest CSI was in the lipid classes including phosphatidyl choline (PC) and phosphatidyl ethanolamine (PE) and the lowest in lysophosphatidyl serine (lyso PS).

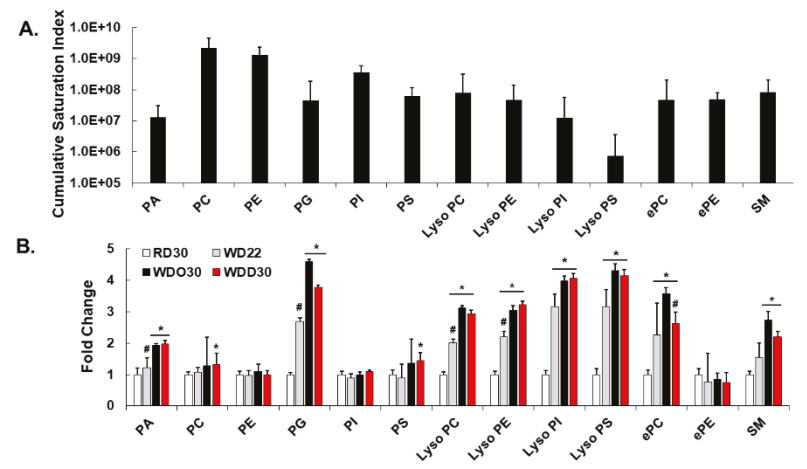

Figure 2. Diet effects on membrane lipids. (A): Cumulative saturation index of lipids in each lipid class. The saturation index (SI) was calculated as follows: one minus (number of double bonds) divided by (number of fatty acyl carbons minus one). The cumulative saturation index was calculated by multiplying the SI by the peak intensity of each lipid species and summing all lipids within each lipid class. (B): Effect of diet on the cumulative saturation index for each lipid class. Results are presented as fold change, mean \pm standard error of the mean (SEM); ${ }^{*}, q<0.05$ vs. RD30; $\#, q<0.05$ vs. WDO30; [ $q$-value is the false discovery rate (FDR) adjusted $p$-value].

The impact of the WD and DHA on CSI is shown in Figure 2B. Since the WD is enriched in SFA and MUFA, we expected a significant increase in the CSI. Accordingly, feeding mice the WD increased the CSI in phosphatidic acid (PA), phosphatidyl glycerol (PG), phosphatidyl serine (PS), all lysophospholipids [(lysophosphatidyl choline (lyso PC), lysophosphatidyl ethanolamine PE (lyso PE), phosphatidyl inositol (lyso PI), phosphatidyl serine (lyso PS)], ether phosphatidyl choline (ePC), and sphingomyelin (SM), but not in PC, PE, phosphatidyl inositol (PI), or ether phosphatidyl ethanolamine (ePE). PA is a precursor to multiple membrane lipids, while PG is a precursor to cardiolipins. Surprisingly, including DHA in the WD significantly lowered the CSI (by 20\%) in only one lipid class, ePC. Yet, a detailed examination of all lipid species within each lipid class revealed assimilation of DHA and its metabolites $(20: 5, \omega 3 ; 22: 5, \omega 3)$ in all lipid classes, except SM (Table S1: diet effects on all lipids). While the WD significantly increased the CSI as a result of increased dietary SFA and MUFA content, DHA and its metabolites had little impact on the overall CSI of most lipid classes.

We next used a statistical approach to establish differences between treatment groups. Accordingly, all lipid data from the UPLC-MS/MS and HPLC-dMRM analysis plus our previous GC analysis [52] was subject to a principal component analysis (PCA) (Figure 3). While four groups were included in our study, the PCA revealed only three clusters. Two clusters (WD22 and WDO30) overlapped indicating that these groups differed little in terms of lipid composition. The lipid composition of the WD22 and WDO30 groups clearly differed from the reference diet group (RD30). Interestingly, the WDD30 group does not overlap with either the RD30 or WD22 and WD30 clusters, reflecting its unique lipid composition. This outcome indicates that $8 \mathrm{wks}$ of DHA treatment does not restore hepatic membrane lipid acyl chain composition to that seen in the RD30 group. 


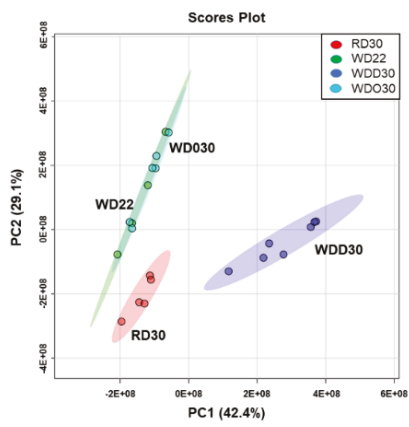

Figure 3. Principal component analysis of diet effects on hepatic lipids. All lipid data collected for this study and our previous study [52] was used in this analysis. The data included fatty acid methyl esters reported previously [52] and all membrane lipid and oxylipin data obtained by UPLC-MS/MS and HPLC-dMRM analysis, respectively. The principal component analysis was carried out using the statistical package in Metabolanalyst 4.0 [66].

Further analysis identified the top 25 highly significant differences $\left(q \leq 3.0 \times 10^{-7}\right)$ in lipid composition amongst the four groups (Figure 4 and Table S2: Lipids significantly affected by diet). A key result of this analysis was that the WD increased 20:4, $\omega 6$ and its metabolites in multiple membrane lipids (PC 38:4, PG 42.8; PE 40:5, lyso PC 20:3; lyso PC 22:4; lyso PE 20:3), but lowered oxylipins derived from linoleic acid (18:2, w6), i.e., 12,13-DiHOME. This finding replicates our previous results documenting the effects of the WD on hepatic lipids derived from WD-fed female $\mathrm{Ldlr}^{-/}$mice [60].

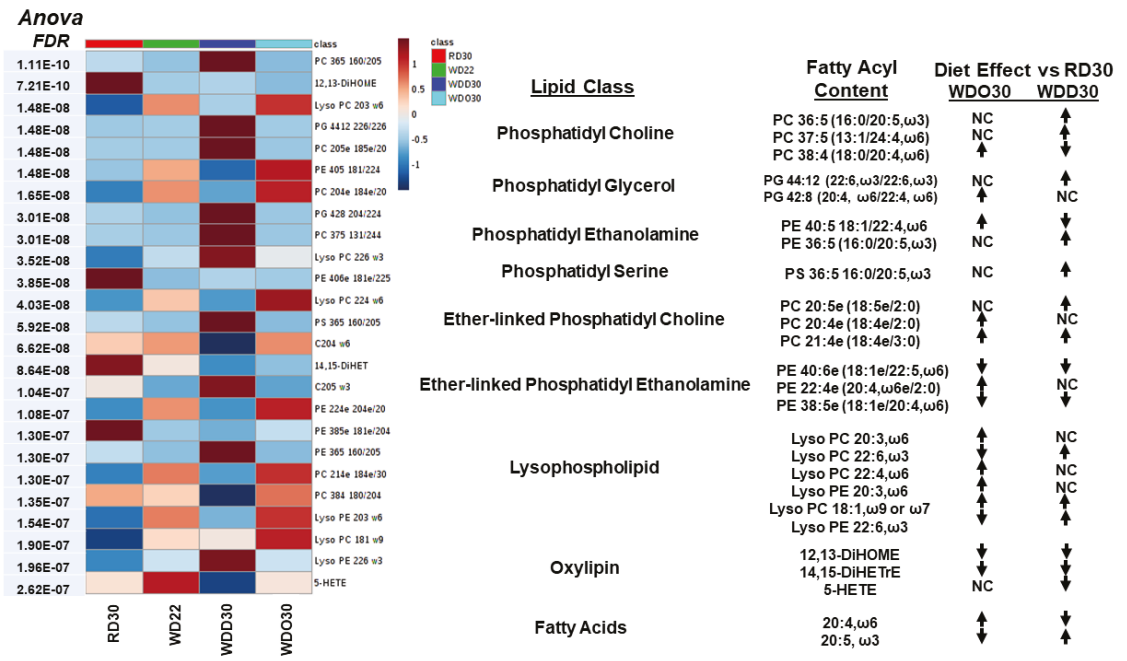

Figure 4. Heat map illustrating diet effects on the top 25 lipid features. As in Figure 3 all lipid data was used to perform an ANOVA (one-way) and a heat map was constructed using the statistical package in Metabolanalyst features [66]. The top 25 highly significant lipid features are illustrated in the heat map. The $q$-value for each lipid is on the left side of the heat map. Columns at the right list specific lipids within each lipid class that were on the heat map. Arrows indicate the effect of the diet WDO30 and WDD30 when compared to the RD30 group (increase, decrease, no change (NC)) (WD supplemented with either olive oil (WDO) or DHASCO (WDD)). 
Addition of DHA to the WD not only increased the abundance of DHA and its metabolites in hepatic phospholipids (PC 36:5, PG 44:12, PS 36.5, lyso PC 22:6, lyso PE 22:6), but also decreased levels of specific membrane lipids containing 20:4, $\omega 6$ and its metabolites (PC 38:4, PE 40:5, PE 38.5). Such effects of dietary $\omega 3$ PUFA on membrane lipid composition are not new. What is new, however, is the impact of WD and DHA on a broad range of hepatic lipids, including phosphoglycerol lipids (PC, PE, PG, PI, PS), ether lipids (ePC, ePE), lysophospholipids (lyso-PC, -PE, -PI, -PS), and sphingolipids. The sphingolipids were the only lipid class significantly affected by WD and DHA, but found to contain no $\mathrm{C}_{20-22}$ PUFA (both $\omega 3$ and $\omega 6$ ) (Table S1: Diet effects on all lipids). As such, DHA mediated effects on SM acyl chain content likely involves DHA regulation of hepatic abundance of SFA and MUFA, as well as the incorporation of these fatty acyls into SM.

\subsection{Diet Effects on Hepatic Non-Esterified Oxylipins}

Intrahepatic non-esterified oxylipins arise from phospholipase-mediated excision of fatty acyls from membrane lipids. These non-esterified fatty acids serve as substrates for cell-specific pathways generating oxylipins that, in turn, serve as regulatory ligands for G-protein receptors (GPR) and nuclear receptors $[61,66]$. Herein, we examined the effect of the WD and DHA on hepatic oxylipins derived from LA, ARA, EPA, and DHA (Figures 5 and 6) and the expression of enzymes involved in oxylipin metabolism (Figure 7); results are summarized in Figure 8.

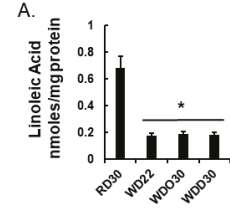

D.

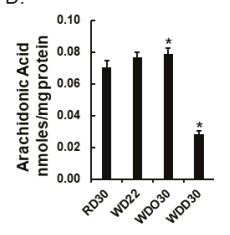

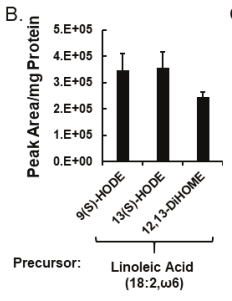

E.

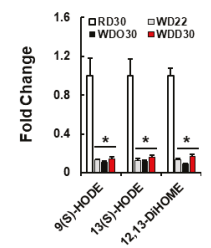

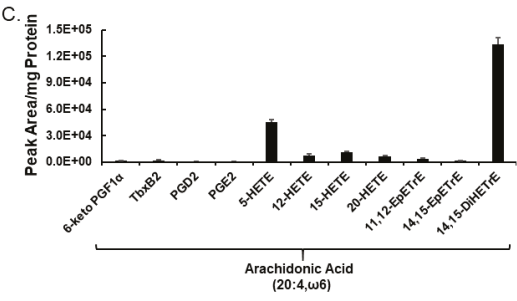

F.

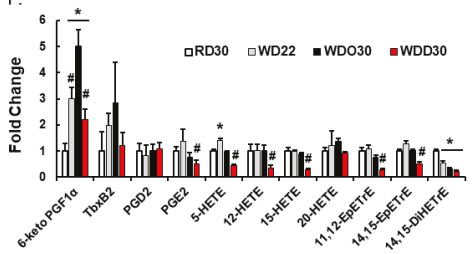

Figure 5. Diet effects on oxylipins derived from $\omega 6$ polyunsaturated fatty acids (PUFA). (A,D): Hepatic content of linoleic acid (LA; 18:2, w6) and arachidonic acid (ARA; 20:4, w6) as determined by gas chromatographic (GC) analysis. Results are presented as nmoles/mg protein, mean \pm SEM. Oxylipins derived from LA (B) and ARA (C) were quantified, by HPLC-dMRM as described in the Materials and Methods Section. Liver samples were derived from the RD group and oxylipin levels are presented as the peak area/mg protein, mean \pm SEM. Effects of diet on specific oxylipins are presented in (Panels E,F) as Fold Change, mean \pm SEM. (Panels E,F) present LAand ARA-derived oxylipins, respectively. *, $q<0.05$ vs. RD30; \#, $q<0.05$ vs. WDO30. 9(S)-HODE, 9(S) hydroxyl octadecadienoic acid; 13(S)-HODE, 13(S) hydroxyl octadecadienoic acid; 12,13-DiHOME, 12,13-dihydroxy octadecenoic acid; 6-keto PGF1 $\alpha$, 6-keto prostaglandin F1 $\alpha$; TbxB2, thromboxane B2; PGD2, prostaglandin D2; PGE2, prostaglandin E2, 5-HETE, 5-hydroxyeicosatrienoic acid; 12-HETE, 12-hydroxyeicosatrienoic acid; 15-HETE, 15-hydroxyeicosatrienoic acid; 20-HETE, 20-hydroxyeicosatrienoic acid; 11,12-EpETrE, 11,12 epoxyeicosatrienoic acid; 14,15-EpETrE, 14,15 epoxyeicosatrienoic acid; 14,15-diHETrE, 14,15-dihydroxyeicosatrienoic acid. 
A.

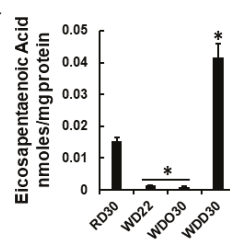

C

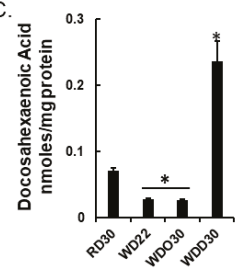

B.

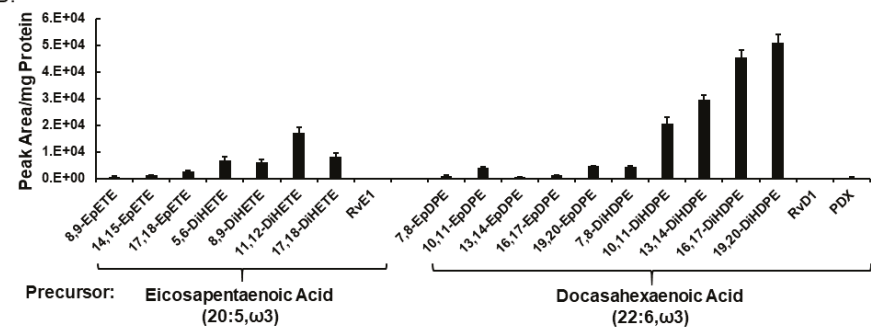

$(22: 6, \omega 3)$

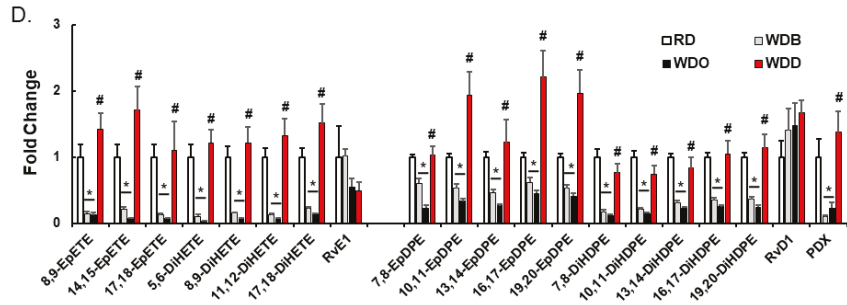

Figure 6. Diet effects on oxylipins derived from $\omega 3$ PUFA. Hepatic content of eicosapentaenoic acid (EPA; 20:5, w3) (Panel A) and docosahexaenoic (DHA; 22:6, w3) (Panel C). Hepatic EPA and DHA were quantified as described in the Materials and Methods Section and presented as the mean \pm SEM of nmoles/mg hepatic protein. (Panels B,D): Effects of diet on EPA and DHA derived oxylipins, respectively. Results are presented as Peak area/mg protein (Panel B) and Fold Change, mean \pm SEM (Panel D). *, $q<0.05$ vs. RD30; \#, $q<0.05$ vs. WDO30. 8,9-EpETE, 8,9-epoxyeicosatetraenoic acid; 14,15-EpETE, 14,15-epoxyeicosatetraenoic acid; 17,18-EpETE, 17,18-epoxyeicosatetraenoic acid; 5,6-DiHETE, 5,6-dihydroxyeicosatetraenoic acid; 8,9-DiHETE, 8,9-dihydroxyeicosatetraenoic acid; 11,12-DiHETE, 11,12-dihydroxyeicosatetraenoic acid; 17,18-DiHETE, 17,18-dihydroxyeicosatetraenoic acid; RvE1, resolvin E1; 7,8-EpDPE, 7,8-epoxydocosapentaenoic acid; 10,11-EpDPE, 10,11-epoxydocosapentaenoic acid; 13,14-EpDPE, 13,14-epoxydocosapentaenoic acid; 16,17-EpDPE, 16,17-epoxydocosapentaenoic acid; 19,20-EpDPE, 19,20-epoxydocosapentaenoic acid; 7,8-DiHDPE, 7,8-dihydroxydocosapentaenoic acid; 10,11-DiHDPE, 10,11-dihydroxydocosapentaenoic acid; 13,14-DiHDPE, 13,14-dihydroxydocosapentaenoic acid; 16,17-DiHDPE, 16,17-dihydroxydocosapentaenoic acid; 19,20-DiHDPE, 19,20-dihydroxydocosapentaenoic acid; RvD1, resolvin D1; PDX, protectin DX.

We previously reported that feeding female $L d l r^{-t}$ mice fed the WD decreased hepatic oxylipins derived from linoleic acid (LA, 18:2, w6), but increased hepatic oxylipins derived from arachidonic acid (ARA, 20:4, w6) [60]. As illustrated in Figure 5, the WD and WDO diets had similar effects on hepatic oxylipins in male $\mathrm{Ldll}{ }^{-}$mice as seen in female mice [60]. Our oxylipin analysis identified $14 \omega 6$ PUFA-derived oxylipins; three from LA and 11 from ARA (Figure 5). Three oxylipins, 12,13-DiHOME; 14,15-DiHETE; and 5-HETE ranked in the top 25 highly significantly lipids affected by the WD (Figure 4). The dihydroxy fatty acid, 12,13-DiHOME, is one of three LA-derived oxylipins, and 14,15-DiHETE is one of eleven ARA-derived oxylipins identified in our analysis (Figures 5 and 8). These dihydroxy oxylipins are generated by the action of a soluble epoxide hydrolase (Ephx2) action on epoxy fatty acids, i.e., 12,13-EpHOME and 14,15-EpETrE, respectively. The LA and ARA derived epoxides are generated by hepatic epoxygenases (Cyp2C; Cyp2J). Highly abundant $\omega 6$ PUFA-derived oxylipins include 9(S)-HODE, 13(S)HODE, 12,13-DiHOME, 5-HETE, and 12,15-DiHETrE, while low abundance oxylipins include 6-keto PGF1 $\alpha$, TBXB2, PGD2, PGE2, and HETEs (12-, 15-, 20-HETE) and 14,15-EpETrE. WD or WDO feeding increased hepatic ARA and significantly increased 6-keto PGF1 $\alpha$ and TBX2B, but had little effect on other 20:4, w6 derived oxylipins (Figure 5C,D).

We identified eight and 12 oxylipins derived from EPA and DHA, respectively, in male mice fed the RD, i.e., RD30 group (Figures 6 and 8). Feeding female $\mathrm{Ldlr}^{--}$mice the WD lowered hepatic levels 
of all $\omega 3$ PUFA-derived oxylipins [60]. Male $L d l r^{-1}$ mice fed the WD or WDO resulted in significantly lower levels of all hepatic $\omega 3$-PUFA-derived oxylipins (Figure 6). The decline in these oxylipins paralleled the WD-mediated decline in hepatic EPA and DHA (Figure 6A,C).

Supplementing the WD with DHA, i.e., WDD30 group, had no effect on hepatic LA or LA-derived oxylipin abundance. The WDD, however, significantly lowered hepatic ARA and all ARA-derived oxylipins, except PGD2 and 20-HETE (Figure 5D,F). The decline in hepatic 20:4, w6-derived oxylipins paralleled the DHA-mediated suppression of hepatic 20:4, w6 content (Figure 5D). Clearly, the WD has a potent effect on hepatic oxylipin type and abundance. Supplementing the WD with DHA had an equally potent effect on hepatic $\omega 3$ PUFA-derived oxylipins by reversing the WD effect on ARA-derived and $\mathrm{C}_{20-22} \omega 3$ PUFA-derived oxylipins.

A.

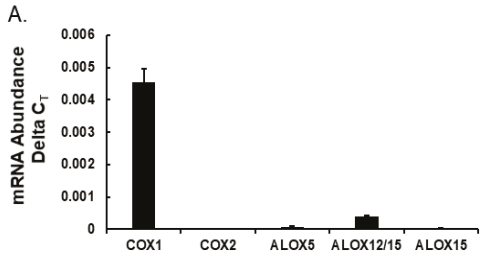

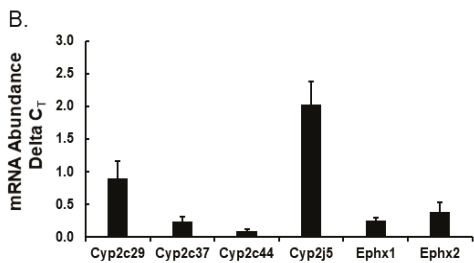

C.

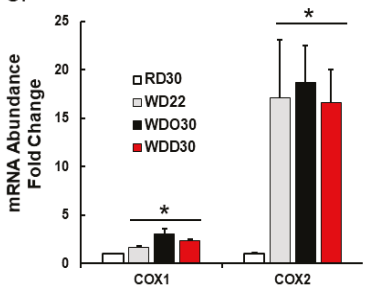

D.

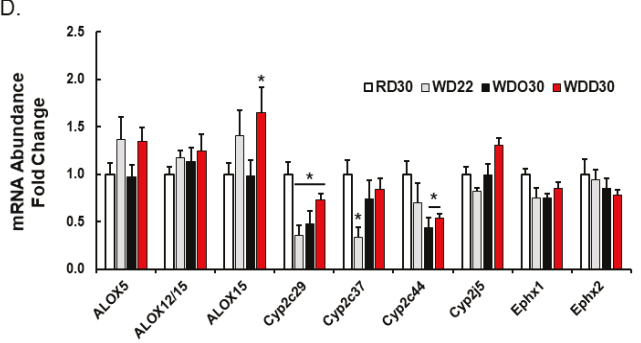

Figure 7. Diet effects on hepatic enzymes involved in oxylipin metabolism. Hepatic RNA was extracted, converted to cDNA and used to quantify transcript abundance using qRTPCR as previously described [52]. The primers used to measure each transcript were previously described [51,60]. Cyclophilin was used as the reference gene. (Top panels $\mathbf{A}, \mathbf{B}$ ): Relative abundance of transcripts encoding enzymes involved in hepatic oxylipin metabolism. Results are presented as delta $\mathrm{C}_{\mathrm{T}}$, mean \pm SEM. (Lower panels C,D): Diet effects on hepatic transcripts encoding enzymes involved in oxylipin metabolism. Results are presented as Fold Change, mean \pm SEM; ${ }^{*}, q<0.05$ vs. RD30. COX, cyclooxygenase; ALOX, arachidonic lipoxygenase; CYP, cytochrome P450, Ephx1, microsomal epoxide hydrolase; Ephx2, soluble epoxide hydrolase.

We next examined the diet effects on hepatic enzymes involved in generating hepatic oxylipins (Figure 7). Cyclooxygenases (Cox1, Cox2) and arachidonic acid lipoxygenases (Alox5, Alox12/15, Alox15) are expressed at low levels in mouse liver (Figure 7A), whereas enzymes generating fatty epoxides (Cyp2C29, Cyp2C37, Cyp2C44, Cyp2J5) and dihydroxy fatty acids (Ephx1, Ephx2) are highly expressed in liver (Figure 7B). The differential expression of these enzymes likely reflects cell specific expression in the liver. For example, Cox1 and Cox2 are expressed in liver, but not in hepatic parenchymal cells, i.e., hepatocytes. These enzymes are likely expressed in resident macrophage (Kupffer cells) and infiltrating leukocytes. Hepatocytes, however, express receptors for Cox products, e.g., EP4, and respond to changes in oxylipins through paracrine mechanisms [67].

Feeding mice the WD and WDO resulted in the induction of both Cox1 and Cox2, but the WD and WDO diets had no significant effects on the Alox subtypes (5-, 12/15-, 15-Alox) (Figure 7). Supplementing the WD with DHA did not attenuate Cox 1 or Cox 2 expression. If Cox 1 and Cox 2 expression parallels Cox 1 and 2 activity, then the decline in Cox-products, e.g., prostacyclin (PGI2, 
precursor of 6-keto PGF1 $\alpha$ ), PGE2, and thromboxane A2 (precursor of TBXB2) cannot be explained by a suppression of enzyme expression. As such, our data suggest that the DHA-mediated decline in Cox products may be due, at least in part, to the DHA-mediated suppression of hepatic ARA levels, particularly in membrane lipids (Figures 4 and 5). Cyp2c29, Cyp2c37 and Cyp2c44 expression was suppressed $\sim 50 \%$ by the WD. Only Cyp2c29 expression was partially restored by the addition of DHA to the diet. Neither WDO nor WDD affected the expression of Cyp2J or Ephx subtypes.

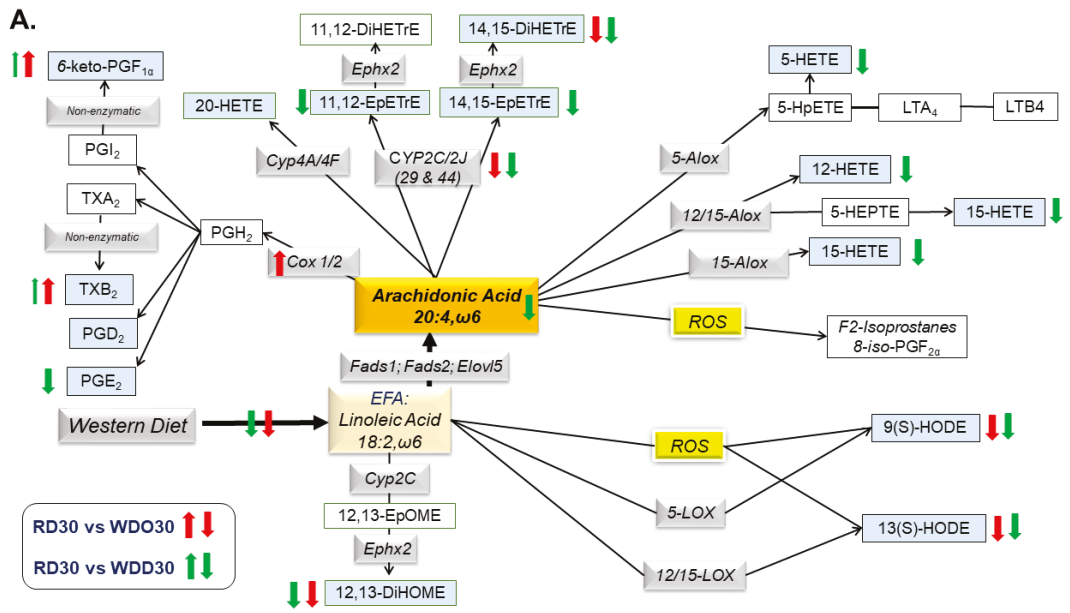

B.

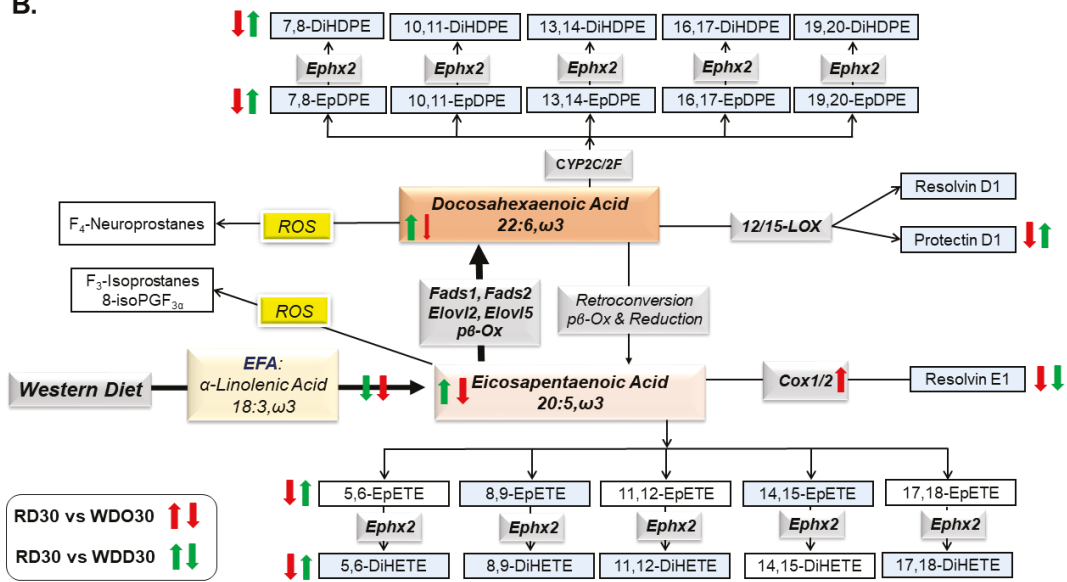

Figure 8. Summary of WD and DHA effects on hepatic oxylipins derived from $\omega 6$ PUFA (A) and $\omega 3$ PUFA (B). The diagrams illustrate the pathway for the conversion of dietary essential fatty acids to $\mathrm{C}_{18-22}$ PUFA and the conversion of PUFA to oxylipins. The pathways are modified from pathways published by Gabbs et al. [61]. Oxylipins highlighted in blue represent oxylipins that were quantified by LC/MS or gas chromatography (Figures 5 and 6). Enzymes involved in oxylipin metabolism are in gray boxes (Figure 7). Red and green arrows are used to represent the effects (increase; decrease) of the WDO versus RD30 (red arrows) and WDD30 versus RD30 (green arrows) on hepatic abundance of fatty acids, oxylipins, and transcripts involved in PUFA and oxylipins metabolism. Thin and thick arrows represent a weak and strong response to diet, respectively. EFA: essential fatty acids; ROS, reactive oxygen species. Fads, fatty acid desaturase; Elovl, fatty acid elongase; $\beta \beta O x$, peroxisomal $\beta$-oxidation; COX, cyclooxygenase; ALOX, arachidonic lipoxygenase; CYP, cytochrome P450, Ephx1, microsomal epoxide hydrolase; Ephx2, soluble epoxide hydrolase. 


\subsection{Associations between Hepatic Lipids and NASH Markers of Inflammation and Fibrosis}

We previously reported that the WD promoted hepatic inflammation and fibrosis, while addition of DHA to the WD blocked disease progression by attenuating expression of inflammation and fibrosis markers [52]. Herein, we asked if changes in specific transcriptomic markers of inflammation and fibrosis were associated with changes in membrane lipids and oxylipins. Accordingly, we used an unbiased statistical approach, i.e., Pattern Hunter in Metabolanalyst 4.0 [66], to identify associations between diet-induced changes in membrane lipids, oxylipins, and NASH pathology, i.e., inflammation and fibrosis (Tables 1 and 2). The transcriptomic data for this analysis was from our previous study [52], the same study used for the current lipidomic analysis. Results are presented as the top 10 positive and negative associations between specific lipids and markers of inflammation (Table 1: osteopontin $(O p n)$, monocyte chemoattractant protein $1(M c p 1)$, cell differentiation $68(C D 68)$ ) and fibrosis (Table 2: collagen 1A1 (Col1A2), tissue inhibitor metalloprotease 1 (Timp1) and lysyl oxidase (Lox)).

\subsubsection{Inflammation}

Lipids positively associated with Opn expression include phosphatidyl glycerol (PG) containing MUFA and PUFA ( $\omega 6>\omega 3$ ) while $50 \%$ of the lipids negatively associated with Opn expression were $\omega 3$ PUFA-derived oxylipins (Table 1). In contrast, lipids positively associated with Mcp1 and CD68 include no PG, but several ether lipids (e.g., PC 16:0e; PC 18:1e) containing predominantly MUFA and short and long chain SFAs. Lipids negatively associated with Mcp1 and CD68 expression include oxylipins (12,13-DiHOME, 14,15-DiHETrE) and membrane phospholipids (PA, PE, PI) containing $\mathrm{C}_{18-22} \omega 3$ and $\omega 6$ PUFA. These association studies indicate that elevated expression of inflammation markers was associated with increased membrane abundance of $C_{18-20}$ MUFA and $C_{20-22} \omega 6$ PUFA, while attenuated expression of these markers was associated with increased membrane content of $\mathrm{C}_{18-22} \omega 3$ and $\omega 6$ PUFA and hepatic levels of $\omega 3$ and $\omega 6$ PUFA derived oxylipins. The epoxygenase and epoxide hydrolase pathways (Cyp2C, Cyp2J and Ephx2) rather than the Cox/Alox and oxidative stress pathways generate the majority of these oxylipins. In addition, lipids associated (positively and negatively) with Opn expression are clearly distinct from the lipids associated with Mcp1 and CD68 expression, suggesting different membrane-associated mechanisms involved in the expression of these inflammation markers.

\subsubsection{Fibrosis}

Lipids positively and negatively associated with the expression of Col1A2 and Timp1 are nearly identical; and include membrane lipids (lyso PA, lyso PE, lyso PC, SM) containing $\mathrm{C}_{14-16}$ SFA, $\mathrm{C}_{18}$ MUFA, and $\mathrm{C}_{18-22} \omega 6$ PUFA, but no $\omega 3$ PUFA. Lipids negatively associated with Col1A2 and Timp1 expression include DHA derived oxylipins (7, 8-DiHDPE; 10, 11-DiHDPE) and membrane lipids (PA, PC, PE, PI) containing $\mathrm{C}_{16-22}$ MUFA and $\mathrm{C}_{18-20} \omega 6$ PUFA. Interestingly, lipids positively and negatively associated with Lox expression are remarkably similar to those associated with Opn expression. PG containing MUFA and $\mathrm{C}_{20-22} \omega 6$ PUFA are positively associated with Lox expression, while $60 \%$ of the lipids negatively associated with Lox expression are oxylipins derived from ARA and EPA. This outcome may reflect common membrane-associated mechanisms associated with Lox and Opn expression. 


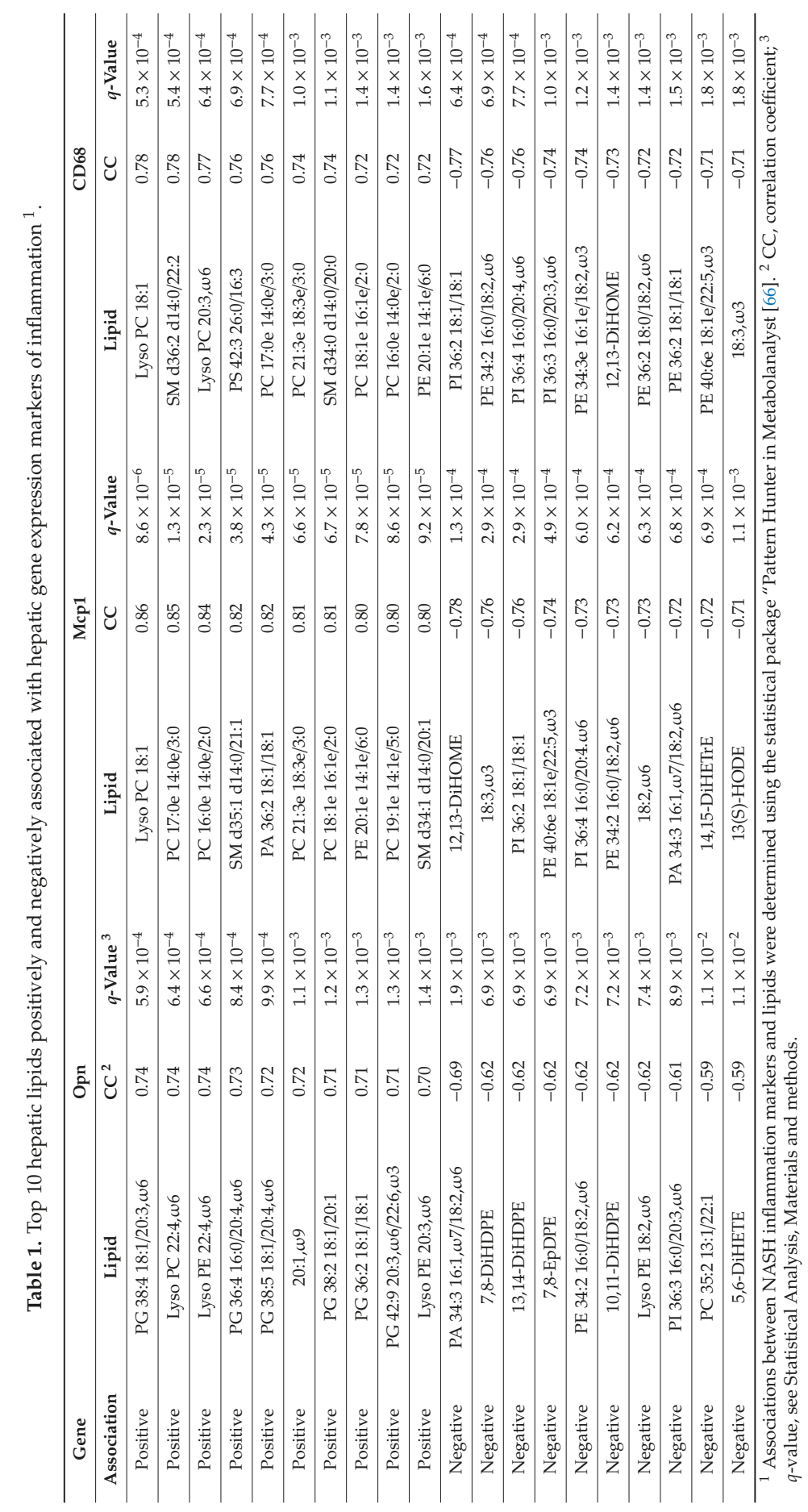




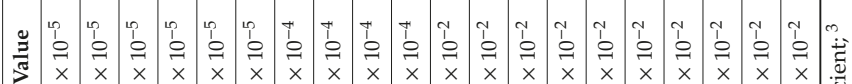

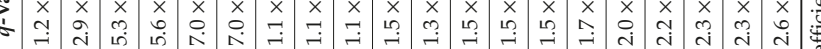

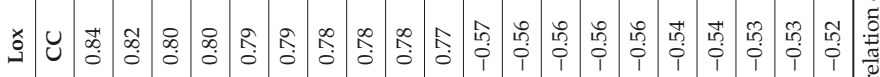

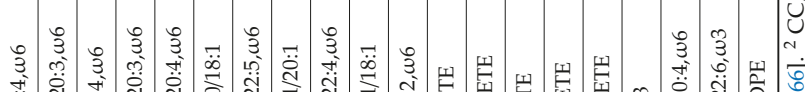

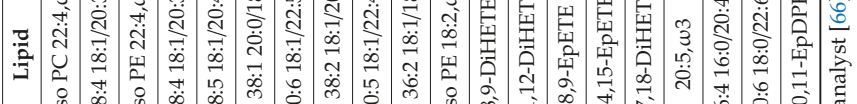

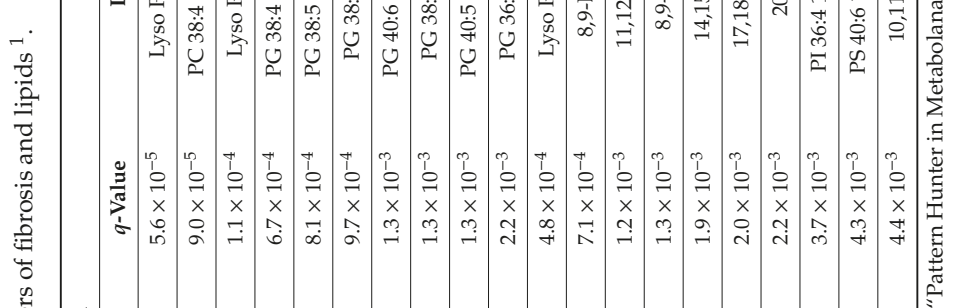

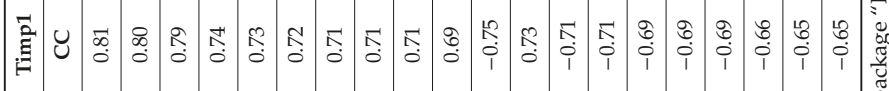

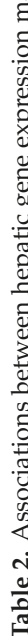

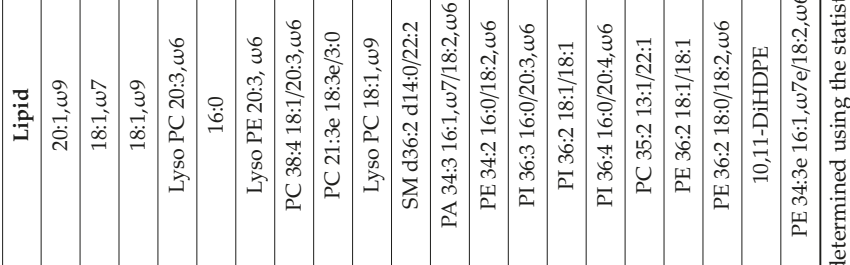

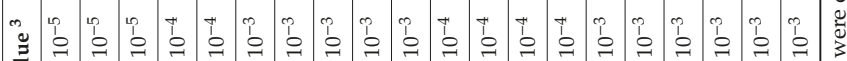

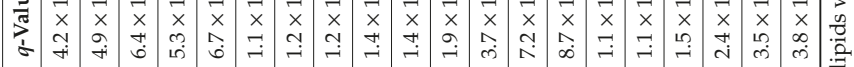

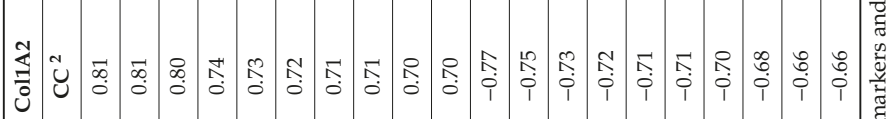

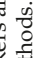

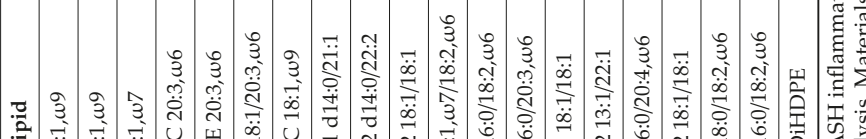

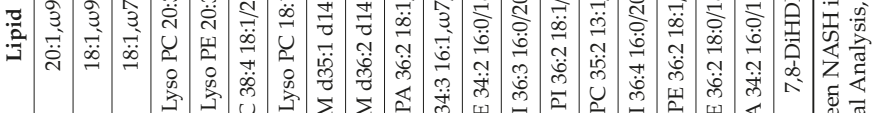

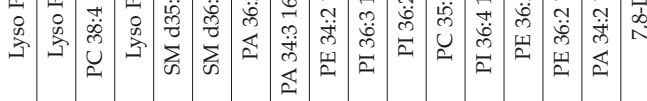

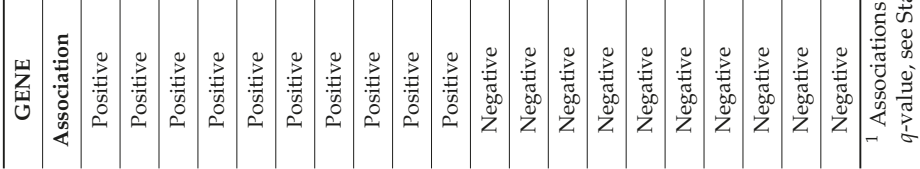




\section{Discussion}

The aim of this study was to use a lipidomic approach to identify potential lipid mediators of inflammation and fibrosis associated with WD-induced NASH and DHA-mediated NASH remission. Accordingly, we identified and quantified hepatic membrane lipids and non-esterified oxylipins in a preclinical mouse model of NASH. Feeding mice the WD significantly increased the saturation index of many, but not all membrane lipids (Figure 2). The WD increased SFA and MUFA in several membrane lipids, i.e., PA, PG, lyso PC, lyso PE, lyso PI, lyso PS, ePC, and SM. Surprisingly, addition of DHA to the WD had little effect on the membrane saturation index, despite the fact that DHA and its metabolites were assimilated into all lipid classes, except SM (Table S1). We suspect the assimilation of DHA and its metabolites into membranes has local effects on membrane fluidity, lipid raft composition, and membrane cholesterol content that potentially affects receptor-mediated mechanisms emanating from membranes [58,59].

On a more granular level, we identified strong associations between diet-induced changes in hepatic membrane lipid composition, membrane derived signaling molecules, and the expression of genes linked to WD-induced hepatic inflammation and fibrosis (Tables 1 and 2). We identified two membrane derived lipid classes that are known to play a role in cell signaling, i.e., lysophospholipids (Lyso PL) and oxylipins. Lyso PLs form in the process in de novo membrane lipid synthesis (Kennedy Pathway) and membrane lipid remodeling (Lands Pathway). Our untargeted lipidomic analysis cannot distinguish between these pathways, nor can it distinguish between acyl chains in the sn1 or sn2 positions of the lyso PLs. Dietary fat content clearly affected the acyl chain composition of these lyso PLs (Figure 4). Oxylipin precursors, i.e., non-esterified fatty acids (NEFA), are generated as a result of membrane remodeling and involves phospholipase activation. NEFA excised from membranes are substrates for several enzymatic pathways that are active in the liver, including cyclooxygenases (Cox (-1 and -2), arachidonate lipoxygenases (Alox (-5, -12/15, -15)), cytochrome P450 class 2 ((Cyp2 (C and J)), and epoxide hydrolases (Ephx 1 and 2) (Figure 8).

Lyso PLs and oxylipins functioning as ligands have the potential to regulated cell function through multiple receptor-mediated mechanisms. Both bioactive lipids regulate cell function through G-protein receptors (GPR) and nuclear receptors. Lyso PLs signal through GPR23, GPR34, GPR44, GPR92, GPR93, and GPR174, while oxylipins signal through GPR for eicosanoids (prostaglandins (PGE2), thromboxanes (TBXA2), and prostacyclins (PGI2)). Lyso PLs and oxylipins also signal through nuclear receptors, e.g., PPAR $\alpha, \beta / \delta, \gamma 1, \gamma 2$ ) [68-75]. Our studies clearly establish that both the WD and DHA have major effects on membrane lipid composition and the type and abundance of hepatic lyso PLs and oxylipins derived from PUFA (Figures 4-8).

We took advantage of our transcriptomic data [52] to identify associations between membrane lipids, oxylipins, and markers of inflammation and fibrosis, key markers of NASH (Tables 1 and 2). Hepatic levels of lyso PC and lyso PE containing $\mathrm{C}_{20-22} \omega 6$ PUFA were positively associated with the expression of inflammation (Opn, CD68) and fibrosis (Col1A2, Timp1, LOX) markers (Tables 1 and 2). As such, changes in the hepatic abundance of lyso PL enriched in $\mathrm{C}_{20-22} \omega 6$ PUFA, acting through membrane GPR and/or nuclear receptors, may contribute to hepatic pathology. Key enzymes involved in lyso PL metabolism include phospholipases (PLA, multiple subtypes) and lysophosphatidyl choline acyl transferases (LpCAT; 4 subtypes). We previously established that hepatic phospholipase (PLA2g6) and LpCAT1 and LpCAT2 were induced by the WD, while DHA, but not EPA, suppressed LpCAT 1 and 2 expression [50]. Thus, DHA has the potential to regulate cellular levels of LpCAT-derived ligands controlling specific G-protein (GPRs) and nuclear receptors.

Oxylipins represent the second group of regulatory lipids examined in this study (Figures 5-8). Prostaglandins and leukotrienes are well-studied oxidation products of PUFA that are generated by cyclooxygenases (Cox) and lipoxygenases (Alox), respectively. The products of these enzymes are short-lived oxidized lipids that bind to and activated G-protein receptors (GPRs) that induce changes in intracellular second messengers, i.e., cAMP and calcium, affecting multiple signaling pathways [76]. These active products are rapidly degraded to relatively inactive compounds. Because 
of the short-lived nature of these active products, the inactive compounds are quantified as surrogates for in vivo synthesis of the bioactive Cox/Alox products [76]. Two 20:4, $\omega 6$-derived oxylipins identified in our analysis include 6-keto-PGF1 $\alpha$ and TXB2; these are degradation products of PGI2 and TXA2, respectively. PGI2 and TXA2 are involved in platelet aggregation, vasodilation, and inflammation, while PGD2 and PGE2 are involved in inflammation and vasodilation [61]. Increased hepatic levels of these products are associated with the induction of expression of Cox1 and Cox 2 mRNAs in response to the WD (Figure 7). Dietary DHA, however, lowers hepatic TBX2, 6-keto-PGF1 $\alpha$, and PGE2. This response parallels DHA-mediated suppression of hepatic ARA levels, as opposed to DHA-mediated suppression of Cox 1 and Cox 2 expression (Figures 5 and 7). Alox products (5 HETE, 12-HETE and 15-HETE) are also lower in livers of WDD-fed mice. Like the Cox products, hepatic levels of Alox products paralleled changes in hepatic ARA.

Other $\omega 6$ PUFA derived oxylipins include 9(S)-HODE and 13(S)-HODE, both of which are derived from linoleic acid by enzymatic (Alox) and non-enzymatic (oxidative stress) pathways. These products affect ER-stress, apoptosis, inflammation, cellular adhesions, and PPAR $\gamma$ function [77]. Since there was little effect of diet on hepatic Alox5, Alox12/15, or Alox15 expression, hepatic oxidative stress likely accounts for the increased hepatic levels of 9(S)-HODE and 13(S)-HODE in response to the WD [50]. The decline in these oxylipins parallel the WD-mediated suppression of hepatic LA content (Figure 5A). Feldstein et al. recently reported increased levels of these oxylipins in NASH patients, when compared to patients with benign steatosis [78]. This finding contrast with our findings and may reflect differences in hepatic oxidative stress management in human versus mouse livers. This group also reported no significant change in 5-HETE, 12-HETE, or 15-HETE in normal, steatotic, or NASH livers of patients. These results are similar to our findings (Figure 5F).

The other class of oxylipins examined included products generated by epoxygenases (Cyp2C, Cyp2J) and a soluble epoxide hydrolase (Ephx2). Like the Cox products, the epoxide products of Cyp2C and CYP2J are bioactive compounds. Moreover, Cyp2C and Cyp2J products are rapidly degraded to dihydroxy fatty acids with low bioactivity [79]. The WD has little effect on the formation of the ARA-derived products, but DHA lowered hepatic levels of the epoxy (11,12-EpETrE; 14,15-EpETrE) and dihydroxy (14,15-DiHETrE) products derived from ARA. Since there was little effect of diet on the expression of the Cyp2C, Cyp2J, and Ephx2 (Figure 7), we attribute the declined in Cyp2C, Cyp2J, and Ephx2 products to DHA-mediated suppression of hepatic ARA content (Figure 5). However, we cannot exclude post-translational mechanisms controlling the activity of these enzymes.

ARA-derived epoxygenase products are anti-inflammatory, pro-resolving bioactive mediators [79]. A decline in the hepatic abundance of these metabolites suggest an increase in hepatic inflammation. However, we previously reported that the WD increased hepatic markers of inflammation while the WD supplemented with DHA suppressed hepatic inflammation [52]. To explain this outcome, our analysis revealed a massive suppression ( $>70 \%$ ) of $\mathrm{C}_{20-22} \omega 3$ PUFA-derived oxylipins (Figures 7 and 8B) in livers of WDO30 fed mice. In WDD30 fed mice, however, the Cyp2C and Cyp2F-derived $\omega 3-P U F A$ oxylipins were restored to levels at or above levels seen in mice fed the RD. Changes in $\mathrm{C}_{20-22} \omega 3$ PUFA-derived oxylipins are inversely associated with transcriptomic markers of hepatic inflammation and fibrosis ( $q \leq 0.026$; Tables 1 and 2$)$. The concept of an inverse association between tissue levels of $\mathrm{C}_{20-22} \omega 3$ PUFA and inflammation is not new [80]. In fact, other investigators using the choline-methionine-deficient rat [81] and mouse [82] models of NAFLD reported a similar inverse association between tissue levels of DHA and liver injury. Our studies extend these observations by showing how specific classes of bioactive lipids are responsive to diet and associated with NASH markers (Figures 4-8; Tables 1 and 2).

The outcome of our lipidomic analysis supports the notion that dietary supplementation with DHA mitigates WD-induced NASH progression, at least in part, by lowering hepatic pro-inflammatory oxylipins derived from $\mathrm{C}_{20-22} \omega 6$ PUFA and increasing hepatic reparative/anti-inflammatory oxylipins derived from $\mathrm{C}_{20-22} \omega 3$ PUFA. While there is limited information on the differential bioactivity of $\omega 3$ PUFA versus $\omega 6$ PUFA-derived Cyp2C and Cyp2J, Lopez-Vicario et al. reported that, when 
compared to $\omega 6$ PUFA-derived epoxides, $\omega 3$ PUFA-derived epoxides were more effective inhibitors of inflammation and autophagy in insulin sensitive tissues, like liver [83]. As such, tissue levels of $\omega 3$ PUFA-derived epoxides may be a good predictor of liver health status in the context of WD-induced NASH. Key next steps will be to identify mechanisms linking specific $\omega 3$ PUFA- and $\omega 6$ PUFA-derived oxylipins to the expression of specific genes involved in hepatic inflammation and fibrosis.

\section{Materials and Methods}

\subsection{Study Design for DHA-Mediated NASH Remission in Male Ldlr ${ }^{-1}$ Mice}

This study was carried out in strict accordance with the recommendations in the Guide for the Care and Use of Laboratory Animals of the National Institutes of Health. All procedures for the use and care of animals for laboratory research were approved by the Institutional Animal Care and Use Committee at Oregon State University (Permit Number: A3229-01). Liver samples used in this lipidomic analysis were obtained from our previously published study assessing the capacity to DHA to promote NASH remission [52]. Briefly, male mice (B6:129S7-Ldlr ${ }^{\mathrm{tm} 1 \mathrm{Her} / \mathrm{J}}$, stock\# 002207 purchased from Jackson Labs) were group housed (4 mice/cage) and maintained on a $12 \mathrm{~h} \mathrm{light/dark} \mathrm{cycle.} \mathrm{Mice}$ were acclimatized to the Oregon State University (OSU) animal facilities for 2 weeks before proceeding with experiments.

At 10 wks of age, mice were fed a chow (Purina Pico Lab diet 5053) and served as a reference diet (RD) group. The RD group was maintained of the RD for the duration of the study, i.e., 30 wks $(\mathrm{RD} 30, n=5)$ (Figure 1). Ldlr ${ }^{-}$mice were also fed the western diet (Research Diets, D12079B). The WD consists of $41 \%$ energy as fat, $43 \%$ energy as carbohydrate, $17 \%$ energy as protein, and $0.15 \% \mathrm{w} / \mathrm{w}$ cholesterol [52]. After 22 wks on the WD, a group of WD-fed mice was euthanized for recovery of blood and liver. This group (WD22, $n=5$ ) served as a baseline for disease progression. The remainder of the WD-fed mice were switched to a diet supplemented with olive oil or DHASCO. DHASCO is a dietary supplement provided by DSM Nutritional Products; it contains DHA in a triglyceride form. DHA represents $\sim 40 \%$ of total acyl chains in DHASCO and DHASCO contains no EPA, DPA (22:5, $\omega 3$ ), ARA, or LA [50]. DHA is present in the diet at $\%$ total calories (WDD30, $n=7$ ). In order to have isocaloric diets, olive oil was added to the WD diet, i.e., WDO30. The WDO30 and WDD30 groups were maintained on their respective diets for $8 \mathrm{wks}$. Mice were then fasted overnight and euthanized for the collection of liver and blood. All samples were stored at $-80^{\circ} \mathrm{C}$ until used for extraction. The design of this study allowed for the assessment of disease progression from 22 to 30 weeks and the capacity of DHA to affect disease progression (Figure 1).

\subsection{RNA Extraction and $q R T P C R$}

Liver RNA was extracted using Trizol (Ambion by Life Technologies, Carlsbad, CA, USA), quantified, and used for qRTPCR as described previously [52]. Primers use for qRTPCR are described in our previous study [60]. Relative quantitation was determined using the delta $C_{T}$ methods using cyclophilin as the reference gene. The delta $C_{T}$ value was used for all statistical analyses.

\subsection{Sample Preparation for Lipidomic Analysis}

Liver lipids were extracted using a biphasic solvent system of cold methanol, methyl tert-butyl ether (MTBE), and water with some modifications [84]. Liver ( 20-25 mg) was transferred to $2 \mathrm{~mL}$ pre-weighted polypropylene tubes containing ceramic beads and of LC-MS-grade cold methanol $(240 \mu \mathrm{L})$. Deuterated lipid recovery standards $\left(5 \mu \mathrm{L}\right.$ of Splash ${ }^{\circledR}$ Lipidomix ${ }^{\circledR}$ Mass Spec Standards (Avanti Polar Lipids, Alabaster, AL, USA) were added to each sample. Samples were homogenized in a Precellys ${ }^{\circledR} 24$ bead-based homogenizer for $2 \mathrm{~min}$ at $1350 \mathrm{rpm}$. Cold MTBE $(750 \mu \mathrm{L})$ was added to the samples, followed by vortexing $(10 \mathrm{~s})$ and shaking $(6 \mathrm{~min})$ at $4{ }^{\circ} \mathrm{C}$. Phase separation was induced by adding LC-MS-grade water $(188 \mu \mathrm{L})$ followed vortexing and centrifugation $(14,000 \mathrm{rpm}, 2 \mathrm{~min})$. The upper organic phase $(300 \mu \mathrm{L})$ was recovered and evaporated using a Labconco centrivap vacuum 
concentrator (Kansas City, MO, USA). Dried lipid extracts were resuspended in a methanol/toluene (9:1, $v / v, 100 \mu \mathrm{L}$ ) mixture containing CUDA (1-cyclohexyl ureido, 3-dodecanoic acid, $50 \mathrm{ng} / \mathrm{mL}$; Cayman Chemical, Ann Arbor, MI, USA) as an additional internal standard. Samples were vortexed (10 s) and centrifuged (14,000 rpm, $2 \mathrm{~min})$ prior to LC-MS/MS analysis.

\subsection{Sample Preparation for Oxylipins Analysis}

Oxylipins were extracted from liver using the approach described by Pedersen et al. [85], with minor modifications. Liver ( 20-25 mg) was transferred $2 \mathrm{~mL}$ pre-weighted polypropylene tubes containing ceramic beads. Cold LC-MS-grade methanol $(35 \mu \mathrm{L})$ and an anti-oxidant solution [0.2 $\mathrm{mg} \mathrm{mL}^{-1}$ solution BHT (butylated hydroxytoluene) in 1:1 methanol:water] (5 $\left.\mu \mathrm{L}\right)$ was added to each sample. Each sample also received $10 \mu \mathrm{L}$ of a deuterated oxylipin recovery standard solution; the standards included 20 deuterated oxylipins (Table S1) in methanol at a concentration of $5 \mathrm{ng} / \mu \mathrm{L}$ each. Ten $\mathrm{mM}$ ammonium formate $+1 \%$ formic acid in isopropanol $(550 \mu \mathrm{L})$ and water $(100 \mu \mathrm{L})$ was added and the tubes were placed in a Precellys ${ }^{\circledR} 24$ bead-based homogenizer for $2 \mathrm{~min}$ at $1350 \mathrm{rpm}$. Samples were centrifuged (9000 rpm for $5 \mathrm{~min}$ ) at room temperature. Supernatants were transferred to a 96-well Ostro Pass Through Sample Preparation Plate (Waters Corp, Milford, MA, USA) and eluted into glass inserts containing $10 \mu \mathrm{L} \mathrm{20 \%} \mathrm{glycerol} \mathrm{in} \mathrm{methanol} \mathrm{by} \mathrm{applying} \mathrm{a} \mathrm{vacuum} \mathrm{(15} \mathrm{mm} \mathrm{Hg}$ ) for $10 \mathrm{~min}$. Eluents were dried by vacuum centrifugation in a Labconco centrivap vacuum concentrator for $2 \mathrm{~h}$ at room temperature. Once dry, samples were reconstituted with $100 \mu \mathrm{L}$ of methanol: acetonitrile (50:50), containing the internal standard (CUDA at $50 \mathrm{ng} / \mathrm{mL})$. Samples were transferred to a spin filter $(0.22$ $\mu \mathrm{m}$ PVDF membrane, Millipore-Sigma, Burlington, MA, USA) and centrifuged $\left(3 \mathrm{~min}\right.$ at $6{ }^{\circ} \mathrm{C}$ at 9000 $\mathrm{rpm}$ ) before transferred to $2 \mathrm{~mL}$ amber LC-MS vials. Extracts were stored at $-20^{\circ} \mathrm{C}$ until analysis by ultra-performance liquid chromatography tandem mass spectrometry (UPLC-MS/MS). The internal oxylipin standards added to the samples (Table S3) were used to correct the recovery of the quantified oxylipins [86].

\subsection{Chromatographic and Mass Spectrometry Conditions for Lipids and Oxylipins Analysis}

\subsubsection{Untargeted Lipidomics}

UHPLC was performed using a Shimadzu Nexera system (Shimadzu, Columbia, MD, USA) coupled to a triple time-of-flight (TOF) ${ }^{\mathrm{TM}} 5600$ mass spectrometer (AB SCIEX, Framingham, MA, USA). Compounds were separated using a Waters Acquity UPLC CSH C18 column $(100 \mathrm{~mm}$ length $\times 2.1 \mathrm{~mm}$ id; $1.7 \mu \mathrm{m}$ particle size) with an additional Waters Acquity VanGuard CSH C18 pre-column (5 mm $\times 2.1 \mathrm{~mm}$ id; $1.7 \mu \mathrm{m}$ particle size) held constant at $65^{\circ} \mathrm{C}$ while utilizing a flow rate of $0.6 \mathrm{~mL} \mathrm{~min}^{-1}$. Resuspended samples were injected at $2 \mu \mathrm{L}$ and $3 \mu \mathrm{L}$ for electrospray ionization (ESI) positive and negative modes, respectively. To improve lipid coverage, different mobile phase modifiers were used for positive and negative mode analysis [87]. For positive mode, $10 \mathrm{mM}$ ammonium formate $+0.1 \%$ formic acid was used, while $10 \mathrm{mM}$ ammonium acetate (Sigma-Aldrich, St. Louis, MO, USA) was used for negative mode. Both positive and negative modes used the same mobile phase composition of (A) 60:40 v/v acetonitrile: water (LC-MS grade) and (B) 90:10 v/v isopropanol:acetonitrile. To enhance solubilization of ammonium formate and ammonium acetate after its addition in the mobile phase, the salts were dissolved first in small volume of water before their addition in the mobile phases $(0.631 \mathrm{~g}$ ammonium formate or $0.771 \mathrm{~g}$ ammonium acetate $/ 1 \mathrm{~mL}$ water/1 L mobile phase). Each mobile phase with modifiers was mixed, sonicated for $15 \mathrm{~min}$ to achieve complete dissolving of modifiers, mixed again, and then sonicated for another $15 \mathrm{~min}$ [88]. The separation was conducted under the following gradient: $0 \min 15 \%$ (B), 0-2 $\min 30 \%$ (B), 2-2.5 $\min 48 \%$ (B), 2.5-11 $\min 82 \%$ (B), 11-11.5 $\min 99 \%$ (B), 11.5-12 $\min 99 \%$ (B), 12-12.1 $\mathrm{min} 15 \%$ (B), and 12.1-15 $\mathrm{min} 15 \%$ (B), at a flow rate of $0.6 \mathrm{~mL} \mathrm{~min}^{-1}$. All samples were kept at $4{ }^{\circ} \mathrm{C}$ throughout the analysis.

All analyses were performed at the high-resolution mode in MS $^{1}(\sim 35,000$ full width at half maximum (FWHM) ) and at the high sensitivity mode $(15,000 \mathrm{FWHM})$ in $\mathrm{MS}^{2}$. Sequential window 
acquisition of all theoretical fragment-ion spectra (SWATH) in positive/negative ion mode was used as the data independent acquisition (DIA) system for all samples. Data dependent acquisition (DDA) on a separate quality control (QC) pool sample was used in order to verify the annotations from SWATH acquisition for the most abundant lipid species. Detailed information of SWATH conditions included in Supplemental Information entitled SWATH parameters for untargeted analysis.

The mass calibration was automatically performed every 6 injections using an APCI positive/negative calibration solution (AB SCIEX) via a calibration delivery system (CDS). Quality control was assured by (i) randomization of the sequence, (ii) injection of QC pool samples at the beginning and the end of the sequence and between each 10 actual samples, (iii) procedure blank analysis, and (iv) checking the peak shape and the intensity of spiked internal standards and the internal standard added prior to injection.

\subsubsection{Targeted Oxylipidomics}

High Performance Liquid Chromatography (HPLC) was performed using a Shimadzu system (Shimadzu, Columbia, MD, USA) coupled to a QTRAP 4000 (AB SCIEX, Framingham, MA, USA). Employing dynamic multi-reaction monitoring (dMRM) we evaluated 39 oxylipins, 17 deuterated oxylipins, CUDA, and the deuterated surrogates eicosapentaenoic acid-d5 (EPA-d5), docosahexaenoic acid-d5 (DHA-d5), and arachidonic acid-d8 (ARA-d8) in a $22 \mathrm{~min}$ LC-run in a targeted approach (Figure S1). For each compound, optimal transitions were determined by flow injection of pure standards using the optimizer application, and transitions were compared to literature values when available for certain compounds. The detailed list of MRM transitions is in Table S4. In the dMRM acquisition mode the triple quadrupole MS system focuses directly on the expected analyte retention time (RT) with a defined detection window instead of user-defined time segments to capture groups of closely eluting compounds. Establishing a constant cycle time for each transition improves peak symmetry and allows for a more accurate quantification of narrow chromatographic peaks. For co-eluting metabolites, compound specific precursor ions and their corresponding fragment ions were used for selective detection and quantification of those compounds. For instance, for 11,12-EpETE (m/z 317 $\rightarrow 195)$ and 12-HETE $(\mathrm{m} / \mathrm{z} 319 \rightarrow 135)$, both elute at RT $16.14 \mathrm{~min}$.

Compounds were separated using a Waters Acquity UPLC CSH C18 column $(100 \mathrm{~mm}$ length $\times$ $2.1 \mathrm{~mm}$ id; $1.7 \mu \mathrm{m}$ particle size) with an additional Waters Acquity VanGuard CSH C18 pre-column (5 $\mathrm{mm} \times 2.1 \mathrm{~mm}$ id; $1.7 \mu \mathrm{m}$ particle size) held constant at $60^{\circ} \mathrm{C}$. The mobile phase and gradient elution conditions were adopted from Pedersen and Newman [85]. In summary, the mobile phase consisted of (A) water (0.1\% acetic acid) and (B) acetonitrile/isopropanol (ACN/IPA) (90/10, v/v) (0.1\% acetic acid). Gradient elution conditions were carried out for $22 \mathrm{~min}$ at a flow rate of $0.15 \mathrm{~mL} \mathrm{~min}{ }^{-1}$. Gradient conditions were: $0-1.0 \mathrm{~min}, 0.1-25 \% \mathrm{~B} ; 1.0-2.5 \mathrm{~min}, 25-40 \% \mathrm{~B} ; 2.5-4.5 \mathrm{~min}, 40-42 \% \mathrm{~B} ; 4.5-10.5 \mathrm{~min}$, $42-50 \%$ B; $10.5-12.5 \mathrm{~min}, 50-65 \%$ B; 12.5-14 min, 65-75\% B; 14-14.5 min, 75-85\% B; 14.5-20 min, 85-95\% B; 20-20.5 $\mathrm{min}, 95-95 \% \mathrm{~B} ; 20.5-22 \mathrm{~min}, 95-25 \%$ B. A $5 \mu \mathrm{L}$ aliquot of each sample was injected onto the column. Limits of detection (LOD) and quantification (LOQ) (Table S3) were calculated based on one concentration point $\left(0.1 \mathrm{ng} \mu \mathrm{L}^{-1}\right)$ for each oxylipin and deuterated surrogate.

\subsection{Data Processing}

\subsubsection{Untargeted Lipidomics}

MS-DIAL (v. 2.80) was the software program used for data processing [89]. This open-source software permits processing of LC-MS data acquired either in MS $^{1}$ only or with accompanying MS/MS information collected in data-dependent or data-independent mode from different MS platforms. We used LipidBlast [90] for lipid identification. Chromatographic peaks were annotated based on different levels of identification [91]. Peak intensities were normalized using the internal standard CUDA and the QC pool sample to correct for differences in injection volume and platform stability 
throughout the fully randomized batch of samples. The SPLASH Lipidomics Mix was used for the precise identification of major lipid classes and to perform relative quantitation.

\subsubsection{Targeted Analysis of Oxylipins}

Oxylipin data obtained by HPLC-dMRM-based analyses was processed using our in-house library on MultiQuant ${ }^{\mathrm{TM}}$ software.

\subsubsection{Statistical Analyses}

Annotated metabolites were used for multivariate statistical analysis. Pathway analysis, principal component analysis (PCA) and heat map plots were generated with MetaboAnalyst 4.0 [66]. The significance of individual metabolites between the treatment groups was assessed with a one-way ANOVA followed by Fisher's post hoc analysis and Holm FDR-correction, with a $q$-value of $<0.05$ indicating significance. If needed, data was logarithmically transformed to correct for unequal variance or non-normal distribution. No outliers were excluded from the statistical analyses. Differences in oxylipins among treatments were analyzed in GraphPad Prism 7.03 (La Jolla, CA, USA). Discovery was determined using the two-stage linear step-up procedure of Benjamini et al., [92], with $q$-value = $5 \%$ (cutoff for FDR $=0.05$ ). Each compound was analyzed individually, without assuming a consistent standard deviation. Figures were generated with GraphPad Prism 7.03 (La Jolla, CA), PowerPoint 2018 (Microsoft, Redmond, WA, USA), and MetaboAnalyst 4.0 [66].

Supplementary Materials: The following are available online at http:/www.mdpi.com/2218-1989/9/11/252/s1, Figure S1. LC-MS/MS chromatogram of 60 transitions in a 22 min LC-run allowing monitoring 39 oxylipins, 17 deuterated oxylipins, CUDA, and the deuterated surrogates eicosapentaenoic acid-d5 (EPA-d5), docosahexaenoic acid-d5 (DHA-d5), and arachidonic acid-d8 (ARA-d8). Analysis were performed on a SCIEX linear ion trap (LIT) QTRAP 4000 using the dMRM method implemented from Pedersen et al., [80]. The use of a quadrupole mass spectrometer with a linear ion trap significantly enhances platform performance by increasing ion capacity, improving injection and trapping efficiencies, and increasing duty cycle, Table S1. Diet effects on all lipids, Table S2. Lipids significantly affected by diet, Table S3. Detailed list of multi-reaction monitoring (MRM) transitions for the deuterated-oxylipins (surrogates) and CUDA (12-[[(cyclohexylamino) carbonyl] amino]-dodecanoic acid) used as internal standards for our analysis. Compounds are ordered based on retention time (RT), Table S4. Detailed list of multi-reaction monitoring (MRM) transitions for the oxylipins contained in our in-house library. Compounds are ordered based on retention time (RT).

Author Contributions: Conceptualization: K.A.L. and D.B.J.; Data curation: M.G.-J., K.A.L., M.H.S. and D.B.J.; Formal analysis: M.G.-J., K.A.L., M.H.S. and D.B.J.; Funding acquisition: D.B.J.; Investigation: M.G.-J., K.A.L., M.H.S. and D.B.J.; Methodology: M.G.-J., K.A.L., M.H.S. and D.B.J.; Project administration: D.B.J.; Resources: D.B.J.; Supervision: D.B.J.; Validation: M.G.-J., K.A.L., M.H.S. and D.B.J.; Visualization: M.G.-J. and D.B.J.; Writing-original draft: M.G.-J. and D.B.J.; Writing-review and editing: M.G.-J., K.A.L., M.H.S. and D.B.J.

Funding: This research was funded by grants from the National Institutes of Health, DK096400 and DK112360. In addition, the Oregon State University Mass Spectrometry Facility received equipment grants from the National Institutes of Health, NIH S10RR022589, S10RR027878.

Conflicts of Interest: The authors declare no conflicts of interest.

\section{References}

1. Bellentani, S.; Scaglioni, F.; Marino, M.; Bedogni, G. Epidemiology of non-alcoholic fatty liver disease. Dig. Dis. 2010, 28, 155-161. [CrossRef] [PubMed]

2. Younossi, Z.; Anstee, Q.M.; Marietti, M.; Hardy, T.; Henry, L.; Eslam, M.; George, J.; Bugianesi, E. Global burden of NAFLD and NASH: Trends, predictions, risk factors and prevention. Nat. Rev. Gastroenterol. Hepatol. 2018, 15, 11-20. [CrossRef] [PubMed]

3. Younossi, Z.M.; Golabi, P.; de Avila, L.; Paik, J.M.; Srishord, M.; Fukui, N.; Qiu, Y.; Burns, L.; Afendy, A.; Nader, F. The global epidemiology of NAFLD and NASH in patients with type 2 diabetes: A systematic review and meta-analysis. J. Hepatol. 2019, 4, 793-801. [CrossRef] [PubMed]

4. Angulo, P.; Lindor, K.D. Non-alcoholic fatty liver disease. J. Gastroenterol. Hepatol. 2002, 17, S186-S190. [CrossRef] [PubMed] 
5. Neuschwander-Tetri, B.A.; Caldwell, S.H. Nonalcoholic steatohepatitis: Summary of an AASLD Single Topic Conference. Hepatology 2003, 37, 1202-1219. [CrossRef]

6. Farrell, G.C.; Larter, C.Z. Nonalcoholic fatty liver disease: From steatosis to cirrhosis. Hepatology 2006, 43, S99-S112. [CrossRef]

7. Cohen, J.C.; Horton, J.D.; Hobbs, H.H. Human fatty liver disease: Old questions and new insights. Science 2011, 332, 1519-1523. [CrossRef]

8. Lauby-Secretan, B.; Scoccianti, C.; Loomis, D.; Grosse, Y.; Bianchini, F.; Straif, K. Body fatness and cancer-viewpoint of the IARC working group. N. Engl. J. Med. 2016, 375, 794-798. [CrossRef]

9. Amarapurkar, D.; Kamani, P.; Patel, N.; Gupte, P.; Kumar, P.; Agal, S.; Baijal, R.; Lala, S.; Chaudhary, D.; Deshpande, A. Prevalence of non-alcoholic fatty liver disease: Population based study. Ann. Hepatol. 2007, 6, 161-163. [CrossRef]

10. Center for Disease Control and Prevention. Adult Obesity Facts. 2015. Available online: http://www.cdc. gov/obesity/data/adult.html (accessed on 21 September 2019).

11. Center for Disease Control and Prevention. Childhood Obesity Facts. 2015. Available online: http: //www.cdc.gov/obesity/data/childhood.html (accessed on 21 September 2019).

12. Spooner, M.H.; Jump, D.B. Omega-3 Fatty Acids and Nonalcoholic Fatty Liver Disease in Adults and Children: Where Do We stand? Current Opinion in Clinical Nutrition and Metabolic Care; Wolters Kluwer: Alphen aan den Rijn, The Netherlands, 2019.

13. Cussons, A.J.; Watts, G.F.; Mori, T.A.; Stuckey, B.G. Omega-3 fatty acid supplementaion decreases liver fat content in polycystic ovary syndrome: A randomized controlled trial employing proton magnetic resonance spectroscopy. J. Clin. Endocrinol. Metabol. 2009, 94, 3842-3848. [CrossRef]

14. Soderberg, C.; Stal, P.; Askling, J.; Glaumann, H.; Lindberg, G.; Marmur, J.; Hultcrantz, R. Decreased survival of subjects with elevated liver function tests during a 28-year follow-up. Hepatology 2010, 51, 595-602. [CrossRef] [PubMed]

15. Ekstedt, M.; Franzen, L.E.; Mathiesen, U.L.; Thorelius, L.; Holmqvist, M.; Bodemar, G.; Kechagias, S. Long-term follow-up of patients with NAFLD and elevated liver enzymes. Hepatology 2006, 44, 865-873. [CrossRef] [PubMed]

16. Adams, L.A.; Lymp, J.F.; St Sauver, J.; Sanderson, S.O.; Lindor, K.D.; Feldstein, A.; Angulo, P. The natural history of nonalcoholic fatty liver disease: A population-based cohort study. Gastroenterology 2005, 129, 113-121. [CrossRef] [PubMed]

17. Chalasani, N.; Younossi, Z.; Lavine, J.E.; Diehl, A.M.; Brunt, E.M.; Cusi, K.; Charlton, M.; Sanyal, A.J. The diagnosis and management of non-alcoholic fatty liver disease: Practice guideline by the American Association for the Study of Liver Diseases, American College of Gastroenterology, and the American Gastroenterological Association. Am. J. Gastroenterol. 2012, 107, 811-826. [CrossRef]

18. Alberti, K.G.; Zimmet, P.; Shaw, J. The metabolic syndrome-A new worldwide definition. Lancet 2005, 366, 1059-1062. [CrossRef]

19. Day, C.P.; James, O.F. Steatohepatitis: A tale of two "hits"? Gastroenterology 1998, 114, 842-845. [CrossRef]

20. LaBrecque, D.; Abbas, Z.; Anania, F.; Ferenci, P.; Gahafoor Kahn, A.; Goh, K.-L.; Hamid, S.S.; Isakov, V.; Lizarzabal, M.; Mojica Pernaranda, M.; et al. Nonalcoholic Fatty Liver Disease and Nonalcoholic Steatohepatitis; World Gastroentrology Organization Global Guidelines: Milwaukee, WI, USA, 2012; pp. 1-29.

21. Tilg, H.; Moschen, A.R. Evolution of inflammation in nonalcoholic fatty liver disease: The multiple parallel hits hypothesis. Hepatology 2010, 52, 1836-1846. [CrossRef]

22. Schuppan, D.; Kim, Y.O. Evolving therapies for liver fibrosis. J. Clin. Invest. 2013, 123, 1887-1901. [CrossRef]

23. Buzzetti, E.; Pinzani, M.; Tsochatzis, E.A. The multiple-hit pathogenesis of non-alcoholic fatty liver disease (NAFLD). Metabolism 2016, 65, 1038-1048. [CrossRef]

24. Angulo, P. Nonalcoholic fatty liver disease. N. Engl. J. Med. 2002, 346, 1221-1231. [CrossRef]

25. Friedman, S.L. Mechanisms of hepatic fibrogenesis. Gastroenterology 2008, 134, 1655-1669. [CrossRef] [PubMed]

26. Koyama, Y.; Brenner, D.A. New therapies for hepatic fibrosis. Clin. Res. Hepatol. Gastroenterol. 2015, 39, S75-S79. [CrossRef] [PubMed]

27. Rosenbloom, J.; Mendoza, F.A.; Jimenez, S.A. Strategies for anti-fibrotic therapies. Biochim. Biophys. Acta 2013, 1832, 1088-1103. [CrossRef] [PubMed] 
28. Vilar-Gomez, E.; Martenez-Perez, Y.; Calzadilla-Bertot, L.; Torres-Gonzalez, A.; Gra-Oramas, B.; Gonzalez-Fabian, L.; Friedman, S.L.; Diago, M.; Romero-Gomez, M. Weight loss through lifestyle modification significantly reduces features of nonalcoholic steatohepatitis. Gastroenterology 2015, 149, 367-378. [CrossRef] [PubMed]

29. Chan, H.L.; de Silva, H.J.; Leung, N.W.; Lim, S.G.; Farrell, G.C. How should we manage patients with non-alcoholic fatty liver disease in 2007. J. Gastroenterol. Hepatol. 2007, 22, 801-808. [CrossRef] [PubMed]

30. Leslie, M. The liver's weight problem. Science 2015, 6243, 18-20.

31. Chalasani, N.; Younossi, Z.; Lavine, J.E.; Diehl, A.M.; Brunt, E.M.; Cusi, K.; Charlton, M.; Sanyal, A.J. The diagnosis and management of non-alcoholic fatty liver disease: Practice guideline by the American Gastroenterological Association, American Association for the Study of Liver Diseases, and American College of Gastroenterology. Gastroenterology 2012, 142, 1592-1609. [CrossRef]

32. Lee, Y.A.; Wallace, M.C.; Friedman, S.L. Pathology of liver fibrosis: A translational success story. Gut 2015, 64, 830-841. [CrossRef]

33. Kappler, R. From crawl to sprint: The race to treat NASH. Life Sci. VC 2015. Available online: https: //lifescivc.com/2015/05/from-crawl-to-sprint-the-race-to-treat-nash/ (accessed on 21 September 2019).

34. Musso, G.; Cassader, M.; Rosina, F.; Gambino, R. Impact of current treatments on liver disease, glucose metabolism and cardiovascular risk in non-alcoholic fatty liver disease (NAFLD): A systematic review and meta-analysis of randomised trials. Diabetologia 2012, 55, 885-904. [CrossRef]

35. Petit, J.M.; Guiu, B.; Duvillard, L.; Jooste, V.; Brindisi, M.C.; Athias, A.; Bouillet, B.; Habchi, M.; Cottet, V.; Gambert, P.; et al. Increased erythrocytes n-3 and n-6 polyunsaturated fatty acids is significantly associated with a lower prevalence of steatosis in patients with type 2 diabetes. Clin. Nutr. 2012, 31, 520-525. [CrossRef] [PubMed]

36. Zheng, J.S.; Xu, A.; Huang, T.; Yu, X.; Li, D. Low docosahexaenoic acid content in plasma phospholipids is associated with increased non-alcoholic fatty liver disease in China. Lipids 2012, 47, 549-556. [CrossRef] [PubMed]

37. Parker, H.M.; Johnson, N.A.; Burdon, C.A.; Cohn, J.S.; O'Connor, H.T.; George, J. Omega-3 supplementation and non-alcoholic fatty liver disease: A systematic review and meta-analysis. J. Hepatol. 2012, 56, 944-951. [CrossRef] [PubMed]

38. Di Minno, M.N.; Russolillo, A.; Lupoli, R.; Ambrosino, P.; Di Minno, A.; Tarantino, G. Omega-3 fatty acids for the treatment of non-alcoholic fatty liver disease. World J. Gastroenterol. 2012, 18, 5839-5847. [CrossRef]

39. Nobili, V.; Bedogni, G.; Alisi, A.; Pietrobattista, A.; Rise, P.; Galli, C.; Agostoni, C. Docosahexaenoic acid supplementation decreases liver fat content in children with non-alcoholic fatty liver disease: Double-blind randomised controlled clinical trial. Arch. Dis. Child. 2011, 96, 350-353. [CrossRef]

40. Shapiro, H.; Tehilla, M.; Attal-Singer, J.; Bruck, R.; Luzzatti, R.; Singer, P. The therapeutic potential of long-chain omega-3 fatty acids in nonalcoholic fatty liver disease. Clin. Nutr. 2011, 30, 6-19. [CrossRef]

41. Glass, L.M.; Dickson, R.C.; Anderson, J.C.; Sufiawinata, A.A.; Putra, J.; Berk, B.S.; Toor, A. Total body weight loss of $\geq 10 \%$ is associated with improved hepatic fibrosis in patients with nonalcoholic steatohepatitis. Dig. Dis. Sci. 2015, 60, 1024-1030. [CrossRef]

42. Sofi, F.; Casini, A. Mediterranean diet and non-alcoholic fatty liver disease. World J. Gastroenterol. 2014, 20, 7339-7346. [CrossRef]

43. Marchesini, G.; Mazzotti, A. NAFLD incidence and remission: Only a matter of weight gain and weight loss. J. Hepatol. 2015, 62, 15-17. [CrossRef]

44. Panera, N.; Barbaro, B.; Della Corte, C.; Mosca, A.; Nobili, V.; Alisi, A. A review of the pathogenic and therapeutic role of nutrition in pediatric nonalcoholic fatty liver disease. Nutr. Res. 2018, 58, 1-16. [CrossRef]

45. Sumida, Y.; Yoneda, M. Current and future pharmacological therapies for NAFLD/NASH. J. Gastroenterol. 2018, 53, 362-376. [CrossRef] [PubMed]

46. Albhaisi, S.; Sanyal, A. Recent advances in understanding and managing non-alcoholic fatty liver disease. F1000Research 2018, 7, 1-11. [CrossRef] [PubMed]

47. Jump, D.B.; Lytle, K.A.; Depner, C.M.; Tripathy, S. Omega-3 polyunsaturated fatty acids as a treatment strategy for nonalcoholic fatty liver disease. Pharmacol. Ther. 2018, 181, 108-125. [CrossRef] [PubMed]

48. Banini, B.A.; Sanyal, A.J. Current and future pharmacologic treatment of nonalcoholic steatohepatitis. Curr. Opin. Gastroenterol. 2017, 33, 134-141. [CrossRef] [PubMed] 
49. McCollough, A.J. Epidemiology of the metabolic syndrome in the USA. J. Dig. Dis. 2011, 12, 333-340. [CrossRef] [PubMed]

50. Depner, C.M.; Philbrick, K.A.; Jump, D.B. Docosahexaenoic acid attenuates hepatic inflammation, oxidative stress, and fibrosis without decreasing hepatosteatosis in a Ldlr(-/-) mouse model of western diet-induced nonalcoholic steatohepatitis. J. Nutr. 2013, 143, 315-323. [CrossRef]

51. Lytle, K.A.; Jump, D.B. Is western diet-induced nonalcoholic steatohepatitis in Ldlr-/- mice reversible. PLoS ONE 2016, 11, e0146942. [CrossRef]

52. Lytle, K.A.; Wong, C.P.; Jump, D.B. Docosahexaenoic acid blocks progression of western diet-induced nonalcoholic steatohepatitis in obese Ldlr-/- mice. PLoS ONE 2017, 12, e0173376. [CrossRef]

53. Simopoulos, A.P. n-3 fatty acid-enriched eggs, lipids, and Western diet: Time for change. Nutrition 1993, 9, 561-562.

54. Cordain, L.; Eaton, S.B.; Sebastian, A.; Mann, N.; Lindeberg, S.; Watkins, B.A.; O’Keefe, J.H.; Brand-Miller, J. Orgins and evolution of the western diet: Health implications for the 21st century. Am. J. Clin. Nutr. 2005, 81, 341-354. [CrossRef]

55. Allard, J.B.; Aghdassi, E.; Mohammed, S.; Raman, M.; Avand, G.; Arendt, B.M.; Jalali, P.; Kandasamy, T.; Prayitno, N.; Sherman, M.; et al. Nutrition assessment and hepatic fatty acid composition in non-alcoholic fatty liver disease (NAFLD): A cross-sectional study. J. Hepatol. 2007, 48, 300-307. [CrossRef] [PubMed]

56. Arendt, B.M.; Comelli, E.M.; Ma, D.W.; Lou, W.; Teterina, A.; Kim, T.; Fung, D.K.H.; McGilvray, I.; Fischer, S.E.; Allard, J.P. Altered hepatic gene expression in non-alcoholic fatty liver disease is associated with lower n-3 and n-6 polyunsaturated fatty acids. Hepatology 2015, 61, 1565-1578. [CrossRef] [PubMed]

57. Spahis, S.; Alverez, F.; Dubois, J.; Ahmed, N.; Peretti, N.; Levy, E. Plasma fatty acid composition in French-Canadian children with non-alsocholic fatty liver disease: Effect of n-3 PUFA supplementation. Prostaglandins Leukot. Essent. Fat. Acids 2015, 99, 25-34. [CrossRef] [PubMed]

58. Jump, D.B. The biochemistry of n-3 polyunsaturated fatty acids. J. Biol. Chem. 2002, 277, 8755-8758. [CrossRef] [PubMed]

59. Jump, D.B.; Tripathy, S.; Depner, C.M. Fatty Acid-regulated transcription factors in the liver. Ann. Rev. Nutr. 2013, 33, 249-269. [CrossRef] [PubMed]

60. Garcia-Jaramillo, M.; Spooner, M.H.; Loehr, C.V.; Wong, C.P.; Zhang, W.; Jump, D.B. Lipidomic and transcriptomic analysis of western diet-induced nonalcoholic steatohepatitis (NASH) in female Ldlr-/- mice. PLoS ONE 2019, 14, E0214387. [CrossRef]

61. Gabbs, M.; Leng, S.; Devassy, J.G.; Monirujjaman, M.; Aukema, H.M. Advances in our understanding of oxylipins derived from dietary PUFAs. Adv. Nutr. 2015, 6, 513-540. [CrossRef]

62. Jump, D.B.; Depner, C.M.; Tripathy, S. Omega-3 fatty acid supplementation and cardiovascular disease. J. Lipid Res. 2012, 53, 2525-2545. [CrossRef]

63. Scorletti, E.; Bhatia, L.; McCormick, K.G.; Clough, G.F.; Nash, K.; Hodson, L.; Moyses, H.E.; Calder, P.C.; Byrne, C.D.; The Welcome Study Investigators. Effects of purified eicosapentaenoic and docosahexaenoic acids in non-alcoholic fatty liver disease: Results from the *WELCOME study. Hepatology 2014, 60, 1211-1221. [CrossRef]

64. Sanyal, A.J.; Abdelmalek, M.F.; Suzuki, A.; Cummings, O.W.; Chojkier, M. No significant effects of ethyl-eicosapentaenoic acid on histologic features of nonalcoholic steatohepatitis in a Phase 2 trial. Gastroenterology 2014, 147, 377-384. [CrossRef]

65. Barter, P.; Ginsberg, H.N. Effectiveness of combined statin plus omega-3 fatty acid therapy for mixed dyslipidemia. Am. J. Cardiol. 2008, 102, 1040-1045. [CrossRef] [PubMed]

66. Chong, J.; Soufan, O.; Li, C.; Caraus, I.; Li, S.; Bourque, G.; Wishart, D.S.; Xia, J. MetaboAnalyst 4.0: Towards more transparent and integrative metabolomics analysis. Nucleic Acids Res. 2018, 46, W486-W494. [CrossRef] [PubMed]

67. Mater, M.K.; Thelen, A.P.; Jump, D.B. Arachidonic acid and PGE2 regulation of hepatic lipogenic gene expression. J. Lipid Res. 1999, 40, 1045-1052.

68. Lopane, C.; Agosti, P.; Gigante, I.; Sabba, C.; Mazzocca, A. Implications of the lysophosphatidic acid signaling axis in liver cancer. Biochim. Biophys. Acta 2017, 1868, 277-282. [CrossRef]

69. Ishii, I.; Fukushima, N.; Ye, X.; Chun, J. Lysophospholipid receptors: Signaling and biology. Ann. Rev. Biochem. 2004, 73, 321-354. [CrossRef] 
70. Gardell, S.E.; Dubin, A.E.; Chun, J. Emerging medicinal roles for lysophospholipid signaling. Trends Mol. Med. 2006, 12, 65-75. [CrossRef]

71. Frasch, S.C.; Bratton, D.L. Emerging roles for lysophosphatidylserine in resolution of inflammation. Prog. Lipid Res. 2012, 51, 199-207. [CrossRef]

72. Tsukahara, T.; Matsuda, Y.; Haniu, H. Lysophospholipid-Related Diseases and PPARgamma Signaling Pathway. Int. J. Mol. Sci. 2017, 18, 2730. [CrossRef]

73. D'Souza, K.; Paramel, G.V.; Kienesberger, P.C. Lysophosphatidic Acid Signaling in Obesity and Insulin Resistance. Nutrients 2018, 10, 399. [CrossRef]

74. Brown, A.J.; Jupe, S.; Briscoe, C.P. A family of fatty acid binding receptors. DNA Cell Biol. 2005, $24,54-61$. [CrossRef] [PubMed]

75. Funk, C.D. Prostaglandins and leukotrienes: Advances in eicosanoid biology. Science 2001, 294, 1871-1875. [CrossRef] [PubMed]

76. Kostenis, E. A glance at G-protein-coupled receptors for lipid mediators: A growing receptor family with remarkably diverse ligands. Pharmacol. Ther. 2004, 102, 243-257. [CrossRef] [PubMed]

77. Graves, J.P.; Gruzdev, A.; Bradbury, J.A.; DeGraff, L.M.; Li, H.; House, J.S.; Hoopes, S.L.; Edin, M.L.; Zeldin, D.C. Quantitative Polymerase Chain Reaction Analysis of the Mouse Cyp2j Subfamily: Tissue Distribution and Regulation. Drug Metab. Dispos. 2015, 43, 1169-1180. [CrossRef] [PubMed]

78. Feldstein, A.E.; Lopez, R.; Tamimi, T.A.; Yerian, L.; Chung, Y.M.; Berk, M.; Zhang, R.; McIntyre, T.M.; Hazen, S.L. Mass spectrometric profiling of oxidized lipid products in human nonalcoholic fatty liver disease and nonalcoholic steatohepatitis. J. Lipid Res. 2010, 51, 3046-3054. [CrossRef] [PubMed]

79. Hasegawa, E.; Inafuku, S.; Mulki, L.; Okunuki, Y.; Yanai, R.; Smith, K.E.; Kim, C.B.; Klokman, G.; Bielenberg, D.R.; Puli, N.; et al. Cytochrome P450 monooxygenase lipid metabolites are significant second messengers in the resolution of choroidal neovascularization. Proc. Natl. Acad. Sci. USA 2017, 114, E7545-E7553. [CrossRef] [PubMed]

80. Calder, P.C. Marine omega-3 fatty acids and inflammatory processes: Effects, mechanisms and clinical relevance. Biochim. Biophys. Acta 2015, 1851, 469-484. [CrossRef] [PubMed]

81. Palladini, G.; Di Pasqua, L.G.; Berardo, C.; Siciliano, V.; Richelmi, P.; Mannucci, B.; Croce, A.C.; Rizzo, V.; Perlini, S.; Vairetti, M.; et al. Fatty Acid Desaturase Involvement in Non-Alcoholic Fatty Liver Disease Rat Models: Oxidative Stress Versus Metalloproteinases. Nutrients 2019, 11, 799. [CrossRef]

82. Stankovic, M.N.; Mladenovic, D.; Ninkovic, M.; Ethuricic, I.; Sobajic, S.; Jorgacevic, B.; de Luka, S.; Vukicevic, R.J.; Radosavljević, T.S. The effects of alpha-lipoic acid on liver oxidative stress and free fatty acid composition in methionine-choline deficient diet-induced NAFLD. J. Med. Food. 2014, 17, $254-261$. [CrossRef]

83. Lopez-Vicario, C.; Alcaraz-Quiles, J.; Garcia-Alonso, V.; Rius, B.; Hwang, S.H.; Titos, E.; Clària, J. Inhibition of soluble epoxide hydrolase modulates inflammation and autophagy in obese adipose tissue and liver: Role for omega-3 epoxides. Proc. Natl. Acad. Sci. USA 2015, 112, 536-541. [CrossRef]

84. Matyash, V.; Liebisch, G.; Kurzchalia, T.V.; Shevchenko, A.; Schwudke, D. Lipid extraction by methyl-tert-butyl ether for high-throughput lipidomics. J. Lipid Res. 2008, 49, 1137-1146. [CrossRef]

85. Pedersen, T.L.; Newman, J.W. Establishing and performing targeted multi-residue analysis for lipid mediators and fatty acids in small clinical plasma samples. Methods Mol. Biol. 2018, 1730, 175-212. [PubMed]

86. La Franko, M.R.; Hernandez-Carretero, A.; Weber, N.; Borkowski, K.; Pedersen, T.L.; Osborn, O.; Newman, J.W. Diet-induced obesity and weight loss alter bile acid concentrations and bile acid-sensitive gene expression in insulin target tissues of C57BL/6J mice. Nutr. Res. 2017, 46, 11-21. [CrossRef] [PubMed]

87. Cajka, T.; Fiehn, O. Increasing lipidomic coverage by selecting optimal mobile-phase modifiers in LC-MS of blood plasma. Metabolomics 2016, 12,1-11. [CrossRef]

88. Cajka, T.; Davis, R.; Austin, K.J.; Newman, J.W.; German, J.B.; Feihn, O.; Smilowitz, J.T. Using a lipidomics approach for nutritional phenotyping in resposne to a test meal containing gamma-linolenic acid. Metabolomics 2016, 12, 1-22. [CrossRef]

89. Tsugawa, H.; Cajka, T.; Kind, T.; Ma, Y.; Higgins, B.; Ikeda, K.; Kanazawa, M.; VanderGheynst, J.; Fiehn, O.; Arita, M. MS-DIAL: Data-independent MS/MS deconvolution for comprehensive metabolome analysis. Nat. Methods. 2015, 12, 523-526. [CrossRef] [PubMed]

90. Kind, T.; Liu, K.H.; Lee, D.Y.; DeFelice, B.; Meissen, J.K.; Fiehn, O. LipidBlast in silico tandem mass spectrometry database for lipid identification. Nat. Methods 2013, 10, 755-758. [CrossRef] 
91. Sumner, L.W.; Amberg, A.; Barrett, D.; Beale, M.H.; Beger, R.; Daykin, C.A.; Fan, T.W.; Fiehn, O.; Goodacre, R.; Griffin, J.L.; et al. Proposed minimum reporting standards for chemical analysis Chemical Analysis Working Group (CAWG) Metabolomics Standards Initiative (MSI). Metabolomics 2007, 3, 211-221. [CrossRef]

92. Benjamini, Y.; Krieger, A.M.; Yekutieli, D. Adaptive linear step-up procedures that control the false discovery rate. Biometrika 2006, 93, 491-507. [CrossRef]

(C) 2019 by the authors. Licensee MDPI, Basel, Switzerland. This article is an open access article distributed under the terms and conditions of the Creative Commons Attribution (CC BY) license (http://creativecommons.org/licenses/by/4.0/). 

Article

\title{
Acupuncture on ST36, CV4 and KI1 Suppresses the Progression of Methionine- and Choline-Deficient Diet-Induced Nonalcoholic Fatty Liver Disease in Mice
}

\author{
Xiangjin Meng ${ }^{1,2}$, Xin Guo ${ }^{1,3, *}$, Jing Zhang ${ }^{1}$, Junji Moriya ${ }^{2}$, Junji Kobayashi ${ }^{2}$, \\ Reimon Yamaguchi ${ }^{4}$ and Sohsuke Yamada ${ }^{1,3}$ \\ 1 Department of Pathology and Laboratory Medicine, Kanazawa Medical University, 1-1 Daigaku, Uchinada, \\ Kahoku, Ishikawa 920-0293, Japan; meng6950@kanazawa-med.ac.jp (X.M.); \\ jing2018@kanazawa-med.ac.jp (J.Z.); sohsuke@kanazawa-med.ac.jp (S.Y.) \\ 2 Department of General Internal Medicine, Kanazawa Medical University, Ishikawa 920-0293, Japan; \\ moriya@kanazawa-med.ac.jp (J.M.); mary@kanazawa-med.ac.jp (J.K.) \\ 3 Department of Pathology, Kanazawa Medical University Hospital, Ishikawa 920-0293, Japan \\ 4 Department of Dermatology, Kanazawa Medical University, Ishikawa 920-0293, Japan; \\ raymon-y@kanazawa-med.ac.jp \\ * Correspondence: tianqi11211216@yahoo.co.jp; Tel.: +81-76-2188021
}

Received: 29 October 2019; Accepted: 6 December 2019; Published: 9 December 2019

\begin{abstract}
Nonalcoholic fatty liver disease (NAFLD) is one of the most common chronic liver diseases worldwide, and its treatment remain a constant challenge. A number of clinical trials have shown that acupuncture treatment has beneficial effects for patients with NAFLD, but the molecular mechanisms underlying its action are still largely unknown. In this study, we established a mouse model of NAFLD by administering a methionine- and choline-deficient (MCD) diet and selected three acupoints (ST36, CV4, and KI1) or nonacupoints (sham) for needling. We then investigated the effects of acupuncture treatment on the progression of NAFLD and the underlying mechanisms. After two weeks of acupuncture treatment, the liver in the needling-nonapcupoint group (NG) mice appeared pale and yellowish in color, while that in the needling-acupoint group (AG) showed a bright red color. Histologically, fewer lipid droplets and inflammatory foci were observed in the AG liver than in the NG liver. Furthermore, the expression of proinflammatory signaling factors was significantly downregulated in the AG liver. A lipid analysis showed that the levels of triglyceride (TG) and free fatty acid (FFA) were lower in the AG liver than in the NG liver, with an altered expression of lipid metabolism-related factors as well. Moreover, the numbers of 8-hydroxy-2'-deoxyguanosine (8-OHdG)-positive hepatocytes and levels of hepatic thiobarbituric acid reactive substances (TBARS) were significantly lower in AG mice than in NG mice. In line with these results, a higher expressions of antioxidant factors was found in the AG liver than in the NG liver. Our results indicate that acupuncture repressed the progression of NAFLD by inhibiting inflammatory reactions, reducing oxidative stress, and promoting lipid metabolism of hepatocytes, suggesting that this approach might be an important complementary treatment for NAFLD.
\end{abstract}

Keywords: nonalcoholic fatty liver disease; acupuncture; imflammation; lipid metabolism; oxidative stress 


\section{Introduction}

Nonalcoholic fatty liver disease (NAFLD) is one of the most common clinicopathological conditions in chronic liver disease and is characterized by an obvious increase in fat deposition in the hepatocytes of the liver parenchyma [1]. Epidemiologically, NAFLD affects approximately $40 \%$ of the population worldwide, and the prevalence is increasing annually all over the world [2]. This disease has been considered to carry a high risk of developing into liver cirrhosis and hepatocellular carcinoma.

However, there are still no effective drugs specifically developed for the treatment of NAFLD, especially in patients with non-alcoholic steatohepatitis (NASH), a severe stage of NAFLD. Although several agents have shown some benefits for patients with NAFLD, even the most promising of such pharmacological agents are associated with significant adverse effects, and none have been approved by the Food and Drug Administration (FDA) for NAFLD therapy [3]. Balancing the benefits and risks in drugs for long-term treatment remains a constant challenge that has hampered the development of therapy strategies for NAFLD [4].

Ectopic fat accumulation in the hepatocytes, a hallmark of NAFLD, is thought to be caused by a complex and multiple mechanism that is still not completely understood but involves several interdependent molecular processes, such as inflammation, lipid metabolism, and oxidative stress [5]. An abnormal lipid metabolism, such as an increased uptake of lipids and the upregulation of de novo lipogenesis in the liver, is the initial trigger of NAFLD. Lipid overload promotes reactive oxygen species (ROS) generation and peroxidation itself, which causes the release of pro-inflammatory cytokines and inflammatory cellular infiltration $[6,7]$. The activation of the nuclear factor- $\mathrm{kB}(\mathrm{NF}-\mathrm{\kappa B})$ inflammatory pathway regulating downstream target genes plays very important roles in the progression of NAFLD. These inflammatory cytokines can recruit and activate Kupffer cells/macrophages and further aggravate liver injury and steatohepatitis formation [8].

Oxidative stress caused by the imbalance between oxidants and antioxidants seems to be one of the most important mechanisms leading to NAFLD liver injury, which plays a fundamental role in the progression from simple steatosis to NASH. The enhanced production of ROS can reportedly lead to necroinflammation and fibrosis through lipid peroxidation induced by astrocyte activation [9].

Acupuncture is a treatment method found in traditional Chinese medicine (TCM). Due to its advantages of low cost, few side effects, and simple operation, the role of acupuncture in disease prevention and treatment has recently attracted attention, and a large body of evidence has shown that acupuncture can induce pathophysiological consequences and alleviate the symptoms of diseases in multiple organs [10-12]. A number of clinical trials have also indicated that acupuncture treatment can improve metabolism conditions and exert beneficial effects on patients with NAFLD [13-15], but the mechanisms underlying its action remain unclear.

According to the theory of TCM, fatty liver formation primarily involves metabolic disorders caused by qi and blood stasis, and acupuncture at certain points of related meridians can improve the metabolism by keeping the body in a balanced state between "Yin" and "Yang" [16]. However, from a modern medicine viewpoint, how acupuncture exerts its therapeutic role and what kind of physiological consequences acupuncture causes at different acupoints of meridians are still unclear.

In the present study, we established a mouse model of NAFLD by administering a methionineand choline-deficient diet (MCD), a classic diet inducing NAFLD [17]. We selected three acupoints of related meridians regulating the metabolism to needle the mice with NAFLD and observed the roles of acupuncture in the progression of NAFLD. Furthermore, we also investigated the pathogenic mechanisms leading to these significant effects through laboratory and molecular biology experiments. Our results suggest that acupuncture might be a useful treatment for NAFLD and provide solid evidence supporting the incorporation of acupuncture into therapy for metabolic syndrome from a modern medicine viewpoint. 


\section{Results}

2.1. Lipid Accumulation was Significantly Reduced in the Livers of AG Mice with NAFLD Induced by an $M C D$ Diet

After two weeks of acupuncture, the livers from the needling-nonapcupoint group (NG) mice were variably pale and yellowish in color. In contrast, the livers from the needling-acupoint group (AG) showed a bright red color (Figure 1A). The mouse body weight and liver weight were significantly lower in AG mice than in those of NG mice $(p<0.0001, n=17)$ (Figure S1). However, the mouse liver/BW ratios were no significant difference between the two groups $(p<0.0001, n=17)$ (Figure 1B).

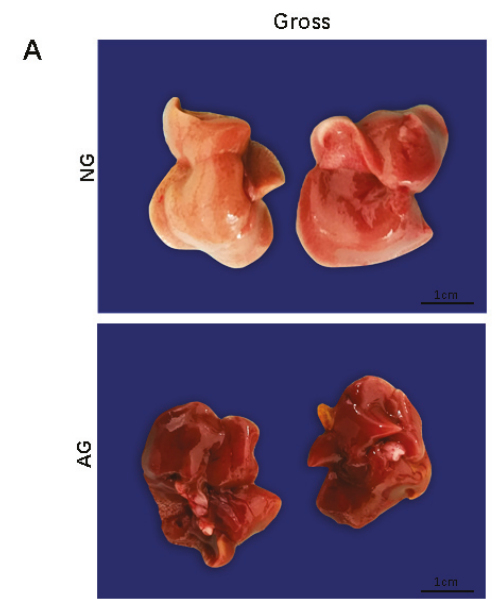

B

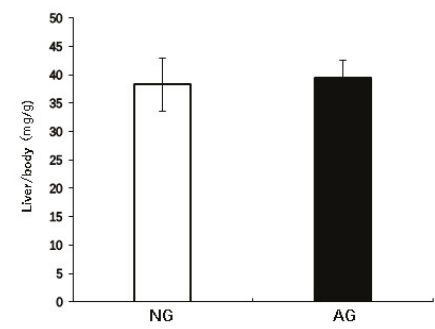

Figure 1. The appearance of the liver and ratio of the liver to the body weight after two weeks of acupuncture. (A) The liver tissues of the NG mice were obviously pale and yellowish in color, and while the tissue of the AG liver showed a bright red color. Bar $=1 \mathrm{~cm}$. (B) There was no marked difference in the ratio of the liver to the body weight between the two groups. Values are shown as the mean $\pm \mathrm{SD}, * p<0.05,{ }^{* *} p<0.001,{ }^{* * *} p<0.0001, n=17$. NG: needling-nonacupoint group, AG: needling-acupoint group, MCD diet: methionine- and choline-deficient diet, HF diet: high-fat diet, NAFLD: nonalcoholic fatty liver disease.

Hematoxylin and eosin (H\&E) staining showed that the AG liver tended to contain fewer lipid droplets and inflammatory foci than those of NG mice, and there was also a significant histological difference between the two livers. Ballooning of hepatocytes, inflammation, and fibrosis were noted in the livers of NG mice. Furthermore, the NASH score in the AG livers was significantly lower than that in the NG livers after acupuncture $(n=17)$ (Figure 2A and Table 1). Oil red-O staining revealed few lipid droplets in the AG mice, while the numbers of lipid-positive hepatocytes and droplets in each hepatocyte were increased in NG mice $(n=17)$ (Figure 2B). These morphology and histology findings indicate that acupuncture can treat the liver damage caused by a high-fat diet. 

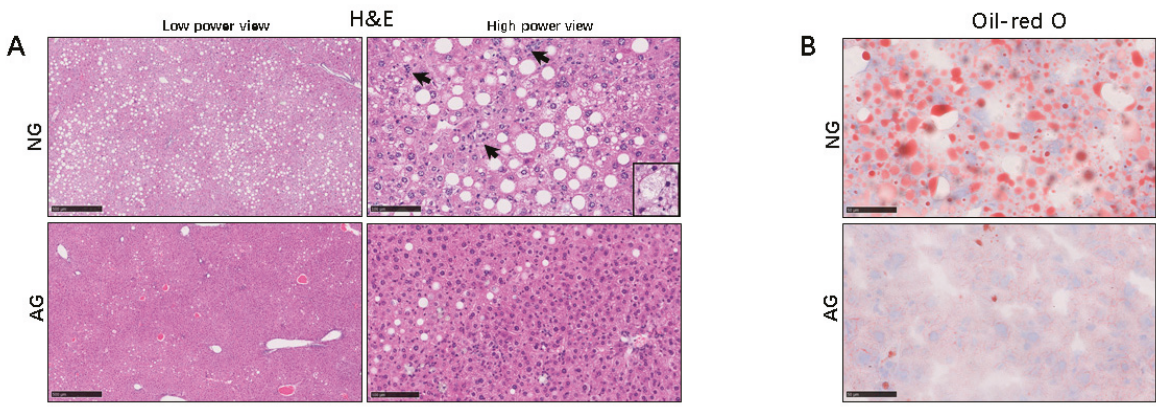

Figure 2. Histological observation of the liver from the two groups of mice. (A) Representative photomicrographs of liver (H\&E). H\&E-stained sections revealed macrovesicular and microvesicular steatosis throughout the entire lobules in the NAFLD livers from NG mice, as well as scattered lobular and perivenular inflammation (arrowhead). (original magnification: $\times 40$ [low]; Bars $=50 \mu \mathrm{m}, \times 200$ [high]; Bars $=100 \mu \mathrm{m}, n=17$ ). (B) Oil Red-O staining revealed a number of lipid droplets accumulated in NG liver, while fewer lipid droplets were observed in the AG liver (original magnification: $\times 400$, Bars $=50 \mu \mathrm{m}, n=17)$. NG: needling-nonacupoint group, AG: needling-acupoint group, H\&E: hematoxylin and eosin, NAFLD: nonalcoholic fatty liver disease.

Table 1. Quantitative scoring of the fat accumulation, inflammation and ballooning in the NAFLD livers of model AG and NG mice.

\begin{tabular}{|c|c|c|c|}
\hline \multicolumn{4}{|c|}{ Steatosis Score } \\
\hline Score & NG & AG & $P$ \\
\hline 0 & 0 & 11 & $<0.001$ \\
\hline 1 & 8 & 6 & \\
\hline 2 & 4 & 0 & \\
\hline 3 & 5 & 0 & \\
\hline \multicolumn{4}{|c|}{ Inflammation Score } \\
\hline Score & NG & AG & $P$ \\
\hline 0 & 0 & 5 & 0.003 \\
\hline 1 & 12 & 12 & \\
\hline 2 & 4 & 0 & \\
\hline 3 & 1 & 0 & \\
\hline \multicolumn{4}{|c|}{ Ballooning Score } \\
\hline Score & NG & AG & $P$ \\
\hline 0 & 5 & 16 & $<0.001$ \\
\hline 1 & 10 & 1 & \\
\hline 2 & 2 & 0 & \\
\hline 3 & 0 & 0 & \\
\hline \multicolumn{4}{|c|}{ NAFLD Score } \\
\hline Score & NG & AG & $P$ \\
\hline $0-3$ & 7 & 17 & $<0.001$ \\
\hline $4-6$ & 8 & 0 & \\
\hline $7-9$ & 6 & 0 & \\
\hline
\end{tabular}

Values are shown as the means \pm standard deviation, ${ }^{*} p<0.05,{ }^{* *} p<0.001,{ }^{* * *} p<0.0001, n=17$. NG: needling-nonacupoint group, AG: needling-acupoint group, NAFLD: nonalcoholic fatty liver disease.

\subsection{Acupuncture Treatment Inhibits the Inflammation Reaction during the Progression of MCD} Diet-Induced NAFLD

IHC for Mac-2 revealed that the AG livers contained a significantly smaller number of infiltrating macrophages (Kupffer cells) than the NG livers (AG $6.7 \pm 2.5$ vs. NG $12.3 \pm 4.7 ; p<0.001, n=17$ ) 
(Figure 3A). In addition, fibrogenesis and stellate cell activation, as determined by IHC for $\alpha$-smooth muscle actin ( $\alpha$-SMA), were not apparent in the AG liver, and the specific and linear expression of $\alpha$-SMA along with the activation of hepatic stellate cells in AG mice was much lower than that in NG mice (AG $18.7 \pm 6.1 .0$ vs. NG 39.4 $\pm 7.5 ; p<0.0001, n=17$ ) (Figure 3B).
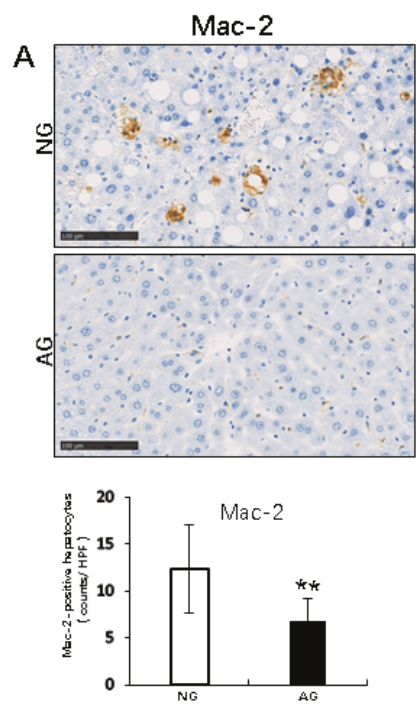
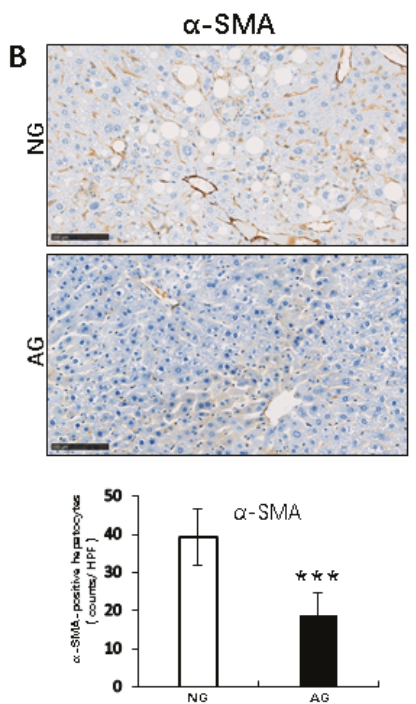

C

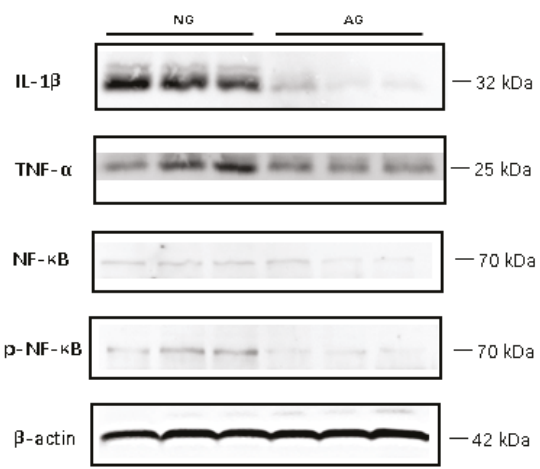

Figure 3. Inflammatory responses in the livers of AG and NG mice. (A) Immunocytochemistry (IHC) showed the number of Mac-2-positive infiltrating macrophages in the NAFLD liver. (B) $\alpha$-SMA-positive activated hepatic stellate cells in the NAFLD liver. Values are shown as the means \pm SD, Bars $=100$ $\mu \mathrm{m} * p<0.05,{ }^{* *} p<0.001,{ }^{* * *} p<0.0001, n=17$. (C) The IL- $1 \beta$, TNF $\alpha$, NF- $\mathrm{kB}$ and $\mathrm{p}-\mathrm{NF}-\mathrm{kB}$ protein expression was determined by Western blotting. Values are normalized $\beta$-actin expression (Western blotting) expression and are presented as means \pm SD. $n=7$. NG: needling-nonacupoint group, AG: needling-acupoint group, IHC: immunocytochemistry, $\alpha$-SMA: $\alpha$-smooth muscle actin, IL-1 $\beta$ : interleukin $1 \beta, \mathrm{TNF} \alpha$ : tumor necrosis factor- $\alpha, \mathrm{NF}-\mathrm{kB}$ : nuclear factor- $\mathrm{kB}, \mathrm{p}-\mathrm{NF}-\kappa \mathrm{B}$ : phospho-nuclear factor- $\mathrm{kB}$.

A Western blot analysis showed that the expression of proinflammatory signaling factors and inflammatory cytokines or transcriptional factor and their receptors, such as interleukin $1 \beta$ (IL-1 $\beta$ ), tumor necrosis factor- $\alpha(\mathrm{TNF} \alpha)$ and the key regulators of inflammation, NF- $\mathrm{kB}$ and phospho-nuclear 
factor-KB (p-NF- $\mathrm{B})$, was significantly lower in the AG livers than in the NG livers. Therefore, acupuncture can promote liver metabolism by inhibiting inflammatory reaction $(n=7)$ (Figure 3 C).

\subsection{Acupuncture Treatment Changed the Lipid Profiles and Regulated Lipid Metabolism in the Liver with} NAFLD Induced by an MCD Diet

The hepatic triglyceride (TG) and free fatty acid (FFA) levels in liver were significantly lower in AG mice than in NG mice (TG: AG $6.7 \pm 3.5 \mathrm{mg} / \mathrm{g}$ vs. NG $11.0 \pm 5.2 \mathrm{mg} / \mathrm{g} ; p<0.05, n=17$; FFA: AG $0.15 \pm 0.15 \mathrm{mEq} / \mathrm{g}$ vs. NG $0.4 \pm 0.2 \mathrm{mEq} / \mathrm{g} ; p<0.01, n=17)$. However, the liver T-cho levels were not significantly different between AG and NG mice (Figure $4 \mathrm{~A})$.
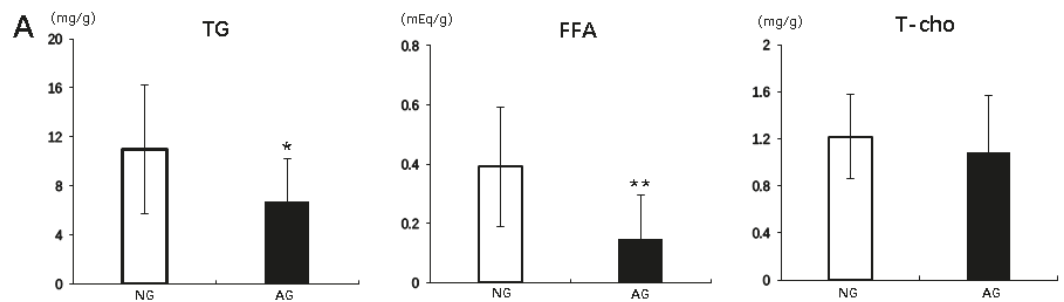

B
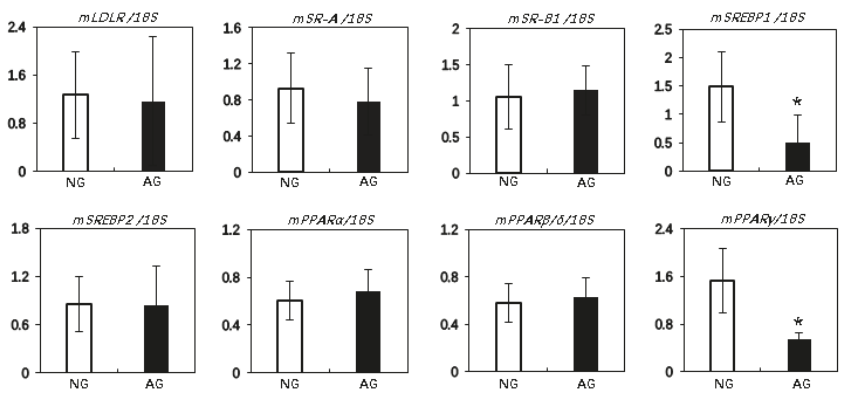

Figure 4. Acupuncture improved the lipid metabolism in the livers of mice with NAFLD. (A) A hepatic lipid analysis in NG and AG mice after two weeks of acupuncture. Values are shown as the mean \pm $\mathrm{SD},{ }^{*} p<0.05,{ }^{* *} p<0.001,{ }^{* * *} p<0.0001, n=17$. (B) Real time PCR revealed that the expression of several proinflammatory signaling factors (SREBP1 and PPAR $\gamma$ ) was significantly lower in the livers of AG mice than in those of NG mice. LDLR, SR-A, SR-B1, and SREBP2 showed no significant difference between the groups, nor did PPAR $\alpha$ or PPAR $\beta / \delta$. Values are normalized by the $18 \mathrm{~S}$ rRNA expression. RT-PCR values are presented as the means \pm SD. ${ }^{*} p<0.05,{ }^{* *} p<0.001,{ }^{* * *} p<0.0001, n=7$. NG: needling-nonacupoint group, AG: needling-acupoint group, TG: triglyceride, T-cho: total cholesterol, FFA: free fatty acid, LDLR: low-density lipoprotein receptor, SR-A: scavenger receptor class A, SR-B1: scavenger receptor class B type 1, SREBP: sterol regulatory element-binding protein, PPAR: peroxisome proliferator-activated receptor.

Real Time Reverse Transcription Polymerase Chain Reaction (RT-PCR) showed that no marked differences between the two groups were observed in the expression of some receptors related to the lipid uptake in the liver, including low-density lipoprotein receptor (LDLR), scavenger receptor class A (SR-A) and scavenger receptor class B type 1 (SR-B1) (Figure 4B). However, the expression of transcription factor sterol regulatory element binding protein 1 (SREBP1), an important transcriptional protein that regulates lipid synthesis with a well-studied function in lipid metabolism [18], was significantly lower in the AG livers than in the NG livers $(p<0.05, n=7)$, while that of SREBP2, which primarily regulates cholesterol biosynthesis, showed no significant difference between the two groups (Figure 4B). The expression of SREBP1 target genes, such as fatty acid synthase (FAS) and stearoyl-CoA 
9-desaturase 1 (SCD1), was also significantly lower in the AG livers than in the NG livers $(p<0.05$, $n=7$ ) (Figure S2B). Peroxisome proliferator-activated receptors (PPARs) are primary modulators in the metabolism of fatty acids in the liver [19] and include PPAR $\alpha, \operatorname{PPAR} \beta / \delta$, and PPAR $\gamma$. The PPAR $\gamma$ RNA expression was significantly lower in AG livers than NG livers $(p<0.05, n=7)$, as well as the the expression of PPAR $\gamma$ target such as adiponectin receptor 2 (AdipoR2). The expression was significantly lower in AG livers than NG livers ( $p<0.05, n=7$ ) (Figure S2B), but the PPAR $\alpha$ and PPAR $\beta / \delta$ RNA expression did not differ significantly between the groups (Figure $4 \mathrm{~B})$. Moreover, the expression of genes involved in hepatic lipid secretion apolipoprotein B (ApoB), apolipoprotein E (ApoE), and microsomal triglyceride transfer protein (MTTP) were significantly higher in the AG livers than in the NG livers $(p<0.05, n=7)$ (Figure S2A).

2.4. Acupuncture Treatment Improved Oxidative Stress Induced by Lipid Accumulation of the NAFLD Liver in Mice

We used IHC to determine the expression of 8-hydroxy-2'-deoxyguanosine (8-OHdG) as a marker for oxidative stress. There were significantly fewer cells positive for 8-OHdG in AG mice than in NG mice after 2 weeks of acupuncture (AG: $104.5 \pm 26.4$ vs. NG: $221.8 \pm 63.9 ; p<0.001, n=17$ ) (Figure 5A).
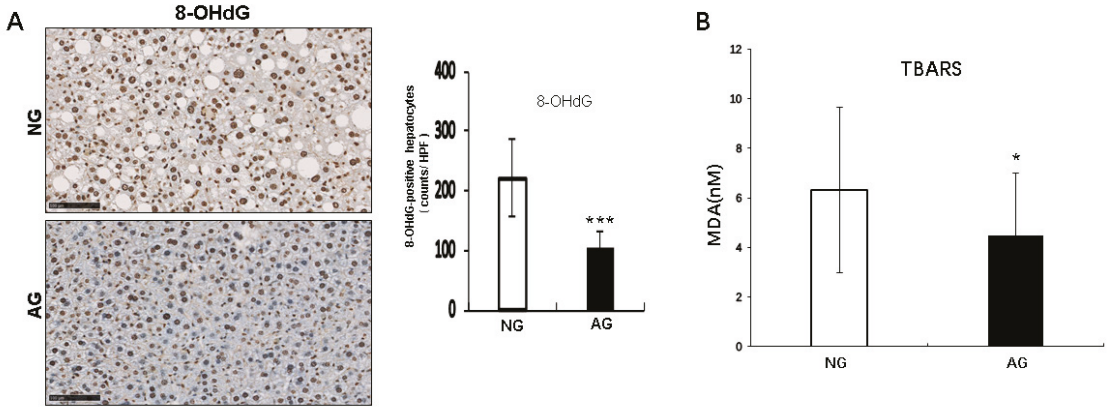

C

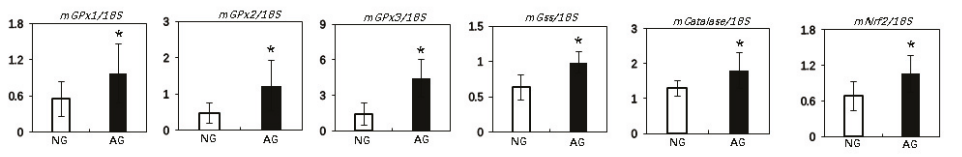

Figure 5. The analysis of hepatic oxidative stress in mice with NAFLD. (A) 8-hydroxy-20deoxyguanosine (8-OHdG) staining revealed significantly fewer accumulated 8-OHdG-positive hepatocytes in the livers of AG mice than in those of NG mice after acupuncture. Values are shown as the mean $\pm \mathrm{SD},{ }^{*} p<0.05,{ }^{* *} p<0.001,{ }^{* * *} p<0.0001, n=17$. (B) The levels of the oxidative stress marker thiobarbituric acid reactive substances (TBARS) in AG mice were significantly lower than in NG mice after acupuncture. Values are shown as the mean $\pm \mathrm{SD},{ }^{*} p<0.05,{ }^{* *} p<0.001,{ }^{* * *} p<0.0001$, $n=17$. (C) Real time PCR showed that the GPx1, GPx2, GPx3, Gss, Catalase, and Nrf2 expression in the liver was significantly higher in AG mice than in NG mice. Values are shown as the means \pm SD. ${ }^{*} p$ $<0.05,{ }^{* *} p<0.001,{ }^{* * *} p<0.0001, n=7$. NG: needling-nonacupoint group, AG: needling-acupoint group, GPx: glutathione peroxidase, Gss: glutathionylspermidine synthetase/amidase, Nrf2: nuclear factor erythroid 2-related factor 2 .

We next measured the liver levels of another oxidative stress marker, thiobarbituric acid reactive substances (TBARS). The TBARS levels were significantly lower in AG mice than in NG mice after 2 weeks of acupuncture (AG $4.5 \pm 2.5 \mathrm{nmol}$ malondialdehyde ([MDA]) protein vs. NG $6.3 \pm 3.3 \mathrm{nmol}$ MDA protein, $p<0.05, n=17$ ) (Figure $5 \mathrm{~B}$ ).

Real time PCR showed that the hepatic expression of several antioxidant enzymes, including glutathione peroxidase 1, 2, and 3 (GPx1, 2, and 3), glutathionylspermidine synthetase/amidase 
(Gss), and catalase and the transcription factor nuclear factor erythroid 2-related factor 2 (Nrf2), were significant higher in AG mice than in NG mice $(p<0.05, n=7)$ (Figure 5C). Therefore, acupuncture may reduce oxidative stress by upregulating the antioxidant expression.

\section{Discussion}

Acupuncture is a vital component of TCM and has a history of more than 2500 years. Given its safety and few side effects, this major TCM has been widely used to treat various diseases and symptoms, especially chronic metabolic illness [20]. Many modern studies have proven the efficacy of acupuncture against a wide range of diseases [21-23]. However, due to lack of understanding of the mechanisms underlying its action, acupuncture is the subject of severe controversy, with its effects proposed to be placebo effects [24]. To clarify these points and the efficacy of acupuncture on the systemic metabolism, it is necessary to determine the molecular mechanism underlying acupuncture's effects on specific tissues and cell metabolism.

Since it is illegal and unethical to carry out laboratory experiments on the human body without need, animal studies of acupuncture are of great value and show obvious advantages regarding research on the metabolic signal pathways compared to clinical studies [25]. Therefore, in this study, we used a mouse model of MCD + HF diet-induced NAFLD to investigate the mechanisms by which acupuncture treatment improves the conditions of this chronic disease.

According to TCM, the three acupoints of Zusanli (ST36), Yongquan (KI1) and Guanyuan (CV4) were selected for needling model mice in order to harmonize the Yin and Yang and dredge the channel of Qi and Blood. Our pathological and experimental results showed that acupuncture treatment significantly attenuated the progression of NAFLD by inhibiting inflammatory reactions, reducing oxidative stress and promoting lipid metabolism of hepatocytes. These results provide solid evidence from a modern medicine perspective supporting the notion that acupuncturing these three acupoints may be beneficial for patients with NAFLD.

Inflammation plays very important roles in the process of NAFLD progression. Injured liver cells can release damage-associated molecular patterns (DAMPs) to promote the activation of the NF- $\mathrm{KB}$ pathway, thus inducing the production of pro-inflammatory cytokines like TNF- $\alpha$ and ILs, which is a key step in the progression from simple steatosis to NASH [26,27]. Many studies have confirmed that acupuncture can downregulate the NF- $\mathrm{kB}$ expression $[28,29]$. Indeed, in the present study, hepatic injury induced by the absence of methionine and choline in the diet clearly upregulated the expression and enhanced the activation of NF- $\mathrm{kB}$, thus increasing the production of downstream factors TNF- $\alpha$ and IL- $1 \beta$ in the livers of NG mice, while acupuncture treatment significantly inhibited the NF- $\mathrm{BB}$ inflammatory signals in the AG mice. These cytokines can contribute to the recruitment and activation of macrophages/Kupffer cells (resident hepatic macrophages) to mediate inflammation [30], which is critical in NASH. A significant increase in macrophages has been shown to occur in the liver tissue of patients with NASH compared to those with simple steatosis [31]. In the present study, with the reduction in the TNF- $\alpha$ and IL-1 $\beta$ expression, much fewer Mac-2- and $\alpha$-SMA-positive cells were observed in the livers of AG mice with simple steatosis than in NG mice showing progression to NASH. These results indicate that acupuncture treatment can improve the pathological progression of NAFLD by inactivating the inflammatory signaling pathways.

The regulatory role of acupuncture in inflammation has also been reported in other studies, but the effects are not the same when needling different acupoints. For example, acupuncture of the Sanyinjiao (SP6) acupoint can increase IL-10 levels [32], while needling Fenglong (ST40) and Neiguan (PC6) acupoints reduced the IL-17 expression [33]. These data imply that although acupuncture treatment at several acupoints can suppresses the inflammation response, different acupoints can achieve the same effect by regulating specific cytokines.

The accumulation of excess lipid in hepatocytes causes organelle failure, such as mitochondrial dysfunction and endoplasmic reticulum stress, and leads to liver injury in patients with NAFLD [34,35]. However, in some NAFLD patients, the accumulation of lipids is not toxic to liver cells, a paradoxical 
effect that is believed to be related to the type of lipid itself. For example, TG reportedly does not seem toxic, but FFA and cholesterol—including its metabolites-are highly toxic to cells [36,37]. In our MCD-induced NAFLD mouse model, the hepatic total cholesterol levels were not markedly different between NG and AG mice, but the TG and especially the FFA levels were markedly reduced in the livers of AG mice compared to the livers of NG mice. Therefore, a significant decrease in the FFA level in the liver may play a more important role in acupuncture treatment for NAFLD than a reduction in TG. The FFA pool in the circulation is the major source of FFA in the liver [38], but no remarkable difference in the hepatic expression of receptors related to the lipid uptake were noted between NG and AG mice. However, we found that some regulators expressions regarding de novo lipogenesis and lipid storage in the liver were significantly decreased and genes involved in hepatic lipid secretion increased in AG mice compared with NG mice, suggesting that the reduction in hepatic lipid deposition after acupuncture treatment is induced by reducing the synthesis and promoting the metabolism of lipids rather than by inhibiting their uptake in the liver altogether.

Mitochondrial dysfunction and endoplasmic reticulum stress caused by the accumulation of lipids, especially FFA, can also result in the increased production of ROS and lead to oxidative stress that promotes inflammatory reactions, the activation of stellate cells and fibrosis in the liver, which have been recognized as important events in the development of NAFLD [39-41]. In the present study, the number of 8-OHdG-positive cells and the level of MDA in the liver were significantly reduced in AG mice compared to NG mice, showing that acupuncture treatment can also improve the oxidative stress status during NAFLD progression. It seems easy to understand that the lower level of hepatic FFA inhibited organelle failure and repressed the increased production of ROS in AG mice. These results should be rational, however, the consideration is probably oversimplified, as we also found expressions of some protective antioxidants to significantly increase in the livers of AG mice (Figure 5C), which may be the real reason for the inhibition of oxidative stress by the accumulation of lipids. Another interesting finding of the present study was that acupuncture treatment had significant regulatory effects on inflammatory reaction, lipid metabolism and redox homeostasis, which was found to be closely associated with changes in the expression of transcription factors related to these signaling pathways, like NF- kB, PPARs, and Nrf2 (Figure 3C, Figure 4B, and Figure 5C respectively). These results remind us that acupuncture may regulate cell metabolism at the expression level. To confirm this suspicion, more molecular mechanism experiments will be needed in the future.

In summary, from this study, we obtained some novel findings: (1) acupuncture on the three acupoints, ST36, CV4 and KI1, can improve pathological process of NAFLD; (2) acupuncture treatment can inhibit inflammatory reactions, reduce oxidative stress and promote lipid metabolism; (3) acupuncture on different acupoints can inhibit inflammatory reactions by regulating specific cytokines; (4) acupuncture treatment have regulatory effects on the expression of transcription factors. Although some exciting results were found in the present study, there are still some limitations to be noted. First, an MCD + HF diet-induced NAFLD model was used to investigate the roles of acupuncture in inhibiting the progression of NAFLD in this study, but the molecular mechanism underlying special diet-induced NAFLD does not totally reflect the pathogenesis of this disease in human, even though some pathological manifestations are consistent between the two entities. Second, although acupuncture treatment has few side effects, since this was the first instance of observing the effects of needling the three acupoints ST36, KI1, and CV4 in mice with NAFLD, and since this treatment was administered daily for only two weeks, we cannot confirm that no side effects would be noted with this approach over a long period of time. Finally, after two weeks of acupuncture treatment, we also found metabolic changes in other tissues and organs aside from the liver, largely related to gastrointestinal absorption, lipid storage and energy metabolism (data not shown), which have a major influence on metabolic syndrome, such as insulin resistance, obesity and fatty liver. However, in the present study, we ignored these other influences temporarily and instead focused on the metabolic changes in the liver after acupuncture treatment. The influence of other factors should therefore be discussed in future studies. 


\section{Materials and Methods}

\subsection{Animals and Experimental Protocol}

Experiments were performed using 8-week-old male C57BL/6 mice weighing approximately $20 \mathrm{~g}$ that were maintained in a temperature- and light-controlled facility with free access to water. Mice were fed an MCD + HF diet (60\% fat; KBT Oriental Corporation, Saga, Japan) for 3 weeks and then given an HF diet for two weeks to maintain their hyperlipidemia. As described previously [17], mice were anesthetized with an injection of ketamine-medetamidine and euthanized by exsanguination. The liver was excised and cut into small pieces, frozen, and fixed in 10\% neutral-buffered formalin for the experiments described below.

\subsection{Acupuncture Manipulation}

The mice were randomly divided into two groups: AG and NG. They were fed an MCD + HF diet for three weeks and then given HF diet for two weeks to maintain their hyperlipidemia. For the needling treatment, three acupoints (AG) or no acupoints (NG) were needled (Figure 6A).
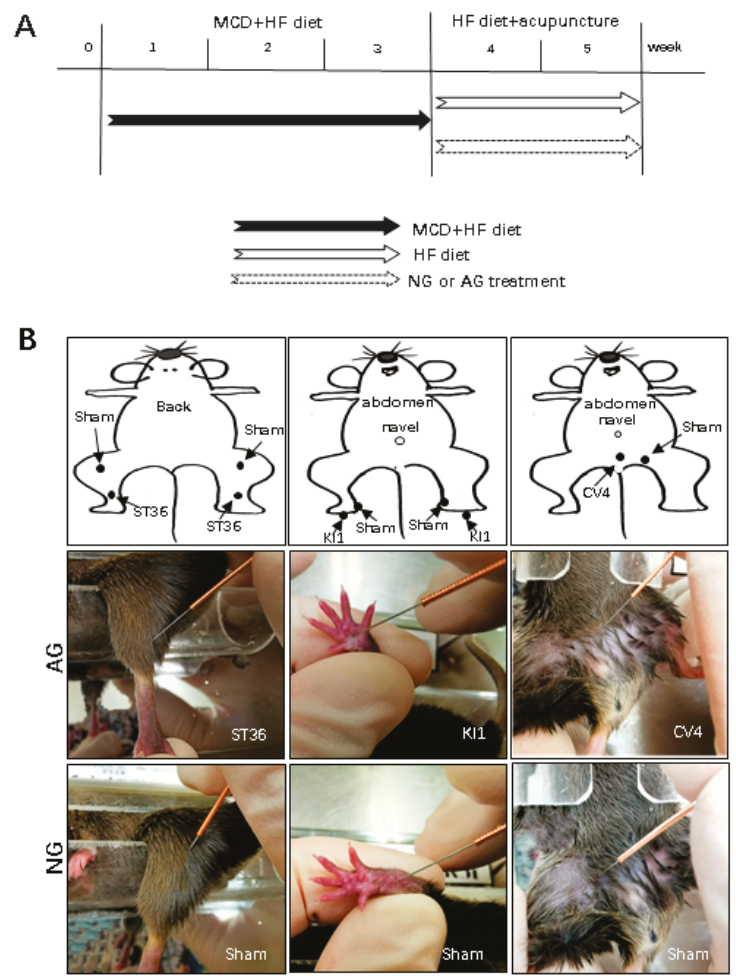

Figure 6. Acupoints and experimental design. (A) Schematic diagram of the experimental design. After being fed an MCD + HF diet for three weeks, mice were needled at acupoints or nonacupoints (sham) under a HF diet. (B) The acupoints and nonacupoints (sham) are shown in the diagrams (upper pictures). The actual acupoints and nonacupoints (sham) in mice are indicated in the middle and lower pictures. NG: needling-nonacupoint group, AG: needling-acupoint group, ST36: Zusanli, CN4: Guanyuan, KI1: Yongquan, sham: nonacupoints, MCD diet: methionine- and choline-deficient diet, HF diet: High Fat diet. 
The ST36 acupoint is located near the knee joint of the hind limb and $1.5 \mathrm{~mm}$ from the distal side of the anterior tibial tubercle. The KI1 acupoint is located in the middle of the hind paw. The pubic symphysis was obliquely stabbed at point $\mathrm{CV} 4$ at the mouse abdomen median line, $10 \mathrm{~mm}$ below the navel $[22,42,43]$ (Figure 6B). AG mice received acupuncture at both sides of ST36 and CV4 with 13-mm needles. Acupuncture was delivered using stainless steel needles (length: $13 \mathrm{~mm}$, diameter: 0.25 mm; Hwatuo, Suzhou Medical Supplies Factory Co., Ltd., Suzhou, China). ST36 and CV4 needling was performed by straightly inserting a stainless steel needle to a depth of $3 \mathrm{~mm}$. KI1 was needled obliquely toward the elbow to a depth of $2 \mathrm{~mm}$. NG mice received nonacupoint needling. All points were rotated slowly at 60 rounds per minute, completed in $2 \mathrm{~min}$, without retaining the needle.

\subsection{Ethics}

The Ethics Committee of Animal Care and Experimentation, Kanazawa Medical University, Japan, approved the protocols. The project code of the approval, 2019-21, was identified on 3 July 2019. Experiments were performed according to the Institutional Guidelines for Animal Experiments and the Law (no. 105) and Notification (no. 6) of the Japanese government. The number of animals used and their suffering were minimized.

\subsection{Histopathology}

After fixation in 10\% neutral-buffered formalin for $24 \mathrm{~h}$, paraffin-embedded liver specimens were systematically cut into sequential $4-\mu \mathrm{m}$-thick sections. For histological analyses of the liver, images of hematoxylin and eosin (H\&E), oil red-O, and immunohistochemistry.

IHC sections were captured and quantified using the NanoZoomer Digital Pathology Virtual Slide Viewer software program (Hamamatsu Photonics Corp, Hamamatsu, Japan). To evaluate the degree of lipid accumulation (steatosis score and lipid accumulation score for the liver), we performed oil red-O staining using frozen liver sections and categorized the tissues into 4 grades, as follows: no lipid droplets (score $=0$ ); lipid droplets in $<33 \%$ of hepatocytes (score $=1$ ); lipid droplets $33 \%-66 \%$ of hepatocytes (score $=2$ ) and lipid droplets in $>66 \%$ of hepatocytes (score $=3$ ). In addition, the degree of liver cell ballooning injury (ballooning score) was classified into three grades as follows: none $($ score $=0)$; few balloon cells $($ score $=1$ ) or many balloon cells/prominent ballooning (score $=2$ ).

\subsection{IHC}

To evaluate the severity of NAFLD, we determined the intensity of inflammation (inflammatory score) using an anti-mouse Mac-2 monoclonal antibody (1:1000; Cedarlane Laboratories Ltd., Burlington, Ontario, Canada). As described previously [44], we counted the number of positive macrophages in 10 randomly selected fields per liver section (original magnification: $\times 200$ ). The NAFLD liver tissues were then classified into four (inflammation score) grades, as follows: no inflammation (score $=0$ ); $<10$ inflammatory foci, each consisting of $>5$ inflammatory cells (score $=1) ; \geq 10$ inflammatory foci (score $=2$ ) or uncountable diffuse or fused inflammatory foci (score $=3)$. We used the HistoMouse ${ }^{\mathrm{TM}}-$ Plus Kit (Invitrogen Corporation, Camarillo, CA, USA) to block endogenous IgG and then stained tissue with a monoclonal mouse anti-human $\alpha$-SMA antibody (1:1000; Dako Cytomation, Carpenteria, CA, USA.). The number of activated stellate cells was then counted in 10 randomly selected fields per section (original magnification: $\times 200$ ), as described previously [44]. To determine the ROS/oxidative stress or expression in hepatocytes after acupuncture, we used an 8-OHdG monoclonal antibody (1:200; Japan Institute for the Control of Aging, Fukuroi, Japan) and quantified the number of hepatocytes positive for either antibodies in 10 randomly selected fields per section (original magnification: $\times 200$ ), as previously described. For IHC studies, we examined at least 1 section from each of 17 mice per experimental group.

All histological and immunohistochemical slides were evaluated by two independent observers (certified surgical pathologists in our department; X.G. and S.Y.) using a blind protocol design (observers 
blinded to the mice treatment data). The agreement between the observers was excellent (more than 95\%) for all antibodies investigated.

\subsection{Western Blotting}

Liver protein samples were separated by electrophoresis on $10 \%$ sodium dodecyl sulfate-polyacrylamide gel electrophoresis (SDS-PAGE) gels and transferred onto Immun-Blot PVDF membranes (Bio-Rad Laboratories, K.K., Tokyo, Japan). The membranes were then incubated overnight at $4{ }^{\circ} \mathrm{C}$ with IL-1 $\beta$ antibody (\#12242; Cell Signaling,), TNF- $\alpha$ antibody (\#11948; Cell Signaling), NF- $\mathrm{KB}$ antibody (\#8242; Cell Signaling), phospho-NF- $\mathrm{kB}$ antibody (\#3033; Cell Signaling) and anti- $\beta$-actin monoclonal antibody (Wako Pure Chemical Co., Osaka, Japan) diluted in Can Get Signal solution 1 (Toyobo, Osaka, Japan), after which the membranes were incubated for $1 \mathrm{~h}$ at room temperature with a horseradish peroxidase-conjugated goat anti-rabbit antibody (Vector Laboratories, Burlingame, CA, USA).

\subsection{Analyses of Lipid Contents from the Liver}

To examine the hepatic lipid profiles, each snap-frozen tissue $(70 \mathrm{mg})$ was homogenized and extracted with chloroform-methanol $(2 / 1 \mathrm{v} / \mathrm{v})$, as described previously [45]. The organic phase was dried and resolubilized in 2-propanol. The TG, FFA, and TCHO levels were then determined using commercial assay kits (Wako Pure Chemical Co.)

\subsection{Real Time Reverse Transcription Polymerase Chain Reaction (RT-PCR)}

Real time PCR was used to analyze the gene expression in the liver. Total RNA was extracted from mouse liver using the extracted by ReliaPrep ${ }^{\mathrm{TM}}$ RNA Tissue Miniprep kit (Promega, Leiden, Netherlands). The whole extraction process was performed under RNase-free conditions in order to prevent RNA degradation. Custom primers and TaqMan probe for gene amplification were purchased from Life Technologies. The mRNA expression of SREBP1, LDLR, SR-A, SREBP2, SR-B1, PPAR $\gamma$, PPAR $\alpha$, PPAR $\beta / \delta$, GPx1, GPx2, GPx3, Gss, catalase, and Nrf2 was analyzed by real time PCR (TaqMan probes Applied Biosystems, Warrington, UK). The relative expression of each gene was normalized to that of $18 \mathrm{~S}$ ribosomal RNA using random primers.

\subsection{The Measurement of the TBARS Levels}

We measured the liver TBARS levels using a TBARS Assay Kit (Cayman Chemical Company, Ann Arbor, MI, USA). Liver tissue specimens were homogenized in 250 RIPA buffer solution. A 100- $\mu \mathrm{L}$ aliquot of the homogenate was added to a reaction mixture containing $200 \mu \mathrm{L}$ of $8.1 \%$ (w/v) SDS, $1.5 \mathrm{~mL}$ of $20 \%(\mathrm{v} / \mathrm{v})$ acetic acid, $\mathrm{pH} 3.5,1.5 \mathrm{~mL}$ of $0.8 \%(\mathrm{w} / \mathrm{v})$ thiobarbituric acid, and $700 \mu \mathrm{L}$ of distilled water. Samples were then boiled for $1 \mathrm{~h}$ at $95{ }^{\circ} \mathrm{C}$ and centrifuged at $1600 \times g$ for $10 \mathrm{~min}$. The absorbance of the supernatant was measure spect rophotometrically at a wavelength of 530-540 $\mathrm{nm}$ [46].

\subsection{Statistical Analyses}

The results are expressed as the means \pm standard deviation (SD). Significant differences were analyzed using Student's t-test, Welch's t-test or a one-way analysis of variance (ANOVA), where appropriate. Values of $p<0.05$ were considered to be statistically significant.

Supplementary Materials: The following are available online at http://www.mdpi.com/2218-1989/9/12/299/s1, Figure S1: The body weight and liver weight after two weeks of acupuncture. (A) The body weight was significantly lower in AG mice than in those of NG mice. Values are shown as the mean $\pm \mathrm{SD},{ }^{*} p<0.05,{ }^{* *} p<$ $0.001,{ }^{* * *} p<0.0001, \mathrm{n}=17$. (B) The liver weight was significantly lower in AG mice than in those of NG mice. Values are shown as the mean $\pm \mathrm{SD},{ }^{*} p<0.05,{ }^{* *} p<0.001,{ }^{* * *} p<0.0001, \mathrm{n}=17$. NG: needling-nonacupoint group, AG: needling-acupoint group, MCD diet: methionine- and choline-deficient diet, HF diet: high-fat diet, NAFLD: nonalcoholic fatty liver disease.; Figure S2: Acupuncture improved the lipid metabolism in the livers of mice with NAFLD. (A) Real time PCR revealed that the expression of ApoB, ApoE and MTTP were significantly higher in the livers of AG mice than in those of NG mice. ApoC-III showed no significant difference between the 
groups. (B)The expression of Fas, SCD1 and AdipoR2 were significantly lower in the livers of AG mice than in those of NG mice. Values are normalized by the $18 \mathrm{~S}$ rRNA expression. RT-PCR values are presented as the means $\pm \mathrm{SD} .{ }^{*} p<0.05,{ }^{* *} p<0.001$. ${ }^{* * *} p<0.0001, \mathrm{n}=7$. NG: needling-nonacupoint group, AG: needling-acupoint group, ApoB: apolipoproteinsB, ApoE: apolipoproteinsE, MTTP: microsomal triglyceride transfer protein, ApoC-III: apolipoproteins C-III, Fas: fat acid syntheas, SCD1: stearoyl-CoA 9-desaturase 1, AdipoR2: adiponectin receptor 2.

Author Contributions: X.M., X.G., and S.Y. conceptualized and designed the experiments; X.M., J.Z., R.Y., and X.G. carried out the experiments; X.M., X.G., J.Z., J.M., and J.K. analyzed the data; X.M. and X.G. wrote the manuscript; X.G., S.Y., and X.M. edited the manuscript.

Funding: This work was supported in part by Grants-in-Aid for Scientific Research 19K16783 to X.G. and 16K08750 to S.Y. from the Ministry of Education, Culture, Sports, Science and Technology, Tokyo, Japan; Grant for Promoted Research from Kanazawa Medical University (S2018-6) (to X.G.).

Acknowledgments: We would like to thank Yuka Hiramatsu and Manabu Yamashita for their expert technical assistance.

Conflicts of Interest: The authors declare no conflict of interest.

\section{References}

1. Marjot, T.; Moolla, A.; Cobbold, J.F.; Hodson, L.; Tomlinson, J.W. Non-alcoholic fatty liver disease in adults: Current concepts in etiology, outcomes and management. Endocr. Rev. 2019, bnz009. [CrossRef]

2. Bugianesi, E.; Gentilcore, E.; Manini, R.; Natale, S.; Vanni, E.; Villanova, N.; David, E.; Rizzetto, M.; Marchesini, G. A randomized controlled trial of metformin versus vitamin E or prescriptive diet in nonalcoholic fatty liver disease. Am. J. Gastroenterol. 2005, 100, 1082-1090. [CrossRef]

3. Neuschwander-Tetri, B.A.; Brunt, E.M.; Wehmeier, K.R.; Oliver, D.; Bacon, B.R. Improved nonalcoholic steatohepatitis after 48 weeks of treatment with the PPAR-gamma ligand rosiglitazone. Hepatology 2003, 38, 1008-1017. [CrossRef]

4. Abd El-Kader, S.M.; El-Den Ashmawy, E.M. Non-alcoholic fatty liver disease: The diagnosis and management. World J. Hepatol. 2015, 7, 846-858. [CrossRef]

5. Zhang, Y.; Cui, Y.; Wang, X.L.; Shang, X.; Qi, Z.G.; Xue, J.; Zhao, X.; Deng, M.; Xie, M.L. PPARalpha/gamma agonists and antagonists differently affect hepatic lipid metabolism, oxidative stress and inflammatory cytokine production in steatohepatitic rats. Cytokine 2015, 75, 127-135. [CrossRef]

6. Pham, D.D.; Bruelle, C.; Thi Do, H.; Pajanoja, C.; Jin, C.; Srinivasan, V.; Olkkonen, V.M.; Eriksson, O.; Jauhiainen, M.; Lalowski, M.; et al. Caspase-2 and p75 neurotrophin receptor (p75NTR) are involved in the regulation of SREBP and lipid genes in hepatocyte cells. Cell Death Dis. 2019, 10, 537. [CrossRef]

7. Liu, Q.; Pan, R.; Ding, L.; Zhang, F.; Hu, L.; Ding, B.; Zhu, L.; Xia, Y.; Dou, X. Rutin exhibits hepatoprotective effects in a mouse model of non-alcoholic fatty liver disease by reducing hepatic lipid levels and mitigating lipid-induced oxidative injuries. Int. Immunopharmacol. 2017, 49, 132-141. [CrossRef]

8. Zhang, B.; Li, M.; Zou, Y.; Guo, H.; Zhang, B.; Xia, C.; Zhang, H.; Yang, W.; Xu, C. NFkappaB/Orai1 Facilitates Endoplasmic Reticulum Stress by Oxidative Stress in the Pathogenesis of Non-alcoholic Fatty Liver Disease. Front. Cell Dev. Biol. 2019, 7, 202. [CrossRef]

9. Murray, P.J.; Allen, J.E.; Biswas, S.K.; Fisher, E.A.; Gilroy, D.W.; Goerdt, S.; Gordon, S.; Hamilton, J.A.; Ivashkiv, L.B.; Lawrence, T.; et al. Macrophage activation and polarization: nomenclature and experimental guidelines. Immunity 2014, 41, 14-20. [CrossRef]

10. Dong, W.; Yang, W.; Li, F.; Guo, W.; Qian, C.; Wang, F.; Li, C.; Lin, L.; Lin, R. Electroacupuncture Improves Synaptic Function in SAMP8 Mice Probably via Inhibition of the AMPK/eEF2K/eEF2 Signaling Pathway. Evid. Based Complement. Altern. Med. 2019, 8260815. [CrossRef]

11. Zhang, Y.Y.; Li, X.H.; Wu, M.X. Effect of electroacupuncture at Wnt/beta-catenin signaling pathway on inhibiting cartilage degeneration in rats with knee osteoarthritis. Zhongguo Zhen Jiu 2019, 39, 1081-1086. [PubMed]

12. Guo, Z.Q.; Huang, Y.; Jiang, H.; Wang, W.B. Randomized clinical trials of early acupuncture treatment of limb paralysis in traumatic brain injury patients and its mechanism. Zhen Ci Yan Jiu 2019, 44, 589-593. [PubMed]

13. Gao, Y.; Chen, R.; Liang, F. Mechanisms of acupuncture for non-alcoholic fatty liver disease: Researches progress and prospects. Zhongguo Zhen Jiu 2018, 38, 109-113. [PubMed]

14. Liang, F.; Koya, D. Acupuncture: Is it effective for treatment of insulin resistance? Diabetes Obes. Metab. 2010, 12, 555-569. [CrossRef] [PubMed] 
15. Wang, H.Y.; Liang, C.M.; Cui, J.W.; Pan, L.; Hu, H.; Fang, H.J. Acupuncture improves hepatic lipid metabolism by suppressing oxidative stress in obese nonalcoholic fatty liver disease rats. Zhen Ci Yan Jiu 2019, 44, 189-194. [PubMed]

16. Dela Peña, A.; Leclercq, I.; Field, J.; George, J.; Jones, B.; Farrell, G. NF-kappaB activation, rather than TNF, mediates hepatic inflammation in a murine dietary model of steatohepatitis. Gastroenterology 2005, 129, 1663-1674. [CrossRef]

17. Nawata, A.; Noguchi, H.; Mazaki, Y.; Kurahashi, T.; Izumi, H.; Wang, K.Y.; Guo, X.; Uramoto, H.; Kohno, K.; Taniguchi, H.; et al. Overexpression of Peroxiredoxin 4 Affects Intestinal Function in a Dietary Mouse Model of Nonalcoholic Fatty Liver Disease. PLoS ONE 2016, 11, e0152549. [CrossRef]

18. Li, W.; Tai, Y.; Zhou, J.; Gu, W.; Bai, Z.; Zhou, T.; Zhong, Z.; McCue, P.A.; Sang, N.; Ji, J.Y.; et al. Repression of endometrial tumor growth by targeting SREBP1 and lipogenesis. Cell Cycle 2012, 11, 2348-2358. [CrossRef]

19. Korbecki, J.; Bobinski, R.; Dutka, M. Self-regulation of the inflammatory response by peroxisome proliferator-activated receptors. Inflamm. Res. 2019, 68, 443-458. [CrossRef]

20. Yang, J.W.; Li, Q.Q.; Li, F.; Fu, Q.N.; Zeng, X.H.; Liu, C.Z. The holistic effects of acupuncture treatment. Evid. Based Complement. Alternat. Med. 2014, 2014, 739708. [CrossRef]

21. Yan, Z.K.; Yang, Z.J.; Chen, F. Effect of electroacupuncture stimulation of "Housanli" (ST 36) and "Zhongwan" (CV 12) on serum leptin and hepatocellular JAK 2-STAT 3 signaling in obese rats. Acupunct. Res. 2015, 40, 1-5.

22. Kim, S.; Zhang, X.; O’Buckley, S.C.; Cooter, M.; Park, J.J.; Nackley, A.G. Acupuncture Resolves Persistent Pain and Neuroinflammation in a Mouse Model of Chronic Overlapping Pain Conditions. J. Pain 2018, 19, 1384 e1-1384 e14. [CrossRef]

23. Li, Z.X.; Zhang, H.H.; Lan, D.C.; Chen, X.Z.; Sun, J. Progress of researches on mechanisms of acupuncture therapy for insulin resistance. Zhen Ci Yan Jiu 2019, 44, 231-234. [PubMed]

24. Fan, X.L.; Yu, M.L.; Fu, S.P.; Zhuang, Y.; Lu, S.F. Effectiveness of Acupuncture in Treatment of Simple Obesity in Animal Models: A Systematic Review and Meta-Analysis. Evid. Based Complement. Altern. Med. 2019, 2019, 5459326. [CrossRef] [PubMed]

25. Wang, L.H.; Huang, W.; Wei, D.; Ding, D.G.; Liu, Y.R.; Wang, J.J.; Zhou, Z.Y. Mechanisms of Acupuncture Therapy for Simple Obesity: An Evidence-Based Review of Clinical and Animal Studies on Simple Obesity. Evid. Based Complement. Altern. Med. 2019, 2019, 5796381. [CrossRef] [PubMed]

26. Machado, M.V.; Cortez-Pinto, H. Non-alcoholic fatty liver disease: What the clinician needs to know. World J. Gastroenterol. 2014, 20, 12956-12980. [CrossRef] [PubMed]

27. Kubes, P.; Mehal, W.Z. Sterile inflammation in the liver. Gastroenterology 2012, 143, 1158-1172. [CrossRef]

28. Zhang, J.; Huang, K.; Zhong, G.; Huang, Y.; Li, S.; Qu, S.; Zhang, J. Acupuncture Decreases NF-kappaB p65, miR-155, and miR-21 and Increases miR-146a Expression in Chronic Atrophic Gastritis Rats. Evid. Based Complement. Altern. Med. 2016, 2016, 9404629. [CrossRef]

29. Kim, S.Y.; Jin, C.Y.; Kim, C.H.; Yoo, Y.H.; Choi, S.H.; Kim, G.Y.; Yoon, H.M.; Park, H.T.; Choi, Y.H. Isorhamnetin alleviates lipopolysaccharide-induced inflammatory responses in BV2 microglia by inactivating NF-kappaB, blocking the TLR4 pathway and reducing ROS generation. Int. J. Mol. Med. 2019, 43, 682-692.

30. Anderson, N.; Borlak, J. Molecular mechanisms and therapeutic targets in steatosis and steatohepatitis. Pharmacol. Rev. 2008, 60, 311-357. [CrossRef]

31. Park, J.W.; Jeong, G.; Kim, S.J.; Kim, M.K.; Park, S.M. Predictors reflecting the pathological severity of non-alcoholic fatty liver disease: comprehensive study of clinical and immunohistochemical findings in younger Asian patients. J. Gastroenterol. Hepatol. 2007, 22, 491-497. [CrossRef] [PubMed]

32. Da Silva, M.D.; Bobinski, F.; Sato, K.L.; Kolker, S.J.; Sluka, K.A.; Santos, A.R. IL-10 cytokine released from M2 macrophages is crucial for analgesic and anti-inflammatory effects of acupuncture in a model of inflammatory muscle pain. Mol. Neurobiol. 2015, 51, 19-31. [CrossRef] [PubMed]

33. Lee, F.Y.; Huo, Z.J.; Zhang, L.; Guo, J.; Chen, H.; Liu, T.; Peng, B.; Hong, P.X.; Peng, Y.Y.; Fan, Y.F.; et al. The Effects of Needling Fenglong (ST40) and Neiguan (PC6) on IL-17 of ApoE-Gene-Knockout Mice's Liver. Evid. Based Complement. Altern. Med. 2014, 2014, 691863. [CrossRef] [PubMed]

34. Browning, J.D.; Horton, J.D. Molecular mediators of hepatic steatosis and liver injury. J. Clin. Investig. 2004, 114, 147-152. [CrossRef] [PubMed]

35. Koo, S.H. Nonalcoholic fatty liver disease: Molecular mechanisms for the hepatic steatosis. Clin. Mol. Hepatol. 2013, 19, 210-215. [CrossRef] 
36. Yamaguchi, K.; Yang, L.; McCall, S.; Huang, J.; Yu, X.X.; Pandey, S.K.; Bhanot, S.; Monia, B.P.; Li, Y.X.; Diehl, A.M. Inhibiting triglyceride synthesis improves hepatic steatosis but exacerbates liver damage and fibrosis in obese mice with nonalcoholic steatohepatitis. Hepatology 2007, 45, 1366-1374. [CrossRef]

37. Caballero, F.; Fernandez, A.; De Lacy, A.M.; Fernandez-Checa, J.C.; Caballeria, J.; Garcia-Ruiz, C. Enhanced free cholesterol, SREBP-2 and StAR expression in human NASH. J. Hepatol. 2009, 50, 789-796. [CrossRef]

38. Donnelly, K.L.; Smith, C.I.; Schwarzenberg, S.J.; Jessurun, J.; Boldt, M.D.; Parks, E.J. Sources of fatty acids stored in liver and secreted via lipoproteins in patients with nonalcoholic fatty liver disease. J. Clin. Investig. 2005, 115, 1343-1351. [CrossRef]

39. Bell, M.; Wang, H.; Chen, H.; McLenithan, J.C.; Gong, D.W.; Yang, R.Z.; Yu, D.; Fried, S.K.; Quon, M.J.; Londos, C.; et al. Consequences of lipid droplet coat protein downregulation in liver cells: Abnormal lipid droplet metabolism and induction of insulin resistance. Diabetes 2008, 57, 2037-2045. [CrossRef]

40. Basaranoglu, M.; Kayacetin, S.; Yilmaz, N.; Kayacetin, E.; Tarcin, O.; Sonsuz, A. Understanding mechanisms of the pathogenesis of nonalcoholic fatty liver disease. World J. Gastroenterol. 2010, 16, 2223-2226. [CrossRef]

41. Tessari, P.; Coracina, A.; Cosma, A.; Tiengo, A. Hepatic lipid metabolism and non-alcoholic fatty liver disease. Nutr. Metab. Cardiovasc. Dis. 2009, 19, 291-302. [CrossRef] [PubMed]

42. Tan, R.; He, Y.; Zhang, S.; Pu, D.; Wu, J. Effect of transcutaneous electrical acupoint stimulation on protecting against radiotherapy- induced ovarian damage in mice. J. Ovarian Res. 2019, 12, 65. [CrossRef] [PubMed]

43. Li, T.; Xu, X.; Chen, B.; Rong, J.; Jiang, H. Photoacoustic imaging of acupuncture effect in small animals. Biomed. Opt. Express 2015, 6, 433-442. [CrossRef] [PubMed]

44. Zhang, J.; Guo, X.; Hamada, T.; Yokoyama, S.; Nakamura, Y.; Zheng, J.; Kurose, N.; Ishigaki, Y.A.-O.; Uramoto, H.; Tanimoto, A.; et al. Protective Effects of Peroxiredoxin 4 (PRDX4) on Cholestatic Liver Injury. Int. J. Mol. Sci. 2018, 19, 2509. [CrossRef] [PubMed]

45. Nabeshima, A.; Yamada, S.; Guo, X.; Tanimoto, A.; Wang, K.Y.; Shimajiri, S.; Kimura, S.; Tasaki, T.; Noguchi, H.; Kitada, S.; et al. Peroxiredoxin 4 protects against nonalcoholic steatohepatitis and type 2 diabetes in a nongenetic mouse model. Antioxid Redox Signal 2013, 19, 1983-1998. [CrossRef] [PubMed]

46. Guo, X.; Noguchi, H.; Ishii, N.; Homma, T.; Hamada, T.; Hiraki, T.; Zhang, J.; Matsuo, K.; Yokoyama, S.; Ishibashi, H.; et al. The Association of Peroxiredoxin 4 with the Initiation and Progression of Hepatocellular Carcinoma. Antioxid. Redox Signal. 2019, 30, 1271-1284. [CrossRef] 



\title{
Metabolomic and Lipidomic Biomarkers for Premalignant Liver Disease Diagnosis and Therapy
}

\author{
Diren Beyoğlu and Jeffrey R. Idle * \\ Arthur G. Zupko's Division of Systems Pharmacology and Pharmacogenomics, Arnold \& Marie Schwartz \\ College of Pharmacy and Health Sciences, Long Island University, 75 Dekalb Avenue, Brooklyn, NY 11201, USA; \\ diren.beyoglu@liu.edu \\ * Correspondence: jeff.idle@liu.edu
}

Received: 13 January 2020; Accepted: 26 January 2020; Published: 28 January 2020

\begin{abstract}
In recent years, there has been a plethora of attempts to discover biomarkers that are more reliable than $\alpha$-fetoprotein for the early prediction and prognosis of hepatocellular carcinoma (HCC). Efforts have involved such fields as genomics, transcriptomics, epigenetics, microRNA, exosomes, proteomics, glycoproteomics, and metabolomics. HCC arises against a background of inflammation, steatosis, and cirrhosis, due mainly to hepatic insults caused by alcohol abuse, hepatitis $\mathrm{B}$ and $\mathrm{C}$ virus infection, adiposity, and diabetes. Metabolomics offers an opportunity, without recourse to liver biopsy, to discover biomarkers for premalignant liver disease, thereby alerting the potential of impending HCC. We have reviewed metabolomic studies in alcoholic liver disease (ALD), cholestasis, fibrosis, cirrhosis, nonalcoholic fatty liver (NAFL), and nonalcoholic steatohepatitis (NASH). Specificity was our major criterion in proposing clinical evaluation of indole-3-lactic acid, phenyllactic acid, $N$-lauroylglycine, decatrienoate, $N$-acetyltaurine for ALD, urinary sulfated bile acids for cholestasis, cervonoyl ethanolamide for fibrosis, $16 \alpha$-hydroxyestrone for cirrhosis, and the pattern of acyl carnitines for NAFL and NASH. These examples derive from a large body of published metabolomic observations in various liver diseases in adults, adolescents, and children, together with animal models. Many other options have been tabulated. Metabolomic biomarkers for premalignant liver disease may help reduce the incidence of HCC.
\end{abstract}

Keywords: metabolomics; lipidomics; biomarker; premalignant; alcoholic liver disease; cholestasis; fibrosis; cirrhosis; NAFL; NASH

\section{The Need for Biomarkers of Premalignant Liver Disease}

Hepatocellular carcinoma (HCC) and intrahepatic cholangiocarcinoma (ICC) are the commonest types of primary liver cancer, and their combined incidence ranks among the highest cancer rates in the world [1]. HCC in particular is a major health problem, with an annual death rate in excess of 500,000 worldwide [2]. HCC in the United States, which comprises $75 \%$ of all primary liver cancers [3], has been attributed primarily to a number of infectious and lifestyle causes. The principal attributable factors among these are alcohol (32.0\% in males, $30.7 \%$ in females), adiposity ( $26.6 \%$ in males, $15.6 \%$ in females), hepatitis C virus (HCV) infection (17.5\% overall), smoking $(9.0 \%$ in males, $8.0 \%$ in females), diabetes (6.9\% in males, $5.5 \%$ in females), and hepatitis B virus (HBV) infection (5.3\% overall). In contrast, in China, HBV (53.8\% overall) is the principal cause, with adiposity a relatively minor contributor ( $7.2 \%$ in males, $4.2 \%$ in females) [4]. These causative factors produce insults to the liver that include inflammation, steatosis, and fibrosis, all of which can progress through various stages, in particular cirrhosis, that can eventually lead to HCC. In recent years, there have been multiple attempts to develop predictive biomarkers of HCC, but many of these have involved the study of HCC cases themselves. Understanding the progression from hepatic insult through premalignant stages to HCC would seem to be the most fruitful means of predicting the development 
of HCC in susceptible individuals. In this review, we examine the investigations into key premalignant stages of HCC and ICC that have employed metabolomics both in patients and in animal models. In particular, we have focused on the metabolomics of alcoholic liver disease (ALD), cholestasis, fibrosis, cirrhosis, nonalcoholic fatty liver (steatosis, NAFL), and nonalcoholic steatohepatitis (NASH). In each case, we evaluated whether the experimental data provide sufficient grounds, especially in terms of specificity, to warrant further development of clinical biomarkers of hepatic premalignancy. Additionally, we considered only metabolites that were upregulated as potential biomarkers for the aforementioned premalignant liver diseases. The references cited in this review were culled from PubMed searches with keywords metabolomics OR metabonomics AND the various disease entities, such as alcoholic liver disease. Some references also arose from the bibliographies cited by publications found in these initial searches.

\section{Hepatic Metabolism}

The human body comprises around 34 trillion cells of which ca. 240 billion (0.7\%) make up the approximately $1.5 \mathrm{~kg}$ of healthy liver, the largest solid organ and the biggest gland in the body. Of the roughly 20,000 human protein-coding genes, $60 \%$ are transcribed in the liver, many of which are not expressed in any other tissue [5]. Studies in mice using single cell transcriptomics revealed that about half of all hepatocyte genes were expressed in a zonal manner, supporting the concept that different liver regions have diverse metabolic functions. This was interpreted as being due to variable microenvironments attributable to gradients of oxygen, nutrients, and hormones [6]. Metabolic reactions that are specific to the liver include de novo synthesis and secretion of the primary bile acids glycocholate, taurocholate, glycochenodeoxycholate, and taurochenodeoxycholate, together with ornithine degradation. Overall, the liver is the most metabolically-active tissue, followed by adipose tissue and skeletal muscle [5]. Parenchymal hepatocytes comprise up to $85 \%$ of the liver volume, with sinusoidal endothelial cells, perisinusoidal stellate cells and phagocytic Kupffer cells, with intrahepatic lymphocytes making up the rest. Strong evidence suggests that different hepatic cell types possess variable gene expression profiles [6-8]. The liver is therefore highly heterogeneous in both gene expression and metabolic function. Assignment of metabolic function to discrete hepatic regions based upon in vivo observations alone is extremely challenging, since metabolic phenotypes vary between cell types and also across the liver. The role of in vitro studies in this regard will be increasingly important as aids to the interpretation of in vivo metabolic phenotyping. For example, laser capture microdissection has been employed as an adjunct to genomic, transcriptomic, and proteomic analyses of liver diseases [9], but so far, rarely for metabolic profiling of liver tissue.

\section{Metabolomics-The What, the How, and the Why}

It is two decades since Jeremy Nicholson and colleagues introduced the concept of metabonomics, with the promise of biomarker discovery from changes in metabolite profiles that result from constitutional differences such as disease or genetics or from exogenous challenges due to drug administration or exposure to toxicants [10]. The initial protocols based upon high-resolution proton nuclear magnetic resonance spectroscopy $\left({ }^{1} \mathrm{H}\right.$ NMR) of body fluids have been supplemented by an array of additional technologies, based mostly on mass spectrometry (MS), which have infiltrated virtually every branch of biology and medicine. The literature currently stands at virtually 30,000 PubMed citations with almost 6000 in 2019 alone. The identification and quantitation of all metabolites in a given organism or biofluid was at first seen as a realistic goal [11]. However, as the biochemical complexity and analytical shortcomings came more into focus, global metabolite quantitation was abandoned, and more realistic definitions emerged, such as, "metabolomics studies the low molecular weight metabolites [e.g., <1.5 kDa] found in cells and organisms, usually through the analysis of plasma/serum, urine or cell culture medium using mainly MS or NMR technologies" [12]. There has also been some confusion regarding the use of the terms "metabolomics" and "metabonomics." Although it has been stated that the difference in terms is not a technical one, and that the terminologies are 
often used interchangeably [13], almost without exception, metabonomics published reports were conducted using NMR rather than MS. Other commonly-used phrases include untargeted and targeted metabolic phenotyping. Untargeted metabolomics is commonly conducted by first separating the biological analytes that have a large range of physicochemical properties using ultraperformance liquid chromatography (UPLC) with either reversed phase (RP) and/or hydrophobic interaction chromatography (HILIC) columns [14]. Interfaced by electrospray ionization (ESI) in either positive (ESI+) and/or negative (ESI-) mode, the UPLC eluate is analyzed by quadrupole time-of-flight mass spectrometry (QTOFMS). This may yield in excess of 5000 ions in each ionization mode, which should not be interpreted as 5000 biological constituents, as many of these features correspond to adducts, dimers, multiply charged species, and fragment ions formed in the electrosprayer. In targeted metabolomics, specific metabolites, for example amino acids or acyl carnitines, are quantitated using stable isotope labeled standards [15]. This is frequently conducted using tandem mass spectrometry, often with a triple quadrupole mass spectrometer (TQMS), rather than a QTOFMS. Another common technology used in metabolomics is gas chromatography-mass spectrometry (GC-MS). This has the benefit of a high confidence in metabolite identification, albeit for a small number of metabolites and a lower throughput than UPLC-QTOFMS. The technologies available for metabolomic analysis have recently been reviewed in detail [16].

In a typical metabolomics experiment, two or more groups of samples are investigated. These could be biofluids from a patient group and age- and sex-matched healthy controls, genetically-modified mice and their wild-type (WT) controls, and persons or experimental animals that have been administered a drug, specific diet, or with some other lifestyle variable (e.g. smoking or particular occupation), compared with a suitable control group. Analysis of the biofluids, usually urine and/or serum/plasma, by MS- or NMR-based methods produces a data table that must first be preprocessed (normalization, scaling, peak picking) prior to multivariate data analysis (MDA). It is first prudent to conduct unsupervised MDA, for example, with principal components analysis (PCA), which reveals the internal structure of the dataset, the principal components of variance, and the existence of any outliers. A number of presentations of the data are common, including the scores plot (with one data point for each sample) and the loadings plot, which for MS methods show the ions responsible for the distribution of samples in the scores plot. If each sample group analyzed clusters and separates from the other group(s), then this leads to supervised analyses such as partial least squares-discriminant analysis (PLS-DA) and orthogonal PLS-DA (OPLS-DA). Unless at least a partial separation of scores was observed in the PCA analysis, there is a danger that the data could be overmodeled using these supervised analyses. The literature is replete with examples of this. The generated loadings plots can be used with various software packages that assist in the identification of metabolites that differ significantly between the test groups. The reader is directed to specific reviews in this area [17-19].

Various estimates of distinct human metabolomes have been reported that were derived using multiple analytical platforms to gain maximum metabolite coverage. The human cerebrospinal fluid metabolome (308 metabolites) [20], the human serum metabolome (4229 "highly probable" metabolites) [21], the human urine metabolome (2651 "confirmed" metabolites) [22], and the human fecal metabolome (>6000 identified metabolites) [23] have all been described. The culmination of these efforts is the human metabolome database (HMDB 4.0) that comprises 114,100 total metabolites that encompass "the complete collection of small molecules found in the human body including peptides, lipids, amino acids, nucleic acids, carbohydrates, organic acids, biogenic amines, vitamins, minerals, food additives, drugs, cosmetics, contaminants, pollutants, and just about any other chemical that humans ingest, metabolize, catabolize or come into contact with" [24]. This still may be the tip of the iceberg. It has been estimated that humans are probably exposed to some 1-3 million discrete chemicals in their lifetimes [11] of which $>25,000$ have already been described in the diet [25].

The lipidome refers to the total number of lipid species present in a cell, tissue, organ, organism, or biofluid such as plasma. Although there is overlap with the human metabolome, the human lipidome is expected to be highly complex due in great part to the varying chain lengths and degrees 
of unsaturation, together with structural isomerism. As of January 2018, there were more than 40,000 lipid structures listed in the LIPID MAPS database [26]. Our conservative estimate is that the human lipidome is made up of at least 100,000 discrete lipid entities.

Based on the foregoing evidence, it is likely that a human metabolome that includes the lipidome may have some 200,000 members. As stated above, there are thought to be $\sim 20,000$ protein coding human genes, although the exact number is yet to be determined. The total number of cellular proteins (proteome) may be 16,000-17,000, similar to the total number of mRNA transcripts (transcriptome) obtained by untargeted RNA sequencing (RNA-seq) [27]. In addition, the existence of a human core proteome of 10,000-12,000 ubiquitously expressed proteins has been postulated, whose primary function is the general control and maintenance of cells [28]. Many of these are enzymes, and therefore contribute to the human metabolome, either through the metabolism of a single specific metabolite or pair of metabolites, such as lactate dehydrogenase or in a pleiotropic fashion, such as the thousands of potential metabolites produced by the human cytochromes P450 [29]. Nevertheless, it has been estimated that there are 1-2 million "protein entities" that are expressed in a cell at a given time as a result of posttranslational modifications (PTMs), such as acetylation, phosphorylation, and glycosylation [30]. To study mechanisms of liver disease through the lens of untargeted proteomics would be an extremely demanding task. However, targeted proteomics in the form of specific protein biomarkers in plasma or serum has a long history. This is because of the availability of commercial antibodies against virtually every protein and this forms the basis of convenient quantitative immunoassays such as ELISA.

The expression of phenotypes, including metabolic phenotypes, from a genomic sequence that is transcribed, spliced, and translated to protein with potential post-translational modifications, is analogous to the information flow involved when listening to a music compact disk or some other digital music format. In the former case, the genome is analogous to the compact disk itself, which without the apparatus for converting it to sound, is simply a digital storage system (Figure 1). This is why the metabolic phenotype is more revealing of the status of a cell, tissue, or organism than a genetic sequence, because it more resembles the musical experience rather than analyzing the so-called pits and lands (Figure 1) on a CD.

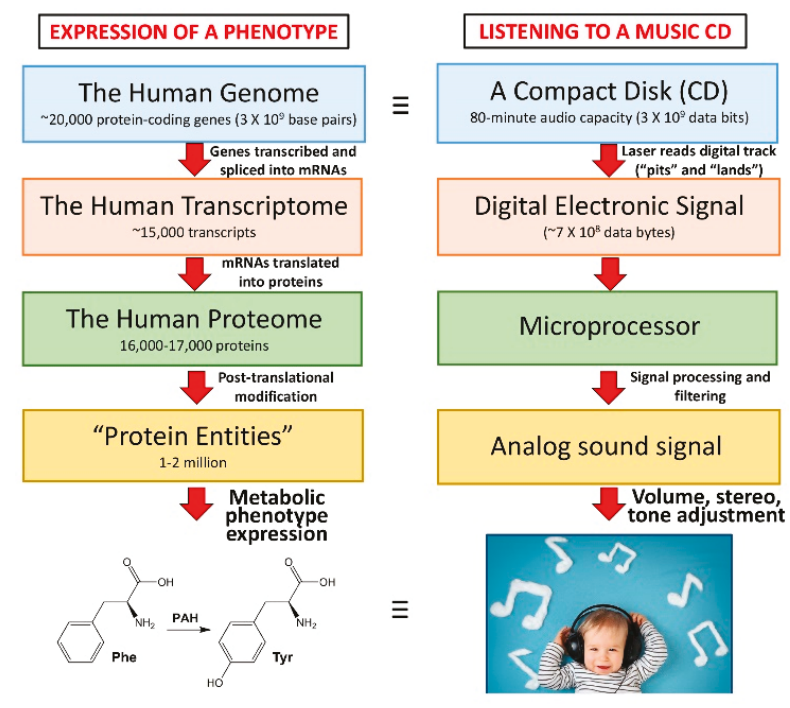

Figure 1. The flow of genetic information vs. digital music data flow.

In terms of generating new knowledge regarding the liver, metabolomics has for some years offered this opportunity. Because the liver is the seat of much of the body's metabolic processes, 
the systemic measurement of metabolites that originate in the liver should provide clear signposts to liver wellbeing or disease. This alone justifies the inclusion of metabolomic protocols in the study of hepatic pathogenesis. As we will demonstrate below, a plethora of such studies has already been reported, but the picture is still not in focus. We will seek to highlight the potential biomarkers that can be determined through metabolomics and that point most directly to disease mechanisms. We will discuss below the shortcomings of the current trend of identifying metabolomic biomarkers as risk factors for liver disease.

\section{Biomarkers-The Good, the Bad, and the Ugly}

A biomarker has been characterized as "a defined characteristic that is measured as an indicator of normal biological processes, pathogenic processes, or responses to an exposure or intervention, including therapeutic interventions." It has also been emphasized that biomarkers can be medical measurements, including physiological measurements, blood tests, molecular analyses of biopsies, genetic or metabolic data, and measurements from images [31]. Blood pressure and blood glucose are commonly determined biomarkers of both pathogenic processes and therapeutic interventions. Neither of these biomarkers point to mechanisms of either disease or therapeutic response. Measurement of the pressure of various parts of the arterial circulation was initiated in the mid-18th century by the English clergyman Stephen Hales [32]. The testing of the color, smell, and taste of urine as indicators of disease goes back at least as far as the ancient Greeks, with diagnostic 'urine charts' dating from the Middle Ages [13]. Determination of blood glucose developed relatively recently and as a substitute to urine taste as a diagnostic biomarker for diabetes [33].

The first metabolic biomarkers that indicated disease mechanisms are contained in the remarkable work of Sir Archibald Garrod (1857-1936) who coined the phrases "inborn errors of metabolism" [34] and "chemical individuality" [35] in the early part of the 20th century. Garrod contended that four diseases, i.e., alkaptonuria, albinism, cystinuria, and pentosuria, were Mendelian autosomal recessive traits, therefore pointing to genetic mechanisms for each. Moreover, he recognized that increased urinary homogentisic acid (HGA; known then as "alkapton acid") in newborn babies with alkaptonuria that stained their diapers black could be further increased by the oral administration of tyrosine or a diet rich in proteins containing aromatic amino acids such as tyrosine and phenylalanine [36]. This led Garrod to propose an impairment in the aromatic ring opening of aromatic amino acids as the mechanism of alkaptonuria. This flew in the face of the contemporaneous "germ theory of disease" that focused on external rather than inborn causes of disease, and maintained that alkaptonuria resulted from a gastrointestinal infection. These ideas hindered the acceptance of Garrod's concepts for many years [37]. Today, we recognize that Garrod's interpretation was correct, and also that mutations in the HGO gene causing a deficiency in hepatic homogentisate 1,2-dioxygenase (EC 1.13.11.5) activity result in an accumulation of HGA and its clinical sequelae such as ochronosis, the yellowish staining of connective tissue by HGA [38]. The major impact of a metabolic biomarker of disease (HGA) is that the mechanism when unmasked can lead to potential therapies of the disease. In the case of alkaptonuria, nitisinone has been shown in several studies to reduce the circulating levels of HGA. Nitisinone is an inhibitor of 4-hydroxyphenylpyruvate dioxygenase (EC 1.13.11.27), the enzyme responsible for the formation of HGA. A daily dose of $2 \mathrm{mg}$ slowed progression of alkaptonuria and arrested ocular and ear ochronosis [39]. This old example of alkaptonuria is a clear-cut prototype for a metabolic biomarker of disease that originates in the liver, which has led to both an understanding of the disease mechanism and its potential treatment. Sadly, many recent examples of liver disease metabolic biomarkers have not lived up to this paradigm.

Alpha-fetoprotein (AFP) was reported in 1956 to be in human fetal serum but not in the serum of healthy adults. The production of AFP by fetal liver largely ceases before birth [40]. The discovery a few years later of AFP in animal models with hepatocellular carcinoma (HCC) [41] led to clinical investigations that associated AFP with HCC. It has been stated that 70\% HCC secrete AFP [42] and up to $40 \%$ of HCC patients may not show elevated serum AFP [43]. This suboptimal sensitivity is 
coupled with specificity issues in relation to premalignant liver diseases such as hepatitis and cirrhosis, together with ovarian and testicular malignancies. Therefore, the clinical interpretation of serum AFP with respect to HCC requires care. Nevertheless, serum AFP is widely used as both a diagnostic and prognostic biomarker for HCC [42-44]. In these regards, it is recognized that it should be replaced with more specific and sensitive biomarkers [43,44]. Neither European nor American guidelines for HCC screening include serum AFP concentration [45].

Although osteopontin (OPN), a protein normally expressed in kidney and bone, has a high sensitivity for the detection of HCC, its elevation can be linked to more than 30 types of cancer [45] and to many other diseases, including diseases of the liver [46]. Its employment as a HCC risk biomarker is clearly inappropriate. We will examine below whether metabolomics can disclose liver disease biomarkers with high sensitivity and especially with high specificity.

Of the biomarkers for liver disease discussed above, the determination of metabolite HGA to diagnose the rare inborn error of metabolism alkaptonuria is by far the most sensitive and specific. Studies in experimental animals in the 1950s suggested that homogentisate 1,2-dioxygenase was expressed in liver, to a lesser extent in kidney, and with little enzyme activity reported for heart, skeletal muscle, brain, intestine, spleen, and blood [47]. Contemporary biochemical and molecular methodologies have recently revealed that homogentisate 1,2-dioxygenase is expressed in human and mouse brain, explaining the various observations of brain pigmentation found in cases of alkaptonuria [48]. Both AFP and OPN are compromised by insufficient specificity, which would require them to be used in combination with other biomarkers for liver disease risk.

\section{Biomarkers of Premalignant Liver Disease}

\subsection{Alcoholic Liver Disease (ALD)}

Excessive alcohol consumption is a global healthcare problem that accounts for almost $1 \%$ of all global deaths and $50 \%$ of all liver cirrhosis-attributable deaths [49]. The spectrum of hepatic lesions includes steatosis, alcoholic steatohepatitis (ASH), alcoholic hepatitis, fibrosis, cirrhosis, and HCC. Alcohol is a principal cause of end-stage liver disease, for which the only curative treatment is transplantation [50]. The insult on the liver by alcohol is closely related to the fact that the liver is the site of most of the metabolism of alcohol. Alcohol dehydrogenase (ADH; EC 1.1.1.1) converts ethanol to acetaldehyde with the generation of NADH reducing equivalents. Subsequent metabolism by acetaldehyde dehydrogenase (ALDH; EC 1.2.1.3) generates further equivalents of NADH. The elevated ratio of $\mathrm{NADH} / \mathrm{NAD}^{+}$due to excess alcohol consumption is responsible for many of the biochemical consequences in the liver. For example, lactic acidosis, hyperuricemia, enhanced lipogenesis, and depressed fatty acid $\beta$-oxidation have long been known to be driven by excess hepatic NADH [51]. However, the influence of ethanol exposure on lipid metabolism is considerably more complicated than redox inhibition of fatty acid $\beta$-oxidation [52].

Much of the understanding of the mechanisms of liver disease have been generated using animal models. In pioneering studies, rats fed a 5\% ethanol diet (36\% total calories) had a plasma glycerolipid profile that mirrored the serum ethanol profile. Relative to paired rats fed a sucrose diet, the ethanol-fed rats displayed a 3-fold increase in total hepatic lipids and an 8-fold greater hepatic triglyceride content [53]. This early work led to the establishment of the Lieber-Decarli experimental alcohol diet [54], which is still widely employed [55]. Binge ethanol administration to mice ( $5 \mathrm{~g} / \mathrm{kg}$ in three divided doses over $36 \mathrm{~h}$ ) has also been used [56], in which case, hepatic $S$-adenosylmethionine (SAM), cysteine, and glutathione were decreased, while hypotaurine and taurine levels were elevated. These findings were interpreted as being due to both oxidative injury and a rapid elevation in cysteine dioxygenase (EC 1.13.11.20) activity, responsible for the production of hypotaurine and taurine. These markers could be attenuated by the co-administration of betaine, thought to be due to the regulation by betaine of hepatic levels of SAM and GSH [56]. Changes in hepatic lipid profiles occurred after chronic feeding of Yucatan micropigs $(20-40 \mathrm{~kg})$ with a $40 \%$ ethanol 
folate-deficient diet. In alcoholic pigs, hepatic triglycerides were elevated with increased desaturation of fatty acids (16:0 to 16:1n7 and 18:0 to 18:1n9) by stearoyl-CoA desaturase (SCD; EC 1.14.19.1) and decreased fatty acid elongation pathway (ELOVL5; EC 2.3.1.199) and phosphatidylethanolamine $N$-methyltransferase (PEMT; EC 2.1.1.17) activity. This latter enzyme attenuation led to a shift from phosphatidylethanolamines to phosphatidylcholines in the liver [57].

The above studies of the effect of alcohol administration were highly targeted, and therefore, limited in their description of the hepatic metabolic phenotype induced by alcohol. They were also limited by the vastly different protocols of ethanol administration. A study in mice was conducted using the Lieber-Decarli diet treated wild-type (WT) and Ppara-null mice (PPAR $\alpha$ is a nuclear receptor that regulates much of lipid metabolism including fatty acid $\beta$-oxidation) [58]. Six months' chronic alcohol exposure led to increased hepatic triglyceride accumulation in the Ppara-null mice. Urines collected from 2 to 6 months were analyzed using an untargeted metabolomic protocol by UPLC-ESI-QTOFMS, and showed differential elevated metabolite profiles for the WT and null mice. In WT mice, the principal elevated urinary metabolites resulting from alcohol administration were ethyl sulfate and ethyl- $\beta$-D-glucuronide, secondary metabolites of ethanol, together with 4-hydroxyphenylacetic acid and its sulfate conjugate. These were also found for the null mice and, in addition, elevated urinary excretion of indole-3-lactic acid was found only in the Ppara-null mice, which was mechanistically related to the administration of ethanol in these animals. In a subsequent and more detailed investigation [59] that used WT and Ppara-null mice with two different strain backgrounds, indole-3-lactic acid and phenyllactic acid were reported as ALD biomarkers, with their formation arising from their corresponding pyruvic acids having been driven by the NADH hepatic overload due to ethanol consumption (Figure 2). The mechanism-based biomarkers also shed light on the development of steatosis, driven by the deficit in $\mathrm{NAD}^{+}$and the hepatic increase in NADH. The redox inhibition of fatty acid $\beta$-oxidation is an initial step of triglyceride and lipid droplet accumulation in the liver [52]. Metabolomic investigations in rats fed the Lieber-DeCarli liquid diet for 2 and 3 months have been conducted using high-field ${ }^{1} \mathrm{H}$ and ${ }^{31} \mathrm{P}$ NMR. These studies reported a two-fold increase in plasma triglycerides and a halving of plasma free fatty acids, mirroring smaller but statistically significant changes in the liver. Both total and free cholesterol were increased two-fold in the liver [60]. Metabolomics has identified specific lipids in serum that were associated with alcohol-induced liver diseases, specifically, $N$-lauroylglycine identified cirrhosis with $100 \%$ sensitivity and $90 \%$ specificity, while decatrienoic acid could evaluate liver disease severity with $100 \%$ sensitivity and specificity [61].

$\mathrm{N}$-Acetyltaurine (NAT) has been reported to be a biomarker of alcohol exposure in mice, arising from metabolism of ethanol to acetaldehyde via ADH and CYP2E1 (EC 1.14.13.n7), and further by ALDH to acetate [62]. NAT is not specific to alcohol exposure, since it has been described as a biomarker of gamma-irradiation in both rats [63] and rhesus monkeys [64]. NAT urinary excretion has been reported in healthy human subjects who drank alcohol (0.66 to $0.84 \mathrm{~g} / \mathrm{kg}$ ) [65]. In blood, NAT concentration as a biomarker of alcohol exposure was of limited value [66]. To date, NAT has not been evaluated with respect to liver disease. 


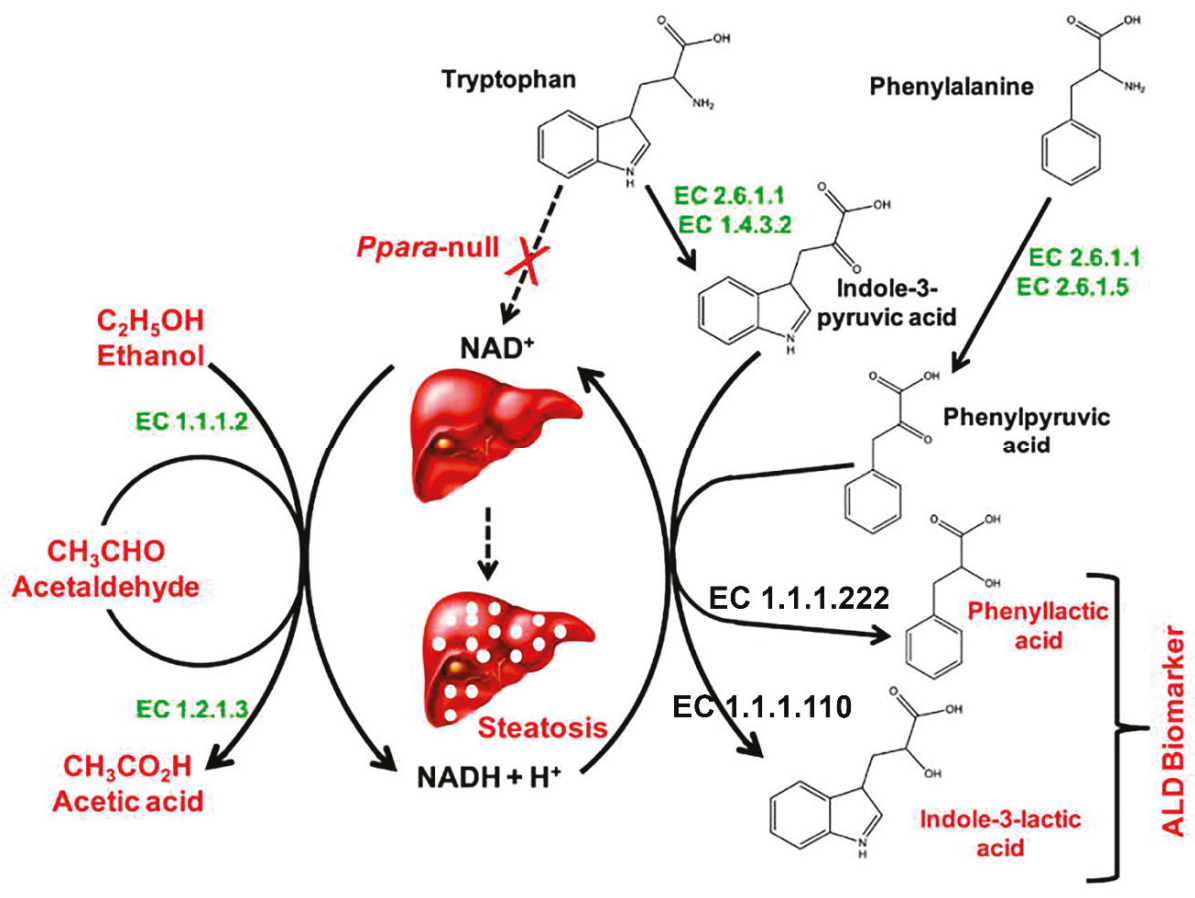

Figure 2. Generation of mechanism-based biomarkers of ALD (adapted from Manna et al., 2011 [59] with permission).

Chronic alcohol exposure in both experimental animals and humans leads to functional perturbations in the intestinal microbiota as determined by metabolomic investigations of intestinal metabolites. A wide range of altered intestinal microbiota metabolites has been reported, including decreased amino acids, changes in steroid, lipid, carnitine, and bile acid metabolism. Short-chain fatty acids (SCFAs) that are produced by bacterial fermentation were lowered by alcohol administration to rats, with the exception of acetate, which is an end-product of ethanol metabolism $[67,68]$. Additionally, saturated long-chain fatty acid (LCFA) biosynthesis by the microbiota is reduced by ethanol administration. These attenuated LCFA metabolites have been shown to contribute to alcohol-associated dysbiosis, influencing ALD [69]. Microbial metabolites combined with reduced levels of Lactobacillus trigger intestinal inflammation and liver disease following alcohol administration highlighting the role of gut microbiome-liver cross talk in ALD [49]. Studies that identified metabolomic and lipidomic biomarkers of alcoholic liver disease are listed in Table 1. 
Table 1. Metabolomic and lipidomic biomarkers of alcoholic liver disease.

\begin{tabular}{|c|c|c|c|c|}
\hline Species & Alcohol Dose & Pathology & Metabolites Reported & Ref. \\
\hline Rat & $\begin{array}{l}20 \% \text { or } 36 \% \text { of total } \\
\text { calories; } 24 \text { days }\end{array}$ & $\begin{array}{l}\text { Hepatomegaly } \\
\text { Fatty infiltration }\end{array}$ & $\begin{array}{c}\text { Plasma triglycerides } \uparrow \\
\text { Plasma phospholipids } \uparrow \\
\text { Hepatic triglycerides } \uparrow \text { 8-fold }\end{array}$ & [53] \\
\hline Rat & $\begin{array}{l}5 \% \text { alcohol } \\
\text { Lieber-DeCarli diet; } \\
\text { 2-3 months }\end{array}$ & $\begin{array}{l}\text { Fatty infiltration } \\
\text { Mild inflammatory } \\
\text { infiltrate; } 3 \text { months } \\
\text { Mild oxidative } \\
\text { stress, } 3 \text { months }\end{array}$ & $\begin{array}{c}\text { Liver triglycerides } \uparrow \\
\text { Liver cholesterol } \uparrow \\
\text { Liver phospholipids and } \\
\text { lysophospholipids } \downarrow\end{array}$ & [60] \\
\hline Rat & $\begin{array}{c}6 \mathrm{~g} / \mathrm{kg} \text { alcohol }+ \\
\text { high-fat diet }\end{array}$ & $\begin{array}{l}\text { Regional laminar } \\
\text { necrosis and edema } \\
\text { around central vein. } \\
\text { Inflammatory } \\
\text { cell infiltrate. }\end{array}$ & $\begin{array}{l}\text { Total of } 37 \text { core ALD } \\
\text { biomarkers identified. } \\
\text { Pathways perturbed } \\
\text { included TCA cycle, } \\
\text { carbohydrate and amino } \\
\text { acid metabolism. }\end{array}$ & [70] \\
\hline Mouse & $5 \mathrm{~g} / \mathrm{kg}$ every $12 \mathrm{~h}$ X 3 & $\begin{array}{c}\text { Serum ALT } \uparrow \\
\text { Hepatic CYP2E1 } \uparrow\end{array}$ & $\begin{array}{c}\text { Malondialdehyde } \uparrow \\
\text { Methionine } \uparrow \\
\text { Hypotaurine } \uparrow \text { Taurine } \uparrow \\
\text { SAM } \downarrow \text { GSH } \downarrow\end{array}$ & [56] \\
\hline $\begin{array}{c}\text { Mouse } \\
129 \text { Sv WT and } \\
\text { Ppara-null }\end{array}$ & $\begin{array}{l}4 \% \text { alcohol } \\
\text { Lieber-DeCarli diet; } \\
2-6 \text { months }\end{array}$ & $\begin{array}{l}\text { Little change after } \\
1 \text { month }\end{array}$ & $\begin{array}{c}\text { Ethylsulfate } \uparrow \\
\text { Ethyl- } \beta \text {-D-glucuronide } \uparrow \\
\text { 4-hydroxyphenylacetic acid } \\
\text { (4HPAA) } \uparrow \text { 4HPAA sulfate } \uparrow \\
\text { in both WT and null. } \\
\text { Indole-3-lactic acid } \uparrow \text { in } \\
\text { null only. }\end{array}$ & [58] \\
\hline $\begin{array}{c}\text { Mouse } \\
129 \text { Sv and } \\
\text { C57BL/6 WT } \\
\text { and Ppara-null }\end{array}$ & $\begin{array}{l}4 \% \text { alcohol } \\
\text { Lieber-DeCarli diet; } \\
1 \text { month }\end{array}$ & $\begin{array}{l}\text { Steatosis in B6 } \\
\text { null mice }\end{array}$ & $\begin{array}{l}\text { Indole-3-lactic acid } \uparrow \text { and } \\
\text { phenyllactic acid } \uparrow \text { in } \\
\text { alcohol-treated Ppara-null } \\
\text { mouse, both } 129 \text { Sv } \\
\text { and C57BL/6 }\end{array}$ & [59] \\
\hline $\begin{array}{c}\text { Mouse } \\
\text { WT and } \\
\text { Cyp2e1-null }\end{array}$ & $\begin{array}{c}2.2 \%, 4.5 \%, 5.4 \% \\
\text { Lieber-DeCarli } \\
\text { semi-solid diet; } \\
21 \text { days }\end{array}$ & $\begin{array}{l}\text { CYP2E1 } \uparrow \text { in WT } \\
\text { Microvesicular and } \\
\text { macrovesicular } \\
\text { steatosis around } \\
\text { central vein; } \\
\text { WT>null }\end{array}$ & $\begin{array}{l}\text { Hepatic and serum } \\
\text { triglycerides } \uparrow \text { in WT only. } \\
\text { Urinary } N \text {-acetyltaurine, } \\
\text { 4HPAA sulfate, ethylsulfate, } \\
\text { ethyl- } \beta \text {-D-glucuronide } \uparrow\end{array}$ & [62] \\
\hline Mouse & $4.896 \mathrm{~g} / \mathrm{kg} ; 7$ days & $\begin{array}{l}\text { ALT } \uparrow \text { AST } \uparrow \\
\text { Focal hepatic } \\
\text { necrosis } \\
\text { Inflammatory } \\
\text { infiltrate }\end{array}$ & $\begin{array}{c}\text { Serum Malondialdehyde } \uparrow \\
\text { GSH } \downarrow \text { GSSG } \uparrow \\
\text { Methylglyoxal } \uparrow\end{array}$ & [71] \\
\hline Mouse & $\begin{array}{l}5 \% \text { alcohol } \\
\text { Lieber-DeCarli diet; } \\
8 \text { weeks }\end{array}$ & $\begin{array}{c}\text { Mild } \\
\text { steatohepatitis } \\
\text { No fibrosis }\end{array}$ & $\begin{array}{l}\text { Correlation between urinary } \\
\text { and fecal metabolites. Many } \\
\text { fecal and urinary } \\
\text { metabolites altered. Amino } \\
\text { acid metabolism perturbed. } \\
\text { Indole-3-lactic acid } \uparrow\end{array}$ & [72] \\
\hline $\begin{array}{c}\text { Mouse } \\
\text { Cramp-null } \\
\text { and WT }\end{array}$ & $\begin{array}{l}5 \% \text { alcohol } \\
\text { Lieber-DeCarli diet; } \\
24 \text { days }\end{array}$ & Not clearly stated & $\begin{array}{c}\text { In alcohol-fed WT, fecal } \\
\text { taurine, } \alpha \text {-aminoisobutyric } \\
\text { acid, nicotinic acid, serine, } \\
\text { SCFAs } \downarrow \\
\text { In alcohol-fed null mice, } \\
\text { only nicotinic acid } \uparrow\end{array}$ & [73] \\
\hline
\end{tabular}


Table 1. Cont.

\begin{tabular}{|c|c|c|c|c|}
\hline Species & Alcohol Dose & Pathology & Metabolites Reported & Ref. \\
\hline Micropig & $\begin{array}{l}40 \% \text { total calories } \\
\text { Folate-depleted diet }\end{array}$ & Not determined & $\begin{array}{c}\text { Hepatic triglycerides } \uparrow \\
\text { SCD pathway } \uparrow \\
\text { ELOVL5 pathway } \uparrow \\
\text { PEMT pathway } \downarrow \\
\text { Phospholipid export } \downarrow\end{array}$ & [57] \\
\hline Human & $\begin{array}{c}100-300 \text { g/day; } \\
10 \text { days } \\
118 \text { g/day; } 11 \text { days, } \\
141 \text { g/day; } 8 \text { days }\end{array}$ & Fatty infiltration & Plasma triglycerides $\uparrow$ & [53] \\
\hline Human & $\begin{array}{c}30 \text { ALD patients } \\
\text { (mean daily alcohol } \\
\text { consumption } 109.7 \\
\text { g/day) vs. } 10 \\
\text { healthy controls }\end{array}$ & $\begin{array}{c}\text { Cirrhosis (80\%) } \\
\text { Decompensated } \\
\text { cirrhosis (DC; } 23 \%)\end{array}$ & $\begin{array}{c}N \text {-Lauroylglycine } \uparrow \\
\text { in cirrhosis. } \\
\text { Decatrienoic acid associated } \\
\text { with disease severity. }\end{array}$ & [61] \\
\hline Human & $\begin{array}{l}30 \text { Alcohol use } \\
\text { disorder (AUD), } 13 \\
\text { alcoholic hepatitis } \\
\text { (AH) and } 16 \\
\text { nonalcoholic controls }\end{array}$ & $\begin{array}{l}\operatorname{ALT} \uparrow(\mathrm{AUD}=\mathrm{AH}) \\
\operatorname{AST} \uparrow(\mathrm{AH}>>\mathrm{AUD})\end{array}$ & $\begin{array}{c}\text { Seven serum oxylipins and } \\
\text { nine fecal oxylipins } \uparrow \\
\text { Results related to } \\
\text { inflammation and } \\
\text { platelet aggregation. } \\
\text { Inflammatory } \omega-6 \text { PUFA } \\
\text { oxylipins counteracted by } \\
\omega-3 \text { bioactive } \\
\text { lipid mediators. }\end{array}$ & [74] \\
\hline Human & $\begin{array}{l}64 \text { AH patients, } \\
26 \text { DC patients } \\
\text { without } \mathrm{AH}\end{array}$ & $\begin{array}{c}\text { AST and GGT } \uparrow \\
(\mathrm{AH}>\mathrm{DC}) . \text { Other } \\
\text { serum markers and } \\
\text { MELD score } \\
\text { AH = DC }\end{array}$ & $\begin{array}{c}\text { Metabolomic signature of } \\
\text { AH claimed but } \\
\text { not disclosed. }\end{array}$ & [75] \\
\hline
\end{tabular}

\subsection{Cholestasis}

Cholestasis is the impaired formation or secretion of bile into the small intestine, and can be classified as intrahepatic or extrahepatic, together with obstructive or nonobstructive. There are many causes of the various manifestations of cholestasis including gallstones, malignancy, and defective bile acid synthesis and secretion [76]. Metabolomics has been employed to attempt to distinguish between the different mechanisms of cholestasis. In the first such study, rat models of inhibited biliary secretion (intrahepatic) and obstructed bile flow (extrahepatic) were employed, and urine was analyzed by ${ }^{1} \mathrm{H}$ NMR. It was concluded that bile acids, valine, and methyl malonate were possible cholestatic biomarkers [77]. These biomarkers did not appear to be specific to cholestatic injury. Another early approach was to use metabolomics to understand the metabolic consequences of perturbed bile acid (BA) homeostasis, as occurs in cholestasis. The farnesoid X receptor (FXR) is a nuclear receptor that regulates genes involved in BA synthesis, metabolism, and transport. Fxr-null and WT mice dosed with the FXR ligands CA or LCA generated metabolites indicative of intrahepatic cholestasis. These included the sulfate and $\beta$-D-glucuronic acid conjugates of $p$-cresol [78], a fermentation product of tyrosine produced by Clostridium difficile in the gut [79], thereby providing further evidence of gut microbiota-liver crosstalk. Other metabolites related to cholestasis included corticosterone and CA metabolites, with the latter being produced by induced CYP3A11 [78]. Furthermore, in LCA-induced experimental intrahepatic cholestasis in mice, TGF $\beta$-SMAD3 signaling mediated the alterations in phospholipid and BA metabolism [80]. In a rat model for cholestasis, mass spectrometry-based targeted metabolomics revealed elevations in urinary taurine and hypotaurine (5- to 9-fold). The largest increases between cholestatic and control rats were for CA, LCA, deoxycholic acid, and ursodeoxycholic acid (10to 23-fold, respectively) [81]. Four independent rat studies that employed the experimental cholestatic 
compound $\alpha$-naphthylisothiocyanate (ANIT) reported that both free and conjugated primary BAs were significantly elevated above controls by ANIT administration [82-85]. It has been demonstrated that several traditional Chinese medicine (TCM) remedies for treating jaundice can reverse the metabolomic fingerprint of ANIT, and therefore, protect against ANIT-induced cholestasis. These treatments include paeoniflorin (from the dried root of Paeonia lactiflora) [83,84], rhubarb [85], Yinchenhao decoction (from the above ground parts of Artemisia annua) [86], chicken bile powder (containing mainly taurochenodeoxycholic acid that is deconjugated in the gut producing the primary BA that is a FXR ligand) [87], Huangqi decoction (a TCM comprising Radix Astragali and Radix Glycyrrhizae) [88], gentiopicroside (from Gentiana rigescens Franch. ex Hemsl.) [89], and Da-Huang-Xiao-Shi decoction [90]. In addition to TCMs, melatonin (100 mg/kg p.o.) has been administered to rats $24 \mathrm{~h}$ after they had received ANIT (25 mg/kg i.p.). This high dose of melatonin (relative to the $4-20 \mathrm{mg} / \mathrm{kg}$ doses used in mouse melatonin studies [91,92]) produced a modest reduction in serum liver enzymes and bilirubin with a less severe liver histology. The metabolomic changes in serum due to melatonin administration were unexceptional and, in part, derived metabolically from melatonin [93]. The mechanism of ANIT-induced cholestasis continues to be investigated using metabolomic tools. The plasma and liver biomarkers described in mice administered ANIT gave rise to the conclusion that the cholestatic liver injury might correlate significantly with hepatocyte necrosis, metabolic disorders, and an imbalance of intestinal microbiome ecology as a result of BA accumulation [94].

A metabolomic investigation has also been reported, whereby regulation of BA metabolism by the nuclear receptor PPAR $\alpha$ and inhibition of NF- $\mathrm{KB} / \mathrm{STAT} 3$ signaling protected against cholestasis induced by ANIT [95]. Furthermore, a lipidomic study of ANIT-induced intrahepatic cholestasis uncovered the role of the aryl hydrocarbon receptor (AHR) in regulating expression of choline kinase (CHK) in mice. Knockout of the Ahr gene significantly reversed ANIT-induced lipid metabolism via Chka expression, and reversed the intrahepatic cholestasis [96]. Vascular protein sorting-associated protein 33B (VPS33B) is involved in the trafficking of intracellular proteins to distinct organelles. Mutations in $V P S 33 B$ are associated with a neonatal syndrome that includes cholestasis (OMIM 208085). Using the lipidomic and metabolomic profiles of hepatic $V p s 33 b$-null male mice, which displayed cholestasis with elevated serum liver enzymes and total bilirubin and total BAs, demonstrated the importance of VPS33B in BA, glycerolipid, phospholipid, and sphingolipid metabolism. In particular, the elevation of hepatic ceramides was thought to influence apoptosis and the progression of cholestasis [97].

Bile duct ligation (BDL) is a nonchemical means to produce experimental cholestasis in rats. Compared with sham operated rats, BDL rats displayed oxidative stress, with diminished serum GSH, total antioxidant capacity, and superoxide dismutase and glutathione peroxidase activities, with upregulated serum malondialdehyde. Changes in certain amino acids, lipids, Krebs cycle intermediates, and lactic acid were signs of the effects of cholestasis on energy metabolism [98]. The BDL cholestasis rat model was shown to generate similar metabolic characteristics as thioacetamide (TAA)-induced cholestasis in rats, with excessive fatty acid oxidation, insufficient glutathione regeneration, and disturbed gut microbiota. These features in both rat models could be reversed by the TCM Huang-Lian-Jie-Du-Decoction [99]. A metabolomic study recently compared three models of chemically-induced cholestasis, using ANIT, 3,5-diethoxycarbonyl-1,4-dihydrocollidine (DDC), or LCA. BAs were increased in all three models, whereas arginine was decreased. Hepatic protoporphyrin IX, a metabolic precursor of heme and cytochrome c, was increased only in the DDC model [100].

Both primary biliary cholangitis (PBC) (previously known as primary biliary cirrhosis) and primary sclerosing cholangitis (PSC) are chronic cholestatic liver diseases. PBC and PSC patients were investigated using targeted profiling of serum BAs. In PBC with cholestasis, total primary BAs (CA and chenodeoxycholic acid) were 13.5-fold higher than noncholestatic donors, in particular, their taurine conjugates (34- to 46.5-fold accumulation) [101]. A similar pattern of elevated free and conjugated primary BAs was reported in another PBC metabolomic investigation. The total secondary BAs (deoxycholic acid and LCA) were not significantly altered in PBC, nor were the 6 $\alpha$-hydroxylated BAs (hyocholic acid and hyodeoxycholic acid). In PSC with cholestasis, primary 
BAs were more abundant and both secondary BAs, and $6 \alpha$-hydroxylated BAs were significantly reduced. The authors recognized that the BA composition of bile requires determination in these two cholestatic diseases [102]. Similar findings were reported in a later study that also included some small changes in free fatty acids and markers of inflammation and oxidative stress [103]. Furthermore, BAs increased during progression of $\mathrm{PBC}$ with a decline in acylcarnitines, such as propionyl and butyryl carnitine [104]. The metabolic signatures of PBC and celiac disease have been compared and contrasted with healthy controls using ${ }^{1} \mathrm{H}$ NMR-based metabolomics on serum and urine. Both diseases showed distinct metabolite patterns, although relatively few metabolites, such as pyruvate, lactate, glutamate, glutamine, hippurate, and trigonelline (a metabolite of niacin also found in coffee) were described [105]. It is unclear whether the differences described were due to dietary factors. Intrahepatic cholestasis of pregnancy (ICP) has an incidence of between 0.1\% (Europe) and 15.6\% (South America) [106]. A urinary metabolomic study of ICP revealed several significant predictive biomarkers of ICP, including the primary BA metabolites glycocholic acid and chenodeoxycholic acid 3-sulfate [107]. In a serum targeted metabolomics ICP study, 60 BAs were detected of which most conjugated BAs were elevated in ICP. Metabolomics was also employed to monitor BAs during treatment with ursodeoxycholic acid [108]. Targeted metabolomics of urinary sulfated BAs was used to define biomarkers for the diagnosis and grading of ICP. Total sulfated BAs were remarkably increased in ICP, particularly those formed from glycine and taurine conjugated BAs. Clear clustering and separation of the PCA and OPLS-DA scores for controls, mild ICP, and severe ICP were reported, and are depicted in Figure 3. In order to better understand how ICP endangers the fetus and the links between fetal BA homeostasis and sulfation capacity, a metabolomic investigation in pregnant swine was conducted. It was found that sulfation played a pivotal role in maintaining BA homeostasis in the fetus. Furthermore, fetal mortality showed an exponential increase in relation to the total BA increase from week 60 to week 90 [109]. A controversial condition related to ICP that is asymptomatic and difficult to distinguish from ICP is asymptomatic hypercholanemia of pregnancy (AHP). A targeted metabolomics study was undertaken in order to establish a differential diagnosis of AHP. Compared to a control group, AHP had several higher urinary BAs and sulfated BAs than controls, and more that were lower in AHP than ICP. Glycocholic acid and tauro- $\omega$-muricholic acid were a potential combination biomarker for AHP, whereas a further combination biomarker involving BA sulfates could distinguish AHP from ICP [110]. Metabolomic profiling of maternal hair was conducted to find predictive biomarkers of ICP. Despite the identification of 105 metabolites in hair, none was associated with ICP [111].

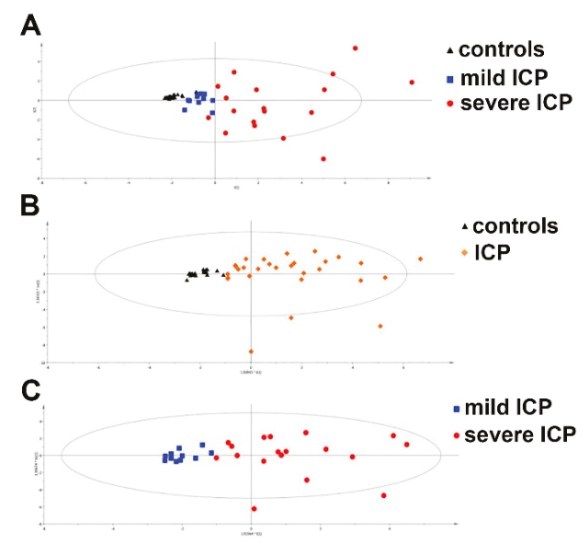

Figure 3. (A) PCA scores plot for controls vs. mild ICP vs. severe ICP; (B) OPLS-DA scores plot for controls vs. ICP; (C) OPLS-DA scores plot for mild ICP vs. severe ICP. Note the data clustering and separation (taken from Li et al., 2018 [112] with permission). 
Cholestasis may also occur in neonates. Infantile hepatitis syndrome (IHS) and biliary atresia (BLA) are the most common in the first three months of life. Using GC-MS metabolomics on urine, it was reported that IHS could be distinguished from BLA with the biomarkers $N$-acetyl-D-mannosamine and $\alpha$-aminoadipic acid [113]. A summary of studies is given in Table 2.

Table 2. Metabolomic and lipidomic biomarkers of cholestasis.

\begin{tabular}{|c|c|c|c|c|c|}
\hline Species & Manipulation/Condition & Pathology & $\begin{array}{c}\text { Analytical } \\
\text { Methodology }\end{array}$ & Metabolites Reported & Ref. \\
\hline Rat & $\begin{array}{l}\text { Inhibition of bile } \\
\text { secretion vs. bile flow } \\
\text { obstruction }\end{array}$ & $\begin{array}{l}\text { Intrahepatic } \\
\text { cholestasis vs. } \\
\text { extrahepatic } \\
\text { cholestasis }\end{array}$ & ${ }^{1} \mathrm{H}$ NMR & $\begin{array}{c}\text { Bile acids } \uparrow \text { Bilirubin } \uparrow \\
\text { vs. Bile acids } \uparrow \text { BCAAs } \uparrow \\
\text { SCFAs } \uparrow\end{array}$ & {$[77]$} \\
\hline Mouse & $\begin{array}{c}\text { Fxr-null vs. WT treated } \\
\text { with FXR ligands CA } \\
\text { and LCA }\end{array}$ & Cholestasis & UPLC-ESI-QTOFMS & $\begin{array}{c}p \text {-Cresol sulfate and } \\
\beta \text {-D-glucuronide } \uparrow \\
\text { Corticosterone metabolites } \uparrow \\
\text { Cholic acid metabolites } \uparrow\end{array}$ & {$[78]$} \\
\hline Rat & $\begin{array}{c}\text { Eisai hyperbilirubinemic } \\
\text { rat }\end{array}$ & Cholestasis & UPLC-TQMS & $\begin{array}{c}\text { Taurine } \uparrow \text { Hypotaurine } \uparrow \\
\text { Unconjugated primary and } \\
\text { secondary bile acids } \uparrow\end{array}$ & {$[81]$} \\
\hline Rat & $\begin{array}{c}\text { ANIT } \\
\text { Methapyrilene } \\
\text { Dimethylnitrosamine }\end{array}$ & Cholestasis & $\begin{array}{c}\text { UPLC-TQMS } \\
\text { GC-MS } \\
\text { UPLC-QTOFMS }\end{array}$ & $\begin{array}{c}\text { Bile acids } \uparrow \text { Arginine } \downarrow \\
\text { Pantothenate } \uparrow \\
\text { Protoporphyrin IX } \uparrow \\
\text { Palmitoyl carnitine } \uparrow \\
\text { Arachidonic, linoleic and } \\
\text { oleic acids } \downarrow\end{array}$ & [82-85] \\
\hline Mouse & $V p s 33 b$-depleted mouse & Cholestasis & UPLC-MS & $\begin{array}{c}\text { Serum bile acids } \uparrow \\
\text { triglycerides } \uparrow \text { and } \\
\text { sphingomyelins } \uparrow\end{array}$ & {$[97]$} \\
\hline Rat & Bile duct ligation (BDL) & Cholestasis & UPLC-QTOFMS & $\begin{array}{c}\text { Phenylalanine } \uparrow \text { Glutamate } \uparrow \\
\text { Tyrosine } \uparrow \text { Kynurenine } \uparrow \\
\text { Lactate } \uparrow \text { LPC }(14: 0) \uparrow \\
\text { Glycine } \uparrow \text { Succinate } \uparrow \text { MDA } \uparrow \\
\text { GSH } \uparrow \text { Valine } \downarrow \text { Isoleucine } \downarrow \\
\text { Citrate } \downarrow \text { Palmitate } \downarrow \text { Taurine } \downarrow \\
\text { LPC }(19: 0) \downarrow\end{array}$ & [98] \\
\hline Rat & TAA or BDL & Cholestasis & ${ }^{1} \mathrm{H}$ NMR & $\begin{array}{c}\text { BDL vs. TAA: } \\
\text { 2-Hydroxybutyrate } \uparrow \\
\text { BCAAs } \uparrow \text { Lysine } \uparrow \text { Arginine } \uparrow \\
\text { Glycine } \uparrow \text { Citrate } \uparrow \\
\text { 2-Oxoglutarate } \uparrow \text { Fumarate } \uparrow \\
\text { Hippurate } \uparrow \\
\text { Phenacetylglycine } \uparrow\end{array}$ & [99] \\
\hline Mouse & ANIT or DDC or LCA & Cholestasis & UPLC-QTOFMS & $\begin{array}{c}\text { Phospholipids } \uparrow \\
\text { Protoporphyrin IX } \uparrow \text { GSH } \downarrow\end{array}$ & {$[100]$} \\
\hline Human & $\begin{array}{l}\text { Primary biliary } \\
\text { cholangitis }\end{array}$ & Cholestasis & UPLC-QTOFMS & $\begin{array}{c}\text { Primary bile acids } \uparrow \\
\text { Phospholipids } \uparrow \text { Oleic and } \\
\text { Linoleic acids } \uparrow\end{array}$ & [101-103] \\
\hline Human & $\begin{array}{l}\text { Intrahepatic cholestasis } \\
\text { of pregnancy (ICP) }\end{array}$ & Cholestasis & HPLC-QTOFMS & $\begin{array}{c}\text { MG(22:5) } \uparrow \operatorname{LPE}(22: 5) \uparrow \\
\mathrm{L}-\text { Homocysteine sulfonic } \\
\text { acid } \uparrow \text { Glycocholic acid } \uparrow \\
\text { Chenodeoxycholic acid } \\
\text { 3-sulfate } \uparrow\end{array}$ & [107] \\
\hline Human & $\begin{array}{l}\text { Hypercholanemia of } \\
\text { pregnancy }(\mathrm{HCP}) \text { vs. } \\
\text { ICP }\end{array}$ & Cholestasis & UPLC-QTOFMS & $\begin{array}{l}\text { Sulfated bile acid pattern } \\
\text { used for differential } \\
\text { diagnosis of HCP and ICP }\end{array}$ & [110] \\
\hline Human & $\begin{array}{l}\text { Infantile hepatitis } \\
\text { syndrome (IHS) vs. } \\
\text { biliary atresia }\end{array}$ & Cholestasis & GC-MS & $\begin{array}{c}N \text {-Acetyl-D-mannosamine } \\
\text { and } \alpha \text {-Aminoadipic acid } \\
\text { used for differential } \\
\text { diagnosis }\end{array}$ & [113] \\
\hline
\end{tabular}

\subsection{Fibrosis and Cirrhosis}

Fibrosis occurs when damage to the liver causing overactive wound healing leads to the formation of scarring or deposition of extracellular matrix proteins including collagen. This process occurs in most chronic liver diseases, and can ultimately lead to cirrhosis and liver failure. Such end-stage liver disease may require transplantation [114]. Fibrosis is staged 0 to 4 by liver biopsy using the METAVIR 
scoring system, F0 = no fibrosis, F1 = portal fibrosis, F2 = periportal fibrosis, F3 = bridging fibrosis, F4 = cirrhosis. Fibrosis is also graded according to the severity of the underlying disease process, activity grades A0 to A3 [115]. Fibrosis and cirrhosis are primarily caused by hepatitis or chronic alcoholism, but can also arise due to nonalcoholic fatty liver disease (NAFLD), including nonalcoholic steatohepatitis (NASH). In compensated cirrhosis, the liver is still able to perform most of its basic functions despite the scarring. Compensated cirrhosis involves Stage 1 (no varices, no ascites) and Stage 2 (varices, no ascites). In decompensated cirrhosis, excessive scarring inhibits basic liver functions and comprises Stage 3 (ascites \pm varices) and Stage 4 (bleeding varices \pm ascites) [116]. The 1-year survival for compensated and decompensated cirrhosis is $87.3 \%$ and $75.0 \%$ and 5 -year survival is $66.5 \%$ and $45.4 \%$, respectively [117]. As the terminal stages of liver fibrosis that can lead to HCC have a high morbidity and mortality with only transplantation as a therapeutic option, there have been extensive studies using metabolomics to define biomarkers for the underlying disease progression.

Relatively few investigations have sought biomarkers of fibrosis using metabolomics. The greatest both quantity and quality of potential biomarker data has been leveraged using mass spectrometry methodologies. Metabolic pathways associated with hepatic fibrosis, specifically, for carbohydrates, amino acids, and lipids, have been reviewed [118]. In a Japanese study that employed CE-TOFMS and LC-TOFMS, the progression of fibrosis in NAFLD was reported to be associated with increased serum concentrations of several metabolites, among them the sulfates of the three steroids etiocholanolone (a major testosterone metabolite), dehydroepiandrosterone (a precursor of androgens and estrogens) and 16 $\alpha$-hydroxy-dehydroepiandrosterone (a precursor of estriol). The first of these sulfates decreased in relation to fibrosis progression from F0/F1 to F4, while the last steroid sulfate increased during fibrosis progression, especially when expressed as a ratio to either of the other two sulfates [119]. Although these steroid sulfates and their ratios appeared to be biomarkers of fibrosis progression in NAFLD, the key biomarker, $16 \alpha$-hydroxy-dehydroepiandrosterone sulfate, has also been reported in serum of patients with breast cancer and endometrial cancer [120]. A Brazilian study in chronic hepatitis $C$ collected large amounts of clinical data on 69 fibrotic patients classified with fibrosis by METAVIR that was significant $(\geq \mathrm{F} 2 ; 42)$, nonsignificant $(<\mathrm{F} 2 ; 27)$, also as advanced $(\geq \mathrm{F} 3 ; 28)$, nonadvanced $(<\mathrm{F} 3$; $41)$, and as cirrhosis $(\mathrm{F} 4 ; 18)$ and noncirrhosis $(<\mathrm{F} 4 ; 51) .{ }^{1} \mathrm{H}$ NMR was used to analyze serum, but not to identify metabolites. The PLS-DA 3-D scores plots showed clustering and separation for F0-F1 vs. F2-F4, F0-F2 vs. F3-F4 with partial separation of F0-F3 vs. F4, leading the authors to hypothesize that their metabolomic strategy could distinguish between significant fibrosis, advanced fibrosis, and cirrhosis [121]. Without knowledge of the altered metabolites central to the metabolomic model used, it is not possible to delineate whether the discriminatory signals arise as biomarkers for the disease process or due to confounding factors such as comorbidities or drug treatment, as commented in another similar case (see below) [122]. A ${ }^{1} \mathrm{H}$ NMR-based metabolomic study was conducted in rats injected i.p. for 8 weeks with CCl4. Seven metabolites were diminished in urine of treated rats compared with controls, namely, 2-oxoglutarate, citrate, dimethylamine, phenacetylglycine, creatinine, and hippurate. Only taurine urinary excretion was found to be significantly elevated in this rat model of fibrosis [123]. A subsequent report from this group found more metabolomic changes in their CCl4 fibrosis rat model. They proposed that the TCM Corydalis saxicola Bunting exhibited antifibrotic effects by regulating ALT, FXR, COX-2, metalloproteinase-1, and angiotensinogen based upon network analysis with their NMR metabolomic data [124], about which we remain skeptical. Shi-Wei-Gan-Ning-Pill (SWGNP) is a multicomponent Tibetan recipe used to treat viral hepatitis, hepatic fibrosis and steatosis, cirrhosis, and HCC. In a study in the CCl4 rat model, SWGNP was also administered at a low, medium, and high dose, equivalent to 3-, 6-, and 12-times the clinical dose, respectively. ${ }^{1} \mathrm{H}$ NMR-based metabolomics was conducted on liver extracts and serum. A total of 39 metabolites were identified in rat liver extracts and 28 in serum. Alterations in energy metabolites suggested that the liver responded to $\mathrm{CCl} 4 \mathrm{crisis}$ by metabolic remodeling from mitochondrial respiration to cytosolic aerobic glycolysis, increased fatty acid $\beta$-oxidation, glycogenolysis, and metabolism of ketone bodies. The medium and high doses of SWGNP significantly decreased the histological scores in the $\mathrm{CCl} 4$ model, together with fibrosis and 
oxidative stress markers. SWGNP also reversed changes in amino acids and nucleosides caused by CCl4. The authors concluded that SWGNP could alleviate liver fibrosis caused by CCl4 [125]. Another Tibetan folk remedy has been investigated in the $\mathrm{CCl} 4$ rat fibrosis model, that of Herpetospermum caudigerum Wall. (HCW), the Himalayan Bitter Gourd, a large climbing plant that grows at an altitude of 1500 to $3600 \mathrm{~m}$, whose dry ripe seeds have been used as a hepatoprotectant. In the CCl4 experiments, HCW was administered at doses of 1 and $3 \mathrm{~g} / \mathrm{kg}$. HCW produced similar effects on fibrosis markers as SWGNP, with the exception that the lower dose was more effective than the higher dose. The metabolomic effects and proposed mechanisms were very similar for HCW [126] to those of SWGNP [125]. The active principles of neither of these TCMs have been identified, except that HCW was said to comprise mainly lignans, coumarins, triterpenes, saponins, phenols, essential oils, amino acids, and trace elements [126]. The underlying antifibrotic mechanisms of these TCM remedies remain unknown, despite the clues provided by metabolomics.

Earliest serum biomarkers of liver cirrhosis (LC) were derived from chronic hepatitis B patients in China, and comprised the four primary bile salts found by UPLC-QTOFMS [127]. However, elevated glycine and taurine conjugated primary bile acids are not specific to LC (see above). A similar population studied using GC-MS identified several elevated metabolic intermediates in cirrhotic serum, including butanoic and hexanoic acid [128]. These two SCFAs are presumably products of the gut microflora (see above). Amino acid D- and L-enantiomers in serum and urine have been examined using two-dimensional gas chromatography-time-of-flight mass spectrometry (GC X GC-TOFMS) in 25 LC patients and 16 controls in Germany. No L-amino acids were significantly higher in the serum of LC patients, although several were significantly higher in controls. In contrast, D-alanine and D-proline were significantly elevated in LC serum, and D-valine, D-leucine, and D-threonine were only detected in LC serum [129]. It is attractive to consider these D-amino acids as candidate biomarkers for LC. However, only D-serine and D-aspartate are considered human tissue-derived, while the rest most likely arise from microbial sources, either in the diet or from the gut microbiota [130]. This may be further evidence of gut microbiota-liver cross-talk in liver disease. Further evidence of this crosstalk is furnished by a Chinese study that examined stool samples by UPLC-ESI-QTOFMS taken from cirrhotic patients (etiologies either HBV, HCV or alcohol; 17) and healthy controls (24). The two groups clustered and separated in both the PCA and PLS-DA scores plots. Several metabolites that were reduced in cirrhotic feces, chenodeoxycholic acid, 7-ketolithocholic acid, urobilin, and urobilinogen. A number of metabolites were more prominent in cirrhotic feces, including amino acids, and long-chain fatty acids and their carnitine esters. These findings were interpreted as due to changes in biliary function and the gut microbiota in cirrhosis leading to fat malabsorption [131]. Another Chinese study claimed that taurocholate was not merely a biomarker for cirrhosis progression, but also actively promoted this progression. Of the 12 BAs targeted using UPLC-TQMS, taurocholate increased 76-fold between LC (32) and HV (27). This was said to be due to increased synthesis. In addition, the promotion of cirrhosis progression by taurocholate was postulated to be due to stellate cell activation via the TLR4 pathway [132].

We have reported a metabolomic and lipidomic investigation of into Swiss HCC patients (20) using UPLC-ESI-QTOFMS and GC-MS, in which LC patients (7) were included together with healthy volunteers $(\mathrm{HV} ; 6)$ and an acute myelogenous leukemia (AML) control group (22). With one exception, all the HCC patients also had LC. Interestingly, LC and HCC clustered together in both the unsupervised (PCA) and supervised (PLS-DA) scores plots, and clearly segregated from the HV and AML clusters. This suggests that the greatest insult to liver metabolism resulted from LC rather than HCC. No elevated biomarkers specific to LC were described, although several were found for HCC (see below) [133]. The investigation by GC-MS of urine from HCV-positive untreated Egyptian patients with LC (40) and HCC (55), together with HV (45) essentially confirmed the findings of metabolomic similarity between LC and HCC patients. With the exception of AFP, serum biochemistry was similar for the LC and HCC. Several urinary metabolites were elevated above HV for both LC and HCC in a similar fashion, including serine, glycine, threonine, and citrate [134]. Although not stated, the HCC patients almost 
certainly also had LC, underlining the difficulties of distinguishing between HCC and LC in studies of this kind for an HCV population. In a Chinese study of LC (20), healthy controls (20) and HCC (59) using UPLC-ESI-QTOFMS, three ions corresponding to canavaninosuccinate (CVS) were virtually absent in LC serum relative to the other groups [135]. CVS is a derivative of aspartate formed from ureidohomoserine; aspartate is further converted to creatine [136]. The extinction of CVS in LC serum is an appealing biomarker for LC, except that it is also massively reduced in plasma of chronic kidney disease patients relative to controls, correlating strongly with the glomerular filtration rate [137]. A US study compared patients with high both liver and kidney disease severity (ascites present, GFR $\leq 60 ; n=34$ ) with those with low liver and kidney disease (ascites absent, GFR $\geq 60 ; n=69$ ) severity. Using UPLC-ESI-TQMS, $34 / 1028$ plasma metabolites were significantly increased in the severe hepatorenal dysfunction group. The greatest change (2.39) was for 4-acetamidobutanoate, the acetylated metabolite of GABA and a product of arginine and proline metabolism (http://www.hmdb.ca/metabolites/HMDB0003681). Pathway enrichment analysis identified glucuronidation and methylation, together with ascorbate and aldarate metabolism, that were linked to hepatorenal dysfunction [138]. Another study in China used both NMR and UPLC-ESI-QTOFMS to analyze serum from LC (42), HCC (43) and HV (18). Several phospholipids and fatty acids together with bilirubin were elevated in LC vs. HV [139], findings similar to those which we had previously reported in Swiss patients [133]. A UK study that employed both NMR and UPLC-ESI-QTOFMS of plasma from 248 subjects examined the differences between surviving and nonsurviving patients with decompensated cirrhosis. NMR profiles of nonsurvivors had increased plasma lactate, tyrosine, methionine and phenylalanine. UPLC-ESI-QTOFMS showed that lysophosphatidylcholines (LPC) and phosphatidylcholines (PC) were downregulated in nonsurvivors. LPC concentrations negatively correlated with the circulating markers of cell death, M30 and M65. Therefore, metabolomic phenotyping ("metabotyping") was said to accurately predict mortality in decompensated cirrhosis, due to LPC and amino acid metabolism dysregulation that reflected hepatocyte cell death [140]. Using LC-MS, a Chinese group profiled 43 steroids in the urine of HV (21), LC (21), and HCC (28) relative to urinary creatinine. The PCA scores plot showed some overlap between these three groups. Many steroids in LC displayed lower urinary excretion than HV controls, including pregnanediol, corticosterone, androsterone, etiocholanolone, dehydroepiandrosterone, and testosterone. In contrast, LC urinary excretion of $16 \alpha$-hydroxyestrone was markedly elevated above HV controls. These findings are consistent with what has been described as a "feminization" phenotype in LC [141]. It is worth noting that these investigators treated the urines with sulfatase and $\beta$-glucuronidase prior to steroid analysis to determine total (free plus conjugated) steroids; therefore, their results are difficult to compare with those cited above where sulfated steroids were quantitated [120]. Using GC-MS, serum from Chinese HBV-positive (49), LC (52) and HCC patients (39), together with healthy controls (61) was analyzed. All four groups clustered and separated in the OPLS-DA scores plot. Of the top 30 discriminating metabolites, serine, succinate, malate, 5-oxoproline, glutamate, phenylalanine, ornithine, citrate, and tyrosine were all elevated in LC relative to controls. Palmitate was proposed as a biomarker for cirrhosis development in HBV hepatitis, with high sensitivity and specificity in ROC analysis. The purpose of this study however was to examine the progression of hepatitis B to HCC via cirrhosis [142]. Interestingly, a review of metabolomic studies of hepatitis B, HBV-related LC and HBV-related HCC clearly shows the overlap in these three groups in upregulated metabolites [143]. Oxylipins are another group of lipids that have been investigated in HBV-related LC and HCC. UPLC-ESI-TQMS was utilized to quantitate 18 omega- 6 fatty acid-derived oxylipins in serum from patients with chronic hepatitis B (34), HBV-related LC (46), HBV-related HCC (38), and healthy controls (50). Compared with healthy controls, LC had statistically significantly elevated 13-HODE, but lower levels of TXB2 [144]. The 13(S)-HODE and 13(R)-HODE enantiomers are produced from linoleic acid by 15-lipoxygenase and are credited with differential effects on cell growth and apoptosis [145]. Unfortunately, it was not determined which enantiomer was elevated in plasma of LC patients [144]. Apparently, patients with HBV-related LC can be classified under the theory of TCM as having one of two typical patterns, Gan Dan Shi Re (GDSR) or Gan Shen Yin 
Xu (GSYX). Serum of cases with GDSR (40), GSYX (41), and those with no obvious pattern (called "Latent Pattern" (LP); 30) were investigated using GC-TOFMS metabolomics. Eight metabolites were specific to the GDSR type of HBV cirrhosis, a separate eight were specific to the GSYX type, and a further 10 metabolites were common to both types. The GDSR metabolites were said to be related to abnormalities in linoleic acid metabolism, while the GSYX metabolites were said to arise from abnormalities in glycine, serine, and threonine metabolism. All these 26 metabolites were potential biomarkers for HBV-related cirrhosis [146].

As mentioned earlier, BLA is a neonatal cholestatic condition and is the most life-threatening cholestatic disorder in children. In a Chinese study, liver samples from BLA (52) and IHS (16) were profiled for amino acids and biogenic amines using UPLC-ESI-TQMS. Several amino acids had higher hepatic concentrations in IHS than in BLA. However, histamine was twice as abundant in BLA as in IHS liver. In addition, the degree of fibrosis from F1/F2 to F4 correlated with histamine concentration. Histamine therefore presents a potential target for preventing fibrosis in BLA [147].

Several investigators have used ${ }^{1} \mathrm{H}$ NMR in the search of biomarkers for liver fibrosis and cirrhosis. For example, a Spanish study of LC with minimal hepatic encephalopathy was conducted by ${ }^{1} \mathrm{H}$ NMR, resulting in elevated glucose, lactate, methionine, trimethylamine $N$-oxide (TMAO), and glycerol [148], none of which is specific to LC or even liver disease. A further Spanish NMR study compared liver biopsies from cirrhosis and chronic hepatitis due to $\mathrm{HCV}, \mathrm{HBV}$, alcohol, and autoimmunity. Elevated in cirrhosis were glutamate and phosphoethanolamine [149]. A UK study used ${ }^{1} \mathrm{H}$ NMR metabolite profiling to compare livers removed from patients with either LC associated with ALD (5) or with NASH (14) with healthy donor transplant livers (16). Cirrhotic livers had significantly increased levels of isoleucine, valine, succinate, lactate, and betaine [150]. Another NMR study was conducted on Chinese patients that included those with HCC. The elevated serum metabolites in LC occurred also when the patients had HCC, with the exception of taurine, namely, acetate, pyruvate, glutamine, $\alpha$-ketoglutarate, glycerol, tyrosine, 1-methylhistidine, and phenylalanine [151]. A French study using ${ }^{1} \mathrm{H}$ NMR examined metabolic differences between alcoholic cirrhotic patients with severe and mild chronic liver failure (CLF) that had been stratified by MELD score. Lactate, pyruvate, glucose, amino acids, and creatinine were significantly higher in patients with severe CLF than mild CLF [152]. These findings cannot be considered as biomarkers of severe CLF, as they are not specific. A Chinese study in compensated cirrhosis (30), decompensated cirrhosis (30), and healthy controls (30) using ${ }^{1} \mathrm{H}$ NMR on serum samples reported that succinate, pyruvate, and phenylalanine increased with cirrhosis progression [153]. Yet, again, these cannot be considered as biomarkers due to their lack of specificity. An earlier Canadian study had been the first to profile metabolites in compensated and decompensated cirrhosis patients with $\mathrm{HCV}$, together with healthy volunteers, but used ${ }^{31} \mathrm{P}$ magnetic resonance spectroscopy performed on the abdomen over the liver. The acquired spectra showed phosphomonoesters (PME), phosphodiesters (PDE), and $\beta$-ATP resonances, the last of which was significantly lower in decompensated cirrhosis vs. the other two groups combined, and the PME/PDE ratio was significantly higher in decompensated cirrhosis than controls. This ratio was interpreted based upon published findings as an indicator of a disturbed endoplasmic reticulum membrane in decompensated cirrhosis [154]. Austrian investigators used high-field ${ }^{1} \mathrm{H}-\mathrm{MRS}$ and ultrahigh-field ${ }^{31}$ P-MRS to examine in vivo the livers of NAFLD patients with little or no fibrosis and NASH patients with advanced fibrosis. The ${ }^{1} \mathrm{H}-\mathrm{MRS}$ lipid signal was massively increased in NASH livers over NAFLD livers and cross-correlated with histology from liver biopsies. The lipid saturation, polyunsaturation, and monounsaturation indices did not differ between NAFLD and NASH livers. Moreover, ${ }^{31} \mathrm{P}-\mathrm{MRS}$ measures of the PME (including phosphoethanolamine) and PDE (including glycerophosphocholine) resonances reflected the severity of fibrosis. Changes in energy metabolism, as reflected by ATP flux, were decreased in advanced fibrosis. This noninvasive real-time profiling technique appeared to be of significant value for investigation of hepatic structure and function [155]. An Italian study combined NMR metabolomics of stool samples with 16S rRNA sequencing of gut microbiota in LC patients (46) and healthy age-matched controls (14). Peripheral blood and liver 
biopsies were also analyzed together with portal blood from seven cirrhotics and caecal biopsies taken during colonoscopy in 17 LC patients and 6 controls. The metagenomics data demonstrated a marked dysbiosis in LC patients. The principally elevated metabolites in LC feces relative to controls were phenylalanine, threonine, butanoate, methanol, cadaverine, and $\alpha$-glucose. Using the metagenomics data, eight pathways were underrepresented and two overrepresented in LC. The authors concluded that intervention with prebiotics/probiotics/synbiotics, diet, or fecal microbiota transplant could support development of new customized treatments for LC patients [156]. Interestingly, partial reversal of dysbiosis and metabolomic profile was reported after splenectomy in LC patients (12) [157]. A combined metagenomics and metabolomic investigation of LC was conducted in China with HV (47), compensated LC (49) and decompensated LC (46). Urine was analyzed by UPLC-ESI-QTOFMS and PCA scores plots for total metabolites and a subset of 75 differential metabolites both separated HV from LC urines, with compensated and decompensated LC clustering together. Six metabolites were reported to be lower in LC urine than in HV urine, but none greater [158]. Another combined metagenomics and metabolomics investigation was conducted to compare Turkish patients on a Mediterranean diet (HV, 46; compensated LC, 50; decompensated LC, 43) with American patients on a Western diet (HV, 48; compensated LC, 59; decompensated LC, 50). In this study, ${ }^{1} \mathrm{H}$ NMR was used for plasma metabolomics, which showed higher lactate concentrations in Turkey vs. USA. There were similar trends between decompensated LC and HV in both Turkey and USA, with reduced lipids and phosphocholines. Correlation networks in cirrhotics showed differences between the beneficial taxa Blautia and Oscillispira in Turkish compared with American patients [159]. The metabolomic differences described in this unique study were disappointing and would have greatly benefitted from analysis using MS-based methodology.

Acute-on-chronic liver failure (ACLF) refers to patients with acute deterioration of liver function in compensated or decompensated but stable cirrhosis. Serum from a group of French compensated and decompensated cirrhosis patients (93) was compared with that from ACLF patients (30) using ${ }^{1} \mathrm{H}$ NMR metabolomics. The latter group showed higher serum lactate, pyruvate, ketone bodies, glutamine, phenylalanine, tyrosine, and creatinine [160], none of which is a specific biomarker. A UK study examined plasma by ${ }^{1} \mathrm{H}$ NMR for stable cirrhotic patients (18), patients with stable cirrhosis during an episode of encephalopathy (18), together with matched controls (17). With the exception of pyruvate, which was significantly higher, glycolysis end-products and gluconeogenesis precursors (pyruvate, alanine, threonine, glycine and aspartate) were significantly lower in cirrhotics with encephalopathy than without and both higher than controls. There was no discernable effect of encephalopathy on branched-chain and aromatic amino acids or on urea cycle intermediates [161]. Yet, again, such NMR-derived metabolites do not show sufficient specificity to be considered as biomarkers. In contrast, a French group compared hepatic encephalopathy (HE) patients (14) with control patients without neurological disease (27) using UPLC-MS analysis of cerebrospinal fluid (CSF) and plasma. A total of 73 metabolites were identified in CSF including amino acids, acylcarnitines, bile acids, and nucleosides. It was further reported that acetylated amino sugars, acetylated amino acids, and metabolites involved in ammonia, amino acid, and energy metabolism were specifically and significantly increased in CSF of HE patients [162]. These findings underscore the superiority of MS-based over NMR-based metabolomics protocols in terms of metabolite identification. Serum analysis by ${ }^{1} \mathrm{H}$ NMR was conducted on a Spanish two groups of HCV patients, one without fibrosis (F0; 30) and the other with cirrhosis (F4; 27). Glucose, citrate, and VLDL1 were significantly elevated, and choline, glutamine, acetoacetate, glycoprotein $N$-acetyl groups, cysteine, histidine, and LDL1 were significantly depressed in the serum of cirrhotic HCV patients. The authors believed that these results provided new biomarkers to distinguish no fibrosis from severe fibrosis (cirrhosis) in $\mathrm{HCV}$ infection [163]. An investigation of Italian patients with chronic HCV attempted to diagnose the degree of fibrosis using ${ }^{1} \mathrm{H}$ NMR on plasma, serum, and urine samples. Remarkably, these investigators did not identify metabolites, but rather, used statistical analysis of their spectra in an attempt to classify and distinguish chronic hepatitis C (little or no fibrosis) from cirrhosis (severe fibrosis) [164]. This study 
has been severely criticized not only on the basis of the lack of metabolite identification, but also for the statistical methods employed for data analysis [122].

Animal models have also been employed. TAA has been administered i.p. to rats to generate experimental fibrosis and cirrhosis. One such study tracked serum and urine by ${ }^{1} \mathrm{H}$ NMR metabolomics over 7 weeks of TAA administration. Liver injury included fibrosis and cirrhosis. TAA was found to increase 2-oxoglutarate and decrease succinate in both serum and urine, while urinary excretion of fumarate, oxaloacetate, and citrate was increased, leading investigators to conclude that TAA impaired the TCA cycle [165]. These and other reported amino acid changes are not specific to fibrosis or cirrhosis. The i.p. administration of dimethylnitrosamine (DMN) to rats produces histologically confirmed fibrosis. UPLC-ESI-QTOFMS metabolomics on serum from control and DMN-treated rats, together with serum from rats treated with DMN together with Yin-Chen-Hao-Tang decoction (YCHT), a TCM long used in the treatment of liver diseases including fibrosis. Biochemical parameters including serum liver enzymes and total bilirubin, together with liver histology, in the YCHT treated rats were intermediate between the controls and the DMN-treated animals. Moreover, several serum lipids, including LPC(18:1), LPC(18:2), oleic acid (18:1), linoleic acid (18:2), arachidonic acid (20:4), and docosahexaenoic acid (22:6; DHA) that were altered by DMN treatment (LPCs $\uparrow$, fatty acids $\downarrow$ ), remained relatively stable with co-administration of YCHT [166]. Despite these lipidomic findings, the antifibrotic mechanism of YCHT remains unclear. Another TCM that has been evaluated in the DMN rat liver fibrosis model is Huangqi Decoction (HQD). In these experiments, 16 individual bile acids were profiled by LC-MS and demonstrated that bile acids were elevated by DMN treatment and that HQD restored these to normal levels. Additionally, gene expression related to bile acid synthesis and transport was examined, and also altered by DMN treatment, but restored by HQD [167].

Carbon tetrachloride $(\mathrm{CCl} 4)$ is another hepatotoxin that can produce liver fibrosis in rats. Its effects upon the serum metabolome of rats has been reported using UPLC-ESI-QTOFMS. The protocol involved 12 weeks twice weekly s.c. injections of $50 \% \mathrm{CCl} 4$ in olive oil at a dose of $5 \mathrm{~mL} / \mathrm{kg}$. Blood biochemistry and liver histology were consistent with liver fibrosis. Of the many prominent metabolites detected, two, i.e., cervonoyl ethanolamide (8,11,14-eicosatrienoyl ethanolamide) and $\beta$-muricholic acid, were defined as biomarker candidates. Pathway analysis proposed that $\mathrm{CCl} 4$ induction of liver fibrosis altered glycerophospholipid metabolism, linoleic acid metabolism, $\alpha$-linoleic acid metabolism, glycine, serine and threonine metabolism, arachidonic acid metabolism, tryptophan metabolism, and aminoacyl-tRNA biosynthesis [168]. This provided a paradigm for chemically-induced liver fibrosis against which other studies could be compared. CCl4 has also been employed to induce decompensated cirrhosis with ascites in rats, using a similar protocol that that described above. In this study, serum and urine were analyzed by Orbitrap UPLC-MS. Aromatic amino acids, alanine, and bile acids were elevated in the CCl4-treated rats, while LPCs, eicosapentaenoic acid, creatine, carnitine, branched-chain amino acids (BCAAs), and arginine were significantly lowered [169].

The TCM used to treat liver fibrosis, Jiaqi Ganxian Granule (JGG), was tested against CCl4-induced hepatic fibrosis in rats. As the mechanism was unknown, detailed UPLC-ESI-QTOFMS metabolomics was conducted on rat serum. Fibrosis markers in serum, namely collagen type IV, procollagen III, hyaluronic acid, and laminin were all significantly increased by CCl4, but normalized by JGG intervention, as was liver histology. Lipid markers that were downregulated by CCl4, but normalized by JGG included sphinganine, dihydroceramide, and monostearoylglycerol. Metabolites that were upregulated by CCl4 but normalized by JGG were the bile acid 3,7-dihydroxy-12-oxocholanoic acid, the phosphatidylinositol PI(18:0/16:0), the ethanolamide metabolite of DHA, LPC(22:6), and PC(20:4/18:2). JGG, therefore, affected sphingolipid and glycerophospholipid metabolism among other pathways [170]. These represent further examples of where metabolomics has informed about the mechanism of action of a TCM on liver disease. A similar study reported in Chinese that Scutellariae Radix decoction, prepared from the root of a flowering plant of the mint family, and baicalin, a flavone glycoside purified from Scutellaria baicalensis, were effective against liver fibrosis in this rat model. UPLC-ESI-QTOFMS analysis showed that several elevated metabolites in fibrotic rat 
urine were ameliorated by the decoction treatment, including, L-tryptophan, 3-methyldioxyindole, 5-hydroxyindoleacetylglycine, kynurenic acid, 4-(2-amino-3-hydroxyphenyl)-2,4-dioxobutanoic acid, methylmalonic acid, and L-leucine. Baicalin treatment also reversed these urinary metabolites with the exception of L-leucine [171]. Another rat model of fibrosis uses dimethylnitrosamine (DMN) i.p. administration over a period of 8 weeks. Cultured bear bile powder (CBBP) has been used as a TCM to treat liver diseases for thousands of years. Using Orbitrap UPLC-MS, it was reported that CBBP co-administration $(65,130$ and $260 \mathrm{mg} / \mathrm{kg}$ ) restored the lowered serum concentrations of eicosapentaenoic and docosahexaenoic acids that occurred when DMN provoked fibrosis. CBBP had the additional effect of inducing the expression of the nuclear receptors PPAR $\alpha$ and PPAR $\gamma$. Moreover, expression of four PPAR $\alpha$-regulated genes involved in fatty acid $\beta$-oxidation (Cpt1b, Cpt2, Mcad, and Hadha) was decreased by DMN treatment but restored by CBBP, suggesting that CBBP may improve fatty acid $\beta$-oxidation. By inducing PPAR $\gamma, \mathrm{CBBP}$ decreased the downstream expression of the inflammatory cytokine IL-6, while also inhibiting activation of hepatic stellate cells, thereby ameliorating fibrogenesis [172]. Further details of the aforementioned studies appear in Table 3.

Table 3. Metabolomic and lipidomic biomarkers of liver fibrosis and cirrhosis.

\begin{tabular}{|c|c|c|c|c|c|}
\hline Species & Manipulation/Condition & Pathology & $\begin{array}{c}\text { Analytical } \\
\text { Methodology }\end{array}$ & Metabolites Reported & Ref. \\
\hline Human & NAFLD & $\begin{array}{l}\text { Fibrosis } \\
\text { progression }\end{array}$ & $\begin{array}{l}\text { CE-TOFMS } \\
\text { LC-TOFMS }\end{array}$ & $\begin{array}{c}\text { F0/F1 } \rightarrow \text { F4 Etiocholanolone } \\
\text { sulfate } \downarrow \\
\text { Dehydroepiandrosterone sulfate } \downarrow \\
16 \alpha \text {-hydroxy-dehydroepiandrosterone } \\
\text { sulfate } \uparrow \\
\text { (all in serum) }\end{array}$ & [119] \\
\hline Human & Fibrosis or Cirrhosis & $\begin{array}{c}\text { Significant fibrosis, } \\
\text { advanced fibrosis, } \\
\text { cirrhosis }\end{array}$ & ${ }^{1} \mathrm{H}$ NMR & $\begin{array}{l}\text { No metabolites reported, only } \\
\text { multivariate model used to } \\
\text { distinguish pathologies. }\end{array}$ & [121] \\
\hline Human & Chronic hepatitis B & Cirrhosis & UPLC-QTOFMS & $\begin{array}{c}\text { Glycocholic acid } \uparrow \\
\text { Glycochenodeoxycholic acid } \uparrow \\
\text { Taurocholic acid } \uparrow \\
\text { Taurochenodeoxycholic acid } \uparrow \\
\text { (all in serum) }\end{array}$ & [127] \\
\hline Human & Chronic hepatitis B & Cirrhosis & GC-MS & $\begin{array}{c}\text { Acetate } \uparrow \text { Hexanoate } \uparrow \text { Butanoate } \uparrow \\
\text { Glucose } \downarrow \text { Sorbitol } \downarrow \\
\text { (all in serum) }\end{array}$ & [128] \\
\hline Human & Causes not stated & Cirrhosis & 2D-GC-TOFMS & $\begin{array}{c}\text { D-Alanine } \uparrow \text { D-Proline } \uparrow \\
\text { D-Valine } \uparrow \text { D-Leucine } \uparrow \\
\text { D-Threonine } \\
\text { (all in serum) }\end{array}$ & [129] \\
\hline Human & HBV, HCV, alcohol & Cirrhosis & UPLC-QTOFMS & 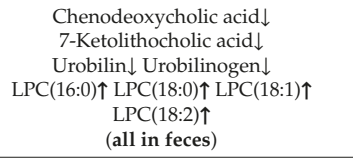 & [131] \\
\hline Human & $\begin{array}{l}\text { HBV, alcohol, PBC, } \\
\text { cryptogenic cirrhosis }\end{array}$ & Cirrhosis & UPLC-TQMS & $\begin{array}{c}\text { Taurocholic acid } \uparrow \\
\text { Taurochenodeoxycholic acid } \uparrow \\
\text { Tauroursodeoxycholic acid } \uparrow \\
\text { Glycocholic acid } \uparrow \\
\text { Ursodeoxycholic acid } \uparrow \\
\text { Chenodeoxycholic acid } \uparrow \\
\text { Cholic acid } \uparrow \\
\text { Taurolithocholic acid } \uparrow \\
\text { Taurodeoxycholic acid } \uparrow \\
\text { Hyodeoxycholic acid } \uparrow \text { Lithocholic } \\
\text { acid } \uparrow \\
\text { Deoxycholic acid } \uparrow \\
\text { (all in serum) }\end{array}$ & [132] \\
\hline
\end{tabular}


Table 3. Cont.

\begin{tabular}{|c|c|c|c|c|c|}
\hline Species & Manipulation/Condition & Pathology & $\begin{array}{l}\text { Analytical } \\
\text { Methodology }\end{array}$ & Metabolites Reported & Ref. \\
\hline Human & Chronic hepatitis C & Cirrhosis & GC-MS & $\begin{array}{c}\text { Proline } \uparrow \text { Serine } \uparrow \text { Glycine } \uparrow \\
\text { Threonine } \uparrow \text { Citrate } \uparrow \text { Xylitol } \downarrow \\
\text { Arabinose } \downarrow \text { Urea } \downarrow \\
\text { (all in urine) }\end{array}$ & [134] \\
\hline Human & Chronic hepatitis B & Cirrhosis & UPLC-QTOFMS & 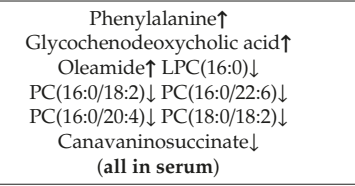 & [135] \\
\hline Human & Hepatorenal syndrome & Cirrhosis & UPLC-TQMS & $\begin{array}{l}\text { 4-Acetamidobutanoate } \uparrow \\
\text { (in plasma) }\end{array}$ & [138] \\
\hline Human & Chronic hepatitis B & Cirrhosis & $\begin{array}{c}{ }^{1} \mathrm{H} \text { NMR } \\
\text { UPLC-QTOFMS }\end{array}$ & 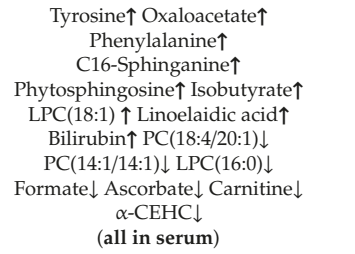 & [139] \\
\hline Human & Causes not stated & $\begin{array}{l}\text { Decompensated } \\
\text { cirrhosis (90-day } \\
\text { mortality vs. } \\
\text { survivors) }\end{array}$ & $\begin{array}{c}{ }^{1} \mathrm{H} \text { NMR } \\
\text { UPLC-QTOFMS }\end{array}$ & 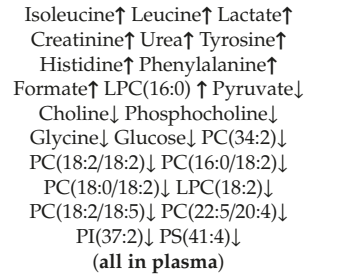 & [140] \\
\hline Human & Causes not stated & Cirrhosis & $\begin{array}{l}\text { UPLC-Orbitrap } \\
\text { MS }\end{array}$ & $\begin{array}{c}16 \alpha \text {-Hydroxyestrone } \uparrow \\
\text { 4-Androstenedione } \downarrow \\
17 \alpha \text {-Hydroxyprogesterone } \downarrow \\
18 \text {-Hydroxycorticosterone } \downarrow \\
\text { Cortisol } \downarrow \text { Cortexolone } \downarrow \\
\text { Allotetrahydrocortisol } \downarrow \\
\text { Deoxycorticosterone } \downarrow \\
\text { Epitestosterone } \downarrow \\
\text { Testosterone } \downarrow \\
\text { Dehydroepiandrosterone } \downarrow \\
\text { Etiocholanolone } \downarrow \\
\text { Tetrahydrodeoxycortisol } \downarrow \\
\text { Androsterone } \downarrow \\
\text { 17 } \alpha \text {-Hydroxypregnenolone } \downarrow \\
\text { Epiandrosterone } \downarrow \\
11 \text {-Oxoetiocholanolone } \downarrow \\
7 \beta \text {-Hydroxy-dehydroepiandrosterone } \downarrow \\
\text { Androstenetriol } \downarrow \\
\text { Androstenediol } \downarrow \\
\text { Pregnanediol } \downarrow \\
\text { (all in urine) }\end{array}$ & [141] \\
\hline Human & Chronic hepatitis B & Cirrhosis (vs. HBV) & GC-TOFMS & $\begin{array}{c}\text { Serine } \uparrow 5 \text {-Oxoproline } \uparrow \\
\text { Phenylalanine } \uparrow \text { Tyrosine } \uparrow \\
\text { Ornithine } \uparrow \text { Citrate } \uparrow \text { Palmitic acid } \uparrow \\
\text { Fructose } \downarrow \text { Glutamate } \downarrow \\
\text { Indole-3-acetic acid } \downarrow \text { arachidonic } \\
\text { acid } \downarrow \text { 2-Deoxy-D-glucose } \downarrow \\
\text { (all in serum) }\end{array}$ & [142] \\
\hline Human & Chronic hepatitis B & $\begin{array}{c}\text { Cirrhosis (vs. } \\
\text { HBV/HV) }\end{array}$ & UPLC-TQMS & $\begin{array}{l}\text { 9,10-DiHOME } \uparrow 13-\mathrm{HODE} \uparrow \mathrm{TXB} 2 \downarrow \\
\text { (all in serum) }\end{array}$ & [144] \\
\hline
\end{tabular}


Table 3. Cont.

\begin{tabular}{|c|c|c|c|c|c|}
\hline Species & Manipulation/Condition & Pathology & $\begin{array}{c}\text { Analytical } \\
\text { Methodology }\end{array}$ & Metabolites Reported & Ref. \\
\hline Human & Chronic hepatitis B & $\begin{array}{l}\text { Cirrhosis (GDSR } \\
\text { and GSYX patterns, } \\
\text { vs. latent } \\
\text { pattern (LP)) }\end{array}$ & GC-TOFMS & $\begin{array}{c}\text { GDSR vs. LP: } \\
\text { Nonanoate } \uparrow \text { Urea } \downarrow \text { Serine } \downarrow \\
\text { 2-Hydroxybutyrate } \downarrow \\
\text { 2-Hydroxyglutarate } \downarrow \\
\text { Phenylalanine } \downarrow \text { Asparagine } \downarrow \\
\text { Citrulline } \downarrow \text { Tyrosine } \downarrow \text { Arabinose } \downarrow \\
\text { Sorbose } \downarrow \text { Fructose } \downarrow \text { Myristate } \downarrow \\
\text { Palmitolate } \downarrow \text { Palmitate } \downarrow \text { Linolate } \downarrow \\
\text { Tryptamine } \downarrow \text { Glycolate } \downarrow \text { Quinate } \downarrow \\
\text { Petroselinate } \downarrow \\
\text { GSYX vs. LP: } \\
\text { 1,5-Anhydrosorbitol } \text { Fructose } \uparrow \\
\text { 2-Hydroxybutyrate } \downarrow \text { Serine } \downarrow \\
\text { Threonine } \downarrow \text { 5-Oxoglutarate } \downarrow \\
\text { 2-Hydroxyglutarate } \downarrow \\
\text { Phenylalanine } \downarrow \text { Asparagine } \downarrow \\
\text { Tyrosine } \downarrow \text { Arabinose } \downarrow \text { Arabitol } \downarrow \\
\text { Nonanoate } \downarrow \text { Glycerate } \downarrow \\
\text { Pipecolate } \downarrow \text { Glutarate } \downarrow \text { Quinate } \downarrow \\
\alpha \text {-Tocopherol } \downarrow \\
\text { (all in serum) }\end{array}$ & [146] \\
\hline Human & $\begin{array}{l}\text { Biliary atresia (BA) and } \\
\text { neonatal hepatitis } \\
\text { syndrome (NHS) }\end{array}$ & Fibrosis F1 to F4 & UPLC-TQMS & $\begin{array}{c}\text { BA/NHS: } \\
\text { Histamine } \uparrow \text { Methionine } \downarrow \\
\text { Phenylalanine } \downarrow \text { Serine } \downarrow \\
\text { Threonine } \downarrow \text { Valine } \downarrow \text { Glutamine } \downarrow \\
\text { Sarcosine } \downarrow \text { Lysine } \downarrow \\
\text { F4>F3>F1/F2 in BA: } \\
\text { Histamine } \uparrow \\
\text { (all in liver homogenates) }\end{array}$ & [147] \\
\hline Human & Alcohol & $\begin{array}{c}\text { Cirrhosis } \pm \\
\text { minimal hepatic } \\
\text { encephalopathy } \\
\text { (MHE) }\end{array}$ & ${ }^{1} \mathrm{H}$ NMR & $\begin{array}{c}\text { MHE+/MHE-: } \\
\text { Lactate } \uparrow \text { Glucose } \uparrow \text { TMAO } \uparrow \\
\text { Glycerol } \uparrow \text { LDL } \downarrow \text { VLDL } \downarrow \\
\text { Isoleucine } \downarrow \text { Leucine } \downarrow \text { Valine } \downarrow \\
\text { Alanine } \downarrow \text { Acetoacetate } \downarrow \text { Choline } \downarrow \\
\text { Glycine } \downarrow \\
\text { (all in serum) }\end{array}$ & [148] \\
\hline Human & $\begin{array}{l}\text { Chronic hepatitis C, } \\
\text { Chronic hepatitis B, } \\
\text { Alcohol, Autoimmunity }\end{array}$ & Cirrhosis & MAS ${ }^{1} \mathrm{H}$ NMR & $\begin{array}{c}\text { Phosphoethanolamine } \uparrow \\
\text { Phosphocholine } \uparrow \text { Glutamate } \uparrow \\
\text { Aspartate } \downarrow \\
\alpha \text {-Glucose } \downarrow \\
\beta \text {-Glucose } \downarrow \\
\text { (all in liver) }\end{array}$ & [149] \\
\hline Human & ALD, NASH & Cirrhosis & ${ }^{1} \mathrm{H}$ NMR & $\begin{array}{c}\text { ALD Cirrhosis: } \\
\text { Isoleucine } \uparrow \text { Valine } \uparrow \\
\text { 1,2-Propanediol } \uparrow \text { Succinate } \uparrow \\
\text { Aspartate } \uparrow \text { Betaine } \uparrow \text { Lactate } \uparrow \\
\text { Glucose } \uparrow \text { Uracil } \uparrow \text { Phenylalanine } \uparrow \\
\text { NASH Cirrhosis: } \\
\text { Leucine } \uparrow \text { Isoleucine } \uparrow \text { Valine } \uparrow \\
\text { 1,2-Propanediol } \uparrow \text { Succinate } \uparrow \\
\text { Aspartate } \uparrow \text { Betaine } \uparrow \text { Lactate } \uparrow \\
\text { Phenylalanine } \uparrow \text { Uracil } \uparrow \text { Uridine } \downarrow \\
\text { Inosine } \downarrow \\
\text { (all in liver) }\end{array}$ & [150] \\
\hline Human & Causes not stated & Cirrhosis & ${ }^{1} \mathrm{H}$ NMR & $\begin{array}{c}\text { Acetate } \uparrow \text { Pyruvate } \uparrow \text { Glutamine } \uparrow \\
\alpha \text {-Ketoglutarate } \uparrow \text { Taurine } \uparrow \\
\text { Glycerol } \uparrow \text { Tyrosine } \uparrow \\
\text { 1-Methylhistidine } \uparrow \\
\text { Phenylalanine } \uparrow \\
\text { N-Acetylglycoproteins } \uparrow \text { LDL } \downarrow \\
\text { VLDL } \downarrow \text { Isoleucine } \downarrow \text { Leucine } \downarrow \\
\text { Valine } \downarrow \text { Acetoacetate } \downarrow \text { Choline } \downarrow \\
\text { (all in serum) }\end{array}$ & [151] \\
\hline
\end{tabular}


Table 3. Cont.

\begin{tabular}{|c|c|c|c|c|c|}
\hline Species & Manipulation/Condition & Pathology & $\begin{array}{c}\text { Analytical } \\
\text { Methodology }\end{array}$ & Metabolites Reported & Ref. \\
\hline Human & Alcohol & $\begin{array}{l}\text { Cirrhosis (mild vs. } \\
\text { severe liver failure) }\end{array}$ & ${ }^{1} \mathrm{H} \mathrm{NMR}$ & $\begin{array}{c}\text { Correlated with severity of } \\
\text { liver failure: } \\
\text { 3-Hydroxybutyrate } \uparrow \text { Alanine } \uparrow \\
\text { Acetate } \uparrow \\
\text { Choline/Phosphocholine } \uparrow \\
\text { (all in serum) }\end{array}$ & [152] \\
\hline Human & Chronic hepatitis B & $\begin{array}{l}\text { Cirrhosis } \\
\text { (compensated vs. } \\
\text { decompensated) }\end{array}$ & ${ }^{1} \mathrm{H} \mathrm{NMR}$ & $\begin{array}{c}\text { Distinguishing between } \\
\text { compensated and } \\
\text { decompensated cirrhosis: } \\
\text { Succinate, Pyruvate, } \\
\text { Phenylalanine, Histidine, Lysine, } \\
\text { Glutamine, Acetone, Glutamate, } \\
\text { Creatine, Alanine } \\
\text { (all in serum) }\end{array}$ & [153] \\
\hline Human & Causes not stated & Cirrhosis & ${ }^{1} \mathrm{H}$ NMR & $\begin{array}{c}\text { Positively correlated with portal } \\
\text { blood proinflammatory cytokines } \\
\text { IL6, TNF } \alpha \text { and IL1 } \beta \text { : } \\
\text { Trimethylamine } \\
\text { Negatively correlated with portal } \\
\text { blood proinflammatory cytokines } \\
\text { IL6, TNF } \alpha \text { and IL1 } \beta \text { : } \\
\text { Acetate, n-Heptanoate } \\
\text { Positively correlated with WBC } \\
\text { and platelet counts: } \\
\text { Threonine, } \alpha \text {-Galactose, } \\
\beta \text {-Glucose } \\
\text { (all in feces) }\end{array}$ & [156] \\
\hline Human & Various liver injuries & $\begin{array}{l}\text { Cirrhosis } \\
\text { (compensated vs. } \\
\text { decompensated) }\end{array}$ & UPLC-QTOFMS & $\begin{array}{c}\text { Lower in LC: } \\
N^{6} \text {-Methyladenosine, } \\
\text { 1-Methyluric acid, } \\
\text { Cinnamic acid, Decenoylcarnitine, } \\
\text { Phenacetylglutamine } \\
\text { (all in urine) }\end{array}$ & [158] \\
\hline Human & $\begin{array}{c}\text { Various etiologies, incl. } \\
\text { HBV, HCV, alcohol, } \\
\text { NASH }\end{array}$ & $\begin{array}{c}\text { Cirrhosis } \\
\text { US vs. Turkish (TR) } \\
\text { population } \\
\text { (dietary) }\end{array}$ & ${ }^{1} \mathrm{H} \mathrm{NMR}$ & $\begin{array}{c}\text { Lactate (Controls and } \\
\text { Decompensated; TR }>\text { US), } \\
\text { Glucose (Controls and } \\
\text { Decompensated; US }>\text { TR ) } \\
\text { (all in plasma) }\end{array}$ & [159] \\
\hline Human & Alcohol & $\begin{array}{l}\text { Acute-on-chronic } \\
\text { liver failure (ACLF) } \\
\text { vs. stable } \\
\text { compensated or } \\
\text { decompensated } \\
\text { cirrhosis (CLF) }\end{array}$ & ${ }^{1} \mathrm{H}$ NMR & $\begin{array}{c}\text { ACLF > CLF: } \\
\text { 3-Hydroxybutyrate, Lactate, } \\
\text { Acetoacetate, Pyruvate, } \\
\text { Glutamine, Glutamate, Creatinine, } \\
\text { Tyrosine, Phenylalanine } \\
\text { (all in serum) }\end{array}$ & [160] \\
\hline Human & Causes not stated & $\begin{array}{l}\text { Stable cirrhosis }(C) \\
\pm \text { encephalopathy } \\
\text { (E) }(C \pm E) \text { and HV }\end{array}$ & ${ }^{1} \mathrm{H} \mathrm{NMR}$ & $\begin{array}{c}\text { C } \pm \mathrm{E}>\mathrm{HV} \text { : } \\
\text { Lactate, Pyruvate, Alanine, } \\
\text { Threonine, Glycine, Aspartate, } \\
\text { Acetoacetate, } \\
\text { 3-Hydroxybutyrate, } \\
\text { Phenylalanine, Tyrosine, } \\
\text { Methionine, Glutamate, } \\
\text { Methylamine, Dimethylamine, } \\
\text { TMAO, Glycerol } \\
\text { C } \pm \mathrm{E}<\mathrm{HV} \text { : } \\
\text { Valine, Glutamine, Histidine, } \\
\text { Arginine } \\
\text { E }>\text { HV: } \\
\text { Leucine, Isoleucine } \\
\text { C }>\text { HV: } \\
\text { Myoinositol } \\
\text { (all in plasma) }\end{array}$ & [161] \\
\hline
\end{tabular}


Table 3. Cont.

\begin{tabular}{|c|c|c|c|c|c|}
\hline Species & Manipulation/Condition & Pathology & $\begin{array}{c}\text { Analytical } \\
\text { Methodology }\end{array}$ & Metabolites Reported & Ref. \\
\hline Human & $\begin{array}{l}\text { Alcohol, HBV or } \\
\text { HCV, NASH }\end{array}$ & $\begin{array}{c}\text { Hepatic } \\
\text { encephalopathy } \\
\text { (HE), cirrhosis (C), } \\
\text { neurological } \\
\text { patients without } \\
\text { liver disease } \\
\text { (NP), HV }\end{array}$ & $\begin{array}{c}\text { UPLC-Orbitrap } \\
\text { MS }\end{array}$ & $\begin{array}{c}\text { HE > NP: } \\
\text { 13x N-Acetyl metabolites, } \\
5 x \text { Glutamate/Glutamine } \\
\text { metabolites, 4x Methionine } \\
\text { metabolites, 4x Phenylalanine } \\
\text { metabolites, 6x Tryptophan } \\
\text { metabolites, 6x Fatty acid } \\
\text { metabolites, Pyruvate, } 5 x \text { Amino } \\
\text { acid derivatives, } 2 \times \text { Dipeptides, } 3 x \\
\text { Bile acids, 3x Nucleoside } \\
\text { derivatives, Dihydrothymine, } 4 x \\
\text { Alcohols and polyols, } \\
\text { Ribitol/Arabitol, Cortisol, } \\
\text { Pyridoxic acid, Phenyl sulfate } \\
\text { HE }<\text { NP: } \\
\text { Alanine, Taurine, Anhydro } \\
\text { sorbitol, Levulinic acid } \\
\text { (both in CSF and plasma) } \\
\text { HE }>\text { C: } \\
\text { 9x N-Acetyl metabolites, } \\
\text { Phenacetylglutamine, } \\
\text { 2x Methionine metabolites, } \\
\text { 2x Phenylalanine metabolites, } \\
\text { 3x Tryptophan metabolites, } \\
\text { 4x Fatty acid metabolites, } \\
\text { Citrulline, 2x Dipeptides, } \\
\text { Taurocholic acid, 3x Nucleosides } \\
\text { and derivatives, Anhydro sorbitol, } \\
\text { 2x Alcohols, Ribitol/Arabitol, } \\
\text { Cortisol, Phenyl sulfate } \\
\text { HE }<\text { C: } \\
\text { Methionine sulfoxide, } \\
\text { Levulinic acid } \\
\text { (all in plasma) }\end{array}$ & [162] \\
\hline Human & Chronic hepatitis C & Fibrosis (F4 vs. F0) & ${ }^{1} \mathrm{H}$ NMR & $\begin{array}{c}\text { F4 vs. F0: } \\
\text { VLDL } \uparrow \text { Citrate } \uparrow \text { Glucose } \uparrow \\
\text { Phenylalanine } \uparrow \text { LDL } \downarrow \text { HDL } \downarrow \\
\text { Choline } \downarrow \text { Acetoacetate } \downarrow \\
\text { Isoleucine/Leucine } \downarrow \\
\text { Creatinine/Creatine } \downarrow \text { Glutamate } \downarrow \\
\text { Glutamine } \downarrow \text { Asparagine } \downarrow \text { Valine } \downarrow \\
\text { Lysine } \downarrow \text { Cysteine } \downarrow \text { Glycerol } \downarrow \\
\text { Arginine } \downarrow \text { Histidine } \downarrow \\
\text { 3-Hydroxybutyrate } \downarrow \\
\text { (all in serum) }\end{array}$ & [163] \\
\hline Rat & TAA & $\begin{array}{l}\text { Fibrosis/Cirrhosis } \\
\text { vs. controls }\end{array}$ & ${ }^{1} \mathrm{H}$ NMR & 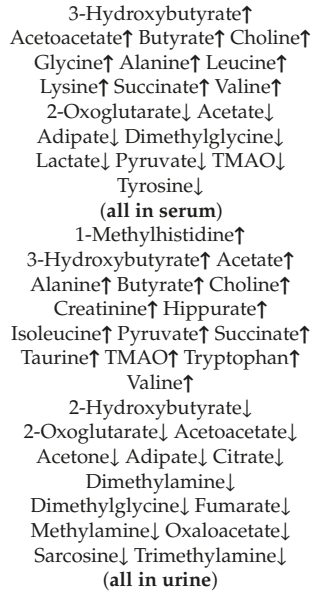 & [165] \\
\hline
\end{tabular}


Table 3. Cont.

\begin{tabular}{|c|c|c|c|c|c|}
\hline Species & Manipulation/Condition & Pathology & $\begin{array}{c}\text { Analytical } \\
\text { Methodology }\end{array}$ & Metabolites Reported & Ref. \\
\hline Rat & Dimethylnitrosamine & Fibrosis & UPLC-QTOFMS & $\begin{array}{c}\mathrm{LPC}(18: 1) \uparrow \mathrm{LPC}(18: 2) \uparrow \\
\mathrm{LPC}(20: 4) \downarrow \mathrm{FA}(22: 6) \downarrow \\
\mathrm{FA}(20: 4) \downarrow \mathrm{FA}(18: 1) \downarrow \mathrm{FA}(18: 2) \downarrow \\
\text { (all in serum) }\end{array}$ & [166] \\
\hline Rat & Dimethylnitrosamine & Fibrosis & UPLC-QTOFMS & $\begin{array}{c}\text { Cholic acid } \uparrow \text { Deoxycholic acid } \uparrow \\
\text { Ursodeoxycholic acid } \uparrow \\
\text { Chenodeoxycholic acid } \uparrow \\
\text { Hyodeoxycholic acid } \uparrow \text { Lithocholic } \\
\text { acid } \uparrow \text { Taurocholic acid } \uparrow \\
\text { Taurodeoxycholic acid } \uparrow \\
\text { Tauroursodeoxycholic acid } \uparrow \\
\text { Taurochenodeoxycholic acid } \uparrow \\
\text { Taurohyodeoxycholic acid } \uparrow \\
\text { Taurolithocholic acid } \uparrow \text { Glycocholic } \\
\text { acid } \uparrow \text { Glycodeoxycholic acid } \uparrow \\
\text { Glycoursodeoxycholic acid } \uparrow \\
\text { Glycochenodeoxycholic acid } \uparrow \\
\text { (all in serum) }\end{array}$ & [167] \\
\hline Rat & $\mathrm{CCl}_{4}$ & Fibrosis & UPLC-QTOFMS & $\begin{array}{c}\text { Cervonoyl ethanolamide } \uparrow \\
\beta \text {-Muricholic acid } \uparrow \\
\text { (all in serum) }\end{array}$ & [168] \\
\hline Rat & $\mathrm{CCl}_{4}$ & $\begin{array}{l}\text { Decompensated } \\
\text { cirrhosis/ascites }\end{array}$ & $\begin{array}{l}\text { UPLC-Orbitrap } \\
\text { MS }\end{array}$ & 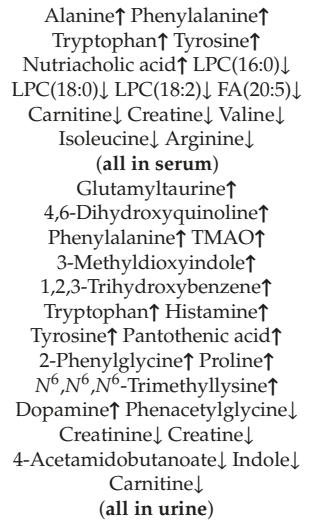 & [169] \\
\hline Rat & $\mathrm{CCl}_{4}$ & Fibrosis & UPLC-QTOFMS & 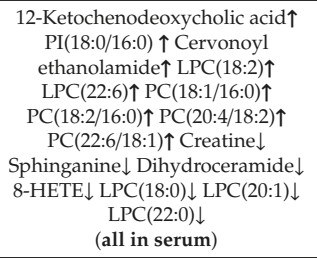 & [170] \\
\hline Rat & Dimethylnitrosamine & Fibrosis & $\begin{array}{c}\text { UPLC-Orbitrap } \\
\text { MS }\end{array}$ & 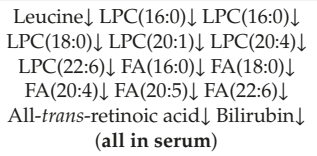 & [172] \\
\hline Rat & $\mathrm{CCl}_{4}$ & Fibrosis & ${ }^{1} \mathrm{H}$ NMR & $\begin{array}{c}\text { 2-Oxoglutarate } \downarrow \text { Citrate } \downarrow \\
\text { Dimethylamine } \downarrow \text { Creatinine } \downarrow \\
\text { Phenacetylglycine } \downarrow \text { Hippurate } \downarrow \\
\text { Taurine } \uparrow \\
\text { (all in urine) }\end{array}$ & [123] \\
\hline Rat & $\mathrm{CCl}_{4}$ & Fibrosis & ${ }^{1} \mathrm{H}$ NMR & $\begin{array}{c}\text { Glucose } \downarrow \text { Lactate } \uparrow \text { Fumarate } \downarrow \\
\text { NADPH } \downarrow \text { Succinate } \uparrow \text { Acetate } \uparrow \\
\text { 3-Hydroxybutyrate } \downarrow \\
\text { UDP-glucose } \uparrow \\
\text { UDP-galactose } \uparrow \\
\text { (in serum and liver) }\end{array}$ & [125] \\
\hline
\end{tabular}




\subsection{NAFL and NASH}

Nonalcoholic fatty liver disease (NAFLD) is the most common liver disorder in Western countries, affecting $17-46 \%$ of adults. NAFLD includes two pathologically-distinct conditions with different prognoses: nonalcoholic fatty liver (NAFL) and nonalcoholic steatohepatitis (NASH). "NAFLD is characterized by excessive hepatic fat accumulation, associated with insulin resistance (IR), and defined by the presence of steatosis in $>5 \%$ of hepatocytes according to histological analysis" [173]. The diagnosis of NAFLD requires the exclusion of chronic alcohol consumption as a cause. NASH is characterized by the presence of steatosis, inflammation, and ballooning degeneration of hepatocytes, with or without fibrosis [174]. NASH can progress to cirrhosis in up to $20 \%$ of cases $[173,174]$. The definitive diagnosis of NASH requires a liver biopsy [173]. A number of biomarkers of NAFLD have been evaluated, including fatty liver index (FLI), NAFLD liver fat score (NAFLD-LFS), hepatic steatosis index (HSI), visceral adiposity index (VAI), and triglyceride $x$ glucose (TyG) index. When steatosis was histologically graded as none $(<5 \%)$, mild $(5-33 \%)$, moderate $(33-66 \%)$, and severe $(>66 \%)$, with the exception of VAI, all biomarkers showed a linear trend across the steatosis grades. The authors concluded, "More research is needed to identify truly independent and quantitative markers of steatosis" [175]. Metabolomics, therefore, has a role to play in delivering biomarkers for steatosis and its progression. Recently, it has been argued that NAFLD patients should be classified into different subtypes dependent upon perturbation of the principal pathways regulating fatty acid homeostasis. Specific serum lipid signatures can be associated with individual mechanisms of progression from steatosis to NASH, and possibly lead to novel and specific NASH therapies [176]. Such metabolomic approaches that help refine definition of the disease phenotype have now been integrated into orthogonal technologies, such as genomics, proteomics, structural biology, imaging [177], and metagenomics.

Investigation of both NAFL and NASH in a metabolomic context is a relatively recent endeavor. Because of the nature of the disease, many investigators have focused on the lipidome. Until a decade ago, the plasma lipidome of NAFLD and whether or not NASH expressed a distinct lipidomic signature were unknown. An early US study examined plasma lipid profiles in both NAFL and NASH compared to healthy controls (HV), and reported significantly increased monounsaturated fatty acids (MUFAs) with an altered pattern of polyunsaturated fatty acids (PUFAs) in both NAFL and NASH. Moreover, the progression of NAFL to NASH was characterized by an increase in the lipoxygenase metabolites 5-HETE, 8-HETE, and 15-HETE. Interestingly, the nonenzymic oxidation product of arachidonic acid, 11-HETE, was significantly increased only in NASH [178]. A Spanish group reported an altered pattern of serum phosphocholines and potentially antioxidant lyso plasmalogens [PC(P-24:0/0:0) and $\mathrm{PC}(\mathrm{P}-22: 0 / 0: 0)]$ in NASH compared to stage 3 hepatic steatosis. Several sphingolipids were also altered in NAFLD compared with healthy subjects. Furthermore, arachidonic acid and glutamate were both decreased in NASH. Metabolic profiling by these authors of an animal model for NAFLD (glycine $\mathrm{N}$-methyltransferase Gnmt-null mice) produced finding consistent with the patient observations [179]. Serum lyso plasmalogens are therefore potential biomarkers for NASH. Another US study of NAFL, NASH and HV found that NAFLD patients had perturbed glutathione metabolism compared to HV, with markedly higher conjugated primary bile acids in plasma. NASH patients displayed lower long-chain fatty acids, higher carnitine and short-chain acyl carnitines, together with several other metabolites. While the metabolomic fingerprints could distinguish NAFL or NASH from HV, they could not distinguish between NAFL and NASH [180]. A ${ }^{1} \mathrm{H}$ NMR-based study in China investigated NAFLD patients and $\mathrm{HV}$, together with mice fed a methionine- and choline-deficient (MCD) diet as a model for NAFLD. Based upon both clinical and animal model findings, four potential biomarkers of NAFLD were proposed: serum glucose, lactate, glutamate/glutamine, and taurine [181]. None of these "usual suspects" provides a basis for evaluating the progression of NAFLD due to lack of specificity. A dietary intervention study in US patients with NAFL examined the effect of insulin sensitivity on the plasma metabolome in NAFL. The pattern of LPCs, in particular LPC(16:0), which was significantly lower in insulin resistant NAFL patients (see Table 4), was put forward to potentially provide biomarkers for NAFL-associated insulin resistance [182]. Serum BA concentrations have also been investigated in 
NASH and reported to be elevated both fasting and after a fatty breakfast designed to contract the gall bladder. Elevated fasting BAs included secondary BAs, which are formed by dihydroxylation of primary BAs by gut microbiota species belonging to the orders Bacteroides, Clostridium, and Escherichia, which may be increased in the dysbiosis associated with NAFLD. Altered patterns of circulating BAs in NASH may contribute to hepatic damage [183]. The pattern of BCAAs and acyl carnitines has been investigated in liver samples from healthy subjects, NAFL, fatty NASH, and nonfatty NASH. Hepatic valine was decreased in NAFL, and all BCAAs and phenylalanine were elevated in NASH, with and without steatosis. Certain carnitine esters were elevated in NAFLD (see Table 4). The findings were interpreted as due to oxidative stress and inflammation in the liver [184]. None of these findings yielded a suitably specific biomarker of NASH or its progression. A US group examined whether or not arachidonic acid-derived eicosanoids could distinguish between NAFL and NASH, since lipotoxicity is a key component of the progression of NAFL to NASH. Several such lipids were altered between NAFL and NASH, including elevated PGE2, 13,14-dihydro-15-keto-PGD2, 11,12-diHETrE, 14,15-diHETrE and attenuated 15-HETE (Table 4). It was reported that 11,12-diHETrE, 13,14-dihydro-15-keto-PGD2 and eicosatetraenedioic acid (20-COOH AA) were the top candidate biomarkers to distinguish NASH from NAFL with an area under the receiver operating characteristic curve (AUROC) of 1 for 11,12-diHETrE and 1 for the combination of 13,14-dihydro-15-keto-PGD2 and 20-COOH AA [185]. If confirmed in other studies, these findings would have great potential as biomarkers for NASH in NAFLD. Another potential distinction between NAFL and NASH are ketone bodies, such as acetoacetate and 3-hydroxybutyrate, which are produced in the liver from fatty acids. NASH patients were found to have lower serum ketone bodies than NAFL patients, with a lower serum total free fatty acid level (Table 4) [186]. Although these findings contribute to an understanding of NASH pathogenesis, they are not useful for the generation of biomarkers of NASH. Examination of increasing severity of NAFL in obese patients revealed $\alpha$-ketoglutarate as the principal marker of NAFL (Table 4) [187]. With a specificity of only $62.5 \%$, $\alpha$-ketoglutarate is unlikely to be a biomarker for NAFL. Patients undergoing bariatric surgery that have a wedge liver biopsy taken routinely during surgery have been investigated with lipidomics and metabolomics. Patients were classified histologically as non-NASH, non-NAFLD, NAFL, and NASH. PNPLA3 I148M (isoleucine $\rightarrow$ methionine) variant was also determined that is more common in NASH. Discovery and validation cohorts were also used. A strong negative correlation was reported between the number of TG double bonds and the TG concentrations in NASH relative to non-NASH livers, for both discovery and validation cohorts. A "NASH ClinLipMet score" was developed based upon (i) clinical variables, (ii) PNPLA3 genotype, (iii) lipidomic data and (iv) metabolomic data. This was highest performing combination biomarker with sensitivity of $85.5 \%$ and specificity of $72.1 \%$ for NASH (Table 4) [188]. In terms of biomarker discovery, the large amount of data were derived only from liver biopsies, and so there are no indications how parts (iii) and (iv) of the aforementioned NASH ClinLipMet score relate to, and can be determined from, serum or plasma. A small clinical study was conducted in liver samples from control, NAFL, and NASH patients in which lipidomic analyses were conducted in liver biopsies. These authors identified a signature comprising 32 lipids that distinguished NASH with $100 \%$ specificity and sensitivity. This signature comprised various phospholipids, sphingolipids, fatty acids, triglycerides, and cholesteryl esters, measured by LC-MS, which we do not believe could represent a viable biomarker for NASH due to its complexity. Furthermore, five fatty acids were identified as accumulating in NASH that were demonstrated to be toxic to HepG2 cells and primary human hepatocytes in culture (see Table 4) [189]. A Chinese urinary metabolomics study compared NAFL patients with normal liver function with NASH patients with abnormal liver function. Many discriminating metabolites were reported (Table 4) [190], although none displayed a large fold-change or was seen as highly specific to NASH. An elegant study was reported containing several large clinical cohorts containing biopsy-proven NASH patients that also had liver fat determined by CT. Serum metabolomics identified the top metabolite associated with liver fat as a mass of $202.1185^{+}$. Databases contained a large number of hits for this mass and so a GWAS strategy was adopted yielding SNPs for the AGXT2 gene whose expressed enzyme produces 
a metabolite, dimethylguanidino valeric acid (DMGV), which matched this mass [191]. In terms of a biomarker for NASH or NAFL, DMGV displayed a wide overlap between control and NASH with approx. $20 \%$ higher mean value for NASH. This is not a viable biomarker and perhaps other ions in their "Top-20" [191] should be investigated. Other investigators chose a single biomarker for progression of NAFL to NASH, i.e. pyroglutamate (5-oxoproline), based upon a serum metabolomic study. 5-Oxoproline had a higher AUROC value than adiponectin, TNF- $\alpha$, or IL-8 [192]. The utility of 5-oxoproline as a biomarker for NASH is doubtful, as it is often found to be elevated in relation to hepatic oxidative stress. We have recently reported a highly statistically significant upregulation of 5-oxoproline in HepG2 cells treated with the experimental anti-HCV drug [193], in the liver of whole-body $\gamma$-irradiated mice [194] and in $\gamma$-irradiated HepG2 cells [195].

An in vivo MRS technique has been applied to patients with biopsy-proven NAFL or NASH. Both high-field ${ }^{1} \mathrm{H}$ and ultra-high-field ${ }^{31} \mathrm{P}$ MRS were employed. Many MRS alterations correlated with NASH, mostly with advanced fibrosis, e.g. phosphoethanolamine/total phosphorus (TP) ratio. ATP/TP declined in advanced fibrosis and ATP flux was lower in NASH [155]. While these rapid noninvasive techniques are useful in a research setting, it is still premature to evaluate their diagnostic potential for NASH. A lipidomic study of patients with chronic hepatitis B virus infection (CHB) with and without NAFLD has been conducted in China. Monounsaturated triacylglycerols (TGs) were found more commonly in NASH patients than non-NASH patients [196]. However, there was considerable overlap between these groups, and examination of the raw data does not support the specificity of monounsaturated TGs, with both saturated and diunsaturated TGs associated with NASH. Patients with steatosis are known to have dysregulation of branched-chain and aromatic amino acids. A metabolomic, transcriptomic and metagenomic study of morbidly obese women with and without steatosis has been reported. The plasma metabolite phenylacetic acid produced from phenylalanine by gut microbiota was the most strongly correlated metabolite to steatosis. Mechanistic studies in human hepatocytes and in mice confirmed this association [197]. Using biopsy-proven patients with normal liver (NL), NAFL, and NASH, serum lipidomics was used to define the pattern of TGs in all three groups. Triglycerides were elevated in the order NAFL $>$ NL $\geq$ NASH. Of the 28 TGs measured, TG(46:0), (48:0), (53:0), (44:1), (48:1), (49:1), (52:1), (53:1), (50:2), (54:5), and (58:2) were always NAFL > $\mathrm{NL}$ and NASH $<$ NAFL. Satisfactory AUROC values were obtained for NAFLD vs. NL. Exclusion of patients with glucose $>136 \mathrm{mg} / \mathrm{dL}$ improved the sensitivity and specificity for NASH vs. NAFL [198]. As with all studies of this nature, there was considerable overlap between the three different clinical states in serum metabolite profiles. A lipidomic investigation in Greece reported differences between NASH, NAFL, and healthy subjects for several lipid groups and for certain free fatty acids in serum (Table 4). The authors proposed that their bioinformatic methods could distinguish between NASH, NAFL, and healthy status based upon the determination of 36 lipids, 61 glycans, and 23 fatty acids. Moreover, the authors stated that they could differentiate with very high accuracy (up to $90 \%$ ) using 10-20 total variables between these three conditions. They also reported that they could robustly discriminate between the presence of fibrosis or not using a model containing 10 lipid species [199]. It is unclear to us at this time how such a complex procedure could be adapted to routine clinical diagnosis.

A large study in Germany measured plasma and urine metabolomic profiles across a wide range of liver fat content (LFC) that had been determined by MRI in 769 selected nondiabetic patients. A wide number of metabolites correlated both positively and negatively with LFC (Table 4). Usual positive associations included BCAAs and aromatic amino acids and their metabolites. A more unusual metabolite correlating with LFC was $7 \alpha$-hydroxy-3-oxo-4-cholestenoate [200], which is a metabolite in the primary bile acid synthesis pathway. Unfortunately, its utility as a potential biomarker for NAFLD is reduced by its occurrence in sterol 27-hydroxylase deficiency, familial hypercholanemia and Zellweger syndrome. A Mexican study targeted 31 acyl carnitines and 7 amino acids in relation to obesity and NAFLD. No biomarkers of NAFLD per se were reported [201]. A search for plasma biomarkers of visceral adipose tissue and hepatic triglyceride content (HTGC) has been reported. A significant number of plasma phospholipids were associated with HTGC (Table 4). Similar findings 
have been reported by other groups. The aromatic amino acids tyrosine and tryptophan were also positively associated with HTGC [202]. No useful biomarkers for NAFL emerged from this study.

As stated earlier, redox changes in the liver can contribute to both steatosis (NAFL) and hyperuricemia (HU). It has been observed that HU often progresses together with NAFLD, and this has stimulated a metabolomic investigation of $\mathrm{HU}, \mathrm{HU}$ that progressed to $\mathrm{HU}$ with NAFLD within one year, HU with NAFLD, and healthy controls. The principal serum changes were upregulated phosphatidic acid and CE(18:0) and downregulated inosine (Table 4) during progression from HU to HU plus NAFLD [203]. Unfortunately, the exact nature of the phosphatidic acid was not given by the authors, although the empirical formula cited corresponded to PA(16:0/16:0), the exact mass given did not, otherwise this could have been a potential biomarker for NAFLD.

A dual investigation in human and mouse liver was conducted in which GC-MS analysis of both human discovery and validation sets found two hepatic metabolites negatively correlated with nonalcoholic steatosis score, i.e., nicotinic acid and hydroquinone. When HFD was supplemented with nicotinic acid or hydroquinone, nicotinic acid prevented fat accumulation in mouse liver and reduced serum ALT (Table 4). The authors discussed the use of nicotinic acid as a lipid lowering agent and the potential of future such studies in identifying novel therapeutic targets for NAFLD [204]. Another dual human and mouse liver investigation conducted a metabolomic and lipidomic analysis of Mat1a-KO and WT mouse liver and serum. MAT1A synthesizes the methylation cofactor $S$-adenosylmethionine. Mat1a-KO mice spontaneously develop steatohepatitis. Based upon the Mat1a-KO metabolome that is associated with NASH, serum of biopsy proven NAFLD patients was also analyzed and compared with Mat1a-KO mouse findings. The metabolomic signature of these mice, comprising high concentrations of triglycerides, diglycerides, fatty acids, ceramides, and oxidized fatty acids, was present in serum of $49 \%$ of NAFLD patients, leading to two subtypes of patient, so-called M-subtype and non-M-subtype. Metabolite patterns also distinguished NAFL from NASH. Potential biomarkers might be used to monitor disease progression and identify novel therapeutic targets [205].

A metabolomic study of Chinese NAFLD patients with and without type-2 diabetes mellitus (T2DM) reported elevated bilirubin, various amino acids, and acyl carnitines, together with oleamide [206]. Many of these metabolic changes were confirmatory of published studies. The elevated acyl carnitines reported are consistent with impaired long-chain fatty acid $\beta$-oxidation. Interestingly, we had previously reported a three-fold elevation of plasma oleamide in $\mathrm{HCV}$-positive patients versus HCV-negative subjects [207]. Apparently, these authors did not test their patients for HCV, despite the high prevalence of HCV in liver disease patients in China [208].

The metabolomics of NAFLD has also been investigated in children and adolescents. A noninvasive breath test was employed to examine 21 volatile organic compounds (VOCs). Compared with children with a normal liver, children with NAFLD had significantly greater breath concentrations of acetaldehyde, acetone, isoprene, pentane, and trimethylamine. It is highly likely that the gut microbiota plays a role in the generation of both acetaldehyde and trimethylamine. We agree with the authors that breath testing represents a potential for screening with diagnostic biomarkers of pediatric NAFLD [209]. However, many of these VOCs may not be specific to NAFLD because of the 17 VOCs identified in the caecal contents of mice, eight, including acetaldehyde, were reported for mice fed either the MCD diet or normal chow [210]. An Italian study recruited children with biopsy-proven NAFLD (64) and matched healthy controls (64). HPLC was used to measure oxidative stress markers that arose from excessive consumption of GSH [211]. In obese Hispanic-American adolescents, with and without NAFLD, untargeted high resolution mass spectrometry demonstrated changes in lipid and amino acid biochemistry with a particular effect on tyrosine metabolism (see Table 4) [212]. The effect of NAFLD with and without obesity, together with small intestine bacterial overgrowth, on the urinary metabolome was examined in Italian children. Data were reported on multiple perturbed host and gut microbiota pathways (Table 4), and in particular, on elevated urine glucose concentrations in NAFLD [213]. Again, none of the reported changes met criteria for a diagnostic biomarker, in particular, the biochemical distinctiveness of the findings. A further Italian study investigated obese adolescents 
with and without NAFLD. Plasma metabolomics established increases in branched-chain and aromatic amino acids, together with certain acyl carnitines in NAFLD subjects (Table 4) [214]. Although one of the elevated metabolites in NAFLD (hydroxydecenoylcarnitine) was an unusual finding, the fold difference between the two groups $( \pm$ NAFLD) was small with large variances and a borderline statistical significance, reducing the opportunity to develop this as a biomarker. Obese adolescents with and without NAFL and with and without metabolic syndrome (MetS) have been studied for their salivary metabolomic changes. Several fatty acids and sugars were reported to differ between these groups (Table 4) [215]. How NAFLD was diagnosed in adolescents, whether by ultrasound or liver enzyme elevations, made a significant difference to the metabolomic findings, especially with lipid profiles, and amino acid and ketone body plasma concentrations [216]. Another NAFLD study in children and adolescents reported changes in certain plasma amino acids and phospholipids (Table 4). These authors generated a model using random forests machine learning with a sensitivity of $73 \%$ and specificity of 97\% for detecting NAFLD. Random forests was applied to a combination of metabolite and clinical data, such as waist circumference, whole-body insulin sensitivity index (based on an oral glucose tolerance test) and blood triglyceride level [217].

Investigations in animal models have been used frequently to understand the mechanisms of NAFLD and to find biomarkers for disease progression. A mechanistic investigation in MCD diet fed mice with NASH, using UPLC-QTOFMS, reported significant decreases in several serum LPCs with marked increases in tauro- $\beta$-muricholate, taurocholate and 12-HETE compared with control mice. These results could be explained by the observed up- and down-regulation of several enzyme and transporter genes. The authors concluded that phospholipid and bile acid metabolism is disrupted in NASH, probably due to enhanced inflammatory signaling in the liver [218]. This group conducted a second study with mice fed MCD, in which they reported an increase in serum oleic and linoleic acids and of nonesterified fatty acids that they attributed to enhanced fatty acid release from white adipose tissue in NASH. They demonstrated that this was due to methionine deficiency and not choline deficiency [219]. Another group fed mice a different NASH-inducing diet based upon lard, cholesterol, and cholic acid. Although this was essentially a proteomic investigation, various key metabolites were measured in liver extracts and found to be altered, including predictable lipid changes, but also perturbations in methionine cycle intermediates (Table 4) [220]. Another strategy for the investigation dietary-induced NASH was reported, whereby livers from mice with a disrupted LDL receptor gene ( $L d l$-null) that had been fed a western diet (WD; $17 \%$ energy as protein, $43 \%$ as carbohydrate, $41 \%$ as fat, and $0.2 \%$ as cholesterol; supplemented with olive oil) were examined. Ldlr-null mice fed regular chow served as controls. WD livers displayed a histology and gene expression profile consistent with NASH. Experiments were conducted by replacing the olive oil supplementation with DHA (22:6n-3). As Table 4 shows, multiple lipid classes were either up- or downregulated by WD + olive oil in this genetic/dietary mouse model of NASH. DHA dietary supplementation was effective at protecting against the effects of WD in this mouse line [221]. The effect of NAFLD progression on hepatic BA pools and 70 genes involved in BA homeostasis have been examined in human liver samples. Expression of CYP7B1 mRNA and protein were highly upregulated in NASH, together with clear changes in glycineand taurine-conjugated BAs away from the classical BA synthesis pathway towards the alternative BA synthetic pathway (Table 4). These findings were interpreted as an attempt by the liver in NASH to minimize hepatotoxicity [222]. Other investigators have used a 16-week high-fat diet (HFD with $60 \%$ calories from fat) in WT mice compared with controls on normal chow (12.7\% calories from fat). This HFD regimen produced NAFLD, which was then investigated by ${ }^{1} \mathrm{H}$ NMR metabolomics in serum, liver and urine. Elevations in serum and liver glucose and lipids were reported, together with a decreased urinary excretion of amino acids (BCAAs, aromatic amino acids), energy metabolites and gut microbiota metabolites [223]. A similar study has been reported in which the mouse sera were analyzed by UPLC-QTOFMS and GC-MS. Glucose was elevated and GSH attenuated after HFD-induced NAFL. Several serum metabolites were altered and related to oxidative stress, inflammation, and mitochondrial dysfunction (Table 4) [224]. Although this was a detailed account of the effects of HFD-NAFLD on 
the metabolome, the findings do not lend themselves readily as biomarkers of NAFLD for reasons of specificity. A different diet feeding regimen has been used to generate NAFLD in the mouse without obesity. This procedure used a high-fat, high-cholesterol, cholate diet (HFDCC) and both liver and plasma were analyzed by GC-TOFMS and UPLC-QTOFMS. Total cholesterol and CE(16:1), (18:1), (18:2), (18:3), (20:1), (20:3), (20:4), (22:5), (22:6) were elevated in liver, together with cholic acid, DGs, TGs, CERs, SMs, LPCs, the PC/PE ratio, while PEs were downregulated. The nonlipid metabolites xylitol, xanthosine, squalene, and phenylethylamine were elevated in liver tissue of HFDCC-fed mice. Citrate, G-1-P, and saccharic acid were all downregulated in these livers. Subtle differences were reported for plasma of HFDCC-fed mice, including elevated total cholesterol, CE(16:1), (18:1), (18:2), (18:3), (20:1), (20:3), (20:4), (22:5), cholic acid, deoxycholic acid, CERs, SMs, and PEs, while FFAs, glycerol, TGs, and LPEs were all diminished in pathological livers [225]. Xanthosine, the ribonucleoside of xanthine, could be a potential biomarker when evaluated in patients. However, it was elevated in liver and its levels in plasma were not reported. Moreover, xanthosine has been reported to be a urinary biomarker for nephropathy in T2DM patients [226] thereby reducing its specificity.

A further means of producing features of NASH in the mouse is with 5-diethoxycarbonyl-1,4dihydrocollidine (DDC). The three mouse strains A/J, C57BL/6J and $\mathrm{PWD} / \mathrm{PhJ}$ were placed on a diet supplemented with $0.1 \%$ DDC or a control diet for 8 weeks. Livers were analyzed for 44 metabolites by targeted MS methods and also subjected to proteomic and RNA-Seq analyses, which showed that many pathways were altered by DDC treatment, in particular, arachidonic acid and $S$-adenosylmethionine metabolism. However, after Bonferroni correction of their findings for multiple comparisons, the following hepatic metabolites were elevated by DDC: putrescine, arginine, citrulline, cAMP, 2-oxoglutarate, asparagine, and glutamate (Table 4). In silico modelling was conducted to understand the effect of DDC on eicosanoid metabolism [227]. Livers from mice fed a HFD were compared with controls in a wide-ranging lipidomics study that analyzed diacylglycerols (DAG), cholesterol esters (CE), phospholipids, plasmalogens, sphingolipids, and eicosanoids. A large number of differences between HFD and controls were observed (Table 4) [228]. Another NASH-generating diet has been employed in mice, that of a high-trans-fat, high-fructose diet (TFD) for 8 weeks (steatosis) and 24 weeks (NASH). These experiments sought to examine flux through the hepatic TCA cycle using ${ }^{13} \mathrm{C}$ NMR-based mass isotopomer analysis, which remained normal during steatosis but was two-fold induced in NASH. In parallel to TCA cycle flux induction, ketogenesis was impaired and hepatic diacylglycerols (DGs), ceramides (CERs) and long-chain acyl carnitines accumulated in the liver (Table 4), suggesting inefficient disposal of free fatty acids. The authors concluded that accumulation of "lipotoxic" metabolites could promote inflammation and the metabolic transition to NASH [229]. As serum or plasma was not analyzed, it is not known whether or not any of the accumulated lipids associated with NASH were also present in the circulation and could be evaluated in patients as potential biomarkers for NAFLD progression.

Correlations between specific gut microbiome species and plasma lipids in mice fed HFD that developed NAFL or NASH. Bacteroides uniformis species decreased while Mucispirillum schaedleri species increased in mice with NASH. Interestingly, Bacteroides uniformis correlated positively with TGs and negatively with FFAs. Mucispirillum schaedleri correlated positively with FFAs, LPC(20:3), LPC(20:4), and DG(16:1/18:2). Mechanistically, it was claimed that Bacteroides uniformis increased specific TGs and decreased hepatic injury and inflammation in diet-induced mice [230]. Clearly, these observations need to be independently evaluated and then investigated in NAFLD patients before potential biomarkers can be proposed.

The $\mathrm{db} / \mathrm{db}$ mouse model of leptin receptor deficiency is currently the most widely-used mouse model of type-2 diabetes mellitus (T2DB). Another means of examining the metabolic pathways associated with NAFL is to reverse the steatosis. Caloric restriction (CR) was applied to obese diabetic $\mathrm{db} / \mathrm{db}$ mice with insulin resistance and steatosis, which were also compared pre- and post-CR to nondiabetic heterozygous $\mathrm{db} / \mathrm{m}$ mice without insulin resistance and steatosis. Compared to $\mathrm{db} / \mathrm{m}$ mice, $\mathrm{db} / \mathrm{db}$ mice had elevated hepatic ketone bodies, lactate, acetate, glutathione, and various 
glycerolipids, in particular, diglycerides and triglycerides, many of which were reversed by CR (Table 4). The transcriptomic findings were consistent with these observations [231]. In addition to the $\mathrm{db} / \mathrm{db}$ mouse, a leptin-deficient obese mouse (ob/ob) has also been developed, which is a model for NAFLD. Homozygous ob/ob mice have been compared with nonsteatotic heterozygous ob/+ mice using high resolution magic-angle spinning (HR MAS) ${ }^{1} \mathrm{H}$ NMR. ${ }^{1} \mathrm{H}$ signals from lipids were highly statistically significantly elevated in ob/ob livers, as expected. Several other molecules involved in betaine ( $N, N, N$-trimethylglycine) metabolism were altered (Table 4) [232].

Rats have also been fed a HFD to induce NASH and serum analyzed by UPLC-QTOFMS. Elevated glucose, triglycerides and cholesterol were indicative of insulin resistance. Altered lipid metabolites involved sphingomyelin (SM), phosphatidylcholine (PC), 13-hydroperoxy-9,11-octadecadienoic acid (13-HpODE), and fatty acids (FA) 20:3, 22:3, 20:1 and phytomonic acid (11,12-methyleneoctadecanoic acid) (Table 4) [233]. This last fatty acid is an unusual finding and, if confirmed, could be evaluated in human samples as a potential biomarker for NASH. Another rat study designed to evaluate the effect of turmeric extract on experimental NASH compared HFD-fed with control-fed rats. UPLC-QTOFMS analysis or serum revealed relatively few upregulated metabolites and a much greater number of downregulated lipids, in particular several steroids, including androgen and corticosteroid metabolites (Table 4) [234]. The only highly statistically significant upregulated metabolite was the fatty acid FA(28:8), which has been described as a marine $\omega-3$ fatty acid [235] derived from dinoflagellate species [236]. If confirmed and a mechanism for its formation in human liver by fatty acid elongases and desaturases can be described, this would represent a potential NASH biomarker. A study was conducted comparing metabolomic profiles of rat and human liver, and, of particular interest, MCD diet-fed rat liver (model for NASH) and liver from NASH patients. Despite the large number of metabolic differences reported between treated and control rat liver and NASH liver and healthy patient liver, very few metabolites corresponded between MCD rat liver and human NASH liver. In fact, in the scores plot presented, healthy rat liver was closer to diseased rat liver than to healthy human liver, which was itself closer to diseased human liver. Asparagine, citrulline, and lysine, together with stearoyl carnitine, were the only metabolites upregulated in both rat MCD liver and human NASH liver (Table 4) [237]. Interestingly, stearoyl carnitine together with $(9 E)$-octadecenoyl carnitine, docosapentaenoic acid and vitamin D2 were elevated in serum of rats fed either HFD (NAFL), MCD diet (NASH), or HFD plus streptozocin (NASH plus T2DM) [238]. These rat observations reduce the potential value of long-chain fatty acyl carnitines, like stearoyl carnitine, as potential biomarkers for clinical NASH or NAFLD progression. Another investigation was conducted in rats focusing on fatty acid profiles in blood cells and the liver of rats fed either a control diet or a HFD/cholesterol diet. Correlations between certain MUFAs and PUFAs were reported for both diets [239]. None of these fatty acids changes were specific enough to be evaluated as biomarkers of NAFLD in patients. Finally, an investigation of the pattern of BAs in serum, liver, and caecal contents was undertaken in rats fed HFD and control diet. Metagenomic analyses established that hyodeoxycholate, which was decreased in both serum and caecal contents of rats fed HFD, was related to the level of the Bacteroidetes phylum. The concentration of cholate that was increased in the caecal contents of rats fed HFD, was correlated with levels of Firmicutes and Verrucomicrobia phyla, but correlated inversely with Bacteroidetes [240]. As the BA pattern appeared to be dependent upon the status of the gut microbiota, the data obtained were not useful for evaluation as biomarkers of NAFLD. 
Table 4. Metabolomic and lipidomic biomarkers of NAFL and NASH.

\begin{tabular}{|c|c|c|c|c|c|}
\hline Species & Manipulation/Condition & Pathology & $\begin{array}{c}\text { Analytical } \\
\text { Methodology }\end{array}$ & Metabolites Reported & Ref. \\
\hline Human & Obesity/metabolic syndrome & $\begin{array}{l}\text { NAFL and NASH } \\
\text { NAFL } \rightarrow \text { NASH }\end{array}$ & HPLC-TQMS & $\begin{array}{c}\mathrm{FA}(14: 0) \uparrow \mathrm{FA}(16: 0) \uparrow \\
\mathrm{FA}(14: 1 \mathrm{n} 5) \uparrow \mathrm{FA}(16: 1 \mathrm{n} 7) \uparrow \\
\mathrm{FA}(18: 2 \mathrm{n} 6) \downarrow \mathrm{FA}(18: 3 \mathrm{n} 6) \uparrow \\
\mathrm{FA}(20: 3 \mathrm{n} 6) \uparrow \\
\mathrm{FA}(22: 6 \mathrm{n} 3) / \mathrm{FA}(22: 5 \mathrm{n} 3) \text { in PC } \\
\text { and PE pools } \downarrow \\
\text { 5-HETE } 8 \text {-HETE } \uparrow \\
\text { 15-HETE } \uparrow \\
\text { (all in plasma) }\end{array}$ & [178] \\
\hline Human & $\begin{array}{l}\text { Nondiabetic. NAFLD } \\
\text { confirmed by liver biopsy }\end{array}$ & NAFLD vs. HV & $\begin{array}{l}\text { UPLC-TQMS } \\
\text { GC-MS }\end{array}$ & $\begin{array}{c}\text { Glycocholate } \uparrow \\
\text { Taurocholate } \uparrow \\
\text { Glycochenodeoxycholate } \uparrow \\
\text { Homocysteine } \uparrow \text { Cysteine } \uparrow \\
\text { GSH } \downarrow \\
\text { Glutamylvaline } \uparrow \\
\gamma \text {-Glutamylleucine } \uparrow \\
\gamma \text {-Glutamylphenylalanine } \uparrow \\
\gamma \text {-Glutamyltyrosine } \uparrow \\
\text { Cysteine-glutathione-disulfide } \downarrow \\
\text { Carnitine } \uparrow \\
\text { Propionylcarnitine } \uparrow \\
\text { 2-Methylbutanoylcarnitine } \uparrow \\
\text { Butanoylcarnitine } \uparrow \\
\text { Tyrosine } \uparrow \text { Glutamate } \uparrow \\
\text { Isoleucine } \uparrow \text { Leucine } \uparrow \\
\text { Valine } \uparrow \text { Taurocholate } \uparrow \\
\text { (all in plasma) }\end{array}$ & [180] \\
\hline Human & $\begin{array}{c}\text { Liver samples from normal } \\
(17), \text { steatosis (4), NASH } \\
\text { (fatty) (14) and NASH (not } \\
\text { fatty) (23) }\end{array}$ & $\mathrm{NAFL} \rightarrow \mathrm{NASH}$ & $\begin{array}{c}\text { UPLC } \\
\text { Orbitrap-MS }\end{array}$ & $\begin{array}{c}\text { Taurocholate } \uparrow \\
\text { Taurodeoxycholate } \uparrow \\
\text { Glycochenodeoxycholate } \uparrow \\
\text { Taurine } \uparrow \\
\text { Cholic acid } \downarrow \\
\text { Glycodeoxycholate } \downarrow \\
\text { (all in liver) } \\
\text { Gene expression data } \\
\text { consistent with the above } \\
\text { (CYP7B1 } \uparrow)\end{array}$ & [222] \\
\hline Human & $\begin{array}{l}\text { Dietary intervention study, } \\
\text { unrelated healthy surgical } \\
\text { liver samples }\end{array}$ & $\begin{array}{c}\text { NAFL ( } 20 \\
\text { insulin-resistant } / 20 \\
\text { insulin sensitive) } \\
\text { vs. control }\end{array}$ & $\begin{array}{l}\text { UPLC-TQMS } \\
\text { GC-MS }\end{array}$ & $\begin{array}{c}\text { Insulin-resistant NAFL vs. } \\
\text { insulin-sensitive NAFL: } \\
\text { Total LPCs } \downarrow \text { LPC(16:0) } \downarrow \\
\text { (all in plasma) }\end{array}$ & [182] \\
\hline Human & $\begin{array}{l}\text { NASH and healthy subjects } \\
\text { given high-fat meal to } \\
\text { stimulate gall bladder } \\
\text { contraction }\end{array}$ & $\begin{array}{l}\text { Fasting and } \\
\text { postprandial serum } \\
\text { from NASH and } \\
\text { healthy subjects }\end{array}$ & UPLC-TQMS & $\begin{array}{c}\text { NASH vs. control } \\
\text { (preprandial): } \\
\text { Total BAs } \uparrow \text { Glyco-BAs } \uparrow \\
\text { Tauro-BAs } \uparrow \\
\text { NASH vs. control } \\
\text { (postprandial): } \\
\text { Mainly Total BAs } \uparrow \\
\text { Glyco-BAs } \uparrow \\
\text { (all in serum) }\end{array}$ & [183] \\
\hline Human & $\begin{array}{c}\text { Normal, Steatosis, NASH } \\
\text { with steatosis, NASH } \\
\text { without steatosis livers }\end{array}$ & $\begin{array}{c}\text { Normal, NAFL, } \\
\text { fatty NASH, } \\
\text { nonfatty-NASH }\end{array}$ & $\begin{array}{c}\text { UPLC } \\
\text { Orbitrap-MS }\end{array}$ & $\begin{array}{c}\text { Control } \rightarrow \text { NAFL: } \\
\text { Acetyl carnitine } \uparrow \\
\text { Lauroyl carnitine } \uparrow \\
\text { Butanoyl carnitine } \uparrow \\
\text { Palmitoyl carnitine } \uparrow \\
\text { Hexanoyl carnitine } \downarrow \text { Valine } \downarrow \\
\text { NAFL } \rightarrow \text { NASH: } \\
\text { Leucine } \uparrow \text { Isoleucine } \uparrow \\
\text { Tyrosine } \uparrow \text { Valine } \uparrow \\
\text { Phenylalanine } \uparrow \\
\text { (all in liver) }\end{array}$ & [184] \\
\hline
\end{tabular}


Table 4. Cont.

\begin{tabular}{|c|c|c|c|c|c|}
\hline Species & Manipulation/Condition & Pathology & $\begin{array}{c}\text { Analytical } \\
\text { Methodology }\end{array}$ & Metabolites Reported & Ref. \\
\hline Human & $\begin{array}{c}\text { Biopsy-proven NAFL, } \\
\text { biopsy-proven NASH and } \\
\text { normal controls with MRI } \\
\text { fat fraction }<5 \%\end{array}$ & $\begin{array}{c}\text { Normal, NAFL and } \\
\text { NASH }\end{array}$ & $\begin{array}{l}\text { UPLC-QTRAP } \\
\text { MS/MS }\end{array}$ & $\begin{array}{c}\text { NAFL } \rightarrow \text { NASH: } \\
\text { PGE2 } \uparrow \\
\text { 13,14-dihydro-15-keto-PGD2 } \uparrow \\
\text { 11,12-diHETrE } \uparrow \\
\text { 14,15-diHETrE } \uparrow \text { 15-HETE } \downarrow \\
\text { [all AA-derived] } \\
\text { (all in plasma) }\end{array}$ & [185] \\
\hline Human & $\begin{array}{l}\text { Obese normal liver, obese } \\
\text { NAFL and obese NASH }\end{array}$ & $\begin{array}{c}\text { Normal, NAFL and } \\
\text { NASH }\end{array}$ & ${ }^{1} \mathrm{H}$ NMR & $\begin{array}{c}\text { LDL-cholesterol } \uparrow \text { Alanine } \uparrow \\
\text { Histidine } \uparrow \text { Phenylalanine } \uparrow \\
\text { Tyrosine } \uparrow \text { Leucine } \uparrow \\
\text { Free fatty acids } \downarrow \text { Citrate } \downarrow \\
\text { 3-Hydroxybutyrate } \downarrow \\
\text { Acetoacetate } \downarrow \\
\text { (all in serum) }\end{array}$ & [186] \\
\hline Human & $\begin{array}{c}\text { Morbid obesity with and } \\
\text { without NAFL }\end{array}$ & $\begin{array}{l}\text { Obesity without } \\
\text { NAFL, mild NAFL, } \\
\text { moderate NAFL, } \\
\text { severe NAFL }\end{array}$ & $\begin{array}{l}\text { UPLC-LITMS } \\
\text { GC-TOFMS } \\
\text { Metabolon, Inc. }\end{array}$ & $\begin{array}{c}\alpha \text {-Ketoglutarate principal } \\
\text { plasma marker with } \\
\text { AUROC of } 0.743 \text {, sensitivity } \\
\text { of } 80 \% \text {, specificity of } 62.5 \% \text {. } \\
\text { (all in plasma) }\end{array}$ & [187] \\
\hline $\begin{array}{l}\text { Human } \\
\text { Mouse }\end{array}$ & $\begin{array}{c}\text { Liver biopsies from patients } \\
\text { with normal liver and } \\
\text { NAFLD } \\
\text { HFD, HFD + nicotinic acid, } \\
\text { HFD + hydroquinone, HFD } \\
+ \text { tert-butylhydroquinone }\end{array}$ & $\begin{array}{l}\text { NASH vs. NAFL } \\
\text { vs. control }\end{array}$ & GC-MS & $\begin{array}{c}\text { Nicotinic acid and } \\
\text { hydroquinone negatively } \\
\text { correlated with steatosis } \\
\text { (NAS) score. } \\
\text { Nicotinic acid } \\
\text { supplementation of HFD } \\
\text { prevented fat accumulation } \\
\text { and improved serum ALT. } \\
\text { (all in liver) }\end{array}$ & [204] \\
\hline Human & $\begin{array}{c}\text { NAFLD, NAFLD + T2DM, } \\
\text { control, evaluated by } \\
\text { ultrasound }\end{array}$ & $\begin{array}{l}\text { NAFLD, NAFLD + } \\
\text { T2DM, control }\end{array}$ & UPLC-QTOFMS & 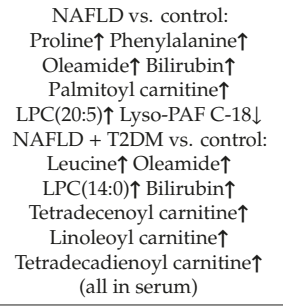 & [206] \\
\hline Human & $\begin{array}{c}\text { Hyperuricemia (HU), } \\
\text { HU+NAFLD, HU } \\
\text { progressed to HU+NAFLD, } \\
\text { healthy controls }\end{array}$ & $\begin{array}{c}\text { HU, initial } \\
\text { HU+NAFLD, } \\
\text { initial } \\
\text { HU } \rightarrow \text { outcome } \\
\text { HU+NAFLD, } \\
\text { healthy controls }\end{array}$ & UPLC-QTOFMS & 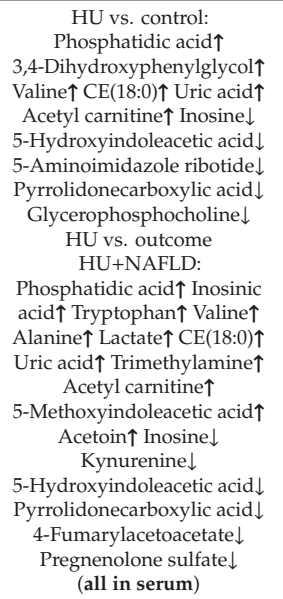 & [203] \\
\hline
\end{tabular}


Table 4. Cont.

\begin{tabular}{|c|c|c|c|c|c|}
\hline Species & Manipulation/Condition & Pathology & $\begin{array}{c}\text { Analytical } \\
\text { Methodology }\end{array}$ & Metabolites Reported & Ref. \\
\hline Human & $\begin{array}{l}\text { Bariatric surgery patients } \\
\text { with wedge liver biopsy } \\
\text { during surgery classified } \\
\text { histologically as non-NASH, } \\
\text { non-NAFLD, NAFL and } \\
\text { NASH. PNPLA3 I148M } \\
\text { variant also determined } \\
\text { (more common in NASH). } \\
\text { Discovery and validation } \\
\text { cohorts used. }\end{array}$ & $\begin{array}{l}\text { non-NASH vs. } \\
\text { non-NAFLD vs. } \\
\text { NAFL vs. NASH }\end{array}$ & $\begin{array}{l}\text { UPLC-QTOFMS } \\
\text { 2D-GC-TOFMS }\end{array}$ & $\begin{array}{l}\text { Strong negative correlation } \\
\text { between number of TG } \\
\text { double bonds to TG } \\
\text { concentrations in NASH } \\
\text { relative to non-NASH for } \\
\text { both discovery and } \\
\text { validation cohorts. A } \\
\text { "NASH ClinLipMet score" } \\
\text { was developed based upon } \\
\text { (i) clinical variables, (ii) } \\
\text { PNPLA3 genotype, (iii) } \\
\text { lipidomic data and (iv) } \\
\text { metabolomic data. This was } \\
\text { highest performing } \\
\text { combination biomarker with } \\
\text { sensitivity of } 85.5 \% \text { and } \\
\text { specificity of } 72.1 \% \text { for } \\
\text { NASH. } \\
\text { (all in liver) }\end{array}$ & [188] \\
\hline Human & $\begin{array}{c}\text { Normal liver, NAFL liver, } \\
\text { NASH liver }\end{array}$ & $\begin{array}{l}\text { NASH vs. NAFLD } \\
\text { vs. control } \\
\text { lipidomics }\end{array}$ & $\begin{array}{l}\text { UPLC-TQMS } \\
\text { GC-MS }\end{array}$ & $\begin{array}{c}\text { Thirty-two lipids } \\
\text { discriminated NASH with } \\
100 \% \text { sensitivity and } \\
\text { specificity. Accumulated } \\
\text { hepatotoxic lipids in NASH } \\
\text { included FA(14:0), FA(16:0), } \\
\text { FA(16:1n-7), FA(18:1n-7) and } \\
\text { FA(18:1n-9). Reduced in } \\
\text { NASH: FA(20:4n-6), } \\
\text { FA(20:5n-3), FA(22:6n-3), } \\
\text { total CER, total SM, total PI, } \\
\text { total PS, total PE, total PC. } \\
\text { (all in liver) }\end{array}$ & [189] \\
\hline Human & $\begin{array}{l}\text { Nondiabetic NAFL patients } \\
\text { with normal liver function, } \\
\text { NASH with abnormal liver } \\
\text { function, healthy controls. }\end{array}$ & $\begin{array}{l}\text { NASH vs. NAFL } \\
\text { vs. control urines. }\end{array}$ & LC-TQMS & $\begin{array}{c}\text { NASH vs. control: } \\
\text { Lysine } \uparrow \text { Valine } \uparrow \text { Citrulline } \uparrow \\
\text { Arginine } \uparrow \text { Threonine } \uparrow \\
\text { Tyrosine } \uparrow \text { Leucine } \uparrow \\
\text { Hippurate } \uparrow \\
\text { 3-Indoleacetate } \uparrow \\
\text { 5-Hydroxyindoleacetate } \downarrow \\
\text { 3-Indoleformate } \downarrow \text { Cortisol } \downarrow \\
\text { NASH vs. NAFL: } \\
\text { Methyl xanthine } \uparrow \\
\text { Tryptophan } \uparrow \\
\text { 3-Indoleacetate } \uparrow \text { Gluconate } \uparrow \\
\text { Proline } \downarrow \\
\text { (all in urine) }\end{array}$ & [190] \\
\hline Human & $\begin{array}{l}\text { Several large clinical cohorts } \\
\text { with CT-defined liver fat } \\
\text { plus NASH patients. }\end{array}$ & NASH vs. controls & $\begin{array}{l}\text { UPLC-Q- } \\
\text { Orbitrap-MS }\end{array}$ & $\begin{array}{c}\text { Top metabolite correlated } \\
\text { with liver fat was } 202.1185^{+} \text {, } \\
\text { which produced } 24 \text { hits in } \\
\text { HMDB. Dimethylguanidino } \\
\text { valeric acid (DMGV) chosen } \\
\text { on basis of GWAS, which } \\
\text { found SNPs for AGXT2 that } \\
\text { produces DMGV. } \\
\text { (all in plasma) }\end{array}$ & [191] \\
\hline Human & $\begin{array}{l}\text { NAFLD criteria met/not met } \\
\text { at baseline, after dietary } \\
\text { manipulation. }\end{array}$ & $\begin{array}{c}\text { Non-NAFLD, } \\
\text { Non-NAFLD } \rightarrow \\
\text { NAFLD, NAFLD } \rightarrow \\
\text { Non-NAFLD }\end{array}$ & UPLC-QTOFMS & $\begin{array}{c}\text { Phospholipid and } \\
\text { sphingolipid changes not of } \\
\text { great statistical significance. } \\
\text { Also lipid groups, not } \\
\text { individual lipids, given only. } \\
\text { (all in serum) }\end{array}$ & [241] \\
\hline
\end{tabular}


Table 4. Cont.

\begin{tabular}{|c|c|c|c|c|c|}
\hline Species & Manipulation/Condition & Pathology & $\begin{array}{c}\text { Analytical } \\
\text { Methodology }\end{array}$ & Metabolites Reported & Ref. \\
\hline Human & $\begin{array}{c}\text { NAFL and NASH based } \\
\text { upon liver biopsy, } \\
\text { healthy controls. }\end{array}$ & $\begin{array}{l}\text { NASH vs. NAFL } \\
\text { vs. controls }\end{array}$ & $\begin{array}{c}\text { HPLC- } \\
\text { Orbitrap-MS }\end{array}$ & $\begin{array}{l}\text { Five metabolites increased } \\
\text { control } \rightarrow \text { NAFL } \rightarrow \text { NASH - } \\
\text { Uracil, } \alpha \text {-Linolenic acid } \\
\text { (all-cis-9,12,15-octadecatrienoic } \\
\text { acid), Glutamate, Glutamine } \\
\text { and 5-Oxoproline, which } \\
\text { was chosen as a biomarker } \\
\text { with a better AUROC for } \\
\text { NASH vs. NAFL, than } \\
\text { adiponectin, } \\
\text { TNF- } \alpha \text {, or IL- } 8 \text {. } \\
\text { (all in serum) }\end{array}$ & [192] \\
\hline Human & $\begin{array}{l}\text { NAFL and NASH confirmed } \\
\text { by liver biopsy }\end{array}$ & NASH vs. NAFL & $\begin{array}{l}\text { High-field }{ }^{1} \mathrm{H} \\
\text { MRS and } \\
\text { ultra-high-field } \\
{ }^{31} \mathrm{P} \text { MRS } \\
\quad \text { (in vivo) }\end{array}$ & $\begin{array}{c}\text { Many MRS alterations } \\
\text { correlated with } \\
\text { NAFL } \rightarrow \text { NASH, mostly with } \\
\text { advanced fibrosis, e.g. } \\
\text { phosphoethanolamine/total } \\
\text { phosphorus (TP) ratio. } \\
\text { ATP/TP } \downarrow \text { in advanced } \\
\text { fibrosis and ATP flux } \downarrow \\
\text { in NASH }\end{array}$ & [155] \\
\hline Human & $\begin{array}{c}\text { Chronic hepatitis B (CHB) } \\
\text { with biopsy-proven NAFLD } \\
\text { and without NAFLD, } \\
\text { healthy controls }\end{array}$ & $\begin{array}{l}\text { CHB +NAFLD vs. } \\
\text { CHB-NAFLD vs. } \\
\text { controls }\end{array}$ & UPLC-QTOFMS & $\begin{array}{l}\text { Most neutral lipids and } \\
\text { ceramides were elevated in } \\
\text { CHB+NAFLD but decreased } \\
\text { in CHB-NAFLD vs. healthy } \\
\text { controls. Monounsaturated } \\
\text { TGs were a good predictor } \\
\text { of NASH, superior to } \\
\text { cytokeratin-18 or ALT. }\end{array}$ & [196] \\
\hline Human & $\begin{array}{l}\text { Hepatic steatosis in } \\
\text { morbidly obese women }\end{array}$ & $\begin{array}{l}\text { Metagenomic } \\
\text { signature of } \\
\text { hepatic steatosis }\end{array}$ & $\begin{array}{l}{ }^{1} \mathrm{H} \text { NMR (urine } \\
\text { and plasma) } \\
\text { UPLC-TQMS } \\
\text { (plasma) }\end{array}$ & $\begin{array}{c}\text { Microbiota metabolite } \\
\text { produced from } \\
\text { phenylalanine, phenylacetic } \\
\text { acid (PAA) associated } \\
\text { with steatosis. }\end{array}$ & [197] \\
\hline Human & $\begin{array}{c}\text { Biopsy-proven subjects with } \\
\text { normal liver (NL), NAFL } \\
\text { and NASH }\end{array}$ & $\begin{array}{l}\text { NASH vs. NAFL } \\
\text { vs. NL discovery } \\
\text { and validation } \\
\text { cohorts }\end{array}$ & UPLC-QTOFMS & $\begin{array}{c}\text { Triglycerides are elevated } \\
\text { NAFL }>\text { NL } \geq \text { NASH. Of the } \\
28 \text { TGs measured, TG(46:0), } \\
(48: 0),(53: 0),(44: 1),(48: 1) \\
(49: 1),(52: 1),(53: 1),(50: 2), \\
(54: 5) \text { and }(58: 2) \text { were always } \\
\text { NAFL }>\text { NL and NASH < } \\
\text { NAFL. } \\
(\text { all in serum) }\end{array}$ & [198] \\
\hline Human & $\begin{array}{c}\text { Large study of } 769 \\
\text { nondiabetic patients with } \\
\text { liver fat content measured } \\
\text { by MRI and correlated with } \\
\text { metabolite profiles of urine } \\
\text { and fasting plasma }\end{array}$ & $\begin{array}{l}613 \text { plasma and } 587 \\
\text { urine samples } \\
\text { across a range of } \\
\text { liver pathologies } \\
\text { (34.7\% with } \\
\text { steatosis) }\end{array}$ & $\begin{array}{l}\text { UPLC-LITMS } \\
{ }^{1} \mathrm{H} \text { NMR }\end{array}$ & $\begin{array}{c}\text { Associations in plasma } \\
\text { with LFC: } \\
\text { BCAAs } \uparrow \text { Aromatic amino } \\
\text { acids } \uparrow \text { Dipeptides } \uparrow \text { Proline } \uparrow \\
\text { Tryptophan } \uparrow \text { Indoleacetate } \uparrow \\
\text { Urate } \uparrow \text { Piperine } \uparrow \\
\text { 7 } \alpha \text {-Hydroxy-3-oxo-cholestenoate } \uparrow \\
\text { Ether-PCs } \downarrow \\
\text { 3-Phenylpropionate } \downarrow \text { Proline } \\
\text { betaine } \downarrow \\
\text { Associations in urine } \\
\text { with LFC: } \\
\text { BCAA derivatives } \uparrow \text { Lactate } \uparrow \\
\text { Isovalerylglycine } \downarrow \\
\text { Isobutyrylglycine } \downarrow \\
\gamma \text {-Glutamylthreonine } \downarrow \\
\text { 4-Vinylphenol sulfate } \downarrow \\
\text { Hippurate } \downarrow \\
\text { Cinnamoylglycine } \downarrow\end{array}$ & $\uparrow$ \\
\hline
\end{tabular}


Table 4. Cont.

\begin{tabular}{|c|c|c|c|c|c|}
\hline Species & Manipulation/Condition & Pathology & $\begin{array}{c}\text { Analytical } \\
\text { Methodology }\end{array}$ & Metabolites Reported & Ref. \\
\hline Human & $\begin{array}{l}\text { NAFLD determined by } \\
\text { hepatic ultrasound }\end{array}$ & $\begin{array}{c}\text { BMI }<25 \text { vs. BMI }> \\
30 \text { vs. BMI }>30 \\
\text { with NAFLD }\end{array}$ & $\begin{array}{l}\text { TQMS for } 31 \\
\text { acyl carnitines } \\
\text { and } 7 \text { amino } \\
\text { acids }\end{array}$ & $\begin{array}{l}\text { Family history predicted } \\
\text { obesity correlating with } \\
\text { amino acids that contributed } \\
\text { to an increase in specific acyl } \\
\text { carnitines. Excess FFAs } \\
\text { related to obesity were } \\
\text { associated with NAFLD. }\end{array}$ & [201] \\
\hline Human & $\begin{array}{l}\text { Patients with normal fasting } \\
\text { glucose. Visceral adipose } \\
\text { tissue (VAT) assessed by } \\
\text { MRI. Hepatic TG content } \\
\text { (HTGC) determined by } \\
\text { proton-MR spectroscopy. }\end{array}$ & $\begin{array}{c}\text { Range of VAT and } \\
\text { HTGC }\end{array}$ & ESI-FIA-MS/MS & $\begin{array}{c}\text { Associated with HTGC: } \\
\text { LPC(14:0), PC(28:1), } \\
\text { PC(30:0), PC(32:1), PC(32:2), } \\
\text { PC(34:1), PC(34:3), PC(34:4), } \\
\text { PC(36:1), PC(36:2), PC(36:3), } \\
\text { PC(36:6), PC(38:3), PC(38:5), } \\
\text { PC(40:4), PC(40:5), SM(22:3), } \\
\text { Tryptophan, Tyrosine } \\
\text { (all in plasma) }\end{array}$ & [202] \\
\hline Human & $\begin{array}{c}\text { NAFL and NASH } \\
\text { determined by liver biopsy } \\
\text { and healthy controls by } \\
\text { ultrasound and liver } \\
\text { enzymes }\end{array}$ & $\begin{array}{l}\text { NASH vs. NAFL } \\
\text { vs. controls }\end{array}$ & $\begin{array}{l}\text { UPLC-Orbitrap-MS } \\
\text { GC }\end{array}$ & $\begin{array}{c}\text { Lipid group trends: } \\
\text { DG: NASH }>\text { NAFL }> \\
\text { healthy } \\
\text { PG: NASH } \approx \text { NAFL }> \\
\text { healthy } \\
\text { PA: NASH } \approx \text { NAFL }> \\
\text { healthy } \\
\text { AcCa: NASH }<\text { NAFL } \approx \\
\text { healthy } \\
\text { CE: NASH }<\text { NAFL }< \\
\text { healthy } \\
\text { LPC: NASH }<\text { NAFL } \approx \\
\text { healthy } \\
\text { SM: NASH }<\text { NAFL } \approx \\
\text { healthy } \\
\text { FA(16:0): NASH }>\text { NAFL }> \\
\text { healthy } \\
\text { FA(16:1n-7cis): NASH }> \\
\text { NAFL }>\text { healthy } \\
\text { FA(18:1n-9cis): NASH }> \\
\text { NAFL }>\text { healthy } \\
\text { FA(18:2n-6): NASH }<\text { NAFL } \\
<\text { healthy } \\
\text { FA(20:4n-6): NASH }<\text { NAFL } \\
<\text { healthy }\end{array}$ & [199] \\
\hline Human & $\begin{array}{l}\text { Children, overweight or } \\
\text { obese, with or without } \\
\text { clinical/radiological signs of } \\
\text { NAFLD }\end{array}$ & NAFLD vs. control & $\begin{array}{l}\text { Selective ion } \\
\text { flow tube mass } \\
\text { spectrometry } \\
\text { (SIFT-MS) }\end{array}$ & $\begin{array}{c}\text { Acetaldehyde } \uparrow \text { Acetone } \uparrow \\
\text { Isoprene } \uparrow \text { Pentane } \uparrow \\
\text { Trimethylamine } \uparrow \\
\text { (all in breath) }\end{array}$ & [209] \\
\hline Human & $\begin{array}{c}\text { Children with } \\
\text { biopsy-proven NAFLD and } \\
\text { matched healthy controls }\end{array}$ & NAFLD vs. control & HPLC & $\begin{array}{c}\text { Homocysteine } \uparrow \text { Cysteine } \uparrow \\
\text { CysGly } \uparrow \text { GSH } \downarrow \\
\text { (all in plasma) }\end{array}$ & [211] \\
\hline Human & $\begin{array}{l}\text { Children with obesity and } \\
\text { NAFL confirmed by MRS } \\
\text { and matched obese controls }\end{array}$ & NAFL vs. control & $\begin{array}{l}\text { UPLC-Q- } \\
\text { Orbitrap-MS }\end{array}$ & $\begin{array}{c}\text { Tyrosine } \uparrow \text { Glutamate } \uparrow \\
\text { Octanoic acid } \uparrow \text { Linoleic } \\
\text { acid } \downarrow \\
\text { (all in plasma) }\end{array}$ & [212] \\
\hline Human & $\begin{array}{l}\text { Children with obesity, } \\
\text { NAFL, NASH and } \\
\text { healthy controls }\end{array}$ & $\begin{array}{l}\text { NASH vs. NAFL } \\
\text { vs. control }\end{array}$ & GC-MS & $\begin{array}{c}\text { 1-Butanol } \uparrow \text { (in NAFL) } \\
\text { 1-Pentanol } \uparrow \text { (in NAFL) } \downarrow \text { (in } \\
\text { NASH) Phenol } \uparrow \text { (in NAFL) } \\
\text { 2-Butanone } \uparrow \text { (in NAFL and } \\
\text { NASH) } \\
\text { 4-Methyl-2-pentanone } \downarrow \text { (in } \\
\text { NAFL) } \uparrow \text { (in NASH) } \\
\text { (all in feces) } \\
\text { Metagenomics also } \\
\text { conducted. Correlations } \\
\text { with NAFLD and certain } \\
\text { VOCs reported }\end{array}$ & [242] \\
\hline
\end{tabular}


Table 4. Cont.

\begin{tabular}{|c|c|c|c|c|c|}
\hline Species & Manipulation/Condition & Pathology & $\begin{array}{c}\text { Analytical } \\
\text { Methodology }\end{array}$ & Metabolites Reported & Ref. \\
\hline Human & $\begin{array}{l}\text { Children with NAFLD, with } \\
\text { or without obesity }\end{array}$ & $\begin{array}{c}\text { Obese - NAFL, } \\
\text { obese + NAFL, } \\
\text { normal weight } \\
\text { healthy controls }\end{array}$ & GC-MS & $\begin{array}{c}\text { NAFL vs. control: } \\
\text { Glucose } \uparrow \\
\text { 1-Methylhistidine } \uparrow \\
\text { Pseudouridine } \uparrow \text { Glycolic } \\
\text { acid } \uparrow \text { Mannose } \downarrow p \text {-Cresol } \\
\text { sulfate } \downarrow \text { Kynurenine } \downarrow \\
\text { Hydroquinone } \downarrow \text { Adipate } \downarrow \\
\text { Phenylacetic acid } \downarrow \\
\text { Small intestine bacterial } \\
\text { overgrowth (SIBO): } \\
\text { Glycolic acid } \uparrow \text { Mannose } \uparrow \\
\text { Valine } \downarrow p \text {-Cresol sulfate } \downarrow \\
\text { Butanoate } \downarrow \text { Adipate } \downarrow \\
\text { (all in urine) }\end{array}$ & [213] \\
\hline Human & $\begin{array}{l}\text { Children with obesity, with } \\
\text { and without NAFLD } \\
\text { assessed by MRI }\end{array}$ & $\begin{array}{l}\text { Obese + NAFLD vs. } \\
\text { obese - NAFLD }\end{array}$ & UPLC-QTRAP-MS & $\begin{array}{c}\text { Isoleucine } \uparrow \text { Leucine } \uparrow \\
\text { Valine } \uparrow \text { C4-carnitine } \uparrow \\
\text { C5-carnitine } \uparrow \\
\text { C14:1-OH-carnitine } \uparrow \\
\text { Tryptophan } \uparrow \text { Lysine } \uparrow \\
\text { Glutamate } \uparrow \text { PC(32:1) } \uparrow \\
\text { (all in plasma) }\end{array}$ & [214] \\
\hline Human & $\begin{array}{c}\text { Children with obesity, with } \\
\text { and without NAFL assessed } \\
\text { by ultrasound (US), with } \\
\text { and without metabolic } \\
\text { syndrome (MetS) and } \\
\text { nonobese controls }\end{array}$ & $\begin{array}{c}\text { Obese + NAFL vs. } \\
\text { obese - NAFL } \\
\text { Obese + MetS vs. } \\
\text { obese - MetS }\end{array}$ & GC-MS & $\begin{array}{c}\text { Obese - NAFL vs. controls: } \\
\text { Palmitate } \uparrow \text { Myristate } \uparrow \text { Urea } \uparrow \\
N \text {-Acetylgalactosamine } \uparrow \\
\text { Maltose } \uparrow \text { Gluconate } \uparrow \\
\text { Isoleucine } \uparrow \\
\text { Hydroxybutanoate } \downarrow \text { Malate } \downarrow \\
\text { Obese + NAFL vs. controls: } \\
\text { Laurate } \uparrow \text { Maltose } \uparrow \\
\text { (all in saliva) }\end{array}$ & {$[215]$} \\
\hline Human & $\begin{array}{l}\text { Adolescents with NAFLD } \\
\text { assessed by US or ALT/AST }\end{array}$ & $\begin{array}{l}\text { US vs. ALT vs. } \\
\text { AST diagnostic } \\
\text { methods }\end{array}$ & $\begin{array}{l}\text { Biochemical } \\
\text { lipid analysis } \\
{ }^{1} \mathrm{H} \text { NMR }\end{array}$ & $\begin{array}{l}\text { Many differences in lipid } \\
\text { profiles, amino acids } \\
\text { (alanine, glutamine, } \\
\text { histidine; BCAAs; aromatic } \\
\text { amino acids) and ketone } \\
\text { bodies (acetate, acetoacetate, } \\
\beta \text {-hydroxybutyrate) } \\
\text { (all in plasma) }\end{array}$ & [216] \\
\hline Human & $\begin{array}{c}\text { Adolescents with obesity } \\
\text { and with or without NAFLD } \\
\text { confirmed by MRI }\end{array}$ & $\begin{array}{l}\text { NAFLD vs. } \\
\text { non-NAFLD }\end{array}$ & $\begin{array}{l}\text { UPLC-Q- } \\
\text { Orbitrap-MS }\end{array}$ & $\begin{array}{c}\text { Leucine/Isoleucine } \uparrow \\
\text { Tryptophan } \uparrow \text { Serine } \downarrow \\
\text { Dihydrothymine } \downarrow \\
\text { LPE(20:0) } \downarrow \text { LPC(18:1) } \downarrow \\
\text { (all in plasma) }\end{array}$ & [217] \\
\hline Human & $\begin{array}{l}\text { Children with or without } \\
\text { NAFLD confirmed by } \\
\text { ultrasound and } \\
\text { liver enzymes }\end{array}$ & $\begin{array}{l}\text { NAFLD vs. } \\
\text { non-NAFLD }\end{array}$ & GC-MS & $\begin{array}{l}\text { 24-h Urinary steroid profiles: } \\
\text { Cortisol (obese controls) } \uparrow \\
\text { Tetrahydrocortisone } \\
\text { (NAFLD) } \uparrow \\
\text { Overall data pointed to } \\
5 \alpha \text {-reductase } \uparrow, \\
\text { 21-hydroxylase } \uparrow \text { and } \\
11 \beta \text {-hydroxysteroid } \\
\text { dehydrogenase } 1 \downarrow\end{array}$ & [243] \\
\hline $\begin{array}{l}\text { Human } \\
\text { Mouse }\end{array}$ & $\begin{array}{l}\text { Morbidly obese, nondiabetic } \\
\text { Gnmt-null vs. WT }\end{array}$ & $\begin{array}{l}\text { NAFL } \rightarrow \text { NASH } \\
\text { NASH vs. control }\end{array}$ & UPLC-QTOFMS & 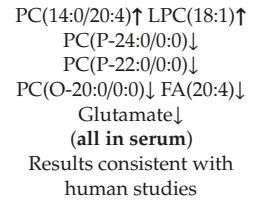 & [179] \\
\hline
\end{tabular}


Table 4. Cont.

\begin{tabular}{|c|c|c|c|c|c|}
\hline Species & Manipulation/Condition & Pathology & $\begin{array}{c}\text { Analytical } \\
\text { Methodology }\end{array}$ & Metabolites Reported & Ref. \\
\hline $\begin{array}{l}\text { Mouse } \\
\text { Human }\end{array}$ & $\begin{array}{l}\text { Methionine and choline } \\
\text { deficient diet (MCD) } \\
\text { HBV-negative, NAFLD } \\
\text { confirmed by liver biopsy }\end{array}$ & $\begin{array}{l}\text { NASH vs. NAFL } \\
\text { vs. control }\end{array}$ & ${ }^{1} \mathrm{H}$ NMR & $\begin{array}{c}\text { Glucose } \uparrow \text { Lactate } \uparrow \\
\text { Glutamate } \uparrow \text { Taurine } \uparrow \\
\text { TG } \uparrow \text { Total cholesterol } \uparrow \\
\text { LDL cholesterol } \uparrow \\
\text { Glucose } \uparrow \text { Lactate } \uparrow \\
\text { Glutamate } \uparrow \text { Taurine } \uparrow \\
\text { (all in serum) }\end{array}$ & [181] \\
\hline $\begin{array}{l}\text { Mouse } \\
\text { Human }\end{array}$ & $\begin{array}{l}\text { Mat } 1 a-K O \text { vs. WT mouse } \\
\text { liver and serum metabolome } \\
\text { NAFL and NASH discovery } \\
\text { and validation cohorts }\end{array}$ & $\begin{array}{c}\text { Mat1a-KO vs. WT } \\
\text { Clustering analysis } \\
\text { into M-subtype } \\
\text { and } \\
\text { Non-M-subtype } \\
\text { based upon mouse } \\
\text { metabolomes }\end{array}$ & UPLC-QTOFMS & $\begin{array}{c}\text { M-subtype NASH } \\
\text { biomarkers: } \\
\text { Amino acids (5), Fatty acyls } \\
\text { (8), Triglycerides (3), } \\
\text { Glycerophospholipids (37), } \\
\text { Sphingomyelins (1) } \\
\text { Non-M-subtype NASH } \\
\text { biomarkers: } \\
\text { Amino acids (1), Fatty acids } \\
\text { (1), Bile acids (1), } \\
\text { Triglycerides (3) } \\
\text { M-subtype patients: } 34 \% \\
\text { NASH } \\
\text { Non-M-subtype patients: } \\
\text { 39\% NASH }\end{array}$ & [205] \\
\hline Mouse & MCD & NASH vs. control & UPLC-QTOFMS & $\begin{array}{c}\text { Tauro- } \beta \text {-muricholate } \uparrow \\
\text { Taurocholate } \uparrow 12 \text {-HETE } \uparrow \\
\text { LPC }(16: 0) \downarrow \text { LPC }(18: 0) \downarrow \\
\text { LPC }(18: 1) \downarrow \\
\text { (all in serum) }\end{array}$ & [218] \\
\hline Mouse & $\begin{array}{c}\text { MCD vs. } \\
\text { choline-supplemented MCD } \\
\text { (MCS) }\end{array}$ & $\begin{array}{l}\text { Differential effects } \\
\text { of methionine and } \\
\text { choline deficiency }\end{array}$ & UPLC-QTOFMS & $\begin{array}{c}\text { MCD vs MCS: } \\
\text { Oleic acid } \uparrow \text { Linoleic acid } \uparrow \\
\text { Total nonesterified fatty } \\
\text { acids } \uparrow \\
\text { (all in serum) }\end{array}$ & [219] \\
\hline Mouse & $\begin{array}{l}\text { NASH-inducing diet }(35 \% \\
\text { lard, } 1.25 \% \text { cholesterol, } 0.5 \% \\
\text { sodium cholate) }\end{array}$ & NAFLD vs. control & HPLC-TQMS & $\begin{array}{c}\text { Glycerol } \uparrow \text { Free cholesterol } \uparrow \\
\text { Esterified cholesterol } \uparrow \\
\text { Putrescine } \uparrow \\
N^{8} \text {-Acetylspermidine } \downarrow \\
\text { Spermine } \downarrow \text { Adenine } \downarrow \\
\text { Adenosine } \downarrow \text { Homocysteine } \downarrow \\
\text { Methylthioadenosine } \downarrow \\
\text { S-Adenosylhomocysteine } \downarrow \\
\text { S-Adenosylmethionine } \downarrow \\
\text { Proteomic findings were } \\
\text { consistent with the above } \\
\text { (all in liver) }\end{array}$ & [220] \\
\hline Mouse & $\begin{array}{c}\text { Ldlr-null mice fed a Western } \\
\text { diet (energy as } 17 \% \text { protein, } \\
43 \% \text { carbohydrate, } 41 \% \text { fat, } \\
0.2 \% \text { cholesterol) + olive oil } \\
\text { (WD + O) }\end{array}$ & $\begin{array}{l}\text { NAFLD/NASH vs. } \\
\text { control }\end{array}$ & $\begin{array}{l}\text { UPLC-LITMS } \\
\text { GC-MS } \\
\text { Metabolon, Inc. }\end{array}$ & $\begin{array}{c}\text { Saturated fatty acids } \uparrow \\
\text { MUFAs } \uparrow \\
\text { Palmitoyl-sphingomyelin } \uparrow \\
\text { Cholesterol } \uparrow \text { n-6 PUFA } \uparrow \\
\text { 12-HETE } \uparrow \text { C }_{20-22} \text { n-3 } \\
\text { PUFA-containing } \\
\text { phosphoglycerolipids } \downarrow \\
\text { 18-HEPE } \downarrow 17,18 \text {-diHETE } \downarrow \\
\text { S-Lactoyl-glutathione } \downarrow \\
\text { (all in liver) } \\
\text { F3-Isoprostanes } \downarrow \\
\text { (in urine) }\end{array}$ & [221] \\
\hline
\end{tabular}


Table 4. Cont.

\begin{tabular}{|c|c|c|c|c|c|}
\hline Species & Manipulation/Condition & Pathology & $\begin{array}{c}\text { Analytical } \\
\text { Methodology }\end{array}$ & Metabolites Reported & Ref. \\
\hline Mouse & $\begin{array}{l}\text { HFD ( } 60 \% \text { calories from fat) } \\
\text { and normal chow }(12.7 \% \\
\text { calories from fat) }\end{array}$ & NAFLD vs. control & ${ }^{1} \mathrm{H}$ NMR & $\begin{array}{c}\text { Glucose } \uparrow \text { Total cholesterol } \uparrow \\
\text { HDL-cholesterol } \uparrow \text { AST } \uparrow \\
\text { ALT } \uparrow \text { Phosphatidylcholine } \uparrow \\
\text { Pyruvate } \downarrow \text { Acetate } \downarrow \text { Lactate } \downarrow \\
\text { Citrate } \downarrow \text { Arginine } \downarrow \\
\text { Ornithine } \downarrow \text { Acetoacetate } \downarrow \\
\text { 3-Hydroxybutyrate } \downarrow \\
\text { Isoleucine } \downarrow \text { Leucine } \downarrow \\
\text { Valine } \downarrow \text { Glutamate } \downarrow \\
\text { Glutamine } \downarrow \text { Tyrosine } \downarrow \\
\text { Phenylalanine } \downarrow \text { Alanine } \downarrow \\
\text { Lysine } \downarrow \text { Glycine } \downarrow \text { Betaine } \downarrow \\
\text { Isobutanoate } \downarrow \\
\text { 1-Methylhistidine } \downarrow \\
\text { (all in serum) } \\
\text { Total cholesterol } \uparrow \\
\text { Triglycerides } \uparrow \text { Fatty acids } \uparrow \\
\text { PUFA/MUFA } \downarrow \\
\text { (all in liver) } \\
\text { Pyruvate } \uparrow \text { Creatinine } \uparrow \\
\text { Taurine } \uparrow \text { Glycine } \uparrow \text { Formate } \uparrow \\
\text { Butanoate } \uparrow \\
\text { Guanidinoacetate } \uparrow \text { Glucose } \uparrow \\
\text { 1-Methylnicotinamide } \uparrow \\
\text { Nicotinamide } N \text {-oxide } \uparrow \\
\text { Acetoacetate } \downarrow \text { Succinate } \downarrow \\
\text { Citrate } \downarrow \text { 2-Oxoglutarate } \downarrow \\
\text { Trimethylamine } \downarrow \\
\text { Trans-aconitate } \downarrow \text { Hippurate } \downarrow \\
\text { Trigonelline } \downarrow \text { Niacinamide } \downarrow \\
\text { Tyrosine } \downarrow \\
\text { 1-Methylhistidine } \downarrow \\
\text { Phenylalanine } \downarrow \\
\text { (all in urine) }\end{array}$ & [223] \\
\hline Mouse & $\begin{array}{l}\text { HFD ( } 60 \% \text { calories from fat) } \\
\text { and normal chow }(13.5 \% \\
\text { calories from fat) }\end{array}$ & NAFLD vs. control & $\begin{array}{c}\text { UPLC-QTOFMS } \\
\text { GC-MS }\end{array}$ & $\begin{array}{c}\text { Methylhippurate } \uparrow \text { Glycerol } \\
\text { 3-phosphate } \uparrow \text { Mannose } \uparrow \\
\text { Ketoleucine } \uparrow \\
\text { 2-Ketohexanoate } \uparrow \\
\text { Hydroxyphenyllactate } \uparrow \\
\text { Succinate } \uparrow \\
\text { Xylose/Ribose/Arabinose } \downarrow \\
\text { Glucuronate } \downarrow \text { Catechol } \downarrow \\
\text { 4-Coumarate } \downarrow \text { Hippurate } \downarrow \\
\text { Taurocholate } \downarrow \\
\text { Glycochenodeoxycholate } \downarrow \\
\text { Glycocholate } \downarrow \text { Histamine } \downarrow \\
\text { (all in serum) }\end{array}$ & [224] \\
\hline Mouse & $\begin{array}{c}\mathrm{A} / \mathrm{J}, \mathrm{C} 57 \mathrm{BL} / 6 \mathrm{~J} \text { and } \mathrm{PWD} / \mathrm{PhJ} \\
\text { strains fed standard diet } \\
\text { with } 0.1 \% \mathrm{DDC}\end{array}$ & $\begin{array}{l}\text { DDC-treated vs. } \\
\text { control }\end{array}$ & UPLC-Q-LITMS & $\begin{array}{c}\text { Putrescine } \uparrow \text { Arginine } \uparrow \\
\text { Citrulline } \uparrow \text { cAMP } \uparrow \\
\text { 2-Oxoglutarate } \uparrow \\
\text { Asparagine } \uparrow \text { Glutamate } \uparrow \\
\text { (all in liver) }\end{array}$ & [227] \\
\hline Mouse & $\begin{array}{l}\text { HFD-fed mice }(42 \% \text { calories } \\
\text { from fat, } 43 \% \text { from } \\
\text { carbohydrates, } 15 \% \text { from } \\
\text { protein) vs. standard chow } \\
\text { (17\% from fat, } 58 \% \text { from } \\
\text { carbohydrates, } 25 \% \text { from } \\
\text { protein) }\end{array}$ & NAFLD vs. control & UPLC-Q-LITMS & $\begin{array}{c}\text { SFA-DAGs } \uparrow \text { MUFA-DAGs } \uparrow \\
\text { PUFA-DAGs } \downarrow \text { SFA-CEs } \uparrow \\
\text { MUFA-CEs } \uparrow \text { PAs } \uparrow \text { PGs } \uparrow \\
\text { SFA-CERs } \uparrow \text { Sphingosine } \uparrow \\
\text { Sphingosine-1-phosphate } \uparrow \\
\text { Dihydrosphingosine } \uparrow \\
\text { Dihydrosphingosine-1-phosphate } \uparrow \\
\text { Galactosylceramide } \downarrow \\
\text { Glucosylceramide } \downarrow \\
\text { Lactosylceramide } \uparrow \\
\text { Globotrioseacylceramide } \uparrow \\
\text { TxB2 } \uparrow \text { PGF2 } \alpha \uparrow \text { All other } \\
\text { eicosanoids } \downarrow \\
\text { Pattern changed from weeks } \\
\text { 16-52. } \\
\text { (all in liver) }\end{array}$ & $\uparrow$ \\
\hline
\end{tabular}


Table 4. Cont.

\begin{tabular}{|c|c|c|c|c|c|}
\hline Species & Manipulation/Condition & Pathology & $\begin{array}{c}\text { Analytical } \\
\text { Methodology }\end{array}$ & Metabolites Reported & Ref. \\
\hline Mouse & $\begin{array}{l}\mathrm{db} / \mathrm{db} \text { leptin } \\
\text { receptor-deficient mice with } \\
\text { insulin resistance and } \\
\text { steatosis subjected to caloric } \\
\text { restriction }(\mathrm{CR}), \mathrm{db} / \mathrm{m} \text { mice } \\
\text { without insulin resistance } \\
\text { and steatosis }\end{array}$ & $\begin{array}{l}\mathrm{db} / \mathrm{db} \text {, pre- and } \\
\text { post-caloric } \\
\text { restriction, } \mathrm{db} / \mathrm{m}\end{array}$ & $\begin{array}{c}{ }^{1} \mathrm{H} \text { NMR } \\
\text { UPLC-QTOFMS }\end{array}$ & $\begin{array}{c}\text { db/db vs. db/m: } \\
\text { Acetone } \uparrow \\
\text { 3-Hydroxybutyrate } \uparrow \\
\text { Lactate } \uparrow \text { Acetate } \uparrow \\
\text { Glutathione } \uparrow \text { Ascorbate } \uparrow \\
\text { Many glycerolipids } \uparrow \\
\mathrm{db} / \mathrm{db}+\mathrm{CR} \text { vs. db/db: } \\
\text { 3-Hydroxybutyrate } \downarrow \\
\text { Ascorbate } \downarrow \\
\text { Many glycerolipids } \downarrow \\
\text { RT-PCR findings consistent } \\
\text { with metabolomic data } \\
\text { (all in liver) }\end{array}$ & [231] \\
\hline Mouse & $\begin{array}{l}\text { Leptin-deficient obese } o b / o b \\
\text { mice and nonsteatotic } o b /+ \\
\text { heterozygous mice }\end{array}$ & $\begin{array}{c}\text { Intact liver tissues } \\
\text { of two mouse lines } \\
\text { compared }\end{array}$ & $\begin{array}{c}\text { HR-MAS }{ }^{1} \mathrm{H} \\
\text { NMR }\end{array}$ & $\begin{array}{c}\text { Many lipid }{ }^{1} \mathrm{H} \text { signals } \\
\text { highly statistically } \\
\text { significantly elevated in } \\
\text { steatotic } o b / o b \text { livers } \\
\text { compared with nonsteatotic } \\
o b /+ \text { livers, as expected. } \\
o b / o b \text { livers vs. } o b /+ \text { livers: } \\
\text { Betaine } \uparrow \text { Phenylalanine } \uparrow \\
\text { Uridine } \uparrow \text { Creatinine } \downarrow \\
\text { Glutamate } \downarrow \text { Glycine } \downarrow \\
\text { Glycolate } \downarrow \text { Trimethylamine } \\
N \text {-oxide } \downarrow \\
N, N \text {-Dimethylglycine } \downarrow \\
\text { ADP } \downarrow \text { AMP } \downarrow\end{array}$ & [232] \\
\hline Mouse & $\begin{array}{l}\text { High-trans-fat high-fructose } \\
\text { diet (TFD) for } 8 \text { weeks } \\
\text { (steatosis) and } 24 \text { weeks } \\
\text { (NASH) }\end{array}$ & $\begin{array}{c}\text { TFD-fed, normal } \\
\text { diet-fed }\end{array}$ & $\begin{array}{c}\text { Fasting hepatic } \\
\text { mitochondrial } \\
\text { flux by }{ }^{13} \mathrm{C} \\
\text { NMR } \\
\text { isotopomer } \\
\text { analysis. } \\
\text { LC-TQMS } \\
\text { lipidomics }\end{array}$ & 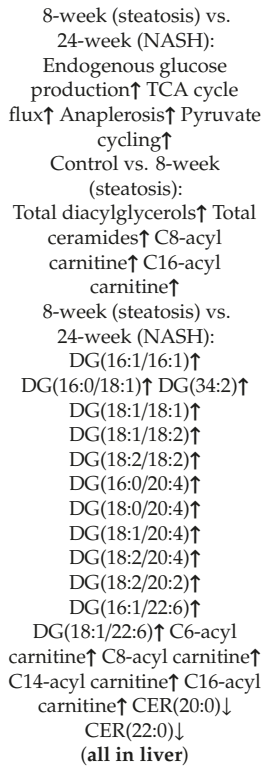 & [229] \\
\hline
\end{tabular}


Table 4. Cont.

\begin{tabular}{|c|c|c|c|c|c|}
\hline Species & Manipulation/Condition & Pathology & $\begin{array}{c}\text { Analytical } \\
\text { Methodology }\end{array}$ & Metabolites Reported & Ref. \\
\hline Mouse & $\begin{array}{c}\text { High-fat, high-cholesterol, } \\
\text { cholate (HFDCC)-fed mice } \\
\text { with NAFLD without } \\
\text { obesity }\end{array}$ & HFDCC vs. control & $\begin{array}{c}\text { GC-TOFMS } \\
\text { UPLC-QTOFMS }\end{array}$ & 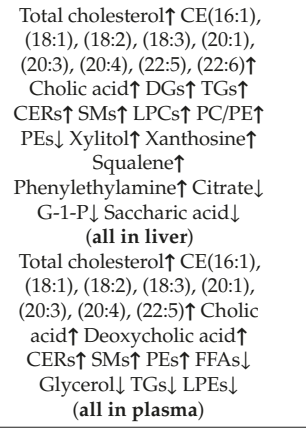 & [225] \\
\hline Rat & HFD-induced NASH & $\begin{array}{l}\text { HFD vs. control } \\
\text { diet }\end{array}$ & UPLC-QTOFMS & 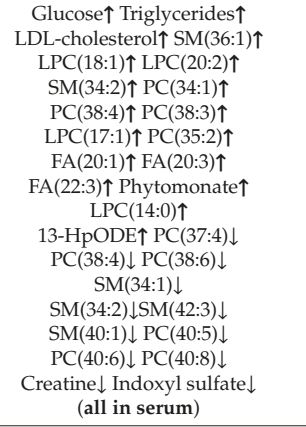 & [233] \\
\hline Rat & $\begin{array}{c}\text { HFD-induced NASH, } \\
\text { positive controls } \\
\text { (methionine plus choline } \\
\text { supplementation), control } \\
\text { diet }\end{array}$ & $\begin{array}{l}\text { HFD vs. positive } \\
\text { control vs. control } \\
\text { diet }\end{array}$ & UPLC-QTOFMS & 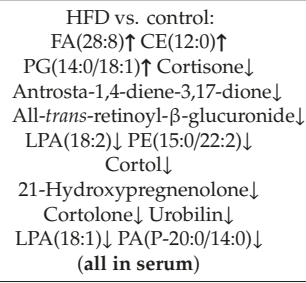 & {$[234]$} \\
\hline $\begin{array}{c}\text { Rat } \\
\text { Human }\end{array}$ & $\begin{array}{l}\text { Rats fed HFD to lead to } \\
\text { steatosis, rats fed MCD diet } \\
\text { to lead to NASH, rats fed } \\
\text { methionine and choline } \\
\text { sufficient diet as } \\
\text { controls } \rightarrow \text { liver samples } \\
\text { NASH (fatty), NASH (not } \\
\text { fatty), steatosis, healthy liver } \\
\text { samples }\end{array}$ & $\begin{array}{l}\text { NASH vs. NAFL } \\
\text { vs. control } \\
\text { (rat and human) }\end{array}$ & $\begin{array}{c}\text { UPLC } \\
\text { Orbitrap-MS }\end{array}$ & $\begin{array}{l}\text { Bile acid metabolomics: } \\
\text { Significant BA profile } \\
\text { differences between rat } \\
\text { MCD and human NASH. } \\
\text { Amino acid metabolomics: } \\
\text { Asparagine } \uparrow \text { Citrulline } \uparrow \\
\text { Lysine } \uparrow \text { comparable } \\
\text { between rat MCD and } \\
\text { human NASH. } \\
\text { Fatty acid, carnitine and } \\
\text { LPC metabolomics: } \\
\text { Stearoyl carnitine } \uparrow \text { only } \\
\text { lipid in both rat MCD and } \\
\text { human NASH }\end{array}$ & [237] \\
\hline Rat & $\begin{array}{l}\text { HFD, MCD diet and } \\
\text { streptozocin (STZ) in rats. } \\
\text { Metabolomics and } \\
\text { transcriptomics on serum } \\
\text { and liver. }\end{array}$ & $\begin{array}{l}\text { NAFL vs. NASH } \\
\text { vs. NAFL + T2DM }\end{array}$ & UPLC-QTOFMS & $\begin{array}{c}\text { Venn diagram for HFD, } \\
\text { MCD and HFD+STZ serum: } \\
\text { Stearoyl carnitine } \uparrow \\
(9 E) \text {-octadecenoyl carnitine } \uparrow \\
\text { docosapentaenoic acid } \uparrow \\
\text { vitamin D2 } \uparrow\end{array}$ & [238] \\
\hline
\end{tabular}


Table 4. Cont.

\begin{tabular}{|c|c|c|c|c|c|}
\hline Species & Manipulation/Condition & Pathology & $\begin{array}{c}\text { Analytical } \\
\text { Methodology }\end{array}$ & Metabolites Reported & Ref. \\
\hline Rat & $\begin{array}{l}\text { HFD/cholesterol diet vs. } \\
\text { normal diet }\end{array}$ & $\begin{array}{l}\text { Stage of steatosis, } \\
\text { inflammation and } \\
\text { fibrosis determined } \\
\text { histologically }\end{array}$ & GC & $\begin{array}{c}\text { Correlation between liver } \\
\text { and blood cell total fatty } \\
\text { acids for control diet: } \\
\text { FA(16:1), FA(22:6), } \\
\text { FA(18:1n-7), FA(22:5) } \\
\text { Correlation between liver } \\
\text { and blood cell total fatty } \\
\text { acids for HFD/cholesterol } \\
\text { diet: } \\
\text { FA(22:6), FA(18:1n-7) }\end{array}$ & [239] \\
\hline Rat & HFD vs. normal diet & $\begin{array}{c}\text { NAFLD } \\
\text { established by } \\
\text { histology and liver } \\
\text { enzymes }\end{array}$ & UPLC-TQMS & $\begin{array}{c}\text { BAs in liver: } \\
\text { Taurocholate } \uparrow \\
\text { Taurohyodeoxycholate } \downarrow \\
\text { Ursodeoxycholate } \downarrow \\
\text { BAs in caecal contents: } \\
\text { Cholate } \uparrow \text { Hyodeoxycholate } \downarrow \\
\text { Muricholate } \downarrow \\
\text { BAs in serum: } \\
\text { Taurocholate } \uparrow \\
\text { Hyodeoxycholate } \downarrow \\
\text { Taurohyodeoxycholate } \downarrow\end{array}$ & [240] \\
\hline
\end{tabular}

Overall Summary

A summary of metabolic, metabolomic, and lipidomic investigations into ALD is given in Table 1. It is clear that the hepatic metabolic phenotypes and therefore biomarkers of both chronic alcohol consumption and ALD are far from being defined. Despite the considerable number of published investigations, large variations in study design, species investigated, experimental methodologies and metabolic findings render a consensus opinion difficult to formulate. Nevertheless, it is becoming clear that various lipid classes may play a role in both ALD etiology and in shaping the resultant hepatic metabolic phenotype. Moreover, the recent attention to gut microbiota-liver cross talk offers new avenues to solving the mechanisms of ALD and providing effective predictive biomarkers. The effects of alcohol on lipid metabolism has recently been reviewed [52], as has the Lieber-DeCarli diet as a model for experimental liver disease [55].

Regarding cholestasis, it is well known to be associated with elevated hepatic and serum bile acids, and can occur in the diseases PBC and PSC, as well as in pregnancy and in the neonatal period. Metabolomics has revealed that not just the expected primary BAs are elevated in these conditions, but also BA removal is enhanced by sulfation, with various sulfate conjugates found in the urine. More mechanistic investigations were generally conducted in rats, predominantly by administration of the hepatotoxin ANIT. This protocol has found particular utility in the screening of TCMs that have been used for centuries to treat jaundice in China. Attesting to the efficacy of these treatments, the metabolomic signature of ANIT-induced cholestasis was attenuated in all cases. In addition, a number of combination biomarkers have been evaluated for the various manifestations of both clinical and experimental cholestasis, but it remains to be seen if any of these are adopted into routine clinical practice (Table 2).

In the case of fibrosis and cirrhosis, a total of 38 studies are summarized in Table 3, i.e., 22 conducted by MS and 16 by NMR. Nine investigations were conducted in rats and 29 in patients and volunteers. Considering first the NMR-based studies, it should be noted that these investigations in general identify in liver samples, serum, plasma, or urine relatively high concentration metabolites, such as Krebs cycle intermediates, amino acids and simple sugars that have been described as "the usual suspects" [244]. This simple fact renders these targets unsuitable as biomarker candidates for the detection or progression of fibrosis because of their ubiquitous nature. Although ${ }^{1} \mathrm{H}$ NMR-based metabolomic studies are seen as having many advantages, such as simplicity, rapidity, and reproducibility, they suffer from modest resolution and sensitivity. MS-based methodologies, in contrast, are able to resolve, identify and quantitate hundreds of molecules in a sample, rather than tens of metabolites by NMR. They have 
the distinct advantage in the realm of the relatively low concentration constituents of the metabolome. As Table 3 demonstrates, fibrosis progression in NAFLD could be evaluated in terms of decreasing serum concentrations of etiocholanolone sulfate (E) and dehydroepiandrosterone sulfate (D), with concomitant increasing concentration of $16 \alpha$-hydroxy-dehydroepiandrosterone sulfate (16). Discovery of these molecules as potential biomarkers was assessed in a validation cohort [119]. The ratios 16/D and 16/E exhibited clear statistically significant trends across F0-1, F2, F3, to F4, with sensitivities/specificities of $81 \% / 80 \%$ and $76 \% / 85 \%$, respectively [119]. More commonly, MS-based methods have shown elevations in specific serum bile acids in human liver cirrhosis $[128,132,135]$, together with fluctuations in a broad range of urinary steroids [141]. Another common finding were alterations in serum phospholipids in both human liver cirrhosis $[135,139,140]$ and in animal models $[169,170,172]$. In both the human and animal model investigations, perturbations in metabolic intermediates, akin to those revealed by NMR, have also been described. We consider that only metabolites that appear unique to fibrosis and/or cirrhosis, such as etiocholanolone sulfate, dehydroepiandrosterone sulfate, and $16 \alpha$-hydroxy-dehydroepiandrosterone sulfate should be evaluated as biomarkers. The unusual metabolite cervonoyl ethanolamide (8,11,14-eicosatrienoyl ethanolamide) was elevated in the rat CCl4 fibrosis model, but has so far not been evaluated in patients with liver fibrosis. However, this fatty acid amide was also elevated in hyperlipidemic rats [245], reducing its potential as a biomarker for fibrosis.

A total of 30 studies involving adult patients, nine involving adolescents or children, and 21 studies that involve mouse or rat investigations of NAFLD, are included in Table 4. Of these, a total of 49 investigations used mass spectrometry-based methodologies and ten used NMR-based methods. A large quantity of literature has described a wealth of metabolomic and lipidomic investigations into steatosis (NAFL) and NASH, together with experiments in laboratory rodent models. In the most part, this accumulated information largely describes potential mechanisms by which the liver accumulates lipid droplets and the transition to an accompanied inflammation that defines NASH. As Table 4 shows, there is a wealth of information regarding up- and down-regulated molecules in plasma/serum, urine and liver itself. The question is: how useful are these data for the generation of biomarkers of NAFL or NASH, or the progression of NAFL to NASH? The metabolic profiles of liver that have been determined in certain investigations are not immediately useful for biomarker evaluation unless serum/plasma or urine was also investigated. The purpose of a biomarker for liver disease is to avoid liver biopsy. The increase in peripheral fatty acids and acyl carnitines are consistent with the known etiology of fatty liver disease. Elevated concentrations of BAs, BCAAs, and aromatic amino acids are also well-known characteristics of these diseases. The issue is specificity, especially as many of the studies involved obese patients and those with diabetes and insulin resistance, all of which factors could confound the NAFLD findings. In a study of nondiabetic patients with steatosis and $\mathrm{NASH}$, an interesting candidate biomarker emerged, $\gamma$-glutamyltyrosine, but unfortunately the change between control subjects and NAFLD patients was small (1.2-1.3-fold, but highly statistically significant) with a number of outliers [180]. Larger fold-changes were observed for acyl carnitines. Lauroyl carnitine was four-fold increased over controls in steatosis and NASH and hexanoyl carnitine was 3.5-fold elevated in NASH but 2.5-fold decreased in steatosis [184]. As no other study reported these acyl carnitine changes in NAFLD, they would need to be independently verified. Nevertheless, acyl carnitine patterns represent potential biomarkers for progression from steatosis to NASH. We have already discussed above $7 \alpha$-hydroxy-3-oxo-4-cholestenoate [200] as a potential biomarker for steatosis. Providing that the patients under investigation were negative for sterol 27-hydroxylase deficiency, familial hypercholanemia and Zellweger syndrome, with which this BA intermediate is also associated, it could be further evaluated as a potential biomarker for steatosis. Regarding NAFL in children and adolescents, almost all patient groups in Table 4 were also obese. These studies did not appear to yield potential biomarkers of pediatric NAFLD.

NAFLD does not have a natural history in almost all laboratory rodent studies; rather, it is induced with specialized diets or occurs in genetically modified mice, such as leptin-deficient obese mice $(\mathrm{ob} / \mathrm{ob})$ or leptin receptor-deficient mice $(\mathrm{db} / \mathrm{db})$ (Table 4$)$. Unusual metabolites such as the 
globotrioseacylceramide Gb3(d18:1/22:1), which was highly statistically significantly elevated in the livers of mice fed a high-fat high-cholesterol diet [228], could not be further evaluated because their serum concentrations were not determined. Another unusual metabolite, phytomonic acid (11,12-methyleneoctadecanoic acid), reported in serum of HFD-induced NASH in rats [233] may also not be useful as human biomarkers of NAFLD, because it may be produced by the gut microbiota, given that its older name is lactobacillic acid. The C 8 and C16 acyl carnitines were elevated in liver tissue of mice fed a high-fructose high-trans-fat diet [229] similar to human findings referred to above [184]. However, again there were no serum/plasma data from which to evaluate the potential of acyl carnitines as biomarkers of human NAFLD. Other data from rats with different NAFLD phenotypes pointed to the elevation of stearoyl and elaidoyl $[(9 E)$-octadecenoyl] carnitine in serum, with palmitoyl and stearoyl carnitine upregulated in liver tissue [238]. In summary, an abundance of metabolomic data from human and animal model studies of NAFLD provide a number of leads for evaluation of biomarkers in independent trials.

Author Contributions: Conceptualization, D.B. and J.R.I.; Literature Analysis, D.B. and J.R.I.; Writing-original draft preparation, D.B. and J.R.I.; Writing-review and editing, D.B. and J.R.I. All authors have read and agreed to the published version of the manuscript.

Funding: This research was funded by the offices of the Senior Vice President for Academic Affairs and the Vice President for Health and Research, Long Island University.

Conflicts of Interest: The authors declare no conflict of interest.

\section{References}

1. Massarweh, N.N.; El-Serag, H.B. Epidemiology of Hepatocellular Carcinoma and Intrahepatic Cholangiocarcinoma. Cancer Control 2017, 24, 1073274817729245. [CrossRef]

2. Severi, T.; van Malenstein, H.; Verslype, C.; van Pelt, J.F. Tumor initiation and progression in hepatocellular carcinoma: Risk factors, classification, and therapeutic targets. Acta Pharmacol. Sin. 2010, 31, 1409-1420. [CrossRef]

3. Altekruse, S.F.; Devesa, S.S.; Dickie, L.A.; McGlynn, K.A.; Kleiner, D.E. Histological classification of liver and intrahepatic bile duct cancers in SEER registries. J. Registry Manag. 2011, 38, 201-205.

4. Baecker, A.; Liu, X.; La Vecchia, C.; Zhang, Z.F. Worldwide incidence of hepatocellular carcinoma cases attributable to major risk factors. Eur. J. Cancer Prev. 2018, 27, 205-212. [CrossRef]

5. Uhlen, M.; Fagerberg, L.; Hallstrom, B.M.; Lindskog, C.; Oksvold, P.; Mardinoglu, A.; Sivertsson, A.; Kampf, C.; Sjostedt, E.; Asplund, A.; et al. Proteomics. Tissue-based map of the human proteome. Science 2015, 347, 1260419. [CrossRef]

6. Ben-Moshe, S.; Itzkovitz, S. Spatial heterogeneity in the mammalian liver. Nat. Rev. Gastroenterol. Hepatol. 2019, 16, 395-410. [CrossRef]

7. Zhang, D.Y.; Goossens, N.; Guo, J.; Tsai, M.C.; Chou, H.I.; Altunkaynak, C.; Sangiovanni, A.; Iavarone, M.; Colombo, M.; Kobayashi, M.; et al. A hepatic stellate cell gene expression signature associated with outcomes in hepatitis C cirrhosis and hepatocellular carcinoma after curative resection. Gut 2016, 65, 1754-1764. [CrossRef]

8. Verhulst, S.; Roskams, T.; Sancho-Bru, P.; van Grunsven, L.A. Meta-Analysis of Human and Mouse Biliary Epithelial Cell Gene Profiles. Cells 2019, 8, 1117. [CrossRef]

9. Aguilar-Bravo, B.; Sancho-Bru, P. Laser capture microdissection: Techniques and applications in liver diseases. Hepatol. Int. 2019, 13, 138-147. [CrossRef]

10. Nicholson, J.K.; Lindon, J.C.; Holmes, E. 'Metabonomics': Understanding the metabolic responses of living systems to pathophysiological stimuli via multivariate statistical analysis of biological NMR spectroscopic data. Xenobiotica 1999, 29, 1181-1189. [CrossRef]

11. Idle, J.R.; Gonzalez, F.J. Metabolomics. Cell Metab. 2007, 6, 348-351. [CrossRef]

12. Beyoglu, D.; Zhou, Y.; Chen, C.; Idle, J.R. Mass isotopomer-guided decluttering of metabolomic data to visualize endogenous biomarkers of drug toxicity. Biochem. Pharmacol. 2018, 156, 491-500. [CrossRef]

13. Nicholson, J.K.; Lindon, J.C. Systems biology: Metabonomics. Nature 2008, 455, 1054-1056. [CrossRef] 
14. Gika, H.; Virgiliou, C.; Theodoridis, G.; Plumb, R.S.; Wilson, I.D. Untargeted LC/MS-based metabolic phenotyping (metabonomics/metabolomics): The state of the art. J. Chromatogr. B Anal. Technol. Biomed. Life Sci. 2019, 1117, 136-147. [CrossRef]

15. Roy, C.; Tremblay, P.Y.; Bienvenu, J.F.; Ayotte, P. Quantitative analysis of amino acids and acylcarnitines combined with untargeted metabolomics using ultra-high performance liquid chromatography and quadrupole time-of-flight mass spectrometry. J. Chromatogr. B Anal. Technol. Biomed. Life Sci. 2016, 1027, 40-49. [CrossRef]

16. Sinclair, K.; Dudley, E. Metabolomics and Biomarker Discovery. Adv. Exp. Med. Biol. 2019, 1140, 613-633. [CrossRef]

17. Bartel, J.; Krumsiek, J.; Theis, F.J. Statistical methods for the analysis of high-throughput metabolomics data. Comput. Struct. Biotechnol. J. 2013, 4, e201301009. [CrossRef]

18. Considine, E.C.; Thomas, G.; Boulesteix, A.L.; Khashan, A.S.; Kenny, L.C. Critical review of reporting of the data analysis step in metabolomics. Metabolomics 2017, 14, 7. [CrossRef]

19. Rosato, A.; Tenori, L.; Cascante, M.; De Atauri Carulla, P.R.; Martins Dos Santos, V.A.P.; Saccenti, E. From correlation to causation: Analysis of metabolomics data using systems biology approaches. Metabolomics 2018, 14, 37. [CrossRef]

20. Wishart, D.S.; Lewis, M.J.; Morrissey, J.A.; Flegel, M.D.; Jeroncic, K.; Xiong, Y.; Cheng, D.; Eisner, R.; Gautam, B.; Tzur, D.; et al. The human cerebrospinal fluid metabolome. J. Chromatogr. B Anal. Technol. Biomed. Life Sci. 2008, 871, 164-173. [CrossRef]

21. Psychogios, N.; Hau, D.D.; Peng, J.; Guo, A.C.; Mandal, R.; Bouatra, S.; Sinelnikov, I.; Krishnamurthy, R.; Eisner, R.; Gautam, B.; et al. The human serum metabolome. PLoS ONE 2011, 6, e16957. [CrossRef] [PubMed]

22. Bouatra, S.; Aziat, F.; Mandal, R.; Guo, A.C.; Wilson, M.R.; Knox, C.; Bjorndahl, T.C.; Krishnamurthy, R.; Saleem, F.; Liu, P.; et al. The human urine metabolome. PLoS ONE 2013, 8, e73076. [CrossRef] [PubMed]

23. Karu, N.; Deng, L.; Slae, M.; Guo, A.C.; Sajed, T.; Huynh, H.; Wine, E.; Wishart, D.S. A review on human fecal metabolomics: Methods, applications and the human fecal metabolome database. Anal. Chim. Acta 2018, 1030, 1-24. [CrossRef]

24. Wishart, D.S.; Feunang, Y.D.; Marcu, A.; Guo, A.C.; Liang, K.; Vazquez-Fresno, R.; Sajed, T.; Johnson, D.; Li, C.; Karu, N.; et al. HMDB 4.0: The human metabolome database for 2018. Nucleic Acids Res. 2018, 46, D608-D617. [CrossRef]

25. Scalbert, A.; Brennan, L.; Manach, C.; Andres-Lacueva, C.; Dragsted, L.O.; Draper, J.; Rappaport, S.M.; van der Hooft, J.J.; Wishart, D.S. The food metabolome: A window over dietary exposure. Am. J. Clin. Nutr. 2014, 99, 1286-1308. [CrossRef]

26. Lydic, T.A.; Goo, Y.H. Lipidomics unveils the complexity of the lipidome in metabolic diseases. Clin. Transl. Med. 2018, 7, 4. [CrossRef]

27. Garcia-Ortega, L.F.; Martinez, O. How Many Genes Are Expressed in a Transcriptome? Estimation and Results for RNA-Seq. PLoS ONE 2015, 10, e130262. [CrossRef]

28. Wilhelm, M.; Schlegl, J.; Hahne, H.; Gholami, A.M.; Lieberenz, M.; Savitski, M.M.; Ziegler, E.; Butzmann, L.; Gessulat, S.; Marx, H.; et al. Mass-spectrometry-based draft of the human proteome. Nature 2014, 509, 582-587. [CrossRef]

29. Zanger, U.M.; Schwab, M. Cytochrome P450 enzymes in drug metabolism: Regulation of gene expression, enzyme activities, and impact of genetic variation. Pharmacol. Ther. 2013, 138, 103-141. [CrossRef]

30. Aslebagh, R.; Wormwood, K.L.; Channaveerappa, D.; Wetie, A.G.N.; Woods, A.G.; Darie, C.C. Identification of Posttranslational Modifications (PTMs) of Proteins by Mass Spectrometry. Adv. Exp. Med. Biol. 2019, 1140, 199-224. [CrossRef]

31. Menetski, J.P.; Hoffmann, S.C.; Cush, S.S.; Kamphaus, T.N.; Austin, C.P.; Herrling, P.L.; Wagner, J.A. The Foundation for the National Institutes of Health Biomarkers Consortium: Past Accomplishments and New Strategic Direction. Clin. Pharmacol. Ther. 2019, 105, 829-843. [CrossRef] [PubMed]

32. Booth, J. A short history of blood pressure measurement. Proc. R. Soc. Med. 1977, 70, 793-799. [CrossRef] [PubMed]

33. Dobson, M. Nature of the urine in diabetes. Med. Obs. Inqu. 1776, 5, 298-310.

34. Garrod, A.E. Inborn Errors of Metabolism; Henry Frowde and Hodder \& Stoughton: London, UK, 1909.

35. Garrod, A.E. The incidence of alkaptonuria: A study in chemical individuality. Lancet 1902, ii, 1616-1620. [CrossRef] 
36. Piro, A.; Tagarelli, G.; Lagonia, P.; Quattrone, A.; Tagarelli, A. Archibald Edward Garrod and alcaptonuria: "Inborn errors of metabolism" revisited. Genet. Med. 2010, 12, 475-476. [CrossRef]

37. Perlman, R.L.; Govindaraju, D.R. Archibald E. Garrod: The father of precision medicine. Genet. Med. 2016, 18, 1088-1089. [CrossRef]

38. Phornphutkul, C.; Introne, W.J.; Perry, M.B.; Bernardini, I.; Murphey, M.D.; Fitzpatrick, D.L.; Anderson, P.D.; Huizing, M.; Anikster, Y.; Gerber, L.H.; et al. Natural history of alkaptonuria. N. Engl. J. Med. 2002, 347, 2111-2121. [CrossRef]

39. Ranganath, L.R.; Khedr, M.; Milan, A.M.; Davison, A.S.; Hughes, A.T.; Usher, J.L.; Taylor, S.; Loftus, N.; Daroszewska, A.; West, E.; et al. Nitisinone arrests ochronosis and decreases rate of progression of Alkaptonuria: Evaluation of the effect of nitisinone in the United Kingdom National Alkaptonuria Centre. Mol. Genet. Metab. 2018, 125, 127-134. [CrossRef]

40. Adinolfi, M. Embryonic antigens. In Encyclopedia of Immunology, 2nd ed.; Delves, P.J., Ed.; Academic Press: Cambridge, MA, USA, 1998; pp. 798-802.

41. Abelev, G.I.; Perova, S.D.; Khramkova, N.I.; Postnikova, Z.A.; Irlin, I.S. Production of embryonal alpha-globulin by transplantable mouse hepatomas. Transplantation 1963, 1, 174-180. [CrossRef]

42. Chan, S.L.; Chan, A.W.H.; Yu, S.C.H. Alpha-Fetoprotein as a Biomarker in Hepatocellular Carcinoma: Focus on Its Role in Composition of Tumor Staging Systems and Monitoring of Treatment Response. In Biomarkers in Disease: Methods, Discoveries and Applications; Patel, V.B., Preedy, V.R., Eds.; Springer Science+Business Media: Dordrecht, The Netherlands, 2017; pp. 623-635. [CrossRef]

43. Wu, M.; Liu, H.; Liu, Z.; Liu, C.; Zhang, A.; Li, N. Analysis of serum alpha-fetoprotein (AFP) and AFP-L3 levels by protein microarray. J. Int. Med. Res. 2018, 46, 4297-4305. [CrossRef]

44. Derosa, G.; Maffioli, P. Traditional markers in liver disease. In Biomarkers in Disease: Methods, Discoveries and Applications; Patel, V.B., Preedy, V.R., Eds.; Springer Science+Business Media: Dordrecht, The Netherlands, 2017. [CrossRef]

45. Lou, J.; Zhang, L.; Lv, S.; Zhang, C.; Jiang, S. Biomarkers for Hepatocellular Carcinoma. Biomark. Cancer 2017, 9,1-9. [CrossRef] [PubMed]

46. Bruha, R. Osteopontin as a biomarker in liver disease. In Biomarkers in Liver Disease: Methods, Discoveries and Applications; Patel, V.B., Preedy, V.R., Eds.; Springer Science+Business Media: Dordrecht, The Netherlands, 2017. [CrossRef]

47. Crandall, D.I.; Halikis, D.N. Homogentisic acid oxidase. I. Distribution in animal tissue and relation to tyrosine metabolism in rat kidney. J. Biol. Chem. 1954, 208, 629-638.

48. Bernardini, G.; Laschi, M.; Geminiani, M.; Braconi, D.; Vannuccini, E.; Lupetti, P.; Manetti, F.; Millucci, L.; Santucci, A. Homogentisate 1,2 dioxygenase is expressed in brain: Implications in alkaptonuria. J. Inherit. Metab. Dis. 2015, 38, 807-814. [CrossRef] [PubMed]

49. Hartmann, P.; Seebauer, C.T.; Schnabl, B. Alcoholic liver disease: The gut microbiome and liver cross talk. Alcohol. Clin. Exp. Res. 2015, 39, 763-775. [CrossRef] [PubMed]

50. Osna, N.A.; Donohue, T.M., Jr.; Kharbanda, K.K. Alcoholic Liver Disease: Pathogenesis and Current Management. Alcohol. Res. 2017, 38, 147-161. [PubMed]

51. Lieber, C.S.; Teschke, R.; Hasumura, Y.; Decarli, L.M. Differences in hepatic and metabolic changes after acute and chronic alcohol consumption. Fed. Proc. 1975, 34, 2060-2074.

52. You, M.; Arteel, G.E. Effect of ethanol on lipid metabolism. J. Hepatol. 2019, 70, 237-248. [CrossRef]

53. Lieber, C.S.; Jones, D.P.; Decarli, L.M. Effects of Prolonged Ethanol Intake: Production of Fatty Liver Despite Adequate Diets. J. Clin. Investig. 1965, 44, 1009-1021. [CrossRef]

54. Lieber, C.S.; DeCarli, L.M. Liquid diet technique of ethanol administration: 1989 Update. Alcohol Alcohol. 1989, 24, 197-211.

55. Guo, F.; Zheng, K.; Benede-Ubieto, R.; Cubero, F.J.; Nevzorova, Y.A. The Lieber-DeCarli Diet-A Flagship Model for Experimental Alcoholic Liver Disease. Alcohol Clin. Exp. Res. 2018, 42, 1828-1840. [CrossRef]

56. Kim, S.J.; Jung, Y.S.; Kwon, D.Y.; Kim, Y.C. Alleviation of acute ethanol-induced liver injury and impaired metabolomics of S-containing substances by betaine supplementation. Biochem. Biophys. Res. Commun. 2008, 368, 893-898. [CrossRef] [PubMed]

57. Zivkovic, A.M.; Bruce German, J.; Esfandiari, F.; Halsted, C.H. Quantitative lipid metabolomic changes in alcoholic micropigs with fatty liver disease. Alcohol Clin. Exp. Res. 2009, 33, 751-758. [CrossRef] 
58. Manna, S.K.; Patterson, A.D.; Yang, Q.; Krausz, K.W.; Li, H.; Idle, J.R.; Fornace, A.J., Jr.; Gonzalez, F.J. Identification of noninvasive biomarkers for alcohol-induced liver disease using urinary metabolomics and the Ppara-null mouse. J. Proteome Res. 2010, 9, 4176-4188. [CrossRef] [PubMed]

59. Manna, S.K.; Patterson, A.D.; Yang, Q.; Krausz, K.W.; Idle, J.R.; Fornace, A.J.; Gonzalez, F.J. UPLC-MS-based urine metabolomics reveals indole-3-lactic acid and phenyllactic acid as conserved biomarkers for alcohol-induced liver disease in the Ppara-null mouse model. J. Proteome Res. 2011, 10, 4120-4133. [CrossRef]

60. Fernando, H.; Bhopale, K.K.; Kondraganti, S.; Kaphalia, B.S.; Shakeel Ansari, G.A. Lipidomic changes in rat liver after long-term exposure to ethanol. Toxicol. Appl. Pharmacol. 2011, 255, 127-137. [CrossRef]

61. Suciu, A.M.; Crisan, D.A.; Procopet, B.D.; Radu, C.I.; Socaciu, C.; Tantau, M.V.; Stefanescu, H.O.; Grigorescu, M. What's in Metabolomics for Alcoholic Liver Disease? J. Gastrointestin. Liver Dis. 2018, 27, 51-58. [CrossRef]

62. Shi, X.; Yao, D.; Chen, C. Identification of N-acetyltaurine as a novel metabolite of ethanol through metabolomics-guided biochemical analysis. J. Biol. Chem. 2012, 287, 6336-6349. [CrossRef]

63. Johnson, C.H.; Patterson, A.D.; Krausz, K.W.; Lanz, C.; Kang, D.W.; Luecke, H.; Gonzalez, F.J.; Idle, J.R. Radiation metabolomics. 4. UPLC-ESI-QTOFMS-Based metabolomics for urinary biomarker discovery in gamma-irradiated rats. Radiat. Res. 2011, 175, 473-484. [CrossRef]

64. Johnson, C.H.; Patterson, A.D.; Krausz, K.W.; Kalinich, J.F.; Tyburski, J.B.; Kang, D.W.; Luecke, H.; Gonzalez, F.J.; Blakely, W.F.; Idle, J.R. Radiation metabolomics. 5. Identification of urinary biomarkers of ionizing radiation exposure in nonhuman primates by mass spectrometry-based metabolomics. Radiat. Res. 2012, 178, 328-340. [CrossRef]

65. Luginbuhl, M.; Rutjens, S.; Konig, S.; Furrer, J.; Weinmann, W. N-Acetyltaurine as a novel urinary ethanol marker in a drinking study. Anal. Bioanal. Chem. 2016, 408, 7529-7536. [CrossRef]

66. Luginbuhl, M.; Konig, S.; Schurch, S.; Weinmann, W. Evaluation of N-acetyltaurine as an ethanol marker in human blood. Alcohol 2017, 65, 11-18. [CrossRef]

67. Xie, G.; Zhong, W.; Li, H.; Li, Q.; Qiu, Y.; Zheng, X.; Chen, H.; Zhao, X.; Zhang, S.; Zhou, Z.; et al. Alteration of bile acid metabolism in the rat induced by chronic ethanol consumption. FASEB J. 2013, 27, 3583-3593. [CrossRef]

68. Xie, G.; Zhong, W.; Zheng, X.; Li, Q.; Qiu, Y.; Li, H.; Chen, H.; Zhou, Z.; Jia, W. Chronic ethanol consumption alters mammalian gastrointestinal content metabolites. J. Proteome Res. 2013, 12, 3297-3306. [CrossRef]

69. Chen, P.; Torralba, M.; Tan, J.; Embree, M.; Zengler, K.; Starkel, P.; van Pijkeren, J.P.; DePew, J.; Loomba, R.; Ho, S.B.; et al. Supplementation of saturated long-chain fatty acids maintains intestinal eubiosis and reduces ethanol-induced liver injury in mice. Gastroenterology 2015, 148, 203-214. [CrossRef]

70. Fang, H.; Zhang, A.H.; Sun, H.; Yu, J.B.; Wang, L.; Wang, X.J. High-throughput metabolomics screen coupled with multivariate statistical analysis identifies therapeutic targets in alcoholic liver disease rats using liquid chromatography-mass spectrometry. J. Chromatogr. B Anal. Technol. Biomed. Life Sci. 2019, 1109, 112-120. [CrossRef]

71. Zhang, T.; Zhang, A.; Qiu, S.; Sun, H.; Guan, Y.; Wang, X. High-throughput metabolomics approach reveals new mechanistic insights for drug response of phenotypes of geniposide towards alcohol-induced liver injury by using liquid chromatography coupled to high resolution mass spectrometry. Mol. Biosyst. 2017, 13, 73-82. [CrossRef]

72. Deda, O.; Virgiliou, C.; Orfanidis, A.; Gika, H.G. Study of Fecal and Urinary Metabolite Perturbations Induced by Chronic Ethanol Treatment in Mice by UHPLC-MS/MS Targeted Profiling. Metabolites 2019, 9, 232. [CrossRef]

73. He, L.; Li, F.; Yin, X.; Bohman, P.; Kim, S.; McClain, C.J.; Feng, W.; Zhang, X. Profiling of Polar Metabolites in Mouse Feces Using Four Analytical Platforms to Study the Effects Of Cathelicidin-Related Antimicrobial Peptide in Alcoholic Liver Disease. J. Proteome Res. 2019, 18, 2875-2884. [CrossRef]

74. Gao, B.; Lang, S.; Duan, Y.; Wang, Y.; Shawcross, D.L.; Louvet, A.; Mathurin, P.; Ho, S.B.; Starkel, P.; Schnabl, B. Serum and Fecal Oxylipins in Patients with Alcohol-Related Liver Disease. Dig. Dis. Sci. 2019, 64, 1878-1892. [CrossRef]

75. Michelena, J.; Alonso, C.; Martinez-Arranz, I.; Altamirano, J.; Mayo, R.; Sancho-Bru, P.; Bataller, R.; Gines, P.; Castro, A.; Caballeria, J. Metabolomics Discloses a New Non-invasive Method for the Diagnosis and Prognosis of Patients with Alcoholic Hepatitis. Ann. Hepatol. 2019, 18, 144-154. [CrossRef] 
76. Keitel, V.; Droge, C.; Haussinger, D. Targeting FXR in Cholestasis. Handb. Exp. Pharmacol. 2019, 256, $299-324$. [CrossRef]

77. Ishihara, K.; Katsutani, N.; Asai, N.; Inomata, A.; Uemura, Y.; Suganuma, A.; Sawada, K.; Yokoi, T.; Aoki, T. Identification of urinary biomarkers useful for distinguishing a difference in mechanism of toxicity in rat model of cholestasis. Basic Clin. Pharmacol. Toxicol. 2009, 105, 156-166. [CrossRef]

78. Cho, J.Y.; Matsubara, T.; Kang, D.W.; Ahn, S.H.; Krausz, K.W.; Idle, J.R.; Luecke, H.; Gonzalez, F.J. Urinary metabolomics in Fxr-null mice reveals activated adaptive metabolic pathways upon bile acid challenge. J. Lipid Res. 2010, 51, 1063-1074. [CrossRef]

79. Passmore, I.J.; Letertre, M.P.M.; Preston, M.D.; Bianconi, I.; Harrison, M.A.; Nasher, F.; Kaur, H.; Hong, H.A.; Baines, S.D.; Cutting, S.M.; et al. Para-cresol production by Clostridium difficile affects microbial diversity and membrane integrity of Gram-negative bacteria. PLoS Pathog 2018, 14, e1007191. [CrossRef]

80. Matsubara, T.; Tanaka, N.; Sato, M.; Kang, D.W.; Krausz, K.W.; Flanders, K.C.; Ikeda, K.; Luecke, H.; Wakefield, L.M.; Gonzalez, F.J. TGF-beta-SMAD3 signaling mediates hepatic bile acid and phospholipid metabolism following lithocholic acid-induced liver injury. J. Lipid Res. 2012, 53, 2698-2707. [CrossRef]

81. Aoki, M.; Konya, Y.; Takagaki, T.; Umemura, K.; Sogame, Y.; Katsumata, T.; Komuro, S. Metabolomic investigation of cholestasis in a rat model using ultra-performance liquid chromatography/tandem mass spectrometry. Rapid Commun. Mass Spectrom. 2011, 25, 1847-1852. [CrossRef]

82. Yamazaki, M.; Miyake, M.; Sato, H.; Masutomi, N.; Tsutsui, N.; Adam, K.P.; Alexander, D.C.; Lawton, K.A.; Milburn, M.V.; Ryals, J.A.; et al. Perturbation of bile acid homeostasis is an early pathogenesis event of drug induced liver injury in rats. Toxicol. Appl. Pharmacol. 2013, 268, 79-89. [CrossRef]

83. Chen, Z.; Zhu, Y.; Zhao, Y.; Ma, X.; Niu, M.; Wang, J.; Su, H.; Wang, R.; Li, J.; Liu, L.; et al. Serum Metabolomic Profiling in a Rat Model Reveals Protective Function of Paeoniflorin Against ANIT Induced Cholestasis. Phytother. Res. 2016, 30, 654-662. [CrossRef]

84. Ma, X.; Chi, Y.H.; Niu, M.; Zhu, Y.; Zhao, Y.L.; Chen, Z.; Wang, J.B.; Zhang, C.E.; Li, J.Y.; Wang, L.F.; et al. Metabolomics Coupled with Multivariate Data and Pathway Analysis on Potential Biomarkers in Cholestasis and Intervention Effect of Paeonia lactiflora Pall. Front. Pharmacol. 2016, 7, 14. [CrossRef]

85. Zhang, C.E.; Niu, M.; Li, R.Y.; Feng, W.W.; Ma, X.; Dong, Q.; Ma, Z.J.; Li, G.Q.; Meng, Y.K.; Wang, Y.; et al. Untargeted Metabolomics Reveals Dose-Response Characteristics for Effect of Rhubarb in a Rat Model of Cholestasis. Front. Pharmacol. 2016, 7, 85. [CrossRef]

86. Sun, H.; Zhang, A.H.; Zou, D.X.; Sun, W.J.; Wu, X.H.; Wang, X.J. Metabolomics coupled with pattern recognition and pathway analysis on potential biomarkers in liver injury and hepatoprotective effects of yinchenhao. Appl. Biochem. Biotechnol. 2014, 173, 857-869. [CrossRef]

87. Li, Y.F.; Wu, J.S.; Li, Y.Y.; Dai, Y.; Zheng, M.; Zeng, J.K.; Wang, G.F.; Wang, T.M.; Li, W.K.; Zhang, X.Y.; et al. Chicken bile powder protects against alpha-naphthylisothiocyanate-induced cholestatic liver injury in mice. Oncotarget 2017, 8, 97137-97152. [CrossRef]

88. Wu, J.S.; Li, Y.F.; Li, Y.Y.; Dai, Y.; Li, W.K.; Zheng, M.; Shi, Z.C.; Shi, R.; Wang, T.M.; Ma, B.L.; et al. Huangqi Decoction Alleviates Alpha-Naphthylisothiocyanate Induced Intrahepatic Cholestasis by Reversing Disordered Bile Acid and Glutathione Homeostasis in Mice. Front. Pharmacol. 2017, 8, 938. [CrossRef]

89. Han, H.; Xu, L.; Xiong, K.; Zhang, T.; Wang, Z. Exploration of Hepatoprotective Effect of Gentiopicroside on Alpha-Naphthylisothiocyanate-Induced Cholestatic Liver Injury in Rats by Comprehensive Proteomic and Metabolomic Signatures. Cell Physiol. Biochem. 2018, 49, 1304-1319. [CrossRef]

90. Zhu, G.; Feng, F. UPLC-MS-based metabonomic analysis of intervention effects of Da-Huang-Xiao-Shi decoction on ANIT-induced cholestasis. J. Ethnopharmacol. 2019, 238, 111860. [CrossRef]

91. Ma, X.; Idle, J.R.; Krausz, K.W.; Tan, D.X.; Ceraulo, L.; Gonzalez, F.J. Urinary metabolites and antioxidant products of exogenous melatonin in the mouse. J. Pineal Res. 2006, 40, 343-349. [CrossRef]

92. Ma, X.; Chen, C.; Krausz, K.W.; Idle, J.R.; Gonzalez, F.J. A metabolomic perspective of melatonin metabolism in the mouse. Endocrinology 2008, 149, 1869-1879. [CrossRef]

93. Yu, H.; Li, Y.; Xu, Z.; Wang, D.; Shi, S.; Deng, H.; Zeng, B.; Zheng, Z.; Sun, L.; Deng, X.; et al. Identification of potential biomarkers in cholestasis and the therapeutic effect of melatonin by metabolomics, multivariate data and pathway analyses. Int J. Mol. Med. 2018, 42, 2515-2526. [CrossRef] 
94. Lin, S.; Wang, T.Y.; Xu, H.R.; Zhang, X.N.; Wang, Q.; Liu, R.; Li, Q.; Bi, K.S. A systemic combined nontargeted and targeted LC-MS based metabolomic strategy of plasma and liver on pathology exploration of alpha-naphthylisothiocyanate induced cholestatic liver injury in mice. J. Pharm. Biomed. Anal. 2019, 171, 180-192. [CrossRef]

95. Dai, M.; Hua, H.; Lin, H.; Xu, G.; Hu, X.; Li, F.; Gonzalez, FJ.; Liu, A.; Yang, J. Targeted Metabolomics Reveals a Protective Role for Basal PPARalpha in Cholestasis Induced by alpha-Naphthylisothiocyanate. J. Proteome Res. 2018, 17, 1500-1508. [CrossRef]

96. Wang, B.L.; Zhang, C.W.; Wang, L.; Tang, K.L.; Tanaka, N.; Gonzalez, F.J.; Xu, Y.; Fang, Z.Z. Lipidomics reveal aryl hydrocarbon receptor (Ahr)-regulated lipid metabolic pathway in alpha-naphthyl isothiocyanate (ANIT)-induced intrahepatic cholestasis. Xenobiotica 2019, 49, 591-601. [CrossRef]

97. Fu, K.; Wang, C.; Gao, Y.; Fan, S.; Zhang, H.; Sun, J.; Jiang, Y.; Liu, C.; Guan, L.; Liu, J.; et al. Metabolomics and Lipidomics Reveal the Effect of Hepatic Vps33b Deficiency on Bile Acids and Lipids Metabolism. Front. Pharmacol. 2019, 10, 276. [CrossRef]

98. Long, Y.; Dong, X.; Yuan, Y.; Huang, J.; Song, J.; Sun, Y.; Lu, Z.; Yang, L.; Yu, W. Metabolomics changes in a rat model of obstructive jaundice: Mapping to metabolism of amino acids, carbohydrates and lipids as well as oxidative stress. J. Clin. Biochem. Nutr. 2015, 57, 50-59. [CrossRef]

99. Wei, D.D.; Wang, J.S.; Duan, J.A.; Kong, L.Y. Metabolomic Assessment of Acute Cholestatic Injuries Induced by Thioacetamide and by Bile Duct Ligation, and the Protective Effects of Huang-Lian-Jie-Du-Decoction. Front. Pharmacol. 2018, 9, 458. [CrossRef]

100. Yang, R.; Zhao, Q.; Hu, D.D.; Xiao, X.R.; Huang, J.F.; Li, F. Metabolomic analysis of cholestatic liver damage in mice. Food Chem. Toxicol. 2018, 120, 253-260. [CrossRef]

101. Lian, J.S.; Liu, W.; Hao, S.R.; Chen, D.Y.; Wang, Y.Y.; Yang, J.L.; Jia, H.Y.; Huang, J.R. A serum metabolomic analysis for diagnosis and biomarker discovery of primary biliary cirrhosis and autoimmune hepatitis. Hepatobiliary Pancreat Dis. Int. 2015, 14, 413-421. [CrossRef]

102. Trottier, J.; Bialek, A.; Caron, P.; Straka, R.J.; Heathcote, J.; Milkiewicz, P.; Barbier, O. Metabolomic profiling of 17 bile acids in serum from patients with primary biliary cirrhosis and primary sclerosing cholangitis: A pilot study. Dig. Liver Dis. 2012, 44, 303-310. [CrossRef]

103. Bell, L.N.; Wulff, J.; Comerford, M.; Vuppalanchi, R.; Chalasani, N. Serum metabolic signatures of primary biliary cirrhosis and primary sclerosing cholangitis. Liver Int. 2015, 35, 263-274. [CrossRef]

104. Tang, Y.M.; Wang, J.P.; Bao, W.M.; Yang, J.H.; Ma, L.K.; Yang, J.; Chen, H.; Xu, Y.; Yang, L.H.; Li, W.; et al. Urine and serum metabolomic profiling reveals that bile acids and carnitine may be potential biomarkers of primary biliary cirrhosis. Int. J. Mol. Med. 2015, 36, 377-385. [CrossRef]

105. Vignoli, A.; Orlandini, B.; Tenori, L.; Biagini, M.R.; Milani, S.; Renzi, D.; Luchinat, C.; Calabro, A.S. Metabolic Signature of Primary Biliary Cholangitis and Its Comparison with Celiac Disease. J. Proteome Res. 2019, 18, 1228-1236. [CrossRef]

106. Ozkan, S.; Ceylan, Y.; Ozkan, O.V.; Yildirim, S. Review of a challenging clinical issue: Intrahepatic cholestasis of pregnancy. World J. Gastroenterol. 2015, 21, 7134-7141. [CrossRef]

107. Ma, L.; Zhang, X.; Pan, F.; Cui, Y.; Yang, T.; Deng, L.; Shao, Y.; Ding, M. Urinary metabolomic analysis of intrahepatic cholestasis of pregnancy based on high performance liquid chromatography/mass spectrometry. Clin. Chim. Acta. 2017, 471, 292-297. [CrossRef]

108. Cui, Y.; Xu, B.; Zhang, X.; He, Y.; Shao, Y.; Ding, M. Diagnostic and therapeutic profiles of serum bile acids in women with intrahepatic cholestasis of pregnancy-a pseudo-targeted metabolomics study. Clin. Chim. Acta. 2018, 483, 135-141. [CrossRef]

109. Wang, P.; Zhong, H.; Song, Y.; Yuan, P.; Li, Y.; Lin, S.; Zhang, X.; Li, J.; Che, L.; Feng, B.; et al. Targeted metabolomics analysis of maternal-placental-fetal metabolism in pregnant swine reveals links in fetal bile acid homeostasis and sulfation capacity. Am. J. Physiol. Gastrointest. Liver Physiol. 2019, 317, G8-G16. [CrossRef]

110. Chen, X.; Zhang, X.; Xu, B.; Cui, Y.; He, Y.; Yang, T.; Shao, Y.; Ding, M. The urinary bile acid profiling analysis of asymptomatic hypercholanemia of pregnancy: A pseudo-targeted metabolomics study. Clin. Chim. Acta 2019, 497, 67-75. [CrossRef]

111. De Seymour, J.V.; Tu, S.; He, X.; Zhang, H.; Han, T.L.; Baker, P.N.; Sulek, K. Metabolomic profiling of maternal hair suggests rapid development of intrahepatic cholestasis of pregnancy. Metabolomics 2018, 14, 79. [CrossRef] 
112. Li, Y.; Zhang, X.; Chen, J.; Feng, C.; He, Y.; Shao, Y.; Ding, M. Targeted metabolomics of sulfated bile acids in urine for the diagnosis and grading of intrahepatic cholestasis of pregnancy. Genes Dis. 2018, 5, 358-366. [CrossRef]

113. Li, W.W.; Yang, Y.; Dai, Q.G.; Lin, L.L.; Xie, T.; He, L.L.; Tao, J.L.; Shan, J.J.; Wang, S.C. Non-invasive urinary metabolomic profiles discriminate biliary atresia from infantile hepatitis syndrome. Metabolomics 2018, 14, 90. [CrossRef]

114. Bataller, R.; Brenner, D.A. Liver fibrosis. J. Clin. Investig. 2005, 115, 209-218. [CrossRef]

115. Goodman, Z.D. Grading and staging systems for inflammation and fibrosis in chronic liver diseases. J. Hepatol. 2007, 47, 598-607. [CrossRef]

116. D'Amico, G.; Garcia-Tsao, G.; Pagliaro, L. Natural history and prognostic indicators of survival in cirrhosis: A systematic review of 118 studies. J. Hepatol. 2006, 44, 217-231. [CrossRef]

117. Fleming, K.M.; Aithal, G.P.; Card, T.R.; West, J. All-cause mortality in people with cirrhosis compared with the general population: A population-based cohort study. Liver Int. 2012, 32, 79-84. [CrossRef]

118. Chang, M.L.; Yang, S.S. Metabolic Signature of Hepatic Fibrosis: From Individual Pathways to Systems Biology. Cells 2019, 8, 1423. [CrossRef]

119. Tokushige, K.; Hashimoto, E.; Kodama, K.; Tobari, M.; Matsushita, N.; Kogiso, T.; Taniai, M.; Torii, N.; Shiratori, K.; Nishizaki, Y.; et al. Serum metabolomic profile and potential biomarkers for severity of fibrosis in nonalcoholic fatty liver disease. J. Gastroenterol. 2013, 48, 1392-1400. [CrossRef]

120. Fishman, J.; Schneider, J.; Hershcope, R.J.; Bradlow, H.L. Increased estrogen-16 alpha-hydroxylase activity in women with breast and endometrial cancer. J. Steroid Biochem. 1984, 20, 1077-1081. [CrossRef]

121. Batista, A.D.; Barros, C.J.P.; Costa, T.; de Godoy, M.M.G.; Silva, R.D.; Santos, J.C.; de Melo Lira, M.M.; Juca, N.T.; Lopes, E.P.A.; Silva, R.O. Proton nuclear magnetic resonance-based metabonomic models for non-invasive diagnosis of liver fibrosis in chronic hepatitis C: Optimizing the classification of intermediate fibrosis. World J. Hepatol. 2018, 10, 105-115. [CrossRef]

122. Abellona, U.M.R.; Taylor-Robinson, S.D. Comments on Gabbani, et al. Metabolomic analysis with (1)H NMR for non-invasive diagnosis of hepatic fibrosis degree in patients with chronic hepatitis C. Dig. Liver Dis. 2018, 50, 209-210. [CrossRef]

123. Wu, F.; Zheng, H.; Yang, Z.T.; Cheng, B.; Wu, J.X.; Liu, X.W.; Tang, C.L.; Lu, S.Y.; Chen, Z.N.; Song, F.M.; et al. Urinary metabonomics study of the hepatoprotective effects of total alkaloids from Corydalis saxicola Bunting on carbon tetrachloride-induced chronic hepatotoxicity in rats using (1)H NMR analysis. J. Pharm. Biomed. Anal. 2017, 140, 199-209. [CrossRef]

124. Liu, X.W.; Tang, C.L.; Zheng, H.; Wu, J.X.; Wu, F.; Mo, Y.Y.; Liu, X.; Zhu, H.J.; Yin, C.L.; Cheng, B.; et al. Investigation of the hepatoprotective effect of Corydalis saxicola Bunting on carbon tetrachloride-induced liver fibrosis in rats by (1)H-NMR-based metabonomics and network pharmacology approaches. J. Pharm. Biomed. Anal. 2018, 159, 252-261. [CrossRef]

125. Feng, X.; Li, M.H.; Xia, J.; Deng Ba, D.J.; Ruan, L.Y.; Xing, Y.X.; Chen, C.; Wang, J.S.; Zhong, G.J. Tibetan Medical Formula Shi-Wei-Gan-Ning-Pill Protects Against Carbon Tetrachloride-Induced Liver Fibrosis-An NMR-Based Metabolic Profiling. Front. Pharmacol. 2018, 9, 965. [CrossRef]

126. Li, M.H.; Feng, X.; Deng Ba, D.J.; Chen, C.; Ruan, L.Y.; Xing, Y.X.; Chen, L.Y.; Zhong, G.J.; Wang, J.S. Hepatoprotection of Herpetospermum caudigerum Wall. against CCl4-induced liver fibrosis on rats. J. Ethnopharmacol. 2019, 229, 1-14. [CrossRef]

127. Yin, P.; Wan, D.; Zhao, C.; Chen, J.; Zhao, X.; Wang, W.; Lu, X.; Yang, S.; Gu, J.; Xu, G. A metabonomic study of hepatitis B-induced liver cirrhosis and hepatocellular carcinoma by using RP-LC and HILIC coupled with mass spectrometry. Mol. Biosyst. 2009, 5, 868-876. [CrossRef]

128. Xue, R.; Dong, L.; Wu, H.; Liu, T.; Wang, J.; Shen, X. Gas chromatography/mass spectrometry screening of serum metabolomic biomarkers in hepatitis B virus infected cirrhosis patients. Clin. Chem. Lab. Med. 2009, 47, 305-310. [CrossRef]

129. Waldhier, M.C.; Almstetter, M.F.; Nurnberger, N.; Gruber, M.A.; Dettmer, K.; Oefner, P.J. Improved enantiomer resolution and quantification of free $\mathrm{D}$-amino acids in serum and urine by comprehensive two-dimensional gas chromatography-time-of-flight mass spectrometry. J. Chromatogr. A 2011, 1218, 4537-4544. [CrossRef]

130. Bastings, J.; van Eijk, H.M.; Olde Damink, S.W.; Rensen, S.S. d-amino Acids in Health and Disease: A Focus on Cancer. Nutrients 2019, 11, 2205. [CrossRef] 
131. Huang, H.J.; Zhang, A.Y.; Cao, H.C.; Lu, H.F.; Wang, B.H.; Xie, Q.; Xu, W.; Li, L.J. Metabolomic analyses of faeces reveals malabsorption in cirrhotic patients. Dig. Liver Dis. 2013, 45, 677-682. [CrossRef]

132. Liu, Z.; Zhang, Z.; Huang, M.; Sun, X.; Liu, B.; Guo, Q.; Chang, Q.; Duan, Z. Taurocholic acid is an active promoting factor, not just a biomarker of progression of liver cirrhosis: Evidence from a human metabolomic study and in vitro experiments. BMC Gastroenterol. 2018, 18, 112. [CrossRef]

133. Patterson, A.D.; Maurhofer, O.; Beyoglu, D.; Lanz, C.; Krausz, K.W.; Pabst, T.; Gonzalez, F.J.; Dufour, J.F.; Idle, J.R. Aberrant lipid metabolism in hepatocellular carcinoma revealed by plasma metabolomics and lipid profiling. Cancer Res. 2011, 71, 6590-6600. [CrossRef]

134. Osman, D.; Ali, O.; Obada, M.; El-Mezayen, H.; El-Said, H. Chromatographic determination of some biomarkers of liver cirrhosis and hepatocellular carcinoma in Egyptian patients. Biomed. Chromatogr. 2017, 31. [CrossRef]

135. Wang, B.; Chen, D.; Chen, Y.; Hu, Z.; Cao, M.; Xie, Q.; Chen, Y.; Xu, J.; Zheng, S.; Li, L. Metabonomic profiles discriminate hepatocellular carcinoma from liver cirrhosis by ultraperformance liquid chromatography-mass spectrometry. J. Proteome Res. 2012, 11, 1217-1227. [CrossRef]

136. Koller, A.; Aldwin, L.; Natelson, S. Hepatic synthesis of canavaninosuccinate from ureidohomoserine and aspartate, and its conversion to guanidinosuccinate. Clin. Chem. 1975, 21, 1777-1782. [CrossRef] [PubMed]

137. Chen, D.Q.; Cao, G.; Chen, H.; Liu, D.; Su, W.; Yu, X.Y.; Vaziri, N.D.; Liu, X.H.; Bai, X.; Zhang, L.; et al. Gene and protein expressions and metabolomics exhibit activated redox signaling and wnt/beta-catenin pathway are associated with metabolite dysfunction in patients with chronic kidney disease. Redox Biol. 2017, 12, 505-521. [CrossRef] [PubMed]

138. Mindikoglu, A.L.; Opekun, A.R.; Putluri, N.; Devaraj, S.; Sheikh-Hamad, D.; Vierling, J.M.; Goss, J.A.; Rana, A.; Sood, G.K.; Jalal, P.K.; et al. Unique metabolomic signature associated with hepatorenal dysfunction and mortality in cirrhosis. Transl. Res. 2018, 195, 25-47. [CrossRef]

139. Liu, Y.; Hong, Z.; Tan, G.; Dong, X.; Yang, G.; Zhao, L.; Chen, X.; Zhu, Z.; Lou, Z.; Qian, B.; et al. NMR and LC/MS-based global metabolomics to identify serum biomarkers differentiating hepatocellular carcinoma from liver cirrhosis. Int J. Cancer 2014, 135, 658-668. [CrossRef]

140. McPhail, M.J.W.; Shawcross, D.L.; Lewis, M.R.; Coltart, I.; Want, E.J.; Antoniades, C.G.; Veselkov, K.; Triantafyllou, E.; Patel, V.; Pop, O.; et al. Multivariate metabotyping of plasma predicts survival in patients with decompensated cirrhosis. J. Hepatol. 2016, 64, 1058-1067. [CrossRef]

141. Dai, W.; Yin, P.; Chen, P.; Kong, H.; Luo, P.; Xu, Z.; Lu, X.; Xu, G. Study of urinary steroid hormone disorders: Difference between hepatocellular carcinoma in early stage and cirrhosis. Anal. Bioanal. Chem. 2014, 406, 4325-4335. [CrossRef]

142. Gao, R.; Cheng, J.; Fan, C.; Shi, X.; Cao, Y.; Sun, B.; Ding, H.; Hu, C.; Dong, F.; Yan, X. Serum Metabolomics to Identify the Liver Disease-Specific Biomarkers for the Progression of Hepatitis to Hepatocellular Carcinoma. Sci. Rep. 2015, 5, 18175. [CrossRef]

143. Hou, Q.; Duan, Z.J. Metabonomic window into hepatitis B virus-related hepatic diseases. World J. Hepatol. 2016, 8, 1-8. [CrossRef]

144. Lu, Y.; Fang, J.; Zou, L.; Cui, L.; Liang, X.; Lim, S.G.; Dan, Y.Y.; Ong, C.N. Omega-6-derived oxylipin changes in serum of patients with hepatitis B virus-related liver diseases. Metabolomics 2018, 14, 26. [CrossRef]

145. Cabral, M.; Martin-Venegas, R.; Moreno, J.J. Differential cell growth/apoptosis behavior of 13-hydroxyoctadecadienoic acid enantiomers in a colorectal cancer cell line. Am. J. Physiol. Gastrointest. Liver Physiol. 2014, 307, G664-G671. [CrossRef]

146. Zhao, C.Q.; Chen, L.; Cai, H.; Yao, W.L.; Zhou, Q.; Zhu, H.M.; Gao, Y.; Liu, P.; Gou, X.J.; Zhang, H. Classification of Gan Dan Shi Re Pattern and Gan Shen Yin Xu Pattern in Patients with Hepatitis B Cirrhosis Using Metabonomics. Evid. Based Complement. Altern. Med. 2018, 2018, 2697468. [CrossRef] [PubMed]

147. Zhou, K.; Xie, G.; Wen, J.; Wang, J.; Pan, W.; Zhou, Y.; Xiao, Y.; Wang, Y.; Jia, W.; Cai, W. Histamine is correlated with liver fibrosis in biliary atresia. Dig. Liver Dis. 2016, 48, 921-926. [CrossRef] [PubMed]

148. Jimenez, B.; Montoliu, C.; MacIntyre, D.A.; Serra, M.A.; Wassel, A.; Jover, M.; Romero-Gomez, M.; Rodrigo, J.M.; Pineda-Lucena, A.; Felipo, V. Serum metabolic signature of minimal hepatic encephalopathy by (1)H-nuclear magnetic resonance. J. Proteome Res. 2010, 9, 5180-5187. [CrossRef]

149. Martinez-Granados, B.; Morales, J.M.; Rodrigo, J.M.; Del Olmo, J.; Serra, M.A.; Ferrandez, A.; Celda, B.; Monleon, D. Metabolic profile of chronic liver disease by NMR spectroscopy of human biopsies. Int. J. Mol. Med. 2011, 27, 111-117. [CrossRef] 
150. Schofield, Z.; Reed, M.A.; Newsome, P.N.; Adams, D.H.; Gunther, U.L.; Lalor, P.F. Changes in human hepatic metabolism in steatosis and cirrhosis. World J. Gastroenterol. 2017, 23, 2685-2695. [CrossRef]

151. Gao, H.; Lu, Q.; Liu, X.; Cong, H.; Zhao, L.; Wang, H.; Lin, D. Application of 1H NMR-based metabonomics in the study of metabolic profiling of human hepatocellular carcinoma and liver cirrhosis. Cancer Sci. 2009, 100, 782-785. [CrossRef]

152. Amathieu, R.; Nahon, P.; Triba, M.; Bouchemal, N.; Trinchet, J.C.; Beaugrand, M.; Dhonneur, G.; Le Moyec, L. Metabolomic approach by $1 \mathrm{H}$ NMR spectroscopy of serum for the assessment of chronic liver failure in patients with cirrhosis. J. Proteome Res. 2011, 10, 3239-3245. [CrossRef]

153. Qi, S.W.; Tu, Z.G.; Peng, W.J.; Wang, L.X.; Ou-Yang, X.; Cai, A.J.; Dai, Y. (1)H NMR-based serum metabolic profiling in compensated and decompensated cirrhosis. World J. Gastroenterol. 2012, 18, 285-290. [CrossRef]

154. Corbin, I.R.; Ryner, L.N.; Singh, H.; Minuk, G.Y. Quantitative hepatic phosphorus-31 magnetic resonance spectroscopy in compensated and decompensated cirrhosis. Am. J. Physiol. Gastrointest. Liver Physiol. 2004, 287, G379-G384. [CrossRef]

155. Traussnigg, S.; Kienbacher, C.; Gajdosik, M.; Valkovic, L.; Halilbasic, E.; Stift, J.; Rechling, C.; Hofer, H.; Steindl-Munda, P.; Ferenci, P.; et al. Ultra-high-field magnetic resonance spectroscopy in non-alcoholic fatty liver disease: Novel mechanistic and diagnostic insights of energy metabolism in non-alcoholic steatohepatitis and advanced fibrosis. Liver Int. 2017, 37, 1544-1553. [CrossRef]

156. Iebba, V.; Guerrieri, F.; Di Gregorio, V.; Levrero, M.; Gagliardi, A.; Santangelo, F.; Sobolev, A.P.; Circi, S.; Giannelli, V.; Mannina, L.; et al. Combining amplicon sequencing and metabolomics in cirrhotic patients highlights distinctive microbiota features involved in bacterial translocation, systemic inflammation and hepatic encephalopathy. Sci. Rep. 2018, 8, 8210. [CrossRef]

157. Liu, Y.; Li, J.; Jin, Y.; Zhao, L.; Zhao, F.; Feng, J.; Li, A.; Wei, Y. Splenectomy Leads to Amelioration of Altered Gut Microbiota and Metabolome in Liver Cirrhosis Patients. Front. Microbiol. 2018, 9, 963. [CrossRef] [PubMed]

158. Shao, L.; Ling, Z.; Chen, D.; Liu, Y.; Yang, F.; Li, L. Disorganized Gut Microbiome Contributed to Liver Cirrhosis Progression: A Meta-Omics-Based Study. Front. Microbiol. 2018, 9, 3166. [CrossRef]

159. Cox, I.J.; Idilman, R.; Fagan, A.; Turan, D.; Ajayi, L.; Le Guennec, A.D.; Taylor-Robinson, S.D.; Karakaya, F.; Gavis, E.; Andrew Atkinson, R.; et al. Metabolomics and microbial composition increase insight into the impact of dietary differences in cirrhosis. Liver Int. 2019. [CrossRef]

160. Amathieu, R.; Triba, M.N.; Nahon, P.; Bouchemal, N.; Kamoun, W.; Haouache, H.; Trinchet, J.C.; Savarin, P.; Le Moyec, L.; Dhonneur, G. Serum 1H-NMR metabolomic fingerprints of acute-on-chronic liver failure in intensive care unit patients with alcoholic cirrhosis. PLoS ONE 2014, 9, e89230. [CrossRef]

161. Dabos, K.J.; Parkinson, J.A.; Sadler, I.H.; Plevris, J.N.; Hayes, P.C. (1)H nuclear magnetic resonance spectroscopy-based metabonomic study in patients with cirrhosis and hepatic encephalopathy. World J. Hepatol. 2015, 7, 1701-1707. [CrossRef]

162. Weiss, N.; Barbier Saint Hilaire, P.; Colsch, B.; Isnard, F.; Attala, S.; Schaefer, A.; Amador, M.D.; Rudler, M.; Lamari, F.; Sedel, F.; et al. Cerebrospinal fluid metabolomics highlights dysregulation of energy metabolism in overt hepatic encephalopathy. J. Hepatol. 2016, 65, 1120-1130. [CrossRef]

163. Embade, N.; Marino, Z.; Diercks, T.; Cano, A.; Lens, S.; Cabrera, D.; Navasa, M.; Falcon-Perez, J.M.; Caballeria, J.; Castro, A.; et al. Metabolic Characterization of Advanced Liver Fibrosis in HCV Patients as Studied by Serum 1H-NMR Spectroscopy. PLoS ONE 2016, 11, e0155094. [CrossRef]

164. Gabbani, T.; Marsico, M.; Bernini, P.; Lorefice, E.; Grappone, C.; Biagini, M.R.; Milani, S.; Annese, V. Metabolomic analysis with (1)H-NMR for non-invasive diagnosis of hepatic fibrosis degree in patients with chronic hepatitis C. Dig. Liver Dis. 2017, 49, 1338-1344. [CrossRef]

165. Wei, D.D.; Wang, J.S.; Wang, P.R.; Li, M.H.; Yang, M.H.; Kong, L.Y. Toxic effects of chronic low-dose exposure of thioacetamide on rats based on NMR metabolic profiling. J. Pharm. Biomed. Anal. 2014, 98, 334-338. [CrossRef]

166. Zhang, H.; Wang, X.; Hu, P.; Zhou, W.; Zhang, M.; Liu, J.; Wang, Y.; Liu, P.; Luo, G. Serum Metabolomic Characterization of Liver Fibrosis in Rats and Anti-Fibrotic Effects of Yin-Chen-Hao-Tang. Molecules 2016, 21, 126. [CrossRef] [PubMed]

167. Song, Y.N.; Zhang, G.B.; Lu, Y.Y.; Chen, Q.L.; Yang, L.; Wang, Z.T.; Liu, P.; Su, S.B. Huangqi decoction alleviates dimethylnitrosamine-induced liver fibrosis: An analysis of bile acids metabolic mechanism. $J$. Ethnopharmacol. 2016, 189, 148-156. [CrossRef] [PubMed] 
168. Yu, J.; He, J.Q.; Chen, D.Y.; Pan, Q.L.; Yang, J.F.; Cao, H.C.; Li, L.J. Dynamic changes of key metabolites during liver fibrosis in rats. World J. Gastroenterol. 2019, 25, 941-954. [CrossRef] [PubMed]

169. Zhang, K.; Zhang, Y.; Li, N.; Xing, F.; Zhao, J.; Yang, T.; Liu, C.; Feng, N. An herbal-compound-based combination therapy that relieves cirrhotic ascites by affecting the L-arginine/nitric oxide pathway: A metabolomics-based systematic study. J. Ethnopharmacol. 2019, 241, 112034. [CrossRef] [PubMed]

170. Wang, G.; Li, Z.; Li, H.; Li, L.; Li, J.; Yu, C. Metabolic Profile Changes of CCl(4)-Liver Fibrosis and Inhibitory Effects of Jiaqi Ganxian Granule. Molecules 2016, 21, 698. [CrossRef]

171. Chang, H.; Meng, H.Y.; Wang, Y.; Teng, Z.; Liu, S.M. [Inhibitory effect of Scutellariae Radix on hepatic fibrosis based on urinary metabonomic]. Zhongguo Zhong Yao Za Zhi 2018, 43, 2140-2146. [CrossRef]

172. Zheng, M.; Li, Y.Y.; Wang, G.F.; Jin, J.Y.; Wang, Y.H.; Wang, T.M.; Yang, L.; Liu, S.Y.; Wu, J.S.; Wang, Z.T.; et al. Protective effect of cultured bear bile powder against dimethylnitrosamine-induced hepatic fibrosis in rats. Biomed. Pharmacother. 2019, 112, 108701. [CrossRef]

173. EASL; EASD; EASO. EASL-EASD-EASO Clinical Practice Guidelines for the management of non-alcoholic fatty liver disease. J. Hepatol. 2016, 64, 1388-1402. [CrossRef]

174. Puri, P.; Daita, K.; Joyce, A.; Mirshahi, F.; Santhekadur, P.K.; Cazanave, S.; Luketic, V.A.; Siddiqui, M.S.; Boyett, S.; Min, H.K.; et al. The presence and severity of nonalcoholic steatohepatitis is associated with specific changes in circulating bile acids. Hepatology 2018, 67, 534-548. [CrossRef]

175. Fedchuk, L.; Nascimbeni, F.; Pais, R.; Charlotte, F.; Housset, C.; Ratziu, V.; Group, L.S. Performance and limitations of steatosis biomarkers in patients with nonalcoholic fatty liver disease. Aliment. Pharmacol. Ther. 2014, 40, 1209-1222. [CrossRef]

176. Mato, J.M.; Alonso, C.; Noureddin, M.; Lu, S.C. Biomarkers and subtypes of deranged lipid metabolism in non-alcoholic fatty liver disease. World J. Gastroenterol. 2019, 25, 3009-3020. [CrossRef] [PubMed]

177. Johnson, C.H.; Ivanisevic, J.; Siuzdak, G. Metabolomics: Beyond biomarkers and towards mechanisms. Nat. Rev. Mol. Cell Biol. 2016, 17, 451-459. [CrossRef]

178. Puri, P.; Wiest, M.M.; Cheung, O.; Mirshahi, F.; Sargeant, C.; Min, H.K.; Contos, M.J.; Sterling, R.K.; Fuchs, M.; Zhou, H.; et al. The plasma lipidomic signature of nonalcoholic steatohepatitis. Hepatology 2009, 50, 1827-1838. [CrossRef] [PubMed]

179. Barr, J.; Vazquez-Chantada, M.; Alonso, C.; Perez-Cormenzana, M.; Mayo, R.; Galan, A.; Caballeria, J.; Martin-Duce, A.; Tran, A.; Wagner, C.; et al. Liquid chromatography-mass spectrometry-based parallel metabolic profiling of human and mouse model serum reveals putative biomarkers associated with the progression of nonalcoholic fatty liver disease. J. Proteome Res. 2010, 9, 4501-4512. [CrossRef] [PubMed]

180. Kalhan, S.C.; Guo, L.; Edmison, J.; Dasarathy, S.; McCullough, A.J.; Hanson, R.W.; Milburn, M. Plasma metabolomic profile in nonalcoholic fatty liver disease. Metabolism 2011, 60, 404-413. [CrossRef] [PubMed]

181. Li, H.; Wang, L.; Yan, X.; Liu, Q.; Yu, C.; Wei, H.; Li, Y.; Zhang, X.; He, F.; Jiang, Y. A proton nuclear magnetic resonance metabonomics approach for biomarker discovery in nonalcoholic fatty liver disease. J. Proteome Res. 2011, 10, 2797-2806. [CrossRef]

182. Lehmann, R.; Franken, H.; Dammeier, S.; Rosenbaum, L.; Kantartzis, K.; Peter, A.; Zell, A.; Adam, P.; Li, J.; $\mathrm{Xu}, \mathrm{G}$; ; et al. Circulating lysophosphatidylcholines are markers of a metabolically benign nonalcoholic fatty liver. Diabetes Care 2013, 36, 2331-2338. [CrossRef]

183. Ferslew, B.C.; Xie, G.; Johnston, C.K.; Su, M.; Stewart, P.W.; Jia, W.; Brouwer, K.L.; Barritt, A.S.t. Altered Bile Acid Metabolome in Patients with Nonalcoholic Steatohepatitis. Dig. Dis. Sci. 2015, 60, 3318-3328. [CrossRef]

184. Lake, A.D.; Novak, P.; Shipkova, P.; Aranibar, N.; Robertson, D.G.; Reily, M.D.; Lehman-McKeeman, L.D.; Vaillancourt, R.R.; Cherrington, N.J. Branched chain amino acid metabolism profiles in progressive human nonalcoholic fatty liver disease. Amino. Acids 2015, 47, 603-615. [CrossRef]

185. Loomba, R.; Quehenberger, O.; Armando, A.; Dennis, E.A. Polyunsaturated fatty acid metabolites as novel lipidomic biomarkers for noninvasive diagnosis of nonalcoholic steatohepatitis. J. Lipid Res. 2015, 56, 185-192. [CrossRef]

186. Mannisto, V.T.; Simonen, M.; Hyysalo, J.; Soininen, P.; Kangas, A.J.; Kaminska, D.; Matte, A.K.; Venesmaa, S.; Kakela, P.; Karja, V.; et al. Ketone body production is differentially altered in steatosis and non-alcoholic steatohepatitis in obese humans. Liver Int. 2015, 35, 1853-1861. [CrossRef] [PubMed] 
187. Rodriguez-Gallego, E.; Guirro, M.; Riera-Borrull, M.; Hernandez-Aguilera, A.; Marine-Casado, R.; Fernandez-Arroyo, S.; Beltran-Debon, R.; Sabench, F.; Hernandez, M.; del Castillo, D.; et al. Mapping of the circulating metabolome reveals alpha-ketoglutarate as a predictor of morbid obesity-associated non-alcoholic fatty liver disease. Int. J. Obes. 2015, 39, 279-287. [CrossRef] [PubMed]

188. Zhou, Y.; Oresic, M.; Leivonen, M.; Gopalacharyulu, P.; Hyysalo, J.; Arola, J.; Verrijken, A.; Francque, S.; Van Gaal, L.; Hyotylainen, T.; et al. Noninvasive Detection of Nonalcoholic Steatohepatitis Using Clinical Markers and Circulating Levels of Lipids and Metabolites. Clin. Gastroenterol. Hepatol. 2016, 14, 1463-1472. [CrossRef] [PubMed]

189. Chiappini, F.; Coilly, A.; Kadar, H.; Gual, P.; Tran, A.; Desterke, C.; Samuel, D.; Duclos-Vallee, J.C.; Touboul, D.; Bertrand-Michel, J.; et al. Metabolism dysregulation induces a specific lipid signature of nonalcoholic steatohepatitis in patients. Sci. Rep. 2017, 7, 46658. [CrossRef] [PubMed]

190. Dong, S.; Zhan, Z.Y.; Cao, H.Y.; Wu, C.; Bian, Y.Q.; Li, J.Y.; Cheng, G.H.; Liu, P.; Sun, M.Y. Urinary metabolomics analysis identifies key biomarkers of different stages of nonalcoholic fatty liver disease. World J. Gastroenterol. 2017, 23, 2771-2784. [CrossRef]

191. O'Sullivan, J.F.; Morningstar, J.E.; Yang, Q.; Zheng, B.; Gao, Y.; Jeanfavre, S.; Scott, J.; Fernandez, C.; Zheng, H.; O'Connor, S.; et al. Dimethylguanidino valeric acid is a marker of liver fat and predicts diabetes. J. Clin. Investig. 2017, 127, 4394-4402. [CrossRef]

192. Qi, S.; Xu, D.; Li, Q.; Xie, N.; Xia, J.; Huo, Q.; Li, P.; Chen, Q.; Huang, S. Metabonomics screening of serum identifies pyroglutamate as a diagnostic biomarker for nonalcoholic steatohepatitis. Clin. Chim. Acta 2017, 473, 89-95. [CrossRef]

193. Keogh, A.; Senkardes, S.; Idle, J.R.; Kucukguzel, S.G.; Beyoglu, D. A Novel Anti-Hepatitis C Virus and Antiproliferative Agent Alters Metabolic Networks in HepG2 and Hep3B Cells. Metabolites 2017, 7, 23. [CrossRef]

194. Golla, S.; Golla, J.P.; Krausz, K.W.; Manna, S.K.; Simillion, C.; Beyoglu, D.; Idle, J.R.; Gonzalez, F.J. Metabolomic Analysis of Mice Exposed to Gamma Radiation Reveals a Systemic Understanding of Total-Body Exposure. Radiat Res. 2017, 187, 612-629. [CrossRef]

195. Wang, M.; Keogh, A.; Treves, S.; Idle, J.R.; Beyoglu, D. The metabolomic profile of gamma-irradiated human hepatoma and muscle cells reveals metabolic changes consistent with the Warburg effect. PeerJ 2016, 4, e1624. [CrossRef]

196. Yang, R.X.; Hu, C.X.; Sun, W.L.; Pan, Q.; Shen, F.; Yang, Z.; Su, Q.; Xu, G.W.; Fan, J.G. Serum Monounsaturated Triacylglycerol Predicts Steatohepatitis in Patients with Non-alcoholic Fatty Liver Disease and Chronic Hepatitis B. Sci. Rep. 2017, 7, 10517. [CrossRef] [PubMed]

197. Hoyles, L.; Fernandez-Real, J.M.; Federici, M.; Serino, M.; Abbott, J.; Charpentier, J.; Heymes, C.; Luque, J.L.; Anthony, E.; Barton, R.H.; et al. Molecular phenomics and metagenomics of hepatic steatosis in non-diabetic obese women. Nat. Med. 2018, 24, 1070-1080. [CrossRef] [PubMed]

198. Mayo, R.; Crespo, J.; Martinez-Arranz, I.; Banales, J.M.; Arias, M.; Minchole, I.; Aller de la Fuente, R.; Jimenez-Aguero, R.; Alonso, C.; de Luis, D.A.; et al. Metabolomic-based noninvasive serum test to diagnose nonalcoholic steatohepatitis: Results from discovery and validation cohorts. Hepatol. Commun. 2018, 2, 807-820. [CrossRef] [PubMed]

199. Perakakis, N.; Polyzos, S.A.; Yazdani, A.; Sala-Vila, A.; Kountouras, J.; Anastasilakis, A.D.; Mantzoros, C.S. Non-invasive diagnosis of non-alcoholic steatohepatitis and fibrosis with the use of omics and supervised learning: A proof of concept study. Metabolism 2019, 101, 154005. [CrossRef] [PubMed]

200. Pietzner, M.; Budde, K.; Homuth, G.; Kastenmuller, G.; Henning, A.K.; Artati, A.; Krumsiek, J.; Volzke, H.; Adamski, J.; Lerch, M.M.; et al. Hepatic Steatosis Is Associated With Adverse Molecular Signatures in Subjects Without Diabetes. J. Clin. Endocrinol. Metab. 2018, 103, 3856-3868. [CrossRef] [PubMed]

201. Romero-Ibarguengoitia, M.E.; Vadillo-Ortega, F.; Caballero, A.E.; Ibarra-Gonzalez, I.; Herrera-Rosas, A.; Serratos-Canales, M.F.; Leon-Hernandez, M.; Gonzalez-Chavez, A.; Mummidi, S.; Duggirala, R.; et al. Family history and obesity in youth, their effect on acylcarnitine/aminoacids metabolomics and non-alcoholic fatty liver disease (NAFLD). Structural equation modeling approach. PLoS ONE 2018, 13, e0193138. [CrossRef]

202. Boone, S.; Mook-Kanamori, D.; Rosendaal, F.; den Heijer, M.; Lamb, H.; de Roos, A.; le Cessie, S.; Willems van Dijk, K.; de Mutsert, R. Metabolomics: A search for biomarkers of visceral fat and liver fat content. Metabolomics 2019, 15, 139. [CrossRef] 
203. Tan, Y.; Liu, X.; Zhou, K.; He, X.; Lu, C.; He, B.; Niu, X.; Xiao, C.; Xu, G.; Bian, Z.; et al. The Potential Biomarkers to Identify the Development of Steatosis in Hyperuricemia. PLoS ONE 2016, 11, e0149043. [CrossRef]

204. Von Schonfels, W.; Patsenker, E.; Fahrner, R.; Itzel, T.; Hinrichsen, H.; Brosch, M.; Erhart, W.; Gruodyte, A.; Vollnberg, B.; Richter, K.; et al. Metabolomic tissue signature in human non-alcoholic fatty liver disease identifies protective candidate metabolites. Liver Int. 2015, 35, 207-214. [CrossRef]

205. Alonso, C.; Fernandez-Ramos, D.; Varela-Rey, M.; Martinez-Arranz, I.; Navasa, N.; Van Liempd, S.M.; Lavin Trueba, J.L.; Mayo, R.; Ilisso, C.P.; de Juan, V.G.; et al. Metabolomic Identification of Subtypes of Nonalcoholic Steatohepatitis. Gastroenterology 2017, 152, 1449-1461. [CrossRef]

206. Chen, Y.; Li, C.; Liu, L.; Guo, F.; Li, S.; Huang, L.; Sun, C.; Feng, R. Serum metabonomics of NAFLD plus T2DM based on liquid chromatography-mass spectrometry. Clin. Biochem. 2016, 49, 962-966. [CrossRef] [PubMed]

207. Semmo, N.; Weber, T.; Idle, J.R.; Beyoglu, D. Metabolomics reveals that aldose reductase activity due to AKR1B10 is upregulated in hepatitis C virus infection. J. Viral Hepat. 2015, 22, 617-624. [CrossRef] [PubMed]

208. Liu, C.R.; Li, X.; Chan, P.L.; Zhuang, H.; Jia, J.D.; Wang, X.; Lo, Y.R.; Walsh, N. Prevalence of hepatitis C virus infection among key populations in China: A systematic review. Int. J. Infect. Dis. 2019, 80, 16-27. [CrossRef] [PubMed]

209. Alkhouri, N.; Cikach, F.; Eng, K.; Moses, J.; Patel, N.; Yan, C.; Hanouneh, I.; Grove, D.; Lopez, R.; Dweik, R. Analysis of breath volatile organic compounds as a noninvasive tool to diagnose nonalcoholic fatty liver disease in children. Eur. J. Gastroenterol. Hepatol. 2014, 26, 82-87. [CrossRef]

210. Reid, D.T.; McDonald, B.; Khalid, T.; Vo, T.; Schenck, L.P.; Surette, M.G.; Beck, P.L.; Reimer, R.A.; Probert, C.S.; Rioux, K.P.; et al. Unique microbial-derived volatile organic compounds in portal venous circulation in murine non-alcoholic fatty liver disease. Biochim. Biophys. Acta 2016, 1862, 1337-1344. [CrossRef]

211. Pastore, A.; Alisi, A.; di Giovamberardino, G.; Crudele, A.; Ceccarelli, S.; Panera, N.; Dionisi-Vici, C.; Nobili, V. Plasma levels of homocysteine and cysteine increased in pediatric NAFLD and strongly correlated with severity of liver damage. Int. J. Mol. Sci. 2014, 15, 21202-21214. [CrossRef]

212. Jin, R.; Banton, S.; Tran, V.T.; Konomi, J.V.; Li, S.; Jones, D.P.; Vos, M.B. Amino Acid Metabolism is Altered in Adolescents with Nonalcoholic Fatty Liver Disease-An Untargeted, High Resolution Metabolomics Study. J. Pediatr. 2016, 172, 14-19. [CrossRef]

213. Troisi, J.; Pierri, L.; Landolfi, A.; Marciano, F.; Bisogno, A.; Belmonte, F.; Palladino, C.; Guercio Nuzio, S.; Campiglia, P.; Vajro, P. Urinary Metabolomics in Pediatric Obesity and NAFLD Identifies Metabolic Pathways/Metabolites Related to Dietary Habits and Gut-Liver Axis Perturbations. Nutrients 2017, 9, 485. [CrossRef]

214. Goffredo, M.; Santoro, N.; Trico, D.; Giannini, C.; D’Adamo, E.; Zhao, H.; Peng, G.; Yu, X.; Lam, T.T.; Pierpont, B.; et al. A Branched-Chain Amino Acid-Related Metabolic Signature Characterizes Obese Adolescents with Non-Alcoholic Fatty Liver Disease. Nutrients 2017, 9, 642. [CrossRef]

215. Troisi, J.; Belmonte, F.; Bisogno, A.; Pierri, L.; Colucci, A.; Scala, G.; Cavallo, P.; Mandato, C.; Di Nuzzi, A.; Di Michele, L.; et al. Metabolomic Salivary Signature of Pediatric Obesity Related Liver Disease and Metabolic Syndrome. Nutrients 2019, 11, 274. [CrossRef]

216. Hartley, A.; Santos Ferreira, D.L.; Anderson, E.L.; Lawlor, D.A. Metabolic profiling of adolescent non-alcoholic fatty liver disease. Wellcome Open Res. 2018, 3, 166. [CrossRef]

217. Khusial, R.D.; Cioffi, C.E.; Caltharp, S.A.; Krasinskas, A.M.; Alazraki, A.; Knight-Scott, J.; Cleeton, R.; Castillo-Leon, E.; Jones, D.P.; Pierpont, B.; et al. Development of a Plasma Screening Panel for Pediatric Nonalcoholic Fatty Liver Disease Using Metabolomics. Hepatol. Commun. 2019, 3, 1311-1321. [CrossRef]

218. Tanaka, N.; Matsubara, T.; Krausz, K.W.; Patterson, A.D.; Gonzalez, F.J. Disruption of phospholipid and bile acid homeostasis in mice with nonalcoholic steatohepatitis. Hepatology 2012, 56, 118-129. [CrossRef]

219. Tanaka, N.; Takahashi, S.; Fang, Z.Z.; Matsubara, T.; Krausz, K.W.; Qu, A.; Gonzalez, F.J. Role of white adipose lipolysis in the development of NASH induced by methionine- and choline-deficient diet. Biochim. Biophys. Acta 2014, 1841, 1596-1607. [CrossRef] [PubMed]

220. Thomas, A.; Stevens, A.P.; Klein, M.S.; Hellerbrand, C.; Dettmer, K.; Gronwald, W.; Oefner, P.J.; Reinders, J. Early changes in the liver-soluble proteome from mice fed a nonalcoholic steatohepatitis inducing diet. Proteomics 2012, 12, 1437-1451. [CrossRef] [PubMed] 
221. Depner, C.M.; Traber, M.G.; Bobe, G.; Kensicki, E.; Bohren, K.M.; Milne, G.; Jump, D.B. A metabolomic analysis of omega-3 fatty acid-mediated attenuation of western diet-induced nonalcoholic steatohepatitis in LDLR-/- mice. PLoS ONE 2013, 8, e83756. [CrossRef]

222. Lake, A.D.; Novak, P.; Shipkova, P.; Aranibar, N.; Robertson, D.; Reily, M.D.; Lu, Z.; Lehman-McKeeman, L.D.; Cherrington, N.J. Decreased hepatotoxic bile acid composition and altered synthesis in progressive human nonalcoholic fatty liver disease. Toxicol. Appl. Pharmacol. 2013, 268, 132-140. [CrossRef] [PubMed]

223. Chao, J.; Huo, T.I.; Cheng, H.Y.; Tsai, J.C.; Liao, J.W.; Lee, M.S.; Qin, X.M.; Hsieh, M.T.; Pao, L.H.; Peng, W.H. Gallic acid ameliorated impaired glucose and lipid homeostasis in high fat diet-induced NAFLD mice. PLoS ONE 2014, 9, e96969. [CrossRef] [PubMed]

224. Lai, Y.S.; Chen, W.C.; Kuo, T.C.; Ho, C.T.; Kuo, C.H.; Tseng, Y.J.; Lu, K.H.; Lin, S.H.; Panyod, S.; Sheen, L.Y. Mass-Spectrometry-Based Serum Metabolomics of a C57BL/6J Mouse Model of High-Fat-Diet-Induced Non-alcoholic Fatty Liver Disease Development. J. Agric. Food Chem. 2015, 63, 7873-7884. [CrossRef]

225. Tu, L.N.; Showalter, M.R.; Cajka, T.; Fan, S.; Pillai, V.V.; Fiehn, O.; Selvaraj, V. Metabolomic characteristics of cholesterol-induced non-obese nonalcoholic fatty liver disease in mice. Sci. Rep. 2017, 7, 6120. [CrossRef]

226. Chen, C.J.; Liao, W.L.; Chang, C.T.; Lin, Y.N.; Tsai, F.J. Identification of Urinary Metabolite Biomarkers of Type 2 Diabetes Nephropathy Using an Untargeted Metabolomic Approach. J. Proteome Res. 2018, 17, 3997-4007. [CrossRef] [PubMed]

227. Pandey, V.; Sultan, M.; Kashofer, K.; Ralser, M.; Amstislavskiy, V.; Starmann, J.; Osprian, I.; Grimm, C.; Hache, H.; Yaspo, M.L.; et al. Comparative analysis and modeling of the severity of steatohepatitis in DDC-treated mouse strains. PLoS ONE 2014, 9, e111006. [CrossRef] [PubMed]

228. Sanyal, A.J.; Pacana, T. A Lipidomic Readout of Disease Progression in A Diet-Induced Mouse Model of Nonalcoholic Fatty Liver Disease. Trans. Am. Clin. Climatol. Assoc. 2015, 126, 271-288. [PubMed]

229. Patterson, R.E.; Kalavalapalli, S.; Williams, C.M.; Nautiyal, M.; Mathew, J.T.; Martinez, J.; Reinhard, M.K.; McDougall, D.J.; Rocca, J.R.; Yost, R.A.; et al. Lipotoxicity in steatohepatitis occurs despite an increase in tricarboxylic acid cycle activity. Am. J. Physiol. Endocrinol. Metab. 2016, 310, E484-E494. [CrossRef]

230. Qian, M.; Hu, H.; Zhao, D.; Wang, S.; Pan, C.; Duan, X.; Gao, Y.; Liu, J.; Zhang, Y.; Yang, S.; et al. Coordinated changes of gut microbiome and lipidome differentiates nonalcoholic steatohepatitis (NASH) from isolated steatosis. Liver Int. 2019. [CrossRef]

231. Kim, K.E.; Jung, Y.; Min, S.; Nam, M.; Heo, R.W.; Jeon, B.T.; Song, D.H.; Yi, C.O.; Jeong, E.A.; Kim, H.; et al. Caloric restriction of $\mathrm{db} / \mathrm{db}$ mice reverts hepatic steatosis and body weight with divergent hepatic metabolism. Sci. Rep. 2016, 6, 30111. [CrossRef]

232. Gogiashvili, M.; Edlund, K.; Gianmoena, K.; Marchan, R.; Brik, A.; Andersson, J.T.; Lambert, J.; Madjar, K.; Hellwig, B.; Rahnenfuhrer, J.; et al. Metabolic profiling of ob/ob mouse fatty liver using HR-MAS (1)H-NMR combined with gene expression analysis reveals alterations in betaine metabolism and the transsulfuration pathway. Anal. Bioanal. Chem. 2017, 409, 1591-1606. [CrossRef]

233. Li, J.; Liu, Z.; Guo, M.; Xu, K.; Jiang, M.; Lu, A.; Gao, X. Metabolomics profiling to investigate the pharmacologic mechanisms of berberine for the treatment of high-fat diet-induced nonalcoholic steatohepatitis. Evid. Based Complement. Altern. Med. 2015, 2015, 897914. [CrossRef]

234. Wang, Y.; Niu, M.; Jia, G.L.; Li, R.S.; Zhang, Y.M.; Zhang, C.E.; Meng, Y.K.; Cui, H.R.; Ma, Z.J.; Li, D.H.; et al. Untargeted Metabolomics Reveals Intervention Effects of Total Turmeric Extract in a Rat Model of Nonalcoholic Fatty Liver Disease. Evid. Based Complement. Altern. Med. 2016, 2016, 8495953. [CrossRef]

235. Van Pelt, C.K.; Huang, M.C.; Tschanz, C.L.; Brenna, J.T. An octaene fatty acid, 4,7,10,13,16,19,22,25-octacosaoctaenoic acid (28:8n-3), found in marine oils. J. Lipid Res. 1999, 40, 1501-1505.

236. Lu, Y.; Chen, Y.; Wu, Y.; Hao, H.; Liang, W.; Liu, J.; Huang, R. Marine unsaturated fatty acids: Structures, bioactivities, biosynthesis and benefits. RSC Adv. 2019, 9, 35312-35327. [CrossRef]

237. Han, J.; Dzierlenga, A.L.; Lu, Z.; Billheimer, D.D.; Torabzadeh, E.; Lake, A.D.; Li, H.; Novak, P.; Shipkova, P.; Aranibar, N.; et al. Metabolomic profiling distinction of human nonalcoholic fatty liver disease progression from a common rat model. Obesity (Silver Spring) 2017, 25, 1069-1076. [CrossRef] [PubMed]

238. Liu, X.L.; Ming, Y.N.; Zhang, J.Y.; Chen, X.Y.; Zeng, M.D.; Mao, Y.M. Gene-metabolite network analysis in different nonalcoholic fatty liver disease phenotypes. Exp. Mol. Med. 2017, 49, e283. [CrossRef] [PubMed] 
239. Maciejewska, D.; Palma, J.; Dec, K.; Skonieczna-Zydecka, K.; Gutowska, I.; Szczuko, M.; Jakubczyk, K.; Stachowska, E. Is the Fatty Acids Profile in Blood a Good Predictor of Liver Changes? Correlation of Fatty Acids Profile with Fatty Acids Content in the Liver. Diagnostics 2019, 9, 197. [CrossRef] [PubMed]

240. Tang, Y.; Zhang, J.; Li, J.; Lei, X.; Xu, D.; Wang, Y.; Li, C.; Li, X.; Mao, Y. Turnover of bile acids in liver, serum and caecal content by high-fat diet feeding affects hepatic steatosis in rats. Biochim. Biophys. Acta Mol. Cell Biol. Lipids 2019, 1864, 1293-1304. [CrossRef] [PubMed]

241. Papandreou, C.; Bullo, M.; Tinahones, F.J.; Martinez-Gonzalez, M.A.; Corella, D.; Fragkiadakis, G.A.; Lopez-Miranda, J.; Estruch, R.; Fito, M.; Salas-Salvado, J. Serum metabolites in non-alcoholic fatty-liver disease development or reversion; a targeted metabolomic approach within the PREDIMED trial. Nutr. Metab. 2017, 14, 58. [CrossRef]

242. Del Chierico, F.; Nobili, V.; Vernocchi, P.; Russo, A.; De Stefanis, C.; Gnani, D.; Furlanello, C.; Zandona, A.; Paci, P.; Capuani, G.; et al. Gut microbiota profiling of pediatric nonalcoholic fatty liver disease and obese patients unveiled by an integrated meta-omics-based approach. Hepatology 2017, 65, 451-464. [CrossRef]

243. Gawlik, A.; Shmoish, M.; Hartmann, M.F.; Wudy, S.A.; Olczak, Z.; Gruszczynska, K.; Hochberg, Z. Steroid metabolomic signature of liver disease in nonsyndromic childhood obesity. Endocr. Connect. 2019, 8, 764-771. [CrossRef]

244. Robertson, D.G. Metabonomics in toxicology: A review. Toxicol. Sci. 2005, 85, 809-822. [CrossRef]

245. Miao, H.; Zhao, Y.H.; Vaziri, N.D.; Tang, D.D.; Chen, H.; Chen, H.; Khazaeli, M.; Tarbiat-Boldaji, M.; Hatami, L.; Zhao, Y.Y. Lipidomics Biomarkers of Diet-Induced Hyperlipidemia and Its Treatment with Poria cocos. J. Agric. Food Chem. 2016, 64, 969-979. [CrossRef]

(C) 2020 by the authors. Licensee MDPI, Basel, Switzerland. This article is an open access article distributed under the terms and conditions of the Creative Commons Attribution (CC BY) license (http://creativecommons.org/licenses/by/4.0/). 
MDPI

St. Alban-Anlage 66

4052 Basel

Switzerland

Tel. +41616837734

Fax +41 613028918

www.mdpi.com

Coatings Editorial Office

E-mail: coatings@mdpi.com www.mdpi.com/journal/coatings

口畐的回

$7+4$

(1)

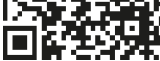



MDPI

St. Alban-Anlage 66

4052 Basel

Switzerland

Tel: +41 616837734

Fax: +41 613028918

www.mdpi.com 